









\section{MISCELLANEOUS PAPERS}

CONNECTED WITH

\section{PHYSICAL SCIENCE.}

BY

\section{HUMPHREY LLOYD, D.D., D.C.L.,} PROVOST OF TRINTTY COLLEGE, DEBLLN;

FORMERLY PROFESSOR OF NATURAL PHLLOSOPHY IN THE CNIVERSITY.

REPRINTED FROX THB TRANSACTIONS OP THB ROYAL IRISH ACADEMY; THB REPORTS OP THE BRITISH ASSOCIATION POR THE ADVANCEMRNT OF SCIENCB; ETC.

\section{ONDON:}

L O N G M N S, GREEN, AND CO. 1877. 
DUBLIN :

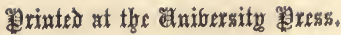




\section{$\varphi_{178}^{5}$ \\ 1877}

\section{$\mathrm{CONTENTS}$}

PAGE.

I. On the Phenomena presented by Light in its passage along the Axes of Biaxal Crystals, . . . . . . . 1

II. Report on the Progress and Present State of Physical Optics, . 19

III. On a new case of Interference of the Rays of Light, . . . 149

IV. On the Light reflected and transmitted by Thin Plates, . 157

V. Observations of the Direction and Intensity of the Terrestrial Magnetio Force in Ireland, · c c c c c . 170

VI. On the position of the Isogonal Lines in Ireland, deduced from the Observations of Captain Sir James Clakke Ross, R. N., .

VII. On a New Magnetical Instrument, for the measurement of the Inclination, and its Changes, . . . . . . . 221

VIII. Remarks on the theory of the Compound Magnetic Needle, . 231

IX. On the Mean Results of Observations, . . . . . 233

X. On the determination of the Horizontal Intensity of the Earth's Magnetic Force in absolute measure, . • • . 246

XI. On the determination of the Total Intensity of the Earth's Magnetic Force in absolute measure, by means of the Dip-circle, . 260

XII. On Earth-currents, and their connexion with the Diurnal Changes of the Horizontal Magnetic Needle, . . . . . 271

XIII. On Earth-currents, in connexion with Magnetic Disturbances, . 295

XIV. On the probable causes of the Earth-currents, . . . . 299

XV. On the direct Magnetic Influence of a distant Luminary upon the Diurnal Variations of the Magnetic Force at the Earth's surface, 
XVI. On the Storm of the 18th of April, 1850, . _ . . . 312

XVII. Notes on the Meteorology of Ireland, deduced from the Observations made in the year 1851, under the direction of the Royal Irish Academy,

XVIII. The Climate of Ireland, and the Currents of the Atlantic. (Lecture delivered before the Dublin Young Men's Christian Association, October 25, 1865), . . . . .

XIX. On the rise and progress of Mechanical Philosophy. (Introductory Lecture delivered in the Philosophy School of Trinity College, in Hilary Term, 1834)

XX. The Applied Sciences, and the mode of Teaching them. (Prælection delivered on the occasion of the Opening of the School of Engineering in the University of Dublin, November 15, 1841),

XXI. Address delivered at a Meeting of the Royal Irish Academy, held April 13, 1846, on the occasion of taking the Chair, . .

XXII. Address delivered at a Meeting of the Royal Irish Academy, held June 26, 1848, on the occasion of the presentation of the Medals awarded by the Council,

XXIII. Address delivered at the Opening Meeting of the British Association for the Advancement of Science, held in Dublin, August 26, 1857,

\section{NOT E S.}

Note to Table VI., p. 289, . . . . . . . . 511

Note to Tables V. and VIII., pp. 286 and 291, . . . . 511 


\section{PLATE $\mathrm{S}$.}

MAGNETIC CHART OF IRELAND . . . opposite page 216

EARTH CURRENTS. Plates 1 and 2 . . . " " 294

meteorology of ireland. Plates 1, 2, 3,

and 4 . . . . . . . . . , 380 


\section{MISCELLANEOUS PAPERS.}

I.-ON THE PHENOMENA PRESENTED BY LIGHT IN ITS PASSAGE ALONG THE AXES OF BIAXAL CRYSTALS.

Transactions of the Royal Irish Academy, Vol. XVII.

IT is well known that when a ray of light is incident upon certain crystals, such as Iceland spar and quartz, it is in general divided into two pencils, of which one is refracted according to the known law of the sines, while the direction of the other is determined by a' new and extraordinary law, first assigned by Huygens.

These laws were long supposed to apply to all doubly-refracting substances; and it was not until the subject was examined by the ablest advocate of the undulatory theory, that the problem of double refraction was solved in all its generality. Setting out from the hypothesis, that the elasticity of the vibrating medium within the crystal is unequal in three rectangular directions, Fresnel has shown that the surface of the wave is not, in general, either a sphere or spheroid, as in the Huygenian law, but a surface of the fourth order, consisting of two sheets; and that the directions of the two refracted rays are determined by tangent planes drawn to these surfaces under known conditions. Such crystals have, in general, two optic axes, and are thence denominated biaxal. When 
the elasticity of the medium is the same in two of the three directions, the equation of the wave-surface is resolvable into two, which represent the sphere and spheroid of the Huygenian law. The two optic axes in this case coincide; and the law of Huygens is thus proved to be a case of a more general law, and shown to belong to uniaxal crystals only. Finally, when the elasticity is the same in all the three directions, the wave-surface becomes a sphere; and the refraction is single, and takes place according to the ordinary law of the sines. This case comprises a few of the crystallized, and most uncrystallized substances.

There are two remarkable cases, however, in this elegant and profound theory, which its author seems to have overlooked, if not to have misapprehended. In a communication made to the Academy at its last meeting, Professor Hamilton has supplied these omissions in the theory of Fresnel, and has been thus led to results in the highest degree novel and remarkable.

To understand these conclusions, it may be useful to revert for a moment to the original theory of Fresnel. The general form of the wave-surface is determined by the equation

$$
\begin{gathered}
\left(a^{2} \cos ^{2} a+b^{2} \cos ^{2} \beta+c^{2} \cos ^{2} \gamma\right) r^{4} \\
-\left[a^{2}\left(b^{2}+c^{2}\right) \cos ^{2} \boldsymbol{a}+b^{2}\left(a^{2}+c^{2}\right) \cos ^{2} \beta+c^{2}\left(a^{2}+b^{2}\right) \cos ^{2} \gamma\right] r^{2} \\
+a^{2} b^{2} c^{2}=0 ;
\end{gathered}
$$

in which $a, \beta, \gamma$, denote the angles made by the radius-vector with the three axes, and $a^{2}, b^{2}, c^{2}$, the elasticities of the medium in these directions. If now we make $\cos \beta=0$ in this equation, so as to obtain the section of the surface made by the plane of $x z$, the result is reducible to the form

$$
\left(r^{2}-b^{2}\right)\left[\left(a^{2} \cos ^{2} a+c^{2} \sin ^{2} a\right) r^{2}-a^{2} c^{2}\right]=0 .
$$

So that the surface intersects the plane of $x z$ in a circle and ellipse, whose equations are

$$
r=b, \quad\left(a^{2} \cos ^{2} \alpha+c^{2} \sin ^{2} \alpha\right) r^{2}=a^{2} c^{2} .
$$

Now $b$, the radius of the circle, being intermediate between $a$ and $c$, the semiaxes of the ellipse, it is obvious that the two curves must intersect in four points, or cusps, as represented in (fig. 1) ; and the angle which the radius-vector $O P$, drawn to the cusp, 
makes with the axis of $x$, is found by eliminating $r$ between the two equations, by which means we obtain

$$
\sin a= \pm \frac{a}{b} \sqrt{\frac{b^{2}-c^{2}}{a^{2}-c^{2}}}
$$

At each of the points thus determined there will be two tangents to the plane section; and consequently the ray $O P$, proceeding within the crystal to one of these points, might be supposed to be divided at emergence into two, whose directions are determined by those of the tangents.

Such seems to have been Fresnel's conception of this case. Professor Hamilton has shown, however, that there is a cusp at each of these points, not only in this particular section, but in every section of the wavesurface passing through the line $O P$; or, in other words, that there is a conoidal cusp on the general wave-surface at the four points of intersection of the circle and ellipse; so

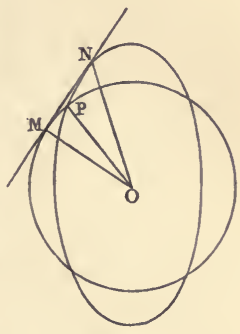

Fig. 1. that there must be an infinite number of tangent planes at each of these points, and consequently a single ray, such as $O P$, proceeding from a point within the crystal to one of these points, must be divided into an infinite number of emergent rays, constituting a conical surface.

It is evident further, that the circle and ellipse will have four common tangents, such as $M N$ (fig. 1). The planes passing through these tangents, and parallel to the third or mean axis, are parallel to the circular sections of the surface of elasticity of Fresnel's theory, or perpendicular to the optic axes. Fresnel seems to have concluded that these planes touched the wave-surface only in the two points just mentioned; and, consequently, that a single ray, incident upon a biaxal crystal in such a manner that one of the refracted rays should coincide with an optio axis, would be divided into two, determined by the points of contact. This result, if verified by experience, would place a remarkable distinction between the phenomena of uniaxal and biaxal crystals; but though the case was examined by M. Biot, no corresponding appearances were observed. 
Professor Hamilton has shown that the four planes of which we have spoken touch the wave-surface, not in two points only, but in an infinite number of points, constituting each a small eircle of contact, whose plane is parallel to one of the two circular sections of the surface of elasticity; and that, consequently, a single ray of common light, incident externally in the abovementioned direction, should be divided within the crystal into an infinite number of refracted rays, constituting a conical surface.

Here, then, are two singular and unexpected consequences of the undulatory theory, not only unsupported by any facts hitherto observed, but even opposed to all the analogies derived from experience. If confirmed by experiment, they would furnish new and almost convincing proofs of the truth of that theory; and if disproved, on the other hand, it is evident that the theory must be abandoned or modified.

Being naturally anxious to submit the undulatory hypothesis to this delicate test, and to establish or disprove these new results of theory, Professor Hamilton requested me to institute a series of experiments with that view. I accordingly applied myself to this interesting research with all the attention which the subject so well merited, and have fortunately succeeded in verifying both cases of conical refraction. The substance I employed in these experiments was arragonite, which is well known to be a biaxal crystal, whose axes are inclined at an angle of nearly $20^{\circ}$. I selected it partly on account of the magnitude of its biaxal energy, and partly also because the optical elements of this mineral have been determined, apparently with great care, by Professor Rudberg; and therefore the results of theory could be applied to it at once without further examination. The specimen I used was one of considerable size and purity, procured for me by Mr. Dollond, and cut with its parallel faces perpendicular to the line bisecting the optic axes.

The first-mentioned species of conical refraction, it has been observed, takes place in air, when a ray of common light is transmitted within the crystal in the direction of the line joining two opposite cusps of the wave. If we suppose such a ray to pass in both directions out of the crystal, it is evident that it must emerge similarly at both surfaces; consequently, the rays which are transmitted along this line within the crystal, and form a diverging cone at emergence at the second surface, must be incident in a 
converging cone at the first. Having therefore nearly ascertained the required direction by means of the system of rings in polarized light, I placed a lens of short focus at its focal distance from the first surface, and in such a position that the central part of the pencil might have an incidence nearly corresponding to the cuspray within. Then looking through the crystal at the light of a lamp placed at a considerable distance, I observed in the expected direction a point more luminous than the space immediately about it, and surrounded by something resembling a stellar radiation. Fearing that this singular appearance might have arisen from some imperfection in the crystal, I transmitted the light in the same manner through several different parts of its substance, and always with the same result. The connexion of the phenomenon with the optio axis was proved by the system of rings which appeared in the same direction, when the light was examined with a polarizing and analyzing plate.

This result is of some interest in itself, independently of its connexion with theory. It has been hitherto supposed that the only method of determining experimentally the direction of the optic axes, in most doubly-refracting substances, consisted in observing the system of coloured rings, which appear around them when the incident and emergent light is polarized. Here, however, we find that common, or unpolarized light, undergoes such modifications in the neighbourhood of one of the optic axes, that the apparent direction of that axis may be at once determined, and with the aid of the simplest contrivance.*

But to examine the emergent cone, it was necessary to exclude the light which passed through the crystal in all but one direction. For this purpose, a plate of thin metal, having a minute aperture, was placed on the surface of the crystal next the eye, and the position of the aperture so adjusted, that the line connecting it with the luminous point on the first surface might be, as nearly as possible, in the direction of the cusp-ray. The exact adjustment to this direction was made by subsequent trial. The phenomenon

- This fact is here mentioned, rather as a matter of curiosity than as one likely to be of practical value in determining the optical elements of crystals. It is to be observed, moreover, that the direction thus determined is that of the normal to the circular section of the ellipsoid of Fresnel's theory; while the rings (there is strong reason to beliove) are related to the normals to the circular sections of the surface of elasticity. 
which presented itself when this disposition was complete was in the highest degree curious. There appeared at first a luminous circle, with a small dark space in the centre; and in this dark central space were two bright points, separated by a narrow and well-defined dark line. These appearances are represented in (figs. $a$ and $b$ ). When the aperture in the plate was slightly

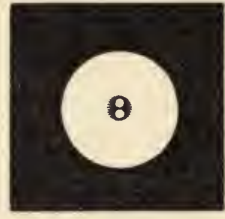

Fig. $a$.

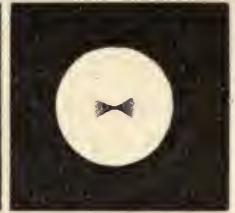

Fig. $b$.

shifted, the phenomena rapidly changed, assuming in succession the forms represented in (figs. $c, d, e$ ). In the first stage of its change, the central dark space became greatly enlarged, and a double sector appeared in the centre. The circle was reduced to about a quadrant, and was separated by a dark interval from the sector just mentioned. This is represented in (fig. c.) The remote sector then disappeared, and the circular arch diminished,

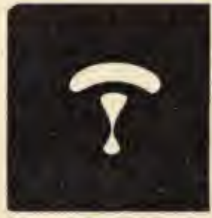

Fig. c.

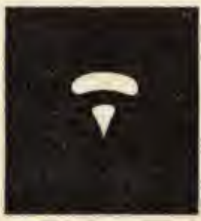

Fig. $d$.

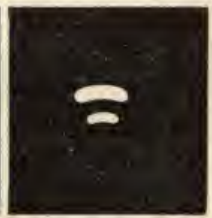

Fig. $e$.

as in. (fig. $d$ ) ; and as the inclination of the internal ray to the cusp-ray was further increased, these two luminous portions merged gradually into the two pencils, into which a single ray is divided in the other parts of the crystal. This change is represented in (fig. $e$ ).

Similar observations were made without the lens, by bringing the flame of the lamp near the first surface of the crystal, and forming the converging cone by covering that surface also with a thin metallic plate, perforated with a minute aperture. In this case the line connecting the two minute apertures was adjusted as before, and the phenomena were the same as in the former 
instance, the rays which passed along this line within the erystal forming a diverging cone at emergence.

In all these experiments the emergent rays were received directly by the eye placed close to the aperture on the second surface. It was obviously desirable, however, to receive them on a screen, and thus to observe the section of the cone at different distances from its summit. After some trials, I effected this with the sun's light, the light of a lamp being too weak for the purpose. The emergent cone being made to fall on a screen of roughened glass, I was enabled to observe its sections at various distances, and therefore with all the advantages of enlargement. The light was sufficiently bright, and the appearance distinct, when the diameter of the section was between one and two inches.

On examining the emergent cone with a tourmaline plate, I was surprised to observe that one radius only of the circular section* vanished in a given position of the axis of the tourmaline, and that the ray which disappeared ranged through $360^{\circ}$ as the tourmaline plate was turned through $180^{\circ}$. Thus it appeared that all the rays of the cone are polarized in different planes.

On examining this curious phenomenon more attentively, I discovered the remarkable law, "that the angle between the planes of polarization of any two rays of the cone is half the angle between the planes containing the rays themselves and the axis."

Having assured myself of the near truth of this law by experiment, I was naturally led to inquire how far it was in accordance with theory ; and on examining Fresnel's theory with this view, I was gratified to find that it led to the very same result.

According to the known rule, the plane of polarization of any one ray of the emergent cone must bisect the angle contained by the planes passing through the corresponding normal to the front of the wave and the two optio axes. Now, it can be easily shown that the normals to the wave, at the cusp, surround one of the optic axes, and are inclined to it all round at small angles. For the tangent of the angle which the normals to the circle and ellipse in the plane of $x z$ make with one another is

$$
\frac{\sqrt{a^{2}-b^{2}} \sqrt{b^{2}-c^{2}}}{a c}
$$

- These sections are not mathematically circular, the line being, in fact, one of the fourth order. 
and it ean be easily shown that the tangent of the angle which the optic axis makes with the normal to the circle, or the cusp-ray, is

$$
\frac{\sqrt{a^{2}-b^{2}} \sqrt{b^{2}-c^{2}}}{b^{2}+a c}
$$

Now, this is about half the former, since $b^{2}=a c$, nearly; and consequently the optic axis nearly bisects the angle contained by the extreme normals in the plane of $x z$. Hence if $A$ and $B$ be the intersections of the two optic axes with the sphere whose centre is at the cusp, and $N$ the intersection of one of the normals at that point with the same (fig. 2), the angle $N A C$ ranges through every magnitude between $0^{\circ}$ and $360^{\circ}$, the are $N A$ being

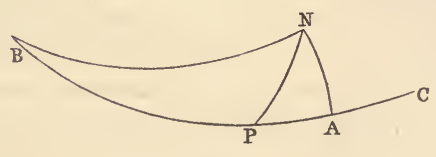

Fig. 2. all the time very small. Let the angle $N A C$ be denoted by $a$, and $N P C$ by $\omega, N P$ being the arch bisecting the angle $N$; then in the triangle $A P N$, we have

$$
\cos \omega=\cos A N \sin a \sin \frac{1}{2} N+\cos \alpha \cos \frac{1}{2} N ;
$$

or, since $A N$ is very small, and therefore $\cos A N=1$, nearly,

$$
\cos \omega=\cos \left(a-\frac{1}{2} N\right), \text { and } \omega=a-\frac{1}{2} N, \text { nearly. }
$$

But, when any side of a spherical triangle is very small in comparison with the other two, the adjacent angles are together equal to $180^{\circ} q \cdot p$. Consequently,

$$
N=a \text {, and } \omega=\frac{1}{2} a \text {, nearly. }
$$

From this it appears that the angle, which the plane of polarization of any ray makes with the plane of the optic axes, is half the angle which the plane passing through the normal and the , near axis makes with the same plane. But this latter angle, it may be easily shown, is very nearly the same as that which the plane passing through the emergent ray and the axis of the cone makes with the plane of the optic axes. Consequently the angle, which the plane of polarization of any ray of the emergent cone 
makes with the plane of the optic axes, is half of that which the plane containing that ray and the axis of the cone' forms with the same plane.

The general phenomena being observed, it remained to examine the magnitude and position of the emergent cone, and to compare the results with those furnished by theory. For this purpose I viewed the aperture in the second plate through a small telescope, which was moved in a plane nearly perpendicular to the axis of the emergent cone; and by noting the points at which the light failed, I obtained the magnitude of the section of the cone made by that plane. The distance of this section from the crystal being then measured, the angle of the cone was obtained from the trigonometrical tables, and was found to be very nearly $6^{\circ}$. I then placed the flame of a wax taper at the centre of the section, and removing the plate from the second surface of the crystal, found the direction of the ray reflected from the surface. A well defined mark was then placed on this line, at a considerable distance, and the angular distance between the centre of the flame and the mark measured by a sextant, whose centre was brought exactly to the place of the crystal. This angle was found to be $31^{\circ} 56^{\prime}$; and consequently the angle of emergence corresponding to the central rays of the cone was $15^{\circ} 58^{\prime}$.

Now to compare these results with those of theory.-It is a well-known principle of the theory of waves that the direction of a ray incident upon or emergent from a crystal, and the normal to the front of the wave, are always in the same plane perpendicular to the surface of incidence or emergence; and the angles which these two lines make with the perpendicular to the surface, are connected by the known law of the sines; the index of refraction being the reciprocal of the normal velocity of the wave, or of the perpendioular upon the tangent plane. Now, at the cusp, there are an infinite number of normals to the wave, and consequently an infinite number of corresponding emergent rays. Of these the two rays in the plane of the optic axes form the greatest angle, and their directions are determined by those of the normals to the circle and ellipse, which constitute the section of the wavesurface in that plane. If then $\rho^{\prime}$ and $\rho^{\prime \prime}$ denote the angles of emergence of these rays, , the angle which the normal to the circle, or cusp-ray, makes with the perpendicular to the surface, $a$ the angle contained by the normals to the circle and ellipse, 
and $p$ the perpendicular from the centre on the tangent to the ellipse at the cusp, we have

in which

$$
\sin \varrho^{\prime}=\frac{1}{b} \sin \iota, \quad \sin \varrho^{\prime \prime}=\frac{1}{p} \sin (\iota-a) ;
$$

$$
\frac{1}{p}=\frac{\sqrt{a^{2}+c^{2}-b^{2}}}{a c}, \quad \tan a=\frac{\sqrt{a^{2}-b^{2}} \sqrt{b^{2}-c^{2}}}{a c} .
$$

Now in arragonite, according to the determination of $M$. Rudberg,

$$
\frac{1}{a}=1.5326, \frac{1}{b}=1.6863, \frac{1}{c}=1.6908 ;
$$

and substituting these values we find

$$
\frac{1}{p}=1.68708, \quad a=1^{\circ} 44^{\prime} 48^{\prime \prime} .
$$

These values being introduced in the first two equations, $\rho^{\prime}$ and $\rho^{\prime \prime}$ will be determined for any given surface of emergence. In this manner Professor Hamilton has found that when $\iota=0$, or the surface of emergence perpendicular to the cusp-ray, $\rho^{\prime}=0$, and $\rho^{\prime \prime}=2^{\circ} 56^{\prime} 51^{\prime \prime}$. And when $\iota=9^{\circ} 56^{\prime} 27^{\prime \prime}$, or the surface perpendicular to the line bisecting the optic axes, $\rho^{\prime}=16^{\circ} 55^{\prime} 27^{\prime \prime}$, and $\rho^{\prime \prime}=13^{\circ} 54^{\prime} 49^{\prime \prime}$. Accordingly, the difference of these angles, $\rho^{\prime}-\rho^{\prime \prime}$, which is the extreme angle of the emergent cone, is in the former case $2^{\circ} 56^{\prime} 51^{\prime \prime}$, " and in the latter $3^{\circ} 0^{\prime} 38^{\prime \prime}$. Also, half the sum of these angles, which is the angle of emergence corresponding to the axis of the cone, is $15^{\circ} 25^{\prime} 8^{\prime \prime}$.

Comparing these with the results of observation, it will be seen that they agree nearly with respect to the mean angle of emergence, the difference amounting only to $33^{\prime}$; whereas the angle of the cone determined by experiment is about double of that furnished by calculation.

I also measured the angle of the cone by tracing the outline of its section on a screen of roughened glass, when the sun's light was employed instead of that of a lamp. The mean diameter of this section being then accurately ascertained, and the distance of the screen from the aperture measured, the angle was given by

- It is easily shown that the sine of the angle of the cone, in this case, is generally expressed by the formula $\frac{\sqrt{a^{2}-b^{2}} \sqrt{b^{2}-c^{2}}}{a b c}$. 
the tables. Measurements taken in this manner gave for the value of the angle, $6^{\circ} 24^{\prime}, 5^{\circ} 56^{\prime}, 6^{\circ} 22^{\prime}$, respectively; and the mean of these is $6^{\circ} 14^{\prime}$, which, like the former measurement, differs very little from the double of the calculated angle.

The results of observation thus appeared to be at variance with those of theory in two important particulars. In the first place, the emergent rays appeared to form a solid cone, instead of a conical surface; and in the next, the magnitude of this cone was about double of the expected magnitude. Conceiving that these discrepancies might probably be owing to the rays which are inclined to the cusp-ray at small angles, and which pass by the edge of the aperture, I determined to ascertain the fact by trying the effects of apertures of various sizes.

I found accordingly that when the aperture was at all considerable, such as that formed by a large-sized pin, two concentric circles were seen to surround the axis, the interior of which had about double the brightness of the exterior annulus. And it was remarkable that the light of the interior circle was unpolarized, while that of the surrounding annulus was polarized according to the law already explained. When smaller apertures were used, the inner circle contracted, the breadth of the exterior annulus remaining nearly the same; until the former was finally reduced to a point in the centre of a fainter circle. When the aperture was still further diminished, a dark space sprung up in the centre, enlarging as the aperture decreased; until finally, with a very minute aperture, the breadth of this central space increased to about $\frac{3}{4}$ ths of the entire diameter.

The phenomena exhibited in these cases assumed the forms represented in figures $(f)$ and $(g)$. (Fig. $h$ ) represents the ap-

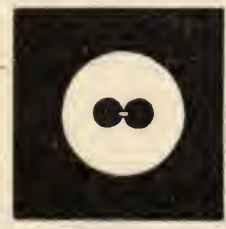

Fig. $f$.

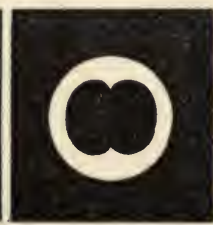

Fig. $g$.

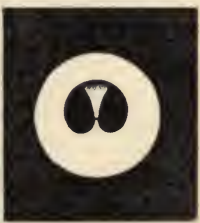

Fig. $h$.

pearance of the section when the line connecting the aperture with the luminous point on the first surface was slightly inclined to the cusp-ray. 
It is easy to render an account of these various appearances. When the aperture $m n$ (fig. 3 ) is at all considerable, the rays $\mathrm{cm}$, $c n$, proceeding to its circumference from a point on the first surface, will be sensibly inclined to the cusp-ray, which we shall suppose to be the line co, connecting the point on the first surface with the

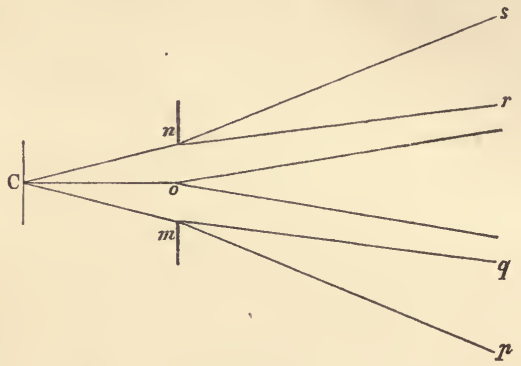

Fig. 3.

centre of the aperture. Consequently the interior refracted rays, $m q, n r$, as well as the exterior, $m p, n s$, will be inclined outuards; and it is obvious that there will be a central bright space, limited by the lines $m q, n r$, each point of which will be illuminated by one interior and one exterior ray. The light in this space, therefore, will have double the intensity of that of the surrounding space; and as the rays which combine to form it are polarized in planes at

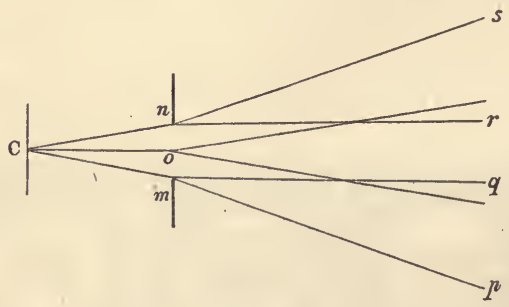

Fig. 4.

right-angles to one another, the resulting light will be unpolarized. When the aperture is diminished, the inclination of the rays $m q$, $n r$, to one another is lessened, until finally they are reduced to parallelism, and the central bright space contracts to a point. This is represented in (fig. 4). When the aperture is still further 
diminished, the rays $m q, n r$, become inclined invards, and cross (fig. 5). It is obvious that beyond the point of intersection there will be a dark space illumined by no ray whatever; and as in the surrounding annulus there is no meeting of rays oppositely polarized, the whole of the light will be polarized, and according to the law already explained. With a yet diminished aperture, the

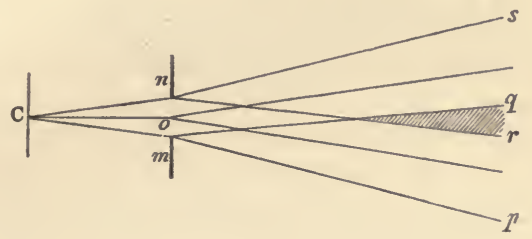

Fig. 5.

rays $m q, n r$, approach to parallelism with the exterior rays, $n s, m p$; and the central dark space enlarges, and approaches to equality with the outer and limiting cone. Thus the annulus of light in any section is diminished indefinitely in breadth, and the cone approaches to a mathematical surface.

Now, if we assume that the divergence of the two refracted rays in this plane, corresponding severally to the rays $\mathrm{cm}, \mathrm{co}, \mathrm{cn}$, is the same, as must be nearly the case, it will follow that the angle of the true cone, which would arise from the single ray $c 0$, is half the sum of the angles of the exterior and interior surfaces of the conical annulus; and that when a bright circle appears in the centre, as is the case with a considerable aperture, the dark space must be considered as negative, and the true angle is half the difference of the observed angles.

From this it follows that when the central bright space is reduced to a point, the true angle is just half the observed. Now this was very nearly the case in the experiments from which the measures were taken; consequently the corrected angle, deduced from these measures, coincides very nearly with that assigned by theory.

Two other measurements, taken since with a more direct reference to this correction, were as follows :-

1. Distance of screen from the aperture on the second surface of the crystal $=19.3$ half inches. Mean diameter of section of 
exterior cone $=1 \cdot 27$. Mean diameter of interior $=0.55$. Corrected angle of cone thence computed $=2^{\circ} 44^{\prime}$.

2 . Distance of screen $=11 \cdot 9$. Mean diameter of section of exterior cone $=0.93$. Mean diameter of interior $=0.41$. Computed angle of cone $=3^{\circ} 14^{\prime}$.

The mean of these two measurements is $2^{\circ} 59^{\prime}$.

Inasmuch as the cusp-ray, within the crystal, corresponds to a cone of rays without, it is evident that there must be a converging cone incident on the first surface, equal to that which diverges from the second. With a view to determine its magnitude, I placed a kind of rough micrometer, consisting of two moveable metallic plates, immediately before the lens; and closed the plates until, on looking through the aperture on the second surface, I could see them touching the circumference of the annular section. The diameters of the interior and exterior circumferences of this section, at the distance of the lens, being thus ascertained, and the focal length of the lens measured, the corrected angle of the cone was found. The mean of three measurements taken in this manner gave for this angle $3^{\circ} 47^{\prime}$. But the methods by which this last result was obtained do not seem susceptible of much accuracy.

Before I conclude this part of the subject, I may observe that an interesting variation in the phenomena is obtained by substituting a narrow linear aperture for the small circular one, in the plate which covers the first surface of the crystal-that surface being close to the lamp. The linear aperture is to be so fixed, that the plane passing through it and the aperture in the plate next the eye shall be the plane of the optic axes. In this case, according to the received theory, all the rays transmitted through the two apertures should be refracted doubly in the plane of the optic axes, so that no part of the line should appear enlarged in breadth on looking through the second aperture; whereas, according to Professor Hamilton's beautiful conclusion from the same theory, the cusp-ray should be refracted in every possible azimuth. I found accordingly that the luminous line was un-dilated, except in the direction corresponding to that of the cusp-ray; and that in the neighbourhood of this direction its boundaries were no longer rectilinear, but swelled out in the form of an oval curve (fig. $i$ ).

When a very minute aperture was used on the surface next the eye, in this experiment, the phenomenon was rendered much more remarkable. The swelling curves in this case were separated by a 
considerable dark interval, and the luminous line was prolonged into this dark space, terminating abruptly near its centre. This

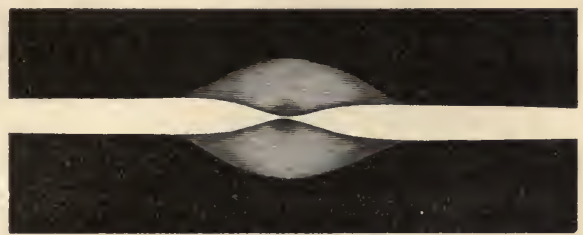

Fig. i.

appearance is represented in (fig. $k$ ). When the plate next the eye was slightly shifted, so that the plane passing through the two

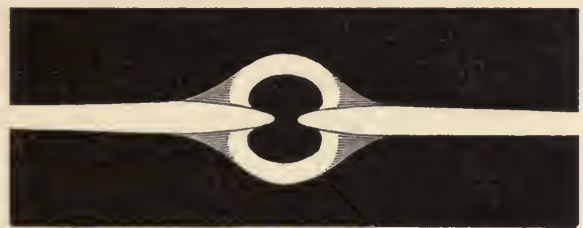

Fig. $k$.

apertures no longer coincided accurately with the plane of the optic axes, the curves rapidly changed, preserving however, in all cases,

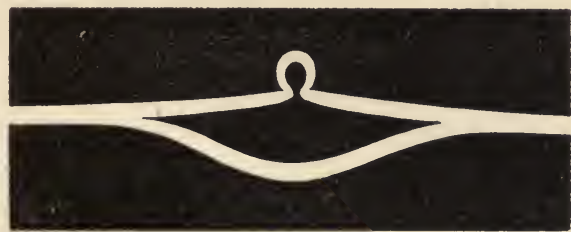

rig. 6.

the form of the conchoid, whose pole was the projection of the axis of the emergent cone, and asymptote the line on the first surface-

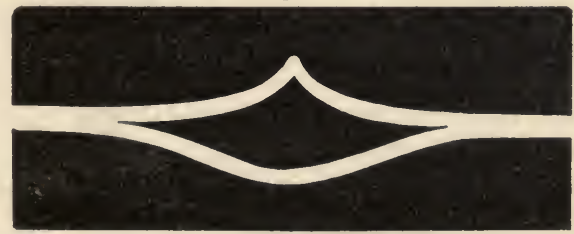

Fig. $m$.

(figs. l, mi). It is easy to show that these results are in accordance with theory. 
The second kind of conical refraction, whose existence has been anticipated by Professor Hamilton, depends (it will be remembered) on the mathematical fact, that the wave-surface is touched in an infinite number of points, constituting a small circle of contact, by a single plane parallel to one of the circular sections of the surface of elasticity. It takes place when a single external ray falls upon a biaxal crystal in such a manner that one refracted ray may coincide with an optic axis. When this is the case, there will be a cone of rays within the crystal, determined by lines connecting the centre of the wave with the points of the periphery of the circle of contact. The angle of this cone is equal to

$$
\operatorname{tang}^{-1} \frac{\sqrt{a^{2}-b^{2}} \sqrt{b^{2}-c^{2}}}{b^{2}}
$$

and its numerical value in the case of arragonite is $1^{\circ} 55^{\prime}$, assuming the values of the three indices as determined for the ray $E$ by Professor Rudberg (see page 10).

As the rays constituting this cone will be refracted at emergence in a direction parallel to the incident ray, they will form a small cylinder of rays in air. This cylinder, it will be seen, is in all cases extremely small; for the diameter of its section made by the surface of emergence subtends an angle of $1^{\circ} 55^{\prime}$ only, at a distance equal to the thickness of the crystal. Hence the experiments required to detect its existence and measure its magnitude demand more care and precision than those already described. The incident light was that of a lamp placed at some distance; and in order to reduce as much as possible the breadth of the incident beam, it was constrained to pass through two small apertures, the first of which was in a screen placed near the flame, and the second perforated in a thin metallic plate adjoining to the first surface of the crystal. Under ordinary circumstances, it is obvious, the incident rays will be divided into two within the crystal, and these will emerge parallel from the second surface. I was able to distinguish these two rays by the aid of a lens; and turning the crystal slowly, so as to vary the incidence gradually, I at length observed that there was a position in which the two rays changed their relative places rapidly, on any slight change of incidence, and appeared at times to revolve round one another, as the incidence was altered. Being convinced that the ray was now near the critical incidence, I changed the position of the crystal, with respect to the incident 
ray, very slowly ; and after much care in the adjustment, I at last saw the two rays spread into a continuous circle, whose diameter was apparently equal to their former interval.

This phenomenon was exceedingly striking. It looked like a small ring of gold viewed upon a dark ground; and the sudden and almost magical change of the appearance, from two luminous points to a perfect luminous ring, contributed not a little to enhance the interest.

The emergent light, in this experiment, being too faint to be reflected from a screen, I repeated the experiment with the sun's light, and received the emergent cylinder upon a small piece of silver-paper. I could detect no sensible difference in the magnitude of the circular sections at different distances from the crystal.

When the adjustment was perfect, the light of the entire annulus was white, and of equal intensity throughout. But when there was a very slight deviation from the exact position, two opposite quadrants of the circle appeared more faint than the other two, and the two pairs were of complementary colours.* The light of the circle was polarized, according to the law which I had before observed in the other ease of conical refraction. In this instance, however, the law was anticipated from theory by Professor Hamilton.

I measured the angle of incidence by a method similar to that already employed for the emergent ray in the former case; and found it to be $15^{\circ} 40^{\prime}$. This determination is, for many reasons, capable of much greater accuracy than the other; and was probably, in this instance, very near the truth.

In order to compare it with the result of theory, it is to be observed that the optic axis is a normal to the wave-surface, and, therefore, the corresponding incident ray will be given by the ordinary law of the sines, the index of refraction being the mean index of the crystal. Now, the angle which the normal to the circular section of the surface of elasticity, or the optic axis, makes with the axis of $x$, or the perpendicular to the surface, is equal to $\tan ^{-1} \sqrt{\frac{b^{2}-c^{2}}{a_{2}-b_{2}}}$; and its numerical value, in the case of arragonite,

* This part of the phenomenon appears to be explained by the non-coincidence of the optic axes for the rays of different colours. 
is $9^{\circ} 1^{\prime}$. We have then $\sin \iota=1.6863 \sin \left(9^{\circ} 1^{\prime}\right)$; from which we find $\iota=15^{\circ} 19^{\prime}$. The difference between this and the observed angle is $21^{\prime}$.

In order to measure the angle of the cone, I was compelled to employ a method somewhat indirect, but, I think, susceptible of considerable accuracy. As the aperture on the first surface of the srystal must have some physical magnitude, it is obvious that, instead of a cone of mathematical rays within the crystal, there will be in all cases a cone of cylindrical pencils, overlapping one another near the point of divergence; and that the diameter of these pencils will be equal to the diameter of the aperture. Now, I tried a number of apertures, until I found one with which these cylindrical pencils just separated at the second surface of the crystal. It is evident that, in this case, the interval between the axes of the cylinders at the surface of emergence is just equal to the diameter of one of them, or to the diameter of the aperture. I had then only to measure the aperture itself. This was effected by the aid of a micrometer divided to the 1-500th of an inch, placed along with the aperture before a compound microscope; and it was found to be $\cdot 016$ of an inch. This therefore was the diameter of the oblique section of the cone made by the surface of emergence : and the diameter of the circular section at the same distance was $\cdot 016 \cos 9^{\circ}$, since the axis of the cone makes an angle of $9^{\circ}$ with the normal to the faces of the erystal. The perpendicular thickness of the crystal was 0.49 of an inch; and therefore the thickness estimated in the direction of the axis of the cone was $\frac{0.49}{\cos 9^{\circ}}$. From these data the angle of the cone was calculated by the tables, and found to be $1^{\circ} 50^{\prime} ;$ a result which differs from the theoretical angle by $5^{\prime}$ only. 


\section{II.-REPORT ON THE PROGRESS AND PRESENT STATE OF PHYSICAL OPTICS.}

Report of the British Association for the Advancement of Science for 1834.

Is the Report which I have the honour to submit to the Association, I have attempted to consider in some detail the present state of our knowledge with regard to the physical theory of light, and the successive advances which have, in late years, been made towards its establishment. The method which. I have thought it expedient to adopt in this review has been to take, in the first instance, a rapid survey of the several leading classes of optical phenomena, which the labours of experimental philosophers have wrought out in such rich profusion, and afterwards to examine how far they are reducible to one or other of the two rival theories which have alone advanced any claim to our consideration. This is, in fact, the only way in which the truth of a physical theory can be established; and the argument in its favour is essentially cumulative.

But in making this comparison it is not enough to rest in vague explanations which may be moulded to suit any theory. Whatever be the apparent simplicity of an hypothesis-whatever its analogy to known laws-it is only when it admits of mathematical expression, and when its mathematical consequences ean be numerically compared with established facts, that its truth can be fully and finally ascertained.* Considered in this point of view, the wave-theory of light seems now to have reached

* C'est en tirant des formules les conséquences les plus subtiles et les plus eloignées des principes, puis allant les vérifier par l'expéricnce, que l'on peut réellement s'assurer si une théorie est vraie ou fausse, et si l'on doit s'y confier comme à un guide fidèle, ou la rejeter comme un systéme trompeur.-Biot, Trailé de Physique, tom. i., p. xiv. 
a point almost, if not entirely, as advanced as that to which the theory of universal gravitation was pushed by the single-handed efforts of Newton. Varied and comprehensive classes of phenomena have been embraced in its deductions; and where its progress has been arrested, it has been owing in a great degree to the imperfections of that intricate branch of analysis by which it was to be unfolded. The principles of the theory of emission, on the other hand, have in comparatively few instances been mathematically expressed and developed; and accordingly this theory presents but rarely those points of contact with experimental truth by which alone it can be judged.

This signal difference in the present state of the two theories has been by some ascribed to a difference in the intellectual power by which they have been worked; and it has been said that had the Newtonian theory been cultivated with the same zeal and talent as the Huygenian, it might have had equal triumphs to boast of. This position, I confess, appears to me altogether untenable. With respect to the implied fact, it may be enough to observe that Newton and Laplace were both engaged on one side of the question; and I believe I may add that among the supporters of the wave-theory of light there are few who have not had to encounter early predilections in favour of the theory of emission. The nature and laws of projectile morement are far more familiar to every lover of mechanical philosophy than those of vibratory propagation; and the triumphant career of the former branch of this science, in its application to the movements of the heavenly bodies, is in itself sufficient to induce everyone to lean to a theory which proposes to account for the phenomena of light on similar principles. As to the opinion itself, it seems highly improbable, to say the least, that two theories so widely separated should run hand in hand in their explanation of phenomena. There is indeed one case, and that a striking one, of this kind:-the fundamental laws of reflexion and refraction are exact and necessary consequences of each of these theories; but I believe their history affords no parallel instance.

An unfruitful theory may, however, be fertilized by the addition of new hypotheses. By such subsidiary principles it may be brought up to the level of experimental science, and appear to meet the accumulating weight of evidence furnished by new phenomena. But a theory thus overloaded does not merit the name. 
It is a union of unconnected principles, which can at best be considered but as supplying the materials for a higher generalization. Its very complexity furnishes a presumption against its truth; for the higher we are permitted to ascend in the scale of physical induction, the more we perceive of that harmony, and unity, and order, which must reign in the works of One Supreme Author. The theory of emission, in its present state, exhibits all these symptoms of unsoundness; but there is something stronger than mere presumption against it. It will appear, I think, upon a fair review, that in almost every instance in which it has been developed, its consequences are at variance with facts; and the proof of its insufficiency seems even stronger than the positive evidence in favour of the rival theory.

In proceeding to the consideration of these arguments, I have found it necessary to deviate from the arrangement which a strictly theoretical view of the subject would naturally suggest. The relation of theory to phenomena, which I propose to consider, obliges me to examine the latter in the groups in which they have been usually brought together, and under which their laws have been investigated. I propose, therefore, to divide the following Report into two parts, of which the first will treat of unpolarized, and the second of polarized light. In the former I shall consider separately

1. The propagation of light, and the principle of interference;

2. The reflexion and refraction of light;

3. Diffraction;

4. The colours of thin and thick plates.

The second part will comprise

1. The polarization of light, and the principle of transversal vibrations;

2. The reflexion and refraction of polarized light;

3. Double refraction;

4. The colours of crystallized plates.

Many subjects of high interest are omitted in this arrangement, as being but remotely connected with the leading object of the present Report. I have left wholly untouched, for this reason, that branch of optical science which is sometimes denominated "mathematical optics," or the development of the fundamental 
laws of reflexion and refraction. The phenomena of vision have been in like manner omitted, as involving also the science of physiology ; and the relations of light to other agents, as heat, electricity, and magnetism, because these relations are as yet little understood, and in the present state of the kindred sciences, the science of light can hope to derive little aid from their examination. These interesting subjects would, each of them, well merit a separate consideration.

\section{Part I. Unpolarized Light.}

\section{Propagation of Light.-Principle of Interference.}

The first property of light which claims our notice is its progressive movement. Light, we know, travels from one point of space to another in time, with a velocity of about 195,000 miles in a second. The inquiry concerning the mode of this propagation involves that respecting the nature of light itself.

There are two distinct and intelligible ways of conceiving such a motion. Either it is the self-same body which is found at different times in distant points of space; or there are a multitude of moving bodies, occupying the entire interval, each of which vibrates continually within certain limits, while the vibratory motion is communicated from one to another, and so advances uniformly. Nature affords numerous examples of each of these modes of propagated movement; and in adopting one or other to account for the phenomena of light, we fall upon one or other of the two rival systems-the theories of Newton and of Huygens.

The Newtonian theory, in the shape in which it is usually presented, is undoubtedly simpler in conception than its rival; but this simplicity is only apparent. Newton himself was far too clear-sighted to suppose that the forces of attraction and repulsion, by which the molecules of light were supposed to be refracted and reflected, were adequate to account for all the phenomena; and it is remarkable that, when he proceeds to speculate on the physical theory of light, he has found it necessary to admit all the apparatus required in the theory of waves. In fact, Newton felt, and distinctly stated, that the vibrations of an ethereal medium were necessary in his hypothesis, ${ }^{*}$ although he denied that those vibra- 
tions constituted light. He has even gone further, and asserted that they were the ehief and essential parts of that hypothesis, the molecules emitted from luminous bodies only performing the office of exciting these vibrations, as stones flung into water produce waves.* On the other hand, the molecules themselves are supposed to be emitted by a vibratory motion of the parts of the luminous body + - the same vibratory movement, though acting with a different energy, in which he supposes heat to consist. It would appear, then, that Newton assumed too much, and that he erred against his own valuable rule-"Causas rerum naturalium non plures admitti debere," \&c. Had he simply left out the molecular part of his hypothesis, and supposed that the vibrations of his ethereal medium were directly excited by those of the luminous body, his theory would have resolved itself into that of Huygens and of Hooke. It may be observed, in connexion with this subject, that Newton seems actually to have admitted the wave-theory with respect to radiant heat; and that he supposed it to be propagated, not by the translation of material particles, but by the vibrations of an ethereal medium. +

The peculiar part of the theory of emission-the supposition that the rays of light are bodies projected with a great velocitywould seem to offer an easy criterion of its truth. If the weight of a molecule of light amounted to one grain, its momentum would equal that of a cannon ball 150 pounds in weight, and moving with the velocity of 1000 feet in a second. The weight of a single molecule may be supposed many millions of times less than this ; but, on the other hand, millions of such molecules may be made to act together, by concentrating them in the foci of lenses or mirrors, and the effects of their impulse might, it was expected, be thus rendered sensible. This easy test of the materiality of light was long since appealed to. The experiments of Homberg seemed to have established the existence of a sensible impulsive effect; but when these experiments were repeated with more caution by Mairan and Dufay, they conducted to the opposite conclusion. The results

* "Were I to assume an hypothesis, it should be this, if propounded more gene.. rally-so as not to deternine what light is, further than that it is something or other capable of exciting vibrations in the ether : for thus it will become so general, and comprehensive of other hypotheses, as to leave little room for new ones to be invented."Bireh's History of the Royal Socuty, vol. iii. p. 249.

† Optics, Query 8.

† Opties, Query 18. 
obtained by Michell at a later period, and with the aid of a more sensitive apparatus than any before employed, seemed to be decisive in favour of the materiality of light.* The effects observed in these experiments, however, have been with much probability referred to aerial currents, produced by unequal temperature, or even to a difference in the elastic force of the air in contact with the opposite surfaces of the body acted on. $\dagger$ The subsequent experiments of Mr. Bennett were made under circumstances far more favourable; and in particular, having been repeated in a vacuum, they are independent of the sources of error now alluded to. Their result was conclusive as to the non-existence of a sensible effect. $\ddagger$

The objection to the materiality of light, arising from its want of sensible momentum, was first urged by Franklin. Horsley attempted to remove the difficulty; $\S$ but his laborious arithmetical calculations only go to prove that the particles of light, if material, must be of extreme minuteness. It must at the same time be confessed that objections of this nature are entitled to little weight. It is easy to attribute to the molecules of light a minuteness sufficient to evade any means that we possess of detecting their inertia by their effects upon other bodies; and in whatever point of view we regard the phenomena of optics, we are forced to contemplate quantities immeasurably smaller than any to which the imagination has been accustomed.

The aberration of the light of the fixed stars, resulting from the motion of the earth and that of light, is an easy consequence of the theory of emission, in which these motions are conceived to subsist independently. In order to account for the phenomenon in the theory of waves, it seems necessary to assume that the ether which encompasses our globe does not participate in its motion; so that the ethereal current produced by this relative motion must be supposed to have a free passage through the solid mass of the earth; or that, in the words of Young, "the luminiferous ether pervades the substance of all material bodies with little or no resistance, as freely perhaps as the wind passes through a grove of trees." " Fresnel has maintained the same opinion, and, startling

* Priestley's History of Optics, p. 387.

+ Young "On the Theory of Light and Colours," Phil. Trans. $180 \mathrm{~L}$.

$\ddagger$ Phil. Trans. 1792.

Ibid. 1770. I.

|| “Experiments and Calculations relatire to Physical Optics,"- Phil. Trans. 1803. 
as the position seems at first, he has very clearly shown that no fair argument can be advanced against it, founded on the opacity of the mass which the ether is supposed to permeate.*

The discoveries of Bradley and Roemer, when compared together, have led to a further and most important conclusion respecting light-namely, that its velocity is one and the same, whatever be the luminous origin, the light of the sun, the fixed stars, the planets and their satellites, being all propagated with the same swiftness. This conclusion must be allowed to present a formidable difficulty in the theory of emission. Laplace has shown that if the diameter of a fixed star were 250 times as great as that of our sun, its density being the same, its attraction would be sufficient to destroy the whole momentum of the emitted molecules, and the star would be invisible at great distances. $\dagger$ With a smaller mass there will be a corresponding retardation; so that the final velocities will be different, whatever be the initial. The suggestion of M. Arago seems to offer the only means of avoiding this difficulty. It may be supposed that the molecules of light are originally projected with very different velocities; but that among these velocities there is but one which is adapted to our organs of vision, and which produces the sensation of light. This supposition seems to be supported by the discoveries of Herschel, Wollaston, and Ritter, respecting the invisible rays of the spectrum; but it does not appear to be easily reconciled with any hypothesis which we are able to frame respecting the nature of vision. This uniformity of velocity, on the other hand, is a necessary consequence of the principles of the wave-theory. The velocity with which vibratory movement is propagated in an elastic medium depends solely on the elasticity of that medium, and on its density ; and if these be uniform in the vast spaces which intervene between the material bodies of the universe-and it is not easy to suppose it otherwise- the velocity must be the same, whatever be the originating source.

The rectilinear motion of light has long been urged in favour of the theory of emission, and against the theory of waves. If light consists in the undulations of an elastio fluid, it has been said, it should be propagated in all directions from every new centre,

" "Sur l' Influence ảu Mouvement terrestre dans quelques Phénomènes d'Optique," Annales de Chimie, tom. ix.

+ Zach, Ephem., iv. 1. 
and so bend round interposed obstacles. Thus, luminous objects should be visible, even when an opaque body is between them and the eye, just as sounding bodies are heard, though a dense body intervene between them and the ear. To this objection, which was first insisted on by Newton, ${ }^{*}$ a full answer has been given. The phenomena of diffraction, and especially the interior fringes in the shadow of narrow opaque bodies, prove that light does bend round obstacles, and deviate perceptibly from the rectilinear course. When the obstacle is of considerable dimensions, the intensity of the light decreases, indeed, very rapidly within the edge of the geometric shadow; so that at a very small distance from that edge it is no longer perceptible. But the darkness does not arise from the absence of luminiferous waves, but from the mutual destruction of those sent there. In fact, if the surface of the wave when it reaches the obstacle be divided into any number of small portions, the motion of the ether at any point behind it is, by the principle of Huygens, the sum of all the motions produced there by these several portions, considered as separate centres of disturbance; and it is easy to show that, when the distance of the point in question from the obstacle is a large multiple of the length of a wave, the magnitude of this resultant must diminish rapidly within the shadow, and the light become insensible when the line drawn from that point to the edge of the screen is inclined at a small angle to the normal to the front of the wave. The accurate calculation of the intensity, in this and other similar cases, has been made by Fresnel by the aid of the principle of interference, and the result is found to agree in the most complete manner with observation. $\dagger$

The same principles apply to the aerial waves which constitute sound; and these too should present analogous phenomena. But the scale is widely different. The length of an aerial wave is more than 10,000 times greater than that of an ethereal undulation; and the distance of the ear from the obstacle must be augmented in the same proportion, in order that the same conclusions may be applicable to the two cases.

According to this account, then, the right-lined propagation of the rays of light is a consequence of the principle of interference, combined with the principle of Huygens. A very different view

* Optics, Query 28.

† "Mémoire sur la Diffraction," Mémoires de l' Inslitut, tom. v. 
of the subject, however, has been presented by M. Poisson, in a memoir on the propagation of motion in elastic fluids read before the French Academy in the year 1823.* The elasticity of the fluid being supposed the same in all directions, the velocity of propagation will be also the same, and consequently the waves spherical. The absolute velocities of the molecules themselves, however, will be very different. M. Poisson finds that when the original disturbance takes place only in one direction, the velocity of the molecules will be indefinitely small in all directions inclined to it at finite angles, so that the motion will not be sensibly propagated except in that direction. This diminution of intensity, he finds, will be greater the more rapid the velocity of propagation; and it is in this manner only, he concludes, that we can account for the rectilinear motion of light in the wave-theory. This conclusion, however, M. Fresnel has shown, is contradicted by the ordinary phenomena of diffraction ; and he has adduced theoretical reasons, drawn from the principle of the coexistence of small motions, to prove that it cannot hold in any fluid whatever, but that the molecules are in all cases disturbed in a sensible manner in directions very much inclined to that of the original vibrations. $\dagger$

The principle of the superposition of small motions, which has been more than once adverted to, is an immediate consequence of the linearity of the original equation of partial differences which determines the law of vibration of an ethereal particle. The complete integral of this question will contain, in general, a term for every distinct original disturbance ; and the total disturbance will be the sum of all the partial disturbances due to each cause acting separately. The partial disturbances may, however, conspire, or be opposed; so that in the case of two such disturbances, for example, the second may have the effect either of augmenting or diminishing the first, and the absolute velocity of the ethereal molecules may be increased, or lessened, or even wholly destroyed by the union. In fact, if the form of the function which expresses the wave-disturbance be assumed to be that by which the law of vibration of the cyeloidal pendulum is represented, the sum of two coexisting disturbances will be a single disturbance of the same form, provided the component undulations have the same length; and the effect of two such coexisting undulations will be a single undulation of the same length, but differing in the position

- Anniles de Chimie, tom. xxii.

† Ibid. tom. xxiii. 
and magnitude of the space of greatest vibration from either of the components. The magnitude of the resulting vibration may be the sum, or difference, of those of the component vibrations, or it may have any value intermediate to these limits. When the component vibrations are equal, the resultant may even vanish altogether; and two lights of equal intensity, when added together, will produce darkness, provided that the interval of retardation of one wave on the other is an odd multiple of the length of half a wave.

This important consequence of the theory of waves-the principle of interference of the rays of light-was first distinctly stated and established by Dr. Thomas Young, although some of the facts by which its truth is experimentally confirmed were known to Grimaldi.* The general calculation of the intensity of the resulting light, for any relative position of the interfering waves, is due to Fresnel, and has been followed out and developed by Sir John Herschel in his valuable Essay on Light. When a beam of homogeneous light is transmitted through two small apertures in a card, or plate of metal, the light will diverge from each as from a new centre. If the two apertures are close together, and the diverging pencils received on a reflecting surface, a series of parallel straight bands is observed, perpendicular to the line connecting the apertures, and separated by intervals absolutely dari. That these alternations of light and darkness are produced by the mutual action of the two pencils, Young proved by the fact that when one of the beams is intercepted, the whole system of fringes instantly disappears, and the dark intervals recover their former brightness.

The experiment of Fresnel is still more satisfactory. In this important and instructive experiment, the fact of interference is placed beyond all question. The two pencils proceed from one common origin, and are separated simply by reflexion at plane surfaces, without any attending circumstance which can, by possibility, be supposed to influence the result. The phenomenon is thus divested of everything non-essential, and it becomes impossible to hesitate about its nature. But the accordance of theory and experiment is maintained, not only in the general features of

* This ingenious philosopher even stated explicitly that an illuminated body may be rendered darker by the addition of light, and adduced a simple experiment in proof of it. Physico-Mathesis de Lumine. Bologna, 1665. 
the phenomenon, but even in its minutest details. The distances of the points of each fringe from the two foci of reflected rays should, according to theory, differ by a constant quantity-that constant being an odd multiple of the length of half a wave for the dark fringes, and an even multiple of the same quantity for the bright ones. Hence the fringes should be propagated in hyperbolic lines, whose foci are the foci of the reflected pencils; and the most accurate measurements have shown that it is so. The constant differences just alluded to are far too minute to be directly measured; but they can be calculated with great accuracy, when the distances of the successive bands from the central one have been obtained. The latter distances have been determined by Fresnel with much nicety by micrometrical measurements; and the lengths of the waves of each species of simple light, thence computed, agree in the most satisfactory manner with the values of the same quantities as deduced from the observation of Newton's rings.

The central fringe is formed at those points in arriving at which the two pencils have traversed equal paths; and as its position is therefore independent of the length of a wave, the rays of all colours will be united there, and the fringe itself will be white, or colourless. Such is the fact, as described by Fresnel himself, and by most observers who have repeated the experiment. Mr. Potter states, however, as the result of his observations, that the central fringe may be seen both black and white, although more frequently the former; and he urges the fact in opposition to the wave-theory.* But it seems premature to draw any inference from such experiments, until the circumstances which have occasioned the variation in the results have been fully investigated and understood.

The interference of the rays of light has, since the decisive experiment of Fresnel, been admitted on all hands; and the phenomena which were previously explained on the Newtonian hypothesis of the "fits of easy reflexion and transmission" are now, by most of the advocates of the Newtonian theory, referred to this simpler and more fertile principle. This principle is, it has been stated, an immediate and necessary consequence of the wave-theory, and its experimental establishment must be regarded as a weighty argument in favour of that theory. It now remains

- Phil. Mag. (3rd Series), vol. ii. p. 280. 
to inquire whether an account can be given of it in the theory of emission.

The molecules of light cannot be supposed to exert any mutual influence; for the regularity of the laws of reflexion and refraction compels us to consider them as independent, and each, separately, the subject of those forces from which, in the theory of emission, these laws are derived. The phenomenon of interference may, however, be plausibly accounted for by the vibrations of the optic nerve, produced by the impulse of the rays of light upon the retina, and by the accordance or discordance of these vibrations when caused by two interfering pencils. On this supposition, which was suggested by Dr. Young himself, the intensity of the light will depend on the relation between the time of vibration of the optic nerve and the interval of the impulses of the succeeding particles. If this interval be equal to the time of vibration, or to any multiple of it, the second impulse will add its effect to that of the first, and the motion be accumulated. It will, on the other hand, be destroyed, if the second impulse follows the first at an interval equal to half that time.

It is here assumed that the emitted particles succeed one another at equal intervals, as will be the case if their emission be owing (as Newton supposed it to be) to a vibratory motion of the parts of the luminous body. But we must assume further that the intervals of emission vary with the nature of the particles, in the light of different colours; or that all the red-making particles (to use an expression of Newton) are emitted at one certain intervalall the blue-making at another-and so for each different species of simple light. Hence the vibrations of the parts of the luminous body must be of different periods for the light of different colours. This is, in truth, a part, and a necessary part, of the theory of waves; but it has no connexion whatever with the principles of the rival theory.

\section{Reflexion and Refraction of Light.}

To account for the phenomena of reflexion and refraction it is supposed, in the Newtonian theory, that the particles of bodies and those of light exert a mutual action; that, when they are nearly in contact, this action is attractive-that at a distance a little greater, the attractive force is changed into a repulsive one, and that these attractive and repulsive forces succeed one another pro- 
bably for many alternations. The absolute values, or intensities, of these forces are different in different bodies; but the form of the law, or the function of the distance by which they are expressed, is assumed to be the same for all. * From these postulates Newton has rigorously deduced the laws of reflexion and refraction. The problem is the first in which the effects of that important class of forces acting only at insensible distances have been submitted to calculation; and the solution is regarded by M. Poisson as forming an era in the history of science.

The reflexion of light at the exterior surface of dense media is ascribed to the repulsive force ; refraction, and internal reflexion, to that inner attractive force which extends up to actual contact. The outermost sphere of action of every body, in this theory, is necessarily attractive, as well as the inmost; for, were it otherwise, no ray could enter, or emerge from, the medium at an extreme incidence. Sir David Brewster has made an ingenious use of this principle to explain the remarkable fact noticed by Bouguer, that water is more reflective than glass at oblique incidences.

But though the theory of emission is perfectly successful in explaining the laws of reflexion and refraction, considered as distinct phenomena, yet it is by no means equally so in accounting for their connexion and mutual dependence. When a beam of light is incident on the surface of any transparent medium, part is in all cases transmitted, and part reflected. The intensity of the reflexion is in general less, the less the difference of the refractive indices of the two media; and accordingly the reflective and refractive forces (if such be the cause of the phenomena) are related to one another in all media, so that one increases or diminishes along with the other. $\dagger$ But how is it that some of the molecules obey the influence of the repulsive force, and are reflected, while others yield to the attractive force, and are refracted? To account "for this Newton was obliged to have recourse to a new hypothesis. The molecules of light are supposed to pass through certain periodical states, called "fits of easy reflexion and trans-

- This assumption is tacitly made by Newton, when he takes the function $\frac{\mu^{2}-1}{8}$ as the measure of the refractive power. See Herschel's "Essay on Light," Encyc. Metrop.

$\uparrow$ The reader will find much novel and interesting matter connected with this subject in a paper by Sir David Brewster, "On the Reflexion and Decomposition of Light at the separating surface of media of the same and of different refractive powers," Phil. Trans. 1829. 
mission," which modify the effects of the attractive and repulsive forces, and in which they are disposed to yield alternately to one or the other. The actual determination of the particle will depend, partly on the phase of the fit, and partly on the obliquity under which it meets the bounding surface. Now the molecules composing a beam of light are supposed to be in every possible phase of their fits when they reach the surface: some of them consequently will be reflected, and others refracted; and the proportion of the former to the latter will depend on the incidence.

As to the fits themselves, Newton thought that they must be referred to a vibratory motion in the ether, excited by the rays themselves, just as a stone flung into water raises waves on its surface. This vibratory motion is supposed to be propagated faster than light itself, and thus to overtake the molecules, and impress upon them the disposition in question by conspiring with or opposing their progressive motion. In one of his queries Newton has even calculated the lesser limit of the elasticity of the ether, as compared with that of air, in order that it should have so great a velocity of propagation.* The hypothesis of Mr. Melville and $\mathbf{M}$. Biot is more in accordance with the spirit of the theory of emission. The molecules of light are supposed, in this hypothesis, to have a rotatory motion round their centres of gravity, which continues along with the progressive motion, and in virtue of which they present attracting and repelling poles alternately during their progress in space. $\dagger$ Boscovich imagined a vibratory motion in the parts of the ray itself, which it received at the moment of emission, and retained in its progress. $\neq$

The theory of the fits has now lost much of its credit, since the phenomena of the colours of thin plates-phenomena which first suggested it to the mind of Newton-have been shown to be irreconcilable with it. The explanation which it gives of the facts now under consideration is, as was observed by Young and Fresnel, inconsistent with the regularity of refraction. In fact, the molecules which are transmitted are not all in the maximum of the fit of transmission, but are supposed to reach the surface in very different phases of this, which may be denominated the positive fit. Now as a change of the fit from positive to negative is,

* Optics, Query 21.

† Phil. Trass. 1753. Traité de Physique, iv. p. 245.

† Philosophia Naturalis Theoria. 
in general, sufficient to overcome altogether the effect of the attractive force, and subject the molecule to the repulsive, it is obvious that the phase of the fit must modify the effects of these forces in every intermediate degree; and that the molecules which do obey the attractive force must have their velocities augmented in different degrees, depending on the phase. Consequently, as the direction of the refracted ray depends on its velocity, the transmitted beam will consist of rays refracted in widely different angles, and will be scattered and irregular.

In some of his writings, Newton attributes the reflexion and refraction of light to a difference in the density of the ether within and without bodies; or rather he refers the attractive and repulsive forces to this, as to a more general principle. The ether is supposed to be rarer within dense bodies than without; and the rays of light, in crossing the bounding surface, are pushed from the side of the denser ether, so that their motion is accelerated if they pass from the rarer to the denser body, and retarded in the opposite case. Reflexion at the surface of the rarer medium is explained on the same suppositions; but to account for the ordinary reflexion by a denser medium, Newton was obliged to introduce new and gratuitous hypotheses respecting the constitution of the ether at the confines of two media in which its density is different.*

The velocity of propagation, in the wave-theory of light, depends solely on the elasticity of the vibrating medium as compared with its density. If, then, a plane wave be incident obliquely on the bounding surface of two media, it is obvious that its several portions will reach that surface at different moments of time; and each of these portions will become the centre of two spherical waves, one of which will be propagated in the first medium with the original velocity, while the other will be propagated in the new medium, and with the velocity which belongs to it. But, by the principle of the coexistence of small motions, the agitation of any particle of either medium is the sum of the agitations sent there at the same instant from these several centres of disturbance; and the surfaces on which they are accumulated at any instant will be the reflected and refracted waves. These surfaces are those which touch all the small spherical waves at any instant. It is easy to see that they are both plane; and that the reflected

- Birch's IIistory of the Royal Society, vol. iii. p. 247.-Optics, Query 19. 
wave is inclined to the surface at the same angle as the incident wave, while the sine of the angle of inclination of the refracted wave is to that of the incident in the constant ratio of the velocities of propagation in the two media.

Such is the demonstration of the laws of reflexion and refraction given by Huygens.* The composition of the grand, or primary wave, by the union of the several secondary or partial waves, in this demonstration, has been denominated the principle of Huygens ; and it is obviously a case of the more general principle of the coexistence of small motions. It easily follows from this mode of composition, that the surface of the primary wave must mark the extreme limits to which the vibratory movement is propagated in any direction, in any given time; so that light, according to this theory, is propagated from any one point to another in the least possible time. This is the well-known law of Fermat, the law of swiftest propagation; and it will readily appear that it holds, whatever be the number of modifications which the course of the light may undergo by reflexion or refraction, as likewise, whatever be the form of the elementary wave.

The demonstration of Huygens has been thrown into an analytical form by Lagrange, + but he has added nothing to its rigour or perspicuity. An important supplement to the demonstration was, however, given by Fresnel. From the reasoning of Huygens it did not appear what became of those portions of the secondary waves which did not conspire in the formation of the grand wave. The crossing of these in all directions ought to give rise to a weak diffused light, filling the entire space between the grand wave and the reflecting or refracting surface; and, in fact, Huygens supposed that such a light did actually exist, but was too feeble to affect the eye. Fresnel has shown, however, that all those portions which do not conspire in the formation of the grand wave, are destroyed by interference; $\ddagger$ so that the formation of one grand wave, by the union of an indefinite number of lesser waves, becomes a precise and definite effect.

The total reflexion of light at the surface of a rarer medium has been urged by Newton against the wave-theory, and the

* Traité de la Lumière.

† " Sur la Théorie de la Lumière d'Huygens."-Annales de Chim., tom. xxi. tom. xxi. 
apparent difficulty seems to have had much weight in inducing him to reject that theory. It is, in fact, not easy to perceive at first view why the disturbance of the ether within the denser medium should not be communicated to the external ether, and a wave be thus propagated to the eye, whatever be the obliquity of the incident wave. To this it may be enough to reply, that the law of refraction itself, in all its generality, is a necessary consequence of the wave-theory; and therefore that the phenomenon of total reflexion, which is a particular case of that law, is likewise accounted for. But the principle of interference furnishes a direct answer to the difficulty. It can be shown that the elementary waves, which are propagated into the rarer medium from the several points of the bounding surface, destroy one another by interference, when the sine of the angle of incidence is greater than the ratio of the velocities of propagation in the two media, or the angle itself greater than the limiting angle of total reflexion.* It is here supposed that the distance from the refracting surface is a large multiple of the length of a wave. The conclusion does not apply to points very near that surface ; and for such points, there is reason to think, the law of refraction is more complicated. Experience shows, in fact, that light may issue from the denser medium, to an appreciable distance, when the incidence exceeds the limiting angle of total reflexion. If two prisms, whose bases are slightly convex, be put together, and the inclination of these bases gradually changed while we look through them, it will be observed that, beyond the limiting angle, the light will still be transmitted in the neighbourhood of the parts in contact. By measuring the breadth of this space, and comparing it to the diameters of the coloured rings, Fresnel found that the interval of the glasses, through which this deviation from the ordinary law of refraction occurred, exceeded the length of the wave. $t$ The analysis of M. Poisson points also to the same result, and it is proved that the second medium will be agitated in the part immediately in contact with the first, this agitation decreasing rapidly, and becoming insensible, at a very minute distance from the surface.

The laws of reflexion and refraction, then, follow from the theory of waves, whether we suppose the vibrating medium in

* See Fresnel, "Sur le Système des Vibrations lumineuses."-Bibliothèque Universelle, tom. xxii.

$\dagger$ Ibid. 
dense bodies to be the body itself, the ether within it, or both conjointly. Euler maintained the first of these opinions, and believed that light was propagated through the gross particles alone, in the same manner as sound. But this hypothesis is contradicted by the most obvious facts; and according to it, as Dr. Young has observed, the refraction of the rays of light in our atmosphere should be a million times greater than it is. Of the other two opinions, Young seems to have held the latter, and to have thought that the molecules of the body formed, together with those of the ether within it, a compound vibrating medium, which was denser than the ether alone, but not more elastic. Others, lastly, attribute the propagation of light in transparent bodies to the vibrations of the ether alone, that fluid being retained by the attraction of the body in a state of greater density within it than in free space.

A very different view of this subject has been recently maintained by Mr. Challis. Assuming that the density of the ether is the same in solid media as in free space-an assumption which he seems to think required by the phenomenon of aberration-this mathematician conceives that the reflexion of light, and its retardation in the denser medium, may be both accounted for by the reflexions which the ethereal waves undergo from the solid. particles of the medium which they encounter in their progress.* He shows, in fact, that the absolute velocities impressed upon the ethereal particles by such reflexion may be resolved into two parts, one of which is propagated uniformly, and is accompanied by a change of density ; while the other is propagated instantaneously, without change of density. $\dagger$ The former of these, he thinks, will account for the reflexion of light, the latter for the diminished velocity of transmission.; This ingenious theory has the advan-

* This manner of conceiving the reflexion of light, in the wave-theory, was that originally entertained by Fresnel, and was put forward in a memoir read to the French Academy in 1819.

† Phil. Mrag., New Series, vol. xi.

‡ The mean effect of these reflexions, Mr. Challis shows, is equivalent to that of a retarding force; and, by a certain supposition respecting its value, he has arrived at the following simple formula for the determination of the ratio of the volocities of propagation in free space and in the medium

$$
\mu^{2}-1=\mu \delta H
$$

in which $\delta$ denotes the density of the medium, and $H$ a constant proportional to he mean retarding effect of a given number of its molecules. For the gases, then, the 
tage of connecting the velocity of propagation in dense bodies directly with their constitution, and so of advancing a step in the process of physical induction. On the other hand, it requires us to admit that the particles of ether and those of gross bodies exert no mutual action of any kind. We know too little of the ether, or of its properties, to deny this, simply because it is unsupported by any of the properties of matter hitherto revealed; but it must at the same time be admitted that the violation of such analogies furnishes an argument of some weight against the theory which demands them.

Whatever supposition we may frame respecting the constitution of bodies, or of the ether within them, in the wave-theory, it must be such that the velocity of propagation is less in the denser medium. In the theory of emission, on the other hand, it is the reverse; so that although it conducts to the same result, it does so by an opposite route. Here, then, the rival theories are at issue upon a matter of fact; and we have only to ascertain this fact, in order to be able to decide between them. This seemed to be accomplished by the reasonings of Young. From the laws of interference it appears that homogeneous light, in its progress in space, passes through certain periodically returning states, the intervals of which are constant in the same medium; while in different media they are proportional to the velocities of propagation, since the number of such intervals in a given quantity of light cannot be supposed to vary. Now, it followed from the experiments of Newton, that the intervals, by which he explained the phenomena of thin plates, were diminished in the denser medium; and as these intervals have been shown by Young to be identical with those deduced from the law of interference, it followed that the velocity of light was slower in the denser medium." Newton had even found the ratio of the magnitudes of the intervals to be the same with that of the sines of incidence and refraction; and this is precisely as it should be on the principles of the wave-theory.

But the retardation of light in the denser medium has beeu

quantity $\frac{\mu^{2}-1}{\delta}$ is nearly constant, whatever be the compression. This result is a very simple consequence of the theory of emission; its experimental truth has been established by MM. Biot and Arago.-Phil. Mag., New Series, vol. vii.

" "Experiments and Calculations relative to Physical Optics."-Phil. Trans., 1803. 
directly established by M. Arago. If two pencils be made to interfere and produce fringes, as in the experiment of Fresnel, and if a thin plate of a denser medium be interposed in the path of one of them, the whole system of fringes will be shifted to one side or the other, according as the light has been accelerated or retarded within the plate. The result of this important and decisive experiment was in favour of the theory of waves.*

The refractive index being equal to the ratio of the velocities of light in the two media, direct or inverse, it follows, whichever theory we adopt, that any change in the velocity of the incident ray must cause a variation in the amount of refraction, unless the velocity of the refracted ray be altered proportionally. Now, the relative velocity of the light of a star is altered by the earth's motion; and the amount of the change is obviously the resolved part of the earth's velocity in the direction of the star. It was therefore a matter of much interest to determine how, and in what degree, this change affected the refraction. By the observation of this effect, it was hoped, we should have an easy and accurate method of determining the constant of aberration; we should be enabled to compare the light of different stars, and detect any difference which might exist in their velocities; and, lastly, we might compare these velocities with that of light emanating from other sources. The experiment was undertaken by M. Arago, at the request of Laplace. $\dagger$ An achromatic prism was attached in front of the object-glass of the telescope of a repeating circle, so as to cover only a portion of the lens. The star being then observed directly through the uncovered part of the lens, and afterwards in the direction in which its light was deviated by the prism, the difference of the angles read off gave the deviation. The stars selected for observation were those in the ecliptic, which passed the meridian nearly at 6 A.M. and 6 P.M., the velocity of the earth being added to that of the star in the former case, and subtracted from it in the latter. No difference whatever was observed in the deviations; and the result was the same whatever vas the origin

* Annales de Chimie, tom. i. See also the account of Mr. Potter's repetition of this experiment.-Phil. Mag., vol. iii. p. 333.

t The idea of detecting a difference in the velocity of the light of the fixed stars, by its effect upon the amount of refraction, seems to have first occurred to Mr. Michell. Such a difference of velocity, he conceived, must necessarily arise from the different attractions of the stars upor the emitted molecules, and he has computed the diminution of the original velocity of emission arising from this cause.-Phil.Trans., 1784. 
of the light.* Fraunhofer has likewise compared the light of several of the fixed stars with respect to its refrangibility. No difference whatever was observed, although the method employed was adequate to the detection of a difference so small as the 10,000 th part of the whole refraction nearly. $\dagger$

This remarkable and unexpected result can be reconciled to the theory of emission, $\ddagger$ as M. Arago has observed, only by the hypothesis already adverted to-namely, that the molecules are emitted from the luminous body with various velocities; but that among these velocities there is but one which is adapted to our organs of vision, and which produces the sensation of light. The wave-theory has been more successful in its explanation. If the ether which encompasses our globe were like its atmosphere, and partook of its motion, the refraction would be precisely the same as if the whole were at rest. This, however, we have seen, cannot be the case; and the phenomena of aberration compel us to admit that the ethereal medium which encompasses the earth is not displaced by its motion. This being assumed, it follows that the ether which is carried along by the refracting medium, is that which constitutes the excess of its density above that of the surrounding ether. On this supposition Fresnel has calculated the length of a wave in the moving medium, and thence also the actual change in the direction of the refracted ray produced by the earth's motion.\$ This change is found to be opposite, and exactly equal to that produced by the same cause in the apparent direction of the ray; so that the ray is actually seen in the same direction as if the earth were at rest, and the apparent refraction is unaltered by the earth's motion. These results, it may be observed, are precisely the same for terrestrial objects, the velocity of wave-propagation being independent of the motion of the luminous body.

* Biot, Astronomie Physique, vol. iii.

+ Edinb. Journ. of Science, viii. p. 7.

¥ M. Prevost has endeavoured to reconcile the experimental result of M. Arago with the ordinary suppositions of the theory of emission, and to show that a change in the relative velocity in the light of the stars, caused by the motion of the refracting plane, does not affect the refraction in the same manner as an equal change in the absolute velocity. "De l'Effet du Mouvement d'un plan refringent sur la Refraction."Geneva Memoirs, vol. i. His reasonings do not appear to be conclusive.

$\S$ The sine of the change is to the sine of the total deviation of the ray in the ratio of the velocity of the earth to that of light. Fresnel's result is much more complicated, but it will be easily seen to reduce itself to this. "Sur l'Influence du Mourement terrestre dans quelques Phénomènes d'Optique." - Annales de Chimie, tom. ix. 
Newton thought that the different refrangibility of the rays of light could be explained by supposing simply that they were bodies of different sizes, the red being greatest and the violet least. It is obvious, however, that this supposition can have no reference to the simple projectile hypothesis. held by his followers, or to the demonstration of the law of refraction given in the Principia. It is connected with that more complex theory, in which the molecules of light are supposed to excite the vibrations of the ether in the bodies which they meet.

M. de Courtivron and Mr. Melville proposed to account for the dispersion of light by a difference in the initial velocity of the molecules, the red being swiftest and the violet slowest. But were such the cause of the phenomenon, the dispersion should be proportionate to the mean refraction. Indeed, the hypothesis was abandoned almost as soon as proposed. Its authors had foreseen the consequence that, in the eclipses of Jupiter's satellites, the colour of the light should vary just before immersion, and after emersion; and the existence of such an effect, in the degree indicated by theory, ${ }^{*}$ was completely disproved by the observations of Mr. Short. + Another consequence of such a difference in the initial velocities of the light of different colours is, that the aberration of the fixed stars should also vary with the nature of the light, and each star appear as a coloured spectrum, whose length is parallel to the direction of the earth's motion.

According to the modern advocates of the theory of emission, the molecules of light are heterogeneous; and the attractions exerted on them by bodies vary with their nature, and are, in this respect, analogous to chemical affinities. This supposition, however, as Dr. Young has justly observed, is but veiling our inability to assign a mechanical cause for the phenomenon.

It is remarkable that Newton himself was the first to suggest that part of the wave-theory, in which the colour of the light is supposed to be determined by the frequency of the ethereal vibra-

* The duration of this change, according to Mr. Melville, should amount to thirtytwo seconds, the velocity of the light of different colours being inversely as their rcfractive indices.-(Phil. Trans., 1753.) This principle, however, as M. Clairaut has shown (Phil. Trans., 1754), is obviously incorrect. It will easily appear that the initial velocities must vary inversely as the quantity $\sqrt{\mu^{2}-1}$, in order to account for dispersion; and that the duration of the expected phenomenon must bo even greater than that assigned by Mr. Melville.

† Phil. Trans., 1753. 
tions, or by the length of the wave;* and the addition has been received by all its supporters. But observation proves that the refractive index, or the ratio of the velocities of propagation, in the two media, is different for the light of different colours. The advocates of the wave-theory, therefore, are forced to conclude that the velocity of propagation in refracting media varics with the length of the wave. Here, then, we encounter a difficulty in this theory, which has been regarded as the most formidable obstacle to its reception. Theory indicates that the velocity of wave-propagation is constant in the same medium, depending solely on the elasticity of the medium as compared with its density. That velocity, therefore, should be the same for light of all colours, as it is found to be for sound of all notes.

Various attempts have been made to solve this difficulty. $\dagger$ Euler thought that the successive waves underwent an increase of velocity arising from their mutual action; and this increase he supposed to vary with their length, the waves of greatest length undergoing the least augmentation of velocity, and being therefore most refracted. $\ddagger$ But the phenomena of coloured rings, as Euler perceived, compel us, on the contrary, to suppose that the lengths of the waves diminish as the refrangibility increases; and he seems himself to have abandoned his first conjecture.

Dr. Young accounted for dispersion by the supposition that the solid particles of the refracting substance vibrate, as well as the particles of the ether within it; and that the former vibrations affect the latter, and affect them differently according to their frequency. Mr. Challis has adopted and developed this hypothesis. According to this author, it has been already observed, the diminished velocity of transmission in the denser medium may be explained by the obstacle which the solid particles of the medium offer to the free movement of the ethereal particles. If the former be supposed to be immovable, the ratio of the velocities of propagation, in free space and in the medium,

* Phil. Trans., 1672.

+ It is scarcely necessary to advert here to the law proposed by M. Rudberg, to connect the lengths of an undulation, or the velocities of propagation, in different media; for this law is purely hypothetical, and its apparent eonsistency with observation has arisen solely from the adaptation of the arbitrary constants which enter the expression.-Annales de Chimie, tom. xxxvi., xxxvii.

$\ddagger$ Opuscula varii Argumenti, tom. i. p. 217. 
will be a simple function of the density of the latter, and in a given medium its value will be constant; but when the particles of the medium vibrate, the value of this ratio will depend also on the length of the wave, and will therefore vary with the colour of the light.*

The solution suggested by Professor Airy is more closely connected with received principles. It is now generally admitted that part of the velocity of sound depends on a change of elasticity which the air undergoes during its vibrations, in consequence of the development of latent heat by compression. If this heat required time for its development, the quantity developed, and therefore the elastic force, must vary with the time of vibration. Consequently, the velocity of propagation should also vary with the time, and be different for waves of different lengths. Professor Airy imagines something similar to this in the case of light; and conceives that the elasticity of the ether, in refracting media, may consequently undergo a change whose amount depends on the time of vibration.

But the explanation offered by Fresnel seems to be the simplest and most natural. The conclusion of analysis-that the velocity of wave-propagation is constant in the same homogeneous medium-is deduced on the particular supposition that the sphere of action of the molecules of the medium is indefinitely small compared with the length of a wave. If this restriction be removed, we have no longer any ground for concluding that waves of different lengths will be propagated with the same velocity. Fresnel states that he has demonstrated that, when the mutual action of the ethereal molecules extends to a sensible distance as compared with the length of a wave, the waves of different lengths will be propagated with different velocities; the elasticity of the medium, and therefore also the relocity, increasing with the length of the wave. $\dagger$ Here, then, the constancy of the velocity of wavepropagation is regarded but as the approximate result of an incomplete analysis. The problem presented itself to M. Cauchy in a similar point of view. In the profound researches of this

* "An attempt to explain theoretically the different Refrangibility of the Rays of Light, according to the hypothesis of Undulations."-Phil. Mag., New Series, vol. viii.

+ This demonstration is more than once referred to by the author, as contained in a note appended to his memoir on double refraction. The note, however, probably by some oversight, hes never been printed. 
mathematician relating to light, the ether is considered as a system of particles solicited by mutual attractions or repulsions; and from the partial differential equations which represent their movement, he had deduced the laws of propagation in crystallized as well as in homogeneous media. These equations, however, were but approximate, and derived from others of greater generality by the omission of the terms containing the higher powers of the displacements, and of their derivatives with respect to the coordinates. Resuming the problem of the propagation of a plane wave, with the aid of the more general equations, he has finally demonstrated the existence of a relation between the velocity of propagation and the length of the wave.*

The opacity of bodies is ascribed by Newton to the discontinuity of their parts, and to the multitude of internal reflexions which the rays of light undergo within them. + We have many reasons for believing this to be the case; but as yet we are far from a complete account of the phenomenon. If the reflexions and refractions, which thus arise at each new bounding surface, be similar to those which take place at the outer surfaces of bodies, the molecules of light will indeed be scattered in every direction, but they should undergo no diminution of velocity. How, then, is it that they do not emerge finally from the body as readily as they entered it, and thus render it visible in all directions, not by a superficial reflexion, but by a secondary emission? To account for the extinction of light, in the theory of emission, we must suppose it united to the body which it enters; and the simplest mode in which we can conceive this union to be brought about is by the direct impact of the molecules of light on those of bodies, whereby they are brought within the sphere of those interior attractive forces to which chemical combinations are referred. This appears to have been the opinion of Newton. "Are not gross bodies and light," says he, "convertible into one another, and may not bodies receive much of their activity from the particles of light which enter their composition? For all fixed bodies being heated emit light, so long as they continue sufficiently hot, and light

* Mémoire sur la Dispersion de la Lumière. - The attention of the Mathematical Section of the British Association was drawn to this theory by Professor Powell, at the last meeting, chiefly in reference to a limitation which seemed to be required in the physical hypothesis.-See Report of Proceedings.

+ Optics, book 2, part 3 . 
mutually stops in bodies as often as its rays strike upon their parts."*

When from the simple fact of absorption we proceed to consider its law, as depending on the nature of the light, the difficulties increase at every step. The intensity of the transmitted light, considered as a function of its refrangibility, appears to be subject to no law, or to a law so complicated as completely to baffle all attempts to embrace it in an empirical rule. The maxima and minima are often actually numberless; and the variable does not reach them gradually, but by what seems to be an abrupt violation of the law of continuity. These apparently capricious changes were observed long since by Dr. Young, in the light transmitted through the common smalt-blue glass. Sir David Brewster has recently directed his attention to the same subject, and examined a great number of coloured bodies with reference to their absorptive properties. He has found, in particular, that a very remarkable definite action is exercised upon the rays of the spectrum by the green liquids obtained by extracting the colouring-matter of the leaves of plants in alcohol; and this action does not cease altogether even when the liquid has become perfectly colourless.t But the absorbing properties of !nitrous-acid gas, observed by the same author, are by far the most remarkable ever noticed. When the light transmitted through this gas is analyzed by a prism, it is found that about 2000 portions of the beam are stopped, and 2000 dark spaces, or abrupt deficiencies of light, appear in the spectrum. These increase in number and magnitude with the temperature of the gas, until, by a sufficient elevation of temperature, this rare body becomes perfectly opaque, and refuses to transmit a single ray of the brightest sunshine. $\neq$ Professor Miller and Professor Daniell have found some analogous properties in other gases. In the spectrum produced by the light transmitted through the vapours of bromine and iodine, more than one hundred dark lines are visible, disposed at equal distances. $\S$

To account for the selection of certain classes of rays by

- Optics. Query 30.

$\dagger$ "On the Colours of Natural Bodies."-Edin. Trass., vol. xii.

+ "On the Lines of the Solar Spectrum," \&c.-Edin. Trans., vol. xii.

\$ French translation of Herschel's Essay on Light, Supplement, p. 455. 
coloured media, in the theory of emission, it seems necessary to suppose that an attractive force is exerted at a distance between the molecules of the body and those of light, and that the absolute value of this force varies with the colour. It does not seem easy to reconcile these suppositions to the Newtonian account of refraction; and the difficulty is still further increased when we proceed to apply the same considerations to the absorption of definite rays, and introduce the hypothesis of specific actions, varying in the most abrupt and irregular manner with the refrangibility of the ray.*

The absorption of light, and the opacity of bodies, were long since urged by Halley as difficulties in the wave-theory. The ether is supposed to penetrate all bodies freely, and why not also the undulatory motion in which light consists? To this difficulty we find a full and complete solution in the principle of interference. When a wave enters a discontinuous substance, it will be broken up, and its parts undergo continued subdivision by internal reflexions; so that when these parts reach the second surface of the body, they are found in every possible phase, and must destroy one another by interference. The phenomenon, as has been observed by Sir John Herschel, is analogous to the impeded propagation of sound in a mixture of gases differing much in elasticity as compared with their density.

The same writer has given an ingenious and natural account of the absorption of specific rays on the principles of the wavetheory, in a paper read before the Association last year. $\dagger$ He considers the molecules of the body and those of the ether as forming, conjointly, compound vibrating systems, which are more disposed to transmit vibrations of some determinate period than others. Other vibrations, however, not in unison with these systems, may be propagated through them. These forccd vibrations, as he calls them, will be obstructed in their progress, and their amplitudes diminished by the mutual influence of the motions of the parts of the systems; and he shows that it is possible to conceive systems, which will be wholly impervious to a vibration of a particular period, while they freely transmit others not differing from

* See Sir David Brewster's Report on Optics.

+ "On the Absorption of Light by coloured Media, viewed in connexion with tho Undulatory Theory." - Phil. Mag., Third Series, vol. iii. 
them materially in their frequency.* But these important and interesting speculations, it must be remembered, are advanced by their author solely with the view of removing an imagined inconsistency between the phenomena of absorption and the mechanical laws of vibratory movement. We are still far from a precise theory of absorption. When such a theory shall have been established, there seems reason to believe that it will bring with it also an insight into the internal constitution of bodies even yet more close than that afforded by the affections of polarized light; and that the laws of molecular action may perhaps, at some future day, be studied in the phenomena of transmitted light.

The properties of solar phosphori, which attracted so much of the attention of experimental philosophers of the last century, seem at first view to favour the account of absorption suggested by the theory of emission, and to arise from the disengagement of the light which had become united to the body. Canton observed that light may remain in these bodies, as it were in a latent state, for several months, until its re-emission is determined by the action of heat. But it must be observed, in the first place, that the feeble light emitted from the phosphori bears a very small proportion to that which they are supposed to receive by absorption. Dessaignes has remarked that most of these substances emit the same kind of light, whatever be the species of light to which they have been exposed. $\dagger$ The same fact has been observed by M. Grotthouss; and other subsequent inquirers; and in some of the diamonds possessing the property of phosphorescence, the most efficacious exciting light is of a different colour from that excited. These facts seem to be inexplicable in the theory of emission. In

* An interesting interference experiment, similar in some respects to that indicated by $\mathrm{Sir}$ Jobn Herschel in this paper, has been recently made by Mr. Kane. A compound tube, whose branches of 9 and $13 \frac{1}{2}$ inches united at the two extremities, was made to sound by the languette of an organ pipe. Each of the tubes, separately, gave its own fundamental note, and all its harmonics; and when a free communication was opened between them, the system gave all the notes of the two series, with the exception of those whose waves were in complete discordance. Thus, the fundamental note of the short tube was stopped altogether, while its octave was given with remarkable clearness; the two waves being in complete discordance in the former case, and in complete accordance in the latter.

† Mém. Inst., tom. xi.

¥ Schweigger's Journal, 1815. - The same observer discovered the curious fact, that the electric current restored the property of phosphorescence, in many cases

- where it appcared to have been destroyed by the action of violent heat. 
the wave-theory, on the other hand, the phenomenon is easily comprehended. As the vibrations of the air excite those of sounding bodies, and communicate to them a motion which continues for some time after the exciting cause has ceased to act, so it must also be with the undulations of the ether. When the body is in unison with the incident light, their vibrations will continue isochronous, and the undulations of the ether excited by the body will be of the same length as those by which it is itself excited. In the other case, the period of vibration, and consequently the length of the wave, will be altered, and the excited and exciting lights will be of different colours. The fact observed by Canton is indeed not so easily explained. Young supposed that the vibrations of the body may be abruptly suspended by cold, and may proceed anew when released from this restraint, like a string which has been stopped and detained in any part of its vibration on either side of the centre.

The fixed lines in the solar spectrum first noticed by Wollaston, and afterwards more minutely traced by Fraunhofer, have lately been examined with great care, and with his usual success, by Sir David Brewster; and he has observed a remarkable coincidence between these lines and the dark bands of the spectrum of the nitrous-acid gas.* Sir David Brewster has also studied, in connexion with the same subject, the definite absorbing effects of the earth's atmosphere. This has been effected by examining the solar spectrum, when the sun was near the horizon; and it has been found that most of the dark bands thus developed belonged to the fixed lines of Fraunhofer, which were thus, as it were, widened and brought out by the absorptive action of the atmosphere. A similar result has been arrived at in other cases, and it has been found that the points of the spectrum on which absorbing bodies exert the strongest specifio actions are generally coincident with the deficient rays of solar light. $\dagger$ This singular connexion gives considerable weight to the speculations of Sir David Brewster respecting the latter phenomena. $\neq$

The observation of the fixed lines in the solar spectrum led Fraunhofer to examine the optical characters of the lights ema-

* "On the Lines of the Solar Spectrum."-Edin. Trans., vol. xii.

+ "On the Colours of Natural Bodies."-Edin. Trans., vol. xii.

$\ddagger$ Report on Optics. 
nating from other sources. He thus arrived at the interesting discovery, that the system of bands in the different species of light which he examined varied with the source; while it was constantly the same in the number of the bands, and their relation to the coloured spaces, in the light of the same source however modified. In the light of Sirius there are three broad bands which have no resemblance to those of solar light. The light of the electric spark, on the other hand, when analyzed by the prism, is found to have several bright lines, of which that in the green is remarkably brilliant. Similar phenomena were observed in the light of artificial flames-the flame of an oil lamp, for example, exhibiting a well-defined bright band between the red and yellow, and another not so distinct in the green.* This, however, is not universally the case. In the red flame of strontia, as was observed by Dr. Faraday and Mr. Talbot, there are a number of red rays separated from each other by dark bands; and in the flame of cyanogen, when similarly analyzed, the violet is found to be divided into three distinct portions, with broad dark intervals. $\dagger$

It is easy to account for the general fact of the deficiency of certain classes of rays in certain lights. When a body violently heated begins to shine, the phenomenon is simply accounted for, in the wave-theory, by an increase in the frequency of its vibrations. In the same manner it seems natural to suppose, generally, that the mechanical agencies at work during combustion accelerate or retard, in various ways, the rate of vibration, and so alter the character of the emitted lights. The light emitted in weak or incipient combustion is generally blue. Sir John Herschel observed that when sulphur burns with a feeble flame, its light contains all the rays of the spectrum, and particularly the blue and violet; while, in vivid combustion, these disappear entirely, and the light is a yellow of almost perfect homogeneity. $\neq$ The various shades of colour in the flame of a common candle-from the deep blue of the lower part, which is found by prismatic analysis to consist of five distinct portions, to the yellowish white in the centre, and thence to the dusky red at the apex of the flameseem to be referrible to the same principle. Fraunhofer and Sir

* Munich Memoirs.

+ Phil. Mag., Third Series, vol. iv. p. 114.

‡"On Absorption of Light in coloured Media."-Edin. Trans., vol. ix. 
David Brewster have both remarked that the flame of oil, urged by the blowpipe, consists chiefly or wholly of yellow rays. The same fact was long since observed by Mr. Melville with respect to the flame of alcohol into which nitre, muriate of soda, and other salts had been introduced;* and Sir David Brewster has found that the quantity of yellow light given out by burning bodies increases with their humidity, the flame of alcohol diluted with water being nearly a homogeneous yellow. $\dagger$ It is more important to remark, however, in illustration of the undulatory view of the phenomenon of emission, that the colour of flames is often found to depend on the presence of something which is itself unaltered in the process of combustion. Thus Mr. Talbot has remarked, that when a small quantity of muriate of lime was placed on the wick of a spirit lamp, it gave out red and green rays during an entire evening, though the salt was not sensibly diminished.+ The absence of definite rays in certain lights, and the fixed lines of the solar spectrum, have been referred by Sir John Herschel to the same principle by which he has explained the absorption of specific rays. $\S$

In what has preceded we have assumed the truth of the received theory with respect to the composition of solar light, and the connexion between the colour of a ray and its refrangibility. This theory, however, has been recently opposed by Sir David Brewster. According to this philosopher, white light consists of but three simple colours-red, yellow, and blue; and the solar spectrum is composed of three overlapping spectra of these colours, the intensity of each of which is greatest at the point where that colour is strongest in the compound spectrum. According to this view, then, all the colours in the solar spectrum are compound, and consist of red, yellow, and blue light, in different proportions. These compound colours cannot be analyzed by the prism, inasmuch as the rays of which they consist at any point of the spectrum have the same refrangibility; and it is only by the different action of absorbing media on their constituent elements that their compound nature can be detected. Each of them may be con-

* Edinb. Essays.

+ On a Monochromatic Lamp, Ihid.

$\ddagger$ Edinb. Journ. of Science, v. 77.

$\S$ Phil. Mag., Third Series, vol. iii. p. 407. 
ceived to consist of a certain quantity of white light, and of an excess of the light of two of the simple colours; and if this excess be absorbed, a white light will be the result, which will be indecomposable by the prism. This result of his hypothesis has been experimentally confirmed by Sir David Brewster.*

These views, if finally established, sever the connexion between the colour of a ray and its refrangibility, laid down by Newton; and the former must be supposed to depend-not on the length of the wave-but on some other element of the vibratory movement.

\section{Diffraction.}

It has been already stated that Newton considered the undulations of an ethereal medium to be a necessary part of his theory, and that that theory, as maintained by its author, differed from the theory of Huygens and of Hooke only by the addition of a new hypothesis. The necessity of something extraneous to the undulations of the ether seems to have been admitted by Newton, mainly to account for the right-lined propagation of the rays of light; and a careful consideration of his optical writings leaves the impression, that had the wave-theory alone appeared to explain this fact, Newton would not have hesitated to embrace it. This explanation has been spoken of in another place, and it has been shown to follow from that theory, that the light which encounters an obstacle must diminish rapidly in intensity within the edge of the geometric shadow. It now remains to consider the other phenomena which arise under these circumstances; and it will be found that the same theory affords the most complete account, not only of their general characters, but even of their numerical details.

In order to understand the theory of shadous, it is necessary to investigate their laws in the simple ease in which the magnitude of the luminous body is reduced to a point. The effects thus presented were first observed by Grimaldi, and they have been since studied as a separate branch of optical science, under the title of diffraction or inflexion. Grimaldi found that when a small opaque body was placed in the cone of light, admitted into a dark cham-

* " On a New Analysis of Solar Light."-Edin. Trans., 1831. 
ber through a very small aperture, its shadow was much larger than its geometric projection, so that the light suffered some deviation from its rectilinear course in passing by the edge. Observing these shadows more attentively, he found that they were bordered with three iris-coloured fringes, which decreased in breadth and intensity in the order of their distances from the edge of the shadow, preserving the same distance from the edge throughout its entire extent, unless where the body terminated in a sharp angle. Similar fringes were observed under favourable circumstances within the shadows of narrow bodies.*

The phenomena of diffraction were subsequently examined by Hooke, and by Newton. The first observations of Newton were but repetitions of those of Grimaldi ; and it is remarkable that he altogether overlooked the important phenomenon of the interior firinges noticed by the Italian philosopher. But to Newton we owe the analysis of the phenomena, so far as they depended on the nature of the light. When the different species of simple light, into which the sun's rays were divided by a prism, were cast in succession on the diffracting body, Newton observed that the fringes formed were broadest in the red light, narrowest in the violet, and of intermediate magnitude in the light of mean refrangibility, so that the iris-coloured fringes which are formed in white light are but the fringes of different colours superposed. But the observations of Newton most closely connected with his physical theory are those in which the light is made to pass between two near knife-edges, whether parallel or inclined. From these observations Newton concluded that the light of the first fringe passed by the edge, at a distance greater than the 800th of an inch, that of the second and third fringes passing at still greater distances. These distances, however, were not the same wherever the fringes were formed; and it appeared to follow from the experiments that the light of the same fringe was not the same light at all distances, but that each fringe was, as it were, a caustio formed by the intersection of the rays passing at different distances from the edge; the portion of the fringe near the knives being formed of light which passed nearest to the edge and was most bent. $\dagger$

To account for these phenomena, Newton supposed the rays of

* Physico-Mathesis de Lumine, Bologna, 1665.

+ Opties, Book iii. 
light to be inflected in passing by the edges of bodies, by the operation of the attractive and repulsive forces which the molecules of bodies were conceived to exert on those of light at sensible distances. Thus, the rays passing by the edges of a narrow opaque body are supposed to be turned aside by its repulsion; and as this force decreases rapidly as the distance increases, the rays which pass at a distance from the body will be less deflected than those which pass close to it. The caustic formed by the intersection of these deflected rays will be concave inwards; and as none of the rays pass within it, it will form the boundary of the visible shadow. To explain the alternations of darkness and light beyond this, Newton appears to have supposed that the attractive and repulsive forces succeed one another for some alternations; and that the molecules composing each ray, in their passage by the body, are bent to and fro by these forces, "with a motion like that of an eel," and are finally thrown off at one or other of the points of contrary flexure. The separation of white light into its elements is explained, by supposing that the rays which differ in refrangibility differ also in inflexibility, the body acting alike upon the less refrangible rays at a greater distance, and upon the more refrangible at a less distance.* In one of his letters to Oldenburgh, $\uparrow$ Newton advances a more refined theory of diffraction. The bending of the ray near the edge of the obstacle he conceived to arise from a variation in the density of the ether in the neighbourhood of the body; and, following the analogy of thin plates, he endeavoured to account for the coloured fringes by the vibrations of the ether, which are propagated faster than the rays themselves, and overtake them at the middle of the curved portion of the trajectory they describe.

It is needless to comment upon the vagueness of these explanations. Newton himself was dissatisfied with them, and the subject fell from his hands unfinished. Still, however, the mere guesses of such a mind as that of Newton must possess a high interest, and we are not to wonder that among his followers more weight should be attached to these explanations than he himself ever gave them. It seems necessary therefore to advert to some of the circumstances of these phenomena, which are not only

* Optics, Book iii., Queries 1, 2, 3, 4 .

+ December 21, 1675. - Birch's History of the Royal Society, vol. iii. 
unexplained by this theory, but which seem moreover irreconcileably at variance with it.

If the phenomena of inflexion be the effects of attractive and repulsive forces emanating from the interposed body, and if these forees are the same with, or even analogous to, those to which the reflexion and refraction of light are ascribed in the theory of emission, it will follow that they must exist in different bodies in very different degrees; so that the amount of bending of the rays, and therefore the position of the diffracted fringes, should vary with the mass, the nature, and the form of the inflecting body. Now it is clearly ascertained, on the contrary, that all bodies, whatever be their nature or the form of their edge, produce under the same circumstances fringes identically the same; and in fact the partial interception of light, caused by the interposition of an obstacle of any kind, seems to be the only condition on which the character of the phenomenon depends. Gravesende seems to have first observed that the nature or density of the body had no effect upon the magnitude of the diffracted fringes; and the fact has since been confirmed in the fullest manner by almost every inquirer in this branch of experimental science. One of the ablest supporters of the theory of emission has admitted that the inflecting forces, if such exist, must be independent of the chemical nature of the inflecting body, and altogether different in their nature from those to which, in the same theory, the phenomena of reflexion and refraction are ascribed.* To ascertain whether the form of the edge had any effect upon the fringes, Fresnel took two plates of steel, the edge of each of which was rounded in one half of its length and sharp in the remaining half, and placed the rounded portion of one edge opposite the angular part of the other, and vice versâ. If, then, the position of the fringes depended on the form of the surface, the effect would thus be doubled, and the fringes appear broken in the middle. They were found, on the contrary, to be perfectly straight throughout their entire length. +

* Biot, Précis élémentaire, vol. ii. p. 473, 3me Edit.

+ Mémoire sur la Diffraction, p. 370. The Bullctin Universel for February, 1828, contains some animadversions on this part of Fresnel's optical labours, in a paper signed by the secretary of the Academ 5 of Sciences of St. Petersburgh, and purporting to be an official reply to some remarks in a former number of the Bulletin on the programme of the prize questions proposed by the Academy. The writers hare con- 
Again, the inflecting forces-though they must be supposed to vary in intensity, with the form and mass of the body, and with the distance of the luminous molecule from the edge-cannot be conceived to depend in any way upon the distance previously traversed by the molecule, before it arrives in the neighbourhood of that edge; so that the magnitude and position of the fringes, in this hypothesis, cannot vary in any way with the distance of the inflecting edge from the luminous point. But this conclusion is the reverse of fact: the fringes dilate in breadth, and their mutual inclination is increased, as the screen approaches the luminous origin. There seems to be but one way of a voiding the inference drawn from this fact against the theory of emission. It may be supposed that the bands have their origin at some sensible distance from the edge of the body, and thus that the obliquity of the incident ray varies as the edge approaches the luminous point. Such was the conjecture of Du Tour, who noticed the fact. Fresnel calculated the breadth of the fringes according to this supposition, and found that the computed and experimental results do not agree.* But, in point of fact, the bands may be supposed without sensible error to have their origin at the edge itself. Fresnel found by direct measurement that the distance of the third band from the edge of the shadow, at its origin, was less than the 100 th part of a millimetre.

The objections just considered seem to apply equally to the hypothesis of Mairan and Du Tour, in which the phenomena of diffraction are referred to the reflexions and refractions of an atmosphere supposed to encompass all bodies. For if such an atmosphere be retained around the body by its attraction,- -and this seems to be the only mode of accounting for its presence,-its density and its form must vary with those of the body itself, and consequently its effects upon the rays of light must vary also. But the experiments of $\mathrm{M}$. Haldat seem to leave no tenable ground for these hypotheses. Every agent has been tried which

founded two experiments of Fresnel which were instituted with different views, and differently reasoned upon. Fresnel's object, in this experiment, was simply to show that the form of the edge produced no effect upon the fringes, as it ought to do if
diffraction arose from attractive or repulsive forces extending to sensible distances
from bodies. Most of the from bodies. Most of the objections urged in the same paper against the wave-theory
arise, in like manner, in misconception.

" "Mémoire sur la Diffraction de la Lumière."-Mém. de l"Institut, tom. v. p. 353. 
could be conceived capable of modifying the attractive force of the body, or the density of the imagined atmosphere, and without effect. The metallio wires and plates which produced the fringes were heated to redness, and cooled down below the freezing-point; they were traversed by voltaio currents, and the charges of powerful batteries transmitted through them; but in whatever manner the condition of the diffracting body was varied, no change whatever was perceived either in the intensity or dimensions of the diffracted fringes.*

Although the phenomena of diffraction were studied by many diligent observerst after the publication of the Optics, no material accession was made to the knowledge of their laws until the principles of the wave-theory were applied to their explanation by Young. The exterior fringes, formed without the shadows of bodies, were ascribed by Young to the interference of two portions of light, one of which passed by the body, and was more or less inflected, while the other was obliquely reflected from its edge, the latter losing half an undulation at the instant of reflexion.+ The fringes formed by narrow apertures were, in like manner, supposed to arise from the interference of the two pencils reflected from the opposite edges; while the interior fringes, within the shadows of narrow bodies, were accounted for by the interference of the pencils which passed on either side of the body at an insensible distance, and were inflected into the shadow. The observed facts closely correspond with the calculated results of this theory ; and in the case last mentioned Young proved that the phenomena admitted of no other explanation. Placing a small opaque sereen on either side of the diffracting body, so as to intercept the portion of light which passed by one of its edges, the bands immediately disappeared, although the light passing by the other edge was unmodified. The same effect was produced, and by the same means, upon the crested fringes of Grimaldi, formed within the shadows of bodies having a rectangular termination.§ Thus the phenomena

* "Sur les Causes de la Diffraction," Annales de Chimie, tom. xli. Similar experiments had been made some time before by Mayer, and with the same result. Güttingen Memoirs, vol. iv.

† Maraldi (Mém. Acad. Par. 1723), Mairan (Ibid. 1738), Du Tour (Mémoires présentes, tom. v.), Mr. Brougham (Phil. Trans. 1796-7), and Mr. Jordan (Now Obsereations coneerning the Inflexion of Light. London, 1795).

+ "On the Theory of Light and Colours." - Phil. Trans., 1802.

$\S$ “ Experiments and Calculations relative to I'hysical Optics."-Phil. Trans. 1804. 
of the fringes, or the alternations of light and darkness, were shown to be cases of the more general principle of interference; and the connexion is now admitted by some of the warmest advocates of the Newtonian theory.* The bending of the light into the shadow, or the fact of inflexion itself, was at first ascribed by Young to the refraction of an ethereal atmosphere encompassing bodies, and decreasing in density with the distance. He afterwards, however, adopted the simpler doctrine of Huygens and Grimaldi, and referred the phenomenon to the fundamental property of waves.

But perhaps the most important of the labours of Young on this subject is that in which he descends into numerical details, and, taking the observations of Newton, as well as his own, calculates the differences of the lengths of the paths traversed by the two pencils, when they destroy or reinforce one another by interference. These intervals he found to constitute an arithmetical progression for the successive bands, the first term of which was the same in the same species of light, whatever be the distance at which the fringes are received, or the other conditions of the experiment. And, finally, comparing these constants with the similar intervals of the two pencils reflected by the surfaces of a thin plate, as deduced from the experiments of Newton, he found that their difference was within the limits of error to which such observations are liable, and that we are warranted in concluding that the two classes of phenomena are to be referred to one simple principle.t It is true that, in these calculations, Young starts from an erroneous principle respecting the lights which form the diffracted fringes by their interference, and he has remarked some discordances in his results which have, no doubt, their origin in that circumstance; but the results of the exact theory are not greatly different from that which he adopted, and the more complete analysis of Fresnel has only tended to confirm the conclusion obtained by Young.

The important experiment of Young, on the disappearance of the fringes in the shadow of a narrow opaque body, when the light passing by one of its edges was intercepted, was that which first led him to the principle of interference. An instructive variation

* Biot, Précis éleméntaire, vol. ii. p. 472, $3^{\text {me }}$ Edit.

+ " Experiments and Calculations relative to Physical Optics."-Phil. Trans. 
in this experiment was made by M. Arago. The interior fringes were found to disappear likewise, when the light passing by one of the edges was transmitted through a plate of some transparent substance; and, by varying the thickness of the interposed plate, M. Arago discovered that the disappearance of the fringes in this case arose from their displacement, the bands being always transferred to the side on which the plate was interposed. From this it followed that the light was retarded in the denser medium.* M. Arago afterwards produced the same modification in the interference bands formed by two mirrors; and the experiment, in this form, is a complete crucial instance, as applied to the two theories of light. The amount of the displacement determines the velocity of light in the medium, and therefore the refractive index, with an accuracy unattainable by any other method. Professor Powell has suggested a very elegant modification of this experiment, which at once establishes the truth of the law that the velocity of light is inversely as the refractive index of the medium traversed. $\dagger$

The experimental laws of the diffracted fringes were next examined by MM. Biot and Pouillet. In the case of a narrow rectilinear aperture-which was that chiefly studied-they found that the deviations produced in the different species of simple light, or the distances of the bands from the axis of the pencil, were in all cases proportional to the lengths of the fits, the magnitude of the aperture remaining the same. The same analogy was preserved in different media, the deviations varying in the inverse ratio of the refractive indices of the media, or in the direct ratio of the fits. $\neq$ M. Pouillet adds, that they were unable to explain these laws, having adopted the theory of emission.§ They are all simple consequences of the wave-theory. The interval of the fits is exactly half the length of a wave; and the true connexion between the place of the fringes and the latter quantity had been already pointed out by Young.

Mayer afterwards studied the phenomena of diffraction, but without adding any new facts to those already known. As to the

* "Sur un Phénomène remarquable qui s'observe dans la Diffraction de la Lumière."-Annales de Chimie, tom. i.

† Phil. Mag., Second Series, vol, xi. p. 6.

‡ Biot, Traité de Physique, tom. iv. Supplement a l'Optique.

\& Elemens de Physique, tom. ii. p. 437. 
theory, he adopted that of Newton, with some modifications. With Newton, he ascribed the inflexion of light into the shadow to the operation of an attractive force; but, unwilling to admit the existence of a repulsive force, he attempted to account for deflexion by the impact of the molecules reflected from the edge against those which passed by it.*

Fresnel at first adopted and developed Young's theory of diffraction, and found that the general laws of the fringes-the dependence of their magnitude upon the length of a wave, and upon the distances of the luminous origin and of the screen-were thus fully explained. It was shown that, as the position of the screen is varied, the successive points at which the same fringe is formed are not in a right line, but constitute an lyperbola; and that when the distance of the luminous origin is lessened, the inclination of these hyperbolic branches, considered as coincident with their asymptots, augments, and the fringes dilate in breadth. $\dagger$ Fresnel, however, was soon dissatisfied with this theory. If the exterior bands had their origin in the interference of the direct and reflected light, their intensity should depend on the curvature of the edge ; it is found, on the contrary, that the fringes formed by the back and by the edge of a razor are precisely alike in every respect. As to the other cases of diffraction, there were many phenomena, and especially those exhibited in Newton's experiment with the two knife-edges, which proved that the rays grazing the edges of the body were not the only rays concerned in the production of the fringes, but that the light which passed by those edges at sensible distances was also deviated, and concurred in their formation. $\ddagger$

Fresnel was thus led to seek a broader foundation for his theory, and the result of his investigations is given in the able memoir which was crowned by the French Academy in 1819. In this memoir the laws of diffraction are derived from the two principles to which the laws of reflexion and refraction are themselves referred-the principle of interference and the principle of Huygens. To apply these principles to the present case, Fresnel supposes the surface of the wave, when it reaches the obstacle, to

\footnotetext{
* Comm. Soc. Göttingensis Recentiores, vol. iv. p. 49.

+ Annales de Chimie, tom. i. p. 239.

† Mémoires sur la Diffraction de la Lumière, p. 368.
} 
be subdivided into an indefinite number of equal portions, and he applies the mathematical laws of interference, unfolded in this memoir, to determine the resultant of all the elementary waves sent by them at the same instant to any point. This resultant is expressed by means of two integrals, which are to be taken within limits determined by the particular nature of the problem. Its square is the measure of the intensity of the light; and it is found that its value has several maxima, and minima, which correspond to the intensities of the light in the bright and dark bands.

The problem of diffraction was thus completely solved; and it only remained to apply the solution to the principal cases, and to compare the results with those of observation. The cases of diffraction selected by Fresnel are: 1st. the phenomena produced by a single straight edge; 2nd. by an aperture terminated by parallel straight edges; and $3 \mathrm{rd}$. by a narrow opaque body of the same form. The agreement of observation and theory is so complete, that the computed places of the several bands seldom differ from those observed by more than the hundredth part of a millimetre, the case of diffraction by narrow apertures alone excepted. The small differences between observation and theory, in this case, Fresnel ascribes to a false judgment of the eye as to the position of the centre of the dark bands, occasioned by the different intensities of the bright bands on either side, the minimum always appearing nearer to the brighter light than it really is. The computed places of the bands, in the first case of diffraction, were found to differ from those deduced from the hypothesis of Young by a small numerical quantity, the distance of the first dark band being less in the former theory, in the ratio of 936 to unity; but small as the difference is, the measures of Fresnel completely decide the question.*

M. Poisson applied Fresnel's integral to the case of diffraction by an opaque circular disc, and arrived at the singular result, that the intensity of the light in the centre of the shadow is precisely the same as if the diso were removed. This remarkable anticipation of theory has been verified by the observation of M. Arago. $\dagger$ Fresnel himself solved the problem in the analogous case of a circular aperture, and arrived at the result, that the intensity of the light of any simple colour, at the central spot, will be the

* Mrémoire sur la Diffiaction de la Lumière, p. 420.

+ Ibid., p. 460 . 
same as that reflected by a plate of air, whose thickness bears a certain simple relation to the radius of the aperture, and to its distances from the luminous origin and from the eye. With homogeneous light, therefore, the illumination of the central spot vanishes periodically, as the distance of the eye from the aperture is varied; and in white light it assumes in succession the most vivid and beautiful hues, coinciding with those of the reflected rings of thin plates. These interesting phenomena were observed about the same time, by Sir John Herschel, and their laws deduced, independently, from observation.*

With the exception of the observations now referred to, no attempt has been made to verify the theory, by comparing the intensity of the light in the fringes with that deduced from the formulæ; and indeed it is obvious that a comparison of this nature is ill calculated to afford any conclusive evidence on the question. Fresnel thought, however, that the expression for the intensity might be indirectly verified, by superposing two sets of fringes (such as the interior and exterior fringes of a narrow opaque body), by means of double refraction, and then examining the position of the new maxima and minima. This ingenious suggestion does not appear to have been acted on.

The intensity of the light in the partial waves sent from each point of the primary wave, considered as a distinct centre of disturbance, will necessarily be different in different directions, depending on the angle which these directions form with the front of the original wave; and to solve the problem of diffraction in its most general form, it would be necessary to know the law of this variation. Fresnel has shown, however, that the rays whose. directions are inclined at sensible angles to the normal to the front of the primary wave, destroy one another by interference; so that the actual effect is produced by rays indefinitely near that normal, and which therefore may be regarded as of equal intensity. The truth of this assumption, however, is disputed by $\mathbf{M}$. Poisson. From his theory of the propagation of motion in fluid media, this mathematician inferred that the absolute velocities of the molecules are insensible in directions making finite angles with the direction of the original vibrations. He concludes, therefore, that these velocities, or the intensity of the light in the 
partial waves, cannot be regarded as sensibly equal in directions inclined to it at very small angles.* Fresnel's reply to this part of M. Poisson's theory has been already referred to. The principle of Huygens itself, which forms the basis of Fresnel's theory, though not denied by M. Poisson, is yet objected to as introducing a needless complication into the question; and indeed it does not seem easy to understand, at first view, why each point of the primary wave in this mode of composition should not give rise to a retrograde as well as to a direct wave. $\dagger$

An objection of a different nature has been raised against Fresnel's theory, derived from its supposed discordance with phenomena. It is a consequence of that theory, when applied to the case of diffraction by a narrow aperture bounded by parallel straight edges, that if a point be taken in the axis of the pencil, whose distances measured from the centre and edge of the aperture differ by half a wave, that point will be the limit within which all the interior fringes are confined; and beyond that point the centre of the image will be alcays white. This result is confirmed by the previous experiments of M. Biot; by the observations of Fresnel himself; and by those of Professors Airy and Powell, by whom they have been since repeated. M. Biot found that the central band was dark and white alternately, to a certain distance from the aperture; after which it was always white. He remarks that when this limit is attained, we may diminish the breadth of the aperture, and even bring its sides into actual contact, without any change in the central band, except its enlargement and consequent diminution of intensity. $\neq$

Newton's celebrated experiment with the two knife-edges has been adduced in opposition to these results. Newton found that when the distance of these edges was the 400 th part of an inch, the light which passed between the knives parted in the middle,

* It may be necessary to state that it was part of M. Poisson's theory, that the vibrations are normal to the wave.

+ See Annales de Chimie, tom. xxii. p. 270; and Airy's Mathematical Tracts, p. 267.

$\ddagger$ Traite de Physique, tom. iv. pp. 749, 760. The description of the phenomenon given by Mayer is very similar: "Prout illa distantia acierum semper magis magisque imminuitur, fascix adeo evanescunt, ita ut denique non nisi fascia media remaneat; sed ad dextram atque sinistram adeo in latitudinem extensa, ut non nisi lumen languidum, a medio spectri initialis utrinque instar caudx cometw sese dilatans, representet." -Göttingen Memoirs, vol. iv. p. 61. 
and left a dark space in the centre.* The experiment has been repeated by Mr. Barton, and with a similar result. $\dagger$ These experiments, however, were made with curved edges; and as Professor Powell has observed, we have no ground for supposing that the phenomenon may not be modified by this change in the conditions under which it is presented. The theory of Fresnel has not been applied to the more complex problem of an aperture with curvilinear edges, and the analytical difficulties of the problem seem to be insuperable. There seems to be some uncertainty, however, with respect to the phenomenon itself. Professor Powell repeated the experiment with edges of various curvatures, and always found that the centre was a point of relative brightness, as compared with other points in the line perpendicular to the length of the aperture. $\neq$ As to Newton's experiment, it seems certain, as the same writer has observed, that we are not acquainted with all its conditions; and it is apparent from many passages that the illustrious observer himself was far from being assured with respect to the real nature and circumstances of these phenomena.

But there is another essential circumstance to be taken into account, in comparing the experiments of Newton with the results of Fresnel's theory. In that theory the origin of light is supposed to be a point; and this condition is practically fulfilled by making the light to diverge from the focus of a lens of high power, the origin of the light in that case being (by the principles of the wave-theory) the minute image of the sun in the focus. In Newton's experiments, however, the sun's light was made to pass through a hole of sensible magnitude; and in the remarkable experiment now referred to, that hole was a quarter of an inch in diameter. The problem of diffraction in this case is one of much greater complexity. It is necessary to determine the joint effect produced at any point of the diffracting aperture by the several indefinitely small portions of a wave transmitted through the ex-

- Optics, Book iii., Obs. vi. and vii.

† Phil. Mag., vol. ii. p. 268.

$\ddagger$ Ibid., p. 429, \&c.

$\S$ "The subject of the third book I have also left imperfect, not having tried all the experiments which I intended when I was about these matters, nor repeated some of those I did try until I had satisfied myself about all their circumstances. To communicate what I have tried, and leave the rest to others for further inquiry, is all my design in publishing these papers." - Optics, Advertisement 1. See also latter part of Obs. 11, Book iii. 
ternal hole; and, considering each of these as a new centre of disturbance, to find their total resultant at any point of the screen on which the fringes are received. The method of solution has been pointed out by Professor Airy; and he has shown that when the external hole is a rectangular parallelogram, and the diffracting aperture of the same form, and similarly placed, the law of illumination at any point of a screen will be similar to that produced by a rhomboidal aperture, in Fresnel's method of observation; the dimensions and distances in the two cases being connected by certain relations.* From these investigations Professor Airy concludes that the size of the external hole could not account for the dark central shadow mentioned by Newton in the sixth observation. $\mathrm{H}_{\theta}$ has confirmed this conclusion by experiment; and employing holes of various magnitudes, he found the central band in all cases bright. The effect recorded by Newton is ascribed by Professor Airy to the influence of contrast on the retina.

A remarkable class of phenomena arise when a lens is placed close to an aperture of any form, and the light received on a screen at its focus, or on an eye-glass at its own focal distance from it. In fact, the phenomena of diffraction are in this manner produced with holes of considerable dimensions, and were observed by Sir W. Herschel, with the undiminished apertures of his great telescopes; the stars being seen encompassed by several dark and bright rings, succeeding one another at equal intervals, when a high magnifying power was employed. But the phenomena become more distinct when the aperture is limited by a diaphragm of moderate size, the diameters of the rings varying inversely as those of the apertures. The effects produced by diaphragms of different sizes and forms have been examined in much detail by Sir John Herschel and M. Arago. $\dagger$

The phenomena produced by minute apertures, when combined with a lens in the manner now spoken of, have been studied with much zeal and success by Fraunhofer. The most remarkable of these phenomena are those produced by a fine grating, such as may be formed by stretching a fine wire between two parallel screws of

* "On the Calculation of Newton's Experiments on Diffraction."-Cambridge Trans., vol. v. part 2.

+ Professor Amici has also noticed some phenomena of the same class. - Sce Ediw. Journal of Science, vol. iv. p. 306. 
equal thread. When such a grating is placed before the objectglass of a telescope, and a narrow slit, whose length is parallel to the wires of the grating, viewed through it, the direct image of the slit is bordered on either side by a succession of richly-coloured diffracted images, which increase in breadth and diminish in brightness as they recede from the centre. The first pair of spectra are separated from the central image by a space absolutely black, and a similar interval occurs between the first and second pair. Fraunhofer observed, under favourable circumstances, thirteen such spectra on either side of the central image. He has measured with great accuracy the angular deviations of the rays of each colour from the axis; and he has found that the experimental laws thus deduced agree in the most complete manner with the results of the principle of interference.* The results are the same, both by theory and experiment, in the case of reflexion from ruled surfaces. $\dagger$

The optical phenomena of gratings are interesting in many points of view. The appearance of lateral spectra, produced by simply intercepting a part of the light, proves that the light actually diverges in all directions from the front of the grand wave where it meets the lens, and that it is to the interference of this light with that intercepted by the grating that we are to ascribe its want of sensible effect under ordinary circumstances. $\neq$ Another very remarkable circumstance of these phenomena is the purity of the light of each simple colour, which is such that the fixed lines

- The angular deviation, $\theta_{n}$, of any ray from the axis is expressed by the formula

$$
\sin \theta_{n}=\frac{n \lambda}{\epsilon}
$$

in which $n$ denotes the order of the spectrum, $\lambda$ the length of an undulation, and $\epsilon$ the interval of the axes of the wires. The value of $\epsilon$ is obtained with great precision, so that the measurement of the angular deviations of the rays of each simple colour affords the most exact data for the determination of the lengths of their waves. Fraunhofer has in this manner computed the lengths of the waves, corresponding to the seven principal fixed lines in the spectrum; and the resulting values are perhaps the most exact optical constants we possess. It is a remarkable consequence of the expression above given, that when $\epsilon$ is less than $\lambda$, the angle $\theta_{n}$ will be imaginary. In this casc, then, there can be no coloured spectra; and it follows that scratches or inequalities on any polished surface, whose interval is less than the length of a wave, do not disturb the regularity of reflexion and refraction.

† Fraunhofer's researches on diffraction are published in the Memoirs of the Bavarian Academy of Sciences, vol. viii. A very full analysis of them is given in the Edinburgh Encyclopadia, art. Optics ; and in Sir J. Herschel's "Essay on Light," Encyc. Metrop.

$\ddagger$ Airy's Math. Traets, p. 331. 
may be discerned in the spectra. The distances of these lines, in the diffracted spectrum, are alvays proportional, whatever be the diffracting substance; while the ratios of their intervals, or the breadths of the coloured spaces, in the spectra formed by refraction, vary with the nature of the prism. This fact appears to be decisive against the Newtonian theory of inflexion, in which inflexion and refraction are referred to the same cause.

The analytical investigation of the problem of diffraction in the cases last alluded to-those, namely, in which a lens is combined with the aperture, and the intensity of the light is sought at any point of a parallel plane passing through the focus-is far more manageable than in most other cases. The general expression of the displacement is at once integrated with respect to one of the variables, and the complete integral can, in many cases, be exactly found. Professor Airy has given the solution of this problem in his valuable tract on the Undulatory Theory," and in applying it to the phenomenon last mentioned has deduced all the appearances observed by Fraunhofer. The remarkable appearance of the sixrayed star, observed by Sir John Herschel, when a triangular diaphragm was placed before the object-glass of a telescope, has been likewise deduced as another case of the same problem.

The same effects, as Fraunhofer observed, were produced by reflexion from grooved surfaces; and their theory is to be referred to the same principles, the light reflected from the surfaces between the grooves interfering in a manner precisely analogous to that admitted through the apertures of the gratings. The colours exhibited by such surfaces under ordinary circumstances were observed by Boyle and Grimaldi; Young showed that they were consequences of the principle of interference, and determined the law of their recurrence depending on the incidence; $†$ and Sir David Brewster seems to have been the first to observe that the spectra formed in these cases of multiplied diffraction approached the solar spectrum in purity far more nearly than the ordinary diffracted bands, or the coloured rings of Newton. These phenomena indicate the superficial structure more unerringly, perhaps, than the most powerful microscopes. Among the most important and beautiful instances of this application of optical science may

* Math. Tracts, p. 321, \&c.

+ " On the Theory of Light and Colours."-Phil. Trans. 1801. 
be ranked the analysis of the colours of mother-of-pearl, ${ }^{*}$ and the investigation of the structure of the crystalline lenses of the eyes of fishes and other animals, by Sir David Brewster. $†$ The same author has also described a new series of periodical colours, which are exhibited by some of the plates of grooved steel constructed by Mr. Barton, and which succeed one another in a plane at right angles to that in which the usual spectra are developed. $\neq$ The theory of this phenomenon remains yet to be developed. In the solution of the analogous problem, given by Professor Airy, a periodical variation in the intensity of the light in the direction of the apertures of the grating is indeed pointed out; but that variation, it is easily seen, will not account for the facts last mentioned.

\section{Colours of thin Plates.}

The earliest observations on record, in which the colours of thin plates were made the subject of experimental research, are those of Boyle.§ This diligent observer remarked the fact, that most transparent substances exhibit colour by reflected light when suificiently reduced in thickness; and that these tints varied in the same substance, and therefore did not depend essentially upon its chemical nature. The observations of Boyle were made on the bubbles of various liquids, and he even succeeded in blowing glass sufficiently thin to exhibit similar phenomena.

The vivid and varying colours of the soap bubble also engaged the attention of Hooke; $\|$ but the most important of the observations of this philosopher, connected with the subject of thin plates, are those recorded in his Micrographia, which was published in the year 1665. In this work he shows that the colours of laminæ of mica are dependent on their thickness, and appear only when that thickness is comprised within certain limits; that when the tint exhibited by a given plate is uniform over its entire surface, the plate is also uniformly thick; and that the colour presented by two plates superposed is different from those of either separately. Hooke has also the merit of producing the phenomena of thin

* Phil. Trans. 1814.

† Ibid. 1833.

$\ddagger$ Phil. Trans. 1829.

Experiments and Observations upon Colours, 1663.

|| Birch's History of the Royal Society, vol. iii. p. 29. 
plates in the instructive form in which their laws have since been studied, namely, by placing two object-glasses in contact; and he found that any transparent fluid introduced between the lenses furnished a succession of colours as well as air; the colour, however, being more vivid, the more the refractive power of the plate differed from that of the glasses within which it was inclosed.

The attention of Newton was soon after directed to the same subject; and his investigations, which ended in the complete discovery of the laws of the phenomena, will ever be considered as a model of experimental inquiry. A convex lens of glass being laid upon a plane surface of the same material, after the manner of Hooke, the bands of the same colour are arranged round the point of nearest approach in concentric circles; and the diameters of these circles will be obviously as the square roots of the thicknesses of the plate of air at the points at which they are exhibited. In order to investigate the relation between the colour and the thickness, then, it was only necessary to measure the diameters of these rings in the different species of simple light; and taking similar measurements when the other circumstances of the phenomena were varied, Newton deduced their laws, as they depended on the substance of the reflecting plate, and on the obliquity of the incident pencil. Newton observed, moreover, that there was a second system of rings formed by transmission. The transmitted rings were found to observe the same laws-with this remarkable exception, that the colour transmitted at any particular thickness of the plate was always complementary to that reflected at the same thickness, so that in homogeneous light, the bright transmitted ring is always found at the same distance from the centre as the corresponding dark one of the reflected system.

The observations of Mariotte, ${ }^{*}$ Mazeas, $\dagger$ and Du Tour, $\ddagger$ have added nothing essential to the laws discovered by Newton. Most of these observations, in fact, related to the colours exhibited by the plate of air inclosed between two plane glasses, and in circumstances, therefore, much less favourable to the analysis of the phenomenon than those selected by Newton. Perhaps the most interesting of the facts noticed by Mazeas are the effects produced

* Traité de la Lumière et des Couleurs.

† Mémoires présentés, tom. ii.

$\S$ Ibid., tom. iv. v. vi.

F 2 
on the coloured bands by the application of heat to the glasses, the colours retreating to the edges of the plates, and the bands diminishing in breadth as the temperature was increased. The same author also found that no sensible change took place in the phenomenon when the air was withdrawn by tho air-pump.

In the observations of $\mathrm{Du}$ Tour, the reflected and the transmitted tints were observed at the same time, the latter being reflected from the second surface of the lower glass, and returning to the eye through the entire system. This latter set of rings is rendered more distinct, when the shadow of an opaque body is passed over the upper surface. In this manner the phenomenon was observed by Sir William Herschel; and it was found that additional sets of rings became visible by increasing the number of reflecting faces. Sir William Herschel observed, likewise, that the primary reflected system was produced when a lens was laid upon a metallic reflector; and he remarks that, in this case, the transmitted system must be conceived to be absorbed by the metal. The same author has described a remarkable set of coloured bands adjacent to the iris, at the limit of total reflexion, when a prism is in contact with a plane surface.* The analysis of this phenomenon has been given by Sir John Herschel in his Essay on Light. $\dagger$

The important observations of M. Arago are the next to demand our notice. $\neq$ Viewing the rings through a rhomboid of Iceland spar, whose principal section was parallel or perpendicular to the plane of incidence, this philosopher observed that the intensity of the light in one of the images varied with the incidence, and that it vanished altogether when the rays made an angle of $35^{\circ}$ with the surface. It was further observed that the same image vanished, and at the same angle, whether the rings were formed by reflexion or transmission. Thus, the light of the transmitted, as well as of the reflected rings, was wholly polarized in the plane of incidence, and at the usual angle for glass. M. Arago has further shown, that the colours of the reflected and transmitted rings are not only complementary, but that their intensities are also precisely the same; for, when the two systems are superposed, they completely neutralize each other.

" "Experiments for investigating the Cause of the Coloured Rings," \&c.-Phil. Trans. 1807, 1809, 1810.

+ Articles 641, 642.

† "Sur les Couleurs des Lames minces."-Mémoires d"Arcueil, tom. iii. 
But the most remarkable of the results obtained by this author relate to the rings formed by the plate of air inclosed between a lens of glass and a metallic reflector. When these were observed in the manner already alluded to, one of the images vanished, as before, at the polarizing angle of glass; while its appearance, at angles above and below the polarizing angle, presented a remarkable contrast. When the incidence was less than this angle, the two images seen through the double refracting crystal differed only in intensity; the dimensions and colours of the rings were the same in both. Beyond the polarizing angle, however, the rings in the two images were of complementary colours; so that if the series in one commenced from a black centre, in the other it began from a white one. The dimensions of the rings of the same order in the two images were also different. Similar phenomena were produced when the thin plate was of a density intermediate to those of the two substances between which it was contained. I shall hereafter have occasion to refer to the observations and deductions of Professor Airy connected with these phenomena.

When the metallic reflector was slightly tarnished, a second system of rings was visible to the naked eye. The formation of these rings depended on the light irregularly dispersed at. the surface of the metal; and they were visible, in whatever manner the eye was placed with respect to the incident light. Their tints were complementary to those of the regular series.

It was soon felt that the phenomena of thin plates were closely connected with some new and fundamental property of light," and that it was in their application to these phenomena that all theories of light were to be judged. For their explanation, it has been already stated, Newton invented his celebrated doctrine of the "fits of easy reflexion and transmission," a doctrine which will always hold a prominent place in the page of philosophical history. Its application is obvious. The ray is in a fit of easy transmission in its passage through the first surface; this is sueceeded by a fit of easy reflexion, and so alternately. On arriving at the second surface, then, the ray will be in a fit of easy transmission or easy

* It is unnecessary to refer to the theories of Sir William Herschel, or of M. Parrot, in both of which the laws of thin plates have been referred to those of reflexion and refraction; or to that of Mayer, who attempted to reduce them to inflexion. None of these theories have had supporters, and they are all of them inconsistent with obvious facts. 
reflexion, according as the interval of the surfaces, or the thickness of the plate, is an even or an odd multiple of the length of the fit. Thus the alternate succession of bright and dark rings in homogeneous light, and the arithmetical progression of the thicknesses at which they are exhibited, are satisfactorily explained. To explain the variation in the dimensions of the rings depending on the nature of the light, it is necessary to suppose that the length of the fits varies with the colour-being greatest in red light, least in violet, and of intermediate magnitude for the rays of intermediate refrangibility. Newton determined the absolute lengths of these fits for the rays of each simple colour, and found that they bore a remarkable numerical relation to the lengths of the chords sounding the octave. These results are even yet referred to as fundamental data in optical inquiries.

To account for the remaining laws Newton was constrained to make new suppositions, and to attribute properties to the fits which seem inconsistent with every physical account which has been given of them. Thus, to explain the dilatation of the rings with the increasing obliquity of the incident pencil, he assumed that the length of the fits augmented with the incidence, and according to a complicated law. This assumption is at entire rariance with the physical theory. If the fits are produced by the vibrations of the ether, which are propagated faster than the rays, and which alternately conspire with and oppose their progressive motion, their lengths should continue the same in the same medium, whatever be the incidence. No attempt, that I am aware of, has been made to reconcile this law with the physical hypothesis of Mr. Melville and M. Biot.

The same may be said of the variation of the dimensions of the rings with the substance of the reflecting plate. Newton found that when a drop of water was introduced between the glasses, the rings contracted; and by comparing their diameters in air and in water, he found that the corresponding thicknesses of the plate were as 4 to 3 , or in the inverse ratio of the refractive indices. It was necessary to suppose, therefore, that in different media, the lengths of the fits varied in the same proportion; and, since in the Newtonian theory the refractive indices are directly as the velocities of propagation, it followed that as the velocities augmented, the spaces traversed by the ray in the interval of its periodical states must diminish, and in the same ratio. 
But the facts observed by M. Arago and Professor Airy seem to overturn altogether this part of the theory of emission. The rings formed by a plate of air, inclosed between a lens of glass and a metallic reflector, vanish altogether when the light is polarized perpendicularly to the plane of incidence, and is incident at the polarizing angle of glass. Under these circumstances, no light is reflected from the upper surface of the plate; but as it is abundantly reflected from the lower, the disappearance of the rings proves that the light reflected from the upper surface is essential to their production. That the light reflected from the lower surface also concurs in their formation, appears from the effects observed by $\mathbf{M}$. Arago, when the metallic plate was tarnished; and we are thus driven to the conclusion, that the phenomena arise from the union and mutual influence of the pencils reflected from the two surfaces.

This mode of explaining the colours of thin plates was pointed out by Hooke, in a remarkable passage in his Micrographia, some years before the subject was taken up by Newton. In this passage he very clearly describes the manner in which the rings of successive orders depend on the interval of retardation of the second "pulse," or wave, on the first, and therefore on the thickness of the plate. But he does not seem to have had any distinct idea of the principle of interference itself; and his conception of the mode in which the colours resulted from this "duplicated pulse" is entirely erroneous. Euler was the next who attempted to connect the phenomena of thin plates with the wave-theory of light; but the attempt, like all the physical speculations of this great mathematician, was signally unsuccessful. Euler thought, in fact, that the colours of thin plates, as well as those of natural bodies, arose from emitted, and not from reflected light. The incident light was supposed to excite the vibrations of the plate, the frequency of which depended on its thickness, in the same manner as the frequeney of the vibrations of the column of air in a tube depends on its length. These vibrations again were believed to excite those of the luminiferous ether, and thus to produce the sensation of various colours, the red corresponding to the less frequent vibrations, and the violet to the most frequent."

The subject remained in this unsatisfactory state until the 
principle of interference was discovered by Young. When this principle was combined with the suggestion of Hooke, the whole mystery vanished. The application was made by Young himself; and all the principal laws of the reflected rings were readily and simply explained, by the interference of the two portions of light which are reflected at the two surfaces of the plate.* In applying this principle, however, Young perceived that the interval of retardation was not simply that due to the difference of the paths traversed by the two pencils; but that one of them must be supposed to undergo a change of phase, amounting to half an undulation, at the instant of reflexion. Young clearly pointed out the accordance of this effect with mechanical principles; and the connexion has been fully confirmed by the more complete investigations of Fresnel. In fact, the two reflexions take place under opposite circumstances, one of the portions being reflected at the surface of a rarer, and the other at that of a denser, medium; and the laws of impact of elastic bodies indicate that the direction of the vibratory movement must be reversed by reflexion in the one case, while in the other it is unchanged. Young had the satisfaction of putting this principle to the test in a remarkable manner. It followed from it that, if the thin plate were of a refractive density intermediate to those of the two media within which it was inclosed, the laws of the phenomenon would be determined by the difference of the paths alone, the reflexion being of the same kind at the two surfaces. Young accordingly predicted that in this case the rings should commence from a white centre, instead of a black one, and the prediction was soon after verified on trial. $\dagger$

The transmitted rings are accounted for, in the wave-theory, by the interference of the direct light with that which has undergone two reflexions within the plate; and it follows from the preceding considerations that their colours must be complementary to those of the reflected system. This origin at once shows the reason of the fact observed by M. Arago, that the light of the transmitted rings is polarized in the plane of reflexion. M. Biot has laboured to reconcile this fact to the theory of emission, with which it appears, at first view, at utter variance. The account which he has given of the phenomenon will, I think, be hardly deemed satisfactory $\ddagger$

" "On the Theory of Light and Colours."-Phil. Trans. 1802.

+ "Account of some Cases of the Production of Colours."-Phil. Trans. 1802.

‡ See Biot's " Traité de Physique," tom. iv. pp. 308, et seq. 
The theory of thin plates, as it came from the hands of Young, was however incomplete. It is obvious that the intensity of the two portions of light reflected from the upper and under surfaces of the plate can never be the same, the light incident on the second surface being already weakened by partial reflexion at the first. These two portions therefore cannot wholly destroy one another by interference ; and the intensity of the light in the dark rings should never entirely vanish, as it appears to do when homogeneous light is employed. M. Poisson was the first to point out, and to remedy, this defect of the theory. It is evident, in fact, that there must be an infinite number of partial reflexions within the plate, at each of which a portion is transmitted; and that it is the sum of all these portions, and not the two first terms of the series only, which is to be considered in the calculation of the effect. Taking up the problem in this more general form, and employing the formula obtained by himself and Young for the intensity of the light reflected and transmitted at a perpendicular incidence, M. Poisson has proved that-at this incidence, and at points for which the thickness of the plate is an exact multiple of the length of half a wave-the intensity of the reflected and transmitted lights will be the same as if the plate were suppressed altogether, and the bounding media in absolute contact; so that when these media are of the same refractive power, the reflected light must vanish altogether, and the transmitted light be equal to the incident.* Fresnel afterwards showed that the result was independent of the expression for the intensity of the reflected light; and by the aid of the property discovered by M. Arago, -namely, that the light is reflected in the same proportion at the first and second surfaces of a transparent plate-he extended the conclusion to all incidences. $t$ The general expression of the intensity of the light in any part of the reflected or transmitted rings has been given by Professor Airy.‡

Here, then, we have reached a point with respect to which the two theorics are completely opposed. According to both, a cer-

* "Sur le Phénomène des Anneaux Colorés."-Annales de Chimie, tom. xxii. p. 337. M. Poisson bas further shown that rings absolutely black will be formed at points corresponding to the bright rings in the ordinary case, when the velocity of propagation within the plate is a mean proportional to the velocities in the bounding media.

† Annales de Chimie, tom. xxiii. p. 129.

$\ddagger$ Math. Tracts, p. 302, \&c. 
tain portion of light is reflected from the first surface of the plate. This, in the Newtonian theory, is left in all cases to produce its full effect; while in the wave-theory it is, at certain intervals, wholly destroyed by the interference of the other pencil, and the dark rings should be absolutely black in homogeneous light. The latter of these conclusions seems to accord with phenomena, while the former is obviously at variance with them. This is clearly shown by an experiment of Fresnel. A prism was laid upon a lens having its lower surface blackened, a portion of the base of the prism being suffered to extend beyond the lens. The light reflected from this portion, according to the Newtonian theory, should not surpass in intensity that of the dark rings. The roughest trial is sufficient to show that the intensity of the light in the two cases is widely different, and to prove that the dark rings cannot arise (as they are supposed to do in the theory of fits) from the suppression of the second reflexion.*

Mr. Potter has applied a new method of " photometry by comparison" to determine the relative intensities of the light in the bright and dark rings of the transmitted system. In this method the ratio of the intensities of the light reflected from two plane glasses is varied, by varying the incidence, until it is judged to be equal to the ratio of the light in the bright and dark rings. The former ratio is then deduced from the incidence by means of an empirical formula. In this manner $\mathrm{Mr}$. Potter concludes that the ratio of the light in the rings, at a perpendicular incidence, is 2.48 for green light, and 3.49 for red. $\dagger$ The ratio deduced from the principles of the wave-theory is about 1.20 in the case of crown glass. But, independently of the uncertainty connected with the empirical law, which is taken by Mr. Potter as the basis of his computation in these deductions, the photometrical method itself seems to be open to objection. It appears to be assumed, in the application of that method, that where the quantity of light incident upon an irregularly reflecting surface is given, the quantity of reflected light will be the same in its entire amount, and in all directions, whatever be the incidence. This seems to be contradicted by obvious facts. There is yet another difficulty in tho application of this method, which appears to leave room for some

\footnotetext{
* Mémoire sur la Diffraction, p. 347.

† Plit. Mag., 3rd Series, vol. i. p. 174.
} 
uncertainty in the results. Where luminous objects are so small that the eye cannot readily distinguish parts, the absolute quantity and the intensity of the light are confounded. I am not aware how far this may have been the case in Mr. Potter's instrument; but it is remarkable that, if we suppose the quantities of light reflected from the two glasses to have been taken as the terms of comparison, the calculated results will accord very closely with theory.*

When a beam of light falls upon two plates superposed, some of the many portions into which it is divided by partial reflexion at the bounding surfaces are often in a condition to interfere and exhibit colour. Thus, when light is transmitted through two parallel plates, slightly differing in thickness, the colour produced will be that corresponding to the difference, and will be independent of the interval of the plates. This phenomenon was observed by Mr. Nicholson, $\uparrow$ and was shown by Dr. Young to arise from the interference of two pencils, one of which is twice reflected within the first glass, and the other twice reflected in the second. Sir David Brewster observed a similar case of interference produced by two plates of equal thickness, slightly inclined, the thickness traversed in the two plates being altered by their inclination. In both these cases, however, the interfering pencils are mixed up with, and overpowered by, the light directly transmitted, and some contrivance is necessary to make the fringes visible. The phenomena are much more obvious in the light reflected by both plates, which, on account of their inclination, is separated from the direct light. It is obvious, in fact, that the direct image of a luminous object seen through the glasses will be accompanied by several lateral images, formed by two, four, six, etc., reflexions. These images Sir David Brewster observed to be richly coloured. The bands are parallel to the line of junction of the two glasses, and their breadth is greater the less the inclination of the plates. $\neq$ The colours in the first lateral image are produced by the interference of the pencils which have undergone two reflexions, one of them being reflected internally by the first plate and externally by the second, while the other is reflected inter- 
nally by the second, and externally by the first. The routes of these portions differ only by reason of the different inclinations at which they traverse the intervals of the surfaces. M. Pouillet has observed a phenomenon of the same kind, when a thick plate of glass is placed above a metallic mirror, and in a direction nearly parallel to its surface.* The interfering rays in this case appear to be those which have undergone two reflexions within the plate, and one at the surface of the mirror, the reflexion from the mirror preceding the others in the case of one pencil, and following them for the other. The routes of two such pencils will slightly differ, owing to the different obliquity under which they traverse the plate.

The remarkable phenomena observed by Mr. Knox, when a double-convex lens was combined with two plane glasses, one adjacent to each surface, have been explained by Young on the same principles. In addition to the rings exhibited by each plate of air, a third system of concentric rings is formed in this case, the dimensions of which are greater than those of either of the primary systems. The diameters of these rings increase indefinitely as those of the primary systems approach to equality; until finally the circles become straight lines when they are equal. $\dagger$ It is easily seen, in fact, that each ring is the locus of the points for which the difference of the thicknesses of the two plates of air is constant; and that this locus is a circle, whose diameter will depend on the curvatures of the surfaces, and on the interval of the centres of the two primary systems. The fringes formed by "double plates" have been observed under another form by $\mathrm{Mr}$. Talbot, when two films of thin blown glass were superposed.

The "colours of thick plates" are perhaps of too unusual occurrence to entitle them to be studied as a separate class of optical phenomena; the attention which they have received is owing to the investigations of Newton. In the experiment of Newton a beam of light is admitted through a small aperture, and received on a concavo-convex mirror with parallel surfaces, the second of which is silvered. When a screen of white paper is then held at the centre of the mirror, having a hole in the middle to allow the beam to pass and repass, a set of broad coloured rings

* Elemens de Physique, tom. ii. p. 478.

† Phil. Trans., 1815, p. 161. 
will be depicted on it, similar to the transmitted rings of thin plates, the diameters of the rings varying inversely as the square roots of the thicknesses of the mirrors. The Duo de Chaulnes observed that similar phenomena were produced when a metallio mirror was substituted for the glass one, and the rays transmitted through a semi-transparent plate of any kind, or even through a screen of gauze placed at a short distance in front of the mirror.* Sir W. Herschel found that the rings could be produced by scattering fine powder in the air before the mirror ; $\dagger$ and M. Pouillet has ascertained that similar rings are formed, when the light incident on the mirror is simply transmitted through an aperture of any form in an opaque screen.‡ More recently, $\mathrm{Mr}$. Whewell and M. Quetelet have observed a set of coloured bands, which are formed when the image of a candle is viewed in a plane glass mirror, the candle being held at a short distance in front of the eye, so that the incident and reflected rays may make a small angle.§ M. Quetelet appears to think, however, that this phenomenon is to be referred to a different class from those last considered.

Newton very ingeniously accounted for the colours observed in his experiments, by the fits of easy reflexion and transmission of that pportion of light which is scattered in all directions at the first surface of the glass; and M. Biot has extended the explanation to the analogous phenomena observed by the Duo de Chaulnes. Young showed that they could be explained by the interference of the two portions of light which are scattered in the passing and repassing of the ray through the refracting surface.ll The complete investigation, as far as relates to the dimensions of the successive rings, is given by Sir John Herschel; and the formula obtained is found to agree precisely with Newton's measures. 9

When the interval between two glasses is filled with different substances, such as water and air, or water and oil, in a finely

* Mém. Acad. Par. 1755.

† Phil. Trans. 1807.

$¥$ Elemens de Physique, tom. ii. p. 476.

\$Correspondance Mathématique, tom. v. p. 6, et.tom. vi. p. 1.

II "On the Theory of Light and Colours."-Phil. Trans.; and Encyel. Brit., Art. Chromatics.

Tा Essay on Light, Art. 679, et seq. 
subdivided state, the portions of light which have traversed them are in a condition to interfere, the interval of retardation depending on the difference of the velocities of light in the two media. Accordingly, coloured rings will be seen when a luminous object is viewed through the glasses; the rings being similar to those usually seen by transmission, but much larger. But when a dark object is behind the glasses, and the incident light somewhat oblique, the rings immediately change their character, and resemble those of the ordinary reflected system; one of the portions in this case being reflected, and therefore suffering a loss of half an undulation. These phenomena were observed and explained by Young," and have been denominated by him the "colours of mixed plates." Young also observed some similar phenomena of colour in an unconfined medium. Thus, when the dust of the lycoperdon is mixed with water, the mixture exhibits a green tint by direct light, and a purple tint when the light is indirect; and the colours rise in the series when the difference of the refractive densities is lessened by adding salt to the water. The interval of retardation in this case depends also on the magnitude of the transparent particle. $\dagger$

In closing the review of this part of the subject, I would observe that any well-imagined theory may be accommodated to phenomena, and seem to explain them, if only we increase the number of its postulates, so as still to embrace each new class of phenomena as it arises. In a certain sense, and to a certain extent, such a theory may be said to be true, so far as it is the mere expression of known laws. But it is no longer a physical theory, whose very essence it is to connect these laws together, and to demonstrate their dependence on some higher principle:-it is an aggregate of separate principles, whose mutual relations are unknown. Thus the cycles and epicycles of the Ptolemaic system represented with fidelity the more obvious movements of the planetary bodies; but when the refinements of astronomical research laid bare new laws, new epicycles were added to the system, until at length its complication rendered it useless as a guide. Such appears to be the present state of the theory of emission; and so

" "Account of some Cases of the Production of Colours."-Phil. Trans. 1802. The Abbé Mazeas noticed many facts which appear to be referrible to the same princi-
ples.-Mémoires présentés, vol. ii.

+ Introduction to Medical Literature, p. 556. 
glaringly does this blemish show itself in that part of the theory which has been last under consideration, that one of its advocates says,- " Reverà illæ vices reflexionis et transitus, cum omnibus additamentis fictitiis, mirabiliores adhue sunt quàm phænomenon ipsum, ad cujus explicationem in usum sunt vocatæ."”* The same attribute appears in the broader divisions of the science. The several classes of phenomena do not flow from the theory as from one common source; but each has its separate and independent head, and its separate and independent data. In the wave-theory, on the other hand, not only the individual laws, but the classes of phenomena are related; and to calculate, numerically, the laws of refraction, the varied phenomena of diffraction, and those of thin plates, we only need to borrow one result from experience,- the length of a wave of light in each medium. There is thus established that connexion and harmony in its parts which is the neverfailing attribute of truth. But powerful as is the weight of this intrinsic evidence in favour of the wave-theory, it has yet stronger claims to our assent. These claims are grounded on the vast body of new phenomena which it explains,-and explains (it is to be remembered) not in a vague and general manner, but in the precise language of analysis, and with an accuracy which the refinements of modern observation have not been able to impugn. It may be confidently said that it possesses characters which no false theory ever possessed before.

\section{Part II.-Polarized Light.}

\section{Polarization.-Transversal Vibrations.}

In the various phenomena which have been hitherto described as taking place when a ray of light encounters the surface of a new medium, it has been assumed that the direction, and the intensity of the several portions into which it is subdivided, are wholly independent of the manner in which the ray is presented to the bounding surface, the direction of the ray remaining unchanged. In other words, it was taken for granted that a ray of light had no relation to space, with the exception of that dependent on its direc-

* Mayer on Newton's Rings._Göttingen Memoirs, vol. v. p. 22. 
tion; - that around that direction its properties were on all sides alike; and that if the ray were supposed to revolve round that line as an axis, the resulting phenomena would be unaltered.

Huygens was the first to observe that this was not always the case. In the course of his researches on the law of double refraction, he found that when a ray of solar light is received upon a rhomb of Iceland crystal, in any but one direction, it is always subdivided into two of equal intensity. But on transmitting these rays through a second rhomb, he was surprised to observe that the two portions into which each of them was subdivided were no longer equally intense;-that their relative brightness depended on the position of the second rhomb with regard to the first; and that there were two such positions in which one of the rays vanished altogether.

From this "wonderful phenomenon," as Huygens justly called. it, it appeared that each of the rays refracted by the first rhomb had acquired properties which distinguished it altogether from solar light. It had, in fact, acquired sides; and it was evident that the phenomena of refraction depended, in some unknown manner, on the relation of these sides to certain planes within the crystal. Such was the conclusion of Newton: "This argues," says he, "a virtue or disposition in those sides of the rays, which answers to, and sympathizes with, that virtue or disposition of the crystal, as the poles of two magnets answer to one another."

This conception was followed out by Malus, whose varied and important discoveries respecting the nature and laws of polarized light have justly placed him in the rank of founder in this most interesting branch of science. The molecules of a polarized ray were supposed by him to have all their homologous sides turned in the same directions. He adopted the term "polarization" to express the phenomenon, and compared the effect to that of a magnet which turns the poles of a series of needles all to the same side. M. Biot has modified the hypothesis of Malus in order to embrace the other phenomena of light, and assumes that there is one line, or axis, similarly placed in each molecule, and that these axes in a polarized ray are all turned in the same direction. The molecules, however, are at liberty to revolve round these axes, and thus to assume different dispositions with respect to the attracting or repelling forces to which they are exposed when they encounter the surface of a new medium. 
The phenomenon of polarization seems to have had much weight with Newton in forcing him to reject the theory proposed by Huygens. "It is difficult," he says, "to conceive how the rays of light, unless they be bodies, can have a permanent virtue in two of their sides, which is not in their other sides, and this without any regard to their position to the space or medium through which they pass."* "Are not all hypotheses erroneous," he adds in another place, "in which light is supposed to consist in pression or motion, propagated through a fluid medium? . . . . Pressions or motions, propagated from a shining body through an uniform medium, must be on all sides alike; whereas by those experiments it appears that the rays of light have different properties in their different sides." $\dagger$ In this objection Newton seems to have fixed his thoughts upon that species of undulatory propagation whose laws he himself had so sagaciously divined. When sound is propagated through air or water, the vibrations of the particles of the fluid are performed in the direction in which the wave advances; and if the vibrations of the ether, which are supposed to constitute light, were of the same kind, the objection would seem to be insuperable. But the case is altered, if, as is now assumed, the vibrations of the ethereal particles be tiansverse to the direction of the ray's progress. And though we were unable to render any account of this hypothesis, or even to show that it is consistent with mechanical principles; yet the numerous olasses of phenomena which it has explained, and the striking and exact manner in which its predictions have been verified on trial, compel us to admit, that if the law to which we have thus reduced so various and such complicated facts be not itself a law of nature, it is at least coordinate with it, in such a sense that we may take it as the representative of actual existence, and reason from it as we would from an established physical law.

The hypothesis of transversal vibrations first occurred to Dr. Thomas Young, who illustrated it by the propagation of undulations along a stretched cord agitated at one of its extremities. Young seems to have been led to this principle while considering the results arrived at by Sir David Brewster, in his researches on the laws of double refraction in biaxal crystals. The principle was soon after raised above the rank of a mere hypothesis, and

* Optics, book iii. Query 29.

+ Query 28 . 
shown to be a necessary consequence of the laws of interference of polarized light, if the theory of waves be admitted at all. It follows, in fact, from the laws of composition of vibrations, that the intensity of the light resulting from the union of two rays oppositely polarized will be constant, and independent of the phase-as was proved to be the case in the experimental researches of MM. Arago and Fresnel-only when the vibrations normal to the wave are evanescent. It appears from the same investigation that the actual vibrations are either parallel or perpendicular to the plane of polarization. As far as the phenomena of interference are concerned, it is indifferent which of these results be assumed to be the fact. But the theory of transversal vibrations itself, when applied to the laws of double-refraction, leads to the conclusion that the vibrations which constitute the ordinary ray in uniaxal crystals are perpendicular to the principal plane; and this being its plane of polarization, Fresnel concluded that the vibrations of a polarized ray are on the surface of the wave, and perpendicular to the plane of polarization.*

The principle of transversal vibrations, thus deduced from the phenomena of interference of polarized light, is easily extended to the case of common or unpolarized light. For when a ray of such light falls perpendicularly upon a double-refracting crystal, it is divided into two polarized pencils, neither of which, it appears from the preceding, can contain vibrations normal to the surface of the wave. If, then, there were any such in the incident ray, they would be destroyed by refraction, and there would ensue a loss of ris riva, and consequently a diminution in the intensity of the light; in other words, the sum of the intensities of the two refracted pencils would be less than that of the incident, which is contrary to observation. In unpolarized light, therefore, as in polarized, the vibrations are only on the surface of the waves; and we must conceive such light to consist of a rapid succession of systems of waves polarized in every possible plane passing through the normal to the front of the wave. The phenomenon of polarization then, in this theory, consists simply in the resolution of the vibrations into two sets, in two rectangular directions, and the subsequent separation of the two systems of waves thus produced.

" "Mémoire sur la Double Refraction."-Mem. Inst., tom. vii. 
The erroneous views of mathematicians on this subject, according to Fresnel, have arisen from the imperfect physical conceptions which they have made the basis of their reasoning. Elastic fluids have been represented as composed of particles in contact, capable -only of condensation and dilatation ; and accordingly the accelerating forces have been conceived to arise solely from the difference - of density of the consecutive shells of the fluid. In this case, it is evident that if any row of particles is displaced in the direction of the connecting line, this row will slide upon the succeeding one, and the motion will be resisted by no elastic force. But when we regard these bodies as they really are-composed of molecules separated by intervals which are probably considerable as compared with their magnitude, and acting on one another according to some law varying with the distance, the whole question is altered. When any row or line of such molecules is similarly displaced, and through a space which is small compared with the separating intervals, the molecules of the succeeding row will be moved in the same direction by the forces which are thus developed with the change of distance; so that the vibrations of the particles composing the first row will be communicated to those of the second, and thus the vibratory motion will be propagated in a direction perpendicular to that in which it takes place.* The rapidity of the propagation will depend on the magnitude of the force developed by the displacement. To account for the fact that there are no sensible vibrations in a direction normal to the wave, we have only to suppose the repulsive force between the moleoules to be very great, or the resistance to compression very considerable ; for in this case, it will be seen, the force which resists the approach of two strata of the fluid is much greater than that which opposes their sliding on one another. Fresnel's views on this subject are contained in a short paper, entitled "Considerations mécaniques sur la polarisation de la lumière, $\uparrow "$ and in his celebrated memoir on double refraction. $\ddagger$

The principle of transversal vibrations, however, has not been

* The existence of transversal vibrations has been fully established in other cases of vibratory motion. M. Savart and Mr. Wheatstone have shown that in many instanees the elementary motions of the molecules of bodies which transmit sound are transverse to the direction of the propagation.

+ Bulletin de la Soc. Philom., 1824.

+ Mímoires de l'Institut, tom. vii. 
received without much discussion; and even to this hour theopinion of the mathematical world is not entirely at rest upon thesubject. In a memoir on the propagation of motion in elasticfluids, read before the Academy of Sciences in the year 1823, M. Poisson arrived at the conclusion that the vibratory motions of the particles finally become normal to the wave, whatever be the direction of the original disturbance.* To this Fresnel replied that the equations of motion of elastic fluids employed by M. Poisson are but a mathematical abstraction, which do not apply to. anything actually existing; that, in fact, these fluids are assumed to be composed of contiguous elements, capable of compression in a degree proportionate to the pressure exerted; that this hypothesis is untrue; and that, although it may accord with the statical properties of these fluids, it can never lead to the discovery of theirdynamical laws. $†$

M. Poisson seems to have felt the full force of this objection; for in his memoirs on the same subject, read to the Academy in the years 1828 and 1830, he has resumed the whole theory, and reared it upon its firmer basis. In the former of these memoirs he has formed the differential equations of equilibrium and motion of elastic bodies, these bodies being supposed to consist of molecules attracting or repelling one another according to some function of the distance. $\neq$ In the latter he proceeds to integrate these equations. generally, and to deduce the laws of propagation of waves at a considerable distance from the origin of disturbance. $\S$ In the case of fluids. he arrives at the conclusion which he had before obtained, namely, that when the distance from the origin of disturbance is very great compared with the length of a wave, the motion of the particles, in any fluid, is normal to the surface of the wave, whatever be the initial motions. He admits, however, that the fundamental equations of the motion of fluids, and therefore also the consequences deduced from them, will probably require modification in the case of very rapid motions, such as those of the luminiferous ether; there being a finite interval of time, whose magnitude depends on

* Annales de Chimie, tom. xxii.

+ Ibid., tom xxiii.

¥ "Mémoire sur l'Equilibre et le Mourement des Corps Elastiques."-Mém. Inst., tom. viii.

" Mémoire sur la Propagation du Mouvement dans les Milieux; Elastiques." Mém. Inst., tom. $x$. 
the nature of the fluid, during which the pressure is not the same in all directions. In the case of very rapid motions this time must be taken into account, and the equations of motion of fluids will no longer be those furnished by the principle of D'Alembert.*

M. Poisson has shown also that a disturbance produced in a limited portion of a solid body will give rise to two vaves, which will be propagated with different velocities. He proves further that, whatever be the initial motions of the disturbed particles, the vibrations in one of these waves will finally be radial, or in the direction of the motion propagated, while those of the other are perpendicular to that direction, or transversal. The first are attended with dilatations proportionate to the absolute velocities of the molecules, and the waves thus propagated are similar to those which take place in fluids. The transversal vibrations, on the other hand, are unaccompanied by any change of density in the medium. M. Poisson does not seem to think that this result can justify the hypothesis of transversal vibrations in the ethereal fluid; though he admits that the properties attributed to the ether are in some respects analogous to those of a solid body.

The propagation of transversal vibrations appears to be now established, as a necessary consequence of dynamical principles, by the able researches of $\mathbf{M}$. Cauchy. $\dagger$ I shall shortly have occasion to allude more particularly to the important conclusions arrived at by this mathematician, in applying the general laws of the propagation of motion in elastic media to the case of light. For the present it will be sufficient to observe that the form of the wavesurface, obtained in the course of these investigations, is a curved surface of three sheets; and that, consequently, a ray of light on entering any medium will be, in general, subdivided into three rays, the directions of the vibrations being determined in each. When the elasticity of the ether, in this medium, is the same in all directions, these three rays will have a common direction, and two of them a common velocity. They' are thus reduced to two-a single and a double ray - coincident in direction, the vibrations of the former being parallel to that direction, and those of the latter perpendicular to it. If the initial vibrations in the system in question are contained in a plane perpendicular to the direction of the

- Annales de Chimie, tom. xliv.

† "Mémoire sur la Théorie de la Lumic̀re."-Mém. Inst., tom. x. 
rays, the single ray will vanish, and the vibrations of the molecules of the double ray will be constantly parallel to the direction of the initial displacements. This condition therefore reduces the three rays to one, which is unpolarized; and as this is known by experience to be the case in media in which the light is propagated in all directions with the same velocity, it follows that the propagation of transversal vibrations is a necessary consequence of the general theory.

Thus the theory of Young and Fresnel has received the strongest possible confirmation; and when we consider the numerous and important conclusions which have been reproduced and confirmed by $\mathbf{M}$. Cauchy in the development of his analysis, it is scarcely possible to believe that there is anything defective in its. principle. There is one important and fundamental difference, however, between the theories of M. Cauchy and Fresnel-a difference which seems to mark the limits to which we have attained in this branch of mathematical physics. According to the latter author, it has been already stated, the vibrations are perpendicular to the plane of polarization, as it is usually defined : according to M. Cauchy they are parallel to that plane. I am inclined to think that the field on which this question between the two theories must be decided is their application to the laws of reflexion of polarized light; and, if so, there seems already reason for believing: that the hypothesis of Fresnel is the true one.

\section{Reflexion and Refraction of Polarized Light.}

Although the phenomenon discovered by Huygens was one of the highest interest in itself, and in its bearings of such importance, in the mind of Newton, as to force him to admit the existence of properties in the rays of light which until then had never been imagined; yet the result remained for more than onehundred years a unique fact in science, and the kindred phenomena-the properties which light acquires in a greater or less degree in almost every modification which it undergoes-remained unnoticed until the beginning of the present century. In the year 1808, while Malus was engaged in his experimental researches on the Huygenian law of double refraction, he discovered the important fact, that when a ray of light is reflected from the surface of glass or water at certain angles, the reflected 
ray acquires all the characters which had been found to belong to one of the pencils produced by double refraction. When received upon a rhomb of Iceland spar, one of the two pencils into which it is generally divided vanished in two positions of the principal section with respect to the plane of reflexion; while in intermediate positions these pencils varied in intensity through every possible gradation.* The same variations were observed when it underwent a second reflexion at the same angle at which the effect was produced by the first; the twice-reflected light being a maximum when the plane of the second reflexion coincided with that of the first, and vanishing altogether when it was perpendicular to it, the whole light in that case passing into the refracted pencil. To represent the intensity of the reflected light, in any position of the plane of the second reflexion with regard to the first, Malus assumed it to vary as the square of the cosine of the angle which these planes formed with one another. $t$ The accuracy of this law has since been verified by the observations of $M$. Arago and others.

From this law it follows that a beam of common light may be represented as composed of two polarized beams of equal intensity, whose planes of polarization are at right angles; for when such a compound beam is received upon a reflecting surface at the polarizing angle, the intensity of the reflected light will be constant, and independent of the position of the plane of reflexion. But though this compound beam so far exhibits the character of common or unpolarized light, it must not be regarded-as it seems to be by many writers-as its physical representative. It appears, in fact, from the theory of the composition of vibrations, that two rays of equal intensity polarized at right angles compound a single ray polarized in a single plane, when the difference of their phases is nothing, or equal to any integer number of semi-undulations; while in intermediate cases the polarization of the resulting light is either circular or elliptic. These indications of theory have been confirmed in the fullest manner by a beautiful experiment of Fresnel.

On pursuing his inquiries Malus found that all other transparent substances impressed upon the reflected light the same modifcation; and that the angle of incidence at which this effect was 
produced, and which he called the angle of polarization, was in general different for every different substance. He ascertained, moreover, the relation between the angles of polarization at the first and second surfaces of the same transparent medium, and found that their sines were in the ratio of the sines of incidence and refraction;-so that when the medium is bounded by parallel surfaces, and the light incident on the first at its polarizing angle, the transmitted portion will meet the second surface also at its polarizing angle, and the light reflected from both will be wholly polarized.* Malus was unable, however, to discover any connexion between the polarizing angle and the other properties of the substances; and he concluded that the power of polarizing light by reflexion, which different bodies possessed at different angles, was wholly independent of their other modes of action upon light.

Sir David Brewster commenced, not long after, an extensive series of experiments, with the view of determining the angles of polarization of different media, and of connecting them by a law. These researches terminated in the discovery of the law-perhaps the most beautiful in the whole range of this interesting sciencethat "the tangent of the angle of polarization is equal to the refractive index." This law, when translated into geometrical language, declares that, when the ray is wholly polarized by reflexion, the angles of incidence and refraction are complementary, so that the reflected and refracted rays form a right angle. The law applies to the case of reflexion from the surface of the rarer, as well as that of the denser medium; and it follows from it that the two angles of polarization at the bounding surface of the same two media are complementary. $\dagger$

Malus observed that when the angle of incidence was either greater or less than the polarizing angle, the properties already described were only in part developed in the reflected pencil.

- Mémoires d'Arcueil, tom. ii. p. 152. M. Arago has extended the same law to the case of partial polarization, and has found that the sines of the angles at which the first and second surfaces of a transparent medium polarized light by reflexion in an equal degree are to one another in the ratio of the sines of incidence and refraction; so that the pencils reflected from the two surfaces of a parallel plate, at any incidence, contain the same proportion of polarized light.

+ "On the Laws which regulate the Polarization of Light by Reflexion from transparent Bodies."-Phil. Trans. 1815. 
Neither of the two pencils, into which it was divided by a rhomb of Iceland spar, ever wholly vanished; but they varied in intensity between certain limits, these limits being closer the more remote the incidence from the angle of complete polarization. From this he naturally concluded that, in these circumstances, a portion only of the reflected pencil had received the modification to which he had given the name of polarization, that portion increasing as the incidence approached the polarizing angle; and that the remaining portion was unmodified, or in the state of common light. In this supposition Malus has been followed by most subsequent philosophers. A different view of the phenomenon of partial polarization has been taken by Sir David Brewster, to which I shall have occasion presently to allude; and he has employed his theory to explain a phenomenon which he seems to have been the first to observe,-namely, that common light may be polarized by a sufficient number of reflexions at any angle, the number of reflexions required to produce the effect being greater the more remote the incidence is from the polarizing angle.*

Examining the transmitted pencil, Malus found that it was partially polarized; and that its plane of polarization was not, like that of the reflected pencil, coincident with the plane of reflexion, but perpendicular to it. $\dagger$ The two portions of light thus polarized in opposite planes he observed to be intimately connected; and in a subsequent memoir he announced the fact that, whenever we produce by any contrivance a ray polarized in any plane, there is produced at the same time a second ray polarized in the opposite plane. These two polarized rays follow separate paths, and their quantities are always proportionate. The connexion, however, is still more strict than was supposed by Malus; for the quantities of polarized light in the reflected and transmitted pencils are not only proportionate, but absolutely equal. This remarkable law was discovered by $\mathbf{M}$. Arago.

When a ray, which is partially polarized by transmission through a plate of glass, is received upon a second plate at the same angle, the portion of common light which it contains undergoes a new subdivision; and so continually, whatever be the 
number of plates. Hence, when that number is sufficiently great, the transmitted light will be, as to sense, completely polarized; and the whole light is thus subdivided into two pencils oppositely polarized, one of which is reflected from, and the other transmitted through, the pile. These facts were also observed by Malus. The laws of the phenomena have since been investigated, in much detail, by Sir David Brewster; and he has arrived at the conclusion, that when a ray of light is transmitted successively through any number of parallel plates, the tangent of the angle at which the polarization of the refracted pencil appears complete is inversely as their number.*

I may now proceed to consider these phenomena in their relation to the two theories of light.

Newton proved that the fundamental laws of reflexion and refraction could be derived from the operation of attractive and repulsive forces exerted by the molecules of body on those of light. The phenomena of polarization, however, show that these forces are exerted in very different degrees, according to the position of the sides of the ray with respect to the plane of reflexion or refraction; and we are now to consider the additional hypotheses which become necessary in the theory of emission in order to render an account of these new facts.

It has been already mentioned that, in the theory of M. Biot, a polarized ray was one in which certain axes (called the axes of polarization) of all the molecules were turned in the same direction. This effect is ascribed to the operation of certain forces emanating from the molecules of the body. These forces M. Biot denominates polarizing forces; and he considers them as distinct from the refleoting and refracting forces, although intimately connected with them. The effect of a polarizing force is to give a rotation to the axes of the molecules; and that which impresses the property of polarization upon the reflected ray is assumed to act in the planeof reflexion. This being supposed, since a ray of common light is polarized by reflexion when incident at a certain angle, we are obliged to admit that, at this angle, the polarizing force turns the axes of polarization of all the molecules, and brings them into the plane of reflexion; and, since this takes place for all the molecules 1814. 
of the reflected ray, such an arrangement of the axes is conceived to be a necessary condition of reflexion at that incidence.

Now, let such a polarized ray fall upon a second reflecting surface at the polarizing angle, and let the plane of the second reflexion be perpendicular to that of the first. Then the axes of polarization of the molecules, in their incidence on the second plate, are perpendicular to the plane of reflexion; and consequently, the polarizing force, acting in that plane, affects equally the two halves of the axis, and cannot therefore turn it into the plane of reflexion,- - a condition which is assumed to be necessary to reflexion at that angle. No light therefore is reflected. But when the plane of the second reflexion is inclined to that of the first at any angle less than $90^{\circ}$, the polarizing force of the second plate no longer acts symmetrically on the two halves of the axes of the molecules: it may therefore turn these axes so as to make them coincide with the plane of reflexion, and thus subject the molecules to the action of the reflecting force. The effect of the polarizing force increases as the inclination of the two planes of reflexion diminishes; and consequently the number of molecules. reflected by the second plate increases likewise.

But here it is necessary to make another supposition. In any position of the plane of the second reflexion with respect to the first, except the perpendicular one, experience proves that a portion of the light is reflected, and another portion refracted. According to this theory, then, some of the molecules obey the polarizing force, and have their axes brought into the plane of reflexion, while others do not. To account for this diversity of effect there must be some diversity of condition in the molecules. themselves. The theory of M. Biot supplies this by attributing to them an oscillatory movement round their axes of polarization, the molecules yielding to the polarizing force, or not, according to the phase of the oscillation in which they are found at the moment they reach the surface.

The force which impresses the property of polarization upon the refracted pencil is supposed by M. Biot to act also in the plane of incidence, its operation however being to turn the axes of polarization of the luminous molecules in a direction perpendiculur to that plane. Thus, when a ray of light traverses the surface of a plate of glass at the polarizing angle, it is subjected to the action of two forces, one tending to bring the axes of polarization of the 
molecules into the plane of incidence, the other to turn them at right angles to it; and the molecules themselves yield to one or other of these forces according to the phases of their fits. For the manner in which this may be supposed to take place we must refer to the Traité de Physique.* The whole quantities of light oppositely polarized by the two forces M. Biot supposes to be equal; but he conceives that the force which polarizes the reflected pencil is exerted on a much greater number of molecules than those which actually undergo reflexion. These molecules, thus polarized in the plane of incidence, enter into the transmitted beamneutralize an equal number of molecules polarized by refraction in the opposite plane,-and compound with them a beam of common light. The whole quantities of light polarized by the two forces being then equal, the remaining portions effectively polarized will still be equal, conformably to the law discovered by M. Arago.

I have endeavoured to present the theory of $\mathrm{M}$. Biot as fully as the limits of the present paper will permit, because it appears to me that the number and the nature of the hypotheses required, in order to render any account of the phenomena of polarization in the theory of emission, furnish in themselves a sufficient argument against it. But let all these be admitted, and how far can we be said to have advanced towards an explanation of the phenomena? The assumed forces and the known laws have not been connected, in any one instance, by the sure processes of mathematical deduction; and we are therefore unable to state how far the explanation offered is competent to express even the general facts, - far less can we calculate them numerically, and compare the results with those of observation.

The first attempt to connect the modifications of reflected light with the theory of waves was made by Dr. Thomas Young. This sagacious philosopher succeeded in solving the problem of reflexion in the case of perpendicular incidence, and showed that the intensity of the reflected light in that case was represented by a simple function of the refractive index. $†$ This formula was afterwards reproduced as the result of a more refined analysis by M. Poisson, in a memoir on the simultaneous motions of two elastic fluids in contact, read to the French Academy in 1817. $\neq$ In that memoir,

* Book vi. chap. i. vol. iv.

† Encyc. Brit. Supp., Art. Chromatics.

† Mém. Inst., tom. ii. 
however, the author had considered only the case of perpendicular incidence, or the law of propagation of a plane wave parallel to the bounding surface of the two media. In a subsequent memoir, to which I have already alluded, and which was read to the Academy in the year 1823, ${ }^{*}$ he has resurned the problem generally, and examined the modifications produced in the intensity as well as the direction of a wave, or series of waves, in passing from one fluid to another of the same elasticity, but of a different density. The expressions obtained for the intensity of the reflected and refracted waves are functions of the angle of incidence, and of the ratio of the velocities of propagation in the two media. When the wave is incident upon the surface of the denser medium, the expression for the intensity of the reflected wave vanishes at a certain angle, whose tangent is equal to the ratio of the velocities of propagation. At this angle, which is the angle of complete polarization, objects should therefore cease to be visible by reflected light; - $\mathrm{a}$, result which is contradicted by all experience, and is only true when the light is polarized in a plane perpendicular to the plane of reflexion. When the wave is reflected at the surface of the rarer medium, there are two expressions for the intensity, for incidences above and below the limiting angle of total reflexion, respectively. There are also in this case two angles of evanescence. These conclusions, which apply to the case of sound as well as light, are sufficient to show the physical inapplicability of the theory.

The theory of waves, however, when combined with the principle of transversal vibrations, has afforded the complete solution of the problem we have been considering. In this development of his theory the character of Fresnel's genius is strongly marked. Our imperfect knowledge of the precise physical conditions of the question is supplied by bold, but highly probable assumptions : the meaning of analysis is, as it were, intuitively discerned, where its language has failed to guide; and the conclusions thus sagaciously reached are finally confirmed by experiments chosen in such a manner as to force Nature to bear testimony to the truth or falsehood of the theory. $\dagger$

* Only a portion of this memoir has been printed in the Memoirs of the Institute, under the title " Mémoire sur le Mouvement de deux Fluides elastiques superposés," tom. $x$.

+ Fresnel's theory of reflexion is contained in a memoir read to the Academy of 
It is evident that the strata of ether in the two media, which are adjacent to the bounding surface, must undergo equal displacements parallel to that surface, inasmuch as one of them cannot slide on the other. Consequently the amplitude of the vibration, resolved in a direction parallel to the surface, must be the same in the two media. Fresnel assumes that this equality at the bounding surface is maintained at all distances; and this furnishes him with one relation among the amplitudes of vibration of the incident, reflected, and refracted waves. A second relation among the same quantities is afforded by the law of the vis viva; but to apply this it is necessary to know the relative densities of the ether in the two media. Here Fresnel assumes that the elasticity of the ether in these media is the same, ${ }^{*}$ but the density different; and this being taken for granted, it follows that the two densities are to one another inversely as the squares of the velocities of propagation, and that therefore their ratio is given when the refractive index is known. The amplitudes of the reflected and refracted vibrations, and therefore also the intensities of the light in the two pencils, are obtained by simple elimination between the equations just mentioned.

The expressions for the intensity of the light in the reflected ray are different, according as the incident light is polarized in the plane of reflexion or in the perpendicular plane. $t$ The intensity of the reflected light in the latter case vanishes when the sum of the angles of incidence and refraction is a right angle; and thus was solved the difficulty, which-in the opinion of Young, pronounced but three years before- "would probably long remain, to mortify the vanity of an ambitious philosophy, completely unresolved by any theory." When common, or unpolarized light, therefore, is

Sciences in the year 1823, entitled, "Mémoire sur la Loi des Modifications que la Reflexion imprime à la Lumière polarisée." An incomplete extract of this memoir was published in the Annales de Chimie, 1825. The original paper was mislaid, and for a time supposed to be lost; it has lately, however, been recovered among the papers of M. Fourier, and has been printed in the 11th vol. of the Memoirs of the Institute.

* Fresnel states that he had solved the problem of reflexion in the general supposition that the two media differ in elasticity as well as density, in the case of rays polarized in the plane of reflexion; and that the resulting formula was the same as that at which he had already arrived on the more limited hypothesis. $-A n$. Chim., tom. $x$ xiii.

+ These two formulæ were first published in the Annales de Chimie, 1821; the second without demonstration. 
incident at an angle whose tangent is equal to the refractive index, the reflected light will be wholly polarized in the plane of reflexion; and the beautiful law of Brewster is among the first fruits of the theory of Fresnel. The remarkable law obtained by M. Arago is also a necessary consequence of the same formulø; and it is easily inferred that the quantities of polarized light in the reflected and refracted pencils are equal, whatever be the incidence.

In the case of perpendicular incidence, these formulø are both reduced to the simple expression obtained by Young and Poisson; and when the incidence is $90^{\circ}$, or the ray grazes the surface, the intensity of the reflected light is equal to that of the incident, or the whole of the light is reflected whatever be the reflecting medium. The latter conclusion has been verified by the observation of the bands produced by the interference of direct light with that which is reflected at an incidence of nearly $90^{\circ}$. The first dark band appears to be perfectly black; and therefore the two lights are, as to sense, of equal intensity.*

We are thus furnished with the solution of a problem which has long baffled the labours of experimentalists,-namely, the determination of the law of intensity of reflected light as dependent on the incidence. The formulø obtained have not been compared with experiment by Fresnel except in the case of two observations of M. Arago, the observations of Bouguer and Lambert being confessedly inaccurate. The result of the comparison alluded to has been given in the Annales de Chimie, $\uparrow$ and the agreement is as satisfactory as can be expected in observations of the kind.

Mr. Potter has recently examined the intensity of the light reflected from diamond and glass of antimony, at various incidences. $\neq$ The photometrical method employed in these observations consisted in comparing the light reflected at any incidence from the substance examined with that reflected from a piece of crownglass, and then varying the incidence on the latter until the intensities are observed to be equal. The intensity of the light reflected from crown-glass at various incidences had been already obtained from a detailed series of experiments, and the results em-

* "On a New Case of Interference."-Tians. Royal Irish Aeademy, vol. xvii.

+ Tom. xvii. p. 190.

† Ihil Mag., Third Series, rol. i. p. 179 ; rol. iv. p. 6. 
bodied in an empirical law, in which the intensity is represented by the ordinate of a rectangular hyperbola, the corresponding abscissa being the sine of incidence. This formula then gives the intensity of the light reflected from crown-glass, and therefore also. from the substance examined, at the corresponding incidences. $\mathrm{Mr}$. Potter concludes, in this manner, that the intensity of the light reflected from diamond at a perpendicular incidence is $9 \cdot 3$, and that from glass of antimony $8 \cdot 2$, the intensity of the incident light being represented by 100 . The intensities calculated from the refractive indices, by the formulæ of Young, Poisson, and Fresnel, are 18.36 , and 13.33 , respectively. This variance in the results of theory and experiment is undoubtedly beyond the limits of the errors of observation; and, were it otherwise, the partial results obtained by $\mathrm{Mr}$. Potter, in these and other experiments of the same nature, agree too closely to permit us to refer the discrepancy to such a source. The principle of the method, however, appears (to say the least) uncertain; and it cannot but be wished that some of the various photometrical methods recently proposed should be applied to the examination of this interesting question.

The formulø of Fresnel supply the account of the remarkable phenomenon observed by M. Arago;-namely, that when Newton's rings are formed between a lens of glass and a metallic reflector, one of the two images into which they are divided by a double-refracting crystal, whose principal section is parallel or perpendicular to the plane of reflexion, changes its character as the incidence passes the polarizing angle of the glass; the colours being the same as in the other image when the incidence is less than the polarizing angle, but complementary to them when it is greater. In fact, when the incident light* is polarized perpendicularly to the plane of reflexion, the amplitude of the reflected vibration (which vanishes at the angle whose tangent is equal to the refractive index) changes sign in passing through zero, being negative when the incidence is less than that angle, and positive when it is greater. Consequently, if the wave reflected from the glass, at the central spot, is in complete discordance with that reflected from the metal in the former case, it will be in complete accordance with it in the latter; and the centre, which before was black, will then be white. For the same reason the whole system.

* The effect is the same whether the light be polarized before or after reflexion. 
will be complementary to that which it was before. Professor Airy was led to anticipate this result from the consideration of Fresnel's expressions, and afterwards verified it on trial,*-apparently without any knowledge of the facts observed by M. Arago. A similar confirmation of the same principles may be obtained by combining, in Fresnel's experiment, a metallie reflector with one of glass. The light being polarized perpendicularly to the plane of reflexion, the central band will be white, when the angle of incidence is below the polarizing angle of the glass; at the polarizing angle, the interference bars will vanish altogether; and beyond that incidence they will reappear with a dark centre, instead of a white one. This method of observation would seem to be peculiarly adapted to the investigation of the change of phase produced by metallic reflexion at various incidences.

By the same considerations Professor Airy was led to expect that when Newton's rings were formed between two transparent substances of different refractive powers-the light being polarized perpendicularly to the plane of incidence,-the rings should be black-centred, when the incidence was less than the polarizing angle of the low-refracting substance, or greater than that of the high-refracting substance; while they should appear with a white centre, when it was intermediate to these angles;-the vibrations of the waves reflected from the two surfaces being of opposite signs in the former case, and of the same sign in the latter. All these expectations were fully confirmed by experiment. $\dagger$ The substances selected by Professor Airy for these observations were plate-glass and diamond,-these substances differing very widely in their refractive powers; and in the course of his experiments he has noticed certain peculiarities in the phenomena, from which he has drawn some highly interesting conclusions respecting the nature of reflexion from diamond. Had this been subjected to the ordinary laws, the reflexion should cease, and the rings disappear, at the polarizing angles of both substances. This however was not the case. The rings did not vanish at the polarizing angle of the diamond; but the first black ring contracted, as the incidence was gradually increased, and finally usurped the place

* "On a Remarkable Modification of Newton's Rings."-Cambridge Trans. 1832.

+ "On the Phenomena of Nowton's Rings, when formed between two transparent strbstances of different refractive powers."-Cambridge Trans. 1832. 
of the central white spot. A portion of the light is therefore still reflected at the maximum polarizing angle of diamond; and it is evident from the phenomenon that the transition from a white to a black centre is owing to a gradual change of phase of the reflected vibration, amounting to nearly $180^{\circ}$, while the coefficient of the vibration itself is not much altered. The diamond therefore has no angle of complete polarization; and Professor Airy concludes that the nature of the reflexion from this singular substance, in the neighbourhood of the angle of maximum polarization, is different from any that has been hitherto described.

Fresnel's theory of reflexion has received experimental confirmation of a different kind, and to an extent which leaves little ground to doubt of its truth. When a ray polarized in any plane falls upon a reflecting surface at any angle, the reflected ray is still polarized, but its plane of polarization is changed-the amount of the change depending on the incidence. The law of this change is at once furnished by the theory of Fresnel; for the tangent of the inclination of the plane of polarization of the reflected ray to the plane of incidence is equal to the ratio of the displacements in the plane of incidence and in the perpendicular plane. The formula thus deduced has been verified in the most complete manner by the observations of Fresnel himself, and more fully since by those of M. Arago and Sir David Brewster.*

The views of the latter philosopher respecting the nature of partially-polarized light are founded upon the phenomenon of the change of the plane of polarization by reflexion. If common light be conceived to consist of two pencils oppositely polarized, in planes inclined $45^{\circ}$ on either side of the plane of reflexion, the effect of reflexion, it is obvious, will be to bring each of these planes nearer to the plane of incidence; so that the planes of polarization of the two pencils will approach each other, and form an acute angle after reflexion. Partially-polarized light, then, according to Sir D. Brewster, consists of two polarized pencils, whose planes of polarization form an acute angle; and no portion of it is in the condition of ordinary light. $\dagger$ This hypothesis receives some support from

* Annales de Chimie, tom. xvii.; Phil. Trans. 1830.

+ Sir David Brewster has computed, on these principles, the 'quantity of light apparentls polarized in the plane of incidence, by a single reflexion at-any angle, adopting Fresnel's expression for the intensity of the reflected ray. The agreement of the 
the explanation which it affords of the effects of successive reflexions. When light thus constituted is received upon a second reflecting surface, in the same plane of incidence, the planes of polarization of the two pencils will be brought nearer, and so continually; until by a sufficient number of reflexions, these planes will, as to sense, coincide with the plane of incidence, and the resulting light will appear to be wholly polarized in that plane.

This ingenious theory seems open to an objection already noticed,-namely, that the light resulting from the union of two oppositely polarized pencils cannot, in all respects, be taken as the physical representative of common or unpolarized light. It also involves this further difficulty, that the positions of the planes of polarization of the two oppositely-polarized portions are entirely arbitrary; and that if they be differently assumed, the results will be physically different. Thus, for example, if the two planes be taken, one coincident with the plane of reflexion itself, and the other with the perpendicular plane, neither of these planes will be changed by reflexion, although the intensities of the corresponding pencils will.

Sir David Brewster has also investigated experimentally the effect of refraction upon the plane of polarization of the refracted ray; and he has found that the law of the change may be expressed by a very simple and elegant formula.* This formula is a necessary consequence of Fresnel's theory, although Fresnel himself does not seem to have observed it. Its discovery by Sir David Brewster adds one to the many instances of rare sagacity by which this philosopher is guided in his experimental inquiries. The partial polarization of light by refraction has been considered by Sir David Brewster in the same memoir. In the investigation of the quantity of polarized light in the refracted pencil, he employs a principle similar to that which he had already applied to the reflected ray; and he arrives at the result that the quantities of

formula with the observations of M. Arago is found to be as near as can be expected in such comparisons. "On the Law of PartialPolarization of Light by Reflexion." $-P$ hil. Trans. 1830.

* $a$ and $a^{\prime}$ being the azimuths of the planes of polarization of the incident and refraeted rays, estimated from the plane of reflexion, and $i$ and $i^{\prime}$ the angles of incidence and refraction,

$$
\cot a^{\prime}=\cot a \cos \left(i-i^{\prime}\right) \text {. }
$$

"On the Laws of the Polarization of Light by Refraction."-Phil. Trans. 1830. 
polarized light in the reflected and refracted pencils are precisely equal, whatever be the incidence, conformably to the law of M. Arago. The effects produced by successive refractions are accounted for on the same principles.

Sir David Brewster seems to have been the first who studied the effects produced by total reflexion upon polarized light; and he observed, in particular, the complementary colours which the light thus reflected furnished when analyzed with a rhomb of Iceland spar.* At this time both he and Dr. Young concurred in thinking that these phenomena arose from the interference of two portions of light, which were reflected at unequal depths; one portion, according to Dr. Young, beginning to be refracted, and being then turned back by the continued exercise of the same power. $\dagger$

Fresnel had likewise observed, at an early period of his inquiries, that when a ray, polarized in a plane inclined at an angle of $45^{\circ}$ to the plane of incidence, undergoes total reflexion, it is in part depolarized; and that this depolarization is rendered completeby two total reflexions at an incidence of about $50^{\circ}$. The reflected light being then circularly-polarized is, according to theory, composed of two equal pencils, one polarized in the plane of incidence, and the other in the perpendicular plane, and differing in their origin by a quarter of a wave. From this it followed that the two pencils into which the incident light may be resolved, polarized in these two planes, are not reflected at the same depth,- or that they have undergone unequal changes of phase at the moment of reflexion, so that after reflexion one of them is in advance of the other. After many ineffectual attempts to discover in what manner this difference of phase depended on the incidence, Fresnel was at length led to the solution of the problem by the discussion of the formulæ for the intensity of the reflected light already noticed.

When the angle of incidence exceeds the angle of total reflexion,- - the light passing from the denser into the rarer medium, -these formula become imaginary. It is evident, however, from the law of the vis viva, that the intensity of the reflected light in this case is simply equal to that of the incident. How, then, are the imaginary expressions to be interpreted? They signify, ac-

* Journ. Royal Inst., vol. iii.

† Suppl. Encyc. Brit., Art. Сивomatics. 
cording to Fresnel, that the periods of vibration of the incident and reflected waves, which had been assumed to coincide at the reflecting surface, no longer coincide there when the reflexion is total; or, in other words, that the ray undergoes a change of phase at the moment of reflexion. The amount of this change is deduced, by a train of the most ingenious reasoning, from the general expressions. Now when a ray, polarized in any azimuth, is incident upon the reflecting surface at an angle greater than the angle of total reflexion, it may be resolved into two-one polarized in the plane of incidence, and the other in the perpendicular plane. The intensities of these two portions will not be altered by reflexion; but their phases will, and each by a different amount. The reflected vibration, therefore, will be the resultant of two rectangular vibrations differing in phase. This vibration, consequently, will be elliptic, and the reflected light will be elliptically-polarized. When the azimuth of the plane of polarization of the incident ray is $45^{\circ}$, the intensities of the resolved portions are equal ; and if, moreover, their difference of phase, after reflexion, is equal to a quarter of an undulation, the ellipse will become a circle, and the light will be circularly-polarized.

Reducing his formulæ to numbers, in the case of St. Gobain glass, Fresnel found that the difference of phase of the two portions of the reflected light amounted exactly to one-eighth of an undulation, when the angle of incidence was $54^{\circ} 37^{\prime}$. Polishing, therefore, a parallelopiped of this glass, whose faces of incidence and emergence were inclined to the other sides at these angles, it followed that a ray incident perpendicularly on one of these faces, and once reflected at each of the sides, should emerge perpendicularly at the opposite face, - the difference of phase in the two portions of the twice-reflected ray amounting to a quarter of an undulation. If, then, the incident ray be polarized in a plane inclined at an angle of $45^{\circ}$ to the plane of reflexion, the emergent light will be circularly-polarized. This was found to be the case on trial; and the parallelopiped thus constructed-and which is known under the name of Fresnel's r/lomb-is of essential service in experiments on circular and elliptic polarization. The results of this remarkable theory have been confirmed by Fresnel by other well-chosen experiments; so that, although the reasoning on which it is based is far from rigorous, there can remain little doubt of its general truth. Fresnel was himself fully aware of the incompleteness of his solu- 
tion, considered in an analytical point of view. In his memoir he has adverted to the method to be adopted in order to obtain an exact solution of the problem, unlimited by any arbitrary hypothesis; and he proposed himself to resume the question. But his. brilliant career of discovery was cut short by an untimely death.

The problem of the reflexion and refraction of polarized light has also engaged the attention of M. Cauchy.* The solution given by this mathematician is derived from a consideration of the conditions which must be fulfilled at the separating surface of the two media; and it assumes that the density of the ether is the same in both. The expressions obtained for the amplitudes of the vibrations in the reflected wave agree with those of Fresnel. The corresponding quantities for the refracted wave differ from those deduced from Fresnel's theory, by the simple inversion of the ratio of the sines of incidence and refraction, which occurs as a factor in both cases; and, thus, although the formulæ are different, their consequences agree in many instances,-as, for example, in the determination of the plane of polarization of the refractecl pencil. It is important to observe, however, that according to the formulæ of M. Cauchy, the velocities of the ethereal molecules in the refracted wave are greater than in the incident; so that the law of the vis viva is violated. This is not the case in Fresnel's. results, which are in fact derived from that law.

The phenomena of metallic reflexion remain yet to be noticed in connexion with this division of the science of light.

The effects produced upon light by reflexion at the surfaces of metals did not escape the scrutiny of Malus. From his first experiments upon the subject, Malus concluded that metals had no effect in polarizing the light. $\mathrm{H}_{\theta}$ soon, however, modified this opinion, and found that the phenomenon of polarization was partially produced, the effect increasing to a maximum as the incidence approached a certain angle. But the most instructive mode of studying these phenomena is to let fall upon the metallic reflector a ray polarized in a plane inclined at an angle of $45^{\circ}$ to the plane of reflexion, and to analyze the reflected pencil by a doublerefracting prism. Proceeding in this manner, Malus found that when the incidence was very small, or very great, the reflected ray was still polarized; while at moderate incidences it was dcpolarized,

* Bulletin Universel, tom. xiv. p. 6. 
and the pencil was divided into two in every position of the rhomb. From these facts Malus concluded that the difference between metals and transparent bodies consisted in this: that the latter reflect all the light which is polarized in one plane, and refract all the light polarized in the opposite plane; while metals, on the other hand, reflect light which is polarized in both planes.

The subject of metallic polarization was next examined by Sir David Brewster; and his labours on this subject constitute the most important addition which has been recently made to our knowledge of the laws of polarized light.* When light reflected at a metallic surface is analyzed by a double-refracting crystal, it is observed to be partially polarized in the plane of reflexion. The effect is greatest in galena, and least in silver; and the angle at which it is a maximum is about $74^{\circ}$, but varies with the metal. By successive reflexions in the same plane, Sir David Brewster found that the proportion of polarized light was increased; and that by a sufficient number of reflexions the light became, as to sense, wholly polarized in the plane of incidence. The number of reflexions required to produce this effect varied widely in different metals.

In order to determine the nature and laws of this phenomenon, it is necessary to examine the effect produced upon polarized light. Adopting, then, the method of Malus, Sir David Brewster found that when a ray of light, polarized in the azimuth of $45^{\circ}$, was received upon a metallic reflector at an incidence greater than $40^{\circ}$, and less than $86^{\circ}$, the reflected light was partly depolarized. The effect produced was greatest at an angle of about $74^{\circ}$; and when the light underwent a second reflexion in the same plane, and at the same angle, it was restored to light polarized in a single plane. This new plane lies always on the other side of the plane of reflexion; and its azimuth varies within the limits $0^{\circ}$ and $45^{\circ}$, being greatest for silver, and least for galena. It is evident, then, that the light produced by a single reflexion eannot be common light. Neither is it plane-polarized light, because it does not vanish in any position of the analyzing rhomb. Sir David Brewster concludes that this light has received a speeies of polarization hitherto unrecognised, intermediate between plane and circular polarization. He calls it elliptic polarization, because the angles of

* "On the Phenomena and Laws of Elliptic Polarization, as exhibited in the Action of Metals upon Light."-Phil. Trans. 1830. 
reflexion at whioh this light is restored to plane-polarized light, in any azimuth of the plane of the second reflexion with regard to the first, may be represented by the variable radii of an ellipse; while these angles are equal in all azimuths in the case of light circularly-polarized.

Sir David Brewster seems to have been led to employ the term "elliptic polarization" in this manner, in his desire to avoid as much as possible all reference to theory. The laws which he has obtained, however, belong to elliptically-polarized light, in the sense in which the term was introduced by Fresnel. It appears, in fact, from the theory of the composition of vibrations, as laid down by this author, that the vibration resulting from the union of two rectilinear and rectangular vibrations will be in general elliptic; so that two oppositely-polarized pencils compound in general a pencil elliptically-polarized-the ellipse becoming a right line, when the difference of phase of the two portions is an integer multiple of $180^{\circ}$. When, therefore, by the effect of reflexion, two such pencils are made to differ $90^{\circ}$ in phase-as Sir David Brewster has shown to be the case when a ray polarized in the azimuth of $45^{\circ}$ is incident at the maximum polarizing angle of the metal-a second reflexion, in the same plane and at the same angle, will raise the difference to $180^{\circ}$, and the resulting light will be plane-polarized. In other parts of his memoir, however, Sir David Brewster seems to acknowledge that theory, for he speaks of elliptic polarization as produced by the interference of two unequal portions of oppositely-polarized light, and even calculates their difference of phase for any incidence.

The identity of the light produced by metallic reflexion with the elliptically-polarized light of the wave-theory seems to be placed beyond all doubt by an observation of Professor Airy. When Newton's rings are formed between glass and metal-the incident light being polarized, and the angle of incidence exceeding the polarizing angle of the glass-it is found that the rings dilate, as the azimuth of the plane of polarization with respect to the plane of reflexion is increased; the dilatation being a maximum when these two planes become perpendicular. In order to account for this fact, Professor Airy has shown that if the vibrations of the incident pencil be resolved into two, one in the plane of incidence, and the other in the perpendicular plane, it is necessary to assume that their phases are unequally changed by reflexion; 
the phases of the vibrations in the plane of reflexion being more retarded than in the perpendicular plane. The two oppositely polarized portions, therefore, will differ in phase after reflexion, and will therefore compound a pencil elliptically-polarized. Professor Airy has observed a similar phenomenon when Newton's rings were formed between diamond and plate-glass, the angle of incidence being a few degrees less than the maximum polarizing angle of diamond; and he concludes that, for such incidences, the nature of reflexion from diamond is analogous to metallic reflexion.

Sir David Brewster has extended his researches on the subject of metallic reflexion to a great variety of cases, and has traced the effects of successive reflexions in the same, or in different planes; and at the same, or different angles. When the light which has been restored to plane-polarized light, by two reflexions in the same plane, and at the maximum polarizing angle, undergoes a third reflexion under the same circumstances, it becomes again elliptically-polarized. By a fourth reflexion it is again restored to planepolarized light, the plane of polarization being, however, brought nearer to the plane of reflexion. This continued approach of the plane of polarization to the plane of reflexion enables the author to explain, according to his peculiar views, the effect of successive reflexions upon common light.

It remains, further, to extend the theory of Fresnel to reflexion at the surface of a medium in which the elasticity of the ether is different in different directions. All that we know on this interesting subject we owe to the unwearied zeal of Sir David Brewster. It had been supposed by Malus, and the opinion seems to have passed current with succeeding philosophers, that the exterior surfaces of erystallized substances acted upon the reflected light exactly in the same manner as the surfaces of ordinary media; or, in the language of the theory of emission, that the reflecting forces extended beyond the limits of the polarising forces of the crystal. Sir David Brewster was led to doubt this opinion; and in the year 1819 he undertook an extensive series of experiments on the subject of crystalline reflexion. One of the first results at which he arrived was, that the angle of complete polarization on the same surface varies with the inclination of the plane of reflexion to the principal section of the crystal; being least when the plane of reflexion coincides with the principal section, and greatest when it is 
perpendicular to it;-and that with different surfaces the variation depended on the inclination of the surface to the axis of the crystal. The difference of the greatest and least angles in the case of Iceland spar, and on one of the cleavage planes of the crystal, was found to amount to more than $2^{\circ}$.

But the effects produced upon the plane of polarization are still more remarkable. On weakening the refleoting force, by causing the reflexion to take place at the surface of contact of the crystal and some fluid-such as oil of cassia - which had nearly the same refractive power, Sir David Brewster found that the ray was no longer polarized in the plane of reflexion; and that the deviation of the plane of polarization from the plane of reflexion depended on the angle which the incident ray formed with the axis of the crystal. This relation Sir David Brewster found to be expressed by the law, - that the sine of half the deviation varied as the square-root. of the sine of inclination of the incident ray to the axis.*

It is much to be desired that the attention of analysts should be directed to the problem of reflexion at the surface of extraordinary media. It is one of the very few important provinces of the science of light, which has not yet yielded its tribute to the wavetheory; and we can hardly conceive a finer subject for the exercise of mathematical and physical skill. $\dagger$

* "On the action of Crystallized Surfaces upon Light."-Phil. Trans. 1819.

+ Since the preceding was written, $\mathrm{Mr}$. M'Cullagh has arrived at an expression for the angle of polarization at the surface of crystallized media, in the case in which the plane of reflexion coincides with one of the principal sections of Fresnel's ellipsoid; and he has found that the law, which he has extended by analogy to all cases, represents with much exactness the observations of Sir David Brewster. If $a$ and $b$ denoto the semiaxes of the elliptic section formed by the intersection of the plane of reflexion with the ellipsoid of indices (or the ellipsoid whose axes coincide in direction with the axes of elasticity of the medium, and are equal to its three principal indices), and $r$ the radias of the same section coinciding with the face of the crystal, the angle of polarization, $\pi$, will be the same at whichsoever side of the perpendicular the ray is incident, its value being given by the formula,

$$
\sin ^{2} x=\frac{1-\frac{1}{r^{2}}}{1-\frac{1}{a^{2} l^{2}}} .
$$




\section{Double Refraction.}

The phenomenon of double refraction was first discovered by Erasmus Bartholinus, in Iceland spar. After a long series of observations, he found that one of the rays within the crystal observed the known law of refraction discovered by Snellius, while the other was bent according to a new and extraordinary law. An account of these experiments was published at Copenhagen in the year 1669, under the title "Experimenta Crystalli Islandici Discliaclastici, quibus mira et insolita refractio detegitur."

The success of Huygens in deriving the laws of ordinary refraction from the hypothesis of waves naturally led him to examine whether these new phenomena could be reconciled to the same theory; and in his desire to assimilate the two classes of phenomena, he was happily led to assign the true law of extraordinary refraction. Huygens had already shown that the direction of the refracted ray, in glass and other uncrystallized substances, could be deduced from the supposition that the ethereal wave within the substance was a sphere, - - or, in other words, that the velocity of undulatory propagation was the same in all directions. One of the rays in Iceland crystal, too, was found to obey the same law; and judging that the law which governed the other, though not so simple, was yet next in simplicity, he assumed the form of its wave to be the spheroid of revolution, the greater and the lesser axis of the generating ellipse being in the ratio of the greatest and least index of refraction. The form of the wave being known, the law of refraction is derived from the principle of the superposition of small motions. Conceive three surfaces having their oommon centre at the point of incidence, and representing respectively the simultaneous positions of three waves diverging from that point, the first in air, the other two within the crystal. Let the inoident ray be produced to meet the air vave, and at the point of intersection let a tangent plane be drawn. Through the line of intersection of this plane with the refracting surface let planes be drawn touching the two refracted waves; - the lines connecting the centre with the points of contact are the directions of the two refracted rays. This beautiful construction, and the other speculations of Huygens on the subject of extraordinary refraction, are contained in the fifth chupter of his Tiraité de la Lumière. 
Huygens was unable to reconcile the existence of a double wave within the crystal with the supposition of a single vibrating nedium; and ho was accordingly forced to assume the existence of two such media-the spherical wave being propagated by the vibrations of the ether alone, while the spheroidal wave arose from the vibrations of the crystal and of the ether jointly.

For the construction of Huygens Newton substituted another, without stating the theoretical grounds on which he formed it, or even advancing a single experiment in its confirmation.* In this unsatisfactory position the problem of double refraction was suffered to rest for nearly a century; and it was not until the period of the revival of physical optics in the hands of Young, that any new light was thrown upon the question. This sagacious philosopher was led by the theory of waves to assume the truth of the law of Huygens; and it was by his advice that Dr. Wollaston undertook the experimental examination $\dagger$ which recalled to it the attention of the scientific world, and ended in its universal admission. The French Institute soon after proposed the question of double refraction as the subject of their prize essay, and the successful memoir of Malus left no doubt remaining as to the accuracy of the Huygenian law. +

The examination of Malus was chiefly directed to the case of Iccland spar; but he made a few similar measurements, also, in quartz, sulphate of barytes, and arragonite. In the first of these erystals he mistook the ordinary for the extraordinary ray; and the faces which he chose for examination in the two latter not happening to be well adapted to the discovery of their properties, he was satisfied with a hasty generalization of the law observed in Iceland spar, and concluded that it belonged to all double-refracting bodies. Malus entered largely, in the same memoir, into several questions connected with the problem of double refraction; and he showed, in particular, that the laws of extraordinary reflexion at the second surfaces of crystals are deducible from the law of Huygens. In a memoir presented to the Institute, in the following year, $\S$ he extended considerably the list of bodies possessing

* Optics, book iii., query 25.

† "On the Oblique Refraction of Iceland Crystal."-Phil. Trans. 1802.

‡ "Théorie de la Double Refraction."-Mém. Inst.

$\S$ "Sur l'Axe de Refraction des Cristaux et des Substances organisées,"-MYém. Inst. 1811. 
the property of double refraction; and arrived at the conclusion that this property belonged to all crystals, excepting those whose primitive form was the cube or regular octahedron. Most organized substances, whether vegetable or animal, were found to possess the same properties.

In Iceland spar the extraordinary refractive index is less than the ordinary. The extraordinary ray consequently is always refracted from the axis of the crystal; and the same law had been supposed to belong to all double-refracting substances. M. Biot made the important discovery that, in many crystals, the extraordinary index was greater than the ordinary, and the extraordinary ray therefore refracted towards the axis. Crystals of the latter kind he called attractive, while those of the former were called repulsive; the extraordinary refraction being ascribed, in the theory of emission, to attractive or repulsive forces which act as if they emanated from the axis.* These crystals are now generally distinguished by the denominations positive and negative. The Huygenian law applies to positive as well as to negative crystals; the spheroid being prolate in the former case, and oblate in the latter.

The construction given by Huygens for the direction of the two refracted rays is, it has been stated, an immediate consequence of the assumed form of the wave-surface. It easily appears, from the principle of Huygens already adverted to, that the same construction will apply in all cases, whatever be the form of the wave, or the law of the velocity of propagation within the crystal; - so that the law of direction is determined when that of velocity is known. A similar connexion between the velocity of the molecule and its path is established, in the theory of emission, by the law - of least action. This principle, we know, holds generally in the motion of a point subjected to the action of attracting or repelling forces; and in applying it to the case of a luminous molecule, acted on by forces emanating from the particles of the body which it meets, we may leave out of consideration the insensible curvilinear portion of the trajectory described in the passage from one medium into another of different density,--provided we assume, with Newton, that the forces exerted by the molecules of body on those of light are sensible only at insensible distances. In this simplification of the problem we have to deal only with straight 
lines and uniform velocities; and when the dependence of these velocities on the directions is assumed, or given, the principle in question furnishes a relation between the directions of the two portions of the trajectory. Such was the problem whose solution was given by Laplace, in his memoir on the motion of light in transparent media ; and he has arrived at two equations in which that solution is completely contained. Laplace applied these results to two cases: one in which the difference of the squares of the velocities of the incident and refracted rays is constant,-and the other in which that difference is equal to a constant quantity, plus another varying as the square of the cosine of the inclination of the refracted ray to the optio axis. In the former of these cases he obtained the known law of Snellius; and the formulæ of refraction at which he arrived in the latter were found to be identical with those furnished by the construction of Huygens.

The velocity of the extraordinary ray, assumed by Laplace, is the reciprocal of the radius-vector of the ellipsoid of Huygens, and therefore the inverse of the assumed velocity in the wave-theory. But Laplace himself has shown that the construction suggested by that theory, and employed by Huygens for the determination of the direction of the refracted ray, resolves itself into the principle of least time, - and that whatever be the form of the wave-surface; and as the law of least action and that of least time are identical, provided the assumed velocities be reciprocal, it ceases to be strange that two such very different methods should lead precisely to the same result. The difference between Huygens and Laplace, as to the mode of deducing the law of extraordinary refraction, is in fact precisely the same as that which existed formerly between Fermat and Maupertuis with regard to the ordinary law of the sines.

This identity of the results afforded by the two theories has since been more distinctly pointed out by $\mathbf{M}$. Ampère. By means of the principle of least action he has arrived at the following general conclusion, whatever be the assumed law of the velocities, - that if from the point of incidence on any extraordinary medium, as centre, two surfaces be described whose radii-vectores are inversely as the velocities of the incident and refracted rays in their directions, and if the incident and refracted rays be pro- 
duced to meet these surfaces, and tangent planes be drawn at the points of meeting, the line of intersection of these planes will be on the separating surface of the two media.* Hence the position of the refracted ray is determined when that of the incident ray is known; and the construction thus supplied for its determination is obviously the generalization of the construction of Huygens already alluded to, if only the radii-vectores be trken in the direct ratio of the velocities, instead of the inverse.

It is obvious, then, that the problem of double refraction, considered as a physical question, resolves itself into the determination of the law of velocities. Newton showed that the constant ratio of the velocities in ordinary media, and therefore the law of the sines, could be explained on the supposition that the luminous molecules are solicited by attracting forces emanating from the molecules of the refracting body, and sensible only at very small distances. The phenomenon of extraordinary refraction, in like manner, was ascribed by Laplace to the operation of similar forces emanating from the molecules of the crystal, - but modified by the form of these molecules and those of light, and by the manner in which they are presented to each other. No attempt, however, has been made in the theory of emission to advance beyond the point at which Newton arrived, and to deduce the velocity of the extraordinary ray in crystallized media from any assumed constitution of the molecular forces ; $\uparrow$ and, indeed, when the condition of polarity is to be superadded to the laws of such forces, the theory seems embarrassed in inextricable difficulties. The refraction which a polarized ray undergoes in a crystal depends upon its plane of polarization; and, by á simple change of that plane, the refracted ray may be converted from an extraordinary to an ordinary ray. The extraordinary force then, it appears from the phenomena, exerts no effect upon a ray polarized parallel to the principal plane. Its effect is greatest upon a ray polarized in the perpendicular plane; and it must be supposed to act in every intermediate

\section{* Mém. Inst. 1815.}

+ Fresnel states, in the commencement of his memoir on double refraction, that Iaplace had derived the velocity of the extraordinary ray, in uniaxal crystals, from the hypothesis of a resultant force acting in a direction perpendicular to the optic axis, and varying as the square of the sine of the angle which the ray makes with that line. I have not been able to discover, in any of Laplace's writings, the discussion thus adverted to. 
degree upon rays polarized in intermediate planes. Now a ray of common light, in the theory of emission, is composed of molecules whose planes of polarization are turned in all azimuths; and these molecules, consequently, should feel the influence of the extraordinary force in every possible degree. Instead, therefore, of two refracted rays, such a ray should be divided into an infinite number, inclined in every possible angle between the limiting directions of the ordinary and extraordinary rays.

It had been hitherto assumed, that no crystal had more than one optic axis. While examining the rings which surround these axes in polarized light, Sir David Brewster made the important discovery that the greater number of crystals possess two optic axes; and he soon after discovered the connexion between these diversities of optical character and the crystalline form.*

The optic axes, however, as Sir David Brewster has shown, cannot be regarded in general as the fundamental axes of the double-refracting medium. $\mathrm{He}$ calls them apparent axes; and considers them as the resultants of others, which he denominates true or polarising axes, and from which the forces which produce the phenomena of polarization and double refraction are conceived to emanate. The polarizing force proceeding from a single axis is measured by the difference of the squares of the velocities of the ordinary and extraordinary rays, and is supposed to vary as the square of the sine of the angle which the direction of the ray within the crystal contains with it; and when two such axes cooperate, it is assumed that the increment of the square of the velocity, arising from their joint action, is equal to the diagonal of a parallelogram whose sides are the increments of the squares of the velocities produced by each separately, and whose angle is double of that formed by the two planes passing through the ray and the axes. $†$ From this hypothesis it followed that two rectangular polarizing axes of equal intensity, and both positive or both negative, compound a single resultant axis at right angles to both. This axis is of the same intensity as the component axes, but of an opposite character; and, accordingly, three equal rectangular axes of the same character balance each other's effects, and have no

* The important relations here alluded to have been already brought under the attention of the Association, in the able Report on Mineralogy, by Mr. Whewell.

+ " On the laws of Polarization and Double Refraction in regularly crystallized Bodies."-Phil. Trans. 1818. 
resultant. Thus, then, the laws of uniaxal crystals, as well as of singly-refracting media, are embraced in this hypothesis The case of two resultant axes is reduoible to that of two unequal polarizing axes; and it has been shown to be a consequence of the rule, that the difference of the squares of the velocities of the ordinary and extraordinary rays within the crystal is proportional to the product of the sines of the angles which the latter makes with the resultant axes. M. Biot was led to the discovery of this beautiful law by analogy,* and he afterwards observed that it was implicitly contained in the law proposed by Sir David Brewster.

The term "polarizing force" seems to have been adopted by Sir David Brewster without any reference to the law which governed the planes of polarization of the two pencils,-a law which, in biaxal crystals, still remained unknown. In the case of uniaxal crystals, it could not fail to be observed, the plane of polarization of one of the pencils contained the direction of the ray and the axis; while that of the other was a plane passing through the ray at right angles to the former. Conceiving that these planes, in biaxal crystals, must be symmetrically placed with respect to the planes passing through the ray and the two axes, M. Biot was led to the simple and elegant law-that the plane of polarization of one of the pencils was that passing through the ray, and bisecting the dihedral angle contained by these planes; while that of the other was perpendicular to the former, or bisected the supplemental dihedral angle. $\dagger$

When a ray of light enters a crystal, the component moleoules are supposed, in the theory of $\mathbf{M}$. Biot, to receive different motions round their centres of gravity, dependent on the nature of the forces exerted upon them by the particles of the body. Sometimes the molecules of the ray are turned by the operation of these forces, so as to have certain lines in each, denominated axes of polarization, all in the same direction; and this arrangement of the molecules is maintained throughout the whole of their future progress. There are other cases, however, according to this author, in which the molecules oscillate round their centres of gravity in certain periods, during their entire progress through the crystal;

* "Mémoiro sur les Lois générales de la Doublo Refraction, \&co," Mém. Inot., tom. iii.

+ Ibid. 
while in others, finally, they receive a motion of continued rotation. To the two latter cases I shall have occasion to advert hereafter.

The phenomena of fixed polarization are ascribed by M. Biot to the operation of certain forces, which he denominates polarizing forces. In the case of uniaxal crystals these forces are supposed to act in the planes containing the two rays and the axis of the crystal,-the ordinary polarizing force tending to arrange the axes of the molecules in the plane containing the ray and the axis, while the extraordinary polarizing force draws them towards the perpendicular plane. If the molecules were similarly circumstanced in every respect, they would necessarily obey the stronger of these forces, and there would be but one plane of polarization. This, however, is supposed not to be the case. Owing to the different phases of their fits, at their incidence upon the crystal, the molecules are disposed to yield more readily to one or other of these forces ; so that when a polarized ray meets a double-refracting medium, some of the molecules fall under the influence of the ordinary polarizing force, and have their axes of polarization turned into the plane containing the ray and the axis of the crystal, while others are actuated by the extraordinary force, and have their axes arranged in the perpendicular plane. The number of molecules which yield to one or other of these forces, or the intensity of the two polarized rays, is supposed to depend on the angle which the plane of primitive polarization makes with the two planes just mentioned. When the plane of polarization coincides with the former, the extraordinary force has no effect, and the ray receives only the ordinary polarization; the converse takes place when the plane of polarization coincides with the perpendicular plane. Similar suppositions were made to account for the phenomena of polarization in biaxal crystals.

Such was the state of the theory of double refraction when the subject was taken up by Fresnel. The law of refraction, we have seen, whether in the theory of emission or in that of waves, was intimately connected with, and dependent on the law of velocities; so that, considered as a physical question, the problem resolved itself into the determination of the latter. With the exception, however, of the reasonings of Young respecting the form of the wave-surface in a medium compressed or dilated in a given direction," no attempt had been made to deduce the velocity of the

* Quarterly Review, vol. ii. 
extraordinary ray from the principles of either theory. Indeed the general law of the velocities was itself unknown, even as an experimental fact, although an important relation between the velocities of the two pencils had been discovered by the labours of Sir David Brewster and M. Biot. But this was not all. It was evident that no physical theory of double refraction could be regarded as complete, which did not at the same time account for the attendant phenomenon of polarization. In this branch of the subject, however, nothing had been accomplished; and all that had been said in explanation of the phenomenon of polarization did not go further than some vague speculations as to its cause. The theory of Fresnel to which I now proceed,- and which not only embraces all the known phenomena, but has even outstripped observation, and predicted consequences which were afterwards fully verified,-will, I am persuaded, be regarded as the finest generalization in physical science which has been made since the discovery of universal gravitation.

Fresnel* sets out from the supposition that the elastio force of the vibrating medium is, in general, different in different directions. This is, in fact, the most general supposition that can be made; and whether we suppose that the vibrating medium is the ether within the crystal, or that the molecules of the body itself partake of the vibratory movement, there will be obviously such a connexion, and mutual dependence, of the parts of the solid and those of the medium in question, that we cannot hesitate to admit for the one what has been already established on the clearest evidence for the other. $t$ Now if a disturbance be produced in a medium so constituted, and any particle displaced from its position of rest, the resultant of the elastio forces which resist the displacement will not, in general, act in the direction of that displacement (as in the case of a medium uniformly elastic), and therefore will not drive the displaced particle directly back to its position of equilibrium. Fresnel has shown, however, that there are three directions at right angles to each other, in any of which, if the particles are displaced, the elastio forces do aot in the direction

" "Mémoire sur la Double Refraction," Mém. Inst., tom. vii.

† M. Savart has shown that the elasticity of crystals, determined by means of their sonorous vibrations, is, in general, different in different directions. The optic axis of Iceland spar is the axis of least elasticity: that of rock crystal is the axis of greatest elasticity. 
of the displacement, whatever be the nature or laws of the molecular action ; and the only assumption which he makes is-that these three directions are parallel all throughout the crystal.* These directions Fresnel denominates axes of elasticity. He conceives that they ought also to be axes of symmetry with respect to the erystalline form; but observes that M. Mitscherlich has noticed some crystals in which this does not hold. $\dagger$ If on each of these axes, and on every line diverging from the same origin, portions be taken which are as the square roots of the elastio forces in their direction, the locus of the extremities of these portions will be a surface which Fresnel calls the surface of elasticity. This surface determines the velocity of propagation of the wave, when the direction of its vibrations is given. For the velocity of undulatory propagation in an elastic medium, being as the square root of the elastio force, must be represented by the radius-vector of the surface of elasticity in the direction of the vibrations.

Now let us conceive a plane wave advancing within the crystal. By the principle of transversal vibrations the movements of the ethereal molecules are all parallel to the wave. But the motion of each displaced particle is resisted by the elastic force of the medium, and that force is, in general, oblique to the direction of the displacement. Fresnel shows, however, that the displacement may be resolved in two directions in the plane of the wave, such that the elastic force called into action by each component will be the resultant of two forces, one of which acts in the direction of the displacement itself, while the other is normal to the wave. The latter, by the principle of transversal vibrations, can produce no effect; and the former will give rise to a wave propagated with a constant velocity. These two directions, he finds, are those of the greatest and least diameters of the section of the surface of elasticity made by the plane of the wave; and if the original displacement be resolved into two, parallel to them, each component will give rise to a plane wave whose velocity of propagation is

* This will be the case, if the homologous lines of the groups of particles are all parallel; an arrangement at once the simplest and most natural, and which appears to be observed in most erystallized bodies. Fresnel admits, however, the possibility of other regular arrangements; and be conceives that the phenomena of circular polarization in rock crystal oblige us to suppose that its molecules are arranged according to some less simple law.

† Sec Bulletin de la Société Phi'omathique, March, 1824. 
represented by that diameter, and the vibrations in each wave will preserve constantly the same direction.

Thus it appears that a polarized plane wave will be resolved into two within the crystal; and these will be propagated with different relocities, and consequently follow different paths. The amplitudes of the component vibrations are as the cosines of the angles which the direction of the original vibration contains with the two fixed rectangular directions; and as the squares of these amplitudes represent the intensities of the two pencils, the law of Malus respecting these intensities follows as an immediate consequence.* Again, the planes perpendicular to these two directions are the planes of polarization of the two pencils ; and it is easily inferred that one of them must bisect the dihedral angle contained by the two planes passing through the normal to the wave, and the normals to the circular sections of the surface of elasticity, while the other is perpendicular to it. This conclusion does not coincide mathematically with the experimental law of M. Biot: but the differences are much within the limits of the errors of observation, and the results of experiment must be regarded as confirmatory of the theory.

The velocity of propagation of a plane wave in any direction being known, the form of the wave-surface diverging from any point within the crystal may be found. For if we conceive an indefinite number of plane waves, which, at the commencement of the time, all pass through the point which is considered as the centre of disturbance, the wave-surface will be that touched by all these planes at any instant. This surface is of the fourth order. Fresnel has deduced its equation, although in an indirect manner; and he has shown that it may be geometrically constructed by means of an ellipsoid whose semiaxes are the same as those of the surface of elasticity. The form of the wave-surface being known, the directions of the two refracted rays are given by the construction of Huygens.

From the construction now alluded to it appears that there

* Young seems to have been the first to observe that the law of the square of the cosine could be derived from the hypothesis of transversal vibrations, (Encyc. Brit., Chromatics, p. 161). The subject of the experimental confirmation of this important law has been recently brought before the French Aeademy by M. Arago, and he has indicated the praetical results which may be derivel from this law in its application to photometry.-Herschel's Essay on Lijht: French Tranisac'ion, Suppl., p. 590. 
are two directions-the normals, namely, to the two circular sections of the ellipsoid,-in which the velocity of the two rays is the same. These directions are called by Fresnel the optic axes, although he sometimes applies this term to the normals to the circular sections of the surface of elasticity, or the directions in which a plane wave is propagated with a single velocity. It thus. appears that crystals have in general two optic axes, and can have no more. When two of the three principal elasticities are equal, the two optic axes unite, and the wave-surface resolves itself intothe sphere and spheroid of revolution. Thus the form of the wave in uniaxal crystals, which Huygens assumed as the most natural, comes out as a simple corollary from the general theory of Fresnel. When, lastly, the three elasticities are all equal, the wave-surfacebecomes a sphere; the velocity is accordingly the same in all directions, and the law of refraction is reduced to the known law of Snellius.

It was easily shown to follow from the general construction, that the difference of the squares of the reciprocal velocities of the two rays, in biaxal crystals, is proportional to the product of the sines of the angles which their common direction within the crystal contains with the two axes; so that the remarkable law of Sir David Brewster and M. Biot is brought under the same theory. But it appeared further, from that theory, that the velocity of neither of the rays is constant, and that the refraction of both is performed according to a new law. This conclusion was at variance with all the received notions upon the subject; and indeed the experiments of M. Biot on limpid topaz* seemed to warrant his. assumption that the refraction of one of the rays followed the ordinary law of the sines. It became, therefore, a matter of much interest to decide this question by accurate experiment. This has been done by Fresnel himself by the ordinary method of prismatio refraction, as well as by the nicer means afforded by the displacement of the diffracted fringes; and the result in both cases has been conclusive in favour of his theory. The numerical data afforded by the observations of $\mathrm{M}$. Biot on topaz enabled Fresnel to compute, according to the principles of that theory, the velocity of the ray in different directions; and the observed variation was found to agree with that deduced.

* SLém. Inst., tom. iii. 
The phenomenon of dispersion, in singly-refracting substances, proves that the elasticity of the vibrating medium varies with the length of the wave. The same thing must take place in doublerefracting media, in which the elasticity is different in different directions; and as we have no reason for supposing that the elasticities should vary in the same proportion in the direction of the three axes of elasticity, it will follow that in general each refractive index will have its appropriate dispersive ratio. Sir David Brewster first showed that this was actually the case, and that Iceland spar and other double-refracting substances had two dispersive powers.* M. Rudberg has recently examined the laws of dispersion in double-refracting media with much eare, following the accurate method of Fraunhofer. He has in this manner determined the greatest and least refractive index, corresponding to the seven principal dark lines of the spectrum, in Iceland spar and rock crystal, and the three principal indices in arragonite and topaz; and has found, in accordance with the discovery of Sir David, Brewster, that the ratio of these indices increased with the refrangibility of the light. $\dagger$ The experiments of M. Rudberg confirm also the fundamental position of Fresnel's theory-namely, that the velocity of a ray in a given medium is the same as long as its plane of polarization is unchanged.

The angle contained by the optio axes, in biaxal crystals, is a simple funotion of the three principal elasticities; and if their ratio vary with the colour of the light, the inclination of the axes must likewise vary. Such a variation has been established by the observations of Sir John Herschel; and it has been found that the inclination of the axes is greater in red than in violet light for some crystals, while in others it is less. $\neq$ In the case of Rochelle salt, the angle between the optio axes of the red and violet rays

\section{- Treatise on Newo Philosophical Instruments, Edin. 1813.}

$\uparrow$ Annales de Chimie, tom. xlviii. For the calculation of the phenomens of double refraction in biaxal crystals, according to Fresnel's theory, it is necessary to know the three principal refractive indices, or the velocities of propagation of rays whose vibrations are parallel to the three axes of elasticity. Beside the researches of M. Rudberg, I do not know that we possess any other in which all these data have been directly determined. It is true that if we know the greatest and least index, and the angle contained by the optic axes, the mean index can be deduced. But the inclination of the optic axes cannot be determined experimentally with the same precision as the other elements.

$\ddagger$ Phil. Trans. 1820. 
amounts to $10^{\circ}$. Generally the position of the three axes of elasticity is invariable, and the optic axes for all colours are confined to one plane; but Sir John Herschel has lately observed that, in borax, the optic axes belonging to different colours lie in different planes; and we are compelled to conclude that the direction of the axes of elasticity in this, and probably in many other crystals, varies with the colour.

The first addition to the theory of Fresnel was made by $\mathbf{M}$. Ampère. The results alluded to are contained in two short papers read to the French Academy in the year 1828, and since embodied into one, and published in the Annales de Chimie.* Fresnel had arrived at the equation which belongs to all the tangent planes of the wave-surface, and had shown in what manner the equation of the surface itself might be thence deduced by differentiation and elimination. This direct process, however, he seemed to think would involve complicated and embarrassing calculations. The method which he substituted for it consisted in verifying the equation, to which he was led by reasonings not altogether rigorous, and proving (by calculations which he found too tedious to transcribe) that it satisfied the conditions already assigned. M. Ampère has supplied the direct demonstration, and deduced the equation of the wave-surface in the manner originally pointed out by Fresnel. From this equation he has derived also the beautiful geometrical construction given by Fresnel, and which the latter had obtained indirectly.

A very concise demonstration of the same theorem, and of the other principal points of Fresnel's theory, was given not long after by Mr. M'Cullagh. $\dagger$ This writer has shown that both the magnitude and direction of the resultant elastic force, called into action by any displacement, may be represented by means of an ellipsoid whose semiaxes are the three principal refractive indices of the medium; and from this ellipsoid, by the aid of a few geometrical lemmas, he has deduced in a clear and simple manner the leading

\footnotetext{
* "Mémoire sur la Détermination de la Surface courbe des Ondes lumineuses," \&c., tom. xxxix.

+ " On the Double Refraction of Light in a crystallized medium, according to the principles of Fresnel," Transactions of the Royal Irish Academy, vol. xvi. A further development of the principles of this memoir has been recently given by the author in the 17th vol. of the same Transactions, under the title "Geometrical Propositions applied to the Wave-theory of Light."
} 
results arrived at by Fresnel. The axes of this ellipsoid coincide in direction with, and are inversely proportional to, the axes of Fresnel's generating ellipsoid; and Mr. M'Cullagh has demonstrated the truth of Fresnel's construction for the wave-surface, by means of a simple geometrical relation between its tangent planes and the sections of the two ellipsoids.

In the third supplement to his "Essay on the Theory of Systems of Rays,"* Professor Hamilton has presented that portion of Fresnel's theory, which relates to the fundamental problem of the determination of the velocity and polarization of a plane wave, in a very elegant analytical form ; and from the velocity and direction of the wave he deduces those of the ray, and therefore the form of the wave-surface, by means of the general relations suggested by his view of mathematical optics.

In this system, of which the author gave a brief sketch at the late meeting of the Association, the laws of reflexion and refraction, ordinary or extraordinary, are comprised in two fundamental expressions, which state that the partial differential coefficients of the first order of a certain function-taken with respect to two final coordinates in the plane which touches the reflecting or refracting surface at the point of incidence, - are not altered by reflexion or refraction. The function here considered is the characteristic function of the author, whose particular form may be considered as characterizing the optical system, and on whose properties, he finds, all the problems of mathematical optics may be made to depend. On the principles of the wave-theory, this function is equal to the undulatory time of propagation of light, from any one assumed point to another, in the same or in a different medium; and the expressions just alluded to signify simply that the components of normal slowness of the wave parallel to the bounding surface, or the reciprocals of the velocities of wave-propagation resolved in the direction of that surface, are not changed by reflexion or refraction. The normal slowness of wave-propagation is, then, of fundamental importance in this theory; and if it be represented in magnitude by a line taken in its direction, there is obtained for its expression a curved surface which, on the principles of Fresnel, is found to be a surface of two sheets, connected with the wave-surface by a remarkable relation of reciprocity. When this relation is com- 
bined with the laws of reflexion and refraction just alluded to, they lead to a very elegant construction for the reflected or refracted ray, which is, in most cases, more convenient than that of Huygens. Thus, when a ray proceeds from air into any crystal, we have only to construct the surfaces of wave-slowness belonging to the two media, and having their common centre at the point of incidence. Let the incident ray be then produced to meet the sphere, which represents the normal slowness of the wave in air; and from the point of intersection let a perpendicular be drawn to the reflecting or refracting surface. This will cut the surface of slowness of the reflected or refracted waves in general in two points. The lines connecting these points with the centre will represent the direction and normal slowness of the waves; while the perpendiculars from the centre on the tangent planes at the same points will represent the direction and slowness of the rays themselves.

This important curved surface presented itself also to M. Cauchy in his able researches on the propagation of waves in elastic media, although he does not seem to have been aware of all its properties. The properties of the same surface, and its use in constructing the direction of a reflected or a refracted ray, were also discovered, independently, by Mr. M'Cullagh, who has recently applied them to the geometrical development of the theory of double refraction.*

The relations between the surface of wave-slowness, and that of the wave, have led Professor Hamilton to the discovery of some new geometrical properties of the latter. These properties are demonstrated by means of certain transformations of the equation of the wave-surface; and it is shown that this surface has four conoidal cusps, at the extremities of the lines of single ray-velocity, at each of which the wave is touched (not by two planes as Fresnel supposed, but) by an infinite number forming a tangent cone of the second degree; while, at the extremities of the lines of single wave-velocity, there are four circles of plane contact, in every point of each of which the wave-surface is touched by a single plane. These singular properties have led Professor Hamilton to antioipate two new laws of refraction-called by him conical refraction, because in each case a single ray is refracted into an infinite number

\footnotetext{
* "Geometrical Propositions applied to the Wave-theory of Light," Transactions of the Royal Irish Academy, vol. xvii.
} 
forming a species of cone. External conical refraction corresponds to the cusp on the wave-surface; and takes place vithout, when a single internal ray coincides with either of the lines of single rayvelocity. Internal conical refraction, on the other hand, takes place within the crystal, when a single ray is incident externally at an angle corresponding to the line of single wave-velocity within. In this latter case, if the crystal be bounded by parallel planes, all the rays of the cone will emerge at the second surface parallel to the ray incident on the first, so as to form a small elliptic cylinder, whose magnitude will depend upon the angle of the cone and the thickness of the crystal. All these remarkable conclusions have been verified in the fullest manner by experiment.*

I shall now proceed to give a brief account of the labours of M. Cauchy in this interesting department of analysis. The researches of this eminent mathematician, on the propagation of motion in elastio media, are scattered through various livraisons of the Exercices de Mathematiques; and he has given a valuable summary of the results of these investigations, as applied to the wavetheory of light, in a memoir read to the French Academy in the year $1830 . \dagger$

Having assigned the general equations of motion of a system of molecules, acting on one another by attracting or repelling forces which vary according to any function of the distance, M. Cauchy observes that it is not necessary to have recourse to their general integrals in order to determine the laws of undulatory propagation. It is sufficient, in fact, to determine the law of propagation of a plane vave. For if we consider a great number of plane waves inclined to one another at small angles, and which are at first superposed in the neighbourhood of the point which is considered as the origin of the disturbance, the vibrations in the elementary waves, to which each of these gives rise, may be supposed too small to affect the sense separately, and these waves become efficacious only by superposition. Consequently the general wave-surface will be the locus of all the points in which the elementary plane waves are superposed; and will therefore be the

* "On the Phenomena presented by Light in its passage along the axes of biaxal Crystals," Transactions of the Royal Irish Academy, vol. xvii.

† "Mémoire sur la Théorie de la Lumière," Mrém. Inst., tom. x. 
surface touched by them all at any instant.* Hence the problem is reduced to the determination of the law of propagation of a plane wave.

M. Cauchy then shows that a disturbance, confined originally to a given plane, will in general give rise to three pairs of plane waves parallel to the original plane, and propagated with uniform velocities,- the two waves of each pair moving with equal velocities in opposite directions. The velocities of propagation of the separate pairs, he proves, may be represented by the reciprocals of the axes of a certain ellipsoid, whose form depends upon the position of the plane wave and upon the nature of the system; and the absolute displacements of the molecules will be parallel to the directions of these axes. Accordingly, a system of plane waves, superposed at first at the point of original disturbance, will be subdivided into three corresponding systems; and these, by their superposition, will generate a curred surface of three sheets, each sheet being touched by all the plane waves of the same system. From these principles it follows that a single ray of light will be, in general, subdivided into three polarized rays; - a ray being said, in this theory, to be polarized parallel to a certain line or plane, when the vibrations of the ethereal molecules are parallel to that line or plane. M. Cauchy does not state the precise physical condition on which the existence of the third ray depends. It would seem, however, that it must arise from the circumstance that the vibration normal to the wave is not absolutely insensible, or that the actual vibrations are not accurately in the plane of the wave. He states that the intensity of this ray will be in all cases very small, and that its observation therefore will be a matter of difficulty; and he promises in a future communication to point out the means of manifesting its existence.

The formulæ, on which the solution of the general problem depends, may be reduced to contain nine constant coefficients depending on the law of distribution of the molecules in space. Three of these represent the pressures sustained in the natural condition of the medium by any three planes parallel to those of the three coordinates; and these (M. Cauchy afterwards concludes)

- M. Poisson does not admit the legitimacy of this conception of the wave-surface: and he thinks that an assemblage of indefinite plane waves, having a small part in common at the origin of the motion, cannot represent the initial condition of a medium disturbed at that point. 
vanish of themselves. When the general theory is applied to the case in which the elasticity is the same in all directions round any line parallel to one of the axes of coordinates, M. Cauchy finds that the nine coefficients. are reduced to five; and that two sheets of the wave-surface become the sphere and spheroid of the Huygenian law, provided that the remaining constants fulfil two assigned equations of condition. In the general case, in which the elasticity is unequal in all directions, he investigates the sections of the wave-surface made by the planes of the three coordinates; and he finds that-for two sheets of that surface-they are reduced to the circle and ellipse of Fresnel's theory, provided that the constants fulfil three assigned equations of condition. The wave-surface itself differs a little from the surface of the fourth order obtained by Fresnel ; but is reducible to it when the excentricities of the ellipses just mentioned are small, as is the case in all known crystals.

Thus the results obtained by M. Cauchy embrace and confirm those of Fresnel; and the mathematical laws of the propagation of light are shown to be particular cases of the more general laws of the propagation of vibratory motion in any elastic medium composed of attracting and repelling molecules. Considered, however, simply with reference to the theory of light, the solution given by M. Cauchy cannot, I conceive, be considered as a complete physical solution. In other words, the phenomena of light are not connected directly with any given physical hypothesis; but are shown to be comprehended in the results of the general theory, in virtue of certain assumed relations among the constants which that theory involves. If, indeed, we were able to assign the precise physical meaning of these equations of condition, we should have nothing more to desire in the general theory of light; for these equations must necessarily express the characteristic properties of the vibrating medium. In this point of view their discussion becomes a subject of the highest interest; and it is probable that the important conclusions of which we have yet to speak may in this manner be confirmed and extended.

These conclusions are contained in a memoir presented to the French Academy by M. Lamé, in the spring of the present year,*

* "Mémoire sur les Lois de l'Equilibre de l'Ether dans les Corps diaphanes." A full account of this paper is given in the Annales de Chimie for March. The momoir itself is not yet published. 
in which the author has proposed to determine the laws according to which the molecules of bodies act on those of the ether, and the molecules of the ether on one another. Setting out from the existence of transversal vibrations, as established by the fact of the non-interference of rays oppositely-polarized, the author supposes a disturbance of the ether to take place in racuum - that is, in a space devoid of all ponderable matter,-and proceeds to consider what will be the result when that disturbance reaches the ether contained in a transparent body. Assuming the property of transversal vibrations noticed by Fresnel, and more explicitly stated by M. Poisson-namely, that they are propagated without any attendant change of density, - M. Lamé then seeks the conditions to be satisfied by the function, which represents the mutual action of the molecules of the ether and those of the solid body, in order that this property may subsist. Introducing, accordingly, this principle into the partial differential equations, which express the laws of the vibratory movement generally, he arrives finally at an equation of condition, from which he concludes that "the action of ponderable matter on the ether varies in the inverse ratio of the square of the distance ; and that the elasticity of the ether itself is proportional to its density."

In order to determine the sign of this action-that is to say, whether it is attractive or repulsive,-it is necessary to integrate the differential equations. After certain transformations of these equations tending to facilitate their examination, he obtains their integral in the case of a single spherical and homogeneous molecule of the body, around which the ether is distributed in spherical shells. The conclusions deduced from this case being combined with the established fact, that the velocity of light is less in transparent bodies than in vacuum, he arrives at the result, that the mean density of the ether is less in the former, or that the action of the molecules of these bodies on those of the ether is repulsive. M. Lamé concludes also from the examination of the same case, that the retardation of the vibratory motion, in penetrating into a dense body, will be greater, the less the length of an undulation, so that the refraction will be greater for waves of shorter length. This he conceives to be the true explanation of the phenomenon of dispersion.

M. Lamé has likewise endeavoured to connect the phenomena of double refraction with an assumed constitution of the ethereal 
fluid. He takes the case in which the ether is supposed to be distributed round the molecules of the body in confocal ellipsoidal shells; and he concludes that a vibratory movement, propagated from vacuum into a body so constituted, will be separated at its entrance into two component movements, which will advance with different velocities. The two component vibrations, he finds, will be at right angles, and parallel to the lines of greatest and least curvature of the elementary ellipsoids. Thus, the bifuroation of a ray of light on entering a crystallized medium, and the opposite polarization of the two pencils, are found to be consistent with a molecular constitution such as that described.

These results are of the highest interest; and will, no doubt, receive an early examination from those engaged in the same department of analysis. Their author seems to be persuaded that his methods will lead him to the mathematical laws of other phenomena, which he conceives to depend, in like manner, on the motions of the ethereal fluid.*

I cannot close this division of the present Report without referring to the phenomena of absorption by crystallized media, although the laws of these phenomena are as yet wholly without the pale of theory. Dr. Wollaston seems to have been the first who noticed any facts connected with this interesting subject. The absorbing properties of crystals were found to vary with the direction : certain crystals of palladium, for example, appearing of a deep red colour when viewed along the axis, and of a yellowish green in a transverse direction. Tourmalines were observed also to possess analogous properties. $\dagger$ Similar observations were afterwards made by $\mathbf{M}$. Cordier and the Count de Bournon.

The next step of any importance in this new field of research

* In a continuation of this memoir, recently read to the French Academy, M. Lamé has considered particularly the mode of vibration of the particles of the ether which are disposed round the ponderable particles of body in concentric spherical shells of decreasing density. Transparent homogeneous bodies are supposed to consist of a multitude of such particles distributed uniformly in space, and at distances incomparably greater than their diameters; and he conceives that the waves propagated from the particles adjoining to the surface of emergence will, by their interference, give rise to phenomena resembling the fixed lines in the spectrum. Ann. Chim., tom. Irii.

† Phil. Trans. 1804. 
was made by Sir David Brewster. This philosopher observed that in some double-refracting crystals, as carbonate of barytes, the two pencils were differently coloured ;" while in others their intensity was widely different. $\dagger$ The unequal absorption of the two pencils is most remarkable in tourmaline, in which it was observed, nearly at the same time, by M. Biot and Dr. Seebeck; and the former philosopher inferred from the phenomena that the more refrangible rays of the spectrum are more easily absorbed by the mineral, when polarized parallel to its axis, than when perpendicularly. $\ddagger$

Sir David Brewster, to whom we owe the greater part of our knowledge on this subject, has shown belong, in a greater or less degree, to most coloured crystals which possess double refraction; and that the absorption of light by such media varies, in general, both with the colour of the light and with the position of the plane of polarization. When a ray of common light therefore enters a plate of such a crystal, the two pencils into which it is divided will be unequally absorbed, and the emergent light will be partially polarized, the difference of the intensities of the oppositely-polarized portions increasing with the thickness of the medium traversed. But the two pencils differ, in general, in colour as well as in intensity; and this difference, in uniaxal crystals, Sir David Brewster found to depend on the inclination of the ray to the axis, vanishing when the ray coincided with the axis, and becoming a maximum when it was perpendicular to it. A ray of common light, therefore, transmitted perpendicularly through a plate of such a crystal, will emerge coloured; and the resulting colour will, in general, vary with the inclination of the surface to the axis. Thus the phenomena of dichroism, observed by Wollaston and others, are reduced to the more general laws of absorption. Analogous properties belong to biaxal crystals, and depend in like manner on the planes of polarization of the two pencils, and on the direction of the ray. These properties Sir David Brewster found could be modified by heat; and were even communicated by such influences to crystals in which they did not naturally reside.

* Edin. Trans., vol. rii.

† Traité de Physique, tom. iv., 313.

†Phil. Trans. 1814.

$\$$ "On the Laws which regulate the Absorption of Polarized Light by doublerefracting Crystals," Phil. Trans. 1819. 
Notwithstanding the important labours of Sir David Brewster, much remains to be done connected with this subject. Sir John Herschel has proposed empirical formula to represent the intensity of the transmitted light as dependent on its direction; and the results of the formulæ present a general accordance with observed facts.* It is much to be desired that these laws should be placed beyond doubt by an extensive series of experiments directed to this specific object. Although the laws of absorption by crystallized media are necessarily more complicated than those of ordinary media, yet they bear an evident and close relation to the well-known laws of double refraction, which seems to hold out a clue to their discovery; and I feel persuaded that it is in the phenomena of dichroism that the physical theory of absorption will first take its rise, and seek its confirmation.

\section{Colours of Crystalline Platrs.}

If a beam of light, polarized by refloxion, be received upon a second reflecting plate at the polarizing angle, it is wholly transmitted when the second plane of incidence is perpendicular to the first. But if between the polarising and analyzing plates, as they are termed, there be interposed a plate of any doublerefracting crystal, a portion of the light is reflected, whose quantity depends on the position of the interposed crystal. In order to analyze the phenomenon, the crystalline plate may be placed so as to receive the polarized beam perpendicularly, and then turned round in its own plane. It is then observed that there are two positions of the plate in which the reflected light totally disappears, just as if no crystal had been interposed. These two positions are those in which the principal and the perpendicular sections of the crystal coincide with the plane of the first reflexion. When the plate is turned round from either of these positions, the light gradually increases; and it becomes a maximum when the principal section is inclined at an angle of $45^{\circ}$ to the plane of the first reflexion. These phenomena were observed by Malus.

The reflected light in these experiments was in all cases $v$ chitc. But M. Arago observed that when the interposed plate is suffi- 
ciently thin-such as the laminæ into which mica or sulphate of lime may be readily divided by cleavage, - the most gorgeous colours appear, which vary with every change of inclination of the plate to the polarized beam. When the plate is perpendicular to the transmitted pencil, and then turned round in its own plane, the tint does not change, but only varies in intensity,being a maximum when the principal section of the crystal is inclined at an angle of $45^{\circ}$ to the plane of primitive polarization, and vanishing altogether when it coincides with that plane, or is perpendicular to it. On the other hand, when the crystal is fixed, and the analyzing plate turned, so as to vary the inclination of the plane of the second reflexion to that of the first, the colours change in the most striking manner; and it is found that the colour reflected, in any one position of the plane of the second reflexion, is always complementary to that reflected in the perpendicular position. The colours disappear altogether when the thickness of the crystalline plate is reduced below a certain limit.*

The experimental laws of these phenomena were investigated with unwearied zeal by M. Biot. $\dagger$ When the light was incident perpendicularly on plates of the same substance, of different thicknesses, the tints were observed to follow the same law as the colours of thin plates; the thicknesses of the crystal at which each tint was developed in perfection being proportional to the thicknesses of the plate of air which gave the same tint in Newton's scale. These thicknesses vary with the nature of the crystal, and are always much greater than the corresponding thicknesses of the uncrystallized plate which exhibit the same tint. Pursuing the same inquiry, afterwards, for oblique incidences, M. Biot found that, in uniaxal crystals, the tint developed was determined by the length of the path traversed by the light within the crystal, and by the square of the sine of the angle which its direction made with the optic axis, jointly. From this law it followed, that if a crystalline plate of moderate thickness be cut perpendicularly to the axis, and a converging or diverging pencil transmitted through it, the lines of equal tint-or the isochromatic lines, as they are sometimes called,will be disposed in concentric circles similar to Newton's rings. 
This phenomenon was observed, under different circumstances, by Sir David Brewster, Dr. Wollaston, M. Biot, and M. Seebeck.

The researches of M. Biot were followed by those of Sir David Brewster. In investigating the law of the tints in biaxal crystals, Sir David Brewster considers the optic axes as the resultants of others which he denominates polarizing axes. The tint developed by a single axis is taken as the measure of its polarizing force, and is assumed to vary as the square of the sine of the angle contained by the ray with it; and when two such axes cooperate, the tint resulting from their joint action is measured by the diagonal of a parallelogram whose sides represent the tints produced by each axis separately, and whose angle is double the angle contained by the two planes passing through them and the ray. This law Sir David Brewster has verified by comparison with the observations of M. Biot on sulphate of lime, and its agreement with phenomena was complete.* When analytically developed by M. Biot, it was found to accord with the beautiful law to which he was himself conducted by analogy - namely, that the tint is measured by the product of the sines of the angles which the direction of the ray within the erystal makes with the optic axes. $\dagger$ From this law it easily followed that the isochromatic lines, in biaxal crystals, will be lemniscates, whose poles are in the apparent direction of the optio axes. $\neq$ This phenomenon was first discovered by Sir David Brewster in topaz. The law has been established in the most complete manner by Sir John Herschel; and he has found that the constant parameter, or the product of

* "On the Laws of Polarization and Double Refraction in regularly crystallized Bodies," Phil. Trans., 1818.

+ From the researches of M. Biot it appeared that the measure of the tint, in uniaxal crystals, observed the same law as that attributed to the difference of the squares of the velocities of the two rays in the theory of emission. The same relation was assumed to hold generally; and thus from the law of the tints in biaxal crystals the relation of the velocities of the two pencils, noticed in the preceding scction, was inferred.

$\mp$ M. Biot has observed an apparent exception to this law in the diopside of the Tyrol, in which the rings are in general unsymmetrical with respect to the two axes. One of the axes presents the ordinary phenomena; but in the neighbourhood of the other there is a remarkable distortion of the rings near their centre, when the crystal. line plate is turned in its own plane. These distortions soemed to observe a regular law, and were the same in all the specimens examined. It may be remarked that the optic axes of this crystal are unsymmetrically placed with respect to the crystalline form, Mém. Inst., tom. x. 
the radii-vectores drawn from any point to the two polos, varies inversely as the thickness of the plate for different plates of the same substance, and increases from one curve to another in the same plate in the ratio of the numbers of the natural series.

To account for these varied phenomena in the hypothesis of emission, M. Biot has imagined his ingenious and beautiful theory of moveable polarization. When a polarized ray of any simple colour enters a crystalline plate, the component molecules are supposed, in this theory, to penetrate at first to a certain depth without losing their primitive polarization; and then to commence a series of regular oscillations round their centres of gravity, the axes of polarization being carried alternately to one side or other of the axis of the crystal, or of the perpendicular line. These oscillations being isochronous, the thickness transversed by the molecule in its motion of translation during each of them is constant, and is assumed to be equal to double the depth to which it has penetrated before commencing its vibrations. The oscillatory movement is supposed to stop, when the molecules repass into air through the second face of the crystal; and the emergent ray has a fixed polarization, the same as if the last oscillation of the molecules had been actually completed at the instant of emergence. Thus a polarized ray which has traversed a thin crystalline plate is ultimately polarized either in the primitive plane, or in a plane inclined to it at an angle equal to double the angle which it forms with the principal section, according as the thickness of the crystal is an odd or an even multiple of a certain length.* The formula deduced from these postulates are found to represent all the more obvious laws of the tints with much fidelity.

This assumed difference between the effects produced by thick and thin crystals has however been completely disproved by the decisive experiments of Fresnel. When two mirrors, slightly inclined, are placed so as to receive the incident light at the polarizing angle, and two laminæ of sulphate of lime of the same thickness are interposed-one in the path of each of the reflected pencils, and so that their principal sections are inclined at angles of $45^{\circ}$ on either side of the plane of primitive polarization, - the phenomena of the interference bands prove in the clearest manner that the light emergent from each consists of two pencils polarized respectively in the principal section, and in the perpendicular

\footnotetext{
* "Sur un nouveau genre d'Oscillation," \&c., Mém. Inst. 1812.
} 
section of the crystals; and that the results differ from those produced by thick crystals only in this, that the two pencils are superposed.* The light resulting from the union of these oppositely polarized pencils has, in certain cases, the properties ascribed to it in the theory of M. Biot; but these properties are immediate and necessary consequences of the laws of interference of polarized light, and of the theory of transversal vibrations.

Let us now inquire what account the wave-theory furnishes of the same phenomena. A ray of light on entering a crystalline plate is divided into two, or, in the language of the wave-theory, a series of waves incident upon the crystal is resolved into two within it, which traverse it in different directions and with different velocities. One of these sets of waves, therefore, will lag behind the other, and they will be in different phases of vibration at emergence. When the plate is thin, the emergent waves are superposed; and as the retardation will then amount only to a few undulations and parts of an undulation, it would appear that we have here all the conditions necessary for their interference, and the consequent production of colour. Such was the sagacious conjecture of Young. And indeed, shortly after the publication of the first researches of M. Biot on the laws of the tints for different thicknesses, it was observed by Young that these tints corresponded accurately to the interval of retardation of the two pencils; so that they were manifestly due to interference. $f$ This correspondence is now made out in the fullest manner. It is an easy consequence of Fresnel's theory of double refraction, that the interval of retardation of the two pencils, in traversing a crystalline plate, is nearly proportional to the length of their path within the crystal multiplied by the product of the sines of the angles which their directions make with the two optic axes; and as this has been found to be the general measure of the tint, it follows that the forms of the isochromatio curves-the lemniscates and the circles, - are all necessary consequences of the wave-theory.

* Sce Report made to the Academy of Sciences, in 1821, on the momoir of Fresnel relative to the colours of crystallized plates, Annales de Chimie, tom. xvii. Indeed, a more obvious objection to M. Biot's theory may be drawn from the fact which he has himself observed; -namely, that the phenomena of colour may be produced by erossing two thick plates of nearly the same thickness, although the thickness in each was sufficient to furnish two images sensibly separated, and thereforo having a faxed polarization.

$\dagger$ Quarterly Revieı, vol. xi. 
But in the first application of the principle of interference to the colours of crystalline plates there arose a difficulty to which the known laws afforded no answer. So far as this explanation went, the phenomena of interference and of colour should be produced by the crystal alone, and in common light, without either polarizing plate or analyzing plate. Such, however, is not the fact; and the real difficulty seemed to be, not to explain how the phenomena are produced, but to show why they are not always produced. It occurred to MM. Arago and Fresnel to inquire how far the state of polarization of the two pencils might modify the known laws of interference; and the results of this inquiry* have happily furnished an account of the difficulty, and completed the solution of the problem. It was found that two rays of light polarized in the same plane interfere, and produce fringes, under the same eircumstances as two rays of common light; - that, when the planes of polarization are inclined, the interference is diminished and the fringes decrease in intensity; - - and that, finally, when the angle between these planes is a right angle, the rays no longer interfere at all. Hence the two rays which emerge from a crystalline plate, being oppositely polarized, eannot interfere; and, to produce the phenomena of colour in perfection, their planes of polarization must be brought to coincidence by the analyzer.

The non-interference of rays oppositely polarized is a necessary result of the mechanical theory of transversal vibrations. Fresnel has shown, on the principles of that theory, that the intensity of the light resulting from the union of two such rays is constant, and equal to the sum of the intensities of the components, whatever be the phases of vibration in which they meet. But though the intensity of the light does not vary with the phase of the component vibrations, the character of the resulting vibration will. It appears from theory that two rectilinear and rectangular vibrations compound a single vibration, which will be also rectilinear when the phases of the component vibrations differ by an exact number of semiundulations; that, in all other cases, the resulting vibration will be elliptic; and that the ellipse will become a circle, when the component vibrations have equal amplitudes, and the difference of their phases is an odd multiple of a quarter of a wave. These results of theory have been completely confirmed

* "Mémoire surr l'Action qus los Rayons de la Lumière polarisée exercent les uns. sur les autre," Annales c'e Chimie, ton. $x$. 
by experiment. When a polarized beam diverging from a luminous origin is transmitted through two rhomboids of Iceland spar of equal thickness, having their principal sections inclined $45^{\circ}$ on either side of the plane of primitive polarization, the emergent light will diverge as if from two near points, and the two portions will be oppositely polarized. It was found by Fresnel and Arago that the light resulting from the union of these pencils was planepolarized, circularly polarized, or elliptically polarized, according to the difference of the paths traversed when they met.

Here, then, we have an account of the facts which seem to have suggested the theory of moveable polarization; and we learn moreover that they are but particular cases of the general result. The light arising from the union of the ordinary and extraordinary pencils, which emerge from the crystalline plate, will be polarized in the primitive plane, or in a plane inclined to it at an angle equal to double the angle which it makes with the principal section, according as the interval of retardation of the two pencils is an even or an odd multiple of half a wave. In all other cases-the thickness of the crystal having any other value than those which exactly answer to these intervals-the resulting light will be elliptically polarized. The ellipse will become a circle, and the light will appear to be completely depolarized, when the two pencils are of equal intensity, and the interval of retardation is an odd multiple of a quarter of a wave. Here, then, is suggested an easy method of putting the theory of moveable polarization to the test. If a plate of sulphate of lime, whose thickness corresponds to such an interval, be placed in a beam of polarized light of some simple colour, so that its principal section is inclined at an angle of $45^{\circ}$ to the plane of primitive polarization, the emergent light should, aecording to the theory of waves, be circularly polarized; and the two pencils into which it is divided by the analyzing rhomb should not vary in intensity during its revolution. According to the theory of moveable polarization, on the other hand, the light should be plane-polarized; and one of the images should vanish when the principal section of the rhomb coincided either with the primitive plane, or with the plane perpendicular to it. This experimentum crucis was tried by M.M. Fresnel and Arago, and the result was just as had been predicted by the ware-theory.* 
In the prosecution of their researches on the laws of interference of polarized light, MM. Fresnel and Arago discovered further that two oppositely polarized rays will not interfere, even when their planes of polarization are brought to coincidence, unless they belong to a pencil the whole of which was originally polarized in one plane; - and that, in the interference of rays which have undergone double refraction, half an undulation must be supposed to be lost or gained, in passing from the ordinary to the extraordinary system. The latter principle is a beautiful and simple consequence of the theory of transversal vibrations. When a vibration in any given direction is resolved into two at right angles, and each of these again into a second pair, in two fixed directions which are also perpendicular, it will easily appear that, of the four components into which the original vibration is thus resolved, the two in one of the final directions conspire, while those in the other are opposed. The tint produced by the interference of the former, therefore, corresponds to the actual difference of routes of the two polarized rays in the plate; while that arising from the latter is that due to the same difference augmented or diminished by half an undulation.

The former of the two laws now mentioned explains the office of the polarizing plate in these phenomena. To account mechanically for the fact of the non-interference of the two pencils, when the light incident upon the crystal is unpolarized, it is necessary to consider such light as a rapid succession of systems of waves polarized in all azimuths; so that if any two planes be assumed at right angles, there will be an equal quantity of light actually polarized in each. Each of these portions, when resolved into two within the crystal, and these afterwards reduced to the same plane of polarization by the analyzing plate, will exhibit the phenomena of interference. But the interval of retardation differs by half a wave in the two cases; the tints produced therefore will be complementary, and the light resulting from their union will be of a uniform whiteness.

We are obliged to admit, therefore, that common light consists of a rapid succession of systems of waves, in each of which the vibrations are different. But the phenomena of interference (which are exhibited by common light) compel us also to admit, as Professor Airy has observed," that the vibrations do not change 
continuously; and that in each system of waves there are probably several hundred successive vibrations, which are all similar,although the vibrations of one system bear no relation to those of another, and the different systems succeed one another with such rapidity as to obliterate all trace of polarization. This per saltum transition from one system of waves, to another in which the vibrations are wholly different, seems a complication in the machinery of light, for which the elegant simplicity of the parts better known has not prepared us; and I cannot but indulge the hope that the hypothesis, which now stands as the representative of experimental laws, may be found to merge in some simpler physical principle.

The laws of interference of polarized light have thus supplied the defective link in the explanation of the colours of crystalline plates first suggested by Young. The magnitudes of the resolved vibrations are known, when the planes of polarization of the two pencils are given with respect to the plane of primitive polarization, and the plane of analyzation; and as the laws of double refraction enable us to find the interval of retardation of these pencils, we have all the data necessary for the computation of the intensity and colour of the light resulting from their interference. This computation has been given by Fresnel, not only for a single plate, but likewise for two plates superposed ;* and the theory has been since more fully developed by Professor Airy. $\dagger$ The results are found to be, in all cases, in exact accordance with the observed facts; and all the circumstances of the coloured rings in uniaxal and biaxal crystals are completely explained.

The form of the rings, or isochromatio curves, depends upon the interval of retardation alone; and the value of this interval had been deduced but approximately. Mr. M'Cullagh has recently given a general and exact method for its calculation, and for the determination of the forms of the rings for any plate of a doublerefracting crystal bounded by parallel planes. This method is made to depend upon the properties of the surface of vave-slocness, of which I have spoken in another place; and it is found that if the incident ray be produced to meet the sphere (which is the surface of wave-slowness for air), and through the point of inter-

+ Cambridge Transactions, 1831, and Math. Tracts. 
section a perpendicular be drawn to the refracting surface, meeting the two sheets of the surface of wave-slowness for the crystal, the interval of retardation of the rays at emergence will be measured by the thickness of the crystal multiplied by the difference of the corresponding ordinates.* By the aid of an expressive notation for the path of a ray, the author has extended his conclusions to the case of a ray which has undergone any number of internal reflexions.

If the double-refracting energy of the crystal were the same for the light of every colour, the colours of the rings should follow exactly the Newtonian scale of tints, and their magnitudes should observe the same laws as those of the rings formed between two object-glasses. This is the case in carbonate of lime, beryl, and some other crystals; and in these, therefore, the colours are similar to those of thin plates. But many remarkable deviations from this law have been observed by Sir John Herschel and Sir David Brewster. Thus, in the common uniaxal apophyllite, it was observed by the former writer, the diameters of the rings are very nearly the same for all the colours of the spectrum; so that the rings of different colours are superposed, and form a succession alternately black and white, which may be traced through a great number of orders. $t$ In this remarkable case, then, the doublerefracting energy of the erystal varies, very nearly, in the subduplicate ratio of the lengths of the waves for the rays of different colours. A very remarkable case of the inversion of the Newtonian scale of tints was observed by Sir John Herschel in some rare varieties of the same mineral. The diameters of the rings, instead of contracting as the refrangibility increases, enlarge, and actually become infinite for rays of mean refrangibility. Having passed through infinity, they again acquire a finite value; and diminish as the refrangibility increases up to the extremity of the spectrum. Here, then, for rays of a certain mean refrangibility the crystal is singly refractive; and as the double refraction changes its character in passing through zero, the crystal is positive for the

- If $y_{i}, y_{0}, y_{e}$ represent the corresponding ordinates of the sphere, and of the two sheets of the surface of wave-slowness for the medium, and $\theta$ the thickness of the crystal, $\theta\left(y_{0}-y_{i}\right), \theta\left(y_{e}-y_{i}\right)$ will be the retardations of the two refracted waves at emergence, and $\theta\left(y_{0}-y_{e}\right)$ will be the interval between them.- "Geometrical Propusitions applied to the Wave-theory of Iight," Trans. Royal Irish Academy, vol. xvii.

† Plil. Trans., 18:0. 
rays of one end of the spectrum, and negative for those of the other.* This singular phenomenon is accounted for on the principles of Fresnel's theory by supposing that the elasticity increases, with the length of the wave, faster in the direction of the axis of the crystal than in the perpendicular direction; so that the difference of these elasticities is positive for the rays of one end of the spectrum, negative for those of the other, and vanishes at some intermediate point.

In biaxal crystals similar deviations take place in the magnitude of the lemniscates corresponding to the different simple colours. But there is here another source of irregularity which is not found in uniaxal crystals. The optie axes vary, in general, with the colour; so that the lemniscates differ also in the position of their poles, and the colours are not the same in different parts of the same ring. Where the optic axes belonging to different colours are in different planes, as Sir John Herschel has observed to be the case in borax, the irregularity produced in the coloured curves is yet more striking.

In all the preceding cases, the laws of double refraction are dependent only on the direction, and are the same all throughout the mass. It is otherwise, however, in many crystals, such as analcime and some varieties of apophyllite. The complicated arrangement of the coloured bands which these substances display in polarized light proves them to consist of several distinct portions, possessing different optical properties; and the phenomena indicate relations among the molecular forces, and principles of aggregation, of which it is difficult in some cases even to form a conception. These remarkable phenomena, and their laws, were discovered by Sir David Brewster. $\dagger$

When a polarized ray traverses a plate of Iceland spar, beryl, or almost any other uniaxal crystal, in the direction of its axis, it suffers no change of any kind; so that when the emergent ray is analyzed by a double-refracting prism, the two pencils into which it is divided are colourless, and one of them vanishes when the principal section of the prism is parallel or perpendicular to

* Cambridge Trans. 1821. Similar properties have been observed by the same author in other crystals, as hyposulphate of lime and vesuvian. From the table of tints exhibited by the latter substance it appears that the most refracted of the two images is the least dispersed.

$\dagger$ Edin. Trans., vuls. ix. \& $\mathbf{x}$. 
the plane of primitive polarization. But when a ray passes in the same manner through a plate of rock-crystal, the phenomena are very different. Two images are given in every position of the prism; and these images are of complementary colours, while the colours change in the most beautiful manner as the prism is turned round in its cell. These phenomena indicate that the plane of polarization has been changed, and differently for the different rays of the spectrum. They were first observed by M. Arago; and he has given an account of his observations in his memoir on the colours of crystalline plates, read to the Institute in the year 1811.

The subject was then taken up by M. Biot, in a paper published in the Mémoires de l'Institut in the year 1812; and the analysis of the phenomenon was completed in a second memoir read in the year 1818*. When a polarized ray of any simple colour passes through a plate of rock-crystal in the direction of the optic axis, it is still polarized after emergence; but its plane of polarization is changed. The angle through which the plane is made to revolve varies with the colour of the light, and with the thickness of the plate, being proportional to that thickness divided by the square of the length of the fit or wave. In some crystals the plane of polarization revolves from left to right, while in others it is turned in an opposite direction; and the crystals themselves are denominated right-handed or left-handed, according as they produce one or other of these effects. When two plates are superposed, the effect produced is, very nearly, the same as that due to a single plate whose thickness is the sum or difference of the thicknesses of the two plates, according as they are of the same or of opposite denominations.

This curious distinction between plates cut from different crystals has been connected by Sir John Herschel with a corresponding diversity in the crystalline form. The ordinary form of the crystal of quartz is the six-sided prism terminated by the sixsided pyramid. The solid angles formed at the junction of the pyramid and the prism are sometimes replaced by small secondary planes, which in the same crystal lean all in the same direction; and it is found that when that direction is to the right (the apex of

\footnotetext{
* "Mémoire sur les rotations que certaines substances impriment aux axes de polarisation des rayons Iumineux."
} 
the pyramid being uppermost), the crystal is right-handed, and that, on the contrary, it is left-handed when the planes lean in the opposite way.* Sir David Brewster has shown that the amethyst, or violet quartz, is actually composed of alternate layers of righthanded and left-handed quartz. It is to the cropping out of the edges of these layers that the undulating appearance peculiar to the fracture of this mineral is owing. The structure itself is displayed in the most beautiful manner in polarized light. $\dagger$

Some liquids, and even gases, have been found by MIM. Biot and Seebeck to possess the same property as quartz, though in a much feebler degree, and to impress a rotation on the plane of polarization of the intromitted ray, which is proportional to the thickness of the substance traversed. These liquids do not lose their rotatory power by dilution with other liquids not possessing the property. They retain it even when raised to the state of vapour; and, in general, the rotatory power is independent of the mode of aggregation, provided the molecular constitution is unchanged. Lastly, when two or more liquids possessing this property are mixed together, the rotation produced by the mixture is the sum of the rotations produced by the ingredients, in thicknesses proportional to the volumes in which they are eombined. From these and other facts, Mr. Biot conoludes that the property of rotatory polarization is inherent in the ultimate particles of bodies, and does not depend on their mutual distance or arrangement.; On the other hand, quartz is found to lose the property when deprived of its crystalline structure. Thus, Sir John Herschel observed that quartz held in solution by potash did not possess the property : and the same thing has been remarked by Sir Darid Brewster with respect to fused quartz.

The phenomena of rotatory polarization in rock-crystal M. Biot ascribed to a continued rotation of the molecules of light round their centres of gravity, produced by the operation of some unknown forces. Fresnel has proved that they arise from the

* Cambridge Trans., vol. i.

+ Edin. Trans., vol. ix.

+ M. Biot has recently extended his researches on this subject to a great rariety of substances, Annales du Museum d'Histoire Naturelle, om. ii. In a memoir read to the French Academy last year he has applied the laws of circular polarization to the analysis of the process of regetation in the grasses: and he has shown, in genoral, the importance of the indications drawn from these phenomena in the researches of organio chemistry.-Institut, Nos. 1 \& 9. 
interference of two circularly polarized pencils which are propagated along the axis with unequal velocities, one revolving from left to right, and the other in the opposite direction. A plane-polarized ray, in fact, is equivalent to two circularly polarized rays of half the intensity, in one of which the vibrations are from left to right, and in the other in the opposite direction. When a plane-polarized ray, therefore, is incident perpendicularly upon a plate of rock-crystal, cut perpendicularly to the axis, it may be resolved into two such circularly polarized rays. These are supposed to be transmitted with different velocities; so that when they assume a common velocity at emergence, one of them is in advance of the other. They then compound a single ray polarized in a single plane; and this plane, it can be shown, is removed from the plane of primitive polarization through an angle proportional to the interval of retardation of the two pencils, and therefore measured by the thickness of the crystal. But this interval varies also with the colour of the light; and we are obliged to suppose that it is the same for a given number of waves, whatever be their length,so that, for a given thickness of the crystal, it varies inversely as the length of the wave. From this supposition it will follow that the deviation of the plane of polarization of the emergent ray is inversely as the square of that length, agreeably to the experimental results of M. Biot.*

The laws of rotatory polarization were thus completely explained; and it only remained to prove the truth of the hypothesis, - that two circularly polarized pencils, whose vibrations are in opposite directions, will actually be transmitted along the axis of quartz with different velocities. This supposition is easily put to the test of experiment, since such a difference of velocities must give rise to a difference of refraction, when the surface of emergence is oblique to the direction of the ray. According to the hypothesis, therefore, a plane-polarized ray, transmitted through a prism of rock-crystal in the direction of the optic axis, should undergo double refraction at emergence; and the two pencils into which it is divided should be circularly polarized. This has been completely verified by Fresnel, by an achromatio combination of right-handed and left-handed prisms arranged so as to double the separation; and he has shown that the two pencils are neither com-

* Annales de Chimie, tom. xxviii. p. 147. 
mon nor plane-polarized light, but possess all the characters which are impressed upon a polarized ray by two total reflexions from glass at an angle of about $50^{\circ}$.

The refraction of quartz, then, in the direction of its optic axis, is wholly different from that of every other known crystal. In other directions, the two pencils into which a single ray is divided were supposed to obey the ordinary laws, and to be plane-polarized in opposite planes. This supposition has been overturned by Professor Airy; ; and it has been shown that the two pencils in quartz are, each of them, elliptically polarized, the elliptical vibrations of the two rays being in opposite directions, and the greater axes of the ellipses being in the principal plane, and perpendicular to it, respectively. The ratio of the axes, in these ellipses, is the same in the two rays, $\uparrow$ but varies with their inclination to the optic axis, being a ratio of equality when the direction of the ray coincides with the axis, and increasing indefinitely with their inclination to that line according to some unknown law. As to the course of the refracted rays, Professor Airy finds that it is still determined by the Huygenian law; but that the sphere and spheroid, which determine the velocity and direction of the two rays, do not touch, as in all other known uniaxal crystals, the latter surface being contained entirely within the former. This position is certainly a startling one. The two sheets of the wave-surface being thus absolutely separated, there is a complete interruption of continuity in passing from the velocity of one ray to that of the other; a result which does not hold in any other case with which we are acquainted. 'It is however necessary to the explanation of the phenomena; for the interval of retardation does not vanish with the inclination of the ray to the axis. Professor Airy has given an elaborate ealoulation, founded on these hypotheses, of the forms of the rings, \&.e., displayed by quartz in plane-polarized and circularly polarized

* "On the Nature of the Light in the two rays produced by the double refraction of Quartz." Cambridge Transactions, 1831.

+ In the Supplement to this paper Professor Airy has explained a highly ingenious method of determining experimentally the relation between the ellipticity and the direction of either of the rays. This method depends upon a remarkable effect which he had been led to expect from theory :-namely, a sudden change of balf an undulation in the interval of retardation, and therefore a change of half an order in the rings when the incident light is elliptically polarized. From the results of some experiments conducted in this method, Professor Airy seems to think that the ratio of the axes in the ordinary ray approaches more nearly to one of equality than in the extraordinary ray. 
light, and in any position of the analyzing plate; and he has found the most striking agreement between the results of calculation and those of observation.

We yet want a mechanical theory which will account for the peculiar form of the wave-surface just alluded to. Fresnel seems to have thought that the difference of the velocities of the two rays in the direction of the axis might be physically explained by an helicoidal arrangement of the molecules of the vibrating medium, which will have different properties according as the helices are right-handed or left-handed. But this hypothesis can hardly be supposed to apply to the case of fluids, in which the property of circular polarization is independent of direction; and we are driven to confess that, with respect to these important laws, physical theory is as yet wholly at fault. The singular relation betweent he interval of retardation and the length of the wave seems to afford the only clue to the unravelling of this difficulty.

The phenomena of depolarization, and of colour, impressed by double-refracting substances upon the transmitted light, are, we have seen, the necessary results of the interference of the two pencils into which the light is divided within them. These properties, then, enable us to discover the existence, and to trace the laws, of double refraction, even in substances in which the separation of the two pencils is too minute to be directly observed. By such means the important discovery has been made, that a double-refracting structure may be communicated to bodies which do not naturally possess it, by mechanical compression and dilatation. Sir David Brewster observed that when pressure was applied to the opposite faces of a parallelopiped of glass, it developed a tint in polarized light, like a plate of a double-refracting crystal; and the tint descended in the scale as the pressure was augmented. Singly-refracting crystals, such as muriate of soda and fluor-spar, acquired the properties of double refraction by the same means.* All this is in perfect accordance with the wave-theory. Owing to the connexion of the vibrating medium with the solid in which it is contained, its elasticity is rendered unequal in different directions by the effect of compression, the maximum and minimum corresponding to the directions of greatest and least 
pressure. Accordingly, the vibrations of the ray on entering the plate are resolved into two in these rectangular directions, and these are propagated with unequal velocities; the colour developed is determined by the interval of retardation. These results of theory were experimentally confirmed by Fresnel, by the method of interferences; and it was found that the velocity with which a ray traversed the glass was greater or less, according as it was polarized parallel or perpendicular to the axis of compression. The bifurcation of the ray at oblique incidences is a necessary consequence of this difference of velocities; but this was also shown by Fresnel by direct experiment. A series of glass prisms were placed together with their refracting angles alternately in opposite directions, and the ends of the alternate prisms powerfully pressed by screws. A ray transmitted through the combination was found to be divided into two oppositely polarized.*

The opposite effects of compression and dilatation may be seen in a thick plate of glass which is bent by an external force. When this body is interposed between the polarizing and analyzing plates, so as to form an angle of $45^{\circ}$ with the plane of primitive polarization, two sets of coloured bands are seen separated by a neutral line; and these vanish altogether when the comjressing force is withdrawn. By crossing the glass with a plate of mica or sulphate of lime, Sir David Brewster found that the parts towards the convex, or dilated side of the neutral line, had acquired a positive double-refracting structure, and those at the concave, or compressed side, a negative one. $\dagger$ The intimate connexion between the double-refracting property, and the internal state of the body as to condensation or rarefaction, is likewise proved by the curious observation of M. Biot,- -that glass, when in a state of sonorous vibration, possesses the power of depolarizing the light.

In these cases of induced double refraction, the phenomena are related to the form of the entire mass ; and are essentially different from those produced by regular crystals, in which the law of elasticity and of double refraction depends solely on the direction, and is the same in all parts of the substance. Sir David Brewster has lately succeeded in communicating a regular double-refracting structure to a mixture of resin and white wax, by pressing it into 
a thin film between two plates of glass. This film had a single axis of double refraction at every point in the direction of the axis of pressure; and the tint developed depended solely on the inclination of the ray to this line. Sir David Brewster has drawn from this phenomenon some highly interesting conclusions respecting the origin of double refraction in regular crystals. He mentions several facts which seem to prove that this property is not inherent in the molecules themselves; and he conceives that it is developed by the unequal pressure caused by the forces of aggregation, which are in general different in the direction of three rectangular axes. Thus the double-refracting properties and the crystalline form are referred to the same agency.*

Sir David Brewster and Dr. Seebeck had before observed the phenomena arising from unequal condensation and rare faction in the case of uncrystallized bodies unequally heated. These phenomena may be studied by applying a bar of hot iron to the edge of a rectangular plate of glass, and placing it in the polarizing apparatus, so that the heated edge may form an angle of $45^{\circ}$ with the plane of primitive polarization. At the end of some time the whole surface of the plate is observed to be covered with coloured bands, the parts near the opposite edges having acquired a positive double-refracting structure, and those near the centre a negative one. The effects are reversed when a plate of glass uniformly heated is rapidly cooled at one of its edges; and all the appearances vanish when the glass acquires the same temperature throughout. $t$ These phenomena may be endlessly varied by varying the form of the glass to which the heat is applied. If now, by any means, the glass be arrested in one of these transient states, it will have acquired a permanent double-refracting structure. This has been accomplished by $M$. Seebeck by raising the glass to a red heat, and then cooling it rapidly at the edges. As the outer parts, which are thus more condensed, assume a fixed form in cooling, the interior parts must accommodate themselves to that form, and therefore retain a state of unequal density. The law of the change of density, and therefore the double-refracting structure, will depend on the external form; and M. Seebeck found, accordingly, that the coloured bands and patches which such bodies display in polarized light, assume a regular arrangement which 
varies with the shape of the mass.* The laws of these phenomena have been completely analyzed by Sir David Brewster; and he has shown that the colours are those of crystallized plates, the direction of the axes, however, being different in different parts of the substance.

As the double-refracting structure is communicated to bodies which do not possess it naturally, by mechanical compression or unequal temperature, - so, by the use of the same means, that structure may be altered in the bodies in which it already resides. Thus Sir David Brewster and M. Biot have found that the double refraction of regular crystals may be altered, and the tints they display made to rise or descend in the scale, by simple pressure. But the changes induced by heat are yet more remarkable. Professor Mitscherlich discovered the important fact that, in general, heat dilates crystals differently in different directions, and so alters their form; and their double-refracting properties have been found to undergo a corresponding change. Thus Iceland spar is dilated by heat in the direction of its axis; while it actually contracts by a small amount in directions perpendicular to it. The angles of the primitive form thus vary, the rhomboid becoming less obtuse, $\uparrow$ and approaching the form of the cube. M. Mitscherlich, accordingly, conjectured that its double-refracting energy must in these circumstances be diminished; and the conjecture was fully verified by experiment. This inquiry has been followed up by M. Rudberg; and the effects of heat on the refractive indices of doublerefracting crystals examined by the direct method of prismatic refraction. In conformity with the observations af M. Mitscherlich, it was found that the extraordinary index in Iceland spar increased considerably with the temperature, while the ordinary index underwent little or no change. In rock crystal, on the other hand, both indices diminished as the temperature augmented, and nearly by the same amount. In arragonite a similar effect was produced on the three principal refractive indices,-the least

* The experiments of M. Scebeck are recorded in Schweigger's Journal, 1814. The depolarizing property of unannealed glass secms to have been first noticed by M. Arago; and was afterwards studied by Sir David Brewster in glass which had been melted and cooled in water.-Phil. Trans. 1814.

t A change of temperature, from the freezing to the boiling-point, produced a shange of $8 \frac{1^{\prime}}{2}$ in the dihedral angles at the extremity of the axis.-Bull. Soc. Philo, March, 1824. 
index, however, undergoing the smallest proportionate diminution.*

The inclination of the optic axes, in biaxal crystals, is a simple function of the elasticities of the vibrating medium in the direction of three rectangular axes; and the plane of the optic axes is that of the greatest and least elasticities. If, then, these three principal elasticities be altered by heat in different proportions, the inclination of the axes will likewise vary; and if, in the course of this change, the difference between the greatest elasticity and the mean, or between the mean and the least, should vanish and afterwards change sign, the two axes will collapse into one, and finally open out in a plane perpendicular to their former plane. All these variations have been actually observed. Professor Mitscherlich found that, in sulphate of lime, the angle between the axes (which is about $60^{\circ}$ at the ordinary temperature) diminishes on the application of heat; that, as the temperature increases, these axes approach until they unite; and that, on a still further augmentation of heat, they again separate and open out in a perpendicular plane. The primitive form of the crystal undergoes a corresponding change, the dilatation being greater in one direction than in another at right angles to it. Sir David Brewster has observed an analogous, and even yet more remarkable property, in glauberite. At the freezing temperature this erystal has two axes for all the rays of the spectrum, the inclination of the axes being greatest in red light, and least in violet. As the temperature rises, the two axes approach, and those of different colours unite in succession; and at the ordinary temperature of the atmosphere, the crystal possesses the singular property of being uniaxal for violet light and biaxal for red. When the heat is further increased, the axes which have united open out in order, and in a plane at right angles to that in which they formerly lay; and at a temperature much below that of boiling water, the planes of the axes for all colours are perpendicular to their first position. $\dagger$ The inclination of the optic axes in topaz, on the other hand, augments with the increase of temperature; and the variation, M. Marx has observed, is much greater in the coloured than in the colourless varieties of this mineral. $\neq$

* Phil. Mag., Third Series, vol. i. 409.

$\dagger$ Edin. Trans., vol. xi.; and Phil. Mag., Third Series, vol. i. 417.

$\ddagger J a h r b$. der Chemie, vol. ix. 


\section{III.-ON A NEW CASE OF INTERFEREYCE OF THE RAYS OF LIGHT.}

Transactions of the Royal Irish Acadcmy, Vol. XVII.

THE experiment of Fresnel, on the interference of the lights proceeding from the same origin, and reflected by two mirrors inclined at a very obtuse angle, has been justly regarded as one of the most important in the whole range of physical optics. The principle of interference itself had, indeed, been stated broadly by Young, and supported by the evidence of phenomena, which, to the unbiassed inquirer, left little to desire. All these phenomena, however, admitted of other possible explanations ; and the advocates of the corpuseular theory of light had recourse to these, rather than admit the truth of a law which afforded such strong support to the undulatory theory. In most of these phenomena, the light was in part intercepted by an obstacle, and it was conceived that, in passing by the edge, the molecular action, which might be supposed to exist between the particles of the body and those of light, was sufficient to account for the facts observed. But, in Fresnel's experiment, the two lights which interfere are regularly reflected by the surfaces of the mirrors, according to the ordinary laws, and are divested of every extraneous circumstance which could, by possibility, be supposed to influence the result. This experiment, accordingly, has materially changed the character of the controversy respecting the nature of light; and the advocates of the Newtonian theory, of the present day, are forced to admit the principle thus rigidly established, and labour only to show how the theory and that principle may be reconciled. 
While examining this important experiment-the adjustment of which is a matter of some delicacy-it occurred to me that the fact of direct interference might be shown in a yet simpler manner, by the mutual action of direct and reflected light. An interference of this kind was assumed by Young to account for some of the phenomena of diffraction; but Fresnel showed that the explanation was incomplete, and that the phenomena in question were caused merely by the interference of the secondary waves, reflexion playing no part in their production. Under these circumstances it is somewhat strange that the fact of the interference of direct and reflected lights should not have been itself submitted to the test of experiment; especially as the character of this interference, if it were found to exist, might be expected to throw some light upon the laws of reflexion itself.

The theory of such interference is easily deduced from the general principles. Let light proceeding from a single luminous origin fall upon a reflecting surface, at an incidence of nearly $90^{\circ}$ : a screen placed at the other side of the reflector will be illuminated, throughout a certain extent, by both direct and reflected lights; and, if the difference of the paths traversed by these lights amounts only to a small multiple of the length of an undulation, the two lights will form fringes by their interference.

Let the intensities of the direct and reflected lights be denoted by $a^{2}$ and $a^{\prime 2}$, and that of the resulting light by $A^{2}$; then, by the theory of the composition of coexisting vibrations, we have

$$
A^{2}=a^{2}+2 a a^{\prime} \cos 2 \pi\left(\frac{\delta^{\prime}-\delta}{\lambda}\right)+a^{\prime 2} ;
$$

$\delta$ and $\delta$ denoting the lengths of the paths traversed by the two waves, from their origin to any given point, and $\lambda$ the length of an undulation.

The intensity of the resulting light will be a maximum, and equal to $\left(a+a^{\prime}\right)^{2}$, at these points for which

$$
\cos 2 \pi\left(\frac{\delta-\delta}{\lambda}\right)=+1, \quad \text { or } \quad \delta^{\prime}-\delta=2 n \frac{\lambda}{2} \text {. }
$$

It will be a minimum, and equal to $\left(a-a^{\prime}\right)^{2}$, when

$$
\cos 2 \pi\left(\frac{\delta^{\prime}-\delta}{\lambda}\right)=-1, \quad \text { or } \quad \delta^{\prime}-\delta=(2 n+1) \frac{\lambda}{2}
$$


$n$ being any number of the natural series $0,1,2,3$, \&c. Bright fringes, therefore, will be formed at all the points included in the former equation, and dark ones at the points corresponding to the latter.

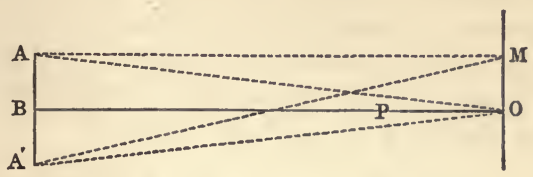

Let $O P$ be the reflector; $O M$ the screen placed in contact with it, and perpendicular to its plane; and let $A$ be the luminous origin, and $A^{\prime}$ its reflected image at an equal distance below the line $O B$. Then, if $M$ be any point whose illumination is required, $\delta=A M$, $\delta^{\prime}=A^{\prime} M$.

Now if $A B$ be denoted by $p, B O$ by $d$, and $O M I$ by $x$, it is obvious that

$$
\delta^{2}=d^{2}+(p-x)^{2}, \quad \delta^{2}=d^{2}+(p+x)^{2} .
$$

Hence, approximately,

$$
\delta=d\left\{1+\frac{1}{2}\left(\frac{p-x}{d}\right)^{2}\right\}, \quad \delta^{\prime}=d\left\{1+\frac{1}{2}\left(\frac{p+x}{d}\right)^{2}\right\} ;
$$

and therefore

$$
\delta^{\prime}-\delta=\frac{2 p x}{d}=2 \tan \alpha \cdot x,
$$

the angle $A O B$ being denoted by $a$. Hence the general expression of the intensity of the light, at any point $M$, is

$$
A^{2}=a^{2}+2 a a^{\prime} \cos \left(4 \pi \tan a \frac{x}{\lambda}\right)+a^{\prime 2} .
$$

Again, substituting for $\delta-\delta$ its value just found, we see that the successive fringes will be formed at the distances given by the formula

$$
x=\frac{1}{4} m \lambda \operatorname{cotan} \alpha ;
$$

in which $m$ is any number of the natural series, its even values giving the places of the bright fringes, and its odd values those of the dark ones. Accordingly, the bright fringes are formed at the distances $0,2 l, 4 l, \& c$. , and the dark ones at the distances intermediate, $l, 3 l, 5 l$, \&c., $l$ being equal to $\frac{1}{4} \lambda \operatorname{cotan} a$ : the successive fringes, therefore, are equidistant. It is obvious that the angle 
a must be very small, or the incidence very oblique, in order that the fringes should have any sensible breadth.

We have hitherto assumed that the light has undergone no change by reflexion, excepting the change of direction. Let us now suppose that the phase of the vibration is accelerated, and let us examine the effect produced in the position of the fringes.

Let the amount of this acceleration be denoted by the angle $\mu \pi$; then the differences of the phases will be

$$
2 \pi\left(\frac{\delta^{\prime}-\delta}{\lambda}\right)-\mu \pi=2 \pi\left(\frac{\delta^{\prime}-\delta-\frac{1}{2} \mu \lambda}{\lambda}\right) .
$$

So that the successive fringes will be formed at the points for which

$$
\delta^{\prime}-\delta-\frac{1}{2} \mu \lambda=\frac{1}{2} m \lambda,
$$

$m$ being any number of the natural series. But we have already found that $\delta-\delta=2 \tan a x$; so that the points in question are given by the formula,

$$
x=\frac{1}{4}(m+\mu) \lambda \operatorname{cotan} \dot{a} ;
$$

the even values of $m$ corresponding to the bright fringes, and the odd values to the dark ones. It is evident from this that the magnitude of the fringes will be unaltered; and that the only effect of the acceleration is to push the entire system from the edge, the amount of the shifting being equal to $\frac{1}{4} \mu \lambda \operatorname{cotan} a$.

In order to submit these results to the test of trial, I employed the apparatus consisting of two moveable metallic plates, which is of so much use in experiments of interference. The plates being closed, so as to form a narrow horizontal aperture, the flame of a lamp was placed behind; and the light thus diverging from the aperture was received, at the distance of about three feet, on a piece of black glass truly polished, and also horizontal. This reflector was then adjusted, so that its plane might pass a little below the aperture; or, in other words, that the light might be incident upon it at an angle of nearly $90^{\circ}$. It is evident, then, that the light thus obliquely reflected will meet the direct light diverging from the aperture under a very small angle, and with a difference in the lengths of their paths which is capable of indefinite diminution. The two lights, therefore, are in a condition to interfere; and I found, accordingly, that when they were received upon an eyepiece, placed at a short distance from the reflector, a very 
beautiful system of bands was visible, in every respect similar to onehalf of the system formed by the two mirrorsin Fresnel's experiment.

The first band was a bright one, and colourless. This was succeeded by a very sharply defined black band; then followed a coloured bright band, and so on alternately. Under favourable circumstances I could easily count seven alternations; the breadth of the bands being, as far as the eye could judge, the same throughout the series, and increasing with the obliquity of the reflected beam. The first dark band was of intense blackness. The darkness of the sueceeding bands was less intense, as they were of higher orders; and after three or four orders, they were completely obliterated by the closing in of the bright bands. At the same time the colouration of the bright bands increased with the order of the band; until, after six or seven alternations, the colours of different orders became superimposed, and the bands were thus lost in a diffused light of nearly uniform intensity. All these circumstances are similar to those observed in Fresnel's experiment, and correspond exactly with the results of theory.

These bands are most perfectly defined when the eyepiece is close to the reflector. Their breadth and colouration increased with the distance of the eyepiece, but remained of a finite and very sensible magnitude, when the latter was brought into actual contact with the edge - a circumstance which distinguishes them altogether from the diffracted fringes formed on the boundary of the shadow.

These fringes appear to me to possess some interest in a theoretical point of view, independently of that which attaches to them as illustrations of an important general law. Depending on the interference of two lights, one of which proceeds directly from the luminous origin, while the other has undergone reflexion, they would seem to afford the means of detecting any difference which might exist in their condition when they meet, and therefore of tracing the modifications produced by reflexion.

There are two circumstances which chiefly demand our attention in the case of reflected light-namely: 1st, the amplitude of the vibration, on which the intensity of the light depends; and 2ndly, the phase. The facts before us seem, to a certain extent, to bear on both these points.

The reasonings of Fresnel, with respect to the intensity of reflected light, are partly of an analogical nature, and very far 
indeed from being strictly demonstrative. Still, however, they have led to conclusions fully borne out by experience, and of the most interesting kind; and we can hardly refuse our assent to doctrines which bear with them such characters of truth. The formula which Fresnel has obtained for the intensity of reflected light has not received any direct confirmation from experiment, except in the case of a few observations made by M. Arago. It results from this formula that the intensity of the reflected light must be equal to that of the incident, or the whole of the light reflected, at the limiting incidence of $90^{\circ}$. Fresnel himself notices this consequence, and adds that we should doubtless find it to be experimentally true, if we could reach this limit. Now the present experiment affords the means of examining this conclusion, and seems fully to establish it. We have already alluded to the intense blackness of the first dark bar, in the phenomena now described. As far as the eye can judge, the intensity of the light is absolutely nothing at the points corresponding to this bar; and. as the intensity of the light in the dark bands is generally expressed by the formula $\left(a-a^{\prime}\right)^{2}$, we are forced to admit that $a=a^{\prime}$, or that the intensities of the direct and reflected lights are equal at this extreme incidence.

With respect to the effect of reflexion upon the phase of vibration, there seems to be some uncertainty in the theory. The phenomena of thin plates compel us to admit that half an undulation is either lost or gained, by the wave reflected from the first or second surface; so that half an undulation must be added to, or subtracted from, the difference in the lengths of the paths traversed by the two waves. That such an effect should take place is in the highest degree probable from theoretical considerations. The light in the one case is reflected from the surface of a denser, in the other from that of a rarer medium; and the mechanical laws, on which Fresnel has founded the doctrine of reflexion, lead us to the conclusion that the displacements of the ethereal particles, in the moment after reflexion, must be of opposite signs in the two cases. This difference in the phase of the vibration is equivalent to a difference of half an undulation in the length of the path.

But it does not seem to be clearly understood to which surface we are to attribute this physical change in the condition of the ray. Dr. Young, indeed, who was the first to state this law, says 
expressly that where "light has been reflected at the surfare of the rarer medium, it must be supposed to be retarded one-half of the appropriate interval." I cannot avoid thinking that the very analogy by which he himself illustrates this point, and still more the reasonings of Fresnel on the subject, lead to an opposite conclusion, and tend to ascribe the effect which is found to take place to reflexion at the surface of the denser medium. In fact, it would appear from Fresnel's conclusions, that the sign of the vibratory movement is in all cases changed by reflexion at the surface of the denser medium, the angle of incidence exceeding the polarizing angle; and it can readily be shown that this change of sign is equivalent to the addition of $\pm \pi$ to the phase.

The present case of interference seems to support this view. It follows, as we have seen, from theory, that if the light undergoes no change of phase by reflexion, the distances of the successive dark fringes from the edge of the shadow will be as the odd numbers, 1, 3, 5, \&c.; so that the distance of the first dark band from the edge will be half the interval between each succeeding pair of dark bands. But it appears, on the contrary, from the phenomena, that the distance is-as far as the eye can judgeexactly equal to the succeeding intervals; or that the bands are all shifted from the edge by the amount of half an interval. 'The phenomena, therefore, require us to suppose that the phase of the reflected wave is accelerated, and that the amount of this acceleration is exactly half a phase, or $\pi$. For the general expression for the shifting of the bands is $\frac{1}{4} \mu \lambda \operatorname{cotan} a$; and as this is found to be equal to $\frac{1}{4} \lambda \operatorname{cotan} \alpha$, it follows that $\mu=1$, or the acceleration equal to $\pi$. It appears then that when light is reflected at the surface of a denser medium, the wave-at the limiting incidence at least-gains half an undulation at the instant of reflexion.

In order to satisfy myself more fully of the effects of reflexion upon the phase, I repeated the experiment with polarized light. The light was polarized, before it reached the aperture in the screen, by transmission through a good tourmaline; and the fringes were observed in various positions of the plane of polarization with respect to the plane of reflexion. I could detect no sensible difference in the position of the fringes under all these changes of circumstance; and, in particular, the distance of the first dark band from the edge of the shadow seemed, as before, to be precisely equal to the intervals of the succeeding 
bands, whether the light was polarized in the plane of incidence, or in the plane perpendicular to it.

This result seems to be just what might be expected from Fresnel's theory of reflexion. From this theory it appears that if $+a$ be the coefficient of the displacement of the incident ray, or the amplitude of the vibration, and $i$ and $i^{\prime}$ the angles of incidence and refraction, the coefficients of displacement of the reflected ray will be

$$
-a \frac{\sin \left(i-i^{\prime}\right)}{\sin \left(i+i^{\prime}\right)}, \text { or }+a \frac{\tan \left(i-i^{\prime}\right)}{\tan \left(i+i^{\prime}\right)^{\prime}}
$$

according as the plane of polarization coincides with the plane of reflexion, or is perpendicular to it. Now the former quantity is always negative, so long as $i$ is greater than $i^{\prime}$, or the ray incident on the surface of the denser medium. Under the same circumstances, the latter quantity is positive or negative, according as $i+i^{\prime}$ is less or greater than $90^{\circ}$, or the angle of incidence below or above the polarizing angle. For very oblique reflexion, then, both displacements are negative; and, therefore, whether the plane of polarization coincides with, or is perpendicular to the plane of reflexion, the wave will undergo a change of half a phase at the instant of reflexion.

From Sir David Brewster's important researches on the nature of metallio reflexion, it appears that a plane-polarized ray, which is incident upon a metallic reflector, becomes elliptically-polarized after reflexion; a result which indicates a difference in the phases of the two resolved vibrations. But it appears further, from the same researches, that this difference of phase varies with the incidence, and vanishes altogether at the extreme incidences; so that at the limiting incidence of $90^{\circ}$, there is either no alteration in the phase of vibration, whether parallel or perpendicular to the plane of reflexion, or that alteration is the same for the two vibrations. From some observation of the fringes produced by the interference of direct light with that reflected from speculum metal, I conclude that the former is the case. 
IV.-ON THE LIGHT REFLECTED AND TRANSMITTED BY THIN PLATES.

Transactions of the Royal Irish Academy, Vol. XXIV.

THe problem of thin plates has been completely solved for the cases in which the incident light is polarized in either of the two principal planes, and therefore also for the case of unpolarized light, which, it is well known, may be regarded as composed of two equal pencils, polarized respectively in these two planes.

There is no difficulty in perceiving in what manner the theory may be extended to light polarized in any plane; but the results become more complicated, from the necessity of including the consideration of phase. When the incident light is polarized in the plane of incidence, or in the perpendicular plane, we have only to seek the magnitude of the resultant vibration, after the successire divisions which it undergoes by reflexion and refraction at the two surfaces of the plate. But when the light is polarized in any other plane, the incident vibration must be resolved into two, in the two principal planes; and for each of these components we must know the phases, as well as the magnitudes of the resultant vibrations, before we can estimate their joint effect. These phases are in general different, and therefore the resulting light is elliptically-polarized.

The preceding, and some other results of theory, were pointed out by the author many years ago; and the general formulx, in which they are included, were at the time laid before the Academy.* In the present communication it is proposed to develop these consequences; and in particular, to deduce the law

* Proccedings of the Royal Irish Academy, June 13, 1842. 
according to which the elliptic polarization varies, both with the thickness of the plate and with the incidence.

It is assumed in this investigation, that in the reflexion and refraction of light at the surface of a transparent medium, the phases of the incident, reflected, and refracted vibrations coincide at the refracting surface. This assumption is that made by Fresnel. Its theoretical truth is indeed now disproved by the more complete analysis of Cauchy, and by the experimental labours of M. Jamin; but its deviation from the phenomena is exceedingly small, except within a small range of incidence in the neighbourhood of the angle of polarization, and the conclusions based upon it are therefore sensibly in accordance with fact, except for the same incidences.

It is unnecessary to add, after what has been just stated, that the elliptio polarization here considered is altogether dictinct, both in its origin and in its laws, from that produced by reflexion at a single surface.

Let us suppose that the incident light is polarized either in the plane of incidence, or in the perpendicular plane. Let $u$ and $u^{\prime}$ denote the ratios of the reflected and refracted vibrations to the incident vibration at the first surface of the plate, and for light falling upon it from without; $(u)$ and $\left(u^{\prime}\right)$ the corresponding ratios for light proceeding in the opposite direction; and $u_{2}$ the ratio of the reflected to the incident vibration at the second surface. Then, the amplitude of the incident vibration being unity, the amplitudes of the vibrations which emerge at the first surface, after one reflexion at the second, $=u^{\prime} u_{2}\left(u^{\prime}\right)$; after three internal reflexions, $=u^{\prime} u_{2}(u) u_{2}\left(u^{\prime}\right)=1$ st portion $\times(u) u_{2}$; after five internal reflexions, $=2$ nd portion $\times(u) u_{2}$, \&c. But

$$
(u)=-u, \quad u^{\prime}\left(u^{\prime}\right)=1-u^{2} .^{*}
$$

These amplitudes, accordingly, form a series in geometric progression, whose first term is $u^{\prime} u_{2}\left(u^{\prime}\right)=u_{2}\left(1-u^{2}\right)$, and whose common ratio is $(u) u_{2}=-u u_{2}$.

But the interval of retardation, after one internal reflexion,

* The truth of these relations is evident from the known formulæ of Fresnel. It has been deduced independently, from very simple general principles, by Professor Stokes, and has been shown by him to hold even in the case of change of phase. See an interesting paper "On the perfect blacknęss of the central spot in Newton's Rings."-Cambridge and Dublin Mathematical Journal, 1849. 
$=2 \tau \cos \theta^{\prime}, \tau$ being the thickness of the plate, and $\theta^{\prime}$ the angle of refraction; and the corresponding difference of phase is

$$
a=\frac{4 \pi}{\lambda} \tau \cos \theta^{\prime}
$$

$\lambda$ being the length of an undulation. The difference of phase, after three internal reflexions, is $2 a$; after five internal reflexions, $3 a$; and so on. Hence, if $\phi$ denote the phase of the vibration reflected at the first surface, at the instant of reflexion, $\phi-a$ will be the phase of the portion which emerges there after one internal reflexion; $\phi-2 a$, after three, \&c. And the sum of all the internally-reflected vibrations will be

$u_{2}\left(1-u^{2}\right)\left[\sin (\phi-a)-u u_{2} \sin (\phi-2 a)+u^{2} u_{2}{ }^{2} \sin (\phi-3 a)-\& c.\right]$; in which, it can be easily shown, the quantity within the brackets is equal to

$$
\frac{\sin (\phi-a)+u u_{2} \sin \phi}{1+2 u u_{2} \cos a+u^{2} u_{2}^{2}} .
$$

Adding $u$ sin $\phi$, - the vibration reflected externally at the first surface of the plate, - the sum of all the reflected vibrations is

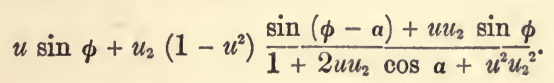

Let this quantity be put under the form

Then we find

$$
P \sin \phi+Q \cos \phi \text {. }
$$

$$
\begin{array}{r}
P=u+u_{2}\left(1-u^{2}\right) \frac{\cos a+u u_{2}}{1+2 u u_{2} \cos \boldsymbol{\alpha}+u^{2} u_{2}{ }^{2}}, \\
Q=\frac{-u_{2}\left(1-u^{2}\right) \sin a}{1+2 u u_{2} \cos \boldsymbol{\alpha}+u^{2} u_{2}{ }^{2}}
\end{array}
$$

Wherefore, the intensity of the resulting light is*

$$
I=P^{2}+Q^{2}=\frac{u^{2}+2 u u_{2} \cos a+u_{3}^{2}}{1+2 u u_{2} \cos a+u^{2} u_{2}^{2}}
$$

and its phase, $\psi$, will be given by the formula,

$$
\tan \psi=\frac{-Q}{P}=\frac{u_{2}\left(1-u^{2}\right) \sin \alpha}{u\left(1+u_{2}^{2}\right)+u_{2}\left(1+u^{2}\right) \cos a} .
$$

* This expression for the intensity has been alıeady obtained by Mr. Airy- "On the Phenomena of Newton's Rings, when formed between two transparent substances of different refractive powers."-Camb. Trans., 1832. 
Substituting $1-\sin ^{2} \frac{a}{2}$ for $\cos a$, the expression for the intensity becomes

$$
I=\frac{\left(u+u_{2}\right)^{2}-4 u u_{2} \sin ^{2} \frac{a}{2}}{\left(1+u u_{2}\right)^{2}-4 u u_{2} \sin ^{2} \frac{a}{2}} .
$$

When the media are the same on the two sides of the plate, $u_{2}=-u$, and the foregoing formula is reduced to the known one,

$$
I=\frac{4 u^{2} \sin ^{2} \frac{a}{2}}{\left(1-u^{2}\right)^{2}+4 u^{2} \sin ^{2} \frac{a}{2}} .
$$

The greatest and least values of $I$, in the general formula, correspond to $\sin \alpha=0$, or $\alpha=m \pi$. When $\alpha=2 m \pi$, the expression is reduced to

$$
I=\left(\frac{u+u_{2}}{1+u u_{2}}\right)^{2} .
$$

When $\alpha=(2 m+1) \pi$, it becomes

$$
I=\left(\frac{u-u_{2}}{1-u u_{2}}\right)^{2} .
$$

When $u$ and $u_{2}$ have the same sign, the former value is a maximum, and the latter a minimum; the rings consequently begin from a bright centre. On the other hand, when $u$ and $u_{2}$ have opposite signs, the former value is a minimum, and the latter a maximum; and the rings begin from a dark centre.

When the incident light is polarized in the plane of incidence, the signs of $u$ and $u_{2}$ are determined solely by the relative refractive densities of the plate, and of the two media which border it on either side. When the refractive density of the plate is greater or less than those of both media, $u$ and $u_{2}$ are of opposite signs, and the rings are dark-centred. On the other hand, when the refractive density of the plate is intermediate to those of the two media on either side, $u$ and $u_{2}$ have the same sign, and the rings are bright-centred. This inversion of the phenomenon was observed by Young. 
When the incident light is polarized perpendicularly to the plane of incidence, the signs of $u$ and $u_{2}$ depend on the incidence, as well as on the relative refractive densities of the media. When the angle of incidence is less than the polarizing angle at either surface, the sign of $u$, or of $u_{2}$, is the same as in the case of light polarized in the plane of incidence; when it is greater, the sign is opposite. Accordingly, when the incidences at both surfaces are less than their respective polarizing angles, the character of the rings will be the same as for light polarized in the plane of incidence. And the same thing is true when the incidences at both surfaces are greater than their respective polarizing angles; for in this case the signs of $u$ and $u_{2}$ are changed simultaneously. But when the incidence at one surface is less, and at the other greater, than their respective polarizing angles, one of the quantities, $u$ and $u_{2}$, changes sign, and the other does not; and the character of the rings will be the opposite to that presented when the light is polarized in the plane of incidence.

It follows, then, that when the refractive density of the plate is greater or less than those of the two media, the rings will be dark-centred at the lower and higher incidences, and bright-centred at the intermediate incidences. This phenomenon was observed by Arago, and explained by Mr. Airy. On the other hand, when the refractive density of the plate is intermediate to those of the two media (the light being still polarized in the perpendicular plane), the rings will be bright-centred at the lower and higher incidences, and dark-centred at the intermediate incidences. This latter ease has not been hitherto noticed.

At the polarizing angles of either surface, $u=0$, or $u_{2}=0$, and the rings vanish.

7. Now let the incident light be polarized in any plane, inclined at the angle $\gamma$ to the plane of incidence; and let the amplitude of the incident vibration $(=1)$ be resolved into two, $\cos \gamma$ and $\sin \gamma$, in the plane of incidence and in the perpendicular plane, respectively. Let $v$ and $v_{2}$ denote the values of $u$ and $u_{2}$ in the case of light polarized in the plane of incidence; $w$ and $v_{2}$ the corresponding values for light polarized perpendioularly ; then the intensities of the light in the two reflected pencils are

$$
I=\frac{v^{2}+2 v v_{2} \cos \boldsymbol{\alpha}+t_{2}{ }^{2}}{1+2 v t_{2} \cos \boldsymbol{a}+v^{2} v_{2}{ }^{2}} \cos ^{2} \gamma, \quad J=\frac{w^{2}+2 u v_{2} \cos \boldsymbol{\alpha}+v_{2}{ }^{2}}{1+2 u v_{2} \cos \boldsymbol{\alpha}+w^{2} v_{2}{ }^{2} \sin ^{2}} \gamma .
$$


And the intensity of the reflected light will be the sum of the intensities of the two component portions.

When the obliquity of the incident pencil is so small, that the squares and products of $v, v_{2}, w, v_{2}$, may be neglected in comparison with unity, the intensity of the reflected light will be, nearly,

$$
\left(v^{2}+2 v v_{2} \cos \boldsymbol{\alpha}+v_{2}^{2}\right) \cos ^{2} \gamma+\left(w^{2}+2 w v_{2} \cos \gamma+w_{2}{ }^{2}\right) \sin ^{2} \gamma .
$$

This expression is independent of the phase, and therefore the intensity is constant for a given incidence, when

$$
v v_{2} \cos ^{2} \gamma+v w_{2} \sin ^{2} \gamma=0, \quad \text { or } \tan ^{2} \gamma=-\frac{v v_{2}}{w w_{2}} \text {. }
$$

It will be easily seen, on substituting for $v, v_{2}, w, v_{2}$, their wellknown values, that the value of $\tan \gamma$ will be real, and therefore the disappearance of the rings possible, only when the angles of incidence at the two surfaces of the plate are, in the one case greater, and in the other less, than the respective polarizing angles.* This is the explanation of the phenomenon observed by Sir David Brewster.

Again, since the values of $v$ and $v_{2}$ are, in general, different from those of $w$ and $w_{2}$, it follows that the phases of the two component vibrations are unequal, and consequently that the resulting reflected light is elliptically-polarized. This consequence of the wave-theory does not appear to have been noticed by observers.

8. We may now proceed to consider more particularly the case in which the media are the same on the two sides of the plate.

In this case, $v_{2},=-v, w_{2}=-w$, and the general expressions for the phases of the two polarized pencils are reduced to

$$
\tan \psi=-\frac{1-v^{2}}{1+v^{2}} \cot \frac{a}{2}, \quad \tan \chi=-\frac{1-w^{2}}{1+w^{2}} \cot \frac{a}{2} .
$$

The difference of phase is given by the formula

$$
\tan (\psi-\chi)=\frac{\left(v^{2}-w^{2}\right) \sin \boldsymbol{\alpha}}{1-\left(v^{2}+w^{2}\right) \cos \boldsymbol{\alpha}+v^{2} w^{2}} .
$$

It follows from this, that $\psi-\chi$ varies with $a$, and therefore with

\footnotetext{
* See the paper above referred to,-Proceedings, vol. ii., p. 268.
} 
the thickness of the plate; and that, in the phenomena of the rings, it will go through all its values within the limits of a single ring.

9. The difference, $\psi-\chi$, generally vanishes, when $\sin a=0,{ }^{*}$ or

$$
\boldsymbol{a}=m \pi .
$$

Accordingly, the difference of phase is nothing both at the bright and at the dark rings; and the light at the former is plane-polarized.

The difference of phase is a maximum, when

$$
\cos \boldsymbol{\alpha}=\frac{v^{2}+w^{2}}{1+v^{2} w^{2}}
$$

and denoting by $\Delta$ the maximum value of $\psi-\chi$, we have

$$
\tan \Delta=\frac{v^{2}-w^{2}}{\sqrt{ }\left\{\left(1-v^{4}\right)\left(1-w^{4}\right)\right\}} .
$$

10. In order to express $a$ and $\Delta$ in terms of the angle of incidence, let us make $\frac{\cos \theta}{\cos \theta}=\rho$; and substituting in Fresnel's formulas, we find

$$
v=\frac{\sin \left(\theta-\theta^{\prime}\right)}{\sin \left(\theta+\theta^{\prime}\right)}=\frac{\mu \rho-1}{\mu \rho+1}, \quad v=\frac{\tan \left(\theta-\theta^{\prime}\right)}{\tan \left(\theta+\theta^{\prime}\right)}=\frac{\mu-\rho}{\mu+\rho} ;
$$

$\mu$ being the refractive index for light entering the plate from the surrounding medium. Making these substitutions, the thickness of the plate corresponding to the maximum difference of phase is determined by the formula,

$$
\cos a=\frac{\left(\mu-\mu^{-1}\right)^{2}+\left(\rho-\rho^{-1}\right)^{2}}{\left(\mu+\mu^{-1}\right)^{2}+\left(\rho+\rho^{-1}\right)^{2}} .
$$

Or, since $\rho^{2}=1+\left(1-\mu^{-2}\right) \tan ^{2} \theta$,

$$
4 \cot ^{2} \frac{a}{2}=\left(\mu+\mu^{-1}\right)^{2}+\left(\rho-\rho^{-1}\right)^{2}=\left(\mu+\mu^{-1}\right)^{2}+\frac{\left(1-\mu^{-2}\right)^{2} \tan ^{4} \theta}{1+\left(1-\mu^{-2}\right) \tan ^{2} \theta} .
$$

Accordingly, the value of $\cot \frac{a}{2}$ increases, from $\frac{1}{2}\left(\mu+\mu^{-1}\right)$ at a perpendicular incidence, to infinity when the incidence is most

- When $\theta=90^{\circ}$, we have $v^{2}=1, w^{2}=1$; and the expression for $\tan (\psi-\chi)$ becomes $\frac{0}{0}$, when $\alpha=2 m \pi$. It appears from the following, that the difference of phase is a maximum in this case. 
oblique; and, in a plate of varying thickness, the points of maximum difference of phase commence near the middle of an interval, and approach indefinitely to the dark rings as the incidence approaches to $90^{\circ}$.

The tangent of the maximum difference of phase is

$$
\tan \Delta=\frac{\left(\mu^{2}-1\right)\left(\rho^{2}-1\right)}{2 \sqrt{ }\left\{\left(1+\mu^{2} \rho^{2}\right)\left(\mu^{2}+\rho^{2}\right)\right\}} ;
$$

or, putting for $\rho^{2}$ its value,

$$
\left.\cot \Delta=\frac{2 \mu^{2}}{\mu^{2}-1} \sqrt{ }\left\{\frac{\mu^{2}+1}{\mu^{2}-1} \cot ^{2} \theta+1\right)\left(\frac{\mu^{2}+1}{\mu^{2}-1} \cot ^{2} \theta+\frac{1}{\mu^{2}}\right)\right\} \text {. }
$$

When $\mu$ is greater than unity, or the plate denser than the surrounding medium, $\Delta=0$, when $\theta=0$. As $\theta$ increases, $\Delta$ increases continuously; until, when $\theta$ approaches to $90^{\circ}, \Delta$ approaches indefinitely to the value,

$$
\cot \Delta=\frac{2 \mu}{\mu^{2}-1} .
$$

This is its greatest value. For ordinary flint glass, and for the extreme red ray, $\mu=1 \cdot 60$, and $\Delta=26^{\circ}$.

When $\mu$ is less than unity, or the plate rarer than the surrounding medium, $\Delta=0$, when $\theta=0$, as before. And $\Delta$ increases as $\theta$ increases, up to the limiting incidence, for which $\theta=\sin ^{-1} \mu$, and $\cot \Delta=\frac{2 \mu}{\mu^{2}-1}$. The greatest difference of phase, therefore, is the same in both cases, and in both corresponds to the extreme incidence at which light is admitted into the plate.*

11. Again, the media being the same on the two sides of the plate, the expressions for the intensities of the two portions of the reflected light, polarized respectively in the plane of incidence and in the perpendicular plane, become

* It is evident that $\cot \Delta=0$, and therefore $\Delta=90^{\circ}$, when the incidence has either of the values given by the formulas

$$
\tan ^{2} \theta=\frac{1+\mu^{2}}{1-\mu^{2}}, \text { and } \tan ^{2} \theta=\mu^{2} \frac{1+\mu^{2}}{1-\mu^{2}} ;
$$

both of which are real when the plate is rarer than the surrounding medium. These values, however, are both greater thsn the limiting incidence, snd the light dces not enter the plate. When the incidence is inten mediate to the two preceding values, $\Delta$ is imaginary. 


$$
I=\frac{4 v^{2} \sin ^{2} \frac{a}{2} \cos ^{2} \gamma}{1-2 v^{2} \cos a+v^{4}}, \quad J=\frac{4 w^{2} \sin ^{2} \frac{\alpha}{2} \sin ^{2} \gamma}{1-2 w^{2} \cos a+v^{4}} .
$$

Let the ratio of the corresponding amplitudes be denoted by $\tan \gamma^{\prime}$; then

$$
\tan \gamma^{\prime}=\tan \gamma \frac{w}{v} \sqrt{\left(\frac{1-2 v^{2} \cos a+v^{4}}{1-2 w^{2} \cos u+v^{4}}\right) .}
$$

The angle $\gamma^{\prime}$ will be the azimuth of the plane of polarization, when the reflected light is plane-polarized.

When the thickness of the plate is that corresponding to the maximum difference of phase, or $\cos a=\frac{v^{2}+w^{2}}{1+v^{2} w^{2}}$, the ratio of the amplitudes becomes

$$
\tan \gamma^{\prime}=\tan \gamma \frac{w}{v} \sqrt{\left(\frac{1-v^{4}}{1-w^{4}}\right) .}
$$

The angles $\Delta$ and $\gamma^{\prime}$ being known, the character of the elliptic polarization is completely determined.

12. We may now proceed to examine the intensity and phase of the transmitted light.

It will be easily seen, by following the same reasoning as before, that the transmitted light consists of an indefinite number of portions, which emerge at the second surface of the plate after $0,2,4, \& c$., internal reflexions; and that the amplitudes of their vibrations form a series in geometric progression, whose first term is $u^{\prime} u_{2}^{\prime}$, and whose common ratio is $-u u_{2}$, in which $u$ and $u_{2}$ denote, as before, the ratios of the amplitudes of the reflected to the incident vibrations, at the first and second surfaces of the plate respectively, and $u^{\prime}$ and $u_{2}^{\prime}$ the corresponding ratios for the refracted vibrations. It is likewise evident that, if $\phi$ denote the phase of the first portion, $\phi-a$ will be that of the second, $\phi-2 a$ that of the third, de., in which

$$
a=\frac{4 \pi}{\lambda} \tau \cos \theta
$$

as before. Consequently the resulting vibration, which is the sum of all these, is

$$
\begin{gathered}
u^{\prime} u_{2}^{\prime}\left\{\sin \phi-u u_{2} \sin (\phi-a)+u^{2} u_{2}{ }^{2} \sin (\phi-2 u)-\mathcal{E} .\right\} \\
=u^{\prime} u_{2}^{\prime} \frac{\sin \phi+u u_{2} \sin (\phi+a)}{1+2 u u_{2} \cos a+u^{2} u_{2}{ }^{2}} .
\end{gathered}
$$


Revolving this, we find

$$
P=u^{\prime} u_{2}^{\prime} \frac{1+u u_{2} \cos \boldsymbol{a}}{1+2 u u_{2} \cos \boldsymbol{\alpha}+u^{2} u_{2}^{2}}, \quad Q=u^{\prime} u_{2}^{\prime} \frac{u u_{2} \sin a}{1+2 u u_{2} \cos a+u^{2} u_{2}{ }^{2}} .
$$

Hence the intensity of the transmitted light is

$$
I^{\prime}=P^{2}+Q^{2}=\frac{\left(u^{\prime} u_{2}^{\prime}\right)^{\prime}}{1+2 u u_{2} \cos \alpha+u^{2} u_{2}^{2}} ;
$$

and the phase is given by the formula

$$
\tan \psi^{\prime}=-\frac{Q}{P}=\frac{-u u_{2} \sin a}{1+u u_{2} \cos \alpha} .
$$

13. When $u$ and $u_{2}$ have the same sign, the greatest and least intensities correspond to $\boldsymbol{a}=(2 m+1) \pi$, and $\boldsymbol{\alpha}=2 m \pi$. They are, respectively,

$$
\left(\frac{u^{\prime} u_{2}^{\prime}}{1-u u_{2}}\right)^{2} \text {, and }\left(\frac{u^{\prime} u_{2}^{\prime}}{1+u u_{2}}\right)^{2} \text {. }
$$

When $u$ and $u_{2}$ have opposite signs, the greatest and least values of $I^{\prime}$ correspond to $a=2 m \pi$, and $a=(2 m+1) \pi$. Hence the bright rings of the transmitted system occur at thicknesses which produce the dark rings of the reflected system, and vice versâ.

These values never vanish, since $u^{\prime}$ and $u_{2}^{\prime}$ are never evanescent; and there are no black rings in the transmitted light.

14. When the incident light is polarized perpendicularly to the plane of incidence, and when the coefficients $u$ and $u_{2}$ are both positive, or both negative, the preceding values of the greatest and least intensities remain unaltered. In other words, the rings exhibit the same character, whether the angle of incidence is less than the polarizing angle at both surfaces, or greater. But when one of these quantities is positive, and the other negative,-i.e. when the incidence at one surface is less than its polarizing angle, and at the other greater, the preceding formulas are transposed, and the bright rings are changed into dark ones, and vice versâ. When $u=0$, or $u_{2}=0$, -i.e. when the light is incident on either surface at its polarizing angle, the preceding values are both reduced to $\left(u^{\prime} u_{2}^{\prime}\right)^{2}$, and there is no variation of intensity caused by interference.

15. It may be easily shown from Fresnel's formulas, that

$$
\left(u^{\prime} u_{2}^{\prime}\right)^{2}=\frac{\sin 2 \theta}{\sin 2 \theta_{2}^{\prime}}\left(1-u^{2}\right)\left(1-u_{2}^{2}\right) \text {. }
$$


When the media are the same on the two sides of the plate, $\theta_{2}^{\prime}=\theta$, and $u_{2}=-u$. In this case, therefore, $u^{\prime} u_{2}^{\prime}=1-u^{2}$, and the general expression for the intensity becomes

$$
I^{\prime}=\frac{\left(1-u^{2}\right)^{2}}{1-2 u^{2} \cos a+u^{4}} .
$$

Comparing this with the expression for the intensity of the reflected light (5), we learn that

$$
I+I^{\prime}=1
$$

or that the intensities of the reflected and transmitted lights are complementary, the maximum of the former corresponding to the minimum of the latter, and vice versâ. This law explains the relations of the reflected and transmitted rings observed by Newton and Arago.

The greatest and least intensities are

$$
1 \text { and }\left(\frac{1-u^{2}}{1+u^{2}}\right)^{2} \text {. }
$$

Accordingly, the intensity of the light in the bright rings is equal to that of the incident light.

16. When the incident light is polarized in any plane, inclined at the angle $\gamma$ to the plane of incidence, the transmitted light is composed of two portions, polarized in the plane of incidence, and in the perpendicular plane, respectively. The phases of these two portions are given by the formulas,

$$
\tan \psi^{\prime}=\frac{-v v_{2} \sin a}{1+v v_{2} \cos a}, \quad \tan \chi^{\prime}=\frac{-w w_{2} \sin a}{1+w w_{2} \cos a} ;
$$

and as these are in general unequal, the light will be ellipticallypolarized. The difference of phase is given by the formula

$$
\tan \left(\psi^{\prime}-\chi^{\prime}\right)=\frac{\left(w w_{2}-v v_{2}\right) \sin \boldsymbol{a}}{1+\left(v v_{2}+w w_{2}\right) \cos \boldsymbol{a}+v v_{2} w w_{2}} .
$$

Hence the ellipticity varies with $a$, and therefore with the thickness of the plate.

The difference, $\psi^{\prime}-\chi^{\prime}$, will be a maximum, when

$$
\cos \boldsymbol{a}=-\frac{v v_{2}+w w_{2}}{1+v v_{2} w w_{2}} .
$$

Substituting in the preceding formula, and denoting the greatest difference of phase by $\Delta^{\prime}$, we have 


$$
\tan \Delta^{\prime}=\frac{w v_{2}-v v_{2}}{\sqrt{\left\{\left(1-v^{2} v_{2}^{2}\right)\left(1-w^{2} v_{2}^{2}\right)\right\}}} .
$$

17. The intensities of the two component pencils are

$$
I^{\prime}=\frac{\left(v^{\prime} v_{2}^{\prime} \cos \gamma\right)^{2}}{1+2 v v_{2} \cos a+v^{2} v_{2}^{2}}, \quad J^{\prime}=\frac{\left(w^{\prime} v_{2}^{\prime} \sin \gamma\right)^{2}}{1+2 w w_{2} \cos a+w^{2} u_{2}{ }^{2}} \text {. }
$$

And denoting the ratio of the amplitudes by $\tan \gamma^{\prime}$, as before,

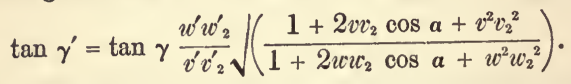

When the thickness of the plate is that corresponding to the maximum difference of phase, this expression is reduced to

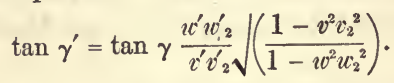

18. When the media are the same on the two sides of the plate, $v_{2}=-v, v_{2}=-w$, and the preceding formulas for the maximum difference of phase are reduced to

$$
\cos a=\frac{v^{2}+u^{2}}{1+v^{2} w^{2}}, \quad \tan \Delta^{\prime}=\frac{v^{2}-w^{2}}{\sqrt{ }\left\{\left(1-v^{4}\right)\left(1-w^{4}\right)\right\}} ;
$$

which are identical with those already obtained for the reflected light. Accordingly, the difference of phase of the two portions of the polarized beam is the same in the reflected and in the transmitted pencils.

In the same case the ratio of the amplitudes is

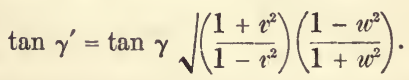

In resuming the theory of the rings in polarized light, I was under the impression that the knowledge of the subject was still confined to the general principles laid down by myself, many years ago. Since the foregoing paper was read, however, I have learned that the problem has been discussed by M. Jamin, in an interesting memoir publisned in the Annales de Chimie in 1822. In this memoir the author has confined himself to the case in which the media are the same on the two sides of the plate. In another respect, he has treated the problem more generally than it has been considered in the foregoing paper, having based it upon the theory of Cauchy, instead of that of Fresnel. He has thus been 
led to detect some curious phenomena of the rings, when the light is polarized perpendicularly to the plane of incidence, which had escaped the notice of preceding observers.

In applying his theory, however, to light polarized in any plane, M. Jamin disregards, as insensible, the additional terms which depend upon the Cauchian phase. His formulas, thus simplified, should therefore accord with those obtained in the present paper for a plate bordered on both sides by the same medium. Such, however, is not the case: the results, in fact, are entirely at variance, M. Jamin's formulas giving plane-polarized light at the extreme incidence, where (according to the theory above given) the departure from plane polarization is greatest. I believe that this discrepancy is accounted for by an error, into which M. Jamin seems to have fallen, in computing the maximum difference of phase of the two components of the light, which are polarized respectively in the two principal planes. 
V.-OBSERVATIONS ON THE DIRECTION AND INTENSITY OF THE TERRESTRIAL MAGNETIC FORCE IN IRELAND, MADE BY THE REV. HUMPHREY LLOYD, M.A., F.R.S., \&c.; BY CAPTAIN EDWARD SABINE, R.A., F.R.S., \&c.; AND BY CAPTAIN JAMIES CLARKE ROSS, R.N., F.R.S., \&c.

Fifth Report of the British Association for the Advancement of Science.

THE observations which form the subject of the present communication were made during the years 1834 and 1835, in compliance with the recommendation of the British Association urged in the first and second Reports of its proceedings. Their main object has been to determine the direction of the lines of magnetic dip and intensity in Ireland, and to make a small, but it was hoped exact, addition to our knowledge of the laws of distribution of the earth's magnetism. The observations are threefold : first, observations of the horizontal part of the earth's magnetic force, as determined by the time of vibration of a needle suspended horizontally, after the method of Professor Hansteen ; secondly, observations of dip, made in the usual manner; and thirdly, observations of dip and intensity at the same time, and with the same instrument, according to the method adopted by Professor Lloyd, and already submitted to the Association.*

\section{Horizontal Intensity.}

The instruments employed in the first series of observations were constructed after the model of that of Professor Hansteen. The needles are cylinders $2 \frac{1}{2}$ inches long, and 13 of an inch in diameter, suspended by a few filaments of the silkworm's thread.

"Fourth Report, p. 557. Transactions of the Royal Irish Academy, Fol. XIII. 
They are inclosed in a small rectangular box, supported upon levelling screws, and having a tubular pillar screwed on at top for the silk suspension. At the bottom of the box is a divided eircle, for the purpose of noting the aro of vibration : the temperature is observed by means of a small thermometer inolosed in such a manner as to avoid contact with the bottom and sides of the box. Before the commencement of the observations, the bottom of the box is to be rendered truly horizontal by means of the levelling screws on which it rests, and of a small spirit level with which it is furnished. The needle being then suspended so as to hang near the bottom, its deviation, if any, from the horizontal position will be detected by its inclination to the surface. It is then to be slightly moved to one side or other in the brass stirrup by which it is supported, until it hangs truly parallel to the lower surface of the box; and when this adjustment is once accurately made, no further alteration will be required, unless the change of dip be considerable.

When an observation is to be made, the needle is raised or lowered by a small roller to which the silk suspension is attached, so that it may hang about midway between the upper and lower surfaces of the box. It is then drawn aside from the magnetic meridian through an are of $25^{\circ}$ or $30^{\circ}$, by a piece of brass wire inserted in the side of the box, and is allowed to oscillate. The registry of the oscillations is commenced when the amplitude of the vibration on either side of the meridian is reduced to $20^{\circ}$, and it is continued during 360 vibrations; the moment of the completion of every 10 th vibration during that interval being noted by a chronometer. The amplitude of the final are, or of the are of the 360 th vibration, is also observed; and the temperature of the air in the box, as indicated by the interior thermometer, is noted at the beginning and end of the observation.

It is obvious that in this manner seven intervals of time are obtained, each corresponding to 300 vibrations, riz. the interval between the 0th and 300th vibration, between the 10th and 310 th, \&o., and betwcen the 60 th and 360th; - and the mean of these is taken as the result. But to this result several corrections must be applied.

1. The time as shown by the chronometer is to be corrected for rate; and accordingly the chronometer's rate must be determined from time to time by comparison with a good timekeeper, or by 
astronomical observations. In the present series the rate was observed at the commencement and end of each group of observations by the former and easier method. The amount of the correction due to rate is in most cases very small, the correction in the time of 100 vibrations corresponding to a daily rate of $2^{\mathrm{s}}$ being less than $0^{8.01}$ with the slowest of the needles employed.

2. Professor Hansteen has applied a correction for the arc of vibration, so as to reduce the time to that corresponding to infinitely small arcs. The correction is investigated on the same principles as that usually applied to pendulum observations. It is however more complicated in its form; for, instead of a single series of vibrations (as in the case of the pendulum), we have here seven distinct series, each commencing from a different arc. The principle, however, seems hardly applicable in the present instance. It is assumed that the successive arcs of vibration decrease in geometric progression, as they must necessarily do if the resistance of the air be proportional to the velocity. This is found to hold good in the vibrations of the pendulum when the ares are very small ; but it is by no means true when they are so considerable as those in which the horizontal magnetic pendulum is made to vibrate. Where, however, the vibrations commence from the same are, and the terminal arc does not much vary, the correction itself may perhaps be disregarded. In the following observations, in which the initial are was $20^{\circ}$, the 360 th or terminal are was generally $2 \frac{1}{2}^{\circ}$, and was in all cases included between the limits $1^{\circ}$ and $4^{\circ}$. In such cases, then, the correction must be, nearly, a constant quantity; its application to the observed times is therefore nearly equivalent to their multiplication by a constant coefficient, and the ratio of the times (with which alone we are concerned in this class of observations) remains unaltered. For these reasons no attempt has been made to introduce a correction for the arcs in the following results; but the terminal ares are given, so as to put the reader in possession of all the circumstances of the observation.

3. By far the most important correction is that due to temperature. If $\mathrm{T}^{\nu}$ be the observed time of 100 vibrations corresponding to the actual temperature $t^{\prime}$, and $\mathrm{T}$ the corrected time corresponding to the standard temperature $t$, the correction is

$$
\mathrm{T}-\mathrm{T}^{\prime}=a \mathrm{~T}^{\prime}\left(t-t^{\prime}\right) ;
$$


$a$ being a constant coefficient whose value is to be determined experimentally for each needle.

The following observations were made with the cylinders $\mathrm{L}(a)$, $\mathrm{L}(b)$, in order to determine the value of the coefficient $a$ for each. The apparatus being inclosed in a large glass bell, the time of 100 vibrations of cylinder $\mathrm{L}(a)$, commencing with the are of $10^{\circ}$, was observed at the mean temperature of the room, and when the air of the bell was heated artificially from below, by means of a spirit lamp. The final are varied between $4^{\circ}$ and $5^{\circ}$. The observations with cylinder $\mathrm{L}(b)$, were made in the bell without the apparatus. In this case no means were taken to observe with any accuracy the are of vibration; and in order to reduce as much as possible any error arising from this source, the observations were continued in each instance until the ares were reduced to the smallest appreciable, and the mean of the last five intervals of 100 vibrations then taken as the result. The chronometer's rate varied from $+0^{8 \cdot} 6$ to $+1^{\mathrm{s}} 4 \mathrm{p}$ per diem, and had therefore no appreciable influence on the results.

Cylinder L $(a)$.

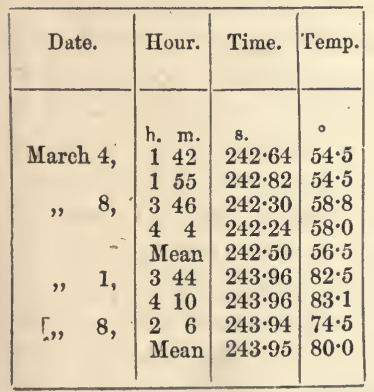

Cylinder L (b).

\begin{tabular}{|c|c|c|c|}
\hline Date. & Hour. & Time. & Temp. \\
\hline March 19.* & $\begin{array}{rr}\text { h. } & \mathrm{m} \\
11 & 7 \\
11 & 40 \\
2 & 42 \\
3 & 5 \\
\text { Mean } \\
12 & 50 \\
1 & 16 \\
\text { Mean }\end{array}$ & $\begin{array}{c}8 . \\
293 \cdot 48 \\
292 \cdot 52 \\
294 \cdot 06 \\
293 \cdot 76 \\
293 \cdot 44 \\
295 \cdot 24 \\
295 \cdot 20 \\
295 \cdot 22\end{array}$ & $\begin{array}{l}\circ \\
53 \cdot 6 \\
55 \cdot 0 \\
60 \cdot 3 \\
59 \cdot 5 \\
57 \cdot 1 \\
80 \cdot 0 \\
83 \cdot 3 \\
81 \cdot 6\end{array}$ \\
\hline
\end{tabular}

The constant coefficient sought is to be calculated from the formula

$$
a=\frac{\mathrm{T}-\mathrm{T}^{v}}{\mathrm{~T}^{v}\left(t-t^{\prime}\right)^{\prime}}
$$

* A series of observations had been made with this cylinder, in the same manner and on the same days as those with cyl. L $(a)$; but the results were unatisfactory, some of them indicating an increase of force with increased temperature. Such contradictory results have been noticed by many observers, and are usually attributed to the disturbing effects of currents of air, determined by inequality of temperature. 
in which $t$ and $t^{\prime}$ are the two temperatures, and $\mathrm{T}$ and $\mathrm{T}^{v}$ the corresponding times of vibration. We find

$$
\begin{aligned}
& \text { Cyl. L }(a) . \quad \mathrm{T}^{\prime}=242^{\mathrm{s} \cdot 50,} \mathrm{~T}-\mathrm{T}^{\prime}=1^{\mathrm{s}} \cdot 45, t-t^{\prime}=23^{\circ} \cdot 5 \\
& a=\cdot 000254 \\
& \text { Cyl. } \mathrm{L}(b) . \quad \mathrm{T}^{\prime}=293^{\mathrm{s} \cdot 44}, \mathrm{~T}-\mathrm{T}^{\prime}=1^{8.78}, t-t^{\prime}=24^{\circ} .5 \\
& a=000248 \text {. }
\end{aligned}
$$

It is to be observed that these cylinders were made at the same time, and were therefore probably tempered to the same degree; and to this circumstance we may, with much probability, ascribe the close agreement in the values of the constant which determines the effects of temperature upon the force of the needle.

No observations were made to determine directly the effects of temperature upon the other needles employed in the course of these observations; and, in correcting the results obtained with them, the coefficient employed by M. Hansteen, viz., $\cdot 00017$, has been that adopted. The standard temperature $(t)$, to which all the results contained in the following pages are reduced, is $60^{\circ} \mathrm{Fahr}$.

4. All that we know of the diurnal variations of the intensity of the horizontal force is due to M. Hansteen and Professor Christie. These writers agree in fixing the hours of minimum intensity at $10 \frac{1}{2}$ A. M. The intensity then increases, and attains its maximum, according to Professor Christie, at about $7 \frac{1}{2}$ P. M. The amount of this maximum is 1.0024 in summer, the minimum intensity being unity; but this amount, as well as the hour of its occurrence, changes with the season. Of the law according to which the force varies between its two limiting values, we know nothing; and it is therefore impossible, in the present state of our knowledge, to apply a correction for these variations. It was proposed to evade this difficulty, in the ensuing observations, by observing at a fixed hour. To this limitation, however, it was found impracticable to adhere, and the results still remain uncertain by the amount of the diurnal change.

5. The variations of the magnetic force give rise to another and still graver class of errors. The least experience in observations of this nature is enough to prove that the horizontal intensity is, from some cause or other, subject to irregular fluctuations; and these fluctuations, like those of the barometer in our climates, are much more considerable than the regular horary changes. It seems probable that these variations in the intensity of the hori- 
zontal force are, like those in its direction, not local phenomena, but occur at the same time at places widely separated. To eliminate them from our results, therefore, it would suffice to have a regular series of observations made at some fixed station, contemporaneous with those made at the different stations; and, if these be not very remote, we may assume that the variation of the observed force at each from its mean amount is the same as that observed at the same time at the fixed station. Unhappily these means of freeing the results from the admixture of what may be called accidental phenomena have not been attended to in the following, or indeed in any similar series of observations, and there is reason to believe that the errors due to this cause are the largest in amount of any by which the present series is affected.

The amount of these fluctuations, from day to day, may be judged of from the following specimen of a series of observations such as that alluded to, commenced by Captain Sabine in the month of June, 1835. The apparatus in which the needle was vibrated was unmoved during the continuation of the series, and the needle remained permanently suspended. The height of the barometer was noted, as well as the temperature; the hour of observation was, nearly, 10 А. м.

Time of 200 Vibrations of a Standard Needle.

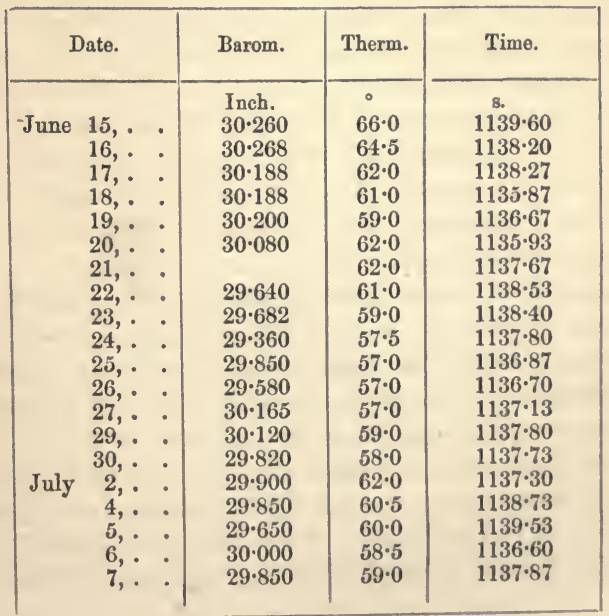


The mean time of 200 vibrations, deduced from these results, is $1137^{\prime \prime} \cdot 66$ at the temperature $60^{\circ} \cdot 6$. But the time observed on the 18th of June is $1135^{\prime \prime} \cdot 87$ at $61^{\circ} \cdot 0$; so that on this day the rate of the needle was less than the mean by $1^{\mathrm{s} \cdot 79}$, a difference which corresponds to an increase of .003 in the horizontal force. The observation of the 5th of July exhibits a difference somewhat greater on the other side.

6. The last source of error which requires to be noticed under this head is the change of the magnetic condition of the needles employed. Independently of the derangements of magnetio equilibrium induced by the presence of iron, or other disturbing causes, it is well known that most needles lose something of their original force. This loss is greatest at first; and the needle, if originally well tempered and then magnetized to saturation, is usually found to arrive at a nearly settled state in about a year. Most of the eylinders employed in the following observations seem to have reached that condition; and the changes of magnetic state which they have exhibited are, except in the case of Cyl. S $(b)$, unimportant. In order to detect any such changes, and to correct for them if they arise, it is necessary to observe at the place chosen as the base of reference, at the termination of each series of observations, as well as at their commencement. If it is then found that the needle has lost any small portion of its force, or if the time of vibration has augmented, the amount of the correction due to each result may be found by assuming the change to have been regular, or proportional to the time elapsed. When the loss is very small, however (as was the case in the observations which form the subject of this paper), the correction may be disregarded, provided we take as the time of vibration at the base of reference the mean of the times observed at the commencement and end of the series.

The needles used in the present series are the cylinders $\mathrm{L}(a)$, $\mathrm{L}(b)$, made by Dollond, and belonging to Mr. Lloyd; cylinder $\mathrm{S}(b)$ belonging to Captain Sabine, and cylinders $\mathrm{R}(c)$ and $\mathrm{R}(d)$ in the possession of Captain James Ross. All the circumstances of the observations are given in the annexed Table. The first, second, and third columns contain the place, day of the month, and hour of the observation. In the fourth column is set down the obserced time of 100 vibrations, or the immediate result of observation divided by 3 . The fifth column contains the terminal arc, 
the initial are being in all cases $20^{\circ}$. In the sixth column is given the chronometer's rate; in the seventh the temperature; and in the eighth the deduced or corrected time. The hour, set down in the third column as the hour of observation, is the mean of the commencement and end; and the recorded temperature is also the mean of those observed at the beginning and end of the observation. It will appear from the preceding that the corrections employed in deducing the corrected from the observed times are those due to temperature and to the rate of the chronometer.

In addition to the observations which follow there were others of an earlier date, made for the purpose of comparing the horizontal intensity at Dublin and Limerick, the two stations with which all the other places in Ireland have been immediately compared. In the observations alluded to, the rate of eylinder $\mathbf{S}(b)$ was observed in the Philosophy School, Trinity College; and the local attraction of the building was determined by subsequent comparisons of the force there with that in the garden of Trinity College, the place which was afterwards selected for all the Dublin observations. These earlier comparisons, as well as some other imperfect ones obtained previously to the autumn of 1834. with two other cylinders, have not been included in the annexed Tables; partly because the needles employed do not seem to be as trustworthy as the rest, but chiefly because of the uncertainties of the double comparison which they involve. 
TABLE I.

Time of Vibration of Cylinder $\mathrm{S}(b)^{*}$.

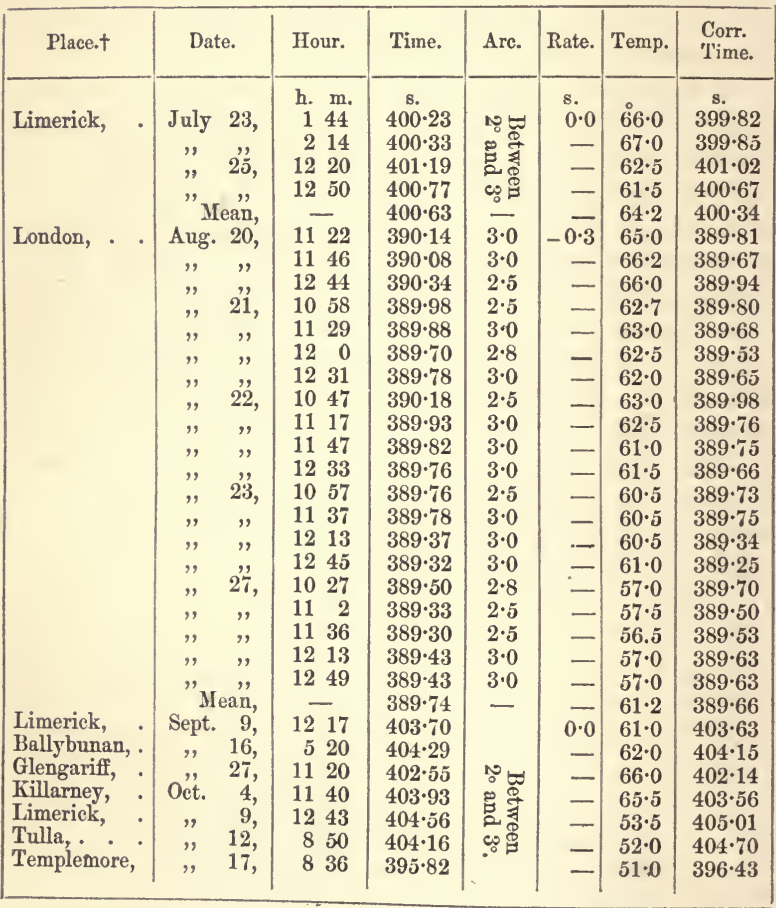

* The observations in London were made by Captain James Ross; all the othors with this needle by Captain Sabine.

† Limerick, garden at Somerville, one mile from town.-London, Regent's Park.Ballybunan, field adjoining the inn.-Glengariff, Mr. Eccles's garden.-Killarney, Mucross demesne.-Tulla, Kiltanon, Mr. Molony's garden.-Templemore, Sir H. Carden's grounds; local attraction suspected. -Clonmel, Darling Hill, garden adjoining the house.-Fermoy, field near the river. 


\section{Table I.-(Continued.)}

\begin{tabular}{|c|c|c|c|c|c|c|c|c|}
\hline Place. & & Date. & Hour. & Time. & Are. & Rate. & Temp. & $\begin{array}{l}\text { Corr. } \\
\text { Time. }\end{array}$ \\
\hline $\begin{array}{l}\text { Clonmel, . } \\
\text { Limerick, } \\
\text { Fermoy, · } \\
\text { Limerick, . } \\
\text { London, . }\end{array}$ & $\dot{\bullet} \cdot$ & 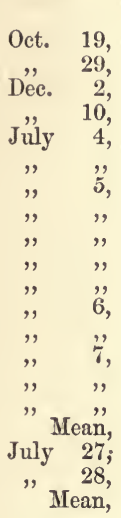 & $\begin{array}{rr}\mathrm{h} . & \mathrm{m} . \\
9 & 0 \\
12 & 6 \\
3 & 45 \\
2 & 45 \\
2 & 43 \\
3 & 14 \\
9 & 54 \\
10 & 21 \\
11 & 13 \\
11 & 46 \\
4 & 48 \\
9 & 53 \\
10 & 30 \\
9 & 32 \\
10 & 6 \\
10 & 43 \\
4 & 0 \\
11 & 58 \\
\end{array}$ & $\begin{array}{c}\text { s. } \\
402 \cdot 16 \\
404 \cdot 16 \\
399 \cdot 93 \\
402 \cdot 67 \\
402 \cdot 36 \\
402 \cdot 38 \\
401 \cdot 84 \\
401 \cdot 73 \\
401 \cdot 02 \\
401 \cdot 50 \\
401 \cdot 74 \\
401 \cdot 36 \\
401 \cdot 96 \\
400 \cdot 96 \\
401 \cdot 09 \\
401 \cdot 53 \\
401 \cdot 62 \\
413 \cdot 54 \\
412 \cdot 83 \\
413 \cdot 18\end{array}$ & 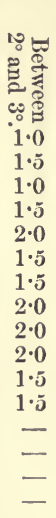 & $\begin{array}{c}8 . \\
0.0 \\
- \\
- \\
- \\
- \\
- \\
- \\
- \\
- \\
- \\
- \\
- \\
- \\
-\end{array}$ & $\begin{array}{l}55 \cdot 0 \\
55 \cdot 5 \\
48 \cdot 0 \\
42 \cdot 0 \\
70 \cdot 0 \\
71 \cdot 0 \\
62 \cdot 0 \\
64 \cdot 0 \\
60 \cdot 0 \\
62 \cdot 0 \\
64 \cdot 0 \\
65 \cdot 0 \\
68 \cdot 0 \\
60 \cdot 0 \\
62 \cdot 0 \\
64 \cdot 0 \\
64 \cdot 3 \\
76 \cdot 2 \\
66 \cdot 8 \\
71 \cdot 5\end{array}$ & $\begin{array}{c}8 . \\
402 \cdot 50 \\
404 \cdot 47 \\
400 \cdot 75 \\
403 \cdot 89 \\
401 \cdot 68 \\
401 \cdot 63 \\
401 \cdot 70 \\
401 \cdot 46 \\
401 \cdot 02 \\
401 \cdot 36 \\
401 \cdot 47 \\
401 \cdot 02 \\
401 \cdot 42 \\
400 \cdot 96 \\
400 \cdot 95 \\
401 \cdot 26 \\
401 \cdot 33 \\
412 \cdot 41 \\
412 \cdot 35 \\
412 \cdot 38\end{array}$ \\
\hline
\end{tabular}

Time of Vibration of Cylinder L $(a)$.* $^{*}$

\begin{tabular}{|c|c|c|c|c|c|c|c|}
\hline Place.t & Date. & Hour. & Time. & Are. & Rate. & Temp. & $\begin{array}{l}\text { Corr. } \\
\text { 'lime. }\end{array}$ \\
\hline $\begin{array}{l}\text { Limerick,. } \\
\text { Ballybunan, } \\
\text { Glengariff, : } \\
\text { Killarney, : } \\
\text { Limerick,. : } \\
\text { Dublin, . : }\end{array}$ & 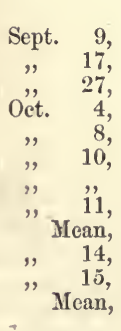 & $\begin{array}{rr}\text { h. } & \mathrm{m} . \\
1 & 23 \\
10 & 35 \\
12 & 0 \\
12 & 15 \\
1 & 14 \\
2 & 31 \\
2 & 50 \\
2 & 4 \\
2 & 22 \\
2 & 16 \\
\end{array}$ & $\begin{array}{c}\text { s. } \\
243 \cdot 26 \\
243 \cdot 87 \\
242 \cdot 29 \\
242 \cdot 46 \\
243 \cdot 16 \\
243 \cdot 71 \\
243 \cdot 81 \\
243 \cdot 52 \\
243 \cdot 68 \\
246 \cdot 23 \\
246 \cdot 30 \\
246 \cdot 26\end{array}$ & $\begin{array}{l}- \\
- \\
- \\
4 \cdot 0 \\
3 \cdot 0 \\
3 \cdot 5 \\
3 \cdot 5 \\
- \\
3 \cdot 5 \\
4 \cdot 0 \\
-\end{array}$ & $\begin{array}{r}8 . \\
0 \cdot 0 \\
- \\
- \\
+15 . \\
+1 . \\
- \\
- \\
- \\
-\end{array}$ & $\begin{array}{l}60 \cdot 0 \\
63 \cdot 0 \\
68 \cdot 0 \\
66 \cdot 0 \\
62 \cdot 0 \\
55 \cdot 2 \\
55 \cdot 0 \\
59 \cdot 0 \\
56 \cdot 4 \\
49 \cdot 5 \\
50 \cdot 7 \\
50 \cdot 1\end{array}$ & $\begin{array}{c}\text { s. } \\
243 \cdot 26 \\
243 \cdot 69 \\
241 \cdot 80 \\
242 \cdot 09 \\
243 \cdot 00 \\
244 \cdot 00 \\
244 \cdot 11 \\
243 \cdot 58 \\
243 \cdot 90 \\
246 \cdot 88 \\
246 \cdot 87 \\
246 \cdot 88\end{array}$ \\
\hline
\end{tabular}

* The first five observations, and those of December 19, 21, 23, 1835, were made by Captain Sabine. All the others with this needle by Mr. Lloyd.

+ Dublin, Provost's garden, Trinity College.-Armagh, grounds of the observatory--Carn an open field near the barracks. - Strabane, an open field adjoining the 


\section{TABLE I.-(Continued.)}

\begin{tabular}{|c|c|c|c|c|c|c|c|}
\hline Place. & Date. & Hour. & Time. & Arc. & Rate. & Temp. & $\begin{array}{l}\text { Corr. } \\
\text { Time. }\end{array}$ \\
\hline 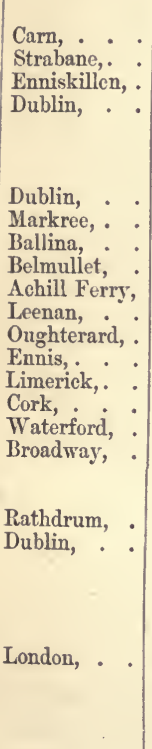 & 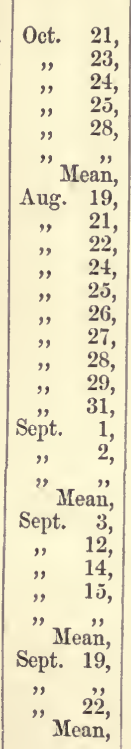 & $\begin{array}{rr}\text { h. } & \mathrm{m} . \\
2 & 57 \\
1 & 22 \\
3 & 45 \\
3 & 24 \\
3 & 0 \\
3 & 26 \\
2 & 39 \\
2 & 35 \\
3 & 2 \\
1 & 50 \\
12 & 0 \\
3 & 58 \\
3 & 16 \\
3 & 42 \\
2 & 52 \\
1 & 46 \\
1 & 55 \\
3 & 15 \\
3 & 35 \\
4 & 8 \\
5 & 5 \\
5 & 29 \\
2 & 36 \\
3 & 2 \\
11 & 48 \\
2 & 44 \\
12 & 43 \\
& \\
\end{array}$ & $\begin{array}{c}8 . \\
247 \cdot 49 \\
248 \cdot 00 \\
247 \cdot 09 \\
243 \cdot 14 \\
243 \cdot 39 \\
243 \cdot 49 \\
243 \cdot 34 \\
243 \cdot 81 \\
249 \cdot 30 \\
249 \cdot 90 \\
250 \cdot 40 \\
249 \cdot 35 \\
248 \cdot 19 \\
246 \cdot 06 \\
244 \cdot 44 \\
243 \cdot 45 \\
241 \cdot 50 \\
242 \cdot 48 \\
241 \cdot 24 \\
241 \cdot 26 \\
241 \cdot 25 \\
243 \cdot 47 \\
243 \cdot 70 \\
243 \cdot 92 \\
243 \cdot 14 \\
243 \cdot 73 \\
243 \cdot 62 \\
236 \cdot 51 \\
236 \cdot 65 \\
236 \cdot 63 \\
236 \cdot 60\end{array}$ & $\begin{array}{l}2 \cdot 5 \\
2 \cdot 5 \\
3 \cdot 5 \\
4 \cdot 0 \\
3 \cdot 5 \\
4 \cdot 5 \\
-\overline{3} \cdot 0 \\
3 \cdot 5 \\
2 \cdot 0 \\
1 \cdot 5 \\
2 \cdot 0 \\
2 \cdot 0 \\
4 \cdot 0 \\
2 \cdot 0 \\
3 \cdot 5 \\
2 \cdot 5 \\
2 \cdot 0 \\
2 \cdot 0 \\
2 \cdot 5 \\
-5 \\
3 \cdot 5 \\
4 \cdot 0 \\
4 \cdot 0 \\
2 \cdot 5 \\
3 \cdot 0 \\
3 \cdot 0 \\
2 \cdot 0 \\
3 \cdot 5 \\
-\end{array}$ & $\begin{array}{c}\text { s. } \\
+\cdot 0 \\
- \\
- \\
- \\
- \\
\overline{+} \\
+\cdot 3 \\
- \\
- \\
- \\
- \\
- \\
- \\
- \\
- \\
- \\
- \\
\bar{E} \\
- \\
- \\
- \\
- \\
\end{array}$ & $\begin{array}{l}50 \cdot 2 \\
51 \cdot 8 \\
38 \cdot 5 \\
45 \cdot 7 \\
53 \cdot 0 \\
53 \cdot 0 \\
50 \cdot 6 \\
72 \cdot 0 \\
69 \cdot 2 \\
71 \cdot 5 \\
69 \cdot 5 \\
69 \cdot 2 \\
59 \cdot 8 \\
57 \cdot 5 \\
75 \cdot 2 \\
67 \cdot 8 \\
69 \cdot 5 \\
69 \cdot 0 \\
66 \cdot 5 \\
66 \cdot 2 \\
66 \cdot 4 \\
63 \cdot 0 \\
57 \cdot 8 \\
60 \cdot 2 \\
63 \cdot 8 \\
63 \cdot 5 \\
61 \cdot 3 \\
68 \cdot 6 \\
69 \cdot 4 \\
72 \cdot 2 \\
70 \cdot 1\end{array}$ & $\begin{array}{c}\text { s. } \\
248 \cdot 10 \\
248 \cdot 51 \\
248 \cdot 42 \\
244 \cdot 01 \\
243 \cdot 82 \\
243 \cdot 92 \\
243 \cdot 92 \\
243 \cdot 06 \\
248 \cdot 71 \\
249 \cdot 16 \\
249 \cdot 79 \\
248 \cdot 76 \\
248 \cdot 18 \\
246 \cdot 19 \\
243 \cdot 49 \\
242 \cdot 96 \\
240 \cdot 90 \\
241 \cdot 92 \\
240 \cdot 83 \\
240 \cdot 87 \\
240 \cdot 85 \\
243 \cdot 27 \\
243 \cdot 81 \\
243 \cdot 89 \\
242 \cdot 89 \\
243 \cdot 50 \\
243 \cdot 52 \\
235 \cdot 98 \\
236 \cdot 07 \\
235 \cdot 89 \\
235 \cdot 98\end{array}$ \\
\hline
\end{tabular}

town.-Enniskillen, field near town.-Markree, demesne of the castle, part surrounded with tall trees.-Ballina, open field near town; no ahelter from sun.-Belmullet, on the beach, at extremity of Broadhaven.-Achill Ferry, on the beach, near ferry.Leenan, field at extremity of Killery harbour.-Oughterard, in a wood near the river.-Ennis, open field near town.-Limerick, garden at Somerville.-Cork, demesne on the banks of river, between Cork and Blackrock.-Waterford, demesne adjoining the river, side opposite town.-Broadway, open field.-Rathdrum, demesne of Avondale; deep wood.-London, Westbourne Green, Harrow Road. 


\section{TABLE I.-(Continued.)}

\begin{tabular}{|c|c|c|c|c|c|c|c|c|}
\hline \multicolumn{2}{|l|}{ Place. } & Date. & Hour. & Time. & Are. & Rate. & Temp. & $\begin{array}{l}\text { Corr. } \\
\text { Time. }\end{array}$ \\
\hline \multirow{4}{*}{\multicolumn{2}{|c|}{ London, . }} & Oct, 23 . & h. $\mathrm{m}$. & $\begin{array}{l}\text { s. } \\
235.09\end{array}$ & $\dddot{3} \cdot 0$ & $\begin{array}{r}8 . \\
+7.8\end{array}$ & 50.4 & 8. \\
\hline & &,$\quad 24$ & 1145 & 235.05 & $3 \cdot 0$ & - & $54 \cdot 5$ & $235 \cdot 35$ \\
\hline & & ", , & 223 & $235 \cdot 12$ & $3 \cdot 5$ & - & $55 \cdot 0$ & $235 \cdot 39$ \\
\hline & & Mean, & - & $235 \cdot 09$ & - & - & $54 \cdot 0$ & 235.42 \\
\hline \multirow[t]{3}{*}{ Dublin, . } & - & Sov. 5, & 146 & $243 \cdot 18$ & $3 \cdot 0$ & - & $54 \cdot 2$ & $243 \cdot 501$ \\
\hline & & " 6 & 1221 & $243 \cdot 35$ & $3 \cdot 0$ & - & $48 \cdot 0$ & $244 \cdot 06$ \\
\hline & & 'Y ", & 155 & $243 \cdot 37$ & $3 \cdot \tilde{J}$ & 一 & $48 \cdot 7$ & $214 \cdot 04$ \\
\hline \multirow{4}{*}{ Limcrick, } & & Mean, & 11 & $243 \cdot 30$ & 一 & - & $50 \cdot 3$ & $243 \cdot 87$ \\
\hline & & Dec. 19 , & $\begin{array}{ll}11 & 40 \\
12 & 19\end{array}$ & $243 \cdot 03$ & - & $+4 \cdot 0$ & $45 \cdot 5$ & $243 \cdot 90$ \\
\hline & & ", 23, & 1117 & $242 \cdot 68$ & - & - & 33.0 & $\begin{array}{l}243 \cdot 01 \\
244 \cdot 32\end{array}$ \\
\hline & & Mean, & - & $242 \cdot 69$ & - & - & $38 \cdot 2$ & $244 \cdot 01$ \\
\hline \multirow[t]{3}{*}{ Dublin, . } & - & Dec. 29, & 131 & $243 \cdot 22$ & $4 \cdot 0$ & $+\tilde{j} \cdot \bar{j}$ & $47 \cdot 6$ & $243 \cdot 96$ \\
\hline & & & 152 & $243 \cdot 18$ & $4 \cdot 0$ & - & $47 \cdot 0$ & 243.95 \\
\hline & & Mean, & - & $243 \cdot 20$ & - & - & $47 \cdot 3$ & 243.95 \\
\hline \multirow[t]{4}{*}{ Dublin, . } & . & Jan. 11, & 1121 & $242 \cdot 67$ & $3 \cdot 0$ & 一 & $33 \cdot 8$ & $244 \cdot 24$ \\
\hline & & , , , & 1144 & $242 \cdot 48$ & $4 \cdot 0$ & - & $34 \cdot 0$ & $244 \cdot 03$ \\
\hline & & "12, & 1012 & $242 \cdot 86$ & $2 \cdot 0$ & 一 & $33 \cdot 8$ & $244 \cdot 43$ \\
\hline & & & & $242 \cdot 67$ & - & 一 & $33 \cdot 9$ & $244 \cdot 23$ \\
\hline
\end{tabular}

Time of Vibration of Cylinder $\mathrm{L}(b) .^{*}$

\begin{tabular}{|c|c|c|c|c|c|c|c|}
\hline Place.t & Date. & Hour. & Time. & Are. & Rate. & Temp. & $\begin{array}{l}\text { Corr. } \\
\text { Time. }\end{array}$ \\
\hline $\begin{array}{l}\text { Limerick, } \\
\text { Ballybunan, } \\
\text { Glengariff, } \\
\text { Killarney, : } \\
\text { Limerick, : } \\
\text { Dublin, . }\end{array}$ & 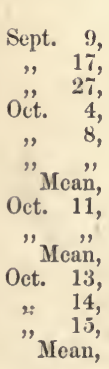 & $\begin{array}{rr}\text { h. } & \text { m. } \\
2 & 14 \\
10 & 3 \\
12 & 24 \\
12 & 38 \\
1 & 38 \\
2 & 5 \\
- & - \\
1 & 34 \\
2 & 50 \\
- \\
1 & 45 \\
1 & 55 \\
1 & 45 \\
\end{array}$ & $\begin{array}{c}8 . \\
292 \cdot 55 \\
292 \cdot 25 \\
293 \cdot 15 \\
292 \cdot 15 \\
292 \cdot 51 \\
292 \cdot 92 \\
292 \cdot 72 \\
292 \cdot 82 \\
292 \cdot 73 \\
292 \cdot 78 \\
294 \cdot 65 \\
295 \cdot 89 \\
295 \cdot 70 \\
295 \cdot 80\end{array}$ & $\begin{array}{l}- \\
- \\
Z \\
4 \cdot 0 \\
4 \cdot 0 \\
- \\
1 \cdot 5 \\
2 \cdot 5 \\
-3 \cdot 0 \\
2 \cdot 5 \\
3 \cdot 0 \\
-\end{array}$ & $\begin{array}{r}8.0 \\
- \\
- \\
+15 . \\
- \\
+\overline{1.0} \\
- \\
- \\
- \\
-\end{array}$ & $\begin{array}{l}60 \cdot 2 \\
62 \cdot 0 \\
68 \cdot 0 \\
68 \cdot 0 \\
62 \cdot 0 \\
62 \cdot 0 \\
62 \cdot 0 \\
62 \cdot 5 \\
58 \cdot 5 \\
60 \cdot 5 \\
59 \cdot 5 \\
51 \cdot 0 \\
52 \cdot 5 \\
51 \cdot 8\end{array}$ & $\begin{array}{c}8 . \\
292 \cdot 54 \\
292 \cdot 10 \\
292 \cdot 57 \\
291 \cdot 57 \\
292 \cdot 31 \\
292 \cdot 72 \\
292 \cdot 52 \\
292 \cdot 63 \\
292 \cdot 84 \\
29 \cdot 2 \cdot 74 \\
294 \cdot 69 \\
296 \cdot 56 \\
296 \cdot 25 \\
296 \cdot 40\end{array}$ \\
\hline
\end{tabular}

* First six observations, and those of 21 st and 23 rd of December, 1835, were made by Captain Sabine: all the others with this needle by Mr. Lloyd.

+ Carlingford, open field east of town. Colcraine, demesne adjoining the town; spot near river surrounded by tall trees; local attraction apparently due to basalt. 


\section{Table I.-(Continued.)}

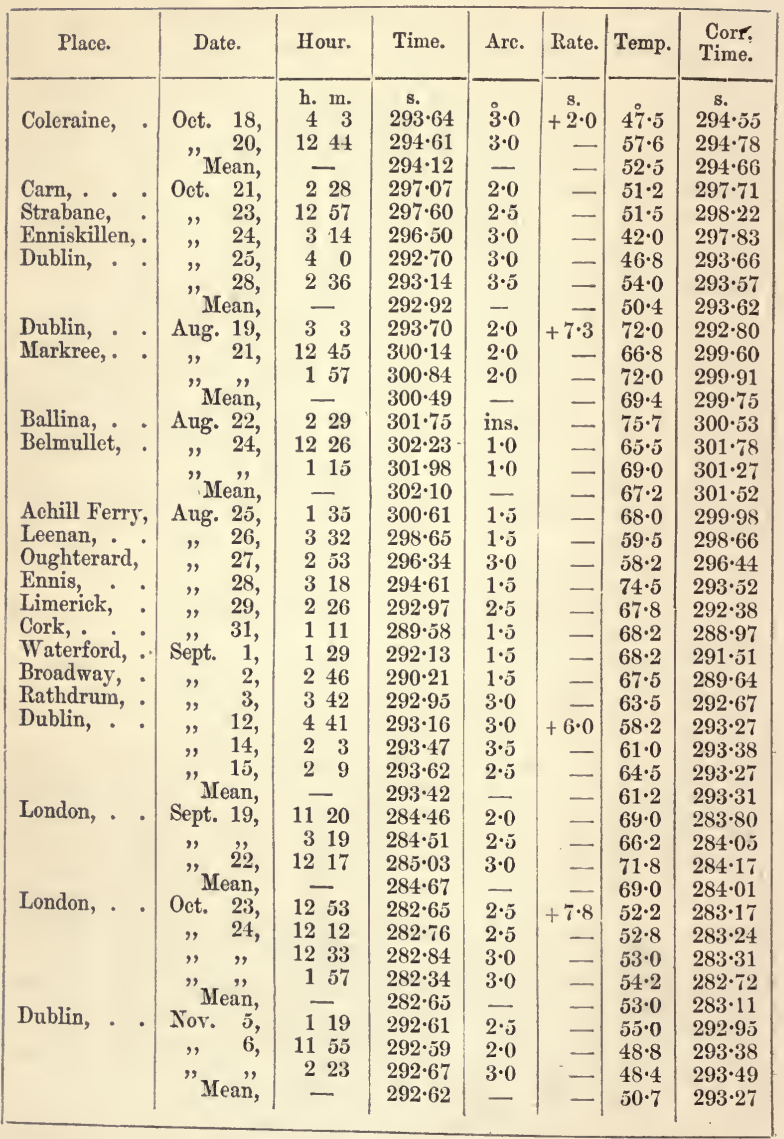




\section{Table I.-(Continued.)}

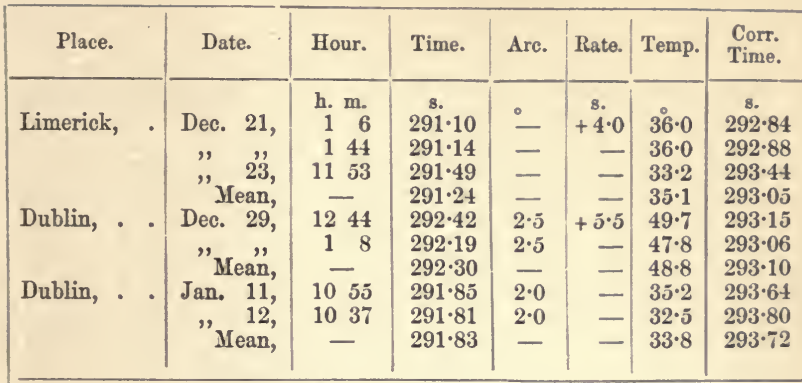

Time of Vibration of Cylinder $\mathrm{R}(c)$.*

\begin{tabular}{|c|c|c|c|c|c|c|c|}
\hline Place. $\dagger$ & Date. & Ifour. & Time. & Are. & Rate. & Temp. & $\begin{array}{l}\text { Corr. } \\
\text { Time. }\end{array}$ \\
\hline London, . . & 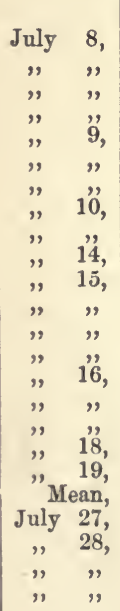 & $\begin{array}{rr}\text { b. } & \mathrm{m} . \\
7 & 34 \\
10 & 9 \\
12 & 20 \\
4 & 17 \\
11 & 26 \\
2 & 15 \\
6 & 10 \\
11 & 7 \\
4 & 47 \\
10 & 41 \\
6 & 51 \\
11 & 11 \\
2 & 31 \\
8 & 24 \\
7 & 39 \\
11 & 35 \\
8 & 2 \\
10 & 14 \\
7 & 35 \\
- & \\
3 & 57 \\
12 & 39 \\
1 & 7 \\
1 & 40\end{array}$ & $\begin{array}{c}8 . \\
440 \cdot 08 \\
440 \cdot 57 \\
441 \cdot 03 \\
441 \cdot 33 \\
441 \cdot 93 \\
441 \cdot 55 \\
441 \cdot 06 \\
441 \cdot 76 \\
441 \cdot 78 \\
441 \cdot 31 \\
440 \cdot 97 \\
443 \cdot 26 \\
443 \cdot 40 \\
440 \cdot 86 \\
441 \cdot 19 \\
442 \cdot 68 \\
441 \cdot 53 \\
443 \cdot 32 \\
441 \cdot 00 \\
441 \cdot 61 \\
453 \cdot 31 \\
454 \cdot 60 \\
454 \cdot 06 \\
454 \cdot 18\end{array}$ & $\begin{array}{l}1.5 \\
1.5 \\
2.0 \\
2.0 \\
1.0 \\
1.0 \\
1.0 \\
1.5 \\
1.2 \\
1.5 \\
1.5 \\
1.0 \\
1.5 \\
1.5 \\
1.0 \\
1.0 \\
1.0 \\
1.0 \\
1.5 \\
-1.5 \\
1.5 \\
2.0 \\
1.5 \\
2.0\end{array}$ & 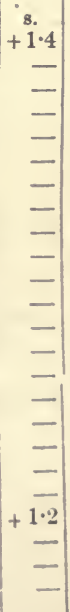 & $\begin{array}{l}58 \cdot 0 \\
60 \cdot 0 \\
64 \cdot 0 \\
66 \cdot 0 \\
57 \cdot 0 \\
61 \cdot 0 \\
61 \cdot 0 \\
64 \cdot 0 \\
62 \cdot 0 \\
58 \cdot 0 \\
54 \cdot 0 \\
66 \cdot 0 \\
70 \cdot 0 \\
55 \cdot 0 \\
52 \cdot 0 \\
64 \cdot 0 \\
57 \cdot 0 \\
65 \cdot 0 \\
48 \cdot 0 \\
60 \cdot 1 \\
68 \cdot 5 \\
60 \cdot 0 \\
59 \cdot 0 \\
59 \cdot 0\end{array}$ & $\begin{array}{c}8 . \\
440 \cdot 22 \\
440 \cdot 56 \\
440 \cdot 72 \\
440 \cdot 88 \\
442 \cdot 15 \\
441 \cdot 46 \\
440 \cdot 97 \\
441 \cdot 45 \\
441 \cdot 62 \\
441 \cdot 45 \\
441 \cdot 42 \\
442 \cdot 81 \\
442 \cdot 64 \\
441 \cdot 23 \\
441 \cdot 78 \\
442 \cdot 37 \\
441 \cdot 75 \\
442 \cdot 93 \\
441 \cdot 89 \\
441 \cdot 59 \\
452 \cdot 65 \\
454 \cdot 59 \\
454 \cdot 13 \\
454 \cdot 25\end{array}$ \\
\hline
\end{tabular}

- Observed by Captain James Ross.

+ London, Westbourno Green, Ilarrow Road.-Limerick, garden at Somerville.Dublin, Provost's Garden, Trinity College.-Markree, demesne of Castle; spot surrounded by lofty trees. 
Table I.-(Continued.)

\begin{tabular}{|c|c|c|c|c|c|c|c|c|}
\hline P'lace. & & Date. & Hour. & Time. & Arc. & Rate. & Temp. & $\begin{array}{l}\text { Corr. } \\
\text { Time. }\end{array}$ \\
\hline \multirow{6}{*}{ Limerick, } & . & & $\begin{array}{l}\text { h. m. } \\
219\end{array}$ & $\begin{array}{c}\text { s. } \\
453 \cdot 84\end{array}$ & $\grave{1} 8$ & $\begin{array}{r}\text { s. } \\
+1 \cdot 2\end{array}$ & $60 \cdot 0$ & $\begin{array}{c}8 . \\
453 \cdot 83\end{array}$ \\
\hline & & Juy 20, & 249 & $453 \cdot 70$ & $2 \cdot 2$ & - & $59 \cdot 0$ & $453 \cdot 77$ \\
\hline & & ", & 322 & $453 \cdot 82$ & $2 \cdot 0$ & - & $60 \cdot 0$ & $453 \cdot 81$ \\
\hline & &, 29 & 110 & $454 \cdot 00$ & $2 \cdot 2$ & 一 & $56 \cdot 0$ & $454 \cdot 30$ \\
\hline & & & 1131 & $453 \cdot 88$ & $2 \cdot 2$ & 一 & $57 \cdot 0$ & $454 \cdot 10$ \\
\hline & & , & 120 & $454 \cdot 04$ & $2 \cdot 0$ & - & $59 \cdot 0$ & $454 \cdot 11$ \\
\hline \multirow{4}{*}{ Dublin, . } & & Mean, & - & $453 \cdot 94$ & - & - & $59 \cdot 8$ & $453 \cdot 95$ \\
\hline & & Aug. 16, & 721 & $453 \cdot 90$ & $2 \cdot 0$ & 一 & $56 \cdot 0$ & $454 \cdot 20$ \\
\hline & & ,, & $\begin{array}{ll}7 & 51 \\
0 & 01\end{array}$ & $453 \cdot 69$ & $2 \cdot 0$ & 一 & $56 \cdot 0$ & $453 \cdot 99$ \\
\hline & & "Yean. & 821 & $453 \cdot 77$ & $2 \cdot 0$ & - & $57 \cdot 0$ & 453.99 \\
\hline \multirow{4}{*}{ Markree, } & & Aug. 19, & 83 & $\begin{array}{l}403 \cdot 79 \\
464 \cdot 39\end{array}$ & $\overrightarrow{1 \cdot 8}$ & - & $54 \cdot 0$ & $\begin{array}{l}404 \cdot 00 \\
464 \cdot 85\end{array}$ \\
\hline & & & 100 & $465 \cdot 17$ & $2 \cdot 0$ & - & 58.0 & $465 \cdot 32$ \\
\hline & & ", & 1030 & 465.02 & $2 \cdot 0$ & 一 & $58 \cdot 0$ & $465 \cdot 17$ \\
\hline & & Mean, & - & $464 \cdot 86$ & - & 一 & $56 \cdot 7$ & $465 \cdot 11$ \\
\hline \multirow[t]{7}{*}{ London,. } & . & Aug. 30 , & 941 & $440 \cdot 72$ & $2 \cdot 5$ & - & $60 \cdot 0$ & $440 \cdot 71$ \\
\hline & & , . , & 1010 & $441 \cdot 52$ & $2 \cdot 0$ & - & $61 \cdot 0$ & $441 \cdot 43$ \\
\hline & & , , , & 1038 & $441 \cdot 72$ & $2 \cdot 0$ & - & $61 \cdot 0$ & $441 \cdot 63$ \\
\hline & & , 31 , & 933 & $440 \cdot 86$ & $2 \cdot 0$ & 一 & $55 \cdot 0$ & $441 \cdot 23$ \\
\hline & &,$\quad$, & 119 & $441 \cdot 41$ & $2 \cdot 0$ & - & $58 \cdot 0$ & $441 \cdot 55$ \\
\hline & & ", ," & 1237 & $442 \cdot 04$ & $2 \cdot 0$ & - & $58 \cdot 0$ & $442 \cdot 18$ \\
\hline & & Mean, & & $441 \cdot 38$ & 一 & - & $58 \cdot 5$ & $441 \cdot 46$ \\
\hline
\end{tabular}

Time of Vibration of Cylinder R $(d) .{ }^{*}$

\begin{tabular}{|c|c|c|c|c|c|c|c|c|}
\hline Place. & & Date. & Hour. & Time. & Arc. & Rate. & Temp. & $\begin{array}{l}\text { Corr. } \\
\text { Time. }\end{array}$ \\
\hline Limerick, & . & 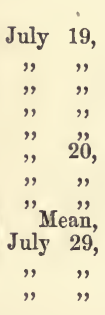 & $\begin{array}{rr}\text { h. } & \mathrm{m} . \\
9 & 44 \\
10 & 30 \\
11 & 44 \\
1 & 31 \\
3 & 6 \\
7 & 19 \\
9 & 54 \\
10 & 39 \\
- & \\
1 & 18 \\
1 & 50 \\
2 & 41\end{array}$ & $\begin{array}{c}\text { s. } \\
437 \cdot 75 \\
438 \cdot 10 \\
438 \cdot 39 \\
438 \cdot 30 \\
439 \cdot 18 \\
438 \cdot 55 \\
439 \cdot 26 \\
440 \cdot 06 \\
438 \cdot 70 \\
449 \cdot 17 \\
450 \cdot 05 \\
449 \cdot 16\end{array}$ & $\begin{array}{l}1 \cdot 0 \\
1 \cdot 0 \\
1 \cdot 2 \\
1 \cdot 0 \\
1 \cdot 0 \\
1 \cdot 0 \\
1 \cdot 0 \\
1 \cdot 0 \\
2 \cdot 0 \\
2 \cdot 0 \\
2 \cdot 0\end{array}$ & $\begin{array}{r}8 . \\
+1 \cdot 4 \\
- \\
= \\
- \\
= \\
+ \\
-\end{array}$ & $\begin{array}{l}58 \cdot 0 \\
59 \cdot 0 \\
62 \cdot 0 \\
64 \cdot 0 \\
66 \cdot 0 \\
60 \cdot 0 \\
61 \cdot 0 \\
63 \cdot 0 \\
61 \cdot 6 \\
60 \cdot 0 \\
61 \cdot 0 \\
62 \cdot 0\end{array}$ & $\begin{array}{c}\text { s. } \\
437 \cdot 89 \\
438 \cdot 17 \\
438 \cdot 23 \\
438 \cdot 00 \\
438 \cdot 72 \\
438 \cdot 54 \\
439 \cdot 18 \\
439 \cdot 82 \\
438 \cdot 57 \\
449 \cdot 16 \\
449 \cdot 96 \\
449 \cdot 00\end{array}$ \\
\hline
\end{tabular}

* Observed by Captain James Ross. 


\section{TABLe I.-(Continued.)}

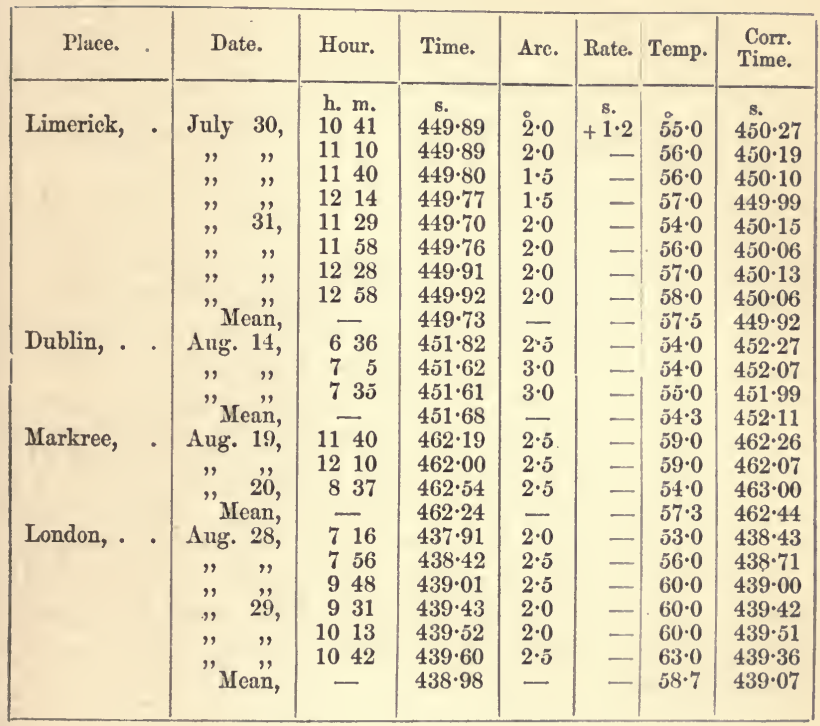

The computed results of the preceding observations are given in Table II. The first and second columns contain the place and the. date of the observations ; the third the designation of the needle employed ; in the fourth column is given the mean time of 100 vibrations, corrected for temperature and for the rate of the chronometer; and the fifth and sixth columns contain the computed values of the horizontal intensity - the numbers in the fifth column being the ratios of the horizontal intensity at the place of observation to that at the station of the observer, and those in the sixth being the ratios of the same force to that at London, to which place all the observations are ultimately referred.

If $\mathrm{T}$ denote the reduced time of 100 vibrations at any place, and $T^{v}$ that at the station with which it is immediately compared, 
and if $h$ and $h^{\prime}$ be the horizontal intensities at the two places, the numbers of the fifth column are computed from the formula

$$
\frac{h}{h^{\prime}}=\left(\frac{T^{\prime}}{T}\right)^{2}
$$

Again, if $h$, denote the horizontal intensity in London, the ratio $\frac{h^{\prime}}{h}$, will be determined in the same manner; and, multiplying by it the numbers in the fifth column, we obtain the values of $\frac{h}{h}$, or the ratios of the horizontal intensity at the places of observation to that at London, as given in the sixth and last column.

The stations with which all the other places in Ireland are immediately compared are Dublin and Limerick; and it will at once appear that, as the ratios of the horizontal force at these stations to that at London enter as factors in all the final results, much accuracy is required in their determination. For this purpose we have three distinct series of observations. In the first and second the intensities of the horizontal force in Dublin and Limerick are directly compared with that in London; and in the third these intensities are compared together. The results of these comparisons, given in Table II., are here put together, so as to be seen at one view.

I. Horizontal intensity in Dublin, the horizontal intensity in London being unity.

$$
\begin{aligned}
& \text { July, Aug., 1835， . . Cyl. R (c). . . Int. }=\cdot 9456 \\
& \text { ". " " . . - } \mathrm{R}(d) . \quad . \quad . \quad-9421 \\
& \text { September, 1835, . . } \quad \text { - L }(a) . \quad . \quad . \quad-\quad-9390 \\
& \text { " } \quad " \quad \text {. . } \quad \text { - L (b). . . - } \quad \text { - } 9376 \\
& \text { Oct., Nov., 1835, . . - L }(a) \text {. . . } \quad \text { - } 9319
\end{aligned}
$$

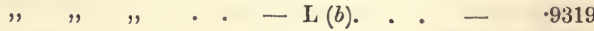

$$
\begin{aligned}
& \text { Mean }=\cdot 9380
\end{aligned}
$$

II. Horizontal intensity in Limerick, the horizontal intensity in London being unity.

$$
\begin{aligned}
& \text { July, Aug., Sept., 1834, Cyl. S (b). . . Int. }=\cdot 9396 \\
& \text { July, 1835, . . . . - - } \\
& \text { July, August, 183j, . } \quad \text { - R }(c) . \quad \text {. . } \quad \text { - } 9461 \\
& \text { " " } \quad \text {. } \quad-\mathrm{R}(d) . \quad . \quad-9513 \\
& \text { Mean }=\cdot 9460
\end{aligned}
$$


III. Horizontal intensity in Limerick, the horizontal intensity in Dublin being unity.

$$
\begin{aligned}
& \text { Sept., Oct., 1834, . . Cyl. L (a). . . Int. }=1 \cdot 0064 \\
& \text { " " . . . - L L (b). . . - } 1.0044 \\
& \text { July, Aug., 1835, . . - R (c). . . - } 1.0005 \\
& \text { Aug., Sept., } " 183 \tilde{0}, \text {. . . }
\end{aligned}
$$

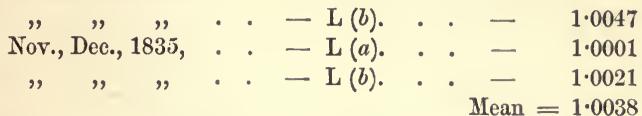

If then $x$ and $y$ denote the horizontal intensities in Dublin and Limerick, that in London being unity, observation gives

$$
x=\cdot 9380, \quad y=\cdot 9460, \quad \frac{y}{x}=1 \cdot 0038,
$$

and it is required to determine the most probable values of $x$ and $y$. To generalize this problem, let the mean results of observation be $a, b$, , and let their weights be $A, B, C$ respectively; so that we have

$$
\begin{aligned}
& x-a=0, \quad \text { weight }=A, \\
& y-b=0, \quad-\quad B, \\
& \frac{y}{x}-c=0, \quad-C:
\end{aligned}
$$

$a$ and $b$ being approximate values of $x$ and $y$, let their true values be $x=\bar{a}+\delta x, y=b+\delta y$,

and let $\frac{b}{a}=c$, Then $\frac{y}{x}=\frac{b+\delta y}{a+\delta x}=c,+a^{-1}\left(\delta y-c^{\prime} \delta x\right)$, the squares and higher powers of the quantities $\delta x$ and $\delta y$ being neglected; so that the preceding equations may be written

$$
\delta x=0, \delta y=0, a^{-1}(\delta y-c, \delta x)+c,-c=0 .
$$

These three equations are to be combined by the method of least squares. We find in this manner

$$
\begin{aligned}
& A \delta x-C c a^{-1}\left(a^{-1} \delta y-c a^{-1} \delta x+c,-c\right)=0, \\
& B \delta y+C a^{-1}\left(a^{-1} \delta y-c, a^{-1} \delta x+c,-c\right)=0 ;
\end{aligned}
$$


from which we obtain, by elimination,

$$
\delta x=+\frac{\frac{B}{A} a c,(c,-c)}{\frac{B}{C} a^{2}+\frac{B}{A} c^{2},+1}, \quad \delta y=-\frac{a(c,-c)}{\frac{B}{C} a^{2}+\frac{B}{A} c^{2},+1} .
$$

If the weights of the three results be regarded as equal, that is, if $A=B=C$, the preceding values become

$$
\delta x=\frac{b(c,-c)^{\prime}}{a^{2}+c_{1}^{2}+1^{\prime}} \quad \delta y=-\frac{a(c,-c)}{a^{2}+c_{1}{ }^{2}+1} .
$$

To apply these results to the present case, we have

$$
\begin{gathered}
a=.9380, \quad b=.9460, \quad c=1 \cdot 0038 ; \\
c,=\frac{b}{a}=1 \cdot 0085, \quad c,-c=\cdot 0047 .
\end{gathered}
$$

And introducing these values into the preceding expressions, we find

$$
\delta x=-\delta y=\cdot 0015
$$

so that the corrected values of $x$ and $y$ are

$$
x=\cdot 9395, \quad y=\cdot 9445 \text {. }
$$


MAGNETIC FORCE IN IRELAND.

TAbLe II.

Intensity of the Horizontal Force.

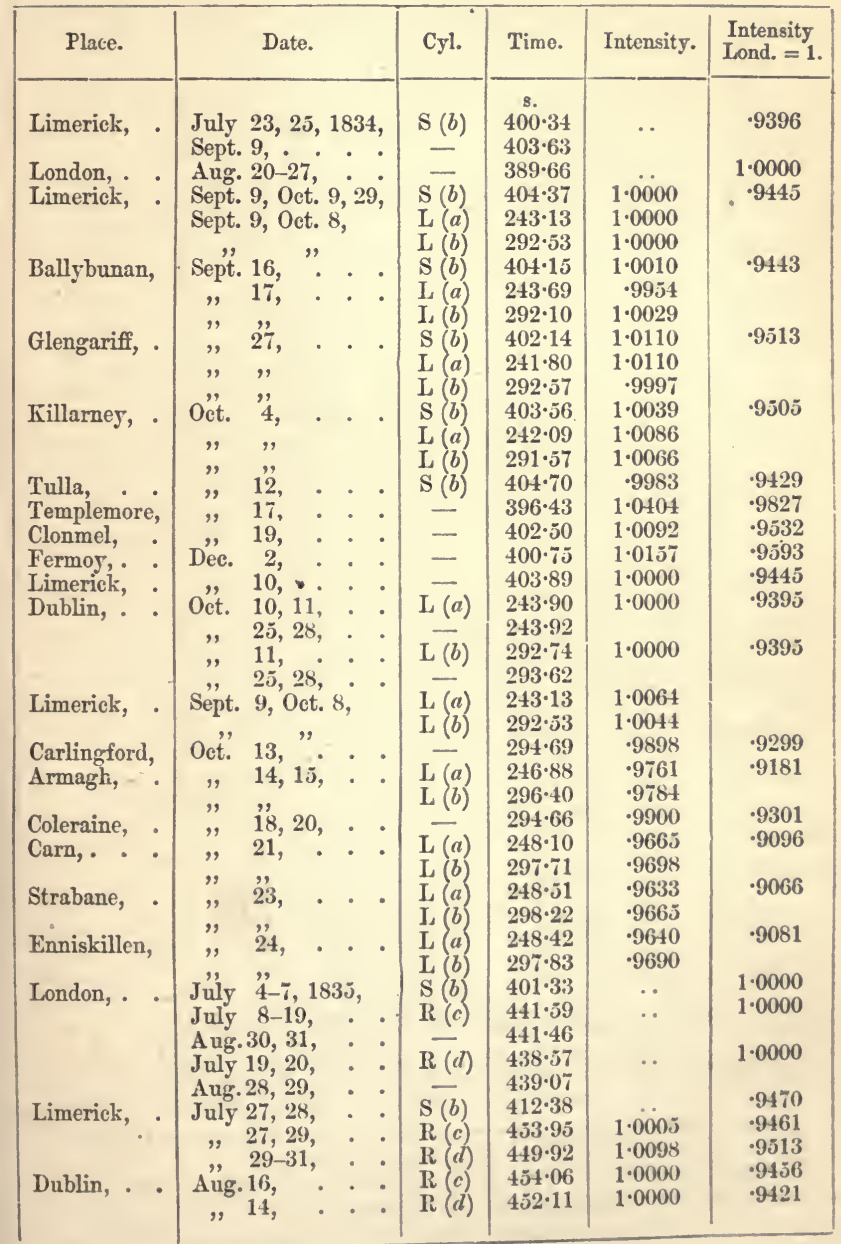




\section{TABLe II.-(Continued.)}

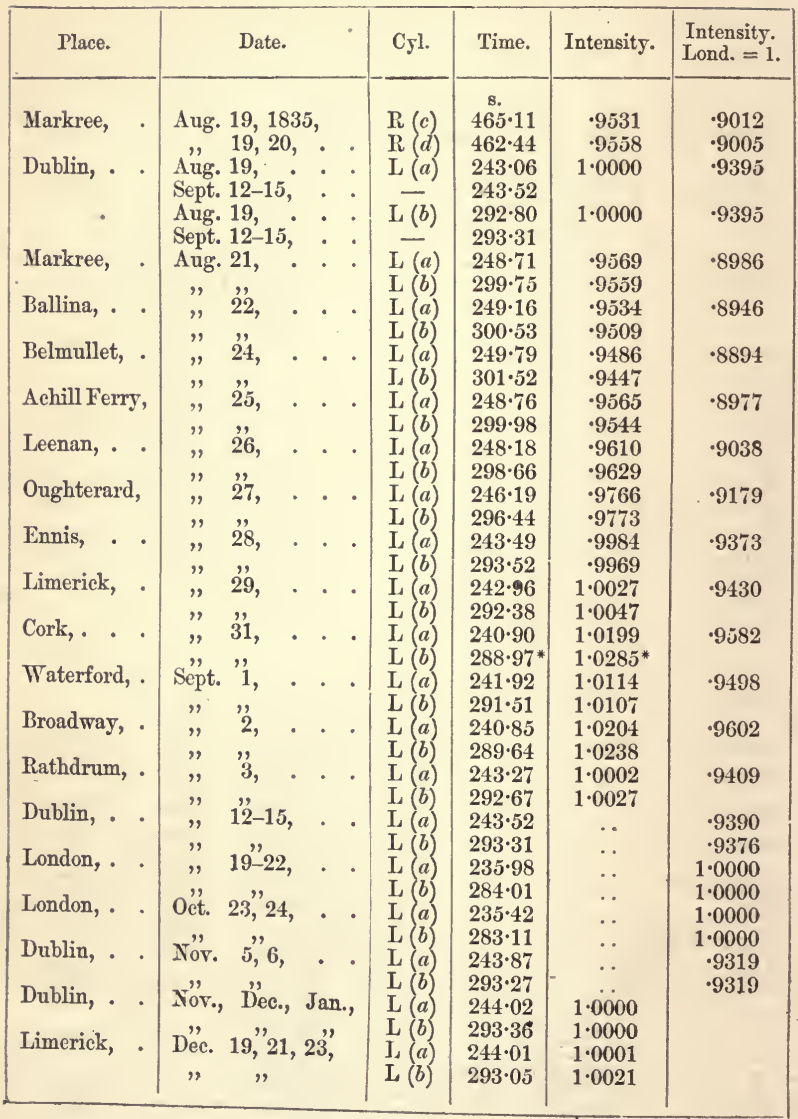

* Disturbing influence suspected in this observation; the result has been therefore omitt 3 in deducing the number in the last column. 


\section{Dip and Intensity.}

All the observations with dipping needles are comprised in the two Tables which follow. The first (Tab. III.) contains the results obtained with needles of the ordinary construction, and used exclusively for the determination of the dip. In the first, second, and third columns are given the place, day of the month, and hour of observation. The fourth column contains the observed inclination (the mean of the usual 8 readings) when the marked end of the needle is a north pole; the fifth contains the similar result of observation with the poles reversed; and the sixth is the mean of these angles, or the resulting dip. The needles employed are Needle L (1) constructed by Robinson, and Needle S (1) made by Dollond; the latter of these is $11 \frac{1}{2}$ inches in length, the former $4 \frac{1}{2}$ inches.

Table IV. contains the observations made for the purpose of determining the dip and intensity at the same time; the latter element being deduced from the direction in which the needle rests under the combined influence of magnetism and gravity, while the former is inferred from the position assumed under the influence of the earth's magnetism alone. Each of these angles of direction is deduced from the usual eight readings, all the reversals being made just as in the ordinary mode of observing the dip, the reversal of the poles of the needle excepted. These angles are given in the fifth and sixth columns of the table; $\zeta$ is the angle which the needle makes with the horizon when unloaded, and $\theta$ the inclination when a small weight is attached to the southern arm at a fixed distance from the centre. The temperature is noted at the commencement and end of each observation, with the view of correcting the value of the force; and the mean temperature is set down in the fourth column of the table. The needles employed in these observations are of the same dimensions as those used for the determinaiion of the dip alone, and are adapted to the same divided circles. Three small holes are drilled close to each other on each arm, at a distance from the centre about two thirds of its length; and much care has been bestowed to make them coincide accurately with the axis of form of the needle. The weight is a small cylinder of brass, which is inserted in one of the holes on the southern arm, the diameter of the cylinder corresponding aceurately to that of the hole. This weight is so adjusted as to bring the needle into a position nearly at right angles to the line of the dip, that being the position in which the resulting value of the force will be leasf affected by the friction of the axle on its supports. 


\section{Table III.}

\section{Observations of Dip.-Needle L (1).*}

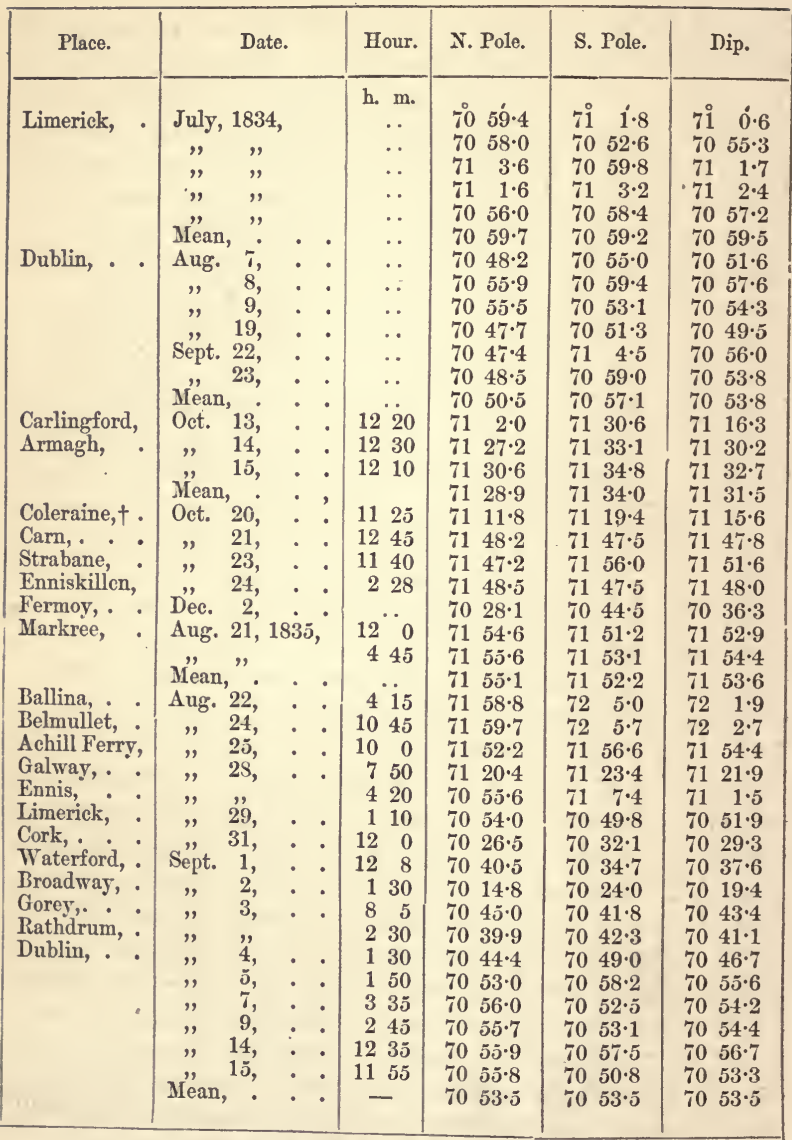

* The observations in Limerick (July, 1834) and that in Fermoy (Dec. 2), were made by Captain Sabine: all the other observations with this needle by. Mr. Lloyd. i Evident local disturbance at this place: Rock, basalt. 
MAGNETIC FORCE IN IRELAND.

TABle III.-(Continued).

Observations of Dip-Needle S (1).*

\begin{tabular}{|c|c|c|c|c|c|}
\hline Place. & Date. & Hour. & N. Pole. & S. Pole. & Dip. \\
\hline $\begin{array}{l}\text { Limerick, · } \\
\text { Glengariff, . } \\
\text { Killarney, · } \\
\text { Tulla, . }\end{array}$ & 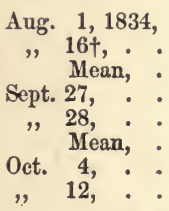 & $\begin{array}{l}\ldots \\
\ldots \\
. \\
\ldots \\
\ldots \\
\ldots \\
\ldots \\
\ldots\end{array}$ & $\begin{array}{lr}71 & 38 \cdot 8 \\
70 & 48 \cdot 5 \\
71 & 13 \cdot 6 \\
70 & 52 \cdot 3 \\
70 & 50 \cdot 4 \\
70 & 51 \cdot 4 \\
71 & 5 \cdot 6 \\
71 & \mathbf{1 6 \cdot 2}\end{array}$ & $\begin{array}{rr}70 & 24 \cdot 3 \\
71 & 22 \cdot 3 \\
70 & 53 \cdot 3 \\
71 & 9 \cdot 0 \\
71 & 14 \cdot 2 \\
71 & 11 \cdot 6 \\
71 & 3 \cdot 4 \\
71 & 15 \cdot 4\end{array}$ & $\begin{array}{rr}71 & 1 \cdot 6 \\
71 & 5 \cdot 4 \\
71 & 3 \cdot 5 \\
71 & 0 \cdot 6 \\
71 & 2 \cdot 3 \\
71 & 1 \cdot 5 \\
71 & 4 \cdot 5 \\
71 & 15 \cdot 8\end{array}$ \\
\hline
\end{tabular}

\section{Table IV.}

Observations of Dip and Intensity.-Needle L (4) + .

\begin{tabular}{|c|c|c|c|c|c|}
\hline Place §̧. ' & Date. & Hour. & Temp. & (६.) & (日.) \\
\hline $\begin{array}{c}\text { London,.. . } \\
\text { Dublin, . . }\end{array}$ & 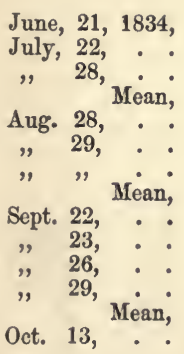 & $\begin{array}{rl}\text { h. } & \mathrm{m} . \\
& . \\
& \quad \\
2 & \ddot{3} \\
2 & 35 \\
12 & 42 \\
1 & 18 \\
2 & \ddot{15} \\
2 & 45 \\
2 & 40 \\
2 & 40 \\
12 & \ddot{5} \\
12 & \end{array}$ & $\begin{array}{l}64 \cdot 0 \\
65 \cdot 0 \\
65 \cdot 0 \\
64 \cdot 7 \\
70 \cdot 0 \\
68 \cdot 5 \\
67 \cdot 7 \\
68 \cdot 7 \\
61 \cdot 0 \\
62 \cdot 5 \\
66 \cdot 2 \\
62 \cdot 5 \\
63 \cdot 0 \\
61 \cdot 2\end{array}$ & $\begin{array}{cr}\because \\
\cdots \\
\because \\
69^{\circ} & 8^{\circ} \cdot 0 \\
69 & 8 \cdot 5 \\
69 & 5 \cdot 6 \\
69 & 7 \cdot 4 \\
71 & 2 \cdot 2 \\
70 & 53 \cdot 8 \\
70 & 0 \\
70 & 44 \cdot 8 \\
71 & 53 \cdot 6 \\
71 & 20 \cdot 6\end{array}$ & $\begin{array}{l}-655 \cdot 0 \\
-\quad 710 \cdot 6 \\
-\quad 621 \cdot 3 \\
-\quad 649 \cdot 0 \\
-1153 \cdot 2 \\
-127 \cdot 4 \\
-1226 \cdot 1 \\
-128 \cdot 9 \\
-\quad 85 \cdot 6 \\
-757 \cdot 0 \\
-759 \cdot 6 \\
-\quad 89 \cdot 5 \\
-757 \cdot 9 \\
-\quad 529 \cdot 4\end{array}$ \\
\hline
\end{tabular}

* All the observations with Needle S (1) were made by Captain Sabine.

+ The needle was rubbed on a hone in the interval between the observations (Aug. 1 and 16); - the marked end most.

+ The observations in Limerick (June, July 1834) were made by Captain Sabine: all the other observations with this needle by Mr. Lloyd.

§London, Sir James South's observatory, Kensington. Limerick, garden af Somerville.-Ballybunan, in the field in front of Captain Raymond's Lodge.-Valentia, 
TABLE IV.-(Continued.)

Observations of Dip and Intensity. - Needle I (4).

\begin{tabular}{|c|c|c|c|c|c|}
\hline Place. & Date. & Hour. & Temp. & $\zeta$ & $\theta$. \\
\hline $\begin{array}{l}\text { Coleraine, : } \\
\text { Carn, } \\
\text { Strabane, } \\
\text { Dublin, : } \\
\text { Dublin, : } \\
\text { Markree, } \\
\text { Ballina, } \\
\text { Belmullet, } \\
\text { Achill Ferry, } \\
\text { Galway, : } \\
\text { Ennis, : } \\
\text { Limeriok, } \\
\text { Cork, : } \\
\text { Waterford, : } \\
\text { Broadway, : } \\
\text { Gorey, : } \\
\text { Rathdrum, : } \\
\text { Dublin, . }\end{array}$ & 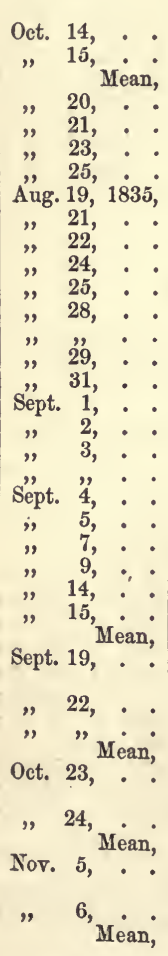 & \begin{tabular}{rr} 
h. & $\mathrm{m}$. \\
1 & 5 \\
12 & 38 \\
12 & 2 \\
1 & 28 \\
12 & 18 \\
3 & 15 \\
1 & 13 \\
3 & 50 \\
3 & 50 \\
11 & 15 \\
10 & 37 \\
8 & 18 \\
4 & 45 \\
1 & 40 \\
12 & 30 \\
12 & 35 \\
2 & 0 \\
8 & 30 \\
3 & 0 \\
1 & 56 \\
2 & 19 \\
4 & 8 \\
3 & 5 \\
1 & 5 \\
12 & 25 \\
\hdashline \\
1 & 0 \\
1 & 10 \\
11 & 32 \\
1 & 45 \\
11 & 33 \\
2 & 25 \\
1 & 16 \\
12 & 22 \\
2 & 32 \\
1 & 15
\end{tabular} & $\begin{array}{l}48 \cdot 8 \\
52 \cdot 0 \\
50 \cdot 4 \\
56 \cdot 3 \\
49 \cdot 5 \\
48 \cdot 8 \\
47 \cdot 0 \\
71 \cdot 5 \\
67 \cdot 0 \\
66 \cdot 5 \\
65 \cdot 5 \\
62 \cdot 0 \\
59 \cdot 0 \\
67 \cdot 2 \\
69 \cdot 2 \\
68 \cdot 5 \\
66 \cdot 2 \\
66 \cdot 8 \\
60 \cdot 0 \\
64 \cdot 7 \\
71 \cdot 8 \\
65 \cdot 5 \\
70 \cdot 0 \\
\cdots \\
62 \cdot 0 \\
67 \cdot 3 \\
\ddot{68} \cdot 0 \\
70 \cdot 8 \\
70 \cdot 0 \\
69 \cdot 6 \\
50 \cdot 5 \\
51 \cdot 6 \\
53 \cdot 8 \\
52 \cdot 0 \\
56 \cdot 2 \\
52 \cdot 8 \\
49 \cdot 0 \\
52 \cdot 7\end{array}$ & $\begin{array}{rr}71 & 19 \cdot 4 \\
71 & 33 \cdot 2 \\
71 & 26 \cdot 3 \\
71 & 12 \cdot 2 \\
71 & 49 \cdot 6 \\
71 & 39 \cdot 4 \\
70 & 54 \cdot 1 \\
70 & 51 \cdot 6 \\
71 & 55 \cdot 6 \\
71 & 51 \cdot 8 \\
71 & 57 \cdot 5 \\
71 & 53 \cdot 2 \\
71 & 17 \cdot 4 \\
70 & 59 \cdot 1 \\
70 & 47 \cdot 5 \\
70 & 33 \cdot 2 \\
70 & 38 \cdot 8 \\
70 & 31 \cdot 6 \\
70 & 43 \cdot 1 \\
70 & 40 \cdot 8 \\
70 & 43 \cdot 6 \\
70 & 52 \cdot 8 \\
70 & 52 \cdot 2 \\
70 & 46 \cdot 2 \\
70 & 53 \cdot 4 \\
70 & 55 \cdot 0 \\
70 & 50 \cdot 5 \\
69 & 5 \cdot 8 \\
69 & 7 \cdot 4 \\
69 & 12 \cdot 4 \\
69 & 13 \cdot 6 \\
69 & 9 \cdot 8 \\
69 & 10 \cdot 6 \\
69 & 2 \cdot 2 \\
69 & 6 \cdot 0 \\
69 & 6 \cdot 3 \\
70 & 49 \cdot 6 \\
70 & 45 \cdot 8 \\
70 & 53 \cdot 9 \\
70 & 49 \cdot 8\end{array}$ & 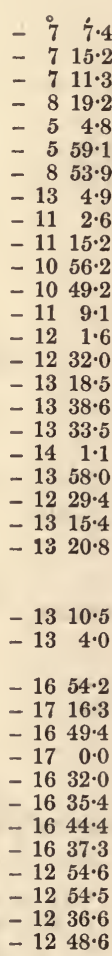 \\
\hline
\end{tabular}

on the sea beach at " the Foot."-Dingle, on the sea beach at Lord Ventry's.-Tulla, Kiltanon, Mr. Molony's demesne.-Youghal, in the garden of the "Devonshire Arms" inn. 
MAGNETIC FORCE IN IRELAND.

TABLe IV.-(Continued.)

Observations of Dip and Intensity.-Needle S (2) *.

\begin{tabular}{|c|c|c|c|c|c|}
\hline Place. & Date. & Hour. & Temp. & $\zeta$. & $\theta$. \\
\hline $\begin{array}{l}\text { Limerick," } \\
\text { Ballybunan, } \\
\text { Valentia, : } \\
\text { Dingle,. : } \\
\text { Tulla, : } \\
\text { Limerick, } \\
\text { Youghal, } \\
\text { Limerick, }\end{array}$ & 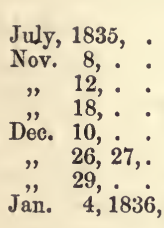 & $\begin{array}{l}\text { h. m. } \\
. . \\
\cdots \\
\cdots \\
\cdots \\
\cdots \\
\cdots \\
\cdots \\
.\end{array}$ & $\begin{array}{l}63 \cdot 0 \\
52 \cdot 0 \\
47 \cdot 0 \\
43 \cdot 0 \\
47 \cdot 0 \\
45 \cdot 0 \\
47 \cdot 0 \\
52 \cdot 5\end{array}$ & $\begin{array}{ll}71 & 16 \cdot 9 \\
71 & 29 \cdot 1 \\
71 & 15 \cdot 0 \\
71 & 17 \cdot 7 \\
71 & 36 \cdot 5 \\
71 & 14 \cdot 6 \\
70 & 49 \cdot 0 \\
& \ldots\end{array}$ & $\begin{array}{lr}\text { - } 159 \cdot 0 \\
\text { - } 1356 \cdot 3 \\
\text { - } 1437 \cdot 3 \\
\text { - } 1345 \cdot 8 \\
\text { - } 14 \quad 46 \cdot 0 \\
\text { - } 15 \quad 29 \cdot 6 \\
\text { - } 16 \quad 0 \cdot 5 \\
-15 \quad 23 \cdot 7\end{array}$ \\
\hline
\end{tabular}

When the observations of dip made at the same station with different needles are compared together, it will be found that they are by no means in accordance. Thus the dip at Limerick in November, $\mathbf{1 8 3 3}$, deduced from four observations with a needle on Meyer's principle, was $71^{\circ} 11^{\prime} \cdot 7$, while the mean of five observations with needle $\mathrm{L}(1)$ at the same place and in the following year was only $70^{\circ} 59^{\prime} \cdot 5$, differing from the former by $12^{\prime}$. When from this difference the amount of the annual change is deducted, the remainder appears to be greater than can be fairly ascribed to the errors of observation. But these diserepancies in the results given by different needles have been placed in the strongest light by the recent observations of Captain James Ross in London. In these observations, which were undertaken with the view of determining the amount of the annual decrease of dip at London, eight different needles were employed, and from eight to ten observations were made with each, the result of each separate observation being a mean of eighty readings.

The results were as follow :

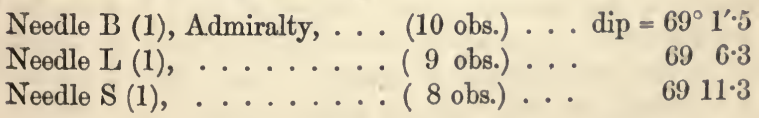

* The observations in Limerick, July, 1835, made by Captains Sabine and Rose ; all the remaining observations with this needle by Captain Sabine. 


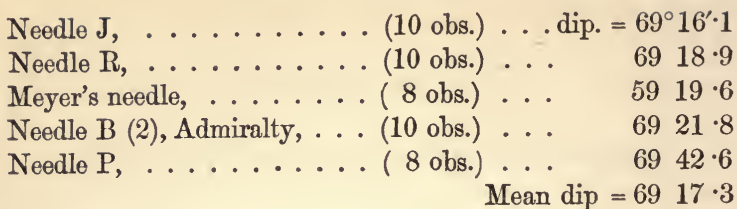

Thus it appears that there is a difference amounting to $41^{\prime}$ in the results of two of the needles used; and that this difference is very far beyond the limits of the errors of observation will appear from the fact that the extreme difference in the partial results with one of these needles B (1) does not amount to four minutes and a half, while with the other $(\mathrm{P})$ the extreme difference is only two minutes. In fact, it so happens that these very needles which differ most widely in their mean results, are those in which the accordance of the partial results is most complete. Of the eight results obtained with needle $(\mathrm{P})$, there is one only which differs from the mean of the eight by a single minute ; and yet the mean of all the observations with this needle differs by more than $20^{\prime}$ from the mean of any of the others, while its excess above the mean of the entire series amounts to $25^{\prime}$.

These differences cannot be ascribed to any partial magnetism in the apparatus; for three of the needles $(J, P$, and $R$ ) were of the same dimensions, and were used with the same circle, and yet their results, as we see, are widely discordant. We must seek, then, in the needles themselves the cause of these perplexing discrepancies, and we are forced to conclude that there may exist, even in the best needles, some source of constant error which remains uncorrected by the various reversals usually made; and that accordingly no repetition of observations with a needle so circunstanced cau furnish even an approximation to the absolute dip. If this error be due to the incomplete adjustment of the needle (such as deviation of centre of gravity from the axle, \&o.), its magnitude will be a function of the dip, and of the force, which may be assumed to be constant where the variations of these elements are not considerable. Hence, to determine its amount for any particular needle, it is necessary to make a careful series of observations with it at some station for which the dip has been accurately determined (from the mean of several needles); and the difference will be 
a constant correction, to be applied to all future results within certain limits.

It fortunately happens that the two ordinary needles used in the present series of observations in Ireland were among those employed by Captain James Ross in London; so that their corrections may be considered to be accurately known. The mean difference of the values of the dip as given by needles $L(1)$ and L (4) having been well determined by observations elsewhere, the results obtained with the latter needle in London may be grouped with those of the former. Thus, the mean of seven observations made with needle L (4), September and October, 1835, when reduced to needle $L(1)$, is $69^{\circ} 9^{\prime} 8$. If then we combine this with the direct result of the nine observations with needle $L(1)$, viz. $69^{\circ} 6^{\prime} 3$ (allowing double weight to each of the latter observations on account of the double number of readings), we find $69^{\circ} 7^{\prime} \cdot 3$ as the mean value of the dip deduced from sixteen observations with the two needles, and reduced to needle $L(1)$ as the standard. Comparing this with the mean result of the eight needles, the correction of needle $\mathrm{L}(1)$ is found to be $+10^{\prime} \cdot 0$.

For the other needles employed in Ireland, we have

$$
\begin{aligned}
& \text { Needle S (1), . . . . correction }=+6^{\prime} \cdot 0 . \\
& \text { Meyer's needle, . . . . . . }-2^{\prime} \cdot 3 \text {. }
\end{aligned}
$$

With respect to Meyer's needle, however, it is to be observed that as the angles from which the dip is deduced differ in general very widely, and as these angles are usually varied in different observations with the same needle, there is a presumption, at least, that every constant error will be removed by repetition, and that the differences of the separate results from the absolute dip will be equal on the positive and on the negative side. This seems to be confirmed by the amount of the final difference in the present instance, which does not appear to be larger than may be fairly ascribed to the errors of observation. It seems better therefore to regard this needle as subject to no constant error.

The degree of confidence to which these determinations are entitled may now be estimated, by applying the corrections so obtained to the observations.made with these needlos at Limerick in 1833 and 1834, the only other station at which they have been all employed. The observations in the former year are reduced to the latter, assuming the annual decrease of dip in Ireland to 
be 3 . The very close agreement of the results must of course be regarded as in a great measure accidental.

\begin{tabular}{|c|c|c|}
\hline & $\begin{array}{l}\text { Observed Dip. } \\
70^{\circ} 59^{\prime} \cdot 5\end{array}$ & $\begin{array}{l}\text { Corrected Dip } \\
71^{\circ} 9^{\prime} \cdot 5\end{array}$ \\
\hline (1), Aug., 1834, & $71 \quad 3 \cdot 5$ & 719.5 \\
\hline Meyer's needle, Nov., 1833, & $7111 \cdot 7$ & $.719 \cdot 7$ \\
\hline
\end{tabular}

We have hitherto spoken only of the needles whose poles are changed in each observation, and which are used exclusively for the determination of the dip. The necessity of a correction in the results obtained with the other needles, whose poles are unaltered, is obvious. By reason of the deviation of the centre of gravity of the needle from the axle, the weight of the needle itself has in all cases a certain moment acting with or against the directive force.

Let $\zeta$, as before, be the inclination of the needle to the horizon when unloaded, and $\theta$ the corresponding angle when the weight is attached, and let $\rho$ denote the ratio of the moment of the needle itself to that of the added weight; then the dip, $\delta$, will be given by the equations*

$$
\begin{aligned}
\delta & =\zeta+\varepsilon ; \\
\sin \varepsilon & =\rho \frac{\cos \zeta}{\cos \theta} \sin (\zeta-\theta) ;
\end{aligned}
$$

in which $\varepsilon$ is the correction sought.

The constant coefficient $\rho$ in the expression for this correction will be known when the corresponding values of the angles $\delta, \zeta$, and $\theta$ are known at some one station. Its value, in the case of Needle IV., has been thus found to be $\cdot 00205$. $\dagger$

It will easily appear, from the second of the preceding formulas, that when the coefficient is so small as that just assigned, the variations in the values of $\varepsilon$, resulting from moderate changes in the angles on which it depends, will be inconsiderable. In the observations in Ireland, for example, the entire change in the amount of the correction is a small fraction of a minute. In this and other similar cases, therefore, the correction may be regarded as constant; and its value may be inferred from any series of simultaneous observations made with the needle to be corrected, and

\footnotetext{
* Transactions of the Royal Irish Academy, Vol. XVII., p. 450!

$\dagger$ Ibid.p. 451.
} 
with some other whose correction is already known. In this manner it has been found that the mean difference of the results of Needles L (1) and L (4) is $\delta_{1}-\delta_{4}=+1^{\prime} \cdot 5$; being somewhat smaller than that assigned above. But if $\delta$ denote the absolute dip, we have already found that $\delta-\delta_{1}=+10^{\prime} 0$; and adding these differences, the correction of Needle L (4) is $\delta-\delta_{4}=+11^{\prime} \cdot 5$.

The correction of Needle $S(2)$ is inferred from the observations made with that needle in Limerick, as given in Table IV.

$$
\begin{aligned}
& \text { Limerick, July, } 1835, \quad \ldots \text { dip }=71^{\circ} 16^{\prime} \cdot 9
\end{aligned}
$$

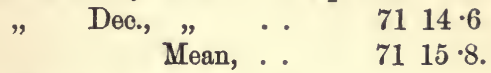

This mean corresponds, in time, to the middle of October, 1835 . But the true dip in Limerick (July, August, 1834) was found to be $71^{\circ} 9^{\prime} .5$; and when reduced to October, 1835 (assuming the annual decrease to be $3^{\prime}$ ), it is $71^{\circ} 6^{\prime} \cdot 0$. The correction of the needle is therefore $-9^{\prime} \cdot 8$.

The corrections of the needles being determined, we may now proceed to deduce the values of the absolute dip at the several places at which observations have been made. These values are given in the following table (Table V.). In the first and second columns are written the place and date of the observation. The third and fourth columns contain the corrected values of the dip, deduced from the results of Tables III. and IV. by the application of the corrections now explained; and the last column contains the mean dip inferred from the two preceding. In taking this mean, double weight has been assigned to the results obtained with needles of the ordinary construction, the number of readings with these needles being double of that made with the needles whose poles are unaltered. 
TaBle V.

Dip._Final Results.

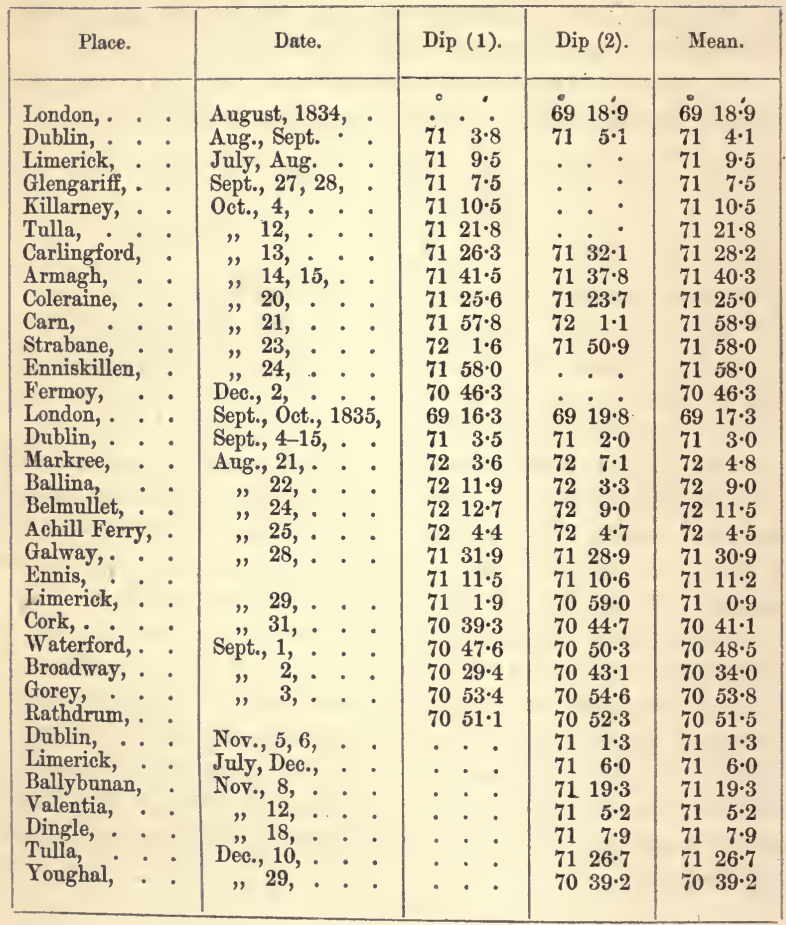

The dip being known, the intensity will be given by the formula

$$
\phi \sin (\delta-\theta)=\beta \cos \theta,
$$

in which $\beta$ is constant, and $\phi$ the measure of the force exerted by the earth on the needle. This force, however, varies with the temperature to which the needle is exposed; and it is necessary to determine the amount of this variation before we can know the relative values of the terrestrial magnetic force at different stations. 
Let $t$, then, be the observed, and $t^{\prime}$ the standard temperature, and let $\phi^{\prime}$ be the value of $\phi$ corresponding to the latter; then

$$
\phi-\phi^{\prime}=-a \phi^{\prime}\left(t-t^{\prime}\right),
$$

in which $a$ is a constant to be determined by observation. For Needle L (4) it has been found that

$$
a=\cdot 00016 .
$$

But we may proceed in another way, which will perhaps be found convenient in practice. We may correct the observed value of $\theta$ by subtracting the change due to temperature; or, in other words, we may reduce the value of $\theta$ to that oorresponding to the standard temperature, and to the standard condition of the needle. For this purpose it is only necessary to find the relation between the corresponding changes of $\phi$ and $\theta$. Differentiating, therefore, the equation (2) with respect to these variables, and dividing the result by the equation itself, we find

$$
\frac{d \phi}{\phi d \theta}=\frac{\cos \delta \sin 1^{\prime}}{\cos \theta \sin (\delta-\theta)^{\prime}}
$$

$d \theta$ being expressed in minutes. Now it is easy to see that the variations of the second member of this equation, arising from changes in the angles $\delta$ and $\theta$ on which it depends, will be inconsiderable for the limited extent of those changes in Ireland. Assuming it to be constant, therefore, its value will be given when we know the corresponding values of $\delta$ and $\theta$ at some one station. Thus, at Dublin, September, 1835 , it was found that

$$
\delta=71^{\circ} 3^{\prime} \cdot 0, \quad \theta=-13^{\circ} 0^{\prime} \cdot 0 ;
$$

from which we find the value of this constant to be $\cdot 00010$. But, since $d \phi=\phi-\phi^{\prime}=-\boldsymbol{a} \phi^{\prime}\left(t-t^{\prime}\right)$, the first member of the equation is

$$
-a \frac{t-t^{\prime}}{\theta-\theta^{\prime}}=-00016 \frac{t-t^{\prime}}{\theta-\theta^{\prime}}
$$

so that the correction is finally

$$
\theta^{\prime}-\theta=+1 \cdot 6\left(t-t^{\prime}\right)^{*} \text {. }
$$

* It is obvious that the coefficient in this correction might have beon determined directly, by observing the angles $\theta$ and $\theta^{\prime}$ corresponding to very unequal temperatures. It did not seem safe, however, to subject the apparatus to the action of high artificial heat, and the thermo-electric currents induced by inequality of temperature would in all probability have sensibly affected the results. 
Now if $\phi_{1}, \delta_{1}$, and $\theta_{1}$ be the values of $\phi, \delta$, and $\theta$ at the station with which the rest are compared, we have

and dividing

$$
\begin{gathered}
\phi \sin (\delta-\theta)=\beta \cos \theta, \\
\phi_{0} \sin \left(\delta_{0}-\theta_{0}\right)=\beta \cos \theta_{0} ; \\
\frac{\phi}{\phi_{0}}=\frac{\cos \theta \sin \left(\delta_{0}-\theta_{0}\right)}{\cos \theta_{0} \sin (\delta-\theta)},
\end{gathered}
$$

which expresses the ratio of the force at the two stations in a form suited to logarithmic calculation.

The following table gives the results of this computation. It contains the place and date of observation; the angle $\theta$ corrected for temperature; and the total intensity at each station, compared in the first instance with Dublin or Limerick, and in the second with London. For the intensity of the magnetic force at Dublin we have the three following determinations:

$$
\begin{array}{rccc}
\text { Aug., Sept., 1834, . . } & \text { Intensity }=1.0194 \\
\text { Sept., 1835, . . . . . } & \Rightarrow & 1.0213 \\
\text { Oct., Nov., 1835, . . } & \text { " } & 1.0211 \\
\text { Mean, . . . } & \# & 1.0206 .
\end{array}
$$

The intensity at Limerick, compared with London, is observed to be 1.0262; and the intensity at the same place, compared with Dublin, is 1.0030. Accordingly, for the determination of the values of the total force at Dublin and Limerick, observation furnishes us with three results, in the two former of which the intensities at these two stations are directly compared with that at London, while in the third they are compared together. To infer from these data, therefore, the most probable values of the force at the two stations, we must proceed precisely as in the analogous problem respecting the horizontal intensities, and we have only to substitute in the formulas already given, ${ }^{*}$ for $a, b, c$, \&c., their particular values. We have then

$$
\begin{aligned}
& a=1 \cdot 0206, \quad b=1 \cdot 0262, \quad c=1 \cdot 0030, \\
& c_{s}=\frac{b}{a}=1 \cdot 0055, \quad c,-c=\cdot 0025 .
\end{aligned}
$$

And since the comparison of Dublin and London is the mean of three distinct comparisons, while each of the other two results is 
inferred from one only, the weights may be assumed as follow :

$$
A=3, \quad B=1, \quad C=1 \text {. }
$$

Substituting these values, therefore, in the formulas alluded to, we find

$$
\begin{gathered}
\delta x=+\cdot 0004, \quad \delta y=-\cdot 0011 ; \\
x=a+\delta x=1 \cdot 0210, \quad y=b+\delta y=1 \cdot 0251 .
\end{gathered}
$$

The numbers in the fifth column of the table are deduced from those in the fourth, by multiplying by one or other of these numbers, -according as the force at the station in question has been compared in the first instance with that at Dublin, or with that at Limerick.

\section{TABLE VI.}

Intensity.-Needle $L$ (4).

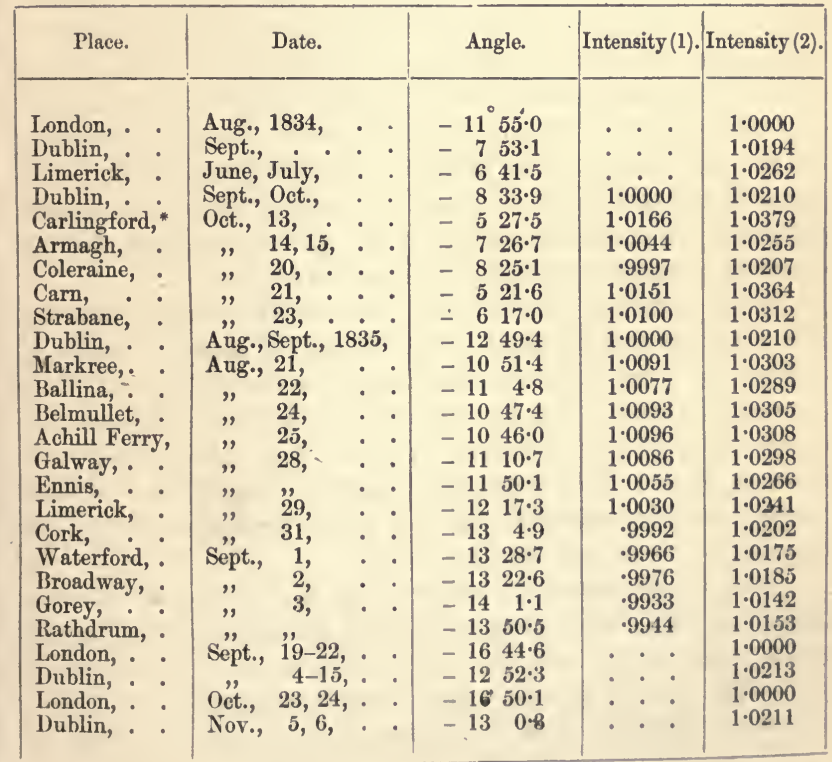

* Evident local disturbance at these two stations. The district about Carlingford 18 intcrsected with trap dykes; Coleraine lies within the basaltic field of the North east of Ireland. 


\section{Table VI.-(Continued.)}

Intensity.-Needle $S(2)$.

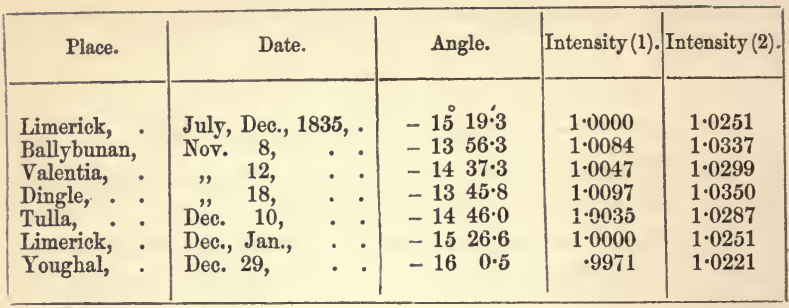

\section{III.-Isodynamic and Isoclinal Lines.}

On a review of the preceding results of observation, it will be seen that they exhibit much irregularity. The errors of observation-in which we are to include the effects of the unsteadiness of the magnetic state of the needles employed, as well as the various other uncertainties arising from the imperfections of our methods of observing-have, of course, their share in these discrepancies; but they are by no means sufficient to explain the whole. The action of the earth on the magnetic needle is itself subject to irregularities, temporary as well as local; and it is to these that the observed anomalies must, in great part, be ascribed.

To the variations of the former kind we have already referred. The direction and intensity of the terrestrial magnetic force, at a given place, are subject to fluctuations, or irregular oscillations round their mean state, the eause of which is as yet little understood ; and it is only by means of simultaneous observations, made at some fixed station within the limits of the district through which these effects take place, that we can hope to ascertain their amount, and to correct for them.

Of the local disturbing causes some are sufficiently obvious. Thus the needle is in general affected by the vicinity of basaltio rocks, owing to the quantity of iron they contain; and instances have been observed in which these rocks were even found to possess magnetic polarity.* But there seem to be grounds for believing

* A remarkable case of this kind has been noticed at Fair Head, on the north coast of Ireland. The magnetic polarity of one of the columns which compose this wonderful façade is said to be so strong as to invert the position of the compass ncedle, when the poles of the same name are made to approach. 
that disturbing actions of a local nature are exerted on a much larger scale. Whether the earth's magnetio force be an inherent property, and the resultant of the forces of all its parts, or whether it be simply the effect of thermo-electrio currents produced by the heating action of the sun, the result must in either case be greatly modified by the configuration of a country, and by the nature of its superficial strata. If this view be just, the greatest irregularities should prevail in those parts of the earth in which the uniformity of surface is broken by hill and valley, and where the strata have been rent and contorted by the uplifting of mountain chains. In Ireland, accordingly, we should expect to find much greater anomalies in the direction and intensity of the magnetic force than in the plains of central Europe; and it must be, consequently, in the same degree more difficult to arrive at general results.

The only mode of escaping from these difficulties was to seek the general result of the entire series of observations, as to the position of the isodynamic and isoclinal lines; and to combine the partial results in such a manner that their deviations-whether local, temporary, or casual-should have the least influence on the final conclusion. Such is the object of the following computations.

Let $\lambda$ and $\mu$ denote the latitude and longitude of any place at which an observation has been made, $\lambda_{n}$ and $\mu_{0}$ the latitude and longitude of the station which is chosen as the origin of the coordinates; then the position of the former place may be fixed with reference to the latter in terms of these quantities. For let $P$ be the pole of the earth, $\boldsymbol{M}$ and $O$ the two places, $P M$ and $P O$ their meridians, and $M Q$ a great circle passing through $M$ and perpendicular to $P O$. It is obvious that the position

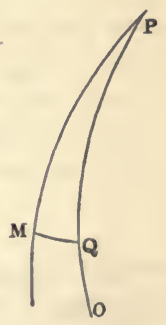
of $M$ will be determined by the rectangular spherical coordinates, $O Q$ and $Q M$. Now in the right-angled triangle $M P Q$, we have

$$
\tan P Q=\tan P M \cos P, \quad \sin M Q=\sin P M \sin P ;
$$

or, denoting the co-ordinates $O Q$ and $Q M$ by $a$ and $\beta$,

$$
\begin{aligned}
& \cot \left(\lambda_{0}+a\right)=\cot \lambda \cos \left(\mu-\mu_{0}\right) \\
& \sin \beta=\cos \lambda \sin \left(\mu-\mu_{0}\right) .
\end{aligned}
$$


When $\mu-\mu_{0}$ is so small as it is within the limits of the present district of observation, we may take

$$
\sin \left(\mu-\mu_{0}\right)=\mu-\mu_{i,}, \quad \cos \left(\mu-\mu_{0}\right)=1, \quad \sin \beta=\beta,
$$

and the preceding equations become

$$
\begin{aligned}
& \alpha=\lambda-\lambda_{0}, \\
& \beta=\left(\mu-\mu_{0}\right) \cos \lambda .
\end{aligned}
$$

This simplification is obviously equivalent to the substitution of the parallel of latitude for the perpendicular to the meridian.

Now let us conceive any line to pass through $O$, making the angle $u$ with the meridian; then, in the same order of approximation, the perpendicular from the point $M$ upon that line will be

$$
p=\beta \cos u-\alpha \sin u ;
$$

and, substituting for $a$ and $\beta$ their values just obtained,

$$
p=\left(\mu-\mu_{0}\right) \cos \lambda \cos u-\left(\lambda-\lambda_{0}\right) \sin u .
$$

It is easy to see in what manner this result may be applied in obtaining equations of condition from the data furnished by observation. The increase of the force, or of the dip, may (throughout the limited area of the present district of observation) be assumed to be proportional to the distance, measured in a direction perpendicular to the line of equal force, or of equal dip. Accordingly, if $u$ be the angle which the line of equal horizontal intensity passing through $O$ makes with the meridian of the place, the difference of the intensities at the two stations will be proportional to $p$, or

$$
h-h_{0}=r p ;
$$

$h$ and $h_{0}$ being the horizontal intensities at the two stations, and $r$ a constant coefficient which determines the rate of increase. Substituting, then, for $p$ its value (C), and making

we have

$$
r \cos u=x, \quad r \sin u=y,
$$

$$
\left(\mu-\mu_{0}\right) \cos \lambda x-\left(\lambda-\lambda_{0}\right) y=h-h_{0} .
$$

The equations of condition deduced from the observations of total intensity, and of dip, will be of a similar form; and the coefficients of the unknown quantities, in the first member of the equations, will be the same. 
The station chosen for the origin of the co-ordinates is Dublin, and it is obvious that there will be as many equations of condition as there are other places of observation. The coefficients of these equations are given in the following table. The first, second, and third columns contain the place of observation, its latitude and its longitude*. The numbers in the fourth and fifth columns are the differences of latitude and longitude (estimated in minutes of latitude) of the place of observation and Dublin, or the values of $\left(\lambda-\lambda_{0}\right)$ and $\left(\mu-\mu_{0}\right) \cos \lambda$; and the numbers in the three remaining columns are the corresponding differences of dip, of horisontal intensity, and of total intensity, which form the second members of the equations. The dip having been observed at Dublin in each of the two years (1834 and 1835), the differences of dip are obtained by subtracting that belonging to the year in which the observation was made at the other station.

\section{TABLE VII.}

\begin{tabular}{|c|c|c|c|c|c|c|c|}
\hline Place. & $(\lambda)$ & $(\mu)$ & $\left(\lambda-\lambda_{0}\right)$ & $\begin{array}{c}\left(\mu-\mu_{0}\right) \\
\cos \lambda\end{array}$ & $\left(\delta-\delta_{0}\right)$ & $\left(h-h_{0}\right)$ & $\left(f-f_{0}\right)$ \\
\hline Carn, . & $5 \delta ْ 15$ & 7 15 & +114 & +34 & $+5 \dot{4} \cdot 9$ & $-\cdot 030$ & $+\cdot 0154$ \\
\hline Coleraine, & 558 & 640 & +107 & +14 & $+21 \cdot 0$ & -.009 & -.0003 \\
\hline Strabane, & $54 \quad 49$ & 728 & +88 & +42 & $+54 \cdot 0$ & $-\cdot 033$ & $+\cdot 0102$ \\
\hline Enniskillen, & 5421 & 738 & +60 & +48 & $+54 \cdot 0$ & -031 & - \\
\hline Armagh, & 5421 & 639 & +60 & +14 & +36.3 & -.021 & $+\cdot 0045$ \\
\hline Belmullet, . & 5420 & 950 & 59 & +125 & $+68 \cdot 5$ & -.050 & $+\cdot 0095$ \\
\hline Markree, & 5414 & 828 & 53 & +77 & $+61 \cdot 8$ & -.039 & $+\cdot 0093$ \\
\hline Ballina,. & 5410 & 93 & 49 & 98 & +66.0 & -.045 & +.0079 \\
\hline Carlingford, & $54 \quad 2$ & 611 & 41 & & $+24 \cdot 2$ & $-\cdot 010$ & $+\cdot 0169$ \\
\hline Achill Ferry, & 540 & 951 & 39 & +127 & $+61 \cdot 5$ & -.042 & $+\cdot 0098$ \\
\hline Leenane,. & 5341 & 940 & 20 & +121 & - & -.036 & - \\
\hline Oughterard, & 5327 & 918 & + & +109 & - & -.022 & - \\
\hline Dublin, . & 5321 & 615 & - & & +0.0 & $+\cdot 000$ & $+\cdot 0000$ \\
\hline Galwa & 5317 & 851 & -4 & 93 & $\begin{array}{r}27 \cdot 9 \\
+\quad 2\end{array}$ & - & $+\cdot 0088$ \\
\hline Rathdrum, . & 5255 & 612 & -26 & 2 & $-11 \cdot 5$ & $+\cdot 001$ & -.0057 \\
\hline Tull & 5253 & 841 & -28 & 88 & $+17 \cdot 8$ & +.003 & $+\cdot 0077$ \\
\hline Ennis, : & $52 \quad 52$ & 854 & $\begin{array}{l}-\quad 29 \\
-\end{array}$ & +96 & $\begin{array}{r}+8.2 \\
+\end{array}$ & $-\cdot 002$ & $+\cdot 0056$ \\
\hline Limerick, $\uparrow$. & 5240 & 836 & -41 & +85 & $+5 \cdot 0$ & $+\cdot 005$ & $+\cdot 0041$ \\
\hline
\end{tabular}

* The latitudes and longitudes of some of the more important stations have been kindly furnished by the officers of the Ordnance Survey. The remainder have been taken from Arrowsmith's map of Ireland.

+ Observations made in the year 1835 give Tulla $\delta-\delta_{0}=+23^{\prime} \cdot 7$; Limeriek, $\delta-\delta_{0}=-2 \cdot 1$. 


\section{TABLE VII.-(Continued.)}

\begin{tabular}{|c|c|c|c|c|c|c|c|}
\hline Place. & ( $\lambda$ ) & $(\mu)$ & $\left(\lambda-\lambda_{0}\right)$ & $\begin{array}{c}\left(\mu-\mu_{0}\right) \\
\cos \lambda\end{array}$ & $\left(\delta-\delta_{0}\right)$ & $\left(h-h_{0}\right)$ & $\left(f-f_{0}\right)$ \\
\hline Clonmel, : & 5220 & 741 & 61 & +53 & - & $+\cdot 013$ & - \\
\hline Ballybunan, & 5235 & 934 & 46 & +121 & $+16 \cdot 3$ & $+\cdot 004$ & $+\cdot 0127$ \\
\hline Broadway, . & 5214 & 620 & 67 & & $-29 \cdot 0$ & $+\cdot 021$ & $-\cdot 0025$ \\
\hline Waterford, . & $52 \quad 12$ & & 69 & +31 & $-14 \cdot 5$ & $+\cdot 010$ & $-\cdot 0035$ \\
\hline Dingle, . & 526 & 1020 & 75 & +150 & $+\quad 4.9$ & - & $+\cdot 0140$ \\
\hline Killarney, & 523 & 931 & 78 & +121 & +6.5 & $+\cdot 011$ & - \\
\hline Fermoy,. & 521 & 834 & 80 & +86 & $-17 \cdot 7$ & +.019 & - \\
\hline Valentia, & 5156 & 1012 & -85 & +146 & +2.2 & - & $+\cdot 0089$ \\
\hline Cork, . & 5154 & 828 & -87 & +83 & $-21 \cdot 9$ & $+\cdot 019$ & $-\cdot 0008$ \\
\hline Youghal, . & 5153 & 751 & -88 & +59 & $-23 \cdot 8$ & - & $+\cdot 0011$ \\
\hline Glengariff, . & 5144 & 933 & $-\quad 97$ & +123 & +3.5 & $+\cdot 011$ & - \\
\hline
\end{tabular}

The equations of condition (E) are of the first dimension with respect to the two unknown quantities they contain, and may be written

$$
a x+b y=c ;
$$

in which the values of $a, b$, and $c$ (or of $\left(\mu-\mu_{0}\right) \cos \lambda, \lambda-\lambda_{0}$, and $h-h_{0}$ ), are given in the preceding table. In order to deduce the most probable values of the two unknown quantities, these equations must be combined by the method of least squares. Accordingly multiplying equation (F) by the coefficient of $x(a)$, and by the weight $(w)$ of the determination which it represents, and adding the results, we have

$$
S\left(v a^{2}\right) x+S(w a b) y=S(w a c) ;
$$

and, performing the same operation with respect to the coefficient of the other unknown quantity,

$$
S(w a b) x+S\left(u b^{2}\right) y=S(w b c) .
$$

These are the two final equations which, by elimination, will furnish the most probable values of the quantities sought.

Let the values of $x$ and $y$, obtained from these equations, be $A$ and $B$; then substituting in $(D)$,

$$
r \cos u=A, \quad r \sin u=B ;
$$


and dividing, we have

$$
\tan u=\frac{B}{A}
$$

by which the direction of the isodynamic line is determined. Again, squaring and adding,

$$
r=\sqrt{A^{2}+B^{2}}
$$

which gives the rate of increase of the force in the normal direction. The lines of absolute intensity, and of dip, will be obtained by a similar process, the only difference being in the values of the second members of the equation (F).

Before we can apply these formulas to the investigation of the lines of horizontal intensity, it is necessary to assign the weights due to each equation of condition, or to the determination which it involves. We shall assume, accordingly, that the weights of the values of $\left(h-h_{0}\right)$, recorded in the preceding Table, are measured by the number of separate comparisons from which they have been deduced,* and we shall have, on this principle,

$$
\begin{aligned}
& \text { Limerick, . . weight }=12 \text {, } \\
& \text { Markree, . . . " }=3 \text {, } \\
& \text { Armagh, . . . }, \quad=2 \text {, }
\end{aligned}
$$

the weights of each of the other determinations being represented by unity.

The values of $a, b$, and $c$ being griven in Table VII., we may now proceed to calculate the coefficients of the equations $(G)$. We find,

$$
\begin{gathered}
S\left(u a^{2}\right)=257431, \quad S(w a b)=+49169, \quad S\left(w b^{2}\right)=116509 \\
S(w a c)=-23 \cdot 598, \quad S(u b c)=+35 \cdot 644
\end{gathered}
$$

And the equations are

$$
\begin{array}{r}
257431 x+49169 y=-23 \cdot 598, \\
49169 x+116509 y=+35 \cdot 644 ;
\end{array}
$$

- For a more correct method of estimating the weights of observed results, the reader is referred to the Report on the Magnetic Isoclinal and Isodynamic lines in the British Islands, Eighth Report of the British Association for the Advancement of Seience, p. $95, \&$ c. 
from which we have, by elimination,

$$
\begin{aligned}
& x=-\cdot 0001633=A ; \\
& y=+\cdot 0003748=B .
\end{aligned}
$$

Finally, substituting these values in equations $(\mathrm{H}, \mathrm{I})$

$$
\tan u=-2 \cdot 2952, \quad u=-66^{\circ} 28^{\prime}, \quad r=-\cdot 000409 . *
$$

The positive branches of the axes of co-ordinates having been assumed to be those which stretch to the north and to the west, it follows that the lines of equal horizontal intensity lie to the east of north, making an angle of $66 \frac{1}{2}^{\circ}$ nearly with the meridian of Dublin. The horizontal intensity decreases as we proceed northward, the decrease being equal to the distance traversed in a direction perpendicular to these lines (estimated in geographical miles, or minutes of latitude) multiplied by the coefficient 000409 . The lines are laid down in the accompanying chart for differences of 0.01 in the value of the intensity, the corresponding intervals of distance being $24 \cdot 4$ geographical miles.

On a comparison of the separate determinations with the resulting lines, it will be observed that the intensities in the northern group are greater than those due to their position, those of the western group less, and those of the south-western again greater. These deviations may, in part, arise from the inexactness of the assumption with which we set out in the computation of the lines, and from the sensible deviation of those lines from parallelism. But they are probably owing in a much greater degree to the disturbing causes to which we have already alluded. The separate results composing each of these groups were for the most part obtained about the same time, and they are therefore probably affected in the same manner, and nearly in the same amount, by the irregular fluctuations in the direction and intensity of the resultant magnetic force. Of these, the changes in the direction of the force are by far the most influential. The relation between the corresponding changes in the dip and in the horizontal intensity is expressed by the formula

$$
\frac{d h}{h}=-\tan \delta \sin 1^{\prime} d \delta ;
$$

* The inclination of the lines of equal horizontal intensity, deduced with the aid of additional observations, is $u=-62^{\circ} 40^{\circ}$, the rate of increase in the normal direction remaining unaltered: Eighth Report, p. 174. 
$d \delta$ being expressed in minutes. Hence when $\delta=71^{\circ} 0^{\prime}$, the change of the horizontal intensity, $\frac{d h}{h}$, corresponding to a change of one minute of dip, is - - 00084 ; and for a variation of 12 in the dip, the corresponding variation of the horizontal force is 01 .

In deducing the lines of dip from observation, it seems advisable to separate the results of the two years. For the weights we shall assume

$$
\begin{aligned}
& \text { Limerick (1834), ․ . weight }=5 \text {, } \\
& \text { Armagh, " . . . },=2 \text {; }
\end{aligned}
$$

the weights of each of the other determinations being unity.

Making the computations for the year 1834, we obtain the following results :

$$
\begin{gathered}
S\left(w a^{2}\right)=86660, \quad S(w a b)=+36129, \quad S\left(w b^{2}\right)=64303, \\
S(w a c)=+11043, \quad S(w b c)=-18633 .
\end{gathered}
$$

The final equations accordingly are

$$
\begin{aligned}
& 86660 x+36129 y=+11043 ; \\
& 36129 x+64303 y=-18633
\end{aligned}
$$

From which we deduce

$$
\begin{aligned}
& x=+\cdot 3228=s \cos v, \\
& y=-\cdot 4705=s \sin v ;
\end{aligned}
$$

in which $v$ denotes the angle which the isoclinal line makes with the meridian of Dublin, and $s$ the coefficient which determines the rate of increase of the dip in the perpendicular direction. Dividing, squaring and adding, we find

$$
\begin{gathered}
\tan v=-1.458, \quad v=-55^{\circ} 33^{\prime}, \\
s=571 .
\end{gathered}
$$

The following are the results of calculation for the year 1835 :

$$
\begin{gathered}
S\left(a^{2}\right)=149922, \quad S(a b)=+31821, \quad S\left(b^{2}\right)=55339 \\
S(a c)=+32157, \quad S(b c)=-18372 \\
\text { P } 2
\end{gathered}
$$


so that the final equations are

$$
\begin{gathered}
149922 x+31821 y=+32157 \\
31821 x+55339 y=-18372 .
\end{gathered}
$$

From these we deduce

$$
\begin{gathered}
x=+\cdot 3250, \quad y=-\cdot 5196, \\
\tan v=-1 \cdot 599, \quad v=-57^{\circ} 59^{\prime}, \\
s=\cdot 613 .
\end{gathered}
$$

It would appear, then, that the angle which the isoclinal lines in Ireland make with the meridian is on the increase-a result which is in conformity with the general progress of these lines, as inferred from a comparison of recent observations with those of an earlier date.*

For the mean of the two years,

$$
\begin{gathered}
x=+\cdot 3239, \quad y=-\cdot 4950, \\
\tan v=-1 \cdot 528, \quad v=-56^{\circ} 48^{\prime}, \\
s=.592 .
\end{gathered}
$$

The lines in the annexed chart are deduced from these last results; and it appears from them that the interval of the lines corresponding to a difference of half a degree of dip is 50.7 geographical miles.

The lines of dip and of horizontal intensity being known, the lines of total intensity may be deduced. For if $f$ denote the total intensity, $h$ its horizontal component, and $\delta$ the dip, as before,

$$
h=f \cos \delta \text {; }
$$

and differentiating, and dividing by the equation itself,

$$
f^{-1} d f=h^{-1} d h+\tan \delta \sin 1^{\prime} d \delta .
$$

Now, if the values of $x$ and $y$ for the lines of dip and of horizontal intensity be denoted by $x_{(\delta)}, x_{(h)}$ and $y_{(\delta)}, y_{(h)}$, and if $x_{(f)}$

* This is further confirmed by the results obtained with the help of additional observations. See Eighth Report of the British Association for the Advancement of Science, p. 118. 
and $y_{(f)}$ be the corresponding quantities for the lines of total intensity,

$$
\begin{aligned}
& d \delta=a x_{(\delta)}-b y_{(\delta),}, \\
& d h=a x_{(h)}-b y_{(h),} \\
& d f=a x_{(f)}-b y_{(f),}
\end{aligned}
$$

in which $a=\left(\mu-\mu_{0}\right) \cos \lambda, b=\lambda-\lambda_{0} ; \mu$ and $\lambda$ being the longitude and latitude of any assumed station, and $\mu_{0}$ and $\lambda_{0}$ those of Dublin. Substituting these values in (I.), it becomes

$$
\begin{aligned}
f^{-1}(a x(f) & \left.-b y_{(f)}\right)=h^{-1}\left(a x_{(h)}-b y_{(h)}\right) \\
+ & \tan \delta \sin 1^{\prime}\left(a x_{(\delta)}-b y_{(\delta)}\right) .
\end{aligned}
$$

But as $a$ and $b$ are entirely independent, their coefficients must be separately equal, and we have

$$
\begin{aligned}
& f^{-1} x_{(f)}=h^{-1} x_{(h)}+\tan \delta \sin 1^{\prime} x_{(\delta)}, \\
& f^{-1} y_{(f)}=h^{-1} y_{(h)}+\tan \delta \sin 1^{\prime} y_{(\delta)} ;
\end{aligned}
$$

so that the values of $x_{(f)}$ and $y_{(f)}$ are found when those of $x_{(h)}, x_{(\delta)}$, $y_{(h)}, y_{(8)}$ are known.

Let the second members of equations (IV.) be denoted, for - abridgment, by $P$ and $Q$, then

$$
\begin{aligned}
& x(f)=t \cos w=f P, \\
& y(f)=t \sin w=f Q ;
\end{aligned}
$$

in which $w$ is the inclination of the line of total intensity to the meridian, and $t$ the coefficient which determines the rate of increase. Dividing the latter by the former, there is

$$
\tan v=\frac{Q}{P} \text {. }
$$

And squaring and adding,

$$
t=f \sqrt{\overline{P^{2}+Q^{2}}}
$$

From the preceding formulas it appears that the direotion of the isodynamic line at any point is dependent on the values of $h$ and of $\delta$ at that point, so that these lines will not be parallel, even though the lines of dip and of horizontal intensity should be so. The deviations, however, will not be considerable within the limits of Ire- 
land; and for our present purpose it will be enough to seek the mean direction of the lines, and the mean rate of increase in the direction perpendicular to them. We must therefore employ in the preceding formulas the values of $f, h$, and $\delta$, corresponding to the mean point of the island, or the point whose latitude and longitude are $53^{\circ} 25^{\prime}$ and $7^{\circ} 55^{\prime},{ }^{*}$ and for which therefore

$$
\lambda-\lambda_{0}=4^{\prime}, \quad \mu-\mu_{0}=100^{\prime} .
$$

Now it has been already found that

$$
\begin{aligned}
& x_{(h)}=-\cdot 0001633, \quad x_{(\delta)}=+\cdot 3239, \\
& y_{(h)}=+\cdot 0003748, \quad y_{(\delta)}=-\cdot 4950 ;
\end{aligned}
$$

and substituting these values in the formulas

we find

$$
\begin{aligned}
& \delta-\delta_{0}=\left(\mu-\mu_{0}\right) \cos \lambda x_{(\delta)}-\left(\lambda-\lambda_{0}\right) y_{(\delta)}, \\
& h-h_{0}=\left(\mu-\mu_{0}\right) \cos \lambda x_{(h)}-\left(\lambda-\lambda_{0}\right) y_{(h),}
\end{aligned}
$$

$$
\delta-\delta_{9}=21^{\prime} \cdot 4, \quad h-h_{0}=-\cdot 0113 .
$$

Consequently,

$$
\delta=71^{\circ} 24^{\circ} \cdot 4, \quad h=\cdot 9282, f=1 \cdot 0295 .
$$

We have now the numerical values of all the quantities which enter the formulas

$$
\begin{aligned}
& P=h^{-1} x(h)+\tan \delta \sin 1^{\prime} x_{(\delta)}, \\
& Q=h^{-1} y(h)+\tan \delta \sin 1^{\prime} y_{(\delta)}
\end{aligned}
$$

and we find on substitution,

$$
P=+\cdot 0001042, \quad Q=-\cdot 0000242 .
$$

Introducing these values in (V.) and (VI.),

$$
\tan w=-\cdot 2322, \quad w=-13^{\circ} 4^{\prime}, \quad t=\cdot 0001102 .
$$

These results, however, are not entitled to much confidence. An attentive consideration of the formulas (IV.) and (V.) will show that the direction of the resultant isodynamic lines will vary

* This point corresponds, almost exactly, to the town of Athlone. 
very widely with moderate variations in the values of $x_{(h)}, x y_{(h)},(\delta)$, $y_{(\delta)}$, on which it depends; or, in other words, that a small error in the position of the lines of dip, or of horizontal intensity, will entail a very great one in that of the lines of total force. Thus, if we were to take for the lines of dip those inferred from the observations of the year 1835 alone, we should find

$$
\begin{array}{ll}
P=+\cdot 0001051 . & Q=-\cdot 0000455, \\
\tan w=-\cdot 4329, & w=-23^{\circ} 25^{\prime} ;
\end{array}
$$

a result differing by more than $10^{\circ}$ from the former. In these latitudes, therefore, very great accuracy is necessary in the determination of the lines of dip and of horizontal force before we can make, in this manner, even an approximation to the direction of the lines of total force. For these reasons the results of the direct method, to which we now proceed, seem to be deserving of more confidence.

In the calculation of the isodynamic lines from the results of observation by the statical method, we shall take the number of observations at each station to represent the weight of the result; we have in this manner

$$
\begin{aligned}
& \text { Limerick, . . . weight }=4 \text {, } \\
& \text { Armagh, . . . } \quad "=2 \text {, } \\
& \text { Youghal, . . . , , }=2 \text {; }
\end{aligned}
$$

the weight of each of the other determinations being unity.

The following are the results of the computation :

$$
\begin{gathered}
S\left(w a^{2}\right)=178390, \quad S(w a b)=+38216, \quad S\left(w b^{2}\right)=96066, \\
S(w a c)=+13 \cdot 3229, \quad S(w b c)=-2 \cdot 2448 .
\end{gathered}
$$

The final equations therefore are

$$
\begin{aligned}
178390 x+38216 y & =+13 \cdot 3229 \\
38216 x+96066 y & =-2 \cdot 2448
\end{aligned}
$$

from which we obtain, by elimination,

$$
x=+\cdot 00008711, \quad y=-\cdot 00005802 .
$$


Consequently,

$$
\tan w=-6661, \quad w=-33^{\circ} 40^{\prime}, \quad t=\cdot 0001047 .^{*}
$$

The lines of total intensity thus deduced are laid down in the annexed chart for differences of $\cdot 005$, these differences corresponding to intervals of $47 \cdot 6$ geographical miles. It will be seen that their direction diverges widely from that of the lines of dip; and although the position of the two classes of lines may need further correction, it does not seem likely that such correction will have the effect of diminishing, at least by any considerable amount, the divergence.

* The mean inclination of the isodynamic lines deduced with the help of additional observations is $u=-35^{\circ} 0^{\prime}$, the rate of increase in the perpendicular direction remaining unaltered. Eighth Rpeort of the British Association for the Advancement of Science, p. 185. 


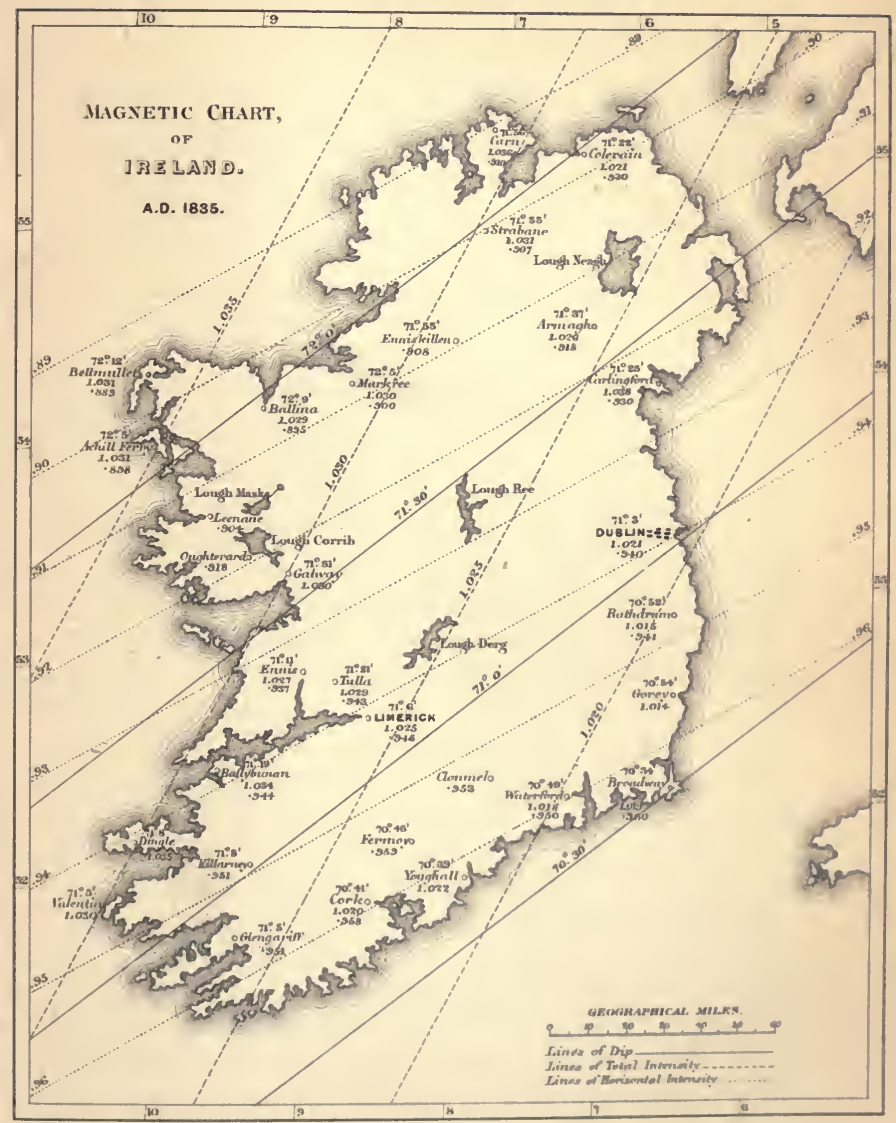



VI.-ON THE POSITION OF THE ISOGONAL LINES IN IRE. LAND, DEDUCED FROM THE OBSERVATIONS OF CAPTAIN SIR JAMES CLARK ROSS, R. $N$.

From the Proceedings of the Royal Irish Academy, 1850.

Is the year 1835 I laid before the British Association, then assembled in Dublin, a Report on the Direction and Intensity of the Terrestrial Magnetic Force in Ireland, based upon observations made by Lieut.-Colonel Sabine, Sir. James C. Ross, and myself. In these observations Mr. Robert Were Fox and Professor Phillips afterwards took part; and the survey was subsequently extended to the whole of the British Islands. The details of this extended survey are given in a Memoir on the Magnetio Isoclinal and Isodynamie lines in the British Islands, drawn up chiefly by Lieut.Colonel Sabine.*

The observations contained in these Reports are limited to the Magnetie Inclination and Intensity. Observations of the Declination, as well as of the other two elements, were indeed made by Sir James Ross; but they have only lately been given to the public in a Memoir by Lieut.-Colonel Sabine, on the lines of Magnetic Declination in the Atlantic. $\dagger$ In this Memoir, the observations referred to are combined with a large mass of other materials, and the position of the isogonal lines inferred from the whole by a graphical process. The Irish portion of these observations is, however, so distinct, and so complete in itself, that it seemed to me desirable that they should be diseussed by the same method which had already been applied to the observations of the other two ele-

\footnotetext{
* Eighth Report of the British Association for the Adeancement of Seience.

†Philosophical Transactions, 1849, Part ii.
} 
ments, in the reports above referred to; such a discussion serving to complete the Magnetic Survey, so far as Ireland is concerned, and to furnish a formula for the Magnetic Declination at any point in the island whose position is known.

The following is the mode of doing this :

If $\delta$ denote the magnetio declination at any place; $\delta_{0}$ that at some near station which is taken as the origin of co-ordinates; and $x$ and $y$ the actual distances (in geographical miles) between them, measured on the parallel of latitude and on the meridian, respectively-or the co-ordinates of position of the former station referred to the latter as an origin; the relation of these quantities is expressed approximately by the equation

$$
\delta-\delta_{0}=M x+N y,
$$

in which $M$ and $N$ represent the increase of deelination corresponding to each geographical mile of distance in the two directions. If $\lambda$ and $\mu$ denote the latitude and longitude of the former station, $\lambda_{\mathrm{o}}$ and $\mu_{0}$ those of the latter,

$$
y=\lambda-\lambda_{\circ}, \quad x=\left(\mu-\mu_{\circ}\right) \cos \lambda .
$$

It is evident, that if $x$ and $y$ be treated as variable, $\delta$ being constant, the preceding equation is that of the locus of all the points of given declination. It is that of a right line, making the angle with the meridian,

$$
\text { ang. }\left(\operatorname{tang}=-\frac{N}{M}\right) \text {; }
$$

and the increase of declination corresponding to each geographical mile of distance, in a direction perpendicular to this line, is

$$
\sqrt{ }\left(M^{2}+N^{2}\right)
$$

It is evident then that, to obtain the values $M \Gamma$ and $N$, observation must give the values of the declination at three, or more, stations. The observations of Sir James Ross were taken at twelve stations, well distributed throughout the island; and as they were all made during the months of October and November, 1838, no correction is required to reduce them to a common epoch. For convenience of reference, they are here extracted from Colonel 
Sabine's Memoir, together with the longitudes and latitudes of the places of observation.

\begin{tabular}{|c|c|c|c|}
\hline Station. & $\lambda$ & $\mu$ & $\delta$ \\
\hline 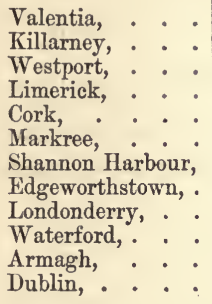 & $\begin{array}{lr}51^{\circ} & 56^{\prime} \\
52 & 2 \\
53 & 48 \\
52 & 40 \\
51 & 54 \\
54 & 14 \\
53 & 14 \\
53 & 42 \\
54 & 59 \\
52 & 15 \\
54 & 21 \\
53 & 21\end{array}$ & $\begin{array}{rr}10^{\circ} & 17^{\prime} \\
9 & 30 \\
9 & 29 \\
8 & 36 \\
8 & 28 \\
8 & 28 \\
7 & 53 \\
7 & 33 \\
7 & 19 \\
7 & 8 \\
6 & 39 \\
6 & 15\end{array}$ & $\begin{array}{lr}28^{\circ} & 42^{\prime} \\
28 & 11 \\
29 & 9 \\
28 & 3 \\
27 & 44 \\
29 & 15 \\
28 & 3 \\
28 & 8 \\
28 & 47 \\
26 & 44 \\
28 & 8 \\
27 & 35\end{array}$ \\
\hline
\end{tabular}

Taking Dublin as the origin of co-ordinates, and substituting the values of $\lambda-\lambda_{\circ}, \mu-\mu_{\circ}$, and $\delta-\delta_{\circ}$, given by this Table, in the equation above given, we obtain eleven equations of condition, from which the values of $M$ and $N$ are obtained by the method of least squares. They are the following:

$$
M I=0^{\prime} \cdot 690, \quad N=0^{\prime} \cdot 585 .
$$

We may now test the accuraey of these numbers, by employing the formula to calculate the values of the declination at each of the eleven stations. The result of this calculation gives, at Waterford, a difference between the observed and calculated values amounting to $34^{\prime}$, an amount which far exceeds the probable error of observation. This difference is, therefore, probably due to some local irregularity of the magnetio force. But, whatever be its cause, it is obvious that it tends to vitiate the general result; and that a nearer approximation to the values of $M$ and $N$ will be obtained by excluding that observation from the computation. We thus obtain, from the remaining ten equations,

$$
M I=0^{\prime} \cdot 689 ; \quad N=0^{\prime} \cdot 527 .
$$

And substituting these values, we find

$$
\text { ang. }\left(\operatorname{tang}=-\frac{N}{M}\right)=-37^{\circ} 25^{\prime} ; \quad \sqrt{ }\left(M^{2}+N^{2}\right)=0^{\prime} \cdot 867 .
$$


Accordingly, the isogonal lines in Ireland lie to the east of north, making an angle of $37^{\circ} 25^{\prime}$ with the meridian of Dublin; and the declination increases as we proceed in the north-westerly direction, the increase being $52^{\prime \prime}$ for each geographical mile, in a direction perpendicular to these lines.*

Finally, the declination at any point of the island, whose longitude and latitude are known, is given by the formula

$$
\delta-\delta_{\circ}=0^{\prime} \cdot 527\left(\lambda-\lambda_{\circ}\right)+0^{\prime} \cdot 689\left(\mu-\mu_{0}\right) \cos \lambda ;
$$

the declination at Dublin, $\delta_{\circ}$, being supposed known. Or, if we substitute for $\cos \lambda$ the value corresponding to the mean latitude $\left(\lambda=53^{\circ} 17^{\prime}\right)$,

$$
\delta-\delta_{\circ}=0^{\prime} \cdot 527\left(\lambda-\lambda_{\circ}\right)+0^{\prime} \cdot 412\left(\mu-\mu_{\circ}\right) .
$$

The mean declination at Dublin, for the year 1850 , is $26^{\circ} 29^{\prime}$ west; and as the yearly value of the secular change of the declination is $-6^{\prime} \cdot 06$, the mean declination, in any not very remote future year, will be given by the formula

$$
\delta_{\circ}=26^{\circ} 29^{\prime}-6^{\prime} \cdot 06 \times n ;
$$

$n$ being the number of years, counted from the present. If greater accuracy be desired, the diurnal and annual variations of the deelination, corresponding to the time of the day and of the year, must be added.

* This result agrees very closely with Colonel Sabine's map of the isogonal lines in the Atlantic, as to the direction of the lines, but gives a more rapid rate of increase. 


\section{VII.-ON A NEW MAGNETICAL INSTRUMENT, FOR THE MEASUREMENT OF THE INCLINATION, AND ITS CHANGES.}

From the Proceedings of the Royal Irish Academy, 1842.

IF a soft iron bar, perfectly devoid of magnetic polarity, be held in a vertical position, it immediately becomes a temporary magnet under the inducing action of the earth's magnetic force, the lower extremity becoming a north pole, and the upper a south pole. Accordingly, if a freely-suspended horizontal magnet, whose dimensions are small in comparison with those of the bar, be situated near, in a plane passing through one of these poles, it will be deflected from the magnetic meridian. The deflecting force is the induced force of the bar, which may be regarded as proportional to the energy of the inducing cause, i.e. to the vertical component of the earth's force; while the counteracting force is the horizontal component of the same force, acting directly on the magnet itself, to bring it back to the magnetio meridian. Thus the magnet will take up a position of equilibrium, under the action of these opposing forces; and this position will serve to determine the ratio which subsists between them. When the right line connecting the centre of the horizontal magnet, and the acting pole of the bar, is perpendicular to the magnetic meridian, the tangent of the angle of deflection will measure the ratio of the two forces, and will therefore be proportional to the tangent of the magnetic inclination. Accordingly, by observing the changes of position of the horizontal magnet, so circumstanced, we can infer those of the inclination itself.

But the iron bar may have (and generally will have) a certain portion of permanent magnetism, which will concur with the in- 
duced magnetism in producing the deflection; and it becomes necessary to institute the observations in such a manner as to be able to eliminate the effects of this extraneous cause. For this purpose we have only to invert the bar, so that the acting pole, which was uppermost in one part of the observation, shall be lowermost in the other. The induced polarity will, under these circumstances, be opposite in the two cases; and the acting force will in one case be the sum of the induced and permanent forces, and in the other their difference.

Let $X$ and $Y$ denote the horizontal and vertical components of the earth's magnetic force, $\boldsymbol{M}$ the intensity of the permanent magnetism in the acting pole, and $m$ the magnetic moment of the suspended magnet. The intensity of the induced magnetism is, by hypothesis, equal to

$$
k Y \text {, }
$$

$k$ being an unknown constant; and when this is of the same name as the permanent magnetism, the intensity of the acting force, at the unit of distance, is

$$
k Y+M \text {. }
$$

Accordingly, the moment of this force to turn the suspended magnet is $(k Y+M) m r \cos u, u$ being the angle of deflection, and $r$ a constant depending on the distance; or, making, for abridgment, $k r=p, M r=q$,

$$
(p Y+q) m \cos u .
$$

But this deflecting force is resisted by the earth's horizontal force, the moment of which to turn the magnet is

\section{$X m \sin u$;}

and the magnet will rest when these moments are equal. Hence the equation of equilibrium is

$$
p Y+q=X \tan u \text {. }
$$

By the same reasoning it will appear, that when the induced and permanent magnetisms are of contrary uames, there is

$$
p Y-q=X \tan u^{\prime} ;
$$

in which $u^{\prime}$ is the new angle of deflection when the bar is inverted. Adding these equations together, and observing that $Y=X \tan \theta$, 
$\theta$ being the inclination, we have

$$
2 p \tan \theta=\tan u+\tan u^{\prime} .
$$

This equation would furnish at once the inclination sought, provided we knew the value of the constant $k$. In order to determine it, we have only to place the iron bar horizontally in the magnetio meridian, its acting pole remaining in the same place as before, but pointing alternately to the north and south. The inducing force is, in this case, the horizontal component of the earth's magnetio force; and it will be readily seen that the equations of equilibrium are similar to (1) and (2), substituting $X$ for $Y$. If therefore $v$ and $v^{\prime}$ denote the angles of deflection in these positions, we have

$$
2 p=\tan v+\tan v^{\prime} ;
$$

and dividing (3) by this,

$$
\tan \theta=\frac{\tan u+\tan u^{\prime}}{\tan v+\tan v^{\prime}} .
$$

Thus, from the deflections produced in these four positions of the bar, we obtain the inclination.

In order to determine the changes of the inclination, it is not necessary to observe the deflections in the horizontal position of the bar. Let equation (1) be differentiated, $X, Y$, and $u$ being all variable, and let the resulting equation be divided by (3). We thus obtain the following equation, from which $p$ and $q$ are both eliminated:

$$
\frac{\Delta Y}{Y}=\frac{2 \Delta u}{\cos ^{2} u\left(\tan u+\tan u^{\prime}\right)}+\frac{2 \tan u}{\tan u+\tan u^{\prime \prime}} \cdot \frac{\Delta X}{X} .
$$

But from the relation $Y=X \tan \theta$, we have

$$
\frac{\Delta Y}{\mathrm{Y}^{-}}=\frac{\Delta X}{X}+\frac{\Delta \theta}{\sin \theta \cos \theta}
$$

and substituting

$$
\frac{\Delta \theta}{\sin 2 \theta}=\frac{\cos u^{\prime} \Delta u}{\cos u \sin \left(u+u^{\prime}\right)}+\frac{1}{\theta} \frac{\sin \left(u-u^{\prime}\right) \Delta X}{\sin \left(u+u^{\prime}\right) X} .
$$

The second term ' of the right-hand member of this equation contains a correction required for the simultaneous changes of the horizontal intensity; but this correstion will be generally small, 
and, when the bar has no permanent magnetism, will vanish altogether. In this latter case, in fact, it appears from (1) and (2) that $u^{\prime}=u$; so that the preceding equation is reduced to

$$
\Delta \theta=\frac{\sin 2 \theta}{\sin 2 u} \Delta u \text {. }
$$

We must remember that, since the angle $u$ in the preceding formulas is the deviation of the suspended magnet from the position which it would assume under the action of the earth alone, its changes are the differences between the observed changes of position of the suspended magnet and the corresponding changes of declination. Let $\boldsymbol{a}$ denote the deviation of the suspended magnet, measured from some fixed line, and $a^{\prime}$ the corresponding angle when the iron bar is removed; then

$$
\text { . } u=a-a^{\prime}, \quad \Delta u=\Delta a-\Delta \alpha^{\prime} .
$$

But $\Delta a=k n, \Delta a^{\prime}=k^{\prime} n^{\prime}$; in which $n$ denotes the number of divisions of the scale of the instrument corresponding to the angle $\Delta a$, $n^{\prime}$ the number corresponding to the angle $\Delta a^{\prime}$ as shown by the declinometer, and $k$ and $k^{\prime}$ the arc-values of a single division in each instrument. Hence

$$
\Delta u=k n-k^{\prime} n^{\prime} .
$$

I now proceed to the construction of the apparatus employed in these measurements.

The magnet is cylindrical ; its length is three inches, and diameter one-fourth of an inch. A mirror is attached to the stirrup by which it is suspended, by means of which the varying position of the magnet may be observed with a telescope at a distance, after the method of Gauss. This mirror is of course vertical ; and it has a motion round a vertical axis, by means of which it may be adjusted to any desired position of the observing telescope. The mirror is circular, and is three-fourths of an inch in diameter. The moveable part, the stirrup to which it is attached, has the form of a cross; and it is rendered vertical by means of three screws, near the extremities of three of the arms of the cross, the heads of which project and hold it. The mirror is maintained in contact with these heads by springs at the back.

The box is octagonal : the interval between the opposite sides is four inches, and that between the top and bottom two inches. The 
top and bottom, and the connecting pillars, are formed of gunmetal; the eight sides are closed by moveable pieces, three of which are of glass, and the rest of ebony. To the top of the box is attached an upright tube of glass, eight inches in length, which incloses the suspension thread. The suspension apparatus at the top of the tube is of the usual construction; the circular piece to which it is attached has a movement of rotation, and its outer surface is graduated to $5^{\circ}$, for the purpose of determining the effect of torsion of the suspension thread.

The base of the instrument is a circle of gun-metal, six inches in diameter, graduated on the edge. The box is connected with this circle by a short conical stem, forming the axis of a second plate, which revolves upon the fixed one. This moveable plate carries two verniers, by which the angle of rotation may be read off to minutes. Two tubular arms, slightly inclined to one another, are attached to this plate; and their other extremities are connected by a cross-piece, which carries a short scale at a distance of eighteen inches from the mirror. This part of the apparatus is employed in determining the total angles of deflection.

The soft iron bar is a cylinder, twelve inches long, and threefourths of an inch in diameter. One of its extremities is inclosed in a hollow cylinder of brass, connected with a horizontal pivot which revolves in a fixed socket. The axis of this pirot being in the line passing through the centre of the suspended magnet, and perpendicular to the magnetic meridian, it is obvious that the bar has a movement of rotation in the plane of the magnetic meridian itself. The distance of the axis of the bar from the centre of the magnet is about five inches; and it is so placed that the induced pole is in the direction of the axis of the pivot, and thus remains fixed during the movement of the bar.

The changes of position of the suspended magnet are observed at a distance by means of a fixed telescope and scale. The scale, whose divisions are reflected by the mirror, is attached above the telescope to a support near the eye-end.

Having explained the principle of this instrument, and given the details of its construction, it remains only that I should describe the observations made for the purpose of testing its performance. I shall pass over for the present those which relate to the absolute inclination, because they have yielded results which can be regarded only as approximations to the truth, and I have not succeeded as 
yet in tracing the errors to their source. It is manifest, however, that an instrument may be a good differential instrument, while it is incapable of yielding absolute results; and there are special reasons why this should be the case with the apparatus now under consideration. Accordingly its failure in the latter respect, even though established, would furnish no ground for despairing of its success in the former.

It is obvious that the apparatus is wholly free from the errors belonging to magnetical instruments moving on a fixed axle; and the only doubt of its performance must relate to the changes of induced magnetism in the iron bar. Thus it might be questioned, before trial, whether such a bar receives in all cases an amount of free magnetism proportional to the inducing force;-whether, again, the minutest changes in the latter are accompanied by corresponding changes in the former;-and whether, lastly, the changes thus produced are instantaneous, or, at least, demand no appreciable time for their development.

In the first experiments which I made, for the purpose of determining these questions, the induced magnetism of the iron bar was altered by means of a permanent magnet, placed in the same right line with the bar, and at a known distance from it. The effect produced upon the position of the suspended magnet being observed, the distance was altered by a known amount, and a new observation taken ; and so on, at many different distances. Then, the law of action of the inducing magnet being known, we may calculate the changes of deflection of the suspended magnet, on the supposition that the changes of the induced force of the bar are proportional to those of the inducing action, and then compare them with the changes of deflection observed. The calculated and observed results of many series of observations, taken in this manner, were found to accord as nearly as the accuracy of the observations themselves allowed.

In making this comparison, however, it is necessary to take into account the effect of the direct action of the fixed magnet upon the suspended one. The axis of the former magnet being not far from the vertical passing through the centre of the latter, its action upon it and upon the iron bar follow, nearly, the same law; so that its direct effects upon the position of the suspended magnet are, very nearly, proportional to those which it produces through the medium of the induced force of the bar. On this principle the 
observed results may be cleared, approximately, of those parts of the changes which are foreign to the question. Still it must be admitted that such a complication of the results tends to weaken their evidence; and it was therefore desirable to obtain further proof, in a manner less exceptionable.

The object being to alter the inducing action according to a known law, and to observe the changes of the induced force, as shown by the position of the suspended magnet, it is manifest that it may be attained by simply varying the angle which the iron bar makes with the direction of the earth's magnetic force, the distance of its pole from the suspended magnet remaining unchanged. In fact, it will be seen, by pursuing the same reasoning as before, that if $R$ denote the total force of the earth, and $\psi$ the angle which the bar makes with its direction, the equation of equilibrium of the suspended magnet is

$$
p R \cos \psi+q=X \tan u,
$$

the line connecting the pole of the bar with the centre of the suspended magnet being, as before, perpendicular to the magnetic meridian. Hence, if the bar be devoid of permanent magnetism (or $q=0$ ), and if the forces $R$ and $X$ remain unchanged during the experiments, we have

$a$ being a constant.

$$
\tan u=a \cos \psi,
$$

In order to observe whether the deflections of the suspended magnet obeyed this law, a small divided circle was attached to the piece upon which the iron bar moved, in such a manner that the axis of the pivot passed through its centre. The circle being fixed, and the bar connected with the moveable arm carrying the vernier, we have the means of determining the angle through which it is moved. The plane of the motion coinciding with the magnetic meridian, the inclination of the bar to the vertical was altered by $5^{\circ}$ between the successive observations of the position of the suspended magnet. The following Tables contain the results of two such series of observations. The first column of each gives the inclination of the bar to the vertical; the second, its inclination $(\psi)$ to the direction of the magnetio force, i.e., the former angle increased by the complement of the magnetic inclination $\left(19^{\circ} 10^{\prime}\right)$. The third column contains the observed readings of the scale, corresponding to the positions of the suspended magnet; the fourth, the differ- 
ences between each of these readings and the reading belonging to the vertical position of the bar, converted into angular measure; the fifth, the actual deflections; the sixth, the calculated deflections, as deduced by the formula given above; and the seventh, the differences.

In order to derive the numbers of the fifth column from those of the fourth, it is necessary to know the deflection corresponding to the vertical position of the bar. This angle is determined by placing the bar vertically, with its acting pole above and below successively, and noting the readings of the horizontal circle, when the same division of the moveable scale, reflected by the mirror, was brought to coincide with the fixed wire of the telescope. The differences between each of these readings, and the similar reading when the bar is removed, are double the deflections corresponding to the two positions of the bar; and, when they are nearly equal, the mean of these deflections may be taken as that due to the induced force.

FIRST OBSERVATION.

Acting end of bar a south pole, reading $=14^{\circ} 8^{\circ}$, deflection $=17^{\circ} 0^{\circ}$

north pole, . . $8251, \quad, \quad=1722$
Bar removed, . 487, mean $=1711$

\begin{tabular}{|c|c|c|c|c|c|c|}
\hline $\begin{array}{l}\text { Inclination } \\
\text { to Vertical. }\end{array}$ & $\psi$ & $\begin{array}{l}\text { Reading } \\
\text { of Scale. }\end{array}$ & $\begin{array}{c}\text { Angular } \\
\text { Differences. }\end{array}$ & $\begin{array}{c}u \\
\text { Observed. }\end{array}$ & $\begin{array}{c}u \\
\text { Calculated. }\end{array}$ & Difference \\
\hline$+14^{\circ} 30$ & $33^{\circ} 40^{\prime}$ & $2 \cdot 2$ & $-1^{\circ} 56^{\prime} \cdot 2$ & $15^{\circ} 14^{\prime} \cdot 8$ & $15^{\circ} 14^{\prime} \cdot 5$ & $+0^{\circ} \cdot 3$ \\
\hline+100 & 2910 & $13 \cdot 2$ & $-112 \cdot 4$ & $15 \quad 58 \cdot 6$ & $\begin{array}{lll}15 & 57 \cdot 2\end{array}$ & $+1 \cdot 4$ \\
\hline $\begin{array}{r}50 \\
+50\end{array}$ & $\begin{array}{ll}24 & 10\end{array}$ & $23 \cdot 1$ & - $\quad 33 \cdot 0$ & $1638 \cdot 0$ & $1637 \cdot 8$ & $+0 \cdot 2$ \\
\hline $\begin{array}{r}00 \\
-\quad 50\end{array}$ & $\begin{array}{ll}19 & 10 \\
14 & 10\end{array}$ & $\begin{array}{l}31 \cdot 4 \\
37 \cdot 5\end{array}$ & $\begin{array}{r}0 \cdot 0 \\
24 \cdot 3\end{array}$ & $\begin{array}{ll}17 & 11 \cdot 0 \\
17 & 35 \cdot 3\end{array}$ & $1736 \cdot 7$ & $-1 \cdot 4$ \\
\hline $\begin{array}{ll}-10 & 0\end{array}$ & 910 & $42 \cdot 8$ & $+\quad 45 \cdot 4$ & $1756 \cdot 4$ & $1754 \cdot 7$ & +1.7 \\
\hline-1330 & 540 & $45 \cdot 8$ & $+\quad 57 \cdot 3$ & $18 \quad 8 \cdot 3$ & $18 \quad 2 \cdot 7$ & $+5 \cdot 6$ \\
\hline
\end{tabular}

SECOND OBSERVATION.

Acting end of bar a south pole, reading $=14^{\circ} 23^{\prime}$, deflection $=16^{\circ} 36^{\prime}$

" north pole, . . $8215, \quad, \quad=1720$

" Bar removed, . . 4735, mean $=1658$

\begin{tabular}{|c|c|c|c|c|c|c|}
\hline $\begin{array}{l}\text { Inclination } \\
\text { to Vertical. }\end{array}$ & $\psi$ & $\begin{array}{l}\text { Reading } \\
\text { of Scale. }\end{array}$ & $\begin{array}{c}\text { Angular } \\
\text { Differences. }\end{array}$ & $\begin{array}{c}u \\
\text { Observed. }\end{array}$ & Calculated. & Difference. \\
\hline $\begin{array}{l}+150^{\circ} \\
+100 \\
+\quad 50 \\
00 \\
-\quad 50\end{array}$ & $\begin{array}{ll}34^{\circ} & 10^{\prime} \\
29 & 10 \\
24 & 10 \\
19 & 10 \\
14 & 10\end{array}$ & $\begin{array}{r}2 \cdot 6 \\
14 \cdot 4 \\
24 \cdot 8 \\
33 \cdot 2 \\
40 \cdot 0\end{array}$ & $\begin{array}{rr}-2 & 1 \cdot 8 \\
-1 & 14 \cdot 8 \\
-\quad 33 \cdot 4 \\
& 0 \cdot 0 \\
+\quad 27 \cdot 1\end{array}$ & $\begin{array}{ll}14^{\circ} & 56^{\prime} \cdot 2 \\
15 & 43 \cdot 2 \\
16 & 24 \cdot 6 \\
16 & 58 \cdot 0 \\
17 & 25 \cdot 1\end{array}$ & 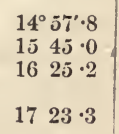 & $\begin{array}{l}-1.6 \\
-1.8 \\
-0.6 \\
+1.8\end{array}$ \\
\hline
\end{tabular}


In the preceding observations a telescope of law power was employed, and the arc-value of a single division of the scale (which was at the distance of eighteen inches from the mirror) was $3^{\prime} \cdot 98$. The differences of the observed and calculated results, therefore, do not in general exceed the amount which may be fairly ascribed to errors of observation; and the accordance is sufficient to establish the fact, that the changes of the induced force of the bar are, within the observed limits, proportional to those of the inducing action. It is important to note also that the changes of the induced force, produced artificially in these experiments, are much greater than any which are likely to arise from the variations of the vertical component of the earth's magnetic foree, and therefore that the experiments may be regarded as severe tests of the performance of the instrument.

The preceding observations further showed, that the changes in the inducing force were instantly followed by their effeets upon the suspended magnet; so that the changes of induced force required no appreciable time for their development. It remained only to ascertain, in a somewhat fuller manner, how far the bar was susceptible of minute magnetic changes, from very small variations of the acting force. For this purpose, a series of readings of the scale was taken, the inclination of the bar to the vertical being altered by half a degree between the consecutive readings. The mean difference of the successive readings was found to agree, very exactly, with the calculated difference; while the partial differences deviated from the mean by an amount not exceeding the limits of error of observation. It may be presumed, therefore, that the changes of the induced force in the iron bar are continuous; and, accordingly, that the sensibility of the instrument is only limited by the optical power, which is applied to observe the changes of position of the suspended magnet.*- In the experiments above described, the arc-value of the divisions of the scale was nearly $4^{\prime}$ : with the modifications since introduced into the reading part of the apparatus, the scale

* Against this conclusion is the fact, that considerable changes in the induced force of the bar seem to be attended with some permanent changes of polarity; and it may be presumed that the same thing will take place, in a proportionate degree, with the minute changes induced by the variations of the earth's force. It remains for future examination to determine how far such permanent changes, if they occur, may impair the accuracy of the results. 
divisions have nearly the same value as in the instrument for the measurement of the declination, so that the readings may be made with certainty to less than the tenth of a minute. The present value of the inclination in Dublin is about $70^{\circ} 50^{\prime}$; and the mean deflection produced by the iron bar in its actual position being about $19^{\circ}$, it follows that the changes of inclination are inferred with the same degree of precision, very nearly, as the observed changes of angle.

The last test to which the instrument was subjected was, to employ it for some time in the regular observation of inclination changes, for which it is destined, and to ascertain how far the mean results of the observations of successive weeks agreed in exhibiting the law of the diurnal wariation. The instrument was accordingly observed for five successive weeks, every second hour during the day and night, and the means calculated, omitting those days in which the series was broken by changes of adjustment during experiment. The curves now laid before the Academy represent the projected results of the observations of each of these weeks, together with that of the mean of the whole. An inspection of them is sufficient to show that the curves of the separate weeks accord with one another, and with the mean, as nearly as can be expected in the results of such limited series, the discordances being only such as are due to the known irregularities in the direction of the earth's magnetic force. 


\section{VIII.-REMARKS ON THE THEORY OF THE COMPOUND MAGNETIC NEEDLE.}

Proceedings of the Royal Irish Academy, 1848.

When two magnetic needles are united by a fixed vertical axis passing through their centres, and perpendicular to both, the moment of the force exerted by the earth upon them is the sum of the moments which it exerts upon each needle separately, and is, therefore,

$$
X\left(M \sin u+M I^{\prime} \sin u^{\prime}\right)
$$

in which $M$ and $M^{\prime}$ denote the magnetio moments of the two needles, $u$ and $u^{\prime}$ the angles which their magnetic axes make with the magnetic meridian, and $X$ the horizontal component of the earth's magnetic force. In the state of equilibrium this moment is nothing; so that if $u_{0}$ and $u_{0}^{\prime}$ denote the corresponding values of $u$ and $u^{\prime}$, there is

$$
M \sin u_{0}+M^{\prime} \sin u_{0}^{\prime}=0 .
$$

Consequently, if two lines be taken from any point of the vertical axis, parallel to the magnetio axes of the two needles, and proportional to their magnetio moments, $M$ and $M \Gamma^{\prime}$, the diagonal of the parallelogram constructed upon them must lie in the magnetio meridian, when the compound needle is at rest.

Again, if we substitute $u=u_{0}+v, u^{\prime}=u_{0}^{\prime}+v$, in the general expression of the statical moment, it becomes, in virtue of (1),

$$
X\left(\not \Gamma \cos u_{0}+I^{\prime} \cos u_{n}^{\prime}\right) \sin v
$$

Hence the compound needle is acted upon as a single needle, 
whose magnetic axis lies in the direction of the diagonal of the parallelogram above mentioned, and whose magnetic moment is

$$
\mu=M \cos u_{0}+M^{\prime} \cos u_{0}^{\prime}
$$

Accordingly, the diagonal of the parallelogram already referred to will represent in magnitude the magnetic moment of the compound needle. For, if the equations (1) and (2) be squared, and added together, and the angle contained by the magnetic axes of the two needles, $u_{0}^{\prime}-u_{0}$, be denoted by $a$, we have

$$
\mu^{2}=M^{2}+2 M M^{\prime} \cos a+M^{\prime 2} .
$$

In the case of the astatic needle, $a=180-\delta, \delta$ being a very small angle, and $\cos a=-\cos \delta=-1+\frac{1}{2} \delta^{2}, q \cdot p$. whence

$$
\mu^{2}=\left(M-M^{\prime}\right)^{2}+M M^{\prime} \delta^{2} \text {. }
$$

Accoraingly, when $M-M I^{\prime}$ is not a very small quantity, the second term may be neglected in comparison with the first, and $\mu=\mathbb{M}-M^{\prime}$, nearly. On the other hand, when $M-M^{\prime}=0$, we have $\mu=M S$. have

Returning to (1), and substituting for $u_{0}^{\prime}$ its value $u_{0}+a$, we

$$
\tan u_{0}=\frac{-\sin a}{\frac{M}{M \Gamma^{\prime}}+\cos a} ;
$$

by which the position of the needle with respect to the magnetic meridian, when at rest, is determined. In the case of the astatic needle, the preceding equation becomes

$$
\tan u_{0}=\frac{-M^{\prime}}{M-M^{\prime}} \cdot \delta \sin 1^{\prime} .
$$

From this we learn,

1. That the tangent of the angle of deviation of the astatic needle from the magnetic meridian varies, cateris paribus, as the angle, $\delta$, contained by the magnetic axes of the two component needles.

2. That however small that angle be, provided it be of finite magnitude, the tangent of the deviation may be rendered as great as we please, and therefore the deviation be made to approach to $90^{\circ}$ as nearly as we please, by diminishing the difference of the moments of the two needles. 


\section{IX.-ON THE MEAN RESULTS OF OBSERVATIONS.}

Transactions of the Royal Irish Academy. Vol. XXII.

1. The problem in which it is sought to determine the daily mean values of the atmospheric temperature or pressure, from a limited number of observed values, is one of fundamental importance in meteorology; and, accordingly, many solutions of it have been proposed by meteorologists. These solutions are derived, for the most part, from the known laws of the diurnal variation of these elements. Many of them are accordingly applicable only to the particular cases considered; while for others, which are really general in their nature, that generality is not established. It is the object of the following investigation to supply this deficiency, and to show in what manner the daily and yearly means may be obtained in all the periodical functions with which we are concerned in magnetism and meteorology.

2. It is known that the mean value of any magnetical or meteorological element, for any day, may be obtained, approximately, by taking the arithmetical mean of any number of equidistant observed values; the degree of approximation, of course, increasing with the number. $\mathrm{A}$ somewhat more exact mean may be deduced, as has been shown by Cotes and Kramp, by combining the equidistant observed values in a different manner; and Gauss has given a method, whereby the values of the integral, $\int_{-a}^{+a} U d x$, may be obtained with still greater accuracy from the observed values of the ordinate, $U$, corresponding to certain definite abscissæ.* But in the case of periodical functions, 
it will appear from what follows that the refinement of Cotes is unnecessary; and, in the case under consideration, there are practical reasons of another kind for adhering to the method of equidistant observations, and which, therefore, deprive us of the advantages of Gauss's method.

3. Any periodical function $U$, of the variable $x$, may be represented by the series

$$
U=A_{0}+A_{1} \sin \left(x+\boldsymbol{a}_{1}\right)+A_{2} \sin \left(2 x+\boldsymbol{a}_{2}\right)+A_{3} \sin \left(3 x+\boldsymbol{a}_{3}\right)+\& c .,
$$

in which the first term, $A_{0}$, is the mean value of the ordinate $U$, and is expressed by the equation

$$
A_{0}=\frac{1}{2 \pi} \int_{-\pi}^{+\pi} U d x
$$

This is the quantity whose value is sought in the present investigation.

It is obvious that the values of $U$ return again in the same order and magnitude when $x$ becomes $x+2 \pi$; so that if $x=a t$, the period is represented by $\frac{2 \pi}{a}$. If then $2 \pi$ be divided into $n$ equal parts, so that the abscissæ of the points of division are $x$, $x+\frac{2 \pi}{n}, x+\frac{4 \pi}{n}, \& c ., x+\frac{2(n-1) \pi}{n}$, the sum of the corresponding ordinates will be

$$
\begin{aligned}
\Sigma(U)=n A_{0}+ & A_{1} \Sigma \sin \left(x+\frac{2 i \pi}{n}+a_{1}\right)+A_{2} \Sigma \sin \left\{2\left(x+\frac{2 i \pi}{n}\right)+a_{2}\right\} \\
& +A_{3} \Sigma \sin \left\{3\left(x+\frac{2 i \pi}{n}\right)+a_{3}\right\}+\& c .,
\end{aligned}
$$

in which $i$ denotes any one of the series of integer numbers, from 0 to $n-1$ inclusive. The multiplier of $A_{m}$, in the general term of this series, is

$$
\begin{gathered}
\Sigma \sin \left\{m\left(x+\frac{2 i \pi}{n}\right)+\boldsymbol{a}_{m}\right\} \\
=\sin \left(m x+\boldsymbol{a}_{m}\right) \Sigma \cos \frac{2 i m \pi}{n}+\cos \left(m x+\boldsymbol{a}_{m}\right) \Sigma \sin \frac{2 i m \pi}{n} .
\end{gathered}
$$

But, when $m$ is not a multiple of $n$,

$$
\Sigma \cos \frac{2 i m \pi}{n}=0, \quad \Sigma \sin \frac{2 i m \pi}{n}=0 ;
$$


and, therefore, the preceding term ranishes. When $m$ is a multiple of $n$,

$$
\Sigma \cos \frac{2 i m \pi}{n}=n, \quad \Sigma \sin \frac{2 i m \pi}{n}=0 ; *
$$

and accordingly the term is reduced to

$$
n \sin \left(m x+\boldsymbol{a}_{m}\right) .
$$

Hence, all the terms of the series vanish, excepting those in which $m=k n, k$ being any number of the natural series, and

$$
\frac{1}{n} \Sigma(U)=A_{0}+A_{n} \sin \left(n x+a_{n}\right)+A_{2 n} \sin \left(2 n x+a_{2 n}\right)+\& c .
$$

That is, the arithmetical mean of the $n$ equidistant ordinates is equal to the sum of the terms of the original series of the order $\mathrm{kn}$, whatever be the value of $x$.

The original series for $U$ being always convergent, the derived series, which expresses the value of $\frac{1}{n} \Sigma(U)$, will be much more so; and, when the number $n$ is sufficiently great, we may neglect all the terms after the first. Hence, approximately,

$$
A_{0}=\frac{1}{n} \Sigma(U) \text {. }
$$

The error of this result will be expressed by the second term

* These results are easily established. The roots of the equation $y^{n}-1=0$, being comprised in the formula $\cos \frac{2 i \pi}{n}+\sqrt{ }(-1) \sin \frac{2 i \pi}{n}$, the $m^{\text {th }}$ power of any one of these roots is $\cos \frac{2 i m \pi}{n}+\sqrt{ }(-1) \sin \frac{2 i m \pi}{n}$; and the sum of the $m^{\text {th }}$ powers of the roots is

$$
\Sigma \cos \frac{2 i m \pi}{n}+\sqrt{ }(-1) \Sigma \sin \frac{2 i m \pi}{n} .
$$

Now, when $m$ is not a multiple of $n$, this sum $=0$, and therofore

$$
\Sigma \cos \frac{2 i m \pi}{n}=0, \quad \Sigma \sin \frac{2 i m \pi}{n}=0,
$$

as above. When $m$ is a multiple of $n$, the sum of the $m^{\text {th }}$ powers of the roots $=n$, and therefore

$$
\Sigma \cos \frac{2 i m \pi}{n}=n, \quad \Sigma \sin \frac{2 i m \pi}{n}=0 .
$$


of the series, $A_{n} \sin \left(n x+a_{n}\right)$, the succeeding terms being, for the same reason, disregarded in comparison; and accordingly the limit of error will be $A_{n}$. Thus, when the period in question is a day, we learn that the daily mean value of the observed element will be given by the mean of two equidistant observed values, nearly, when $A_{2}$ and the higher coefficients are negligible; by the mean of three, when $A_{3}$ and the higher coefficients are negligible; and so on.

4. The coefficient $A_{2}$ is small in the series which expresses the diurnal variation of temperature; and, consequently, the curve which represents the course of this variation is, nearly, the curve of sines. In this case, then, the mean of the temperatures at any turo equidistant or homonymous hours is, nearly, the mean temperature of the day. The same thing holds with respect to the annual variation of temperature; and the mean of the temperatures of any two equidistant months is, nearly, the mean temperature of the year. These facts have been long known to meteorologists.

5. The coefficient $A_{3}$ is small in all the periodical functions with which we are concerned in magnetism and meteorology; and, therefore, the daily and yearly mean values of these functions will be given, approximately, by the mean of any three equidistant observed values.

In order to establish this, as regards the daily means, I have calculated the coefficients of the equations which express the laws of the mean diurnal variation of the temperature, the atmospheric pressure, and the magnetic declination, as deduced from the observations made at the Magnetical Observatory of Dublin during the year 1843. The observations were taken every alternate hour during both day and night; and the numbers employed in the calculation are the yearly mean results corresponding to the several hours. The origin of the abscissæ is taken at midnight.

6. The following is the equation of the diurnal variation of temperature :

$$
\begin{aligned}
U-A_{0}= & +3^{\circ} \cdot 60 \sin \left(x+239^{\circ} \cdot 0\right)+0^{\circ} \cdot 70 \sin \left(2 x+67^{\circ} \cdot 2\right) \\
& +0 \cdot 26 \sin (3 x+73 \cdot 5)+0 \cdot 03 \sin (4 x+102 \cdot 7) \\
& +0 \cdot 14 \sin (5 x+258 \cdot 6)+0 \cdot 09 \sin (6 x+180 \cdot 0) .
\end{aligned}
$$

Hence, the error committed in taking the mean of the temperatures 
at any two equidistant hours as the mean temperature of the day, is expressed nearly by the term

$$
0^{\circ} \cdot 70 \sin \left(2 x+67^{\circ} \cdot 2\right)
$$

and, consequently, cannot exceed $0^{\circ} \cdot 70$. To obtain the pairs of homonymous hours, whose mean temperature corresponds most nearly with that of the day, we have only to make $\sin \left(2 x+67^{\circ} \cdot 2\right)=0$, which gives for $x$ the values

$$
x=56^{\circ} \cdot 4, \quad x=146^{\circ} \cdot 4,
$$

corresponding to the times

$$
t=3^{h} 46^{m}, \quad t=9^{h} 46^{m} .
$$

Accordingly, the best pairs of homonymous hours, so far as this problem is concerned, are $3^{h} 46^{m}$ A. м. and $3^{h} 46^{m}$ P. M., or $9^{h} 46^{m}$ A.M. and $9^{h} 46^{m}$ P. ..

The error committed, in taking the mean of the temperatures at any three equidistant hours as the mean temperature of the day, is, very nearly,

$$
+0^{\circ} \cdot 26 \sin \left(3 x+73^{\circ} \cdot 5\right)
$$

andcan not, therefore, exceed $0^{\circ} \cdot 26$. The best hours are those in which the angle, in the preceding expression, is equal to $180^{\circ}$ or $360^{\circ}$. The corresponding values of $x$ are

whence

$$
x=35^{\circ} \cdot 5, \quad x=95^{\circ} \cdot 5 ;
$$

$$
t=2^{h} 22^{m}, t=6^{h} 22^{m} .
$$

Accordingly, the best hours of observation are

and

$$
2^{h} 22^{m} \text { A.M., } 10^{h} 22^{m} \text { A.M., } 6^{h} 22^{m} \text { P.M. ; }
$$

$$
6^{h} 22^{m} \text { A.M., } 2^{h} 22^{m} \text { P.M., } 10^{h} 22^{m} \text { P.M. }
$$

By taking the mean of any fou equidistant observed ralues, the limit of error will, of course, be less. Its amount, which is the coefficient of the fourth term of the preceding formula, is only $0^{\circ} .03$; and, accordingly, the mean temperature of the day is inferred from the temperatures observed at any four equidistant hours with as much precision as can be desired. 
7. The law of the diurnal variation of the atmospheric pressure is contained in the following equation :

$$
\begin{aligned}
U-A_{0}= & +\cdot 0024 \sin \left(x+244^{\circ} \cdot 3\right)+\cdot 0089 \sin \left(2 x+144^{\circ} \cdot 4\right) \\
& +\cdot 0008 \sin (3 x+27 \cdot 9)+\cdot 0006 \sin (4 x+78 \cdot 5) \\
& +\cdot 0001 \sin (5 x+228 \cdot 7)+\cdot 0002 \sin (6 x+180 \cdot 0)
\end{aligned}
$$

The second term in this formula being the principal one, the mean of the pressures observed at any tico equidistant hours, so far from approaching the mean daily pressure, may recede from it by the greatest possible amount within the limits of the diurnal variation. The error committed, in taking the mean of the pressures observed at three equidistant hours as the mean daily pressure, is, very nearly,

$$
+\cdot 0008 \sin \left(3 x+27^{\circ} \cdot 9\right)
$$

and cannot therefore exceed $\cdot 0008$. It is needless to inquire into the least value of this quantity, which is in all cases less than the probable error.

8. The law of the diurnal variation of the magnetic declination is expressed by the equation

$$
\begin{aligned}
U-A_{0}= & +3^{\prime} \cdot 29 \sin \left(x+65^{\circ} \cdot 7\right)+2^{\prime} \cdot 08 \sin \left(2 x+224^{\circ} \cdot 5\right) \\
& +0^{\prime} \cdot 63 \sin (3 x+71 \cdot 7)+0^{\prime} \cdot 30 \sin (4 x+237 \cdot 5) \\
& +0^{\prime} \cdot 13 \sin (5 x+114 \cdot 7),
\end{aligned}
$$

the coefficient of the last term being evanescent. Hence the error to which we are liable, in taking the mean of the declinations observed at any three equidistant hours as the mean of the day, is, very nearly,

$$
+0^{\prime} \cdot 63 \sin \left(3 x+71^{\circ} \cdot 7\right)
$$

and cannot exceed $0^{\prime} 63$. This term vanishes, and the mean of the three observed values will deviate from the true daily mean by an amount less than the errors of observation, when

that is, when

$$
\begin{aligned}
& x=36^{\circ} \cdot 1, \text { or } x=96^{\circ} \cdot 1 ; \\
& t=2^{h} 25^{m}, \text { or } t=6^{h} 25^{m} .
\end{aligned}
$$

Accordingly, the best three hours of observation, for the elimination of the diurnal variation of the declination, are

and

$$
2^{h} 25^{m} \text { A.м., } 10^{h} 25^{m} \text { A.м., } 6^{h} 25^{m} \text { P.м. ; }
$$

$$
6^{h} 25^{m} \text { A.M., } 2^{h} 25^{m} \text { P.M., } 10^{h} 25^{m} \text { P.M. ; }
$$


which coincide, almost exactly, with the best hours for the determination of the mean temperature.

By taking the mean of the declinations observed at any four equidistant hours, as the mean of the day, the limit of error is reduced to $0^{\prime} \cdot 30$.

9. It appears from the preceding, that any three equidistant observations are sufficient to give the daily mean values (and, therefore, also the monthly and yearly mean values) for each of these elements, with nearly the requisite precision; and that, by a suitable choice of the hours, the degree of accuracy may be augmented as much as we please. But, in determining the particular hours for a continuous system of observations, this should not be made the primary ground of selection. The error of the daily means being in all cases reduced within narrow limits by the method already explained, we should choose the particular hours which correspond nearly to the maxima and minima of the observed elements, so as to obtain also the daily ranges. This condition will be fulfilled in the case of the magnetic declination, very nearly, by the hours

$$
6 \text { A.м., } 2 \text { р.м., } 10 \text { P.M. ; }
$$

which will, moreover, give nearly the maximum and minimum of temperature, and of the tension of vapour, together with the maximum pressure of the gaseous atmosphere.* And, if we add the intermediate hours, $10 \mathrm{~A}$. M. and 6 P. M., we shall have, nearly, the principal maxima and minima of the two other magnetic elements. Accordingly, for a limited system of magnetical and meteorological observations, at places for which the epochs of maxima and minima do not differ much from those at Dublin, the best hours of observation appear to be

$$
6 \text { А.м., } 10 \text { А.м., } 2 \text { р.м., } 6 \text { р.м., } 10 \text { р.м. }
$$

The conditions of the problem are altered, if at any place the laws of the diurnal variation have been already obtained from a more extended system of observations. In this case the mean of the day may be inferred from observations taken at any hours whatever,

* The ternary combination above proposed possesses the further advantage of coinciding, nearly, with one of those deduced above, as the most favourable for the determination of the mean temperature and mean declination. The errors of the resulting means are found by making $x=90^{\circ}$ in the third terms of the general formulas; and we thus find the error of temperature $=-0^{\circ} \cdot 07$, while that of the declination $=-0^{\circ} \cdot 20$. 
by the addition of a known correction; and the hours of observation should therefore be chosen chiefly, if not exclusively, with reference to the diurnal range of the observed elements.

10. The next question which presents itself for consideration, with respect to the daily means, is one which affects more nearly the reduction of the observations hitherto made at Dublin. In the extended system prescribed by the Council of the Royal Society in 1839, and followed at the Magnetical Observatory of Dublin during the four years eommencing with 1840, observations were directed to be taken twelve times, at equal intervals, throughout the day-namely, at the even hours of Göttingen mean time. In a system of observations so frequent, and extending over so considerable a time, blanks must unavoidably occur; and the question which presents itself here is-in what way are the daily means to be deduced in such a case?

It has been shown that the effect of the regular diurnal variation may be nearly eliminated, and the mean of the day obtained, by taking the mean of three equidistant observed values. For the elimination of the irregular ehanges, however, the number of observations combined should be as great as possible; and in the ease of the magnetic elements, in which these changes are often very considerable, this condition is an important one.

Now it is obvious that the twelve results of any day may be resolved into two groups of six equidistant results, or into three groups of four, or into four groups of three. Hence, when one result is wanting in the day, the mean may be inferred either from one group of six results, from two groups of four, or from three of three. The last of these combinations, containing nine separate results, is, of course, to be preferred. When two results are wanting, the mean may be inferred from one group of four results, or from two groups of three; of which the latter combination, containing six results, is to be preferred. When three results are wanting, the mean of the day can be inferred (in general) only from one group of three; and when more than three are wanting, that mean cannot be generally obtained.

11. What has been said above applies to the irregular changes of short period-such, especially, as those to which the magnetic elements are subject. But there are also irregular changes of longer duration (as, for example, those produced in the atmospheric pressure by the passage of the greater aerial waves), which 
complicato the problem, inasmuch as a different process is required for their elimination.

In the reduction of the magnetical and meteorological observations made at the Observatory of Dublin, the civil day is adopted; and the observations being made at the odd hours of Dublin mean time, very nearly, the epoch of the mean of all the twelve results is mean noon. But in the case of deficient observations, the epoch of the mean, inferred from the remaining observations, may deviate one or more hours from noon; and its amount, therefore (as compared with the mean reduced to noon), is affected by an error equal to the change which the observed element undergoes in that time. In the case of the atmospherie pressure, this error is often very considerable, and much exceeds that due to the changes of whose elimination we have hitherto spoken.

The law of the changes here referred to being unknown, we can only deal with them on the assumption that their course is uniform throughout the space of a day; and this assumption will, probably, seldom err much from the truth. Upon this principle, the effect of the irregular change will be eliminated by taking the mean of two or more results equidistant from noon (that is, the mean of a forenoon and afternoon result corresponding to the hours $x$ and $12-x$, or any combination of such means); and we have only to consider in what manner this process can be combined with the elimination of the regular diurnal change.

Let the mean of the four equidistant observed values commencing with the $n^{\text {th }}$ hour be denoted, for brevity, by $\mathrm{IV}_{n}$; then the epochs of the means $\mathrm{IV}_{1}, \mathrm{IV}_{3}, \mathrm{IV}_{5}$, are 10 A.M., noon, and 2 P.M., respectively; so that the two conditions are satisfied by the combinations

$$
\frac{1}{9}\left(\mathrm{IV}_{1}+\mathrm{IV}_{5}\right) \text {, and } \mathrm{IV}_{3} \text {. }
$$

In like manner, the means of any three equidistant observed values being denoted by $\mathrm{III}_{n}$, the epochs of the means $\mathrm{III}_{1}, \mathrm{III}_{s}, \mathrm{III}_{s}$, III $_{\text {7, are }} 9$ A. M., 11 A.M., 1 P. M., and 3 P.M. respectively; so that both conditions are satisfied by the combinations

$$
\frac{1}{2}\left(\mathrm{III}_{1}+\mathrm{III}_{7}\right) \text {, and } \frac{1}{2}\left(\mathrm{III}_{3}+\mathrm{III}_{5}\right) \text {. }
$$

12. When, from the number and disposition of the blanks, none of these combinations can be had, and therefore both changes (regular and irregular) cannot be eliminated, we must attend chiefly to 
that which is greater in amount. For the purpose of comparing their magnitude, I have taken the differences of the successive daily means, for the declination, the atmospheric pressure, and the temperature, as deduced from the observations of the year 1843; and have calculated the square-root of the mean of the squares of these differences. The results, which may be taken as the measures of the irregular changes from day to day, are the following:

\section{Mean Fluctuation from Day to Day.}

$\begin{array}{llll}\text { Magnetic declination, . . . Fluctuation } & =1^{\prime} \cdot 04 . \\ \text { Atmospheric pressure, . . . } & , & =0 \cdot 214 . \\ \text { Atmospheric temperature, . . } \quad, \quad & =3^{\circ} \cdot 07 .\end{array}$

Similarly, if we take the differences of the yearly means corresponding to the successive hours of observation, and combine them in the same way, we obtain the mean two-hourly fluctuations, arising from the regular diurnal change. These numbers are the following :

\section{Mean Fluctuation in two Hours.}

$\begin{array}{lll}\text { Magnetic declination, . . . Fluctuation } & =2^{\prime} \cdot 04 . \\ \text { Atmospheric pressure, . . . } \quad ~ & =0 \cdot 0065 . \\ \text { Atmospheric temperature, . . } \quad, \quad & =1^{\circ} \cdot 46 .\end{array}$

These numbers, compared with the twelfth part of the former, serve to measure the relative magnitude of the regular and irregular changes to which the elements are subject in the same time. We thus find that, in the case of the magnetic declination, the irregular change (which is less than $\frac{1}{20}$ th part of the regular) may be safely neglected; and we have only to attend to the diurnal changes, and to the irregular changes of short period. The daily means are, therefore, to be deduced from one of the combinations of Art. 10, giving the preference to that which contains the greatest number of individual results.

In the case of the atmospheric temperature, the irregular change (which is less than one-fifth part of the regular) is small; and we must attend chiefly to the latter. The mean of the day is, therefore, to be inferred from one of the combinations of Art. 10, giving the preference to those of Art. 11, whoe epoch is noon.

In the case of the atmospherio pressure, on the contrary, the irregular change (which is triple the regular) is the more important. The mean of the day is, therefore, to be deduced from any 
combination whose epoch is noon, giving, however, the preference to one of those of Art. 11, in which the diurnal change is also eliminated.

13. I now proceed to consider the reduction of the monthly means, in the case of deficient observations.

For the purpose of determining the regular diurnal variation of any magnetical or meteorological element, it is necessary to take the mean of an adequate number of separate results corresponding to each hour of observation, so as to eliminate the irregular and accidental changes. The results usually so combined are those of each month. Their number is, in general, sufficient for the purpose above mentioned; while, on the other hand, the course of the diurnal change is sufficiently different from one month to the next, to demand a separate determination.

But in the case of deficient observations, the monthly means of the results corresponding to each hour will not exhibit, in general, the true course of the diurnal change without a correction. If a result be wanting at one hour of a day, in which all the results are much above the mean, it is obvious that the monthly mean corresponding to that hour will be too small, as compared with the means of the other hours; while, on the other hand, it will be too great when all the results of the day in question are below the mean. The error will be greater, the greater the variation of the element observed from day to day. In the case of the atmospheric pressure, it is so considerable, that the uncorrected monthly means afford no approximation to the law of the diurnal change, in the case of deficient observations.

The remedy which first suggests itself, in such a case, is to omit all the results of a day in which one or more are wanting. This process is inartificial and unsatisfactory. The veeight of the mean is diminished in the proportion of the number of observations combined; and it is therefore important to employ all the observed results in its deduction, provided we can obtain a correction. Such a correction is easily found.

14. Let $x$ denote the observed value of any element, at any hour on any day; and let $a$ denote its mean value for that day; then

$$
x=a+\xi,
$$

in which $\xi$ is the magnitude of the diurnal variation corresponding to the hour in question. Let there be $n$ days of observation to be 
combined; then, summing the $n$ results, dividing by $n$, and denoting the mean values by $\underline{x}, \underline{a}$, and $\underline{\xi}$,

$$
\underline{x}=\underline{a}+\underline{\xi} .
$$

Now, at any particular hour of any day, let one of the results be wanting; and let $a^{\prime}$ denote the mean for that day; summingthe $n-1$ results,

$$
S_{n-1} x=S_{n} a-a^{\prime}+S_{n-1} \xi .
$$

And dividing by $n-1$,

$$
\underline{x}=\frac{S_{n} a-a^{\prime}}{n-1}+\underline{\xi}=\underline{a}+\frac{\underline{a}-a^{\prime}}{n-1}+\underline{\xi} ;
$$

whence

$$
\underline{x}+\frac{a^{\prime}-\underline{a}}{n-1}=\underline{a}+\underline{\xi}
$$

The correction, therefore, is $+\frac{a^{\prime}-a}{n-1}$.

Similarly, if $p$ results be wanting, we find

$$
\underline{x}+\frac{S a^{\prime}-p \underline{a}}{n-p}=\underline{a}+\underline{\xi},
$$

in which $S a^{\prime}$ denotes the sum of the means of the days on which the deficiencies occur. Hence, the correction to be applied to the observed mean, $x$, deduced from the $n-p$ values, is $+\frac{S a^{\prime}-p \underline{a}}{n-p}$.

15. The preceding correction depends, as might have been anticipated, on the difference of the daily means, for the days of deficient observations, and the mean daily mean. With the view of determining its probable amount, I have taken the differences between the mean of each day, and the mean of the month, for the declination, the atmospheric pressure, and temperature, as deduced from the observations of the year 1843; and have calculated the square root of the mean of the squares of these differences, or

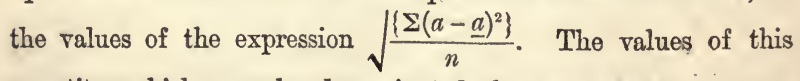
quantity, which may be denominated the mean daily crror', are the following: 


\section{Mean Daily Error.}

$\begin{array}{lrl}\text { Magnetic declination, . . Daily error } & =0^{\prime} \cdot 95 . \\ \text { Atmospheric pressure, . . } & " & =0 \cdot 301 . \\ \text { Atmospheric temperature, . } & " & =4^{\circ} \cdot 25 .\end{array}$

Now, the mean value of $n$ in each month (Sundays being omitted) is 26 . Hence the mean correction, in the case of a single deficient observation, is, for the magnetic declination, $0^{\prime} \cdot 04$; for the atmospheric pressure, 0.012 ; and for the temperature, $0^{\circ} \cdot 17$. In the case of the two meteorological elements, and especially in that of the atmospheric pressure, the correction is too considerable to be overlooked; in the case of the magnetio declination, and probably also in that of the other magnetic elements, it may be disregarded. 
X.-ON THE DETERMINATION OF THE HORIZONTAL INTENSITY OF THE EARTH'S MAGNETIC FORCE IN ABSOLUTE MEASURE.

\section{Transactions of the Royal Irish Academy. Vol. XXI.}

THE attention of mathematicians and experimentalists has been, for some time past, directed to the means of determining the intensity of the earth's magnetic force in absolute measure. These means consist, it is well known, in observing the time of vibration of a freely-suspended horizontal magnet, under the influence of the earth alone, and then employing the same magnet. to act upon another, which is also freely suspended, and noting the effects of its action combined with that of the earth. From the former of these observations we deduce the product of the horizontal component of the earth's magnetic force into the moment of free magnetism of the first magnet, and from the latter, the ratio of the same quantities; and, the product and the ratio being thus known, the two factors are absolutely determined. The former part of this process involving no difficulty which may not be overcome by due care in observing, we shall confine our attention, in the present communication, to the latter.

Two methods have been proposed for this second observation, one by Poisson, and the other by Gauss. The method of Poisson consisted in observing the time of vibration of the second magnet, under the combined action of the first and of the earth, the acting magnet having its axis in the magnetic meridian passing through the centre of the suspended magnet. In the method of Gauss, which is now universally adopted, we observe the position of equilibrium of the second magnet, resulting from the action of the same forces. The acting magnet being placed transversely with 
respect to the suspended one, the latter is deflected from the meridian, and the amount of this deflection serves to determine the ratio of the deflecting force to the earth's force. The position chosen by Gauss for the deflecting magnet is that in which its axis is in the right line passing through the centre of the suspended magnet, and perpendicular to the magnetic meridian, in which case the tangent of the angle of deflection is equal to the ratio of the two forces. From this ratio it remains to deduce that of the magnetic moment of the deflecting bar to the earth's force.

The difficulty of this process arises from the form of the expression of the force of the deflecting bar. This force being expressed by a series descending according to the negative odd powers of the distance, with unknown coefficients, it is erident that observation must furnish as many equations of condition, corresponding to different distances, as there are terms of sensible magnitude in the series; and from these equations the unknown quantities are to be deduced by elimination. Now, the greater the number of unknown quantities thus eliminated, the greater will be the influence of the errors of observation on the final result; and if, on the other hand, the distance between the magnets be taken so great, that all the terms of the series after the first may be insensible, the angle of deflection becomes very small, and the errors in its observed value bear a large proportion to the whole.

It-fortunately happens, that at moderate distances (distances not less than four times the length of the magnets) all the terms beyond the second may be neglected. The expression for the tangent of the angle of deflection is thus reduced to two terms, one of which contains the inverse cube of the distance, and the other the inverse fifth power; that is, if $u$ denote the angle of deflection, and $D$ the distance,

$$
\tan u=\frac{Q}{D^{3}}+\frac{Q^{\prime}}{D^{3}} ;
$$

in which $Q$ and $Q^{\prime}$ are unknown coefficients, the former of which is double of the ratio sought. Accordingly, the method recommended by Gauss consists in observing the angles of deflection, $u$ and $u^{\prime}$, at two different distances, $D$ and $D^{\prime}$, and inferring the coefficient $Q$ by elimination between the two resulting equations of condition. 
The object of the present Paper is to point out the means by which the quantity sought may be obtained, without elimination, from the results of observation at one distance only; and thus not only the labour of observation be diminished, but (which is of more importance) the accuracy of the result increased. Before entering on this, however, it will be expedient to ascertain the amount of the probable error in the received method.

The coefficient of the first term, obtained by elimination between the two equations of condition above alluded to, is

$$
Q=\frac{D^{\prime 5} \tan u^{\prime}-D^{5} \tan u}{D^{\prime 2}-D^{2}} .
$$

The distances being greater than four times the length of the magnets, the angles of deflection are small, and there is, approximately, $\tan u=u \tan 1^{\prime}, \tan u^{\prime}=u^{\prime} \tan 1^{\prime}, u$ and $u^{\prime}$ being expressed in minutes; and making $D^{\prime}=q D$, the preceding expression becomes

$$
Q=D^{3} \tan 1^{\prime} \frac{q^{5} u^{\prime}-u}{q^{2}-1}
$$

The probable errors of $u$ and $u^{\prime}$ are equal; and, by a well-known theorem of the calculus of probabilities, the probable error of $Q$ is

$$
\Delta Q=D^{3} \tan 1^{\prime} \frac{\sqrt{q^{10}+1}}{q^{2}-1} \Delta u .
$$

In determining the ratio of this error to the quantity itself, we may observe that there is, approximately, $q^{3} u^{\prime}=u$, and

$$
Q=D^{3} \tan 1^{\prime} . u ;
$$

and dividing the formula last found by this,

$$
\frac{\Delta Q}{Q}=\frac{\sqrt{q^{10}+1}}{q^{2}-1} \frac{\Delta u}{u} .
$$

It appears, from the preceding theorems, that the value of $\Delta Q$, corresponding to a given value of $\Delta u$, varies with the assumed ratio of the distances, $q$; and that, in order to apply the method most advantageously, this ratio must be taken in such a manner that the probable error, $\Delta Q$, shall be the smallest possible. This condition gives

$$
\frac{d}{d q}\left(\frac{\sqrt{q^{10}+1}}{q^{2}-1}\right)=0
$$


whence we obtain the following equation for the determination of $q$ :

$$
3 q^{10}-5 q^{8}-2=0 .
$$

In order to solve this equation, we may observe that, $q$ being greater than unity, the last term of the equation may, in a first approximation, be neglected in comparison with the others; so that we have, approximately,

$$
3 q^{2}-5=0, \quad q=\sqrt{\frac{5}{3}}=1 \cdot 3 .
$$

And setting out from this value, we find, by any of the known methods of approximation,

$$
q=1 \cdot 32 ;
$$

or $1 \frac{1}{3}$, very nearly. Accordingly, the smaller distance, $r$, being determined by the condition that the third term of the series shall be insensible, the greater distance should be $1.32 r$.

If we substitute this value of $q$, in the expression for $\frac{\Delta Q}{Q}$ above obtained, we find

$$
\frac{\Delta Q}{Q}=5 \cdot 563 \frac{\Delta u}{u} ;
$$

from which we can calculate the least probable error corresponding to any given angle of deflection, the probable error of reading being known.*

Now let us suppose the term containing the fifth power of the distance to vanish, in the expression for the deflecting force. The value of $Q$ will then be reduced to

$$
Q=D^{3} \tan u
$$

* In the Dublin Magnetical Observatory the deflecting bar hitherto employed is 12 inches in length, and the least deflecting distance therefore 4 feet. The deflection produced by it at this distanee is about $3^{\wedge} 56^{\prime}$; and the probable error of observation does not exceed $5^{\prime \prime}$. Hence, in this case,

$$
\frac{\Delta Q}{Q}=\frac{5 \cdot 563}{236 \times 12}=\cdot 0020 \text {. }
$$

The absolute intensity, $X$, varics inversely as the square root of $Q$; so that

$$
\frac{\Delta X}{X}=\frac{1}{2} \frac{\Delta Q}{Q} \text {. }
$$

Consequently, the resulting probable error in the determination of the absolute intensity, made according to the usual method, is, at this Observatory, about the rovoth part of the entire quantity. 
in which we may $\operatorname{take} \tan u=u \tan 1^{\prime}$, as before. Hence there is

$$
\frac{\Delta Q}{Q}=\frac{\Delta u}{u}
$$

and the probable error is less than in the usual method in the ratio of 1 to 5.563 , even when the latter is employed in the manner most conducive to accuracy. Accordingly, if by any means the coefficient of the inverse fifth power of the distance can be annihilated, or rendered so small that the term shall have no sensible influence, the accuracy of the results will be increased. more than five-fold, and, at the same time, the observations being taken at one distance only, the labour of observation will be halved.

The same advantages will be gained if, the coefficient of the inverse fifth power of the distance retaining a sensible value, the ratio of the two coefficients may be known à priori. Let

$$
Q^{\prime}=h Q,
$$

$h$ being a known quantity. In this case the expression for the tangent of the angle of deflection becomes

$$
\tan u=\frac{Q}{D^{3}}\left(1+\frac{h}{D^{2}}\right) ;
$$

and the coefficient sought is obtained, from the result of observation at a single distance, by the formula

$$
Q=\frac{D^{3} \tan u}{1+h D^{-2}} \text {. }
$$

It is evident that the probable error of $Q$ thus obtained, arising from an error in the observed deflection, is the same as in the case last considered, and therefore between five and six times less than in the ordinary method.

The object of the following investigation is to point out the means of attaining these advantages.

Let the axis of the deflecting magnet be supposed to lie in the right line joining the centres of the two magnets, and let the axis of the suspended magnet make the angle $\psi$ with that line. Then, if $X$ and $Y$ denote the forces exerted by the deflecting magnet upon any element of free magnetism, $m$, of the suspended magnet, in the direction of the line connecting it with the centre of the 
deflecting magnet, and in the line perpendicular to it, respectively, their moment to turn the magnet round its point of suspension will be

$$
r\{X \sin (\phi+\psi)+Y \cos (\phi+\psi)\} ;
$$

$r$ denoting the distance of the particle $m$ of the suspended magnet from its centre, and $\phi$ the angle which the line connecting this particle and the centre of the deflecting magnet makes with the axis of the latter. Now, I have elsewhere shown* that, if we include the terms involving the fifth power of the distance, the values of $X$ and $Y$ are

$$
\begin{aligned}
& X=\frac{2 m}{a^{3}} \cos \phi\left\{M+\frac{M I_{3}}{a^{2}}\left(5 \cos ^{2} \phi-3\right)\right\}, \\
& Y=\frac{m}{a^{3}} \sin \phi\left\{M+\frac{3}{2} \frac{M M_{3}}{a^{2}}\left(5 \cos ^{2} \phi-1\right)\right\} ;
\end{aligned}
$$

$a$ being the distance of the particle $m$ of the suspended magnet from the centre of the deflecting magnet, and $M$ and $M_{3}$ denoting, respectively, the integrals corresponding to $\int m r d r, \int m r^{3} d r$, for the deflecting magnet, taken between the limits $r= \pm \frac{1}{2}$ length. Now

$$
\sin \phi=\frac{r}{a} \sin \psi ;
$$

so that, extending the approximation to the term involving the fifth power of the distance only, we must make $\sin \phi=0, \cos \phi=1$, in the coefficient of that term. The preceding expressions are thus reduced to

$$
X=\frac{2 m}{a^{3}}\left(M \cos \phi+\frac{2 M \Gamma_{3}}{a^{2}}\right), \quad Y=\frac{m}{a^{3}} M \sin \phi ;
$$

and, substituting, the moment of these forces to turn the magnet is

$$
\frac{m r}{a^{3}}\left\{M\left(3 \sin \phi \cos \phi \cos \psi+\left(2-3 \sin ^{2} \phi\right) \sin \psi\right)+\frac{4 M r_{3}}{a^{2}} \sin \psi\right\} ;
$$

or, eliminating $\phi$ by the relation between it and $\psi$,

$$
\frac{m r}{a^{3}} \sin \psi\left\{M\left(2+3 \frac{r}{a} \cos \psi-3 \frac{r^{2}}{a^{2}} \sin ^{2} \psi\right)+\frac{4 M_{3}}{a^{2}}\right\} .
$$


Now, if $D$ denote the distance between the centres of the two magnets,

$$
a^{2}=D^{2}+r^{2}-2 D r \cos \psi .
$$

Wherefore, developing the inverse powers of $a$ in series ascending according to the powers of $\frac{r}{D}$, stopping at the inverse fifth power of the distance, and substituting in the expression for the moment above given, it becomes

$$
\frac{m r}{D^{3}} \sin \psi\left\{M\left(2+9 \cos \psi \frac{r}{D}+6\left(5 \cos ^{2} \psi-1\right) \frac{r^{2}}{D^{2}}\right)+\frac{4 M_{3}}{D^{2}}\right\}
$$

This being the moment of the force exerted by the deflecting magnet upon a single particle, $m$, of the suspended magnèt, the moment of the force exerted upon the entire magnet is obtained by multiplying by $d r$, and integrating between the limits $r= \pm l$, $l$ being half the length of the suspended bar.* The magnetism being supposed to be distributed symmetrically on either side of the centre of the suspended magnet, and the axis of suspension to pass through that centre, we have

$$
\int_{-l}^{+l} m r^{2} d r=0
$$

Accordingly, denoting the integrals $\int_{-l}^{+l} m r d r, \int_{-l}^{+l} m r^{3} d r$, by $\boldsymbol{I I}^{\prime}$ and $M r_{3}^{\prime}$, the expression for the moment of the whole force is

$$
\frac{2 M M^{\prime}}{D^{3}} \sin \psi\left\{1+\left(2 \frac{M I_{3}}{M}+3\left(5 \cos ^{2} \psi-1\right) \frac{M_{3}^{\prime}}{M^{\prime}}\right) \frac{1}{D^{2}}\right\} \text {. }
$$

When there is equilibrium, this moment must be equal to that of the force exerted by the earth upon the suspended magnet. Let $X$ be now taken to denote the horizontal component of the earth's magnetic force. The moment of the force exerted by that component upon the particle $m$ of the suspended magnet is

$$
m X r \sin u \text {; }
$$

* We have here assumed that the effect of a magnet is the same, with respect to any point at a moderate distance, as if the magnetic elements in each section perpendicular to its axis were all concentred in the axis; or, in other words, that the integration with respect to the other dimensions of the bar introduces no new term into the integral. There is no difficulty in proving that such is the case, the magnetic elements being supposed to be distributed symmetrically with respect to the axis. 
$u$ denoting the deviation of the axis of the magnet from the direction of the force. Multiplying by $d r$, therefore, and integrating, the total moment is

\section{$X I^{\prime} \sin u$.}

Hence the equation of equilibrium is

$$
X \sin u=\frac{2 M I}{D^{3}} \sin \psi\left\{1+\left(2 \frac{M I_{3}}{M}+3\left(5 \cos ^{2} \psi-1\right) \frac{M I_{3}^{\prime}}{M^{\prime}}\right) \frac{1}{D^{2}}\right\} .
$$

There are two cases of this solution which demand our consideration.

In the method of Gauss, the deflecting magnet is perpendicular to the magnetic meridian, and therefore $\psi=90^{\circ}-u$. In this case, then, the preceding equation becomes

$$
X \tan u=\frac{2 M}{D^{3}}\left\{1+\left(2 \frac{M I_{3}}{M I}-3 \frac{M I_{3}^{\prime}}{M I^{\prime}}+15 \sin ^{2} u \frac{M \Gamma_{3}^{\prime}}{M^{\prime}}\right) \frac{1}{D^{2}}\right\} .
$$

Accordingly, the term containing the fifth power of the distance is composed of two parts, one of which is constant, while the other varies with the angle of deflection; so that, if there were no means of determining a priori the values of the ratios $\frac{M I_{3}}{M I}, \frac{M I_{3}^{\prime}}{M I^{\prime}}$, three equations of condition would be, in strictness, required for the determination of the three unknown quantities; -namely, the coefficient of the inverse cube of the distance, and the two parts of the coefficient of the inverse fifth power. However, the distance being greater than four times the length of the magnet, the angle of deflection, $u$, is always small, and the term involving the square of its sine may be neglected in comparison with the others. Accordingly, if we make, for abridgment,

$$
\frac{2 M I}{X}=Q, \quad 2 \frac{M I_{3}}{M I}-3 \frac{M I_{3}^{\prime}}{\boldsymbol{I}^{\prime}}=h,
$$

the expression for the tangent of the angle of deflection is reduced to the form

$$
\tan u=\frac{Q}{D^{3}}\left(1+\frac{h}{D^{2}}\right) .
$$

In the method of deflection employed by Professor Lamont, the deflecting bar is always perpendicular to the suspended bar. 
In this case, therefore, $\psi=90^{\circ}$, and the equation of equilibrium is reduced to

$$
X \sin u=\frac{2 M}{D^{3}}\left\{1+\left(2 \frac{M_{3}}{M}-3 \frac{M I_{3}^{\prime}}{M^{\prime}}\right) \frac{1}{D^{2}}\right\},
$$

and the equation is the same as that to which it is reduced in the former case, the sine of the angle of deflection being substituted for the tangent. It appears from the result, that this method is to be preferred to the former, not only because the angle of deflection is greater, cat. par., but also because the variable part in the coefficient of the inverse fifth power of the distance is strictly evanescent.

It remains now to inquire in what manner the quantity $h$, which expresses the ratio of the two coefficients, may be known à priori; and whether that quantity can be made to vanish, by any simple relation between the acting magnets.

For this purpose we must know, at least approximately, the law of magnetic distribution, or the function of $r$ by which $m$ is represented. Almost the only knowledge which we possess on this subject is that derived from the researches of Coulomb. From these researches M. Biot has inferred that the quantity of free magnetism, in each point of a bar magnetized by the method of double touch, may be represented by the formula

$$
m=A\left(\mu^{l-r}-\mu^{l+r}\right) ;
$$

$\mu$ being a quantity independent of the length of the magnet, and $A$ a function of $\mu$ and $l$. M. Biot has further shown, that when the length of the magnet is small, the relation between $m$ and $r$ is approximately expressed by the simple formula

$$
m=m^{\prime} \frac{r}{l}
$$

the curve of intensities becoming, in that case, very nearly a right line passing through the centre of the magnet.

Employing, then, this approximate formula, we have

$$
\begin{aligned}
& M=\int_{-l}^{+l} m r d r=\frac{m^{\prime}}{l} \int_{-l}^{+l} r^{2} d r=\frac{2}{3} m^{\prime} l^{2} ; \\
& \boldsymbol{M}_{3}=\int_{-l}^{+l} m r^{3} d r=\frac{m^{\prime}}{l} \int_{-l}^{+l} r^{4} d r=\frac{2}{5} m^{\prime} l^{4} .
\end{aligned}
$$


The ratio of these quantities is independent of $m^{\prime}$, and we have simply

$$
\frac{M I_{3}}{M}=\frac{3}{5} l^{2}
$$

Finally, substituting in the value of $h$ above given, and designating the half lengths of the deflecting and the suspended magnets by $l$ and $l^{\prime}$, respectively,

$$
h=\frac{3}{5}\left(2 l^{2}-3 l^{\prime 2}\right) ;
$$

a quantity whose value may be exactly known, independently of experiment. This quantity vanishes, when $l^{2}=\frac{3}{2} l^{2}$, or

$$
l=1 \cdot 224 l^{\prime} \text {; }
$$

a result which is independent of the magnetic state of the bars.*

As the preceding results depend, in part, upon an empirical law of magnetic distribution, which is only approximately true in the case of small magnets, it seemed desirable to obtain a confirmation of their accuracy by direct experiment. The nature of such confirmation will be immediately understood from the form of the relation between the angle of deflection and the distance. For since, in the method of deflection employed by Gauss, $D^{3} \tan u$ $=Q\left(1+h D^{-2}\right)$, the function $D^{3} \tan u$ will be constant for all values of $D$, when $h=0$; while, if the coefficient of the fifth power of the distance has a sensible value, it will vary with $D$, its values forming a decreasing or increasing series, as $D$ increases, according as $h$ is positive or negative. Hence we have only to observe the deflections produced at different distances, when the two magnets have the relative lengths pointed out above, and to compare the results with those obtained under other circumstances.

Several series of experiments were accordingly made in the beginning of the present month, in some of which the lengths of the two magnets were the same, while in others they were in the

*If the centre of the deflecting magnet be in the magnetic meridian passing through the centre of the suspended magnet, and its axis perpendicular to the same line, we find, by a process similar to that above given, that the condition to be fulfilled, in order that the term involving the fifth power of the distance may vanish, is

$$
\frac{M M_{3}}{M}=4 \frac{M_{3}^{\prime}}{M \Gamma^{\prime}}
$$

and, accordingly, that the corresponding relation between the lengths of the two magnets is, in that case,

$$
l=2 l \text {. }
$$


deduced ratio of the number 1:224 to 1 . The form of the magnets was cylindrical, their lengths being 3 inches, and $3 \frac{2}{3}$ inches, and their diameter $\frac{3}{10}$ of inch. The suspended bar was hung by two fibres of untwisted silk, and inclosed in a small wooden box with glazed front. The deflections were observed by means of a mirror attached below the magnet, which reflected the divisions of a scale placed at a distance of nearly six feet from it. As the utmost precaution was required in the experiments, the use of a copper ring or metallio box was dispensed with, and the arc of vibration reduced by means of a magnet, which was always replaced carefully in the same position after use. The deflecting magnet was placed on the east and west sides of the suspended one, at distances varying from 15 to 30 inches. The distances were observed by the help of a standard scale, a line at the middle of the magnet being made to coincide with the image of the division of the scale, reflected from its polished side. The observations were made beginning with the longest, and proceeding to the shortest distance at one side, and back again in the reverse order at the other, so that. the two observations at the same distance were taken at times equally remote from the middle epoch.

The angles of deflection were calculated by the formula

$$
\tan 2 u=\frac{1}{2}\left(n_{e}-n_{w}\right) k ;
$$

where $n_{e}$ and $n_{v}$ denote the observed readings of the scale, with the marked end of the deflecting bar to the east and to the west respectively. The value of the constant $k$ is given by the formula

$$
k=\frac{a}{d}\left(1+\frac{H}{F}\right) ;
$$

$a$ denoting the length of one division of the scale, $d$ its distance from the mirror, and $\frac{H}{F}$ the ratio of the torsion force to the magnetio force. In the present instance, $a=\cdot 038935$ of an inch; $d=68.52$ inches; and $\frac{H}{F}=\cdot 000345$. Hence $\log k=6.75467$; and the angle corresponding to one division of the scale was consequently

$$
\frac{1}{8} \tan ^{-1} k=58^{\prime \prime} \cdot 623 \text {. }
$$

The following Tables exhibit the results of the observations. The first column of each contains the distances of the magnets, in 
feet; the second, the values of $\frac{1}{2}\left(n_{e}-n_{w}\right)$, these values being the means of those obtained with the deflecting magnet on the east and on the west side of the suspended magnet; the third column contains the calculated values of $u$; and the fourth those of $D^{3} \tan u$. In the observations of I., II., and III., the lengths of the deflecting and suspended magnets were in the ratio of the numbers 1.224 to 1 ; in those of IV. and V., the lengths of the two magnets were equal.

I.-Magnet away, Scale reading $=496.5 \ldots 494 \cdot 5$.

\begin{tabular}{|c|c|c|c|}
\hline$D$ & $\frac{1}{2}\left(n_{e}-n_{w}\right)$ & \multicolumn{1}{|c|}{$u$} & $D^{3} \tan u$ \\
\hline $1 \cdot 5$ & $351 \cdot 05$ & $5^{\circ} 38^{\prime} 32^{\prime \prime} \cdot 5$ & $\cdot 33344$ \\
$2 \cdot 0$ & $146 \cdot 92$ & 22313 & $\cdot 33347$ \\
$2 \cdot 5$ & $75 \cdot 12$ & 11321 & $\cdot 33343$ \\
\hline
\end{tabular}

II.-Magnet away, Scale reading $=495 \cdot 9 \ldots 495 \cdot 0$.

\begin{tabular}{|c|c|c|c|}
\hline$D$ & $\frac{1}{2}\left(n_{e}-n_{w}\right)$ & $u$ & $D^{3} \tan u$ \\
\hline $1 \cdot 5$ & $350 \cdot 61$ & $55^{\circ} 38^{\prime} \quad 7^{\prime \prime} \cdot 5$ & $\cdot 33302$ \\
\hline $2 \cdot 0$ & $146 \cdot 71$ & $\begin{array}{lll}2 & 23 & 1\end{array}$ & $\cdot 33300$ \\
\hline $2 \cdot 5$ & 75.06 & $11317 \cdot 5$ & $\cdot 33317$ \\
\hline
\end{tabular}

III.-Magnet away, Scale reading $=496 \cdot 4 \ldots 494 \cdot 3$.

\begin{tabular}{|c|c|c|c|}
\hline$D$ & $\frac{1}{2}\left(n_{e}-n_{w}\right)$ & $u$ & $D^{3} \tan u^{\circ}$ \\
\hline 1.4167 & 417.24 & $6^{\circ} 40^{\prime} 16^{\prime \prime}$ & $\cdot 33254$ \\
1.8750 & $177 \cdot 89$ & 25313 & $\cdot 33242$ \\
2.5000 & 74.96 & 11312 & $\cdot 33275$ \\
\hline
\end{tabular}

IV.-Magnet away, Scale reading $=494 \cdot 4 \ldots 494 \cdot 6$.

\begin{tabular}{|c|c|c|c|}
\hline$D$ & $\frac{1}{2}\left(n_{e}-n_{w}\right)$ & w6 & $D^{3} \tan u$ \\
\hline $1 \cdot 25$ & $389 \cdot 70$ & $6^{\circ} 14^{\prime} 42^{\prime \prime} \cdot 5$ & $\cdot 21373$ \\
\hline 1.50 & $223 \cdot 95$ & $\begin{array}{lll}3 & 37 & 38\end{array}$ & $\cdot 21393$ \\
\hline $2 \cdot 00$ & $94 \cdot 38$ & $\begin{array}{llll}1 & 32 & 7 & 5\end{array}$ & $\cdot 21444$ \\
\hline $2 \cdot 50$ & $48 \cdot 45$ & $\begin{array}{lllll}0 & 47 & 19 & \cdot 5\end{array}$ & $\cdot 21510$ \\
\hline
\end{tabular}


V.-Magnet away, Scale reading $=495 \cdot 7 \ldots 498 \cdot 3$.

\begin{tabular}{|c|c|c|c|}
\hline$D$ & $\frac{1}{2}\left(n_{e}-n_{v}\right)$ & $u$ & $D^{3} \tan x$ \\
\hline $1 \cdot 25$ & $368 \cdot 46$ & $5^{\circ} 54^{\prime}, 52^{\prime \prime} \cdot 5$ & $\cdot 20233$ \\
\hline 1.50 & $211 \cdot 99$ & $\begin{array}{lll}3 & 26 & 8\end{array}$ & $\cdot 20261$ \\
\hline 1.75 & $133 \cdot 41$ & $\begin{array}{lll}2 & 10 & 6\end{array}$ & $\cdot 20292$ \\
\hline $2 \cdot 00$ & $89 \cdot 39$ & 12716 & $\cdot 20313$ \\
\hline $2 \cdot 2 j$ & $63 \cdot 00$ & $\begin{array}{lll}1 & 1 & 32\end{array}$ & $\cdot 20390$ \\
\hline $2 \cdot 50$ & $45 \cdot 99$ & $\begin{array}{llll}0 & 44 & 5 & 5\end{array}$ & $\cdot 20420$ \\
\hline
\end{tabular}

These results verify the conclusions to which we have arrived above. The values of the function $D^{3} \tan u$ are constant for all distances in the first three series, the differences in the resulting values being less than the probable errors of observation; and, consequently, the coefficient of the inverse fifth power of the distance is insensible. In the fourth and fifth series, on the other hand, in which the lengths of the magnets are equal, the values of this function form an increasing series, as $D$ increases; and therefore, in this case, the coefficient of the inverse fifth power of the distance has a sensible negative value.

We may further employ these results to test the accuracy of our conclusions, by deducing from them the values of the two coefficients, in the expression for the tangent of the angle of deflection, and comparing their ratio with that furnished by theory. It is needless to make this computation for the numbers of the first three series; for it is manifest from the results, that the second coefficient is insensible, as theoretically it should be. From the results of IV. we deduce, by the method of least squares,

$$
Q=\cdot 2148, \quad h Q=-\cdot 0017, \quad h=-\cdot 0078 .
$$

We obtain, in like manner, from the results of $\mathrm{V}$.,

$$
Q=\cdot 2037, \quad h Q=-\cdot 0022, \quad h=-\cdot 0110 .
$$

And the mean of the resulting values is -.0094 . Now, in these two series, the length of each of the magnets was three inches; that is, $l=l^{\prime}=\cdot 125$, the half lengths being expressed in feet. Substituting these values in the expression for $h$, it becomes

$$
h=-\cdot 0094,
$$

agreeing exactly with the mean of the experimental values." 
We are therefore justified in concluding that, in the case of small magnets, the ratio of the two coefficients may be inferred it priori, by the formula

$$
h=\frac{3}{5}\left(2 l^{2}-3 l^{\prime 2}\right) ;
$$

and, consequently, that the coefficient $Q$, or $2 \frac{I I}{X}$, may be obtained, from the result of observation at a single distance, by the formula

$$
Q=\frac{D^{3} \tan u}{1+h D^{-2}} \text {. }
$$

It follows from this, as we have seen, that when $l^{\prime}=1 \cdot 224 l, l=0$, and the value of $Q$ is reduced to

$$
Q=D^{3} \tan u \text {. }
$$

Postscript. - While the preceding pages were passing through the press, I received a memoir from Professor Lamont, on the determination of the earth's magnetic force in absolute measure, in which the author has proposed various modifications in the existing method, and has considered, with great minuteness of detail, the many corrections which are required in the immediate results of observation. Some of the conclusions of the present Paper are, I find, thus anticipated;-in particular, the form of the equation of equilibrium of the suspended magnet, for the case in which the axes of the two magnets are at right angles; and the ratio of the coefficients of the terms in that formula, which Professor Lamont has deduced in the case of what he calls a simple magnet,-that is, the imaginary magnet in which the attractive and repulsive forces are supposed to emanate solely from two points or poles. The value, so inferred, he naturally regarded as a mere approximation.; and he has accordingly not thought of employing it, as is proposed in the present Paper, to supersede experiment, and thus evade the errors resulting from the process of elimination. 
XI.-ON THE DETERMINATION OF THE TOTAL INTENSITY OF THE EARTH'S MAGNETIC FORCE IN ABSOLUTE MEASURE, BY MEANS OF THE DIP-CIRCLE.

Transactions of the Royal Irish Academy. Vol. XXIII.

THE force exerted by the earth upon a magnet is usually found by suspending the bar horizontally, and observing its time of vibration. The result thence obtained is the product of the horizontal component of the earth's magnetic force by the magnetic moment of the magnet; and before we can know the value of either of the factors which compose it, observation must furnish another result in which they are combined differently. This is effected, in the method of Gauss, by using the same. magnet to deflect another, similarly suspended, and by observing the angles of deflection at known distances: this observation gives the ratio of the magnetic moment of the deflecting magnet to the horizontal component of the earth's force, and the two factors are therefore absolutely known.

This method, although much improved by the labours of Lamont and others, has one insurmountable imperfection. The total force must be inferred from its horizontal component, by multiplying by the secant of the inclination. The relative error of the deduced force, arising from a given error of inclination, varies therefore as the tangent of that angle, and when the inclination approaches to $90^{\circ}$, it becomes very considerable. The method is, accordingly, unsuited to the high magnetic latitudes.

I was induced to consider the means of supplying this defect some years ago, upon the occasion of the Aretic Expeditions of 1845 and 1848; and I then suggested another process, by which 
the total intensity might be found directly, without the intervention of its horizontal component. In the Paper in which it was explained," it was shown that the ordinary dip-circle may be employed in both parts of the observation. Subsequent considerations, however, derived from the probable errors of observation, led me to propose that the dip-circle should be employed only in one part of this process, and that the observation should be completed by the known methods.

The present communication is intended to show in what manner this complication may be avoided, and the original proposal carried out. It is of great importance to the scientific traveller that the instruments which he has to carry should be reduced, as far as possible, in number and in weight, and that their adjustments should be few and simple; and it is believed that these objects are attainable by the method explained in this Paper. Before entering into details, it will be convenient to revert to the theoretical principles on which the method is founded.

If $X$ and $Y$ denote the horizontal and vertical components of the Earth's magnetic force, $m$ the magnetic moment of the needle acted on, and a the azimuth of the plane in which it moves, measured from the magnetic meridian, the effective forces exerted upon it are

$$
m X \cos a, \quad m Y \text {; }
$$

and their moment to turn the needle is

$$
m(Y \cos \eta-X \cos a \sin \eta),
$$

$\eta$ denoting the actual inclination of the needle to the horizon. This moment is opposed by that of the added weight, or by $W r$, $W$ being the weight, and $r$ the radius of the pulley by which it acts $; \dagger$ and the equation of equilibrium is therefore

$$
m(Y \cos \eta-X \cos a \sin \eta)=u r .
$$

When the needle is removed, in the second part of the process,

* Proccedings of the Royal Irish Academy, January 24, 1848.

+ It is here supposed that the weight is attached to a fine thread passing round a light pulley, whose centre is on the axis of the cylindrical axle of the needle, in the manner proposed by Mr. Fox. If the weight be attached to the southern arm of the needle, at a fixed point, its moment is $\operatorname{cor} \cos \eta$. 
and applied to deflect another substituted in its place, the moment of its force to turn the latter is

$$
m m^{\prime} U \text {; }
$$

in which $m^{\prime}$ is the moment of free magnetism of the second needle, and $U$ a function of the distance of the centres of the two needles, and of certain integrals depending on the distribution of free magnetism in them. The moment of the earth's magnetic force, opposed to this, is of the form already assigned, in which we have only to substitute $m^{\prime}, \eta^{\prime}$, and $a^{\prime}$, for $m, \eta$, and $a$. Hence the equation of equilibrium is

$$
Y \cos \eta^{\prime}-X \cos a^{\prime} \sin \eta^{\prime}=m U ;
$$

the quantity $m^{\prime}$ disappearing from the result. The magnetic moment of the deflecting needle, $m$, is eliminated from equations. (1) and (2) by multiplication; and we thus obtain a single relation between the intensity of the earth's magnetic force, the observed angles $a, \eta, a^{\prime}, \eta^{\prime}$, and the quantities $v, r$, and $U$. Hence the magnetic intensity will be determined when these are known.

There are three obvious cases of these formulas, each of which suggests a different method for the determination of the terrestrial magnetic intensity.

1. When the planes in which the needles move coincide with the magnetic meridian, or $a=0$, and $a^{\prime}=0$, the left-hand members of (1) and (2) are reduced to $m R \sin (\theta-\eta), R \sin \left(\theta-\eta^{\prime}\right) ; R$ denoting the total force, and $\theta$ the inclination. Wherefore, by multiplication, we have

$$
R^{2} \sin (\theta-\eta) \sin \left(\theta-\eta^{\prime}\right)=U(r) .
$$

2. When the planes in which the needles move are perpendicular to the magnetic meridian, or $a=90^{\circ}$, and $a^{\prime}=90^{\circ}$, the left-hand members of (1) and (2) become, respectively, $m I \cos \eta$, $Y \cos \eta^{\prime} ;$ whence

$$
Y^{2} \cos \eta \cos \eta^{\prime}=C_{\imath \imath)^{\prime}}
$$

3. Finally, the equilibrium may be produced, in both cases, by turning the instrument in azimuth until the free needle stands vertically. In this case $\eta=90^{\circ}, \eta^{\prime}=90^{\circ}$, and the left-hand members become $-m X \cos a,-X \cos a^{\prime}$; whence

$$
X^{2} \cos a \cos a^{\prime}=U i r r .
$$

Thus we may apply this principle to the determination of the total intensity, or to that of either of its two components. 
In comparing the foregoing methods, it is to be observed that the third fails when the inclination approaches to $90^{\circ}$, on account of the magnitude of the error of $R$ resulting from a given error of $\theta$, when the total force is deduced from its horizontal component. In like manner, and for the same reason, the second method fails in the vicinity of the magnetic equator, or line of no inclination. 'The first alone is applicable at all parts of the earth's surface, and I proceed to consider it more in detail.

The observed angles, $\eta$ and $\eta^{\prime}$, are liable to error, the friction of the needles on their supports causing them to rest in positions slightly different from those due to the acting forces. The probable errors of $\eta$ and $\eta^{\prime}$, due to this cause, vary with the angles themselves. To determine their magnitude in any case, we hare

$$
m R \sin (\theta-\eta)=F,
$$

$F$ being the moment of the deflecting force; and when friction is taken into account,

$$
m R \sin (\theta-\eta+\Delta \eta)=F+f
$$

$f$ denoting the moment of friction, and $\eta-\Delta \eta$ the new angle of equilibrium. Developing the latter equation, and subtracting the former,

$$
m R \cos (\theta-\eta) \Delta \eta=f ;
$$

the angle $\Delta \eta$ being expressed in parts of radius. Hence, $\cos (\theta-\eta) \Delta \eta$ is constant with a given instrument, and at a given point of the Earth's surface.

To find the probable error of the force corresponding to the error of the observed angle, we must differentiate the equation of equilibrium, $m R \sin u=F$, with respect to $R$ and $u$, where $\imath=\theta-\eta$; and we have

But

$$
\Delta R \sin u+R \cos u \Delta u=0 .
$$

$$
u=\frac{1}{9}\left(\eta_{1}-\eta_{2}\right)
$$

$\eta_{1}$ and $\eta_{2}$ being the observed angles of inclination under the two opposite actions of the deflecting force. Hence the probable crror of $u$ is

$$
\Delta u=\frac{1}{2} \sqrt{\Delta \eta^{2}{ }_{1}+\Delta \eta^{2}{ }_{2}}=\frac{1}{\sqrt{2}} \Delta \eta ;
$$


since $\Delta \eta_{2}=\Delta \eta_{1}$. Accordingly, the second term of the preceding equation becomes $\frac{1}{\sqrt{ } 2} R \cos u \Delta \eta=\frac{1}{\sqrt{ } 2} \frac{f}{m}$; and we have

$$
\Delta R=\frac{-f}{m \sqrt{ } 2 \sin u} .
$$

We learn, then, that the probable error of the force varies inversely as the sine of the angle of deflection; and that it is therefore requisite for accuracy that this angle should be considerable. There is no difficulty in augmenting the angle of deflection as much as we please in the first part of the process, in which the magnet is deflected by a weight; but in the second the case is different, and with the slender needles to be employed as deflectors, a large deflection can only be obtained by placing the deflecting needle at a very short distance from the moveable one. The most convenient arrangement appears to be to attach the deflecting needle to the moveable arm of the divided circle which carries the verniers, and at right angles to the wires of the microscopes.* So attached, it must always be rendered perpendicular to the deflected needle in the course of the observation, although in a different plane.

The form of the function denoted by $U$, in this position, is easily found.

Let the distances of any points of the axes of the deflecting and deflected magnets from their respective centres be denoted by $r$ and $r^{\prime}$, and let $\mu$ and $\mu^{\prime}$ denote the quantities of free magnetism at these points, contained in the slices perpendicular

- To obtain the value of $\Delta R$ by observation, we must substitute for $f$ its value given above. But when $\eta=\theta$, or when the ncedle is undeflected, $f=m R \Delta \theta$; wherefore

$$
\Delta R=\frac{-R \Delta \theta}{\sqrt{2 \sin u}}
$$

In the instrument with which I made trial of this method, the length of the needles was $3 \frac{1}{2}$ inches, and the angle of deflection produced, in the position of the deflecting needle here described, was $24^{\circ} 10^{\prime}$. But the probable error of a single reading of the inclination, obtained by repetition-the needle being lifted off the agate planes between the successive readings-was $1^{\prime} \cdot 6$; and if four readings (which is a very usual number) be taken, the probable error of their mean will be one-half of this. In this case, therefore, $\Delta \theta=0^{\prime} \cdot 8$; and the probable error of the deduced force, computed by the preceding formula, is $\Delta R=.0004 R$. 
to the axes whose thicknesses are $d r^{\circ}$ and $d r^{\prime}$. Then the force exerted by the former upon the latter is

$$
\frac{\mu \mu^{\prime} d r d r^{\prime}}{z^{2}},
$$

$\approx$ denoting their mutual distance. The portion of this force contained in the plane of the deflected magnet, and perpendicular to its axis, is

$$
\frac{\mu \mu^{\prime} d r d r^{\prime}}{z^{2}} \times \frac{r^{\prime}}{z}
$$

and the moment of this force to turn the magnet is obtained by multiplying by $r^{\prime}$. But

$$
z^{2}=D^{2}+r^{2}+r^{\prime 2},
$$

$D$ being the distances of the centres of the two magnets; and accordingly the total moment of the acting forces is

$$
\iint \frac{\mu r d r \cdot \mu^{\prime} r^{\prime} d r^{\prime}}{\left(D^{2}+r^{2}+r^{\prime 2}\right)^{\frac{3}{2}}}
$$

Expanding the denominator, and making, for abridgment,

$$
\begin{aligned}
& \int \mu r d r=m_{1}, \quad \int \mu r^{3} d r=m_{3}, \quad \int \mu r^{5} d r^{r}=m_{5}, \quad \text { Sc., } \\
& \int \mu^{\prime} r^{\prime} d r^{\prime}=m_{1}^{\prime}, \quad \int \mu^{\prime} r^{\prime 3} d r^{\prime}=m_{3}^{\prime}, \quad \int \mu^{\prime} r^{\prime 2} d r^{\prime}=m_{5}^{\prime}, \quad \text { \&.e, }
\end{aligned}
$$

in which the integrals are to be taken between the limits $r= \pm l$, $r^{\prime}= \pm l^{\prime}, l$ and $l^{\prime}$ being half the lengths of the two magnets, this becomes

$$
\frac{1}{D^{3}}\left(m m^{\prime}-\frac{3}{2}\left(m_{3} n \iota^{\prime}+m m_{3}^{\prime}\right) \frac{1}{D^{2}}+\frac{3 \cdot 5}{2 \cdot 4}\left(m m_{5} m \iota^{\prime}+2 m_{3} m_{3}^{\prime}+m m_{5}^{\prime}\right) \frac{1}{D^{4}}+\& c .\right\},
$$

or $m m^{\prime} U$, in which

$$
U=\frac{1}{D^{3}}\left(1-\frac{3}{2}\left(\frac{m_{3}}{m}+\frac{m_{3}^{\prime}}{m^{\prime}}\right) \frac{1}{D^{2}}+\frac{15}{8}\left(\frac{m_{5}}{m}+2 \frac{m_{3}}{m} \frac{m_{3}^{\prime}}{m^{\prime}}+\frac{m_{3}^{\prime}}{m^{\prime}}\right) \frac{1}{D^{4}}+\mathcal{E} .\right\}
$$

Now it is to be observed, that the rariations of the ratios $\frac{m_{3}}{m}, \frac{m_{5}}{m}$, \&c., arising from the variations of $\mu$, are of a lower order of magnitude than that of $m$, and may be disregarded in their effect upon the value of $U .^{*}$ On the supposition that the quantity

* This circumstance was first pointed out by Dr. Lamont. 
of free magnetism, at any point of a magnet, is proportional to the distance from the centre, or that $\mu=k r$, we have

$$
m=\frac{2}{3} k l^{3}, \quad m_{3}=\frac{2}{5} k l^{5}, \quad m_{5}=\frac{2}{7} k l^{7},
$$

and when $k$ becomes $k+\delta k$, these values will all be altered proportionally, and consequently the ratios $\frac{m_{3}}{m}, \frac{m_{5}}{m}$, \&c., will be absolutely unchanged; and the same thing is manifestly true, if the quantity of free magnetism be supposed to vary as any simple power of the distance, whether integer or fractional.

This is a point of considerable importance in reference to the method now proposed. For it follows that, at a given distance between the two needles, the function $U$ may be regarded as constant; and therefore that, even when $U$ is unknown, the value of $R$ will be relatively determined, by a process which is independent of the changes induced by time in the magnetic moments of the needles employed. Accordingly, if the value of the force be found at any one place, by any independent means, it will be absolutely known at all ; and it is only necessary that the observer should include in his series an observation at some base-station, at which the absolute value of the force is determined simultaneously by the ordinary method.

I now proceed to show, however, that the value of the constant $U$ may be found by deflection, by the instrument itself, and without any subsidiary apparatus; and that the method may therefore be rendered rigorously absolute. It is obvious that the ordinary process is inapplicable in this case, owing to the large number of terms which acquire a sensible value, in the value of the function $U$, and the consequent difficulty and uncertainty of the elimination : moreover, the position which has been adopted for the deflecting needle will not admit of the required alteration of distance.

Now here I premise, that it is not necessary that the usual deflection distance should be one of the series employed in deducing the coefficients of the inverse powers of the distance in the value of $U$ : it is not even requisite that the relative positions of the two magnets should be the same in the two cases. For if the value of the corresponding function be found, for any other position, and at any distance, that of $U$ will be known by a comparison of the deflections produced. Accordingly, I"propose 
to determine, in the first place, the value of the corresponding function in a different relative position of the two magnets, and by means of deflections at the usual distances; and thence to conclude that of $U$ in the position of the magnets here employed.

In using the dip-circle for this purpose, it will be found most convenient to adopt the third of the methods above described, in which the equilibrium is produced by turning the instrument in azimuth until the deflected magnet becomes vertical; for in this case the deflecting magnet is always horizontal, and can be placed in the usual position with respect to the deflected magnet without difficulty. For this purpose the apparatus is provided with a gunmetal bar, the middle of which is broad, and has a rectangular aperture which enables it to pass over the box containing the deflected magnet: this bar rests horizontally on two supports fixed outside the box, on the level of the agate planes. The deflecting magnet is to be placed on this support at different known distances, and on each side of the deflected magnet, its axis being in the plane in which the latter moves; and the apparatus is to be turned in azimuth until the deflected needle is rertical. In this case equation (2) becomes

$$
-X \cos a=m V
$$

in which $V$ is of the form

$$
V=\frac{1}{D^{3}}\left(1+\frac{p}{D^{2}}+\frac{q}{D^{4}}+\& c \cdot,\right.
$$

where

$$
p=2 \frac{m_{3}}{m}-3 \frac{m_{3}^{\prime}}{m^{\prime}} \quad q=3 \frac{m_{5}}{m}-15 \frac{m_{3}}{m} \frac{m_{3}^{\prime}}{m^{\prime}}+\frac{45}{8} \frac{m_{5}^{\prime}}{m^{\prime}} .
$$

Let $V_{1}, V_{2}, V_{3}$, \&c., denote the values of $V$ corresponding to the distances $D_{1}, D_{2}, D_{3}$, \&c. ; and $a_{1}, a_{3}, a_{3}$, Ec., the corresponding azimuths observed; then it is obvious that

$$
V_{2} \cos \alpha_{1}-V_{1} \cos \alpha_{2}=0, \quad V_{3} \cos \alpha_{1}-V_{1} \cos a_{3}=0, \quad \text { E. },
$$

from which equations the values of the coefficients $p, q$, \&c., are obtained by elimination in the usual manner. Hence $V$ is completely determined.

Now let the deflecting magnet be removed from the horizontal bar, and placed in its ordinary position between the microscopes; and let the observation be repeated, the instrument being turned 
in azimuth until the deflected needle is vertical. Then, if $\boldsymbol{a}_{0}$ denote the corresponding azimuth, we have

$$
-X \cos a_{0}=m U
$$

whence there is

$$
U=V \frac{\cos a_{0}}{\cos \alpha}
$$

Thus the uncertainty of the result, arising from the smallness of the angle of deflection at the usual distances, is removed from the regular series of observations, and is thrown upon the determination of the constant, which may be made at leisure, whenever convenient, and may be repeated as often as is required for accuracy.

In speaking of $U$ as constant, and independent of the changes of the magnetic moments of the needles, I have expressly limited the statement to those small and regular losses of magnetism which occur in time. It would not be safe to extend the assumption to the case of the larger changes brought about abruptly by concussion, or by any other accidental cause; and still less to those in which the magnetic distribution of the needles is altered by contact with, or proximity to, magnetic bodies. In such cases (the occurrence of which is easily detected), the value of the constant $U$ should be re-determined.

It may be useful to add a few words respecting the order of the observations.

The apparatus should be furnished with three needles, all of the same size, viz., $3 \frac{1}{2}$ inches in length. One of these (which we shall denote by the letter $\mathrm{A}$ ) is to be employed in observations of inclination: the other two, B and C, are to be used in the observations of intensity,- $\mathrm{B}$ being the loaded needle, which is also used as a deflector, and $\mathrm{C}$ the deflected needle. The two latter needles should not have their poles reversed, nor be touched with a magnet. order :-

The observations may be conveniently taken in the following

1. Needle A is to be placed on the agate planes, the other two needles being removed, and a complete observation of inclination taken in the four usual positions of the needle and limb, and with the poles of the needle direct and inverted. 
2. Needle A is then to be removed, and the loaded needle, B, substituted; and its inclination to the horizon, $\eta$, is to be observed in the four positions of the needle and limb. The deviation of this needle from the position due to the earth's magnetic force alone is $u=\theta-\eta$, the angle $\eta$ being positive when measured at the same side of the horizontal line with $\theta$, and negative in the contrary case.

3. Needle B is now to be removed from the agate planes to its supports between the microscopes, and needle $\mathrm{C}$ substituted; and the inclination of the latter to the horizon is then to be observed in one position of the needle and limb. The observation is to be repeated with the north end of needle B turned in the opposite direction, by the revolution of the moveable arms which carry the microscopes; half the difference of the readings in the two positions is the angle of deflection, $u^{\prime}$.

The total intensity is given by the formula

$$
R=\sqrt{\frac{U u r}{\sin u \sin u^{\prime}}}
$$

which is fitted, without any artifice, for logarithmic computation.*

In strictness a correction is required for the effect of the change of temperature of needle $B$, in the two observations in which it is employed; but as one of these observations may be made to follow the other quiekly, and as the needle may be placed in both in nearly the same circumstances, the correction may generally be disregarded. It will be necessary, however, that this needle, when employed as a deflector, should be protected from the heat of the observer's body by a small case of glass or of metal.

The method here proposed appears to offer the following advantages to the travelling observer:globe.

1. It is applicable, with equal accuracy, at all parts of the

2. It dispenses with the employment of a separate instrument

* If the weight be attached to the loaded needlo at a fixed point, the formula becomes

$$
R=\sqrt{\frac{U w r \cdot \cos \eta}{\sin u \sin u^{\circ}}} .
$$


for the determination of the magnetic intensity, and with the separate adjustments required in erecting it.

3. The constants to be determined-the magnitude of the added weight, and the radius of the pulley by which it acts, - can be ascertained with more ease and certainty than those which are required in the method of vibrations, and are less liable to subsequent change.

4. The observations themselves are less varied in character than the usual ones, and may be completed in a shorter time. 
XII.-ON EARTH CURRENTS, AND THEIR CONNEXION WITH THE DIURNAL CHANGES OF THE HORIZONTAL MAGNETIC NEEDLE.

Transactions of the Royal Irish Academy. Vol. XXIV.

1. Whex the discovery of Oersted had made known the connexion which subsists between magnetism and current electricity, the idea occurred to many that the magnetism of the earth-or, at least, its diurnal fluctuation,- -was the result of electric currents traversing its crust. This idea gained much force from the fact, soon after discovered by Seebeck, that electric currents are generated when heat is applied to a circuit composed of different metals; and it was supposed that the phenomena were thus traceable to the thermal agency of the sun, operating in succession upon the conducting substances of which the earth's crust is composed.

The most explicit statement, and chief support of this hypothesis, is contained in a memoir by Professor Christie, published in the Philosophical Transactions for 1827. In this memoir it is maintained that the phenomena of the diurnal variation of the horizonal magnetic needle correspond with those which are produced experimentally, by the application of heat to a globe composed of two metals; and the author was even led by his experiments to anticipate the fact, at that time unknown, that at opposite sides of the equator the poles of the needle, having the same name as the latitude, are always deflected in the same direction.

I propose, in the present memoir, to examine this theory apart from the hypothesis as to the origin of the currents; and to show, 
that the diurnal variation of the horizontal magnetic needle is due to electric currents traversing the earth's crust.

2. The first and most important step towards the result above referred to has been made by Mr. Barlow. In a Paper* communicated by him to the Royal Society in the year 1848, he established the important fact, that a wire, whose extremities are connected with the earth at two distant points, is unceasingly traversed by electric currents, the intensity of which varies with the azimuth of the line joining the points of contact with the ground. The direction of these currents was proved to be the same at both extremities of the same wire, and was shown to depend on the relative positions of the Earth-connexions, while it was wholly independent of the course followed by the wire itself. The currents cease altogether when either of the contacts with the earth is interrupted. From these facts Mr. Barlow concluded that "the currents are terrestrial, of which a portion is conveyed along the wire, and rendered visible by the multiplying action of the coil of the galvanometer."

Mr. Barlow further observed that, apart from sudden and occasional changes, the general direction of the needle of the galvanometer appeared to exhibit some regularity. He was thus led to institute a series of observations for fourteen days and nights simultaneously on two telegraphic wires, one connecting Derby and Rugby, and the other connecting Derby and Birmingham, the positions of the needles in both circuits being recorded every five minutes, day and night. From these observations he concluded-

"1. That the path described by the needle consisted of a regular diurnal motion, subject to disturbances of greater or less magnitude.

" 2 . That this motion is due to electric currents passing from the northern to the southern extremities of the telegraph wires, and returning in the opposite direction.

" 3 . That, exclusive of the irregular disturbances, the currents flowed in a southerly direction from about 8 or 9 A.M. until the evening, and in a northerly direction during the remainder of the twenty-four hours."

* "On the spontaneons Electrical Currents observed in the wires of the Electric Telegraph."-Phil. Trans., 1848. 
Mr. Barlow was thus led to examine whether any relation subsisted between these movements and the daily changes of the horizontal magnetic needle. And having made, for this purpose, a series of simultaneous observations with a delicate declinometer, he came to the conclusion that, although generally the currents flow southwards during that part of the day in which the variation of the horizontal needle is vesterly, and northwards when the variation is easterly, "yet simultaneous observations showed no similarity in the paths described by the magnetio needle and the galvanometer."

An examination of the galvanometric observations above referred to led me, some time since, to an opposite conclusion; and at the last meeting of the British Association I stated my conviction, founded on these observations, that the earth-currents, whose continuous flow Mr. Barlow has the merit of establishing, would eventually explain the variations of terrestrial magnetism, both periodic and irregular. I now proceed to state some of the grounds of this conviction; and in the present paper I hope to show that the diurnal changes of the earth-currents correspond with those of the horizontal component of the earth's magnetic force.

4. Before proceeding to the detailed examination of this correspondence, it is necessary to advert to a difference between the things compared, which will account for the method employed in the comparison. The cause of this difference will be adverted to hereafter.

When we examine the curves, ${ }^{*}$ in which Mr. Barlow has represented the course of the galvanometrio deflections caused by the earth-currents, we observe that the regularity of that course is continually interrupted by rapid reciprocating movements, in which the needle oscillates from one side to the other of the zero, alternately. These movements are very different in magnitude at different times: thus, May 29 was a day of considerable movement; May 25, one of comparative rest. These movements are similar to those of the magnetometers, with which we are familiar; but they are much more rapid, and bear a larger proportion to the regular changes. Among the days of observation whose results are given in this diagram, it fortunately happens that one,-viz., the day commencing May 28, 10 P. M. (Göttingen time), was a "term-day" of the magnetie system, during which the magnetometers were observed every five minutes at the Greenwich Obser-

- Philosophical Transnctions, 1818, Diagram, No. 1. 
vatory, and consequently at the same intervals of time as the needles of the galvanometers in Mr. Barlow's observations. I find, upon an examination of the two records, that there were on that day, on the average, $5 \cdot 1$ alternations of the galvanometer needles in the hour, the corresponding number of alternations of the magnetometers being only $3 \cdot 1$. The numbers are very nearly the same for the two galvanometers of the two lines of telegraph, as well as for the two magnetometers of the Greenwich Observatory. And the disproportion in the magnitude of the deflections, as compared with the amount of the regular daily changes, appears to be even more considerable.

The frequency and the magnitude of the deflections may both be taken into account, by adding together the alternate changes, without regard to sign, and dividing the sum by the regular daily changes. I have selected for this calculation the observations made during the six hours commencing at 3 A. M. on May 29, that being a period of comparative disturbance. The sum of the changes of the galvanometer needle during that period, on the Derby and Rugby line, was equivalent to 571 divisions of the instrument, the mean daily range for the entire week being 11.4 divisions: and the ratio $=50$. The corresponding ratio, for the galvanometer of the Derby and Birmingham line, is somewhat smaller. The sum of the changes of the Greenwich declinometer during the same period was only 57 minutes, the mean daily range being 12.4 minutes. In like manner, the sum of the changes of the horizontal force (in parts of the whole) was $\cdot 0158$, the mean daily range being $\cdot 0034$. The ratio is accordingly the same for the two magnetic elements, and its amount is $4 \cdot 6$, or less than one-tenth of the corresponding ratio in the case of the galvanometric changes. We learn therefore that the rapid changes of the earth-currents are much greater, in proportion to the regular daily changes, than the corresponding movements of the magnetometers.

The physical interpretation of the foregoing facts will be considered in the sequel. For the present, I shall merely notice the consequence which appears to flow immediately from them-namely, that little or no accordance is to be expected, in comparing the individual results of the two classes of measures. I have verified this conclusion by a comparison of Mr. Barlow's diagram, for the period above referred to, with the corresponding Greenwich observations. 
5. For the foregoing reasons, the magnetometric observations are here compared, not with the single corresponding deflections of the galvanometer needles, but with the means of several. The observations instituted by $\mathrm{Mr}$. Barlow on the two lines of telegraph were taken at intervals of five minutes, and were continued for fourteen consecutive days, commeneing May 17, 1847. In the Tables given in his Paper, the individual results are grouped into means corresponding to the beginning of each hour; so that each number of the Table is the result of twelve distinct readings. The means so obtained are, notwithstanding, more affected by irregularities than the single readings of the magnetometers corresponding to the same epochs; and I have found it advisable to group the results still further, by taking the mean of each pair of succeeding hourly means. The following Tables, accordingly, contain the means of the results belonging to the hours, 1 A.M. and 2 A.M., 3 A.M. and 4 A.M., \&.c.; they therefore correspond to the epochs, $1^{\mathrm{h}} 30^{\mathrm{m}}, 3^{\mathrm{h}} 30^{\mathrm{m}}$, \&c., each number being the mean of twenty-four single readings. The positice numbers indicate currents proceeding towards Derby; and the negative, currents in the contrary direction. It is only necessary to observe further, that of the fourteen days of observation, four, viz., May 17, 23, 24,30 , are very incomplete, and are therefore omitted altogether in this place:-

\section{TABLe I.}

Intensity of the Currents traversing the Wire connecting Derby and Rugby.

\begin{tabular}{|c|c|c|c|c|c|c|}
\hline \multirow{2}{*}{ MAY. } & \multicolumn{6}{|c|}{ A. M. } \\
\hline & $1^{\mathrm{h}} 30^{\mathrm{m}}$ & $3^{\mathrm{h}} 30^{\mathrm{m}}$ & $5^{\mathrm{h}} 30^{\mathrm{m}}$ & $7^{\mathrm{h}} 30^{\mathrm{m}}$ & $9^{\mathrm{h}} 30^{\mathrm{m}}$ & $11^{\mathrm{h}} 30^{\mathrm{m}}$ \\
\hline $\begin{array}{l}18 \\
19 \\
20 \\
21 \\
22 \\
25 \\
26 \\
27 \\
28 \\
29\end{array}$ & $\begin{array}{l}+1.9 \\
+4.6 \\
+0.6 \\
+3.2 \\
-1.3 \\
+1.8 \\
-0.2 \\
-2.1 \\
-2.9\end{array}$ & $\begin{array}{l}-3.5 \\
+1.4 \\
+0.3 \\
+2.5 \\
+3.6 \\
+5.0 \\
+0.6 \\
+1.4 \\
+0.1\end{array}$ & $\begin{array}{l}-1.7 \\
-0.8 \\
+1 \cdot 2 \\
+1.0 \\
+5.5 \\
+3.1 \\
+5.0 \\
+2.4 \\
-0.4\end{array}$ & $\begin{array}{r}+1.8 \\
+\quad 4.5 \\
+\quad 0.2 \\
+\quad 2.8 \\
+\quad 1.8 \\
+\quad 2.2 \\
+\quad 4.7 \\
+10.3 \\
+\quad 2.8\end{array}$ & $\begin{array}{l}+4.9 \\
-0.5 \\
-1.5 \\
+1.1 \\
-2.3 \\
-0.6 \\
-1.7 \\
+1.9 \\
+5.9 \\
-2.1\end{array}$ & $\begin{array}{l}-1.4 \\
-\quad 3.8 \\
-\quad 2.6 \\
-\quad 2.4 \\
-\quad 5.2 \\
-\quad 3.2 \\
-4.4 \\
-2.5 \\
-4.1 \\
-12.2\end{array}$ \\
\hline Means & +0.9 & $+1 \cdot 3$ & $+1 \cdot 7$ & $+3 \cdot \dot{s}$ & +0.5 & $-4 \cdot 2$ \\
\hline
\end{tabular}


Tarie I.-continued.

\begin{tabular}{|c|c|c|c|c|c|c|}
\hline \multirow{2}{*}{ MAY. } & \multicolumn{6}{|c|}{ P. M. } \\
\hline & $1^{\mathrm{h}} 30^{\mathrm{m}}$ & $3^{\mathrm{h}} 30^{\mathrm{m}}$ & $5^{\mathrm{h}} 30^{\mathrm{m}}$ & $7^{\mathrm{h}} 30^{\mathrm{m}}$ & $9^{\mathrm{h}} 30^{\mathrm{m}}$ & $11^{\mathrm{h}} 30^{\mathrm{m}}$ \\
\hline 18 & $-7 \cdot 5$ & $-8 \cdot 3$ & $-8 \cdot 8$ & $-1 \cdot 5$ & $+3 \cdot 5$ & $+6 \cdot 1$ \\
\hline 19 & -0.1 & -0.4 & ․ & ․ & ․ & $\because:$ \\
\hline 20 & $-2 \cdot 7$ & $-1 \cdot 9$ & $-2 \cdot 1$ & $-8 \cdot 0$ & $+1 \cdot 3$ & $+5 \cdot \bar{j}$ \\
\hline 21 & $-2 \cdot 2$ & $-2 \cdot 4$ & $-2 \cdot 0$ & $-5 \cdot 2$ & $-3 \cdot 4$ & $+2 \cdot 6$ \\
\hline 22 & -1.8 & $-1 \cdot 2$ & $-1 \cdot 7$ & $-2 \cdot 2$ & $-2 \cdot 3$ & $-1 \cdot 1$ \\
\hline 25 & -2.7 & +0.8 & +0.6 & 0.0 & $-1 \cdot 0$ & $+1 \cdot 4$ \\
\hline 26 & $-\quad 3 \cdot 9$ & +0.7 & $+4 \cdot 4$ & $+3 \cdot 1$ & $+1 \cdot 8$ & -0.1 \\
\hline 27 & $-4 \cdot 3$ & -0.5 & $+0 \cdot 6$ & $-4 \cdot 7$ & +6.5 & $+4 \cdot 7$ \\
\hline 28 & $-7 \cdot 8$ & -0.9 & $-2 \cdot 9$ & $+4 \cdot 2$ & $+3 \cdot 5$ & $+1 \cdot 9$ \\
\hline 29 & $-10 \cdot 6$ & $-6 \cdot 6$ & -6.9 & $-1 \cdot 0$ & $-0 \cdot \tilde{\delta}$ & $-3 \cdot 5$ \\
\hline Means & $-4 \cdot 4$ & $-2 \cdot 1$ & $-2 \cdot 1$ & $-1 \cdot 7$ & $+1 \cdot 0$ & +1.9 \\
\hline
\end{tabular}

Table II.

Intensity of the Currents traversing the Wire connecting Derby and Birmingham.

\begin{tabular}{|c|c|c|c|c|c|c|}
\hline \multirow{2}{*}{ MAY. } & \multicolumn{6}{|c|}{ A. M. } \\
\hline & $1^{\mathrm{h}} 30^{\mathrm{m}}$ & $3^{\mathrm{h}} 30^{\mathrm{m}}$ & $5^{\mathrm{h}} 30^{\mathrm{m}}$ & $7^{\mathrm{h}} 30^{\mathrm{m}}$ & $9^{\mathrm{h}} 30^{\mathrm{m}}$ & $11^{\mathrm{h}} 30^{\mathrm{m}}$ \\
\hline $\begin{array}{l}18 \\
19 \\
20 \\
21 \\
22 \\
25 \\
26 \\
27 \\
28 \\
29\end{array}$ & $\begin{array}{l}+0.3 \\
+3.6 \\
+8.7 \\
+2.1 \\
+9.7 \\
+1.0 \\
+1.8 \\
-0.8 \\
-1.7 \\
-2.4\end{array}$ & $\begin{array}{l}-9 \cdot 7 \\
+6 \cdot 0 \\
-2 \cdot 6 \\
+1 \cdot 7 \\
+8 \cdot 5 \\
+2 \cdot 4 \\
+3 \cdot 4 . \\
+0.3 \\
+0.4 \\
-1 \cdot 1\end{array}$ & $\begin{array}{l}-5 \cdot 7 \\
-7 \cdot 0 \\
-1 \cdot 1 \\
+5 \cdot 0 \\
+5 \cdot 2 \\
+3 \cdot 0 \\
+1 \cdot 2 \\
+2 \cdot 3 \\
+1 \cdot 4 \\
+1 \cdot 4\end{array}$ & $\begin{array}{l}+5 \cdot 7 \\
+9 \cdot 7 \\
+7 \cdot 2 \\
+1.5 \\
+9 \cdot 9 \\
+0.4 \\
+0.8 \\
+2.4 \\
+8.4 \\
+7.8\end{array}$ & $\begin{array}{l}+6.6 \\
-0.6 \\
+1.1 \\
+7.6 \\
-6.0 \\
-0.6 \\
-1.6 \\
+0.5 \\
+5.1 \\
+1.4\end{array}$ & $\begin{array}{r}-10.0 \\
-10.3 \\
-\quad 8.5 \\
-\quad 9.0 \\
-17.5 \\
-\quad 3.1 \\
-\quad 4.7 \\
-\quad 2.5 \\
-\quad 3.7 \\
-\quad 5.7\end{array}$ \\
\hline Means & $+2 \cdot 2$ & +0.9 & $+0 \cdot 6$ & +5.4 & $+1 \cdot 4$ & $-7 \cdot 5$ \\
\hline \multirow{2}{*}{ MAY. } & \multicolumn{6}{|c|}{ P. Mr. } \\
\hline & $1^{\mathrm{b}} 30^{\mathrm{m}}$ & $3^{\mathrm{h}} 30^{\mathrm{m}}$ & $5^{\mathrm{h}} 30^{\mathrm{m}}$ & $7^{\mathrm{h}} 30^{\mathrm{m}}$ & $9^{\mathrm{h}} 30^{\mathrm{m}}$ & $11^{\mathrm{h}} 30^{\mathrm{m}}$ \\
\hline $\begin{array}{l}18 \\
19 \\
20 \\
21 \\
22 \\
25 \\
26 \\
27 \\
28 \\
29\end{array}$ & $\begin{array}{r}-17 \cdot 4 \\
-\quad 9 \cdot 2 \\
-11 \cdot 6 \\
-11 \cdot 3 \\
-\quad 9 \cdot 3 \\
-\quad 3 \cdot 2 \\
-\quad 5 \cdot 5 \\
-\quad 5 \cdot 0 \\
-\quad 8 \cdot 0 \\
-\quad 4 \cdot 2\end{array}$ & $\begin{array}{r}-21 \cdot 1 \\
-10.8 \\
-\quad 9.9 \\
-12.8 \\
-\quad 7 \cdot 3 \\
-\quad 0.6 \\
-\quad 3 \cdot 6 \\
-1 \cdot 8 \\
-2.9 \\
-\quad 2.6\end{array}$ & $\begin{array}{l}-10 \cdot 6 \\
-18.2 \\
-\quad 6.1 \\
-\quad 7 \cdot 0 \\
-8 \cdot 9 \\
-1 \cdot 1 \\
+\quad 2 \cdot 3 \\
-\quad 2 \cdot 1 \\
-6.1 \\
-\quad 3.0\end{array}$ & $\begin{array}{r}-4.2 \\
-13.0 \\
-10.0 \\
-16.7 \\
-\quad 9.3 \\
-\quad 1.5 \\
+\quad 0.3 \\
-7.7 \\
+\quad 1.4 \\
+\quad 2.5\end{array}$ & $\begin{array}{l}+3 \cdot 7 \\
+2 \cdot 4 \\
+2.9 \\
-5 \cdot 2 \\
-8 \cdot 6 \\
-1 \cdot 7 \\
+0.1 \\
+4 \cdot 4 \\
+0 \cdot 7 \\
+2 \cdot 2\end{array}$ & $\begin{array}{r}+5.6 \\
+\quad 9.0 \\
+11.6 \\
+\quad 6.4 \\
+\quad 2.2 \\
+\quad 1.0 \\
-\quad 2.1 \\
+\quad 4.2 \\
+\quad 1.6 \\
+\quad 0.8\end{array}$ \\
\hline Means & $-8 \cdot 5$ & $-7 \cdot 3$ & $-6 \cdot 1$ & $-5 \cdot 8$ & +0.1 & $+3 \cdot 6$ \\
\hline
\end{tabular}


The means corresponding to each epoch are given in the lowest lines of the preceding Tables. On examining them, it will be seen that the law of the diurnal changes in the force and direction of the currents is very systematic. In both lines the current flows southucards from $11^{\mathrm{h}} 30^{\mathrm{m}}$ A.M. to $7^{\mathrm{h}} 30^{\mathrm{m}}$ P.M. inclusive; and northwards at the remaining epochs. The maximum of the southerly current occurs at $1^{\mathrm{h}} 30^{\mathrm{m}}$ P.M., and that of the northerly rurrent at $7^{\mathrm{h}} 30^{\mathrm{m}}$ A.M.

6. Let us now compare these results with those deducible from the diurnal changes of the magnetic declination and horizontal force, on the assumption that the forces which produce the latter are due to electric currents traversing the upper strata of the earth in a horizontal direction.

Let $\eta$ denote the disturbing force by which the north pole of the magnet is urged to the eastuard of its mean position; $\xi$, that by which it is impelled northward. Then, on the assumption above referred to, the force of the current in the magnetio meridian, flowing northward, $=a \eta, a$ being an unknown constant; and that of the current perpendicular to the magnetic meridian, flowing castward, $=-a \xi$. Hence, the force of the current in any direction, making the angle $\varepsilon$ with the magnetic meridian measured to the east of north, is

$$
f=a(\eta \cos \varepsilon-\xi \sin \varepsilon) .
$$

The quantities $\eta$ and $\xi$ are given, in terms of the horizontal component of the earth's magnetic force, by the readings of the two horizontal magnetometers. For it is evident that

$$
\eta=X \sin \left(\psi-\psi_{0}\right)=X \delta \psi \sin 1^{\prime},
$$

$X$ being the horizontal component of the earth's magnetio force, and $\psi$ the magnetic declination; and

$$
\xi=X-X_{0}=\delta X .
$$

7. Now, to reduce the preceding formula into numbers, we have $\varepsilon=a-\psi$, in which $a$ is the azimuth of the line connecting the two stations, measured from the true meridian castward. The observations of Sir James Ross, at Derby, give

$$
\psi=-22^{\circ} 25^{\prime} ;
$$


and for the line connecting Derby with Rugby,

$$
a=-13^{\circ} \gamma^{\prime}, \quad \varepsilon=+9^{\circ} 18^{\prime} .
$$

Introducing these values in the expression for $f$,

$$
f=a X\left(\cdot 000287 \delta \psi-0 \cdot 16 \frac{\delta X}{X}\right)
$$

The following Table gives the values of the quantity within the brackets in this formula, for the days on which the force of the currents was observed, and for the even hours of Göttingen mean time, the unit being $\frac{1}{10000}$. The values of $\delta \psi$, and $\frac{\delta X}{X}$ are obtained from the Greenwich observations.*

* In my first calculation of the Intensity of the Currents, communicated to the Academy on the 11th Norember, I employed the mean values of $\delta \psi$ and $\frac{\delta X}{X}$ for the month of May, as deduced from observations made at the Dublin Magnetical Observatory in the years 1840-1843. My reason for this was, that in the year 1848, which was stated in Mr. Barlow's Paper to have been the year of his galvanometric observations, the daily observations at the Dublin Observatory were not sufficiently numerous for the required comparison; while the Greenwich published observations were then limited to term-days, and days of unusual disturbance. But I soon after learned from Mr. Barlow himself, that his observations were made in 1847 , and that the date in the heading of his Tables had been misprinted by oversight. And as the Greenwich observations were taken twelve times in the day in 1847, I have recalculated the forces from the simultaneous elements thus furnished. The comparison is, of course, in every way more satisfactory than the former. 


\section{Table III.}

Calculated Values of the Intensity of the Earth-Currents, in the Line connecting Derby and Rugby.

\begin{tabular}{|c|c|c|c|c|c|c|}
\hline \multirow{2}{*}{ MAY. } & \multicolumn{6}{|c|}{ A. M. } \\
\hline & $1^{\mathrm{h}} 20^{\mathrm{m}}$ & $3^{\mathrm{h}} 20^{\mathrm{m}}$ & $5^{\mathrm{h}} 20^{\mathrm{m}}$ & $7^{\mathrm{h}} 20^{\mathrm{m}}$ & $9^{\mathrm{h}} 20^{\mathrm{m}}$ & $11^{\mathrm{h}} 20^{\mathrm{m}}$ \\
\hline $\begin{array}{l}18 \\
19 \\
20 \\
21 \\
22 \\
25 \\
26 \\
27 \\
28 \\
29\end{array}$ & $\begin{array}{l}+13 \cdot 8 \\
-\quad 2.9 \\
+12.6 \\
-\quad 0.8 \\
+\quad 7 \cdot 2 \\
+\quad 4 \cdot 7 \\
+14.7 \\
-\quad 0.1 \\
-\quad 2 \cdot 6 \\
+4.0\end{array}$ & $\begin{array}{l}+10 \cdot 7 \\
+\quad 0 \cdot 5 \\
+9 \cdot 1 \\
+6 \cdot 8 \\
+14 \cdot 1 \\
+\quad 7 \cdot 9 \\
+16 \cdot 5 \\
+\quad 2 \cdot 8 \\
+12 \cdot 0 \\
+\quad 7 \cdot 1\end{array}$ & $\begin{array}{l}+12 \cdot 0 \\
+4 \cdot 3 \\
+21 \cdot 7 \\
+7 \cdot 9 \\
+7 \cdot 8 \\
+19 \cdot 2 \\
+16 \cdot 5 \\
+13 \cdot 7 \\
+9 \cdot 8 \\
+28 \cdot 3\end{array}$ & $\begin{array}{r}+9.4 \\
+9.5 \\
+16.5 \\
+14 \cdot 7 \\
+14.9 \\
+20.8 \\
+17 \cdot 2 \\
+19 \cdot 0 \\
+23 \cdot 1 \\
-\quad 3.8\end{array}$ & $\begin{array}{l}+2 \cdot 6 \\
-\quad 0.3 \\
+\quad 7 \cdot 0 \\
+9 \cdot 5 \\
+11 \cdot 8 \\
+10 \cdot 6 \\
+\quad 0.5 \\
+11 \cdot 3 \\
+\quad 8 \cdot 1 \\
+\quad 2.9\end{array}$ & $\begin{array}{l}-14 \cdot 2 \\
-21 \cdot 6 \\
-17 \cdot 7 \\
-12 \cdot 8 \\
-13 \cdot 1 \\
-5 \cdot 1 \\
-19 \cdot 7 \\
-10 \cdot 9 \\
-7 \cdot 7 \\
-17 \cdot 8\end{array}$ \\
\hline Means & $+5 \cdot 1$ & $+8 \cdot 8$ & $+14 \cdot 1$ & $+14 \cdot 1$ & $+6 \cdot 4$ & $-14 \cdot 1$ \\
\hline \multirow{2}{*}{ MAY. } & \multicolumn{6}{|c|}{ P. M. } \\
\hline & $1^{\mathrm{h}} 20^{\mathrm{m}}$ & $3^{\mathrm{h}} 20^{\mathrm{m}}$ & $5^{\mathrm{h}} 20^{\mathrm{m}}$ & $7^{\mathrm{h}} 20^{\mathrm{m}}$ & $9^{\mathrm{h}} 20^{\mathrm{m}}$ & $11^{\mathrm{h}} 20^{\mathrm{m}}$ \\
\hline $\begin{array}{l}18 \\
19 \\
20 \\
21 \\
22 \\
25 \\
26 \\
27 \\
28 \\
29\end{array}$ & $\begin{array}{l}-27 \cdot 4 \\
-24 \cdot 2 \\
-17 \cdot 7 \\
-19 \cdot 8 \\
-22 \cdot 6 \\
-16 \cdot 2 \\
-29 \cdot 4 \\
-19 \cdot 6 \\
-20 \cdot 1 \\
-23 \cdot 0\end{array}$ & $\begin{array}{r}-20 \cdot 5 \\
-\quad 9 \cdot 0 \\
-\quad 4 \cdot 8 \\
-\quad 8 \cdot 6 \\
-\quad 6 \cdot 0 \\
-\quad 8 \cdot 4 \\
-19 \cdot 3 \\
-9 \cdot 6 \\
-10 \cdot 3 \\
-13 \cdot 2\end{array}$ & $\begin{array}{l}-2.9 \\
-\quad 1.5 \\
-\quad 2.7 \\
+\quad 0.9 \\
-\quad 3.0 \\
-\quad 3.8 \\
-11.0 \\
-\quad 1.2 \\
-\quad 5.0 \\
-8.6\end{array}$ & $\begin{array}{l}-5.9 \\
-3.9 \\
+1.5 \\
+7.9 \\
-1.5 \\
-0.8 \\
-1.9 \\
-5.7 \\
-0.7 \\
-7.1\end{array}$ & $\begin{array}{l}-4.2 \\
-0.3 \\
+1.3 \\
+1.6 \\
+1.2 \\
-0.5 \\
-0.9 \\
-1.9 \\
+2.6 \\
-1.4\end{array}$ & $\begin{array}{l}-3.5 \\
+\quad 5.5 \\
+1.7 \\
+3.8 \\
+2.1 \\
+8.1 \\
+3.6 \\
+12.9 \\
+3.1 \\
+2.7\end{array}$ \\
\hline Means & $-22 \cdot 0$ & $-11 \cdot 0$ & $-3 \cdot 9$ & $-1 \cdot 8$ & $-0 \cdot 3$ & $+4 \cdot 0$ \\
\hline
\end{tabular}

8. For the line connecting Derby and Birmingham, we have

$$
\boldsymbol{a}=+33^{\circ} 27^{\prime}, \quad \varepsilon=+55^{\circ} 52^{\prime} ;
$$

and, introducing these values, the formula becomes

$$
f=a X\left(.000163 \delta \psi-0.83 \frac{\delta X}{X}\right)
$$


The values of the quantity within the brackets, for the same days and hours, are given in the following Table :-

\section{TABLe IV.}

Calculated Values of the Intensity of the Earth-Currents, in the Line connecting Derby and Birmingham.

\begin{tabular}{|c|c|c|c|c|c|c|}
\hline \multirow{2}{*}{ MAY. } & \multicolumn{6}{|c|}{ A. M. } \\
\hline & $1^{\mathrm{h}} 20^{\mathrm{m}}$ & $3^{\mathrm{h}} 20^{\mathrm{m}}$ & $5^{\mathrm{h}} 20^{\mathrm{m}}$ & $7^{\mathrm{h}} 20^{\mathrm{m}}$ & $9^{\mathrm{h}} 20^{\mathrm{m}}$ & $11^{\mathrm{h}} 20^{\mathrm{m}}$ \\
\hline $\begin{array}{l}18 \\
19 \\
20 \\
21 \\
22 \\
25 \\
26 \\
27 \\
28 \\
29\end{array}$ & $\begin{array}{l}+13.8 \\
-\quad 7.5 \\
+11.7 \\
+\quad 1.6 \\
+0.5 \\
+1.3 \\
+3.8 \\
+\quad 2.4 \\
-\quad 9.0 \\
+\quad 3.8\end{array}$ & $\begin{array}{l}+11.1 \\
-\quad 6.3 \\
-\quad 0.3 \\
+4.5 \\
+6.3 \\
+4.2 \\
+10.5 \\
+\quad 1.8 \\
+8.7 \\
+8.2\end{array}$ & $\begin{array}{l}+13.4 \\
-3.4 \\
+12.4 \\
+10.0 \\
+3 \cdot 7 \\
+11 \cdot 8 \\
+10.4 \\
+8 \cdot 1 \\
+3.7 \\
+8.1\end{array}$ & $\begin{array}{l}+25.4 \\
+\quad 8.2 \\
+10 \cdot 4 \\
+18 \cdot 5 \\
+5 \cdot 1 \\
+14.4 \\
+12.9 \\
+17 \cdot 2 \\
+23.5 \\
+25.8\end{array}$ & $\begin{array}{r}+24.2 \\
+17 \cdot 7 \\
+25.2 \\
+24.5 \\
+23 \cdot 4 \\
+12 \cdot 1 \\
+3 \cdot 0 \\
+12 \cdot 7 \\
+27 \cdot 8 \\
+23.5\end{array}$ & $\begin{array}{l}+8 \cdot 9 \\
-\quad 1 \cdot 3 \\
+\quad 2 \cdot 5 \\
+4 \cdot 7 \\
-\quad 3 \cdot 9 \\
+\quad 4 \cdot 2 \\
-\quad 9 \cdot 1 \\
+1 \cdot 3 \\
+16.1 \\
-10.0\end{array}$ \\
\hline Means & $+2 \cdot 2$ & $+4 \cdot 9$ & $+7 \cdot 8$ & $+16 \cdot 1$ & $+19 \cdot 4$ & $+1 \cdot 3$ \\
\hline \multirow{2}{*}{ MAY. } & \multicolumn{6}{|c|}{ P. Ar. } \\
\hline & $1^{\mathrm{h}} 20^{\mathrm{m}}$ & $3^{\mathrm{h}} 20^{\mathrm{m}}$ & $5^{\mathrm{h}} 20^{\mathrm{m}}$ & $7^{\mathrm{h}} 20^{\mathrm{m}}$ & $9^{\mathrm{h}} 20^{\mathrm{m}}$ & $11^{\mathrm{h}} 20^{\mathrm{m}}$ \\
\hline $\begin{array}{l}18 \\
19 \\
20 \\
21 \\
22 \\
25 \\
26 \\
27 \\
28 \\
29\end{array}$ & $\begin{array}{r}-14 \cdot 5 \\
-19 \cdot 0 \\
-7 \cdot 9 \\
-16 \cdot 0 \\
-18 \cdot 7 \\
-12 \cdot 1 \\
-23 \cdot 3 \\
-14 \cdot 8 \\
-6 \cdot 8 \\
-17 \cdot 6\end{array}$ & $\begin{array}{r}-18 \cdot 0 \\
-\quad 7 \cdot 8 \\
-4 \cdot 3 \\
-16 \cdot 3 \\
-11 \cdot 9 \\
-11 \cdot 3 \\
-19 \cdot 7 \\
-9 \cdot 1 \\
-9 \cdot 2 \\
-10 \cdot 8\end{array}$ & $\begin{array}{r}-17 \cdot 1 \\
-\quad 6 \cdot 3 \\
-17 \cdot 6 \\
-\quad 8 \cdot 0 \\
=5 \cdot 3 \\
-11 \cdot 1 \\
-15 \cdot 8 \\
-5 \cdot 8 \\
-12 \cdot 2 \\
-16 \cdot 1\end{array}$ & $\begin{array}{r}-11 \cdot 4 \\
-\quad 8 \cdot 0 \\
-13.9 \\
-\quad 6 \cdot 4 \\
-\quad 3 \cdot 2 \\
-10 \cdot 2 \\
-\quad 8 \cdot 3 \\
-23.9 \\
-\quad 4 \cdot 0 \\
-15.2\end{array}$ & $\begin{array}{r}-10.9 \\
-\quad 2.4 \\
-\quad 4.7 \\
-\quad 2.9 \\
-1.4 \\
-\quad 6.2 \\
+\quad 2.5 \\
-14.4 \\
+\quad 2.5 \\
-1.6\end{array}$ & $\begin{array}{l}-\quad 9 \cdot 3 \\
+\quad 1 \cdot 1 \\
+\quad 5 \cdot 5 \\
-\quad 3 \cdot 3 \\
-\quad 0.5 \\
-\quad 2 \cdot 3 \\
+\quad 2 \cdot 0 \\
+10.6 \\
+\quad 3.7 \\
-\quad 2 \cdot 6\end{array}$ \\
\hline Means & $-15 \cdot 1$ & $-11 \cdot 8$ & $-11 \cdot 5$ & -10.5 & $-4 \cdot 0$ & +0.5 \\
\hline
\end{tabular}

9. The mean results corresponding to the several hours, in these four Tables, are projected in curves in Plate I., figs. 1 and 2. As we are concerned only with relative values in both cases, the ranges of the observed and computed results have been previously 
equalized, by multiplying the former by constant coefficients. The curres of both lines exhibit a general resemblance to the course of the diurnal variation of the declination; but the influence of the horizontal force is also very evident, especially in the afternoon branch of the curve, where its effect is to retard the return from the minimum at 1 P. м. This effect is much greater in the Derby and Birmingham line than in that of Derby and Rugby, the azimuth of the former line, measured from the magnetic meridian, being much greater than that of the latter. The agreement of the calculated with the observed curves is probably as close as could be expected in the results of so short a series; and we seem entitled to conclude that the diurnal movements of the two magnetometers are justly accounted for by electric currents traversing the upper strata of the earth.

10. Upon a closer examination of the two sets of curres, however, there are found some differences to which it is necessary to advert. In the first place, the turning points of the calculated curves are generally later than those of the observed, by about one hour. Thus, in the Derby and Birmingham line, the maximum of the observed force occurs about $8^{\mathrm{h}} 0^{\mathrm{m}}$ A. M., and the minimum about $12^{\mathrm{h}} 40^{\mathrm{m}}$ р. м. The corresponding times for the calculated force are $9^{\mathrm{h}} 0^{\mathrm{m}}$ A. M., and $1^{\mathrm{h}} 40^{\mathrm{m}}$ P. M., nearly. We shall presently find grounds for believing that time may possibly be required, in order that the current may produce its full magnetic effect.

11. Another discrepancy is, that the calculated curve is, for the most part, above the observed, especially in the Derby and Birmingham line. This will be evident if the two curves be referred to the same axis of abscissæ. It is probably to be accounted for by the fact, that the zero from which the magnetic deflections are measured is not the true one, corresponding to the absence of deflecting force. As we have no means of determining the latter, we are accustomed to take the mean position for the entire day, or the mean of the readings taken at equal intervals, as the point from which the deflections are measured. But there is reason to believe that this is not the true position of rest, corresponding to the absence of all disturbing force. The comparative quiescence of the magnets, during the early hours of the morning, seems to indicate that they are then near their true positions of equilibrium; and this indication is confirmed by the galvanometric curves, the zero-line, which corresponds to the absence of all current, 
dividing the area of the diurnal curve unequally, and being nearer to the night observations than to those of the day. From the last line in Tables I. and III., we find that the mean of all the daily observations of the galvanometers is neyative on both lines, or below the true zero. On the other hand, the mean of the night observations is positive, or above the zero; and the same thing is true of that portion of the night (from 1 A. M. to 5 A. M.) during which the magnetic changes are smallest. From these facts it would seem to follow that the true zero of the magnetometric observations lies between the mean of the day and the mean of the hours of magnetic repose. This conclusion, however, it must be remembered, is derived from the observations of a single fortnight only; and, in the absence of fuller knowledge, we should not be justified in changing the origin usually employed.

Thus magnetometric observations furnish merely differential results; and we are ignorant even of the relative values of the effects, and therefore unable to compare them accurately with their physical causes, whether real or supposed. It is true that, if the galvanometric and the magnetometric results were completely identified, the zero of the latter could readily be obtained by their comparison. For if $\eta_{0}$ and $\xi_{0}$ be the values of $\eta$ and $\xi$; corresponding to the true zero, measured from the mean of the day, it is plain that, for the hours at which the current changes sign on any line,

$$
\eta_{0} \cos \varepsilon-\xi_{0} \sin \varepsilon=\eta \cos \varepsilon-\xi \sin \varepsilon .
$$

If therefore these hours be known for two lines, which differ considerably in azimuth, and also the corresponding values of $\boldsymbol{\eta}$ and $\xi$, we should have two equations for the determination of the two unknown quantities, $\eta_{0}$ and $\xi_{0}$. It is obvious, however, that this process cannot be legitimately employed in the comparison and identification itself.

12. In order to see how far the correspondence, which we have found in the mean results, is traceable in separate days, I projected in curves the observed and calculated values of the intensity of the currents on the Derby and Birmingham line, as given in Tables II. and IV., for the five days commencing May 2.5. The accordance of the two curves is very remarkable, every altermation in direction in one of them having its counterpart in the other.

13. It remains to say a few words of the manner in which the 
electric currents may be supposed to operate in producing the magnetic effects.

An electric current, traversing the earth's crust in a horizontal direction, may affect a horizontal magnetic needle above its surface in two ways. For the current may either act directly upon the needle, according to the known laws of electro-magnetio action; or it may induce temporary magnetism in the earth itself, which will thus affect the needle differently from before. I believe, with Dr. Lamont, that the former hypothesis is inadmissible, at least as regards the principal part of the observed effect. In addition to the reason assigned for this by Dr. Lamont, I may adduce the known similarity in the course of the magnetio changes over constderable portions of the earth's surface, a similarity incompatible with the supposition that the magnet is directly acted on, to any great extent, by the subjacent current. We must suppose, therefore, that the earth is acted on, to a considerable depth, by the wave of currents which sweep over its surface, and which alter by induction the magnetism of the subjacent mass, and that the effect produced upon the freely suspended magnet at its surface is the result of this induced change. On this supposition, the magnetio phenomena, whose laws we are considering, are the indirect effects (not of the subjacent current merely, but) of the entire wave traversing an extended portion of the earth's surface. We can thus understand the cause of the similarity in the more rapid magnetic changes to which we have adverted; and we may even frame some idea of the depth acted on by the superficial ourrent, from the geographical limits of the phenomena.

Upon this supposition, also, we are prepared to expect differences in the laws of the observed and computed currents, such as have been above noticed. For the galvanometric measures belong only to currents at the place of observation; while the magnetio changes are, by hypothesis, the mean results of currents occupying a considerable portion of the earth's surface. Hence, also, it follows that the complete identification of the two classes of phenomena can only be made by the help of simultaneous observations of earth-currents at numerous points in an extended district.

14. Before concluding this part of the subject, I must refer briefly to the previous investigations of Dr. Lamont connected with it, so far as they have been yet made public.

In a letter, dated July 29,1861 , which was read by the 
Astronomer Royal at the last meeting of the British Association, Dr. Lamont stated that he had found "that electric currents, or (as they may be more properly termed) electric wares, varying in direction and intensity, are constantly passing at the surface of the earth, and that these waves correspond perfectly with the variations of terrestrial magnetism." The correspondence here referred to seems to relate to the epochs of the two classes of changes, and not to their amount. For in the latest account of Dr. Lamont's researches, of which I am aware, and which is contained in a letter to Professor De la Rive, dated October 30, 1861, the writer states that the galvanometer indicates, not the earth-current itself, but its momentary changes. In fact the needle of the galvanometer, in his observations, appears to have been affected only during a rapid increase or decrease of the earth-current, and to return to zero when the current became equable. It is obvious that, if such were the case generally, there could be no correspondence, such as has been pointed out in the preceding pages, between the magnitude of the magnetic changes and the deflections of the galvanometer. It is stated, in fact, by Dr. Lamont, that in such circumstances the galvanometer does not vary much from its mean position, even when the magnetometers have changed considerably; and he expresses his doubt whether the constant part of the action of the earth-current can be observed at all with our present means.

Dr. Lamont ascribes the singular effect above described to the double conductor, the current moving (as he believes) at first in the wires, and afterwards diffusing itself in the earth below. I venture to suggest that it may be due to some disturbing cause, which operates more powerfully (in relation to the principal effect) in short than in long wires. No effect of a similar kind appears to have occurred in the observations of $\mathrm{Mr}$. Barlow, which were made upon telegraphic wires of great length. And, on the other hand, it is a fact well known to telegraphists, that the currents, produced by chemical action upon the terminal plates, interfere with the primary current much more in short, than in long wires. In confirmation of the same view, I may mention the fact observed at many points of the earth during the remarkable aurora of August and September, 1859,-namely, that the proper earthcurrent was strongest, coteris paribus, in the longest lines of telegraph. 
15. The connexion of the diurnal changes of the horizontal needle with earth-currents being assumed, we may reason from the former to the latter, and infer the laws which govern the diurnal changes of the earth-currents from the known laws of the related phenomena.

We have already seen that the intensity of the current in the magnetic meridian (flowing northeard) $=a_{\eta}$; and that of the current perpendicular to the magnetic meridian (flowing east$w(a r d)=-a \xi$. Hence, if $\rho$ denote the intensity of the resultant current, and $\phi$ the angle which it makes with the magnetic meridian, measured to the east of north-

$$
\tan \phi=\frac{-\xi}{\eta} ; \quad \rho=a \sqrt{\eta^{2}+\xi^{2}} ;
$$

in which $\eta=X \delta \psi \sin 1^{\prime}$, and $\xi=\delta X, X$ being the horizontal component of the earth's magnetic force, and $\psi$ the magnetic declination*. The azimuth of the direction of the current, measured from the true meridian in the same direction, is

$$
\omega=\phi+\psi \text {. }
$$

Substituting in these formulas the mean values of $\delta \psi$ and $\delta X$ at Dublin, for summer, winter, and for the entire year, we obtain the daily changes of $\rho$ and $\omega$, given in the following Table. The values of $\delta \psi$ and $\delta X$ were observed at Dublin during the four years $1840-43$, twelve times in the day, namely, at the alternate hours. The values corresponding to the intermediate hours are obtained from them by the usual formulas of interpolation.

* The preceding combination of the daily changes of the declination and horizontal intensity, as well as the graphical representation of the results, seems to have been first employed by Professor Hansteen, to represent the laws of the disturbing force, to which these changes are due. The only difference between the two representations is, that the azimuth of the disturbing force at any hour is $90^{\circ}$ greater than that of the earthcurrent. Hence, if the diagrams of Plate II. be turned $90^{\circ}$ forward in azimuth, they represent the daily changes of the horizontal disturbing force. 
TABLE V.

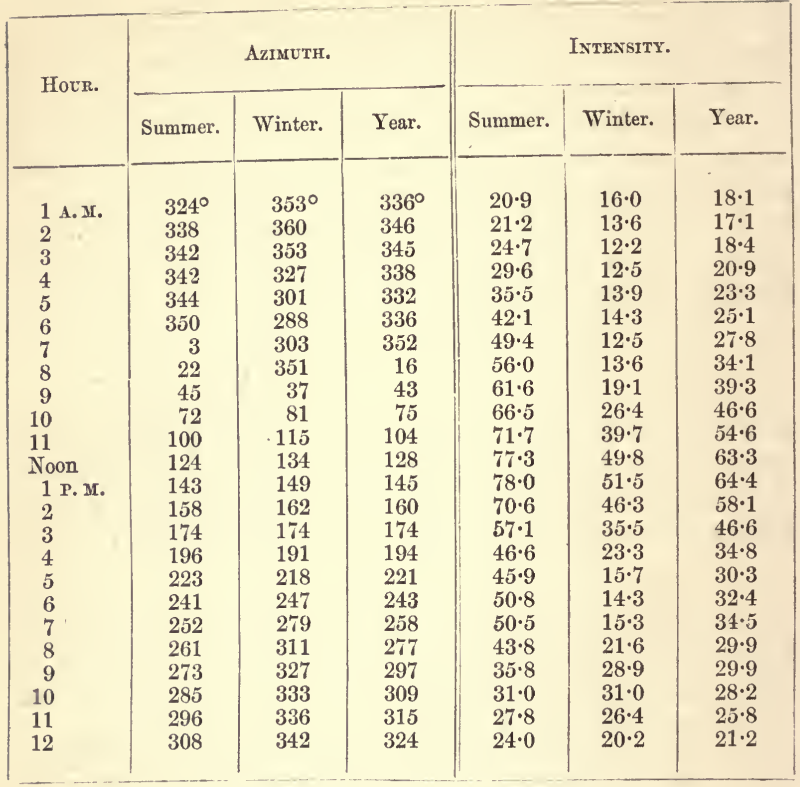

16. The numbers in the fourth and seventh columns of the foregoing Table are graphically represented in the annexed diagram (Plate II., fig. 1), which accordingly exhibits the law of the changes for the entire year, the radius-vector of the curve measuring the intensity of the current, and the angle which it makes with the meridian, its azimuth. The corresponding hours are indicated on the perimeter of the curve, the afternoon hours being distinguished by brackets. It will be seen that in the early hours of the morning, namely, from 1 A. м. to 6 A. M., inclusive, the direction of the current changes little; its mean azimuth for that period is $\mathrm{N} .21^{\circ} \mathrm{W}$. At 7 A. M. the current begins to move eastward, and its direction is due north at about $7^{\mathrm{h}} 15^{\mathrm{m}}$. At $10^{\mathrm{h}} 25^{\mathrm{m}}$ A.x., the azimuth of the resultant current is $90^{\circ}$, or its direction is to the east; at $3^{\mathrm{h}} 15^{\mathrm{m}}$ P. .., it becomes south; and at 
$7^{\mathrm{h}} 35^{\mathrm{m}}$ P. M., it is vest. Finally, after midnight it reaches its stationary position to the west of north.

The order of the changes is similar in summer and in winter. The principal differences lie in the times of reaching the azimuths $0^{\circ}$ and $270^{\circ}$, the direction of the current being in the former azimuth about $1 \frac{1}{2}$ hours earlier in summer than in winter, and in the latter about two hours later. The time of the afternoon meridian passage is nearly the same at the two seasons.

The intensity of the current is greatest in the south and east. The maximum intensity occurs at 1 P. M., the azimuth of the current being then S. $35^{\circ} \mathrm{E}$. There is a secondary maximum about 7 P. M., preceded by a secondary minimum about 5 P.м.

A curious inversion, or fold of the curve, takes place during the night, between 10 P.M. and 6 A.M. The nocturnal minimum occurs between 1 A.M. and 2 A.M. ; and the direction of the current is then nearly opposite to that of the maximum at 1 Р. м.

17. In the foregoing deductions, it is to be borne in mind, in conformity with the remarks of Art. 13, that the current referred to is not that actually subjacent to the place of observation, but the resultant, for that place, of all the currents occupying a considerable portion of the earth's surface. The reasoning, in fact, relates to the magnetic effects of the currents, rather than to the currents themselves, and the immediate subject of calculation is the intensity of the magnetic disturbing force, and the normal to its direction.

It is further to be remembered, that the conclusions are affected by the uncertainty to which we have before adverted (Art. 11), respecting the zero, or origin, from which the magnetio deflections are to be measured. It is probable, however, that this uncertainty does not materially affect the results, except at those hours at which the magnetic variations are small, and when, of course, any given change in the amount of the deflections bears a much larger proportion to the whole.

18. Although the laws of the diurnal ehanges of the magnetio elements at different points of the globe have much in common, they present nevertheless marked differences. Corresponding differences must, therefore, be expected in the diurnal changes of the earth-currents at different places ; and, consequently, the general laws to which they are subject can only be known by a comparison of the results at many places widely distributed over the globe. I have accordingly thought that it would reward the labour to make 
a calculation, similar to the foregoing, for places at which hourly, or two-hourly, observations of the two magnetic elements have been made for any considerable period. The results of these calculations are given in the following Tables.

Table VI. contains the names of the places, together with their geographical co-ordinates, and magnetic elements, the positive sign denoting north latitude, east longitude, and easterly declination. The years in the sixth column of the Table are those of the hourly observations which have been employed in the calculation. The absolute values of the magnetic declination and intensity, in the fourth and fifth columns, correspond to the mean time of the period. The observations were taken, in all cases, at the full hours of Göttingen mean time; but the corresponding local time is indicated in the last column of the Table, the numbers being the minutes to. be added to, or subtracted from, the Tabular hours.

Tables VII. and VIII. contain the calculated values of the azimuth and intensity of the current, at the several hours of observation, for each of the foregoing stations.*

* The diurnal changes of declination and horizontal intensity, employed in the calculation, are contained in the following works:-

1. "Magnetical and Meteorological Observations made at the Royal Observatory, Greenwich (1844-47)."

2. "General Results of the Makerstoun Observations."-Edin. Trans., vol. ix.

3. "Resultate des Magnetischen Observatoriums in München."-Abhandlungen der $K$. Akadesnie der Wissenschaften, München, band v.

4. "Resultate aus den Magnetischen Beobachtungen zu Prag."-Denkschriften der Kaiserlichen Akademie, Wien, band viii.

5. " Annales de l'Observatoire Physique Centrale de Russie (1850-55).",

6. "Observations made at the Magnetical and Meteorological Observatories at Toronto, Hobarton, St. Helena, and the Cape of Good Hope."

7. "Magnetic and Meteorological Observations made at the Girard College, PhiIadelphia." 


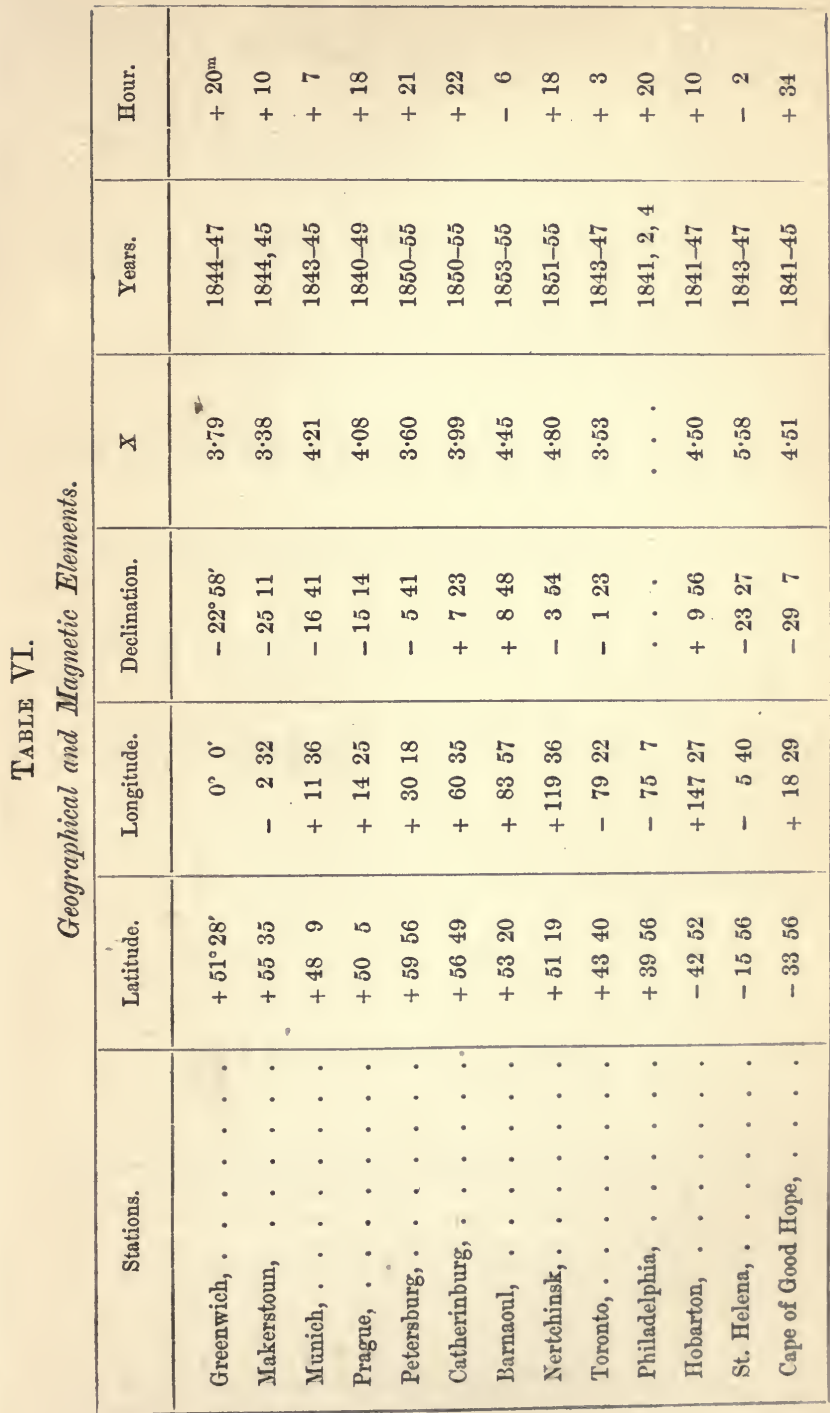




\begin{tabular}{|c|c|c|}
\hline \multirow{14}{*}{ 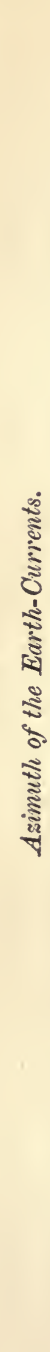 } & 竞 & ล일 \\
\hline & 离 & 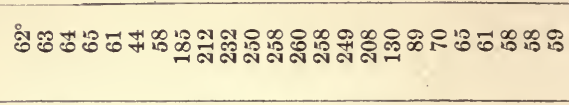 \\
\hline & 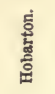 & 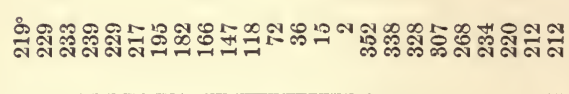 \\
\hline & 要 & 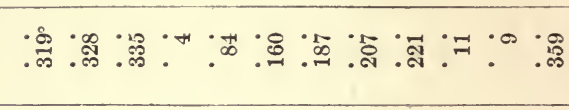 \\
\hline & 悹 & 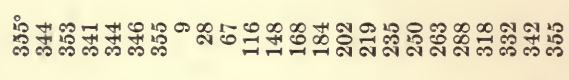 \\
\hline & 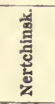 & 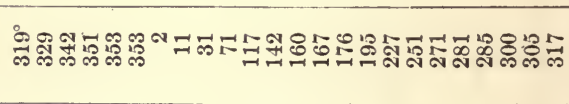 \\
\hline & 营 & 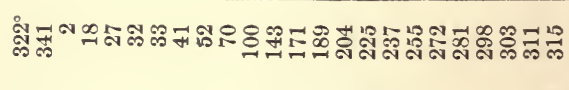 \\
\hline & 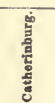 & 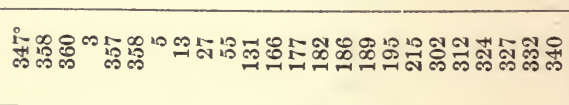 \\
\hline & 竞 & 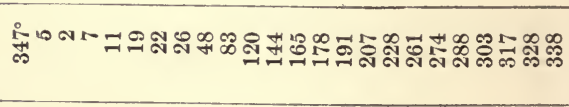 \\
\hline & 恚 & • \\
\hline & 竎 & 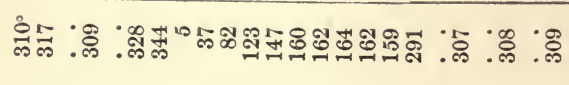 \\
\hline & 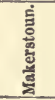 & 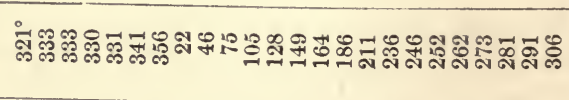 \\
\hline & 这 & 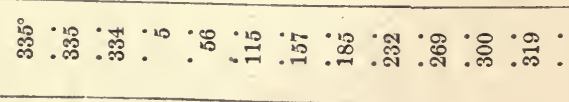 \\
\hline & $\stackrel{\vec{g}}{g}$ & 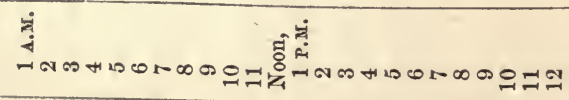 \\
\hline
\end{tabular}




\begin{tabular}{|c|c|}
\hline 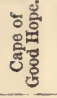 & 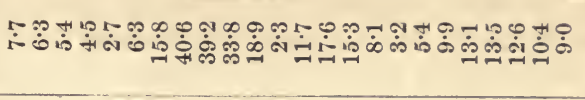 \\
\hline 离 & 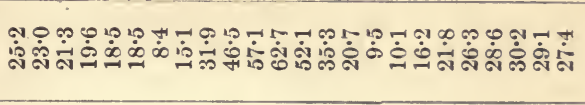 \\
\hline 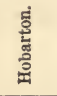 & 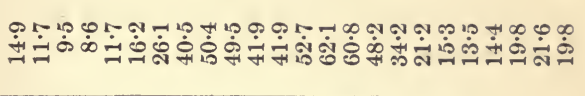 \\
\hline 递 & 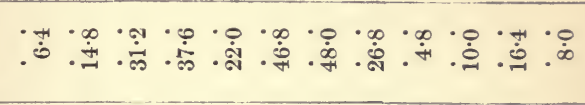 \\
\hline 客 & 以 \\
\hline 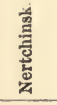 & 舟 \\
\hline 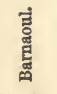 & 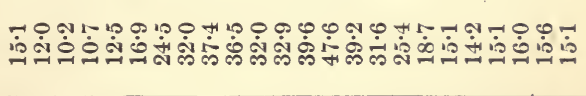 \\
\hline 鄫 & 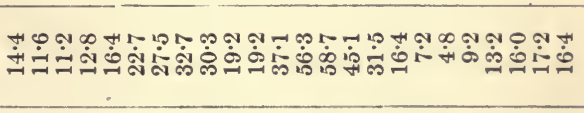 \\
\hline 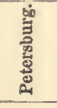 & 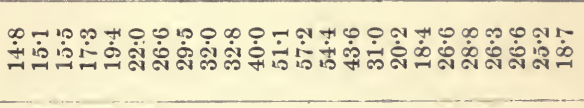 \\
\hline 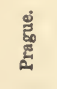 & 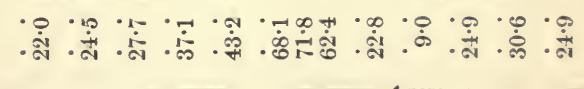 \\
\hline 苛 & 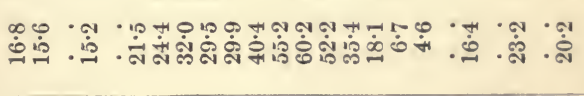 \\
\hline 要 & 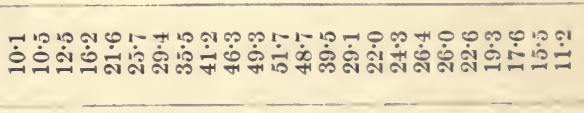 \\
\hline 迆 & 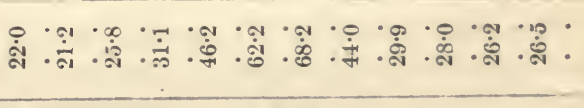 \\
\hline 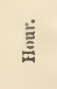 & 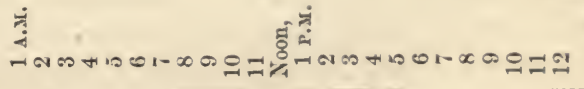 \\
\hline
\end{tabular}


19. From a review of the preceding, we are enabled to see what are the laws of the earth-currents common to all, or to the greater number of the places of observation; and to ascertain in what manner, and to what extent, these laws are departed from in particular instances. And from a consideration of the physical circumstances of the localities in which these deviations occur, we may, in some cases at least, be enabled to trace them to their probable origin.

I. In the first place, then, it will be seen that at most of the stations in the northern hemisphere the direction of the current changes, throughout the day, according to the same general law. This will be evident from the annexed Table, which contains the hours at which the direction of the current passes through the cardinal points at the undermentioned stations.

TAble IX.

Direction of the Currents.

\begin{tabular}{|c|c|c|c|c|c|c|c|c|c|}
\hline Station. & & \multicolumn{2}{|l|}{ N. } & \multicolumn{2}{|c|}{ E. } & \multicolumn{2}{|c|}{ S. } & \multicolumn{2}{|c|}{ W. } \\
\hline $\begin{array}{l}\text { Greenwich, . } \\
\text { Dublin, - } \\
\text { Petersburg, } \\
\text { Catherinburg, } \\
\text { Barnaoul, . } \\
\text { Nertchinsk, } \\
\text { Toronto,. . }\end{array}$ & 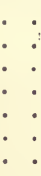 & $\begin{array}{lc}7 \mathrm{~h} & 0^{\mathrm{m}} \\
7 & 15 \\
3 & 20 \\
3 & 20 \\
2 & 50 \\
7 & 5 \\
7 & 25\end{array}$ & $\begin{array}{l}\text { A.x. } \\
\text {, } \\
", \\
, \\
, \\
, \\
,\end{array}$ & $\begin{array}{ll}10^{\mathrm{h}} & 30^{\mathrm{r}} \\
10 & 25 \\
10 & 30 \\
10 & 50 \\
10 & 35 \\
10 & 45 \\
10 & 30\end{array}$ & $\begin{array}{c}\text { A.s. } \\
\text {," } \\
", \\
", \\
, \\
, \\
,\end{array}$ & $\begin{array}{lr}3^{\mathrm{h}} & 0^{\mathrm{n}} \\
3 & 15 \\
2 & 30 \\
2 & 0 \\
1 & 25 \\
3 & 30 \\
1 & 50\end{array}$ & $\begin{array}{l}\text { P.M. } \\
\text { ", } \\
\text { ", } \\
\text {," }\end{array}$ & $\begin{array}{lr}7^{\mathrm{h}} & 25^{\mathrm{m}} \\
7 & 35 \\
7 & 0 \\
7 & 0 \\
6 & 45 \\
7 & 15 \\
7 & 20\end{array}$ & $\begin{array}{l}\text { Mr. } \\
", \\
", \\
", \\
", \\
\text { ", }\end{array}$ \\
\hline
\end{tabular}

From this Table we learn that the times of passage of the current through the east, south, and west points, vary within narrow limits. The means are :-

Mean time of easterly current, . . $10^{\mathrm{h}} 36^{\mathrm{m}}$ A.M.

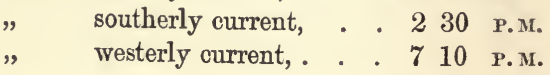

The mean time of the south meridian passage is earliest at Barnaoul, and latest at Nertchinsk. The times of the north meridian passage differ much more widely, the mean epoch being $3^{\mathrm{h}} 10^{\mathrm{m}}$ A.M. for the east of Europe, and for the west of Asia, and $7^{\mathrm{h}} 10^{\mathrm{m}} \mathrm{A} . \mathrm{M}$. for the west of Europe, the east of Asia, and America. 
II. In the southern hemisphere,- - so far, at least, as may be inferred from the phenomena at Hobarton,- - the rotation of the currents is in the opposite direction to that in the northern. This will be best seen by a comparison of the annexed diagrams (Figs. 1 and 2), which represent the directions of the currents at the several hours at Toronto, and at Hobarton, places which have equal latitudes north and south.
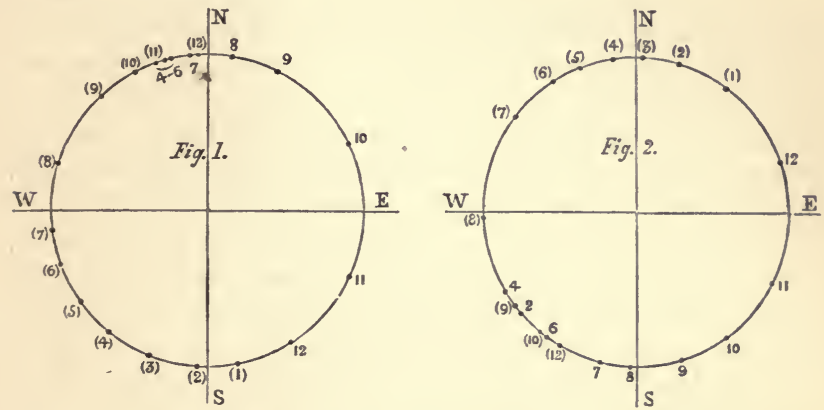

It appears, therefore, that the point to which the earth-current is directed in all cases follows the sun, although at unequal intervals at the different periods of the day.

III. The hour at which the force of the current is greatest, in the northern hemisphere, ranges between noon and 2 P. x., being earliest in the British Islands, and latest in Western Siberia. Its change-with longitude will be understood from the following :-

Western Europe, hour of maximum $=12^{\mathrm{h}} 30^{\mathrm{m}}$ P. M.

\begin{tabular}{|c|c|c|c|}
\hline Central Europe, & " & " & 1 \\
\hline Eastern Europe, & "' & ", & 135 \\
\hline Western Siberia, & " & ", & \\
\hline Central Siberia, & " & " & 15 \\
\hline Eastern Siberia, & יפ & " & 140 \\
\hline Canada, & „ & , & 115 \\
\hline
\end{tabular}

The mean time of the maximum is $1^{\mathrm{b}} 25^{\mathrm{m}} \mathrm{P} . \mathrm{M}$.

IV. The minimum of current intensity during the night takes place at an interval of about twelve hours from the epoch of the maximum; and the directions of the greatest and least currents are, in nearly all cases, exactly opposite. 
20. The foregoing are the general features of the diurnal variation of the earth-currents, as inferred from the changes of direction and intensity of the horizontal magnetic force. But while the phenomena have much in common, there is at the same time great diversity in the details. This will be evident upon inspection of the diagrams of Plate II., which represent the diurnal changes of the currents at the different stations. The most remarkable peculiarities are those presented at Munich and Prague in Europe, at Catherinburg in Siberia, at Philadelphia in the United States, and at St. Helena and the Cape of Good Hope in the southern hemisphere.*

21. The great diversity which, in the midst of order, we have thus seen to prevail in the diurnal changes of the earth-currents, cannot be wondered at, when we consider the endless variety which exists in the distribution of land and water on the earth's surface, as well as in the configuration of the land itself, and in the materials of which it is composed; for all these circumstances affect, in a material degree, the conductibility of the superficial strata. In some of the instances above referred to, we have found a probable connexion between these physical circumstances and the observed facts. We are thus encouraged to hope, that the complex phenomena of the diurnal change may at some future time be completely unravelled, and the peculiar features which it presents at each place traced to their causes. Meanwhile, it would be of great importance to determine more precisely the influence of lines of coast, of mountain chains, and of other geographical and physical conditions, by short series of observations of the diurnal changes of the two magnetic elements at well-selected stations. Such observations would add little to the labour or expense of our numerous exploring expeditions, while they would further in an important degree the knowledge of Terrestrial Magnetism.

\footnotetext{
* The remarks in the original Paper upon these diversities, and upon their probable causes, have been here omitted.
} 
FIG:'I.

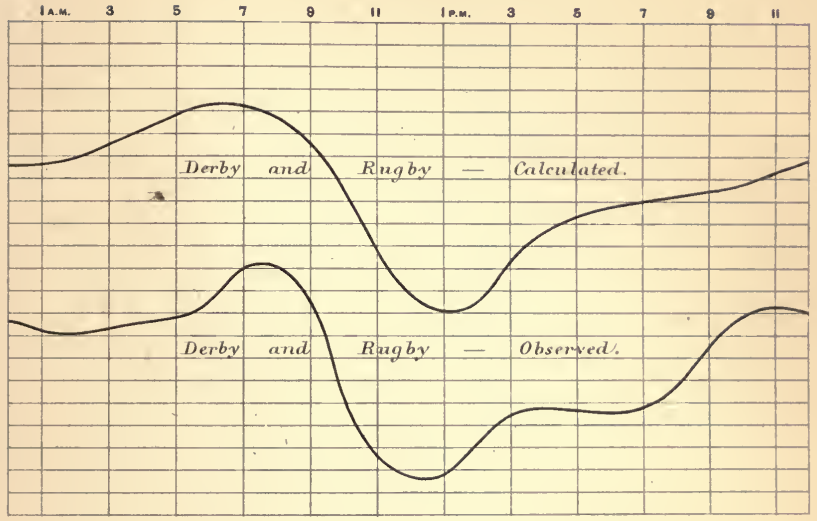

FIG: 2.

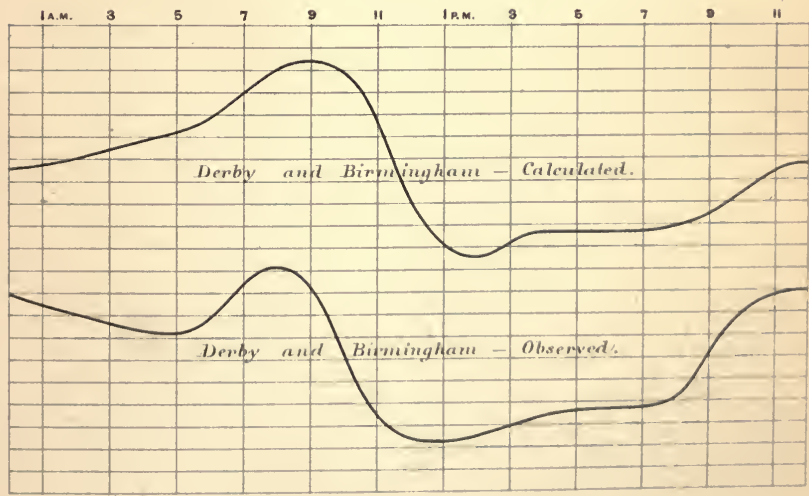



XIII.-ON EARTH-CURRENTS IN CONNEXION WITH MAANETIC DISTURBANCES.

Proceedings of the Royal Irish Academy, 1862.

Is a Paper recently communicated to the Academy, the author showed that the regular diurnal changes 'of the horizontal component of the earth's magnetic force were probably due to electric currents traversing the earth's crust, these currents operating as disturbing forces, which cause the magnets to deviate from their mean positions according to known laws. This relation being established, the diurnal laws of the earth-currents may be inferred from their effects. It was thus concluded that the azimuth and the intensity of the currents varied throughout the day, according to fixed laws depending upon the hour-angle of the sun. At difierent parts of the globe these laws were found to exhibit certain well-marked features in common; while their differences were probably accounted for by the geographical and physical characters of the region in which they occur. The author now proceeds to extend the same inquiry to the currents which produce the magnetic disturbances.

It has been shown, by the labours of Kreil, Sabine, and others, that the disturbances of the magnetio elements are subject to periodical laws, depending upon the hour, which are constant for a given place, and for a given season of the year. The sums of the changes produced by these disturbances, at each hour of observation, have been calculated by General Sabine for three of the British Colonial Observatories. The corresponding quantities have been deduced by Dr. Lamont, for Munich; by Mr. Broun, for Makerstoun, in Scotland; and by the author, for Dublin. We possess, in addition to the foregoing, similar results at Lake Athabasca, in British North America, deduced by Colonel Iefroy 
from observations made by himself, which, although derived from a shorter series of observations, are of the highest scientific value. For these places, therefore, it only remains to combine the results of the declination and horizontal intensity, by the method which has been already applied to the regular changes of the same elements.

The result of this calculation, applied to the Dublin observations, shows that the direction of the disturbance-current at that place observes a mean law, not very dissimilar to that which governs the regular diurnal current. Its azimuth rotates, during the day, in the same direction as the sun, its direction pointing almost exactly to the luminary. The direction is east about 5 A. м.; south, about noon; and west, at 6 P. .. The current is easterly from 9 P. м. to 9 A. M., inclusive, and westerly during the remainder of the 24 hours. The mean azimuth of the easterly current, measured from the north eastward, is $40^{\circ} 15^{\prime}$; that of the westerly is $230^{\circ} 18^{\prime}$. If the mean directions of the easterly and westerly currents be assumed to be in the same right line, the mean azimuths will be $\mathrm{N} .45^{\circ}$ E., and S. $45^{\circ} \mathrm{W}$. This result agrees, in a very remarkable manner, with those obtained by $\mathrm{Mr}$. Barlow and Mr. Walker from the direct measures of the intensity of the earth-currents, as observed on days of disturbance in several of the telegraphic lines of England; and the agreement must be regarded as an additional proof of the dependence of the magnetic changes upon earth-currents.

The phenomena at Makerstoun are very similar to those at Dublin; and the epochs of the passage of the current through the cardinal points are nearly the same.

At Toronto, in Canada, the current is wholly easterly, the mean azimuth being $81^{\circ} 25^{\prime}$. On the other hand, at Athabasca, the current is easterly from 12 Р. м. to 6 A. M., inclusive, and westerly during the remainder of the 24 hours. The sums of the easterly and westerly changes for the entire day balance one another, the easterly currents being as much greater in magnitude as they are less in duration. Their mean azimuths are $110^{\circ} 18^{\prime}$ and $290^{\circ} 56^{\prime}$.

At St. Helena the direction of the current is easterly throughout the day, the mean azimuth being $70^{\circ} 53^{\prime}$. The direction is singularly constant, the greatest deviation from the mean being only $10^{\circ}$. The phenomena at the Cape of Good Hope closely resemble those at St. Helena. The direction of the current is easterly at 
every hour, excepting 5 A. M. when there is a slight westerly movement. The mean azimuth is $77^{\circ} 54^{\prime}$.

It thus appears that at some places-as in the British Islandsthe mean direction of the disturbance current rotates through the entire compass in the course of the day; while at others-as Munich, Toronto, St. Helena, and the Cape of Good Hope-it is easterly throughout the day. While, therefore, there is a periodicity in the easterly and westerly currents depending on the hour, we are obliged to infer that there is, at the same time, some cause constantly operating which tends to produce an easterly current.

The mean azimuth of this current appears to be connected with the magnetic meridian of the place, to which it is nearly perpendicular. This will appear from the following Table of the mean azimuths of the disturbance-currents at the northern stations, measured from the astronomical and from the magnetical meridians, respectively:-

\begin{tabular}{|c|c|c|}
\hline Places. & Az. (Astron.) & Az. (Magn.) \\
\hline Dublin, . . . . & $45^{\circ}$ & $72^{\circ}$ \\
Makerstoun, . . . & 51 & 76 \\
Munich, . . . . & $52 \cdot 5$ & 69 \\
Toronto, . . . . & $81 \cdot 5$ & 83 \\
Athabasca, . . . & 110 & 81 \\
\hline
\end{tabular}

The mean azimuth (magnetic) for the five stations is E. $14^{\circ} \mathrm{N}$.

The mean azimuth at the two stations in the southern hemisphere is E. $11^{\circ} \mathrm{S}$., deviating nearly as much to the south, as that of the northern stations deviates in the opposite direction. It thus appears that while the principal current is easticard in both hemispheres, there is also a meridional current tending northward in the northern hemisphere, and southward in the southern. Its intensity is between one-fourth and one-fifth of that of the other component.

These results are wholly at variance with the hypothesis imagined by M. de la Rive in explanation of the phenomena of magnetic disturbances, according to which the disturbance-current flows from north to south only.*

* The discrepancy of M. de la Rive's hypothesis with the phenomena of the earthcurrents, as observed in the British Islands, has been already pointed out by Mr. Walker. It is even more marked at other parts of the globe. 
The diurnal changes of the intensity of the disturbance-currents present features equally marked. In order to perceive them clearly, it may be convenient to examine separately the meridional currents, and those at right angles to the magnetic meridian.

The meridional currents are developed chiefly at the European stations, and at Toronto, in Canada: at Athabasca, and at the southern stations, they are comparatively small. The northerly maximum occurs at Toronto, at 9 P. M., at Munich at 10 P. M., and at Dublin at 11 P. M. Its epoch at Makerstoun is between 9 P. M. and 11 P. м. The southerly maximum occurs at 8 A. M., very nearly, at the four stations. Thus the epochs are nearly at the same hours of local time, notwithstanding the differences of longitude.

A similar result appears from an examination of the currents at right angles to the magnetic meridian. Thus, in the northern hemisphere, the easterly maximum occurs between 2 A. M. and 4 A. M., and the westerly maximum (or easterly minimum) between 3 P. м. and 5 P.м. The two epochs are precisely the same at Makerstoun and at Toronto, places which differ more than five hours in longitude.

The corresponding epochs for the two stations in the southern hemisphere in like manner agree with one another. The easterly maximum occurs between 6 P. м. and 7 P. м. at St. Helena and at the Cape of Good Hope, and the easterly minimum between 5 A. M. and $6 \mathrm{~A} . \mathrm{M}$. It is deserving of remark that these epochs do not differ considerably from those of the opposite movements in the northern hemisphere, the easterly extreme in the one corresponding nearly with the westerly extreme in the other. A similar opposition in the phenomena of the regular diurnal change in the two hemispheres was pointed out by the author on a former occasion, and there seems good reason to suppose that the two facts are physically related.

It appears, then, that the principal epochs of the disturbancecurrents are connected with the sun's hour-angle, and are independent of the longitude of the place at which they occur.

The foregoing relations appear to be of a very general nature, and such as to afford a distinct basis for physical theory. 


\section{XIV.-ON TḦE PROBABLE CAUSES OF THE EARTH- CURRENTS.}

Proceedings of the Royal Irish Academy, 1862.

Is a former communication to the Academy, I endeavoured to prove that the diurnal changes of the horizontal needle were the results of electric currents traversing the earth's crust. The existence and continuous flow of such currents had been established, as I believe, by the observations of Mr. Barlow made on two of the telegraphic lines of England; and it only remained to show that their laws corresponded with those of the magnetic changes.

In that communication I refrained from offering any conjecture as to the origin of the currents themselves. Every speculation of this kind must remain a pure hypothesis, until it can be confronted and compared with facts; and the magnetic phenomena presented at different points of the earth's surface are so diversified, that a wide collection of the facts is necessary in order to form the basis of any sound physical theory. For these reasons, I have deemed it the more proper course to ascertain the lacs of the diurnal changes of the earth-currents at many places, so far as they may be inferred from the magnetio phenomena, before proceeding to the consideration of their causes.

It has been shown, in the Paper referred to, that the earthcurrents, as inferred from the changes in the two horizontal components of the magnetio force, observe certain general laws, which are common to all the stations at which these changes have been observed; while, on the other hand, their departures from a common type are various and considerable. We thus learn that the phenomena are produced by a common cause, the effects of which are greatly modified by the physical peculiarities of the parts of the 
earth where they are observed. The following are the principal features of the phenomena common to all, or to most of the places of observation.

I. The point to which the resultant earth-current is directed follows the sun, although not at a uniform rate, throughout the day. In the northern hemisphere its direction is eastuard, on the average, at $10^{\mathrm{h}} 30^{\mathrm{m}}$ A. M. ; southucard, at $2^{\mathrm{h}} 30^{\mathrm{m}}$ P. м. ; and vestward, at 7 P. M.

II. The intensity of the current is greatest between noon and 2 P.M., the mean time of the maximum in the northern hemisphere being about $1^{\mathrm{h}} 30^{\mathrm{m}}$ P.M. The intensity of the current is least at an interval of about twelve hours from the epoch of the maximum; and the direction of the current of least intensity is, in nearly all cases, opposite to that of the greatest.

III. There are two subordinate maxima, separated from the principal maximum by intervening minima. The morning maximum occurs, on the average, at $8^{\mathrm{h}} 30^{\mathrm{m}} \mathrm{A}$. $\mathbf{~ r}$. It may be traced in the diurnal curves of the American and Siberian stations, and in those of the Cape of Good Hope and Hobarton. The current is then northerly in the northern hemisphere, and southerly in the southern. The evening maximum occurs at about 10 P.M., and is observed at almost all the stations.

The foregoing facts leave no doubt that the sun is the primary cause of the currents; and the only question is as to the mode of its agency. Upon this point I concur with Dr. Lamont in believing the electrical currents (or waves) on the earth's surface to be due to disturbances of equilibrium of statical electricity; but I regard these derangements of equilibrium to be simply the effects of solar heat, and not (as Dr. Lamont believes) the results of an electrical force emanating directly from the sun.

It is well known that the earth and the atmosphere are, in ordinary circumstances, in opposite electrical states-the electricity of the earth being negative, and that of the atmosphere positive. It is also known that the electricity of the air increases rapidly with the height, a few feet-and in some cases even a few inchesbeing sufficient to manifest a difference of electrical tension. The rate of this increase is very different at different periods of the day, the difference appearing to be due to the greater or less conductibility of the lower strata of the atmosphere, causing a greater or or less interchange of the opposite electricities. 
Now, we have in this machinery, as it appears to me, means fully adequate to the production of the observed effects. If it be assumed that the sun produces these changes by its calorific action, the effects at any given place will depend upon the relative temperatures of the neighbouring portions of the earth's surface. The earth being, in its normal state, negatively electrical, the negative electricity will be greatest-or the positive electricity least,-at the parts most heated ; and there will, consequently, be a flow of electricity to those parts from the place of observation. Thus the varying azimuth of the current, which is directed towards the most heated parts of the earth's surface, is explained. The maximum intensity of the current, at $1^{\mathrm{h}} 30^{\mathrm{m}} \mathrm{P}$. M. is also accounted for, that being the period of the day when the solar calorific action is most intense. It should be noted, however, that the magnitude of the effect will depend, not on the absolute temperature, but on its relative increase. It is, accordingly, greatest at those parts of the earth at which the increment of temperature corresponding to a given distance is greatest.

The secondary maxima are probably due to the recombination of the atmospheric and terrestrial electricities, through the medium of vapour in the lower regions of the atmosphere. The effects of this recombination in producing horizontal currents in the earth's crust will, of course, be differential only, and will depend on the excess of the positive electricity thus transported at the places on the same meridian which are nearer to the equator. In confirmation of this view it may be observed, that the epochs correspond with those of the maxima of atmospheric electricity, as deduced by Quetelet from the observations made under his direction at Brussels, the morning maximum of atmospherio electricity in summer occurring at 8 A.M., and the evening maximum at 9 P.M.

The phenomena hitherto described are such as would take place if all the parts of the earth's crust were similarly constituted, and therefore similarly acted on by the solar rays. In order to be able to explain the diversity which exists in the magnetic phenomena at different places, we must know something more of the nature of the solar action, and of the mode in which electricity is developed by it.

The speculations respecting the origin of atmospherio and terrestrial electricity are various. Thus De Saussure believed that this electricity was dereloped by evaporation, the rapour taking 
the positive electricity, and the water the negative; and this hypothesis, with some limitations, has been very generally admitted by physicists. On the other hand, M. de la Rive is of opinion that the origin of this electricity is to be sought in the chemical actions which he supposes to be going on in the interior of the solidified crust of the earth; and he thinks that evaporation acts merely by transporting one of the separated electricities, and carrying it into the higher regions of the atmosphere. But whatever be the correct view as to the force which develops the electricity, it seems to be granted that the separation of the two electricities (in the earth and the atmosphere) is the consequence of evaporation, the vapour carrying with it the positive electricity, and the vaporizing body retaining the negative. It follows from this, that the effect produced will vary greatly with the distribution of land and water, and will be greatest, cceteris paribus, where they come into juxtaposition along the coasts of the great continents, especially where the coast-lines are in, or near, the meridian. The evaporation from the surface of the sea being much greater than from the land, the electricity will be most deficient at the former. Hence there will be a flow of electricity from land to sea, which will combine with, and often mask, that due to the sun's position alone.

Now this seems to be what happens. The most marked instance of the phenomenon which we possess is that afforded by the diurnal changes of the currents at St. Helena. There the currents (as I have already shown) flow from the coast of Africa during the hottest portion of the day, and tovards it during the night. The influence of the form of the coast seems to be shown in the diurnal curve of the Cape of Good Hope, by the existence of three maxima, of which the principal is directed from the land, and the two subordinate along the lines of coast. At Hobarton, in Van Diemen's Land, the same influence appears in the extension of the southern lobe of the curve, which is there nearly equal to the northern.

I have since calculated the direction and intensity of the currents at the Indian stations, and I find that the curves follow nearly the type of the St. Helena curve. Thus, at Singapore, for which place we possess the results of observation during the three years 1843-1845, the maximum of current intensity takes place between $10 \mathrm{~A} . \mathrm{Mr}$. and 11 A.M., and its direction is S. $80^{\circ} \mathrm{W}$. At Madras, so far as may be inferred from the observations of a single month, the maximum takes place at noon; and the direction 
of the current is then nearly the same as at Singapore. At Simla, in the Himalaya, the maximum occurs also at noon; but the direction of the current of greatest intensity is more southerly, its mean yearly direction being S. $47^{\circ} \mathrm{W}$. This is precisely what should happen according to the hypothesis, this being nearly the direction of the line drawn to the nearest point of the coast.*

The variation in the epoch of the maximum intensity of the current, at different places, is also in accordance with the same principles; that epoch being earliest in islands, or places nearly encompassed by sea, and latest in the interior of the great continents. Thus it occurs at noon at St. Helena, and in the southern parts of the peninsulas of Hindostan and the Malaya; while it takes place at 2 P.м. at Catherinburg and Barnaoul, in the interior of Siberia. This accords with the laws of the sun's calorific action.

It will be seen, upon an inspection of the diurnal curves of the earth-currents, $\dagger$ that at most of the northern stations, as well as at Hobarton in the southern, the easterly currents are greater than the vesterly. I believe this effect to be due to the disturbance-currents, which (as I have already shown) have an easterly tendency. This preponderance of the easterly currents, however, is found to be greater at places-such as Greenwich, Dublin, Makerstoun, and Toronto-which are near an eastern coast, than at those places-such as Petersburg, Catherinburg, and Barnaoul-which are in the interior of the continent. The results, therefore, so far confirm the supposition above made.

There are, unfortunately, very few places situated near the western shore of a great continent, at which continued observations of the two magnetic elements have been made. At Sitka, on the western coast of North America, the results confirm the view above stated,- - the westerly currents being there greater than the easterly.

There are probably other circumstances in the configuration

* These additional results oblige me to abandon the conclusion formerly derived from a more limited induction, that the direction of the current of greatest intensity is connected with the magnetic meridian of the place. From the facts which we now possess, it would appear that the currents affect a meridional direction in the higher latitudes, while they are nearly parallel to the equator within the tropics. This will be seen in a striking manner by comparing the directions of the maximum current in India, above given, with those of the Russian stations in the northern part of the Asiatic Continent.

+ Transactions of the Royal Irish Academy, Vol. XXIV. 
and structure of the earth's surface which influence the direction and magnitude of the currents; but I incline to think that the principal one is that above stated, viz. the distribution of land and water in the vicinity of the place of observation. It may be, also, that this cause is sufficient to account for some of the peculiarities in the form of the diurnal curve noticed in my former communication, and there referred to other causes. Thus, it is not improbable that the persistent direction of the current at Munich, there referred to the influence of a mountain range, may be, in fact, the result of the proximity of the Adriatic Gulf, which lies nearly in the direction of the persistent current.

In the preceding remarks I have referred only to the regular diurnal changes. I believe that the irregular are produced by the same forces, but operating in a somewhat different manner. The regular currents are produced, as I conceive, chiefly by the separation of the two electricities by evaporation, under the action of the sun; while the disturbance-currents are caused by their rapid recombination, through the medium of moisture, in the lower strata of the atmosphere.* In connexion with this view, I may refer to the fact which has been established by an examination of the mean effects of the magnetic disturbances, $\uparrow$ namely, that the epochs of the maxima of the disturbance-currents depend, in their mean values, upon the sun's hour-angle, and are independent of the longitude of the place. This result is in accordance with the hypothesis which ascribes these currents to changes in the sun's calorific agency, and to the meteorological effects which these engender.

In the limits within which it is necessary to confine this abstract, I have been able only to refer to some of the leading facts in confirmation of the hypothesis which I have ventured to propose ; and I am obliged to omit altogether all reference to the objections which will probably be raised against it. There is, however, one fact which appears at first sight to offer a formidable difficulty to its reception, and which it seems necessary to notice here. The regular magnetic changes are greater in summer than

* This hypothesis as to the cause of magnetic disturbances is due to M. de la Rive; but his views respecting the laws of the resulting currents are, as I have elsewhere shown, inconsistent with the phenomena. The regular diurnal changes of terrestrial magnetism are ascribed by M. de la Rive to a direct electrical action emanating from
the sun.

$\dagger$ Prccedings of the Royal Irish Acqdcmy, April 28, 1862. 
in winter; while with the electrical tension, and its changes, it is the reverse. This objection, however, disappears when it is viewed more closely. The physical quantity measured by our electrometers is not the absolute electric tension, but its rariation with the height; while the electric changes which engender terrestrial currents are the variations as depending on horizontal distance. It is easily conceivable that these should not correspond. In fact, it is natural to suppose that in summer the zero-plane, which separates the two electricities, should rise considerably; and thus that the variations for a given increase of altitude (which probably diminish with the distance from that plane) should lessen, although the absolute tensions, as well as the changes in horizontal distance, may be greater.

It would be of importance, in reference to this inquiry, to institute electrical observations of a totally different kind from any which we now possess, and to measure the differences of tension as depending on horizontal distance. There seems to be no diffculty in the way of such observations,- at least none greater than those which present themselves in the ordinary observations of atmospheric electricity; and the results would probably do more to clear up the physical aspect of these complex and interwoven phenomena than any other observational means. 
XV.-ON THE DIRECT MAGNETIC INFLUENCE OF A DISTANT LUMINARY UPON THE DIURNAL VARIATIONS OF THE MAGNETIC FORCE AT THE EARTH'S SURFACE.

Philosophical Magazine, March, 1858.

Ir has been usual to ascribe the ordinary diurnal variations of the terrestrial magnetic force to solar heat, whether operating directly upon the magnetism of the earth, or generating thermo-electric currents in its crust. The credit of these hypotheses has been somewhat weakened by the discovery of a variation which is certainly independent of any such cause, namely, the lunar variation of the magnetic elements; while at the same time new laws of the solar diurnal change have been established, which are deemed to be incompatible with the supposition of a thermic ageney. There has been, accordingly, a tendency of late to recur to the hypothesis, that the sun and moon are themselves endued with magnetism, whether inherent or induced; and it is therefore of some importance to determine the effects which such bodies would produce at the earth's surface, and to compare them with those actually observed.

I have endeavoured, in what follows, to solve this question, on the assumption that the supposed magnetism of these luminaries is inherent. The result will show the insufficiency of the hypothesis to explain the phenomena; and will therefore bring us one step nearer to their explanation, by the removal of one of their supposed causes.

Let $x, y, z$ be the coordinates of any point of a fixed magnet, referred to three rectangular axes passing through its middle point ; $a, b, c$ those of a distant magnetic element, $m$; and, $e$ their 
mutual distance. Then, if $\mu$ denote the quantity of free magnetism contained in the element $d s$ of the magnet at the point $(x, y, z)$, the force exerted by $\mu$ on $m$ is

$$
\frac{m \mu d s}{e^{2}}
$$

and its resolved portions in the directions of the three axes of coordinates are

$$
\frac{m(a-x) \mu d s}{e^{3}}, \quad \frac{m(b-y) \mu d s}{e^{3}}, \quad \frac{m(c-z) \mu d s}{e^{3}} .
$$

Let the magnitudes of the lines connecting the points $(a, b, c)$ and $(x, y, z)$ with the origin be denoted by $u$ and $s$, and let the angle contained by their directions be $\omega$. Then

$$
e^{2}=u^{2}-2 u s \cos \omega+s^{2} ;
$$

and if $s$ be so small in comparison with $u$ that the squares and higher powers of $\frac{s}{u}$ may be neglected,

$$
e^{-3}=u^{-3}\left(1+\frac{3 s}{u} \cos \omega\right) \text {. }
$$

Again, if $a, \beta, \gamma$ denote the angles contained by the axis of the magnet with the three axes of coordinates,

$$
x=s \cos a, \quad y=s \cos \beta, \quad z=s \cos \gamma .
$$

Substituting these values in the expressions for the components of the force above given, integrating, and observing that $\int \mu d s=0$, we have, for the components of the total force exerted by the magnet on the magnetic element,

$$
\begin{aligned}
& \frac{M m}{u^{3}}\left(3 \frac{a}{u} \cos \omega-\cos \alpha\right), \\
& \frac{M m}{u^{3}}\left(3 \frac{b}{u} \cos \omega-\cos \beta\right), \\
& \frac{M m}{u^{3}}\left(3 \frac{c}{u} \cos \omega-\cos \gamma\right) ;
\end{aligned}
$$

in which we have put, for abridgement, $\boldsymbol{M I}=\int \mu$ scls. The angle $\omega$ is connected with $\alpha, \beta, \gamma$ by the relation

$$
\text { u. } \cos \dot{\omega}=a \cos a+b \cos \beta+c \cos \gamma \text {. }
$$

Now let the point $(a, b, c)$ be on the earth's surface, and lot us $\mathrm{x} 2$ 
suppose, for simplicity, that the acting magnet is in the plane of the equator. Let that plane be taken as the plane of $(x, y)$, and the line connecting the centre of the magnet and that of the earth as the axis of $x$. Then, if the distance of the acting magnet be considerable, relatively to the earth's radius, $b$ and $c$ are small in comparison with $a$, and we may neglect the small quantities of the second order, $\frac{b^{2}}{a^{2}}, \frac{c^{2}}{a^{2}}, \frac{b c}{a^{2}}$. Wherefore, substituting for $\cos \omega$ its value, the components of the acting force become

$$
\begin{aligned}
& \frac{M m}{a^{3}}\left(2 \cos \alpha+\frac{3 b}{a} \cos \beta+\frac{3 c}{a} \cos \gamma\right), \\
& \frac{M m}{a^{3}}\left(-\cos \beta+\frac{3 b}{a} \cos \alpha\right), \\
& \frac{M m}{a^{3}}\left(-\cos \gamma+\frac{3 c}{a} \cos \alpha\right) .
\end{aligned}
$$

Now, if $D$ denote the distance of the centre of the magnet from the centre of the earth, $r$ the earth's radius, $\lambda$ the latitude of the point $(a, b, c)$ on its surface, and $\theta$ the angle contained by the meridian passing through it with that containing the acting magnet,

$$
a=D-r \cos \lambda \cos \theta, \quad b=r \cos \lambda \sin \theta, \quad c=r \sin \lambda .
$$

Hence the maximum values of $\frac{b}{a}$ and $\frac{c}{a}$ are equal to $\frac{r}{a}$; and if we disregard the terms containing them in comparison with the rest, the preceding values are reduced to

$$
2 \frac{M m}{D^{3}} \cos a, \quad-\frac{M m}{D^{3}} \cos \beta, \quad-\frac{M m}{D^{3}} \cos \gamma .
$$

Now, in place of a single magnet, let there be an indefinite number distributed in any manner throughout the entire magnetic body ; and let us make, for abridgement,

$$
\Sigma(M \cos \alpha)=P, \quad \Sigma(M \cos \beta)=Q, \quad \Sigma(M \cos \gamma)=R .
$$

Then, if the radius of this body be small in comparison with its distance, we may neglect the variations of $D$, and we shall have, for the three components of the acting forces,

$$
X=\frac{2 m P}{D^{3}}, \quad Y=\frac{-m Q}{D^{3}}, \quad Z=\frac{-m R}{D^{3}} .
$$


In order to determine the effect of these forces upon a freely suspended horizontal magnet at the earth's surface, we must resolve $X$ and $Y$ in the direction of the tangent, and of the radius, of the parallel of latitude. The resolved forces are, respectively,

$$
X \sin \theta+Y \cos \theta, \quad X \cos \theta-Y \sin \theta .
$$

Again, resolving the forces $Z$ and $X \cos \theta-Y \sin \theta$ in the direction of the tangent to the meridian, and in that of the radius of the earth, we have finally the three components, viz. :

$X \sin \theta+Y \cos \theta$, directed eastward;

$Z \cos \lambda+(X \cos \theta-Y \sin \theta) \sin \lambda$, directed northward;

$-Z \sin \lambda+(X \cos \theta-Y \sin \theta) \cos \lambda$, vertical, towards centre.

Of these, the latter has no effect upon the horizontal magnet. The moment of the two former to turn it is

$(X \sin \theta+Y \cos \theta) \cos \delta-\{Z \cos \lambda+(X \cos \theta-Y \sin \theta) \sin \lambda\} \sin \delta$, $\delta$ denoting the magnetic declination; or, substituting for $X, Y, Z$ their values,

$$
\begin{gathered}
\frac{m}{D^{3}}\{\cos \delta(2 P \sin \theta-Q \cos \theta)-\sin \delta(2 P \cos \theta+Q \sin \theta) \sin \lambda \\
-R \cos \lambda \sin \delta\} .
\end{gathered}
$$

But the moment of the earth's magnetism, opposed to this, is

$$
H \Delta \delta \sin 1^{\prime} \text {, }
$$

in which $H$ denotes the horizontal component of the earth's magnetic force. Wherefore

$$
\begin{aligned}
\Delta \delta=\frac{1}{D^{3} H} \sin 1^{\prime} & \{\sin \theta(2 P \cos \delta-Q \sin \lambda \sin \delta) \\
& -\cos \theta(2 P \sin \lambda \sin \delta+Q \cos \delta)+R \cos \lambda \sin \delta\} .
\end{aligned}
$$

At the equator, this is reduced to

$$
\Delta \delta=\frac{1}{D^{3} H \sin 1^{\prime}}\{\cos \delta(2 P \sin \theta-Q \cos \theta)+R \sin \delta\} .
$$

To determine the effect of the magnetio body upon the horizontal component of the earth's magnetic force, we must resolve 
the horizontal parts of the disturbing forces, viz., $X \sin \theta+Y \cos \theta$, acting eastward, and $Z \cos \lambda+(X \cos \theta-Y \sin \theta) \sin \lambda$, acting northward, in the direction of the magnetic meridian. We have thus

$$
\Delta H=
$$

$(X \sin \theta+Y \cos \theta) \sin \delta+\{Z \cos \lambda+(X \cos \theta-Y \sin \theta) \sin \lambda\} \cos \delta$ $=\frac{1}{D^{3}}\{\sin \theta(2 P \sin \delta+Q \sin \lambda \cos \delta)+\cos \theta(2 P \sin \lambda \cos \delta-Q \sin \delta)$ $-R \cos \lambda \cos \delta\}$

and at the equator,

$$
\Delta H=\frac{1}{D^{3}}\{\sin \delta(2 P \sin \theta-Q \cos \theta)-R \cos \delta\} .
$$

Lastly, if $V$ denote the vertical component of the earth's magnetic force, we have

$$
\begin{aligned}
\Delta V & =-Z \sin \lambda+(X \cos \theta-Y \sin \theta) \cos \lambda \\
& =\frac{1}{D^{3}}\{(2 P \cos \theta+Q \sin \theta) \cos \lambda+R \sin \lambda\} ;
\end{aligned}
$$

a result which, as might have been anticipated, is independent of the magnetic declination. At the equator

$$
\Delta V=\frac{1}{D^{3}}(2 P \cos \theta+Q \sin \theta)
$$

From the foregoing we learn :-

1. That the effect of a distant magnetic body on each of the three elements of the earth's magnetic force consists of two parts, one of which is constant throughout the day, while the other varies with the hour-angle of the luminary.

2. Each of these parts varies inversely as the cube of the distance of the magnetic body.

3. The variable part will give rise to a diurnal inequality, having one maximum and one minimum in the day, and subject to the condition

$$
\Delta_{\theta}+\Delta_{\pi+\theta}=0 .
$$

The third of these laws does not hold, with respect either to the solar-diurnal or to the lunar-diurnal variation. Thus, in the solardiurnal variation of the declination, the changes of position of the 
magnet throughout the night are comparatively small, and do not correspond, with change of sign only (as required by the foregoing law), to those which take place at the homonymous hours of the day. 'The phenomena of the lunar-diurnal variation are even more opposed to the foregoing law, the variation having two maxima and two minima of nearly equal magnitude in the twenty-four lunar hours, and its values at homonymous hours having for the most part the same sign. Hence the phenomena of the diurnal variation are not caused by the direct magnetic action of the sun and moon.

It is true that if we proceed another step in the approximation, and include in the values of the disturbing forces the terms containing the first powers of $\frac{b}{a}, \frac{c}{a}$, the former will produce in the resulting values of $\Delta \delta, \Delta H$, and $\Delta V$, terms containing $\sin 2 \theta, \cos 2 \theta$, and giving rise therefore to a semidiurnal inequality. But the coefficient $\frac{3 r}{D}$, by which these terms are multiplied, amounts in the case of the sun to $\frac{1}{8000}$ only, while in that of the moon it is about $\frac{1}{20}$; and the magnitude of the semidiurnal inequality should bear to that of the diurnal the ratios designated by these small fractions. The facts are altogether opposed to this result. The coefficient of the solar-diurnal inequality of the declination at Dublin, in the mean of the entire year, is $3^{\prime} \cdot 52$, while that of the semidiurnal is $2 \cdot 13$,-nearly two-thirds of the former. In the case of the lunardiurnal variation, the semidiurnal inequality exceeds the diurnal. 
XVI.-ON THE STORM OF THE 18Th OF APRIL, 1850.

Proceedings of the Royal Irish Academy, 1850.

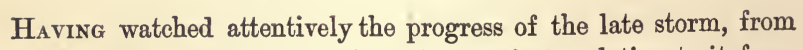
a favourable position, and collected some facts relative to it from the records of the Observatory, and from other sources, I avail myself of the present opportunity to lay them before the Academy. The phenomena were of a nature so unusual (I may say unexampled) in these climates, that it is desirable that some notice of them, however imperfect, should be placed on record; and the present summary of facts is offered, chiefly in the hope that it may serve as a nucleus to a more complete one. I shall limit myself, mainly, to those which have an immediate scientific bearing.

The morning of the 18th was fine in Dublin, with bright sunshine, light cirrous clouds being scattered loosely over the sky; at ten o'clock these became diffused, and the sky was evenly, but lightly overcast.

From the tracings of the self-registering anemometer, erected in Trinity College, it appears that on the 17 th, and during the morning of the 18th, the wind blew gently from the south-west. Towards noon, on the latter day, it gradually veered to the south, and continued at that point until the arrival of the storm. This veering of the wind, however, appears to have been confined to the lower current; the direction of the upper current, as estimated by the motion of the clouds, appeared to be nearly south-west.

The first indications of the approach of the storm were observed soon after three o'clock. Massive cumuli were seen in the western and south-western portions of the horizon. These became denser as they approached, until they formed a mass of an ash-grey 
colour, projected on a sky of a paler tint, while the rugged outliers from the mass, of the peculiar form which indicates a high degree of electrical tension, showed plainly that a storm was approaching. About half-past three o' clock it burst forth. The flashes of lightning (generally forked) succeeded one another with rapidity, and at length the roar of the thunder seemed continuous. Some persons who observed the phenomena from a distance were able to distinguish the two strata of oppositely electrical clouds, and to see the electrical discharges passing between them.

Hitherto the wind was light, and there was that peculiar closeness in the air which is the result of high temperature and excessive humidity. Shortly before four o'clock the rain commenced; this was followed almost immediately by discharges of hail, and at four, P. M., the terrific tornado, which was the grand and peculiar feature of this storm, reached us.

This gale, which appears to have been a true whirlwind, first sprung up from the south-east, driving the hail before it impetuously. It then suddenly, and apparently in an instant, shifted to the point of the compass diametrically opposite, and blew with increased violence from the north-west. The noise about this time of the shifting of the wind was terrific, and arose (as is conjectured respecting similar tropical phenomena) from the confused conflict of hail in the air. The size of the hailstones, as well as the vehemence of the gale, appeared to be greater during the second phase of the storm than the first. These masses, many of which were as large as a pigeon's egg, were formed of a nucleus of snow or sleet, surrounded by transparent ice, and this again was sacceeded by an opaque white layer, followed by a second coating of ice. In some of them I counted five alternations.

In less than ten minutes the tornado had passed. The wind returned to a gentle breeze from the south-west, the clouds dispersed, and the weather became beautiful. All the phenomena,the direction of the gale perpendicular to that in which the stormcloud was advancing, and the sudden reversal of that direction,seem to prove that it was a tornado, whose centre passed directly over the place of observation. It is evident, on comparing the direction of the wind when the whirl first reached this part of the town, with that of the progressive motion of the vortex itself, that its rotatory motion was retrograde, or in an opposite direction to that of the hands of a watch with its face upward. It is deserving 
of notice also, that in the northern hemisphere this is the invariable direction of the cyclones, or great revolving storms, to which the attention of meteorologists has been directed by Colonel Reid and Mr. Redfield. The late storm was, however, different from a cyclone, both in the dimensions of the vortex and in the causes from which it originated. The horizontal section of the cyclone where it meets the earth is often 500 miles in diameter; and the vortex is supposed to be the effect of two crossing currents of air, which generate a movement of rotation. In the tornado (to which species the late storm belonged) the vortex is of much smaller dimensions, and is produced by rapidly ascending currents of air, caused by the heating of a limited portion of the earth's surface under the action of the sun's rays. In the temperate zones, accordingly, it is never produced in winter.

The evidence relating to the direction of the gale, and its changes, as it passed over the College Park, is very complete and satisfactory. In the park, and garden adjoining, nineteen trees were rooted up and prostrated, eleven of them being trees of large size. Of these ten have fallen from the south-east, or under the action of the first half of the gale, and nine from the north-west. Their bearings have been accurately taken; and the general result is, that the mean direction of the south-east gale, as indicated by that of the trees, is S. $56^{\circ} \mathrm{E}$., and that of the north-west gale $\mathrm{N} .53^{\circ} \mathrm{W}$. I believe that these results are even more accurate than those furnished by the anemometer; and they prove that in this locality the direction of the wind was exactly reversed, and, therefore, that the centre of the vortex passed over the College.

A remarkable circumstance connected with the direction of the fallen trees is their great uniformity, the individual directions seldom differing more than $10^{\circ}$ from the mean. This is an indirect evidence of the great violence of the gale; and it proves, moreover, that the transition from the south-east to the north-west wind was immediate. There is greater regularity in the direction of the trees fallen from the north-west than in those which have been blown down from the opposite quarter. This may have arisen partly from the greater violence of the gale in the former direction; but it is partly also due to the circumstance that the trees which fell from the north-west are generally larger, and in a less inclosed portion of the ground. It may be mentioned also, that the trees which fell from the north-west generally lie to the 
southward of the others; there are, however, two large trees in the garden lying side by side, but in directions diametrically opposed.

It has been stated that in the College Park the shifting of the wind amounted to $180^{\circ}$; and it has been inferred that the centre of the vortex passed over that spot. From what has been said as to the nature of, the phenomenon, it will follow that in other localities, over which the vortex did not pass centrally, the wind must have shifted through different points of the compass, and through angles smaller in proportion to their distances from the centre. Thus, on the southern side of the line described by the centre of the vortex, the change of the wind should be from south to west, and on the northern side of the same line from east to north. We are not yet in possession of facts which bear upon this point; but from the limited dimensions of the vortex, and the consequent smallness of the distance necessary to produce such a variation, it is probable that evidence bearing upon it may be obtained. I shall only observe that, in seeking and comparing such evidence, care must be taken not to confound eddies arising from local obstructions with the general direction of the current.

The hours of observation at the Magnetical Observatory are 7 A. M., 10, 1 P.м., 4, 7, 10. The observations of the barometer, and of the dry and wet thermometers, made at these hours on the day of the storm, are the following:-

\begin{tabular}{|c|c|c|c|}
\hline Hour. & Barometer. & Dry Therm. & Wet Therm. \\
\hline $7 \mathrm{A.M}$. & $29 \cdot 944$ & 49.5 & $47 \cdot 4$ \\
\hline 10, & $29 \cdot 952$ & $54 \cdot 7$ & $50 \cdot 5$ \\
\hline 1 Р. M. & $29 \cdot 964$ & $58 \cdot 6$ & $52 \cdot 0$ \\
\hline $4 \quad$ & $29 \cdot 930$ & $56 \cdot 0$ & $52 \cdot 3$ \\
\hline 7 & $29 \cdot 944$ & $52 \cdot 6$ & $52 \cdot 0$ \\
\hline $10 "$ & $29 \cdot 936$ & $51 \cdot 0$ & $49 \cdot 6$ \\
\hline
\end{tabular}

The fall of rain, and melted hail, in Trinity College during the storm amounted to 0.596 of an inch; but it is probable that much of the hail was driven out of the receiver of the gauge by the wind.

It will be seen that the barometric fluctuation is small. It is stated, however, that a sudden and considerable fall of the barometer took place shortly before the storm. From tho obser- 
vations above given, at 1 P. M. and 4 P. M., it will be seen that the barometric equilibrium, if so disturbed, was soon restored.

I have collected, from the newspapers and other sources, such information as I could obtain respecting the area of the city visited by the gale, but it is as yet incomplete. It appears, however, that the diameter of the vortex was not very different from the length of the city from north to south; the gale having been limited by the Circular-road in these two directions. Hail fell, however, abundantly beyond the limits of the gale. Thus, at the gardens of the Royal Dublin Society, at Glasnevin, the damage done by the hail was very great; but it was limited to the roofs of the houses, the hail having fallen perpendicularly. The amount of the rain and melted hail registered there was 1.7 inches in 35 minutes.

Further information is wanting to enable us to determine exactly the progressive movement of the centre of the vortex. We are informed by the newspapers that a storm similar to that which visited Dublin, although not so severe, took place at Mullingar, about an hour and a half previously. If this be the same storm, the direction of the progressive movement must have been nearly from west to east, and its velocity about thirty miles an hour. This direction accords with that given by the observed limits of the storm on the northern and southern sides of the city; but it seems to have been modified, at the surface of the earth, by the lower current. The velocity of the rotatory movement was, of course, vastly greater than that of the progressive; but we have no direct measure of its amount.

The damage done in Dublin has been principally in the destruction of glass caused by the hail; but many chimneys have been thrown down, and many roofs dismantled, by the gale. The estimated amount of the loss sustained, as ascertained by the Metropolitan Police, is $£ 27,800$. Many houses were struck by the lightning; but, happily, there was no loss of life from that cause.

There seemed to have been a disturbance of electrical equilibrium, accompanied by rain, in many remote parts of Ireland on the same day. 
XVII.-NOTES ON THE METEOROLOGY OF IRELAND, DEDUCED FROM THE OBSERVATIONS MADE IN THE YEAR 1851, UNDER THE DIRECTION OF THE ROYAL IRISH ACADEMIY.

\section{Transactions of the Royal Irish Academy, Vol. XXII.}

THE science of meteorology is, perhaps more than any other, dependent upon co-operation and upon method. Individual observers may investigate successfully certain detached meteorological problems, such as the laws of the diurnal and annual changes of temperature, pressure, and humidity, at a given place; but little progress can be made in climatology, or in the knowledge of the greater movements of the atmosphere, and their relation to the non-periodic variations of temperature and pressure, without the co-operation of many observers distributed over a large area, and acting upon a common plan.

For this task the voluntary association of individuals is insufficient. However zealous such persons may be, it is not possible to bind them to that uniformity of system without which little can be effectively done. Observations taken at different hours, or by different methods, can never be compared satisfactorily; and any comparison will involve an amount of labour in the processes of reduction which may render them impracticable. In addition to this, certain rules of observation are imposed by the conditions of some of the great problems of meteorology ; and no co-operation in which these. rules are deviated from can contribute to thcir solution.

For these and other reasons it is desirable that, in every country, such observations should be provided for by the Government, and placed under the direction of one of its official departments. And 
there can be no doubt of the services which meteorology, properly studied, may be made to contribute to those interests which it is the duty of every Government to promote. The health of man, the operations of agriculture by which he procures his food, and many other of his material interests, are dependent upon climatological relations, which must be known and studied before they can be applied. Every one acknowledges the fact, that the salubrity of a district, and its adaptation (or the reverse) to particular human constitutions, is intimately connected with its meteorological conditions. And the same thing is true of all organized beings, and especially of those which are subservient to the uses of man. Thus, the question of the naturalization of exotic plants is, mainly, a meteorological problem, dependent upon the climatological relations of the region to which the plant is indigenous, and of that to which it is to be transferred; and the importance of obtaining accurate data for its solution will be recognised, when it is borne in mind that, in Europe, most of the plants useful to man belong to this class, and that those hitherto acclimatized probably bear a very small proportion to the whole. Lastly, the processes of cultivation, to which these vegetables are to be subjected, are also connected in an intimate manner with meteorological knowledge. We may instance this connexion in the operations of irrigation, and of drainage, both of which are dependent upon the knowledge of the amount of rain-fall in the district to be operated on.

It is true that meteorological science has been hitherto comparatively barren in such applications; and the fact itself, with many persons, would be accepted as evidence that abstract and practical knowledge are wholly separate and unconnected. But, when properly understood, it leads to a different conclusion. Superficial knowledge in this science can indeed yield but few practical results; and those by whom such results have been hitherto sought have expected to find them at the surface. There are indeed eases-such, for example, as the one last referred to-in which the connexion between meteorological science and its applications is obvious and simple, and in which, accordingly, that connexion has been traced and made use of. But in general it is otherwise. In a subject so complex as the laws which govern the aerial envelope of the earth, and where so many causes are in operation, practical applications can be obtained only from mature theoretical knowledge. Thus, it may be shown that the knowledge of the 
phenomena of temperature, requisite for the determination of the possible geographical limits of a single species of plants, is by no means inconsiderable; ${ }^{*}$ and when to this we add the consideration of the various other agencies which are at work in the atmosphere, all influencing vegetable life, it is plain that we are not in a condition to deduce any useful result connected with the distribution of species, until we have mastered a much larger amount of theoretical knowledge than is usually brought to bear in such deductions.

It would seem, therefore, to be the duty of the Government of every civilized state to provide the statistical data which have so many important bearings upon the material welfare of the people, and in the form best fitted for their discussion and examination. And to the lover of truth itself, for its own sake, the fulfilment of this duty would, fortunately, supply the wants of science in the most complete and satisfactory manner.

In many countries, accordingly, provision has been made by their respective Governments for the collection and diseussion of meteorological data upon a uniform and well-digested plan. The Government of Prussia appears to have taken the lead in this important labour. Its example has been followed by those of Russia, Austria, Bavaria, and Belgium ; and the names of Dove, Kupffer, Kreil, Lamont, and Quetelet, to whom the superintendence of these observations has been intrusted, afford the surest warrant of their successful prosecution. $\dagger$ But perhaps the most important undertaking of this nature is the recent organization of a system of meteorological observations at sea by the Government of the United States. There are, at the present time, nearly 1000 masters of ships, belonging to the navy and merchant services of

* For each plant there is a lower limit of temperature, below which it will cease to vegetate; while, in order that it may blossom and bear fruit, it must receive, between the two seasons of this minimum temperature, a certain amount of heat beyond this limit which is constant for each species. It is upon this integral of effectire heat, as has been shown by De Candolle, that the existence of the species depends. For information on this and other subjeets connected with the applications of neteorology, see the interesting introduction, by M. Martins, to the Ammuaire Meteorologique de la France.

+ The results of many of these series have been already published. Professor Dove has published the results of the observations made in Prussis in the years 1848 and 1849. The observations made at the Russian observatories have been published from time to time by M. Kupffer, in the Recueil des Cbservations faites dans l' Empire de Rusaic. The results of the Bavarian observations have been given by Dr. Iamont, in the Annalen der Meteorologie; and those of the Belgian system, in the admimble series of papers drawn up by M. Quetelet, Sur le Climat de Belgique. 
the United States, engaged in such observations; and the discussion of the results, by Lieutenant Maury, has led to many consequences of great value to the sciences of meteorology and hydrography, and rich in practical applications ta navigation. The Government of the United States has earnestly sought the co-operation of the Governments of the several maritime nations of Europe in this enterprise, and the demand has led to a Conference at Brussels, for devising a uniform system of meteorological observations at sea. This Conference, held in August and September last, was attended by individuals representing the respective Governments of Belgium, Denmark, France, Great Britain, the Netherlands, Norway, Portugal, Russia, Sweden, and the United States.

Impressed with the conviction that it was the duty of each country to take its part in these labours, and especially in the investigation of its own climatology, the Council of the Royal Irish Academy directed their attention, early in the year 1850, to the object of organizing a uniform system of meteorological observations in Ireland. And the peculiarity of the climate of this island perhaps more than balances the smallness of its extent, in giving an interest to the investigation. Situated as it is at the north-western extremity of Europe, and exposed to the full influence of the northern branch of the gulf stream which sweeps its western shores, its winter temperature is as high as that of the southern shores of the Euxine; while, on the other hand, the great precipitation of vapour, due to the same cause, gives it a summer heat as low as in parts of Finland.

The questions whose solution was aimed at by this measure, are thus stated by the Council in their second Report :-

1. The distribution of temperature, humidity, and rain, as affected by geographical position and by local circumstances; and the other phenomena of climate.

2. The effect of season (combined with the influences already referred to) upon the distribution of temperature, and the varying position of the isothermal lines from month to month.

3. The non-periodic variations of pressure, temperature, and humidity, and their connexion with the course and direction of the aerial currents.

4. The phenomena and laws of storms, whether revolving or otherwise. 
5. The periodical winds prevailing during certain seasons, and their modifications, from geographical position or local causes.

6. The course and rate of progress of atmospherio waves.

Concurrently with the meteorological observations, it was determined to institute an extended series of observations on the phenomena and laws of the tides around the coasts of Ireland, the results of which will be laid before the Academy by Mr. Haughton. The observations of the former class having been intrusted by the Council to my care, for reduction and discussion, I now proceed to lay before the Academy their principal results. It will be necessary, however, in the first instance to describe the plan of observation itself.

Stations.-The meteorological stations are:-

1. The Coast-guard stations at Portrush,Buncrana, Donaghadee, Courtown, Dunmore East, Castletownsend, Cahirciveen, and Kilrush; and, for observations of sea temperature only, those of Cushendall and Bunown. At all of these the observations were taken, with the permission of the Lords of the Treasury and of the Comptroller-General, by the boatmen belonging to the Coast-guard Service, the individuals having been specially selected for the duty by the inspecting officers, and having been instructed in the mode of observing by members of the Council of the Academy.

2. The Light-houses at Killough, Inishgort, and Killybegs, where, with permission of the Ballast Board, the observations were made by the light-keepers, instructed as before.

3. The Astronomical Observatories of Armagh and Markree, where the observations were taken by the Observatory assistants, with the permission of Dr. Robinson and Mr. Cooper; the Magnetical Observatory of Dublin, where they were made with the permission of the Board of Trinity College; and the stations at Portarlington and Athy, at which they were undertaken by Dr. Hanlon and Alfred Haughton, Esq.

In addition to these, the Academy has received observations, made upon the prescribed plan, from the Royal Observatory of Dublin, and from the Queen's Colleges at Belfast and Galway, which could not conveniently be included in the following discussions, not having extended over the whole of the period discussed. The observations at the Royal Observatory, and at the Queen's College, Belfast, commenced in April 18.51, and have been conti- 
nued to the present time; the necessity for their omission is the more to be regretted, as they appear to have been made with every possible care.

The positions of the several stations, together with the heights (in feet) of the cisterns of the barometers above the mean sea level," are given in the annexed Table. They are shown in Plate $\mathbf{r}$.

\section{Positions of the Meteorological Stations.}

\begin{tabular}{|c|c|c|c|c|}
\hline Station. & Lat. & Long. & $\begin{array}{c}\text { Height } \\
\text { above } \\
\text { Sea. }\end{array}$ & Locality. \\
\hline Portrush, . . & $55^{\circ} 13^{\prime}$ & $6^{\circ} 41^{\prime}$ & 29 & Coast-guard Station. \\
\hline Buncrana, . & 558 & 727 & 48 & Do. \\
\hline Donaghadee, · & 5438 & 533 & 16 & Do. \\
\hline Killybegs, . • & 5434 & 827 & 20 & Light-house. \\
\hline Armagh, . . & 5421 & 639 & 211 & Observatory. \\
\hline Killongh, • • & 5413 & 540 & 23 & Light-house. \\
\hline Markree, . . & 5414 & 828 & 132 & Observatory. \\
\hline Westport, · & 5350 & 937 & 17 & Light-house. \\
\hline Dublin, . • . & 5321 & 615 & 19 & Magnetical Observatory. \\
\hline Portarlington, . & $53 \quad 9$ & 712 & 230 & Dr. Hanlon's residence. \\
\hline Athy, . . . & 530 & 658 & 200 & Mr. Haughton's residence. \\
\hline Courtown, . . & 5239 & 613 & 34 & Coast-guard station. \\
\hline Kilrush, . . & 5238 & 930 & 19 & Do. \\
\hline Dunmore, . & 528 & 659 & 66 & Do. \\
\hline Cahirciveen, & 5156 & 1013 & 52 & Do. \\
\hline Castletownsend, & 5133 & 99 & 18 & Do. \\
\hline
\end{tabular}

* At Portarlington and Athy these heights have been taken from the Contour Maps of the Ordnance Survey, and must, therefore, be considered as only approximate: at all the other places they have been obtained by actual levelling from the nearest Ordnance bench-marks. 
The instruments were furnished by the Academy to the Coastguard and Light-house stations, and were constructed under the direction of the Council, and upon a common plan. They consist of a barometer ; a pair of ordinary thermometers (dry and wet bulb); a pair of self-registering thermometers; a wind-vane; Lind's anemometer; a rain-gauge; and (at the Coast-guard stations) a thermometer adapted to the observation of sea temperature. The thermometers were previously compared in Dublin with the standards belonging to the Magnetical Observatory, and their errors exactly determined. The barometers were compared with the Dublin standard, after they were placed at the several stations, by means of good portable barometers; and the heights of the cisterns above the sea were ascertained by levelling. All this was done by members of the Council, under whose superintendence the instruments were erected.

The four thermometers at every station were inclosed in a shallow box with a sloping roof, and wire-gauze front. $\Lambda$ vertical gnomon was fixed at most of the stations in the window-sill of the guard-house, for the purpose of determining the time of noon; and the observers were furnished with a Table of the equation of time, computed for the year 1851, and for the mean longitude of Ireland. The barometers were put up, generally, in the guard-house of the station.

Plan of Observation.-It is probable that over a tract of country so limited as this island, the distribution of temperature, humidity, and rain, does not vary materially from one year to another; and that, consequently, a tolerable approximation to the laws of this distribution may be obtained from the results of a single year, if every precaution be adopted to insure the perfect comparability of the results. It was arranged, accordingly, that the observations should be continued at the Coast-guard stations until the end of the year 1851, в0 as to embrace a period of at least one year, reckoned from the time when the observers had acquired the power of observing with accuracy. The monthly means for this year may be reduced to their absolute mean values, by the help of the more extended series of observations made in Dublin, by which the deviations of any monthly result from its absolute mean value is sufficiently known.

The Committec, upon whom the duty of superintending these 
arrangements devolved, were desirous that the plan of observation should be the least onerous that could lead satisfactorily to the results aimed at. One of the principal of these-the determination of the movements of masses of air, whether in storms, or in the displacement of atmospherio waves,-demands, as has been said, that the observations should be taken at equal intervals of time; and the only condition imposed by the other meteorological problems is, that these times should be so chosen as to furnish the daily means of the elements sought. Now any three observations, taken at equal intervals throughout the day, are sufficient to eliminate the diurnal variation, and therefore to give the daily means of all the meteorological elements; and undoubtedly, where such a system is practicable, the observations should be taken at 6 A. M., 2 P.м., and 10 P. M., which has been shown to be preferable to any other eighthourly group, for meteorological purposes.

At the Coast-guard stations, however, such a plan of observation would have been incompatible with the regular duties of the men; and it was advisable to adopt a less complete system, which might be followed at all the stations, and in which interruptions were not likely to occur. Fortunately, two observations in the day, taken at equal intervals, are sufficient to give the daily means of all the meteorological elements, excepting the atmospheric pressure; and, as the diurnal variation of the pressure is very small-much smaller than its irregular fluctuations in these latitudes, -it may be disregarded, and the objects for which the present system was instituted may be attained by taking two observations in the day, at homonymous hours.

The best pair of homonymous hours, for the determination of the mean temperature, and nearly also for that of the mean humidity, are $9^{\mathrm{h}} 46^{\mathrm{m}}$ A.M., and $9^{\mathrm{h}} 46^{\mathrm{m}}$ P.M. Limiting themselves to the exact hours, the Committee might accordingly have chosen either 9 A.M. and 9 P.м., or 10 A.M. and 10 P.M.; the former pair was adopted, its superior convenience seeming to outweigh the advantage of the latter in accuracy.

For the fuller elucidation of some of the questions proposed, it was further arranged that hourly observations should be taken at all the stations for twenty-four hours, at the equinoxes and solstices, according to the plan laid down by Sir John Herschel. It was likewise provided, that hourly observations should be taken occasionally, under special circumstances, such as storms, unusual disturbances of barometric equilibrium, \&c. 
For further details of the plan of observation, the reader is referred to the "Instructions" prepared by the Council of the Academy. I now proceed to the results of the observations.

\section{Temperature of the Air.}

Corrections. - It has been already stated, that the thermometers employed in measuring the temperature and humidity of the air were carefully compared with a standard thermometer, and their errors noted. When the errors differed by more than $0^{\circ} \cdot 2$ in different parts of the scale, the instrument was rejected; when they did not, the mean of the observed errors was adopted as a constant error for the whole scale of the instrument.

It has been stated that the mean of the temperatures observed at 9 A.M. and 9 P.M. is, very nearly, the mean of the entire day. The small corrections required, in order to reduce the former to the latter, are obtained from the bi-hourly observations of temperature made at Dublin in the years 1840-1843. The following Table contains the results of that series, and gives the mean differences between the temperature at each hour of observation, and that of the entire day :- 


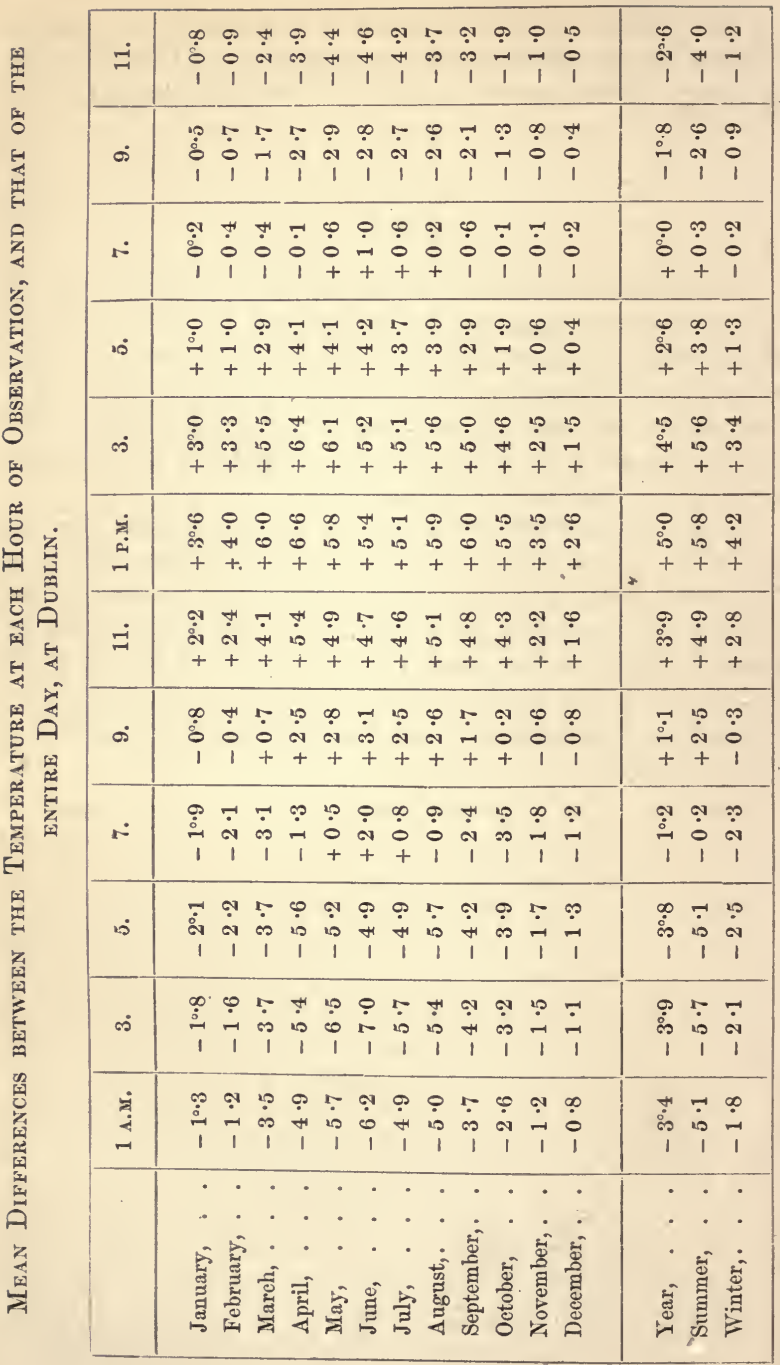


From the preceding Table we obtain the following corrections, which are to be applied to the means of the observed temperatures at 9 A.M. and 9 P.M., in order to reduce them to the mean of the day :-

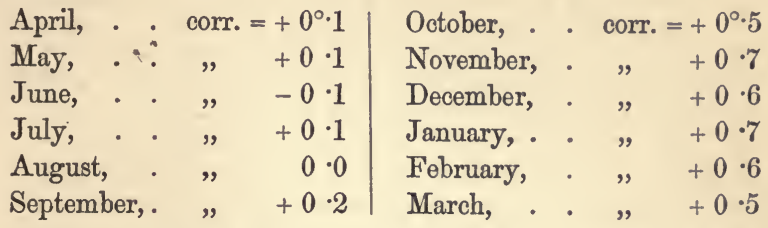

It hence appears that the correction is nearly constant throughout the summer, and throughout the winter months, respectively. The mean summer correction is $+0^{\circ} .1$; the mean winter correction $+0^{\circ} \cdot 6$.

Mean Monthly Temperatures. - The mean temperatures have been obtained, at all but three of the stations, from the observations at 9 A.M. and 9 P.M., by the application of the preceding corrections. At Markree the observations were taken at 10 A.M. and 10 P.M. ; and the reducing numbers are therefore somewhat different, and smaller in amount. At Portarlington and Athy the observations were taken but once in the day, namely, at 9 A.M. ; and at these stations, accordingly, the mean temperatures are inferred from the maximum and minimum temperatures, as given by the self-registering thermometers. The formula employed is that of Kæmtz, viz. :-

$$
\text { mean temp. }=\min +a(\max .-\min .)
$$

The mean value of the coefficient," as deduced from the observations at the observatories of Armagh, Markree, and Dublin, is $a=0 \cdot 41$.

The following Table contains the resulting values of the mean temperature for the several months of the year 1851 :-

* The coefficient in Kæmtz's formula appears to vary considerably at different places, both in its mean amount, and in the law of its variation from month to month. At Armagh and Markree its greatest value is in December, and its least in July ; at Dublin, it is tho reverse. I have taken above the mean of the yearly values for the three stations. 


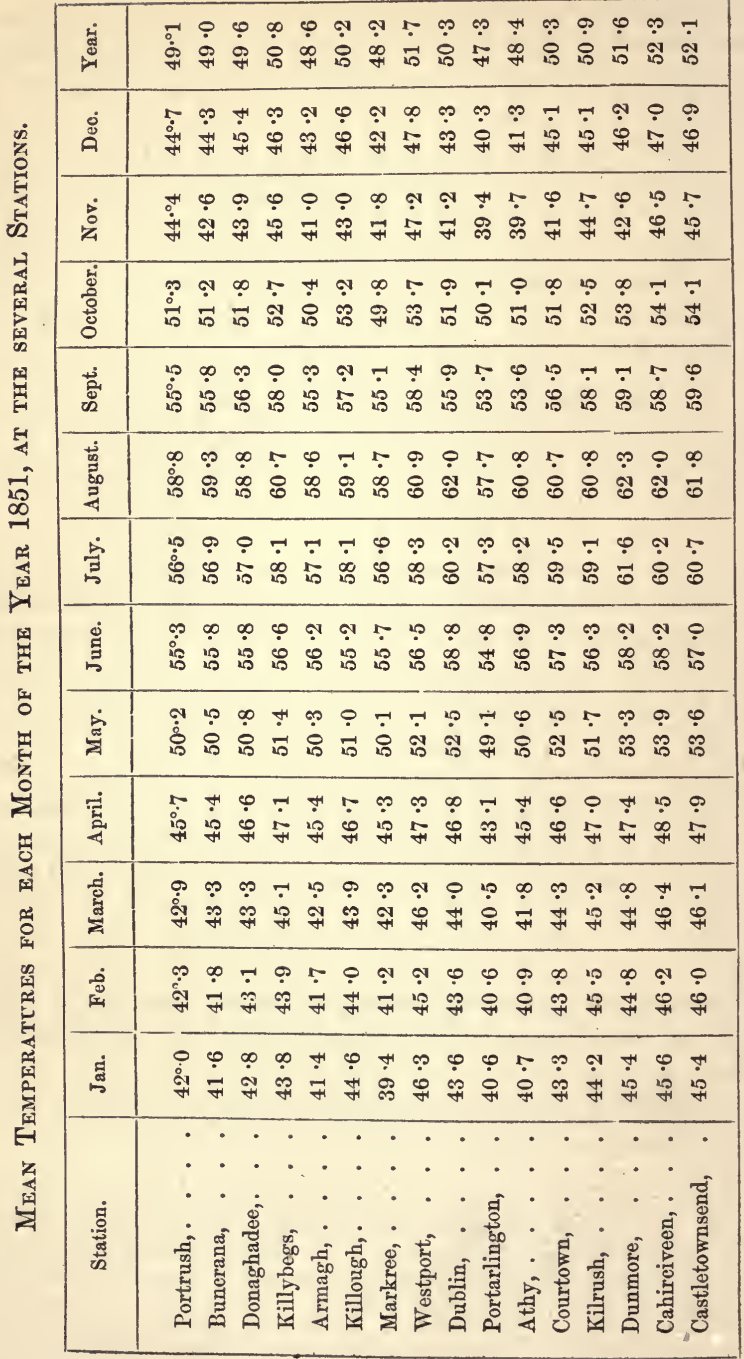


Before we proceed to discuss the mean temperatures in the several months of the year 1851, it is important that we should know the absolute mean temperatures at some one station, and thereby the deviations from the means in the several months of the year in question. Over a tract of country so limited as Ireland, these deviations, will not differ much in different localities; and therefore, knowing them for one station, we are enabled to reduce the results of the single year, with probably sufficient exactness, to their absolute mean values at all the rest.

The absolute mean temperatures of the several months are known, at Dublin, by means of the series of observations made during twelve years at the Magnetical Observatory. The monthly mean temperatures, deduced from that series, are given in the following Table. From the year 1840 to 1843 , inclusive, the daily means are those of twelve equidistant hours; from 1844 to 1850 , inclusive, they are inferred from the temperatures observed at 10 A.M. and 10 P.M. ; and in 1851, from those at 9 A.M. and 9 P.M. In the last line of the Table are given the deviations of the monthly means in 1851, from the mean monthly means, as deduced from the twelve years.

It will be seen from the Table, that the temperature in the months of January, February, and October, 1851, was higher than the average temperature, while, in November, it was considerably lower. The mean temperature of the entire year was only $0^{\circ} \cdot 3$ above the average.

The depression of temperature in the month of November is a remarkable case of those non-periodio fluctuations to which the attention of meteorologists has been drawn by Professor Dove. This fluctuation appears to have proceeded from north-east to southwest, and to have been nearly obliterated when it reached the western coast of the island. At the northern and eastern stations the unusual cold began on the 24th day of the month ; at the southern and western it commenced on the 26th and 27th. It reached its maximum about the 30th, and ceased about the 3rd of December. When we compare the mean temperatures of November and December at Killough, Dublin, Courtown, and Dunmore, on the eastern coast, with those at Killybegs, Westport, Kilrush, and Cahirciveen, on the western, we observe that the tomperature of November is less than that of December by $3^{\circ} \cdot 3$ at the former stations, while the defect is only $0^{\circ} \cdot 6$ at the latter. 


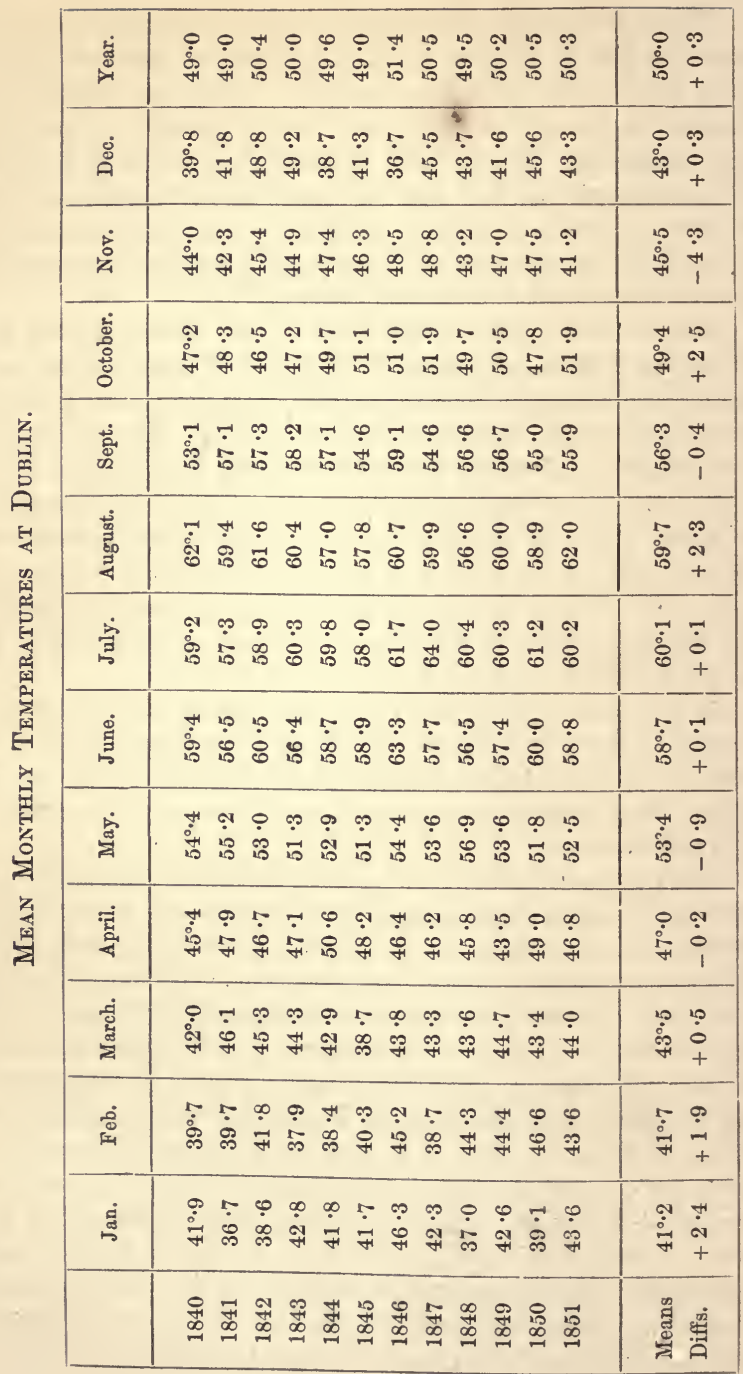


Upon a comparison of the mean yearly temperatures of the several stations, we observe that those of the inland stations are in defect, as compared with the corresponding coast stations. Thas the mean temperature of Armagh (48.6) is less than that of Donaghadee by $1^{\circ}$, and less than that of Killough by $1^{\circ} \cdot 6$. The mean temperature of Markree $\left(48^{\circ} \cdot 2\right)$ is less than that of Killybegs by $2^{\circ} \cdot 6$, and than that of Westport by $3^{\circ} .5$. The mean temperatures of Portarlington and Athy $\left(47^{\circ} \cdot 3\right.$ and $\left.48^{\circ} \cdot 4\right)$ are in like manner in defect, when compared with those of Dublin and Courtown, and by an intermediate amount. I shall return to this subject hereafter, and merely notice it at present for the purpose of observing that no satisfactory conclusion can be drawn as to the dependence of temperature upon geographical position, unless the inland and coast stations be compared separately.

Confining ourselves for the present to the coast stations, which are the most numerous and the most widely distributed, we observe that there is an increase of mean annual temperature in proceeding from north to south of the island, the mean temperature of Portrush and Buncrana being $49^{\circ} \cdot 0$, and that of Dunmore, which is nearly on the intermediate meridian, $51^{\circ} \cdot 6$. Similarly there is an increase of temperature in proceeding from east to west, the mean temperature of Killough and Dublin being $50^{\circ} .2$, and that of Westport, which is nearly on the intermediate parallel, $51^{\circ} .7$.

But for an accurate determination of the rate of increase of temperature in the two directions, it is necessary to combine the results by the method of least squares. For this purpose let $t$ denote the observed mean temperature of any month, at any given station; $T$ the probable temperature of the same month at an assumed central station; and let the distances (in geographical miles) of the former from the latter, measured on the meridian and parallel of latitude to the north and west, respectively, be denoted by $y$ and $x$; then, if $V$ and $U$ be the increase of temperature corresponding to a single mile in each direction,

$$
t=T+U x+V y .
$$

There will be a similar equation for each station; and combining them by the method of least squares, we shall obtain the most probable values of the unknown quantities $T, U$, and $V$.

The simplest mode of employing this method in the present instance is to take, as the arbitrary contral station, that whose 
latitude and longitude are the arithmetical means of the latitudes and longitudes of the stations of observation. The resulting equations are thus reduced to the following :-

$$
\begin{gathered}
n T=\Sigma(t), \\
U \Sigma\left(x^{2}\right)+V \Sigma(x y)=\Sigma(x t), \\
U \Sigma(x y)+V \Sigma\left(y^{2}\right)=\Sigma(y t) .
\end{gathered}
$$

For the reason already stated, I shall employ in this calculation only the results obtained at the coast stations. These are, in the order of latitude, Portrush, Buncrana, Donaghadee, Killybegs, Killough, Westport, Dublin, Courtown, Kilrush, Dunmore, Cahirciveen, Castletownsend. The mean latitude and longitude of these stations are $53^{\circ} \cdot 29^{\prime}$, and $7^{\circ} \cdot 39^{\prime}$ respectively. And we find

$$
\Sigma\left(x^{2}\right)=39094, \quad \Sigma(x y)=-22569, \quad \mathrm{Z}\left(y^{2}\right)=65811 .
$$

Substituting and eliminating between the second and third equations, we obtain-

$$
\begin{aligned}
& U=\cdot 0000319 \Sigma(x t)+\cdot 0000109 \Sigma(y t) ; \\
& \nabla=\cdot 0000109 \Sigma(x t)+\cdot 0000189 \Sigma(y t) .
\end{aligned}
$$

By these formulas the values of $T, U$, and $V$, for each month are calculated. They are given in the following Table.

The values of $U$ and $V$ being known, the positions of the isothermal lines are determined. The inclination of the isothermal lines to the meridian, measured from north to west, $u$, and the rate of increase of temperature in the direction perpendicular to them, $W$, are known by the formulas

$$
\tan u=\frac{V}{U}, \quad W=\sqrt{ }\left(U^{2}+V\right)^{2} .
$$

The values of $u$ and $W$ for the several months are given in the Table. 
ON THE METEOROLOGY OF IRELAND.

Elememts of Monthly Isothermal Lines.

\begin{tabular}{|c|c|c|c|c|c|c|c|}
\hline \multirow{2}{*}{\multicolumn{2}{|c|}{$\approx$}} & \multicolumn{2}{|c|}{$T$} & \multirow{2}{*}{$U$} & \multirow{2}{*}{$V$} & \multirow{2}{*}{$W$} & \multirow{2}{*}{$u$} \\
\hline & & 1851. & Mean. & & & & \\
\hline January, . & - & $44^{\circ} \cdot 1$ & $41^{\circ} \cdot 7$ & $+\cdot 0080$ & $-\cdot 0102$ & $\cdot 0130$ & $52^{\circ}$ \\
\hline February, & - & $44 \cdot 2$ & $42 \cdot 3$ & $+\cdot 0093$ & $-\cdot 0119$ & .0151 & 52 \\
\hline March, . & - & $44 \cdot 6$ & $44 \cdot 1$ & $+\cdot 0131$ & $-\cdot 0064$ & $\cdot 0146$ & 26 \\
\hline April,. . & . & $46 \cdot 9$ & $47 \cdot 1$ & $+\cdot 0043$ & $-\cdot 0070$ & $\cdot 0082$ & 59 \\
\hline May, . & - & $52 \cdot 0$ & $52 \cdot 9$ & $+\cdot 0012$ & $-\cdot 0139$ & $\cdot 0140$ & 85 \\
\hline June, . & - & $56 \cdot 8$ & $56 \cdot 7$ & $-\cdot 0031$ & $-\cdot 0109$ & .0114 & 106 \\
\hline July, · & - & $58 \cdot 9$ & $58 \cdot 8$ & $-\cdot 0049$ & $-\cdot 0202$ & $\cdot 0208$ & 104 \\
\hline August, . & - & $60 \cdot 6$ & $58 \cdot 3$ & $+\cdot 0029$ & $-\cdot 0121$ & $\cdot 0124$ & 77 \\
\hline September, & - & $57 \cdot 4$ & $57 \cdot 8$ & $+\cdot 0101$ & $-\cdot 0090$ & $\cdot 0135$ & 42 \\
\hline October, . & - & $52 \cdot 7$ & $50 \cdot 2$ & $+\cdot 0059$ & $-\cdot 0070$ & $\cdot 0092$ & 50 \\
\hline November, & . & $44 \cdot 1$ & $48 \cdot 4$ & $+\cdot 0304$ & $+\cdot 0077$ & $\cdot 0313$ & -14 \\
\hline December, & - & $45 \cdot 7$ & $45 \cdot 4$ & $+\cdot 0103$ & $-\cdot 0017$ & $\cdot 0104$ & 9 \\
\hline Year, . & . & $50 \cdot 7$ & $50 \cdot 3$ & $+\cdot 0073$ & $-\cdot 0085$ & $\cdot 0112$ & $49^{3}$ \\
\hline
\end{tabular}

We see then that, on the mean of the whole year, the isothermal lines are inclined to the meridian by the angle $\mathrm{N} .49^{\circ} \mathrm{W}$.; and that the temperature increases in a direction perpendicular to these lines, by:0112 of a degree for each geographical mile, or at the rate of 1 degree for 89 miles. The increase of temperature, in proceeding from north to south, is $V=\cdot 0085$, or $1^{\circ}$ in 118 geographical miles; the corresponding increase, in proceeding from east to west, is $U=\cdot 0073$, or $1^{\circ}$ in 137 geographical miles.

We learn further, that the mean annual isothermal lines furnish a very inadequate representation of the progression of temperature; and that when we follow the course of these lines from month to month, we find them to vary within very wide limits. The extreme positions of these lines, as given in the preceding Table, are those for the months of June and November. But the result obtained for the latter month must, I think, be regarded as anomalous, on account of the irregularity in the distribution of temperature already 
noticed; and, rejecting it, the extreme positions correspond to the two solstitial months. They are the following:-

$$
\begin{array}{lll}
\text { June, . . } & u=\mathrm{N} .106^{\circ} \mathrm{W} ., & W=\cdot 0114, \\
\text { December, } & u=\mathrm{N} . \quad 9 \mathrm{~W} ., & W=\cdot 0104 ;
\end{array}
$$

so that the direction of the isothermal lines varies through an angle of $97^{\circ}$ in the course of the year, being nearly parallel to the meridian in December, and nearly perpendicular to it in June. (See Plate I.)

We may now employ the formula

$$
t=T+U x+V y,
$$

to deduce the probable temperature at any place, and compare it with that actually observed; we shall thus find the effect [due to local causes. Making this calculation for the four inland stations,

\begin{tabular}{|c|c|c|c|c|c|}
\hline Month. & & Armagh. & Markree. & Portarlington. & Athy. \\
\hline January, & . . & $1^{\circ} \cdot 8$ & $4^{\circ} \cdot 4$ & $3^{\circ} \cdot 6$ & $3^{\circ} \cdot 5$ \\
\hline February, & . . & $1 \cdot 5$ & $2 \cdot 7$ & $3 \cdot 7$ & $3 \cdot 4$ \\
\hline Mareh, . & . . & $1 \cdot 3$ & $2 \cdot 4$ & $4 \cdot 1$ & $2 \cdot 7$ \\
\hline April, . & . . & $1 \cdot 0$ & $1 \cdot 4$ & $3 \cdot 9$ & $1 \cdot 6$ \\
\hline May, . & . . & $0 \cdot 9$ & $1 \cdot 3$ & $3 \cdot 1$ & $1 \cdot 8$ \\
\hline June, . & . . & $0 \cdot 1$ & $0 \cdot 5$ & $2 \cdot 2$ & $0 \cdot 3$ \\
\hline July, . & . . & $0 \cdot 9$ & $1 \cdot 2$ & $2 \cdot 0$ & $1 \cdot 4$ \\
\hline August, & . . & $1 \cdot 3$ & $1 \cdot 4$ & $3 \cdot 1$ & $0 \cdot 1$ \\
\hline September, & . . & $1 \cdot 3$ & $2 \cdot 2$ & $3 \cdot 7$ & $3 \cdot 8$ \\
\hline October, & . . & $1 \cdot 7$ & $2 \cdot 7$ & $2 \cdot 6$ & $1 \cdot 8$ \\
\hline November, & . . & $2 \cdot 4$ & $3 \cdot 5$ & $4 \cdot 1$ & $3 \cdot 4$ \\
\hline December, & . . & $2 \cdot 1$ & $3 \cdot 7$ & $3 \cdot 3$ & $4 \cdot 2$ \\
\hline Year, . & - : & $1 \cdot 4$ & $2 \cdot 3$ & $3 \cdot 4$ & $2 \cdot 3$ \\
\hline
\end{tabular}
we obtain the results given in the following Table:-

Excess of Calculated Temperatures at Inland Stations. 
We learn that the defect of temperature due to inland position is, as might have been expected, least in summer and greatest in winter. A small part of this defect is due to elevation; but it is easily eliminated. The mean height of the instruments at the coast stations above the level of the sea is 30 feet. We have, therefore, only to subduct this from the known heights at the inland stations, and to correct for the difference of level at the rate of $1^{\circ} \mathrm{Fahr}$. for 276 feet, which is the mean of the determinations made by $\mathrm{Mr}$. Welsh in his balloon ascents, for the lower portion of the atmosphere lying beneath the great vapour plane. The mean yearly results at the four inland stations, thus corrected, are as follows:-

\begin{tabular}{|c|c|c|c|c|}
\hline & $\begin{array}{c}\text { Observed } \\
\text { Defect. }\end{array}$ & $\begin{array}{c}\text { Height } \\
\text { above Sea. }\end{array}$ & Correction. & $\begin{array}{c}\text { Reduced } \\
\text { Defect. }\end{array}$ \\
\hline Armagh,. . . & $1^{\circ} \cdot 4$ & 211 & $-0^{3} \cdot 7$ & $0^{\circ} .7$ \\
\hline Markree,. . . & $2 \cdot 3$ & 132 & -0.4 & $1 \cdot 9$ \\
\hline Portarlington, . & $3 \cdot 4$ & 230 & $-0 \cdot 7$ & $2 \cdot 7$ \\
\hline Athy, . . . & $2 \cdot 3$ & 200 & -0.6 & $1 \cdot 7$ \\
\hline & & & \multicolumn{2}{|c|}{ Mean $=1^{\circ} \cdot 8$} \\
\hline
\end{tabular}

Diurnal Ranges of Temperature.

Climatology depends upon the ranges of temperature (whether diurnal, monthly, or annual), no less than upon mean values; and their investigation is accordingly a necessary part of the present inquiry. In the present series of observations, the diurnal ranges of temperature are given by means of the results obtained with selfregistering thermometers. These results are the least satisfactory portion of the whole series. It is well known that the ordinary self-registering thermometers are extremely apt to get out of order, the maximum, by the index becoming entangled in the mercury, and the minimum, by the distillation of the spirit into the upper part of the tube; and although the observers were carefully instructed in the mode of remedying these derangements, no one (I believe) who has handled such instruments will wonder that men previously unaccustomed to them should have sometimes failed, in what is in all cases a somewhat delicate operation. 
But there is another source of error affecting the maximum thermometer, which it is still more difficult to avoid. If the instrument be exposed to the influence of radiation for any portion of the day, however short, it will, from its construction, retain the impression made upon it; and, consequently, if the abnormal temperature to which it has been thus subjected exceeds the greatest temperature of the air in the day, an erroneous result will be recorded. The difficulty of guarding thermometers completely from such influences is well known; and although some trouble was taken to insure this protection, the observations themselves show that it was not effective at all the stations. I have, accordingly, been compelled to reject a portion of the results obtained with the maximum thermometer, as defective from this cause.

In the following Table are given the diurnal ranges deduced from the maximum and minimum temperatures, combined in yearly and half-yearly periods, retaining only those stations at which one or other of the two half-years is complete :-

Diurnal Rajges (Half-yearly and Yearly Means).

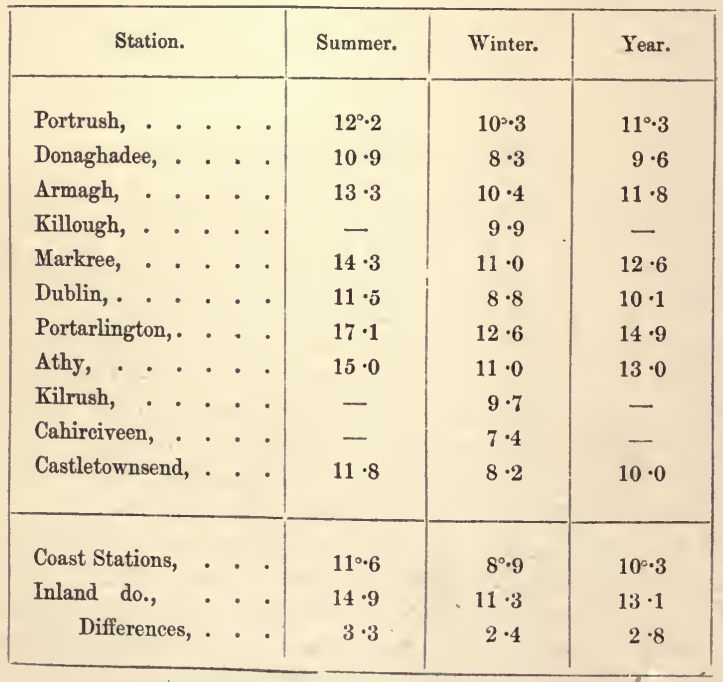


From the mean results of the preceding Table, we learn that the diurnal range is greater at the inland than at the coast stations, the mean excess being 2.8 degrees. The excess is greater in summer than in winter, being $3^{\circ} .3$ in the former, and $2^{\circ} .4$ in the latter season. .

We are now in a position to refer to one, at least, of the practical inferences which may be deduced from the preceding results.

The climatological conditions connected with temperature, which favour the prevention or cure of pulmonary diseases, are, firstly, a high winter temperature; and secondly, a small amount of diurnal range. It has been already stated that Ireland is well circumstanced as to these conditions; let us now inquire which is its most favourable region as respects them.

The months of lowest temperature in Ireland, and which are on that account the most trying to the sufferers from the diseases referred to, are those of December, January, February, and March. During these months the mean temperature varies very little in Ireland, the mean range at Dublin varying from $41^{\circ} .7$ in January, to $45^{\circ} .4$ in March, or only 3.7 degrees. Now the mean direction of the isothermal lines for these four months is $\mathrm{N} .37^{\circ} \mathrm{W}$.; so that the highest mean temperature for these months is to be found on the south-western coast, not far from Valentia.

The second condition above mentioned, although not frequently taken into account, is, perhaps, still more important. In proof of this it may be mentioned that in Norway, which is remarkable for the small amount of the diurnal range of temperature, consumption is uncommon, even in the highest latitudes; while in parts of Sweden, where this condition does not hold, it is prevalent. Now, we learn from the preceding Table that, among the stations at which observations were made in 1851 , the winter diurnal range of temperature is least at Cahirciveen. Both conditions, therefore, point to the south-western coast of Kerry as the region in Ireland most favourable to patients affected with these formidable maladies.

I am not in possession of any statistical data bearing upon this question, and am therefore unable to say how far the conclusion thus drawn is borne out by facts. 


\section{Temperature of the Sea.}

Provision was made that the temperature of the sea should be observed at all the places at which tidal observations were taken. For this purpose each station was furnished with a thermometer, having its bulb inclosed within a small reservoir of copper, for the double purpose of guarding it from accident, and of protecting it (by means of the contained water) from rapid changes of temperature, when it was lifted into the air for observation. The observer was instructed to note its indications twice in the day, at intervals of about twelve hours, the thermometer being attached to a pole, and plunged to the depth of about one foot in deep water. The diurnal change of the temperature of the sea being very small, it is completely eliminated by two such observations. At many of the stations the instrument was lost, or broken, in the attempt to use it during boisterous weather. We are, therefore, only in possession of the results from six stations, which are contained in the following Table.

In the last two lines of the Table are given the mean results of the six stations, and the differences between them and the mean of the entire year. These numbers accordingly exhibit the law of the annual variation of sea temperature, around the coasts of Ireland ; and the remarkable regularity in their progression shows that, even from the results of a single year, we obtain a close approximation to the actual law. 
ON THE METEOROLOGY OF IRELAND.

339

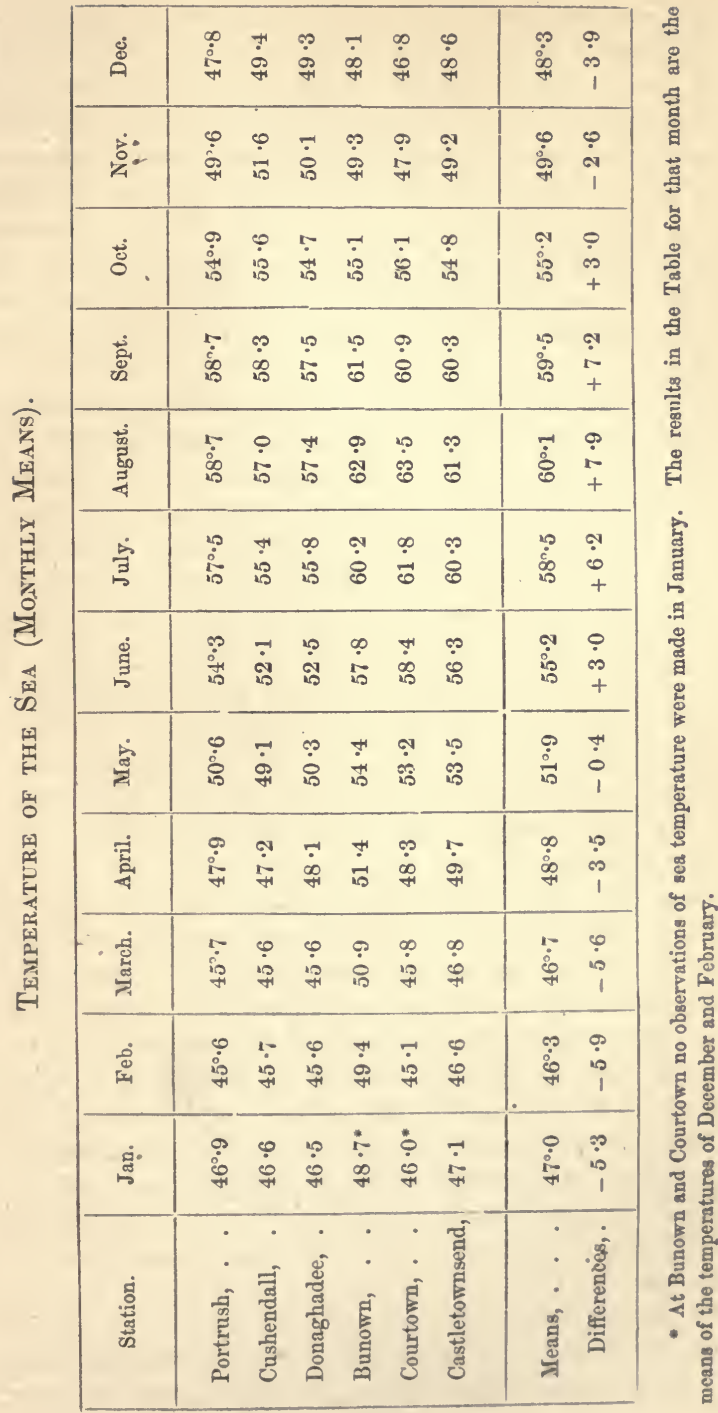


We learn from these numbers that the annual variation of the sea-temperature, at the surface, differs considerably from that of the air above it, the difference consisting chiefly in a retardation of the epochs of maximum and minimum. Thus the minimum temperature occurs in the middle of February, and the maximum in the middle of August,-or about a month after the corresponding epochs of the temperature of the air. The annual range is also, as might have been expected, considerably less than that of the air. These results accord sufficiently well with the conclusions drawn by Kæmtz, from a comparison of the results of many voyagers.

But the most interesting result is that concerning the relation between the temperature of the sea at the surface, and that of the superincumbent air. Upon this subject the greatest discordance exists in the statements of different observers. According to Humboldt, the mean temperature of the Atlantic Ocean, at the surface, is in all cases higher than that of the atmosphere above it. This conclusion is confirmed by the observations of Peron and Fitzroy, and is contradicted by those of Irving, Forster, and Kotzebue. From an elaborate discussion of the observations of many voyagers, Kæmtz infers that the temperature of the sea is less than that of the air over the land in the lower latitudes, while in the higher latitudes it is greater. The original conclusion of Humboldt, however, seems to be placed beyond all doubt by the recent observations of Captain Duperrey, which appear to be more numerous, and taken with more precautions to insure accuracy, than any preceding. It seems now to be generally admitted that, in the temperate and polar regions, the temperature of the sea is higher than that of the air; and the only question that remained was as to the tropics. Now the observations of Duperrey were made all round the globe, between $10^{\circ} \mathrm{N}$. and $10^{\circ} \mathrm{S}$. latitude; and they were taken at intervals of four hours, so as completely to eliminate the effects of the diurnal change. From these observations it appears that the temperature of the sea is higher than that of the air within the zone already mentioned, the mean excess in the Atlantic being $0^{\circ} .83$ Fahr., and in the Great Ocean about half that amount.

The present observations possess much interest in connexion with these questions. In order to perceive their bearing, I have, in the Table which follows, given the half-yearly and yearly 
means of the sea-temperature at the several stations, together with the differences between them and the corresponding means of the temperature of the air. At Cushendall and Bunown no observations of the temperature of the air were actually made; and for these stations, consequently, the latter means are caloulated from the isothermal lines.

Temperature of the Sea (Yearly and Half-yearly Means).

\begin{tabular}{|c|c|c|c|c|c|c|}
\hline & \multicolumn{2}{|c|}{ Summer. } & \multicolumn{2}{|c|}{ Winter. } & \multicolumn{2}{c|}{ Year. } \\
\cline { 2 - 7 } Station. & Temp. & Excess. & Temp. & Excess. & Temp. & Excess. \\
\hline & & & & & & \\
\hline Portrush, . . & $54^{\circ} \cdot 6$ & $+1^{\circ} \cdot 0$ & $48^{\circ} \cdot 4$ & $+3^{\circ} \cdot 8$ & $51^{\circ} \cdot 5$ & $+2^{\circ} \cdot 4$ \\
Cushendall, . & $53 \cdot 2$ & $-1 \cdot 0$ & $49 \cdot 1$ & $+4 \cdot 4$ & $51 \cdot 1$ & $+1 \cdot 7$ \\
Donaghadee, . & $53 \cdot 6$ & $-0 \cdot 6$ & $48 \cdot 6$ & $+3 \cdot 6$ & $51 \cdot 1$ & $+1 \cdot 5$ \\
Bunown, . . & $58 \cdot 0$ & $+2 \cdot 4$ & $50 \cdot 2$ & $+3 \cdot 2$ & $54 \cdot 1$ & $+2 \cdot 8$ \\
Courtown, . . & $57 \cdot 7$ & $+2 \cdot 2$ & $47 \cdot 9$ & $+3 \cdot 0$ & $52 \cdot 8$ & $+2 \cdot 6$ \\
Castletownsend, & $56 \cdot 9$ & $+0 \cdot 1$ & $48 \cdot 9$ & $+1 \cdot 5$ & $52 \cdot 9$ & $+0 \cdot 8$ \\
\hline Mean diff., & & $+0^{\circ} \cdot 7$ & & $+3^{\circ} \cdot 3$ & & $+2 \cdot 0$ \\
\hline
\end{tabular}

It appears from the last line of this Table, that the temperature of the sea is, upon the mean of the entire year, $2^{\circ} \cdot 0$ higher than that of the air above the coast. The exeess is $3^{\circ} \cdot 3$ in winter, and $0^{\circ} .7$ in summer. There appears also to be considerable diversity in the amount of the excess at the different stations; it is greatest, on the mean of the entire year, at Bunown, and least at Castletownsend.

This excess of the temperature of the sea above that of the air furnishes the explanation of the fact already noticed,-namely, the diminution of the temperature of the air in proceeding from the coasts inland; for it is obvious that the air in the vicinity of the sea must have its temperature raised by contact with the water.

It follows also, that the absolute excess of sea-temperature considerably exceeds that above stated. Thus, we have seen, the 
temperature of the sea, on the average of the entire year, exceeds that of the air over the coasts by $2^{\circ} \cdot 0$; while the latter temperature exceeds that of the air inland (for the same latitude and longitude) by $1^{\circ} .8$. The total excess of the sea-temperature above that of the air amounts, therefore, to $3^{\circ} \cdot 8$ Fahrenheit.

This excess, which appears to be much greater than has been observed elsewhere, is to be ascribed, mainly, to the influence of the gulf-stream upon the temperature of that part of the ocean which bathes our shores. But there is likewise another cause which undoubtedly contributes to the effect. It has been shown by Mayer and Joule, that heat is generated by the friction of fluids in motion, and the latter experimentalist has established the important physical law, that there is a definite relation between the heat so produced, and the mechanical power expended by the moving mass. Mr. Rankine has applied this principle to explain the fact, observed by M. Renou, - namely, that the temperature of the river Loire at Vendôme is higher than that of the air above it; and it is obvious that a similar explanation is applicable to the phenomenon under consideration. There is no doubt as to the reality of the cause; the only question is as to the magnitude of the effect to be ascribed to it. That such effect is, at all events, sensible, I infer from two circumstances. The first of these is, that the phenomenon of the excess of seatemperature appears to be general, and must, therefore, be the effect of some general cause; the second is, that on the coasts of Ireland there is no sensible difference between the amount of the excess on the eastern and on the western shores.

Should the effect of this cause be found to be sensible, and its amount be determined, our views of the cycle of meteorological phenomena would be much enlarged. The elevation of temperature rarefies the air ; the denser air flows in to supply the partial vacuum, and wind is produced; and finally, this wind, both by its own motion, and by that of the ocean which is so subject to its power, restores again the heat which had been converted. Thus the normal condition of temperature is preserved, not only throughout the changes which render it latent and sensible, in the generation and condensation of vapour, but also in its conversion into mechanical power, and its reproduction, in the phenomena of the tempest and of the billowy sea. 


\section{Barometric Pressure.}

An attempte was made to correct the barometric results, by a careful comparison of the several instruments with the Dublin standard, by means of portable barometers, and by the reduction of the results to the sea-level, calculated at the rate of $\cdot 0011$ of an inch for each foot of altitude. These results are, however, incomplete, no comparisons having been made of the barometers at the four inland stations. For this reason, and also because of the uncertainty attending the comparison of barometers by means of portable instruments, I have thought it necessary to seek th. corrections by a comparison of the observed results themselves. I comparisons of this kind, where the stations are widely separater it seems necessary to employ the means of a somewhat extender series of observed results, during which the fluctuations of baro metric pressure are small. I have accordingly selected for the purpose the monthly means of May, July, and September, in which months there was but little variation of barometric equilibrium. The defects of the means at each station, compared with those at Dublin, for these months, are given in the following Table, and the last column contains the inferred corrections, which are equal to the mean differences $+\cdot 021$, the added number being the reduction of the Dublin results to the sea-level. 
Barometric Corrections.

\begin{tabular}{|c|c|c|c|c|c|}
\hline \multirow{2}{*}{\multicolumn{2}{|c|}{ Station. }} & \multicolumn{3}{|c|}{ Defect from Dublin. } & \multirow{2}{*}{$\begin{array}{c}\text { Correction } \\
\text { by } \\
\text { Comparison. }\end{array}$} \\
\hline & & May. & July. & Sept. & \\
\hline Portrush,. . & . . & $\cdot 058$ & $\cdot 070$ & $\cdot 056$ & $+\cdot 082$ \\
\hline Buncrana, . & . . & $\cdot 120$ & $\cdot 100$ & .088 & $+\cdot 124$ \\
\hline Donaghadee, & . . & .052 & $\cdot 062$ & .054 & $+\cdot 077$ \\
\hline Killybegs, · & . . & .056 & $\cdot 032$ & $\cdot 080$ & $+\cdot 077$ \\
\hline Armagh, . . & . . & .252 & $\cdot 257$ & $\cdot 248$ & $+\cdot 273$ \\
\hline Killough,. . & . . & .058 & $\cdot 054$ & $\cdot 068$ & $+\cdot 081$ \\
\hline Markree, . . & . . & $\cdot 134$ & $\cdot 130$ & $\cdot 155$ & $+\cdot 161$ \\
\hline Westport, & . . & $\cdot 046$ & .058 & $\cdot 114$ & $+\cdot 094$ \\
\hline Dublin, . • & . . & $\cdot 000$ & $\cdot 000$ & $\cdot 000$ & $+\cdot 021$ \\
\hline Portarlington, & . . & $\cdot 254$ & $\cdot 268$ & $\cdot 309$ & $+\cdot 298$ \\
\hline Athy, . . . & . . & $\cdot 325$ & $\cdot 304$ & 317 & $+\cdot 336$ \\
\hline Courtown, . & - . & $\cdot 022$ & $\cdot 008$ & .014 & $+\cdot 036$ \\
\hline Kilrush, . . & . . & $\cdot 090$ & $\cdot 062$ & 089 & $+\cdot 101$ \\
\hline Dunmore, . & . . & $\cdot 078$ & $\cdot 056$ & 076 & $+\cdot 091$ \\
\hline Cahirciveen, . & . . & $\cdot 062$ & $\cdot 034$ & .085 & $+\cdot 081$ \\
\hline Castletownsend, & 1, & .016 & $\cdot 006$ & .054 & $+\cdot 046$ \\
\hline
\end{tabular}

Applying the foregoing corrections, we obtain the numbers of the following Table. In order to perceive more clearly the simultaneous variations in the distribution of pressure, I have, in the last four lines of the Table, combined the stations, and their results, into four groups, as hereafter described in treating of the observations of wind-force :- 
ON THE METEOROLOGY OF IRELAND.

\begin{tabular}{|c|c|c|}
\hline 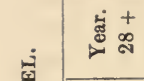 & 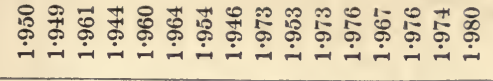 & 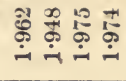 \\
\hline 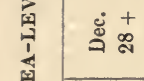 & 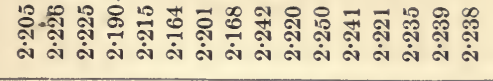 & 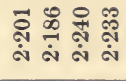 \\
\hline $\begin{array}{l}\dot{8}+ \\
\dot{z} \\
\dot{a}\end{array}$ & 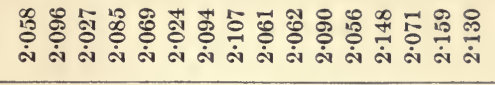 & 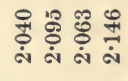 \\
\hline$\stackrel{+}{+\infty} \stackrel{\infty}{\circ}$ & 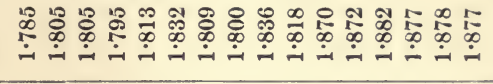 & 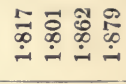 \\
\hline 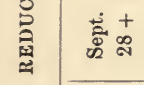 & 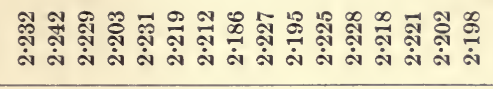 & 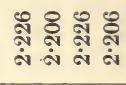 \\
\hline 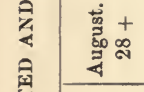 & 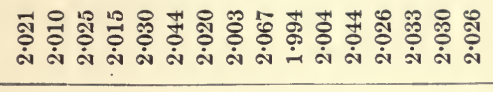 & 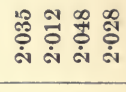 \\
\hline 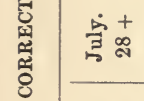 & 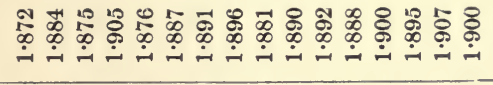 & 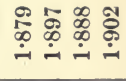 \\
\hline 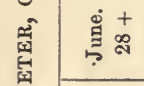 & 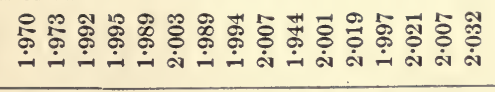 & 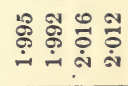 \\
\hline 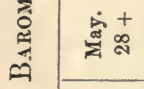 & 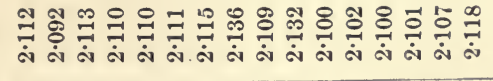 & 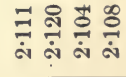 \\
\hline 芯 & 离 & 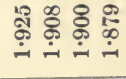 \\
\hline 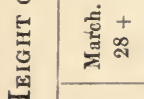 & 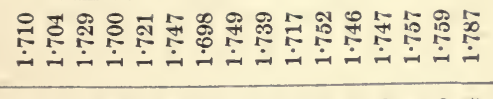 & 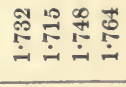 \\
\hline$\stackrel{+}{0}+$ & 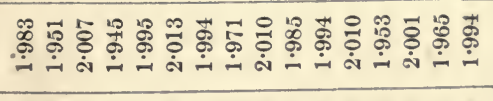 & 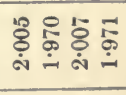 \\
\hline 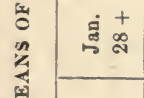 & 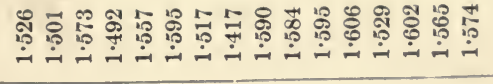 & 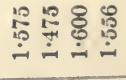 \\
\hline 营 & 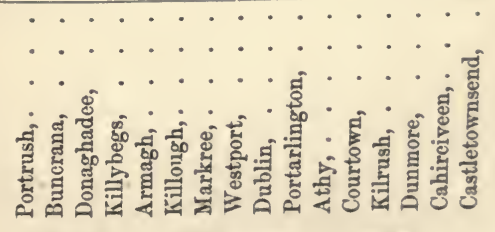 & 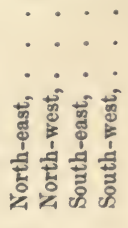 \\
\hline
\end{tabular}


The phenomena of the distribution of pressure are very clearly shown in the foregoing Table. It will be seen from it that, on the average of the entire year, there is an excess of pressure in the south of the island, and a defect in the north, the minimum being at the north-western extremity. This excess of pressure in the south is shown in the means for the several seasons of summer, autumn, and winter, respectively ; and the cause of it will, I think, hereafter appear upon the discussion of the phenomena of storms. In the separate months, the points of greatest and least pressure vary somewhat irregularly; but they are, in nearly every month, at opposite extremities of the island. Thus, in January, the maximum pressure is in the south-east, and the minimim in the northwest; and so for the others. This circumstance is what should have been expected à priori; and it affords satisfactory evidence of the general accuracy of the results themselves.

\section{Direction and Force of the Wind.}

Direction of Wind.-The direction of the wind was observed, at most of the stations, by means of the ordinary wind-vane. Much care was taken, not only in placing these instruments truly in azimuth, but also in selecting positions for them which seemed least exposed to eddies or other local irregularities. At Armagh and Dublin the direction of the wind was recorded continuously, by means of self-registering anemometers.

The following Tables give the number of times, out of 100 , in which the wind blew from each of the eight points at the several stations, for the summer and winter half-years respectively, and for the entire year. The winds from the intermediate points, when observed, were divided equally between the two adjacent principal points :- 
Frequency of the several Winds (Summer).

\begin{tabular}{|r|r|r|r|r|r|r|r|r|}
\hline Station. . & N. & N.E. & E. & S.E. & S. & S.W. & W. & N.W. \\
\cline { 1 - 5 } Portrush, . . & 20 & 5 & 6 & 6 & 24 & 15 & 11 & 13 \\
Buncrana, . . & 13 & 8 & 6 & 12 & 11 & 19 & 12 & 19 \\
Donaghadee, . & 23 & 6 & 5 & 13 & 11 & 14 & 16 & 11 \\
Killybegs, . . & 15 & 9 & 14 & 7 & 8 & 13 & 21 & 14 \\
Armagh, . . & 12 & 9 & 7 & 6 & 16 & 19 & 18 & 15 \\
Killough, . . & 11 & 7 & 14 & 12 & 16 & 18 & 8 & 14 \\
Markree, . . & 14 & 5 & 4 & 17 & 14 & 15 & 10 & 21 \\
Westport, . . & 10 & 10 & 14 & 10 & 3 & 3 & 32 & 19 \\
Dublin,. . . & 2 & 10 & 12 & 13 & 8 & 23 & 11 & 19 \\
Portarlington, & 5 & 28 & 2 & 11 & 7 & 14 & 13 & 21 \\
Athy, . . . & 13 & 1 & 2 & 12 & 16 & 12 & 25 & 19 \\
Courtown, . . & 13 & 17 & 5 & 7 & 8 & 23 & 15 & 13 \\
Kilrush. . . & 12 & 10 & 14 & 8 & 8 & 13 & 19 & 17 \\
Dunmore, . . & 15 & 8 & 14 & 5 & 7 & 18 & 8 & 16 \\
Cahirciveen, . & 11 & 9 & 12 & 9 & 12 & 18 & 16 & 14 \\
Castletownsend, & 8 & 9 & 11 & 12 & 2 & 37 & 15 & 6 \\
\hline
\end{tabular}

Frequency of the several Winds (Winter).

\begin{tabular}{|l|r|r|r|r|r|r|r|r|}
\hline Station. & N. & N. E. & E. & S.E. & S. & S.W. & W. & N.W. \\
\cline { 1 - 5 } Portrush, . . & 15 & 3 & 4 & 7 & 35 & 23 & 9 & 4 \\
Buncrana, . . & 10 & 5 & 5 & 10 & 15 & 27 & 15 & 13 \\
Donaghadee, . & 9 & 7 & 4 & 7 & 14 & 25 & 26 & 9 \\
Killybegs, . . & 11 & 6 & 8 & 9 & 13 & 19 & 18 & 15 \\
Armagh, . . & 6 & 5 & 2 & 5 & 26 & 35 & 12 & 10 \\
Killough, . . & 9 & 4 & 4 & 5 & 18 & 25 & 12 & 24 \\
Markree, . . & 12 & 4 & 4 & 19 & 19 & 23 & 9 & 10 \\
Westport, . . & 13 & 4 & 12 & 10 & 6 & 8 & 26 & 21 \\
Dublin, . . . & 2 & 1 & 2 & 14 & 14 & 38 & 14 & 13 \\
Portarlington, & 5 & 11 & 1 & 4 & 10 & 19 & 20 & 30 \\
Athy, . . . & 7 & 2 & 1 & 15 & 28 & 11 & 23 & 13 \\
Courtown, . . & 7 & 5 & 4 & 4 & 18 & 23 & 24 & 16 \\
Killrush, . . & 10 & 9 & 10 & 6 & 16 & 23 & 13 & 14 \\
Dunmore, . . & 16 & 4 & 3 & 5 & 14 & 20 & 22 & 16 \\
Cahirciveen, . & 8 & 8 & 13 & 12 & 12 & 20 & 17 & 10 \\
Castletownsend, & 13 & 5 & 3 & 6 & 5 & 35 & 21 & 13 \\
& & & & & & & \\
\hline
\end{tabular}


Frequency of the several Winds (Year).

\begin{tabular}{|c|c|c|c|c|c|c|c|c|}
\hline Station. & N. & N.E. & E. & S.E. & S. & S.W. & w. & N.W. \\
\hline Portrush, · & 17 & 4 & 5 & 6 & 30 & 19 & 10 & 8 \\
\hline Buncrana, . & 12 & 7 & 6 & 11 & 13 & 23 & 14 & 16 \\
\hline Donaghadee, . & 16 & 7 & $4^{\prime}$ & 10 & 13 & 19 & 21 & 10 \\
\hline Killybegs, . • & 13 & 8 & 11 & 8 & 10 & 16 & 20 & 15 \\
\hline Armagh, : : & 9 & 7 & 4 & 5 & 21 & 27 & 15 & 12 \\
\hline Killough, · · & 10 & 5 & 9 & 8 & 17 & 21 & 10 & 19 \\
\hline Markree, · . & 13 & 4 & 4 & 18 & 17 & 19 & 10 & 16 \\
\hline Westport, . & 11 & 7 & 13 & 10 & 4 & 5 & 29 & 20 \\
\hline Dublin, . . . & 2 & 6 & 7 & 14 & 11 & 31 & 13 & 16 \\
\hline Portarlington, & 5 & 19 & 1 & 7 & 9 & 16 & 16 & 25 \\
\hline Athy, . . & 10 & 2 & 1 & 13 & 22 & 12 & 24 & 16 \\
\hline Courtown, . . & 10 & 11 & 4 & 6 & 13 & 23 & 19 & 14 \\
\hline Kilrush, · . & 11 & 9 & 12 & 7 & 12 & 18 & 16 & 16 \\
\hline Dunmore, . & 15 & 6 & 9 & 5 & 11 & 19 & 20 & 16 \\
\hline Cahirciveen, · & 9 & 8 & 13 & 10 & 12 & 19 & 17 & 12 \\
\hline Castletownsend, & 10 & 7 & 7 & 9 & 3 & 36 & 18 & 9 \\
\hline
\end{tabular}

The following are the mean results for the whole island:-

$$
\text { N. N. E. E. S. E. S. S. w. W. N. w. }
$$

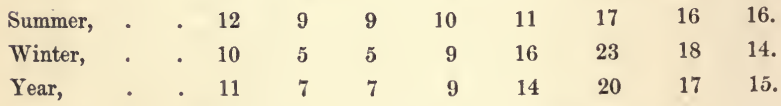

We learn from them that, in the year 1851, the wind blew, on the average of the entire year, most frequently from between $\mathrm{S}$. W. and W., and least frequently from between N. E. and E. The same thing holds also for the summer half-year, the point of maximum frequency being, very nearly, W.S. W., and that of minimum frequency E.N.E. In the winter half-year the point of maximum frequency is more nearly $\mathrm{S}$. W., that of the minimum being as before. The ratio of the numbers representing the greatest and least frequency is greater in winter than in summer. 
It is not necessary to enter more minutely into the discussion of the numbers of the preceding Tables, as it is probable that the results of a single year, as to the frequeney of the several winds, will deviate considerably from the means of several. I may observe, however, that they afford some indications of a law of distribution, depending upon the aspect of the coast. Thus, on comparing the numbers denoting the frequency of any particular wind at the several stations, with their mean for the whole island, it would seem that easterly winds are slightly in excess on the western coast, and westerly winds on the eastern. In other words, there appears to be a preponderating tendency of the wind to blow from the land, at each place, as compared with the mean of all. It will remain for future inquiry to ascertain whether this holds good in other years, and is, therefore, to be referred to a general law. If so, it is probably the effect of the land and sea breezes, the former preponderating in the average of the winds at 9 A. M. and 9 P. M.

Pressure of the Wind.-For the measurement of the pressure of the wind, a Iind's anemometer was furnished to each station. The difficulty of obtaining accurate results with this little instrument arises, partly, from the smallness of its indications, and, partly, from the oscillations of the fluid in the tube; the latter are so considerable as to render the instrument of little value, except in the hands of a patient and somewhat practised observer. After some trial, accordingly, it was deemed advisable that the force of the wind should be in all cases estimated, and that the use of Lind's anemometer should be limited to that of furnishing a cheek upon this estimation in the case of the stronger winds.

The first thing to be determined, then, was the choice of a scale of force. The scales in use are various: in one of them there are four degrees of wind-force; in another six; and in a third (the Admiralty scale) there are ticelve. The last of these appears to be too minute for the ordinary powers of unaided estimation, and the first not sufficiently so. The intermediate scale (from 0 to 6 ) was accordingly adopted; and it appears to be further recommended by the circumstances, -1 , that it is the subdivision most generally used on the Continent; and 2, that, as its numbers represent the same degrees of wind-force with the alternate numbers of the $\Lambda$ dmiralty scale, the latter are readily 
convertible into the former. The six degrees of wind-force were designated as follow :-1. Light breeze; 2. Moderate breeze; 3. Strong breeze; 4. Moderate gale; 5. Strong gale; 6 . Storm.

In order to know the amount of confidence which may be placed in such observations, it is necessary to determine how far, in respect of accuracy, six degrees of wind-force can be estimated, the observations being supposed to be made by practised observers. And to be able to apply the observations, we must further know what are the pressures and velocities of the wind corresponding to the several terms of the scale. For these purposes I made a somewhat extended series of observations, estimating the force of the wind according to the prescribed scale, and, at the same time, measuring its velocity by means of Robinson's anemometer. The following Table gives the mean results of these observations. The numbers in the first column are the terms of the scale; those in the second are the corresponding times of 100 revolutions of the instrument, expressed in seconds ; * the third column contains the corresponding velocities of the wind, in feet per second; and the fourth the calculated velocities, deduced as hereafter described.

* Dr. Robinson has shown (Trans. R.I. A., vol. xxii., p. 167) that the velocity of the wind is to that of the centres of the hemispherical cups, as 3 to 1 . But $r$ being the length of the horizontal arms of the instrument, measured to these centres, the circumference of the circle described by them is $2 \pi r$; and if $r$ be expressed in feet, and $n$ be the number of revolutions performed in a second, their velocity is $2 \pi r \times n$. The corresponding velocity of the wind therefore is $V=6 \pi r \times n$. In the instrument in my passession the radius is $5 \cdot 5$ inches. Hence $2 r=\frac{11}{12}$, and substituting for $\pi$ its numerical value, $V=8.64 \times n$.

Instead of noting the number of revolutions, and parts of a revolution, performed in a given time, I have found it convenient to observe the time of performing 100 revolutions. I have had the instrument accordingly provided with a little hammer, which is pressed against the registering wheel by a spring, and which, being raised by a projecting pin at one point of its circumference, falls again with a sharp noise when this has passed. The interval between two such strokes of the hammer, therefore, is the time of one whole revolution of the registering wheel, or of 100 revolutions of the arms. Accordingly, a chronometer being held close to the ear, the whole observation is effected by the help of that organ. The velocity of the wind in this case is given by the formula $V=\frac{864}{T}, T$ being the observed time of 100 revolutions. 
Velocities of the Wind corresponding to the Terms of the Scale $(0-6)$.

\begin{tabular}{|c|c|c|c|}
\hline$n$ & $T$ & $V$ (observed). & $V$ (calculated). \\
\hline I. & $71^{8}$ & 12 & 12 \\
II. & 35 & 25 & 23 \\
III. & 25 & 35 & 35 \\
IV. & 20 & 43 & 46 \\
V. & $16 \cdot 8$ & 51 & 58 \\
VI. & 11.6 & 75 & 70 \\
\hline
\end{tabular}

We see that the terms of the estimated scale correspond, nearly, to an arithmetical progression of velocities, and not of pressures. This fact has been already noticed by Dr. Robinson.

The common difference in this series, which is equal to its first term, is obtained from the numbers of the third column by means of the formula $V=n V_{1}$. The following are the deduced values:

$\begin{array}{crrr}\text { I. } & V_{1}=12 \cdot 0 . & \text { IV. } & V_{1}=10 \cdot 8 . \\ \text { II. } & 12 \cdot 5 . & \text { V. } & 10 \cdot 2 . \\ \text { III. } & 11 \cdot \% . & \text { VI. } & 12.5 .\end{array}$

The mean of these values is $V_{1}=11 \cdot 6$. The calculated values of $V$, contained in the last column of the foregoing Table, are, accordingly, obtained from the formula

$$
V=11 \cdot 6 \times n ;
$$

their agreement with the observed values is sufficient to establish the assumed law.

As a verification of the preceding result, I took also a tolerably extended series of measurements of the pressures of the wind, corresponding to the highest term of the scale, with Lind's anemometer. Their mean gave 2.06 inches for the reading of the instrument corresponding to that term; and the corresponding pressure on one square foct of surface, computed in the proportion of $5 \cdot 20$ pounds to the inch, is 10.7 pounds. Hence, the pressure belonging to the unit of the scale is $P_{1}=0.30$. The corresponding velocity is inferred from the formula $V^{2}=437 P$. Its value is $V_{1}=11 \cdot 5$; a result which agrees very closely with that already deduced from Robinson's anemometer.

The results hitherto given rest only on my own estimations; it remains to see how far they accord with those of other observers. 
I have selected for this purpose the results of the observations with Lind's anemometer, made at Portrush and Donaghadee by two of the best of the Coast-guard observers, and have placed my own beside them, for comparison. The results, converted into pressures (expressed in pounds on the square foot) are contained in the following Table. The numbers in the last column are the calculated pressures, deduced from the formula

$$
P=P_{1} n^{2},
$$

$n$ being the number of the term of the scale, and $P_{1}(=0.30)$ the pressure corresponding to the first term.

Pressures of the Wind corkesponding to the Terms of the Scale $(0-6)$.

\begin{tabular}{|r|c|c|c|c|}
\hline Term. & Dublin. & Portrush. & Donaghadee. & Calculated. \\
\cline { 1 - 3 } I. & 0.5 & 0.4 & 0.5 & 0.3 \\
II. & 1.3 & 1.3 & 1.1 & 1.2 \\
III. & 3.0 & 2.9 & 2.9 & 2.7 \\
IV. & 4.2 & 5.3 & 5.3 & 4.8 \\
V. & 7.0 & 7.3 & 7.9 & 7.5 \\
VI. & 10.7 & - & - & 10.8 \\
\hline
\end{tabular}

It will be seen, that the differences of the corresponding numbers at the three stations are small, and that their means agree very well with the calculated pressures. It seems, therefore, to be fully proved that the velocity of the wind may be estimated to six degrees, by practised observers, with sufficient accuracy.

In the following Table are given the results of the observed wind-force for the entire year, and for its two principal divisions. The excess of the force in winter appears at all the stations, excepting Dublin, Portarlington, and Athy.* At these three stations, also, the force of the wind is below the average.

The mean force for the entire year is $1 \cdot 76$, corresponding to a velocity of 20.4 feet per second. The force is, of course, greater in winter than in summer, the mean force for the winter half-year being $1 \cdot 87$, and that for the summer half-year $1 \cdot 65$.

* Buncrana is likewise an exceptional case; but the exception is there probably due to inaccuracy of observation. 
Mean Force of the Wind for the Summer and Winter Half-years, and for the whole Year.

\begin{tabular}{|c|c|c|c|c|c|c|}
\hline Station & & & & Summer. & Winter. & Year. \\
\hline Portrush, - & • & . & . . & $1 \cdot 68$ & 1.85 & $1 \cdot 77$ \\
\hline Buncrana, . & • & . & . . & $2 \cdot 15$ & $2 \cdot 15$ & $2 \cdot 15$ \\
\hline Donaghadee, . & • & . & . . & $1 \cdot 50$ & 1.82 & 1.66 \\
\hline Killybegs, . . & • & . & •. & $1 \cdot 40$ & 1.75 & 1.58 \\
\hline Armagh, . . & • & . & . . & $1 \cdot 23$ & $1 \cdot 78$ & $1 \cdot 51$ \\
\hline Killough, . . & • & . & . . & $1 \cdot 60$ & 1.92 & $1 \cdot 76$ \\
\hline Markree, . & • & . & . . & $1 \cdot 68$ & $1 \cdot 82$ & $1 \cdot 75$ \\
\hline Westport, & & . & . . & $2 \cdot 20$ & $2 \cdot 78$ & $2 \cdot 49$ \\
\hline Dublin, . . . & • & . & . . & $1 \cdot 33$ & $1 \cdot 32$ & $1 \cdot 33$ \\
\hline Portarlington, . & . & . & . . & $1 \cdot 42$ & $1 \cdot 27$ & $1 \cdot 34$ \\
\hline Athy, . . . & & . & . . & $1 \cdot 42$ & $1 \cdot 20$ & $1 \cdot 31$ \\
\hline Courtown, & & . & . . & $1 \cdot 40$ & $1 \cdot 70$ & 1.55 \\
\hline Kilrush, . . & & . & . . & $1 \cdot 72$ & $2 \cdot 02$ & $1 \cdot 87$ \\
\hline Dunmore, . & & . & •. & $1 \cdot 88$ & $2 \cdot 03$ & 1.96 \\
\hline Cahirciveen, . & & . & . . & $1 \cdot 80$ & $2 \cdot 17$ & 1.98 \\
\hline Castletownsend, & & . & . . & 1.93 & $2 \cdot 38$ & $2 \cdot 16$ \\
\hline
\end{tabular}

If, to eliminate local irregularities, we combine the preceding results in groups, according to the arrangement hereafter described, we find the following values for the mean forces of the entire year:-

$$
\begin{array}{ll}
\text { North-east, . . 1.64. } & \text { North-west, . . } 1.94 . \\
\text { South-east, . . 1.61. } & \text { South-west, . . } 2 \cdot 00 .
\end{array}
$$

From this it appears that the mean force of the wind is considerably greater in the west of the island than in the east, the ratio being somewhat greater than that of $1 \cdot 2$ to 1 . There is but little difference between the forces in the northern and southern portions of the island.

\section{Cyclonic Movements.}

In analyzing the phenomena of rotation, the first step was to note those cases in which the mean directions of the wind, in any two districts, differed by $90^{\circ}$, or upwards. It was soon perceived, that no conclusion could be drawn as to a general movement of the atmosphere, when the wind was very moderate, the direction being 
then greatly influenced by local causes. Accordingly, excluding those cases in which the wind did not exceed a light breeze at most of the stations, the remainder were examined in detail, by laying down the simultaneous directions of the wind upon a series of skeleton charts prepared for the purpose; and there was no difficulty in ascertaining, by the inspection of these charts, the existence or non-existence of rotatory movement. The same means sufficed to determine, very nearly, the position of the centre of the vortex at each epoch; and the places of the centre being thus found, for epochs distant by intervals of twelve hours, the direction and velocity of its progressive movement are ascertained.

The position of the centre of the vortex at any instant may be determined, more accurately, by calculation. Thus, if $y$ and $x$ denote the distances (in geographical miles) of the place of observation from any assumed central point, measured on the meridian, and on the perpendicular to the meridian, respectively; $y_{0}$ and $x_{0}$ the corresponding co-ordinates of the centre of the vortex; and $\theta$ the angle which the direction of the wind at the point $(y, x)$ makes with the meridian, measuring from north to east:

$$
y-y_{0}+\left(x-x_{n}\right) \tan \theta=0,
$$

the direction of the wind being perpendicular to the line connecting the points $(y, x)$ and $\left(y_{0}, x_{0}\right)$. Now, all the quantities in this equation are given, excepting $y_{n}$ and $x_{0}$; so that, if the direction of the wind be accurately known at two stations, the co-ordinates of the centre of the vortex may be completely determined. The irregularities due to local causes, and the errors of observation themselves, forbid this; and, in order to lessen their influence, it is necessary to know the direction of the wind at several stations. There will then be as many equations of the preceding form as there are places of observation; and the unknown quantities, $y_{0}$ and $x_{0}$, are to be determined by combining these equations by the method of least squares.

It is found, that the centre of the vortex is also the point of least barometric pressure, and that the pressure increases regularly with the distance from it. Hence the position of the centre may be inferred from the barometric observations alone. The positions thus determined have been found to coincide in all cases, very nearly, with those deduced from the observed directions of the wind. 
The following are the well-marked instances of aerial rotation which.have.occurred in Ireland in the course of these observations. No case has been included in the enumeration, in which the simultaneous directions of the wind did not differ, at two points, by at least $90^{\circ}$; and thus, probably, many cases of cyclonic movement are passed over, in which the centre of the vortex was remote.

1850. Oct. 6, 7.-Cyclone and storm, moving from S.W. to N.E., with a velocity of about 290 geographical miles per diem. (Plate II., figs. 1, 2, 3.)

Oct. 6, 9 A. M.-Centre of the vortex on the south-western coast of Ireland, west of Kilrush. Least pressure at Cahirciveen. Mean velocity of the wind $=25$ feet per second; greatest do. (on the west coast) $=45$ feet. The atmosphere at the northern stations unaffected by the vortex at this epoch.

Oct. 6, 9 P.м.-Centre of the vortex over the north of Ireland, a few miles north of Killybegs. Absolute barometrio minimum $(=28.836)$ at Killybegs ; increase of pressure in 100 miles $=0.30$ inch. Mean velocity of wind $=35$ feet per second; greatest do. $($ Markree $)=70$. Southern stations unaffected by the vortex.

Oct. 7, 9 A.M.-Centre on south-western coast of Scotland. Least pressure at Donaghadee. Mean velocity of wind $=45$ feet per second; greatest do. (north coast) $=60$ feet. Hail fell at Markree; wind amounting to a gale in the north, in the evening of the same day.

The diameter of the vortex may be estimated with tolerable precision in this case, by measuring from the centre to the limits of the region affected by the movement; it was about 280 geographical miles.

Oct. 22, 23.-An interesting and instructive case of conflicting currents generating a rotatory movement. 'The velocity of the wind was uniform throughout the island, and was from 30 to 35 feet per second. (Plate II., figs: 4, 5, 6.)

Oet. 22, 9 P. M.-Wind from N. W. in the north of Ireland, and from S. W. in the south-east. The central point of junction of these carrents was over the channel, to the north-east of Dublin. Least pressure at Donaghadee.

Oct. 23,9 A. м.-A distinct rotatory movement, whose centre was a little to the north-east of the point of junction above referred 
to, not far from Donaghadee. Least pressure at Donaghadee, as before.

Oct. 23, 9 P. м.-Rotatory movement continued. Centre of vortex had moved from S.W. to N.E., at the rate of about 100 miles per diem. Absolute minimum of pressure $(=29 \cdot 360)$ at Donaghadee; increase of pressure in 100 miles $=0 \cdot 10$ inch.

Nov. 18, 19.-A cyclone, with violent storm, crossing the island from W.S. W. to E.N.E. (Plate III., figs. 1,2,3.) The movement of the centre of the vortex appears to have been curvilinear, and to have varied considerably in velocity. Between 9 P. M. of the 18 th, and 9 A.M. of the following day, its path was from S. W. to N. E., and its velocity about 320 miles per diem; in the succeeding twelve hours its course was nearly from W. to E., with a greatly diminished velocity. The mean velocity of the wind, throughout the storm, was from 45 to 50 feet per second.

Nov. 18, 9 P. M.-Centre of the vortex on the south-western coast, about 30 miles to the north of Cahirciveen. Least pressure at Kilrush. Maximum velocity of wind (in south of island) $=60$ feet per second.

Nov. 19, 9 A.M.-At this epoch the wind was blowing from N. at Killybegs, and from S. at Donaghadee; it was blowing from S. E. at Portrush, and from N. W. at Castletownsend; from S. S. E. at Armagh, and from N. N. W. at Markree. The centre of the vortex was therefore over Ireland at that time, and between the stations above mentioned, its exact position being about 15 miles to the west of Armagh. Absolute minimum of pressure $(=28.248)$ at Armagh; increase of pressure $=0.31$ inch. Maximum velocity of wind (in south) $=65$ feet per second.

Nov. 19, 9 P. M.-Centre over the Channel, to the south-east of Donaghadee. Absolute minimum of pressure $(=28.410)$ at Donaghadee ; increase of pressure $=0 \cdot 28$ inch. Maximum velocity of wind (in south) $=55$ feet per second.

We have seen that the centre of the vortex was between Armagh and Markree at 9 A.M. of the 19th; and, as the direction of its progressive movement was not far from the line connecting these places, it must have passed nearly centrally over both. Hence we should expect there the peculiar phenomena-the lull of the wind, and the sudden reversal of its direction-which are observed to occur at places in the path of the centre of a cyclone. I shall, 
therefore, briefly describe the series of changes at these two stations. The observations at Armagh are taken from the records of the selfregistering anemometer, which were, of course, continuous ; those at Markree were made at short intervals.

At Armagh the wind began to blow at 7 P. M. of the 18th, with a velocity of 32 feet per second. The maximum velocity, with the exception of a short squall* at 5 A. M., occurred at 7 A. M. of the 19th, and amounted to 43 feet per second. From this time the wind abated rapidly almost to a calm, its velocity at noon amounting only to 6 feet per second; but at 3 Р. м. it rose again, with a velocity of 22 feet. The initial direction of the gale was from the E. S. E. From 9 P. M. on the 18th, to 1 A. M. on the 19 th, it veered to S., at which point it continued for several hours, including the period of greatest foree of the gale. At 11 A.M. its direction had returned to S. E., and it then suddenly shifted to W. N. W., altering through $160^{\circ}$ in 24 minutes. The minimum of pressure took place at $11^{\mathrm{h}} 30^{\mathrm{m}}$, at the close of this movement; its amount was $27 \cdot 930$ inches. $\dagger$

At Markree the gale commenced at $4^{\mathrm{h}} 30^{\mathrm{m}}$ P.M. of the 18th, with a rapidly falling barometer. At 7 Р. м. the wind abated to a breeze, the barometer still falling. It recommenced at $10 \mathrm{P.M}$.

- During the squall, which lasted only three minutes, the velocity reached 90 feet per second.

+ The following are the anemometric observations above referred to. The direction is measured from S. through W. to $\mathrm{N}$.; the velocity is expressed in miles per hour. On the 19 th, from 4 A.M. to 8 A.M., the direction-registering pencil was thrown out of gear, but there appears to have been no change of any magnitude in the interval:-

\begin{tabular}{|c|c|c|c|c|c|c|c|c|}
\hline \multirow[b]{2}{*}{ Hour. } & \multicolumn{2}{|c|}{ Nov. 18, A.M. } & \multicolumn{2}{|c|}{ Nov. 18, P.M. } & \multicolumn{2}{|c|}{ Nov. 19, A.м. } & \multicolumn{2}{|c|}{ Nov. 19 , P.M. } \\
\hline & Vel. & Dir. & Vel. & Dir. & Vel. & Dir. & Vel. & Dir. \\
\hline 0 & $12 \cdot 2$ & $28^{\circ} \cdot 8$ & $4 \cdot 8$ & $212^{\circ} \cdot 3$ & $23 \cdot 4$ & $335^{\circ} \cdot 2$ & $4 \cdot 1$ & $124^{\circ} \cdot 0$ \\
\hline 1 & $8 \cdot 3$ & $28 \cdot 2$ & $5 \cdot 3$ & $243 \cdot 6$ & $24 \cdot 4$ & $354 \cdot 3$ & 10.5 & $104^{-4}$ \\
\hline 2 & $5 \cdot 9$ & $47 \cdot 8$ & $8 \cdot 7$ & $276^{\prime} \cdot 2$ & $22 \cdot 2$ & $353 \cdot 4$ & $12 \cdot 0$ & $93 \cdot 9$ \\
\hline 3 & $7 \cdot 2$ & $49 \cdot 8$ & $7 \cdot 8$ & $274 \cdot 5$ & $23 \cdot 6$ & $345 \cdot 6$ & $15 \cdot 4$ & $90 \cdot 2$ \\
\hline 4 & $8 \cdot 1$ & $48 \cdot 1$ & $14 \cdot 7$ & $278 \cdot 8$ & $23 \cdot 2$ & - & $16 \cdot 9$ & $108 \cdot 8$ \\
\hline 5 & $3 \cdot 8$ & $41 \cdot 3$ & $16 \cdot 1$ & $281 \cdot 7$ & $25 \cdot 3$ & - & $13 \cdot 8$ & $136 \cdot 7$ \\
\hline 6 & $2 \cdot 9$ & $75 \cdot 0$ & 16.6 & $281 \cdot 9$ & $27 \cdot 4$ & - & $15 \cdot 1$ & $138 \cdot 3$ \\
\hline 7 & $3 \cdot 7$ & $162 \cdot 3$ & $22 \cdot 0$ & $283 \cdot 5$ & $29 \cdot 4$ & - & $16 \cdot 2$ & $151 \cdot 1$ \\
\hline 8 & 1.5 & $162 \cdot 0$ & $19 \cdot 2$ & $285 \cdot 5$ & $29 \cdot 0$ & - & $16 \cdot 6$ & $153 \cdot 6$ \\
\hline 9 & $2 \cdot 3$ & $212 \cdot 4$ & $19 \cdot 3$ & $282 \cdot 1$ & $17 \cdot 6$ & $339 \cdot 8$ & 15.8 & $151 \cdot 2$ \\
\hline 10 & $4 \cdot 9$ & $205 \cdot 4$ & $17 \cdot 6$ & $321 \cdot 3$ & $14 \cdot 6$ & $320 \cdot 6$ & $9 \cdot 2$ & $147 \cdot 5$ \\
\hline 11 & $5 \cdot 9$ & $209 \cdot 2$ & $20 \cdot 1$ & $330 \cdot 6$ & $7 \cdot 6$ & $324 \cdot 5$ & $11 \cdot 8$ & $159 \cdot 5$ \\
\hline
\end{tabular}


from the S. E. ; and at 3 A. M. on the 19th it appears to have attained its maximum. At 6 A. м. the wind again abated; and at 7 A. м. there was a calm. The minimum pressure took place at this time, and amounted to $28 \cdot 170$ inches. At $9 \mathrm{~A}$. $\mathrm{M}$. the wind rose again from the N.N.W., but not with such force as before; and in the afternoon there was a strong gale again.*

From these facts it is evident, that the centre of the vortex passed nearly over Markree at 7 A. M., and over Armagh at $11^{\mathrm{h}} 30^{\mathrm{m}}$ A.M. At Donaghadee, which is nearly in the prolongation of the line connecting the two former places, the wind ceased at 1 P. M., and recommenced at 5 P.м. ; so that the vortex passed nearly centrally over this station at about 3 P. M. From these data we learn that the cyclone moved from W. S. W. to E. N. E. ; and that the velocity of the progressive movement was then about 12 miles per hour.

The dimensions of the vortex may likewise be collected from the same data. The interval between the commencement of the storm, and the passage of the centre, at Armagh, was $16 \frac{1}{2}$ hours ; and, the velocity being 12 miles an hour, the radius of the vortex

* The following are the extra observations at Markree above referred to. The numbers in the column headed "Barometer," are the excesses above 28 inches :-

\begin{tabular}{|c|c|c|c|c|}
\hline Date. & Hour. & Bar. & Ther. & Remarks. \\
\hline \multirow{7}{*}{ Nov. 18 , } & $4^{\text {h }} 30^{\mathrm{m}}$ & $1 \cdot 124$ & $46^{\circ} \cdot 8$ & Blowing a gale: storm and rain began about noon. \\
\hline & & 1.002 & $48 \cdot 8$ & $\begin{array}{l}\text { Blowing a gale; storm and rain began about noon. } \\
\text { Ditto; rain. }\end{array}$ \\
\hline & 7 & 0.926 & $49 \cdot 5$ & Strong breeze; heavy rain. \\
\hline & 8 & 0.835 & $50 \cdot 5$ & Ditto; ditto. \\
\hline & 10 & 0.652 & $53 \cdot 7$ & Gale. \\
\hline & 11 & $0 \cdot 577$ & $54 \cdot 0$ & Wind rising to gale; mizzling rain. \\
\hline & 12 & 0.538 & $52 \cdot 5$ & Strong breeze; ditto. \\
\hline \multirow[t]{10}{*}{ Nov. 19, } & 3 А.M. & $0 \cdot 315$ & $50 \cdot 5$ & Wind higher than at any previous time. \\
\hline & & 0.120 & $46 \cdot 5$ & Gale ; mizzling rain. \\
\hline & 6 & $\begin{array}{l}0.027 \\
0.009\end{array}$ & $45 \cdot 5$ & Strong breeze. \\
\hline & $\begin{array}{l}7 \\
8\end{array}$ & $\begin{array}{l}0.009 \\
0.023\end{array}$ & $\begin{array}{l}47 \cdot 3 \\
48 \cdot 2\end{array}$ & $\begin{array}{l}\text { Calm. } \\
\text { Light breeze; mizzling rain. }\end{array}$ \\
\hline & 9 & 0.067 & $45 \cdot 8$ & $\begin{array}{l}\text { Light breeze ; mizzling rain. } \\
\text { Gale. }\end{array}$ \\
\hline & 10 & $0 \cdot 124$ & $46 \cdot 0$ & Strong breeze; wind N. N. W. \\
\hline & 3 P.x. & 0.303 & $48 \cdot 8$ & Strong breeze. \\
\hline & & $0 \cdot 483$ & $49 \cdot 5$ & Strong gale. \\
\hline & 10 & 0.533 & $48 \cdot 0$ & Gale from N. W. ; light showers. \\
\hline & 1130 & 0.578 & $49: 0$ & Strong gale. \\
\hline \multirow[t]{4}{*}{ Nov. 20, } & 10 A.M. & $\begin{array}{l}1 \cdot 084 \\
1 \cdot 128\end{array}$ & $48 \cdot 4$ & Ditto; showers. \\
\hline & 130 & $\begin{array}{l}1 \cdot 128 \\
1 \cdot 227\end{array}$ & $\begin{array}{l}48 \cdot 3 \\
47 \cdot 5\end{array}$ & $\begin{array}{l}\text { Ditto. } \\
\text { Ditto. }\end{array}$ \\
\hline & 3 & $1 \cdot 282$ & $46 \cdot 5$ & Ditto; heavy rain. \\
\hline & 5 & $1 \cdot 376$ & $44 \cdot 8$ & Moderate gale. \\
\hline
\end{tabular}


was about 200 miles. The magnitude of the nearly quiescent portion of air in the centre of the vortex is better defined. At Armagh the lull lasted from three to four hours; at Markree three hours; and at Donaghadee four hours. The diameter of the quiescent central portion was, therefore, about 40 miles.

We may now refer to some particulars connected with this gale, which appear to merit attention-although probably, in the present state of our knowledge on this subject, we should not be justified in offering any suggestions in explanation.

Among the first of these are the abnormal variations in the rotatory movement, especially along the track of the centre. The most curious of these irregularities is that of the direction. At Armagh this began to change rapidly at 9 P. M. of the 18th. At 9 р. м. it was E. S. E. ; at 10 P. м., S. E. ; at midnight, S.S. E. ; and at $1 \mathrm{~A} . \mathbf{M}$. on the 19 th, S. At this latter point it remained for several hours; and the direction then retrograded through an arc of about $45^{\circ}$. At 9 A. M. on the 19 th it was S.S. E. ; and at 11 A. м. it came back to S. E., after which the sudden shift to W.N.W., already noticed, took place.

The next point which seems to merit notice is the fact, that the force of the gale was considerably greater to the south of the line of passage of its centre, than on that line itself, or to the north of it. Thus, at Killiney, where I made frequent observations during the gale, I found the maximum velocity to be 80 feet per second; at Armagh it was little more than half that amount.

It has been already mentioned that the greatest force of the storm occurred at Armagh and Markree, before the epoch of miuimum pressure, the interval at both places being about four hours and a half. A similar interval took place at Killiney, but in the opposite direction, the epoch of greatest intensity following that of least pressure by four hours and a half.

The last point which appears to demand notice is the fact, that there was a considerable interval between the epochs of the greatest intensity of the storm at Dublin and at Killiney, places only ten miles apart. The greatest force of the gale, at Dublin, took place between 1 P. M. and 2 P. M. ; at Killiney it oceurred between 5 P. M. and $6 \mathrm{P} . \mathrm{M}$. There is a similar interval between the times of minimum pressure at the two places, the least height of the barometer occurring later at Killiney than at Dublin by two or three hours. 
These differences are probably connected with the difference of altitude of the places of observation.

1851. Jan. 15, 16. - A remarkable case of a double cyclone with storm, and a double minimum of pressure. (Plate III., figs. $4,5,6$.) The first of the two vortices crossed the island from $\mathrm{S}$. to $N$. on the 15th, and the second traversed the north-western portion of it, from S. W. to N.E., on the following day. The velocity of the former is not well determined; that of the latter was about 270 miles per diem. The mean velocity of the wind was from 30 to 35 feet per second on the former day, and from 55 to 60 on the latter.*

Jan. 15, A. M.-Centre of vortex about 20 or 30 miles south of Dunmore. Absolute minimum of pressure $(=28.718)$ at Dunmore; increase of pressure $=0.15$ inch. Maximum velocity of wind (west coast) $=60$ feet per second.

Jan. 15, 9 P. M.-Centre of vortex appears to have been at this time a few miles north of Buncrana; the cyclonic movement was,

* The following extra observations were taken at Markree :-

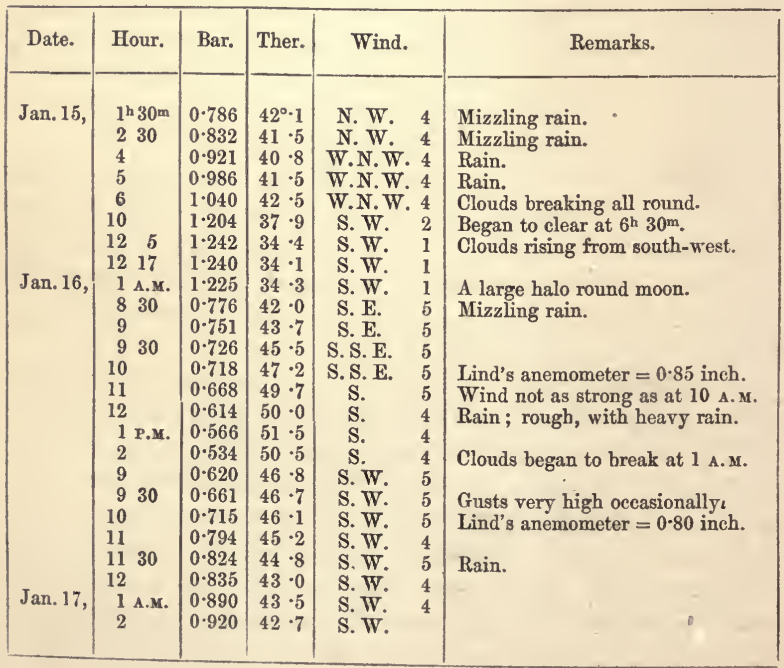


however, not distinctly marked, probably owing to the influence of the second cyclone. Least pressure at Buncrana. Velocity of wind uniform throughout the island.

Jan. 16, 9 A. M.-Centre of second vortex to the south-west of Westport. Least pressure at Westport.

Jan. 16, 9 P. м.-Centre about 20 miles west of Buncrana. Absolute minimum of pressure (= 28.671) at Buncrana ; increase of pressure $=0.20$ inch.

Jan. 30, 31.-A very interesting cyclone traversing the western portion of the island, in direction from N. to S. nearly, at the rate of about 150 miles per diem. The wind light, the mean velocity being about 20 feet per second.

Jan. 30,9 P. м.-Centre of vortex over north-western portion of the island, a little to the north of Killybegs. Least pressure at Killybegs. Maximum velocity of wind (in south-west) $=40$ feet per second.

Jan. 31, 9 A. M.-Centre a little to the eastward of Westport. Absolute minimum of pressure $(=29 \cdot 032)$ at Westport ; increase of pressure $=0 \cdot 10$ inch. Maximum velocity of wind (in south-west) $=25$ feet per second. Lightning observed in north in evening of this day and day preceding.

March 18.-A cyclone, with storm, traversing the island from S. to N., at the rate of about 200 miles per diem.

March 18, 9 A. м.-Centre of vortex near Markree. Absolute minimum of pressure $(=29.328)$ at Armagh ; increase of pressure $=0 \cdot 10$. Mean velocity of the wind $=45$ feet per second; greatest do. (west coast) $=50$ feet.

March 18, 9 P. M.-Centre of vortex north of the island. Absolute minimum of pressure $(=29 \cdot 371)$ at Portrush ; increase of pressure $=0 \cdot 13$ inch. Mean velocity of the wind $=35$ feet per second; greatest do. (north-west) $=50$ feet.

March 19, 9 A. м.-Rotatory movement broken up, and wind lessened. Barometer fell, and wind rose again to a gale in the evening; greatest velocity (north-west) $=65$ feet per second.

March 25.-A distinct rotatory movement at 9 A. M. of this day, the centre of which was a little to the north of Westport. 
Absolute minimum of pressure $(=29.408)$ at Westport; increase of pressure $=0.13$ inch. The velocity of wind uniform, and about 30 feet per second. The wind was very light at the preceding and subsequent observations, so that the progressive movement of the vortex cannot be traced.

June 11, 12.-Cyclone crossing the island from S. W. to N. E., with a velocity of about 260 miles per diem.

June 11, 9 P. M.-Centre of the vortex a little to the west of Cahirciveen. Least pressure at Cahirciveen. Mean velocity of wind $=40$ feet per second.

June 12, 9 A. M.-Centre over the island, between Kilrush and Westport. Absolute minimum of pressure $(=29 \cdot 347)$ at Kilrush; increase of pressure $=0.04$ inch. Mean velocity of wind $=25$ feet yer second.

June 12, 9 P. м.-Centre over the channel, to the east of Killough. Least pressure at Dublin. Mean velocity of wind $=20$ feet per second.

July 27, 28.-Cyclone traversing the western coast, in direction from S. S. W. to N. N. E. Velocity of wind $=30$ feet per second.

July 27, 9 A. M. - Centre of vortex west of Cahirciveen. Least pressure at Cahirciveen. Greatest velocity of wind in south-west. The wind at the north-eastern stations uninfluenced by the vortex.

July 27, 9 P. м.-Centre south of Westport. Absolute minimum of pressure $(=29.559)$ at Markree ; increase of pressure $=0 \cdot 10$ inch. Velocity of wind uniform.

July 28, 9 A. M.-General current from S. W. ; mean velocity $=30$ feet per second.

August 23, 24.-Well-defined cyclone advancing in a curvilinear path, the movement of the centre being at first from N.W. to S.E., and afterwards from S. W. to N.E.

Aug. 23, 9 P. M.-Centre of the vortex north-west of the island. Least pressure at Buncrana. Mean velocity of wind $=25$ feet per second. Lightning along the whole of the eastern coast during the day.

Aug. 24, 9 A. M.-Centre near Armagh. Absolute minimum of pressure (=29.439) at Armagh; increase of pressure $=0.13$ inch. 
Mean velocity of wind $=35$ feet per second; greatest do. (south) = 55 feet. The centre of the vortex appears to have passed over Donaghadee about noon. At 9 A. M. the direction of the wind at that place was E.S. E. ; at 12 (noon) W.S. W.; and at $1^{\text {h }} 30^{\text {m }}$ P. x. W. N. W., the shift being accompanied by strong gales and heavy rain.

Aug. 24, 9 P. м.-Centre north-east of the island. Least pressure at Donaghadee. Mean velocity of wind $=25$ feet per second.

September 29, 30.--Interesting cyclone and storm, crossing the island from S. S. W. to N.N. E., with a velocity of about 270 miles per diem. (Plate IV., figs. 1,2,3.) Mean velocity of wind on the 29 th $=45$ feet per second.*

Sept. 29, 9 A. м.-Centre of vortex off the south-western coast, to the west of Cahirciveen. Force of wind greatest at the same station at 3 A. M. ; but the barometer continued to fall until noon, when the pressure was $28 \cdot 970$. Increase of pressure $=0.22$ inch. Greatest velocity of wind (north-west) $=60$ feet per second.

Sept. 29, 9 P. м. - Centre over the island, about midway between Kilrush and Dublin. Absolute minimum of pressure $(=29.030)$ at Markree; increase of pressure $=0.12$ inch. Least pressure in

* The following extra observations were taken at Markree:-

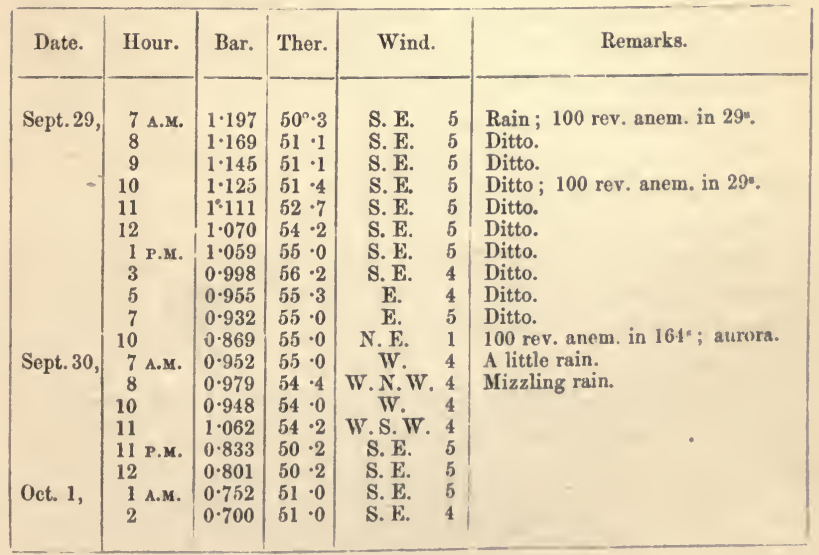


south-east at 6 P. м. Greatest velocity of wind (north-east) $=55$ feet per second.

Sept. 30, 9 A. M.-Centre near Malin Head, at northern extremity of the island. Absolute minimum of pressure $(=29.020)$ at Portrush ; increase of pressure $=0.15$ inch. Mean velocity of wind $=35$ feet per second; greatest do. (north-west) $=55$ feet.

Sept. 30, Oct. 1.-Cyclone moving apparently in curvilinear path, its course being at first from W. to E., until it reached the centre of the island, and afterwards from S. S. W. to N. N. E. Mean velocity of wind between 25 and 30 feet per second.

Sept. 30, 9 P. M.-General southerly current. Centre of vortex to the west of the island; least pressure on west coast. Greatest velocity of wind (on west coast) $=45$ feet per second.

Oct. 1, 9 A. M.-Centre of vortex over the island, between Kilrush and Courtown. Absolute minimum of pressure (=28.838) equally distant from Dublin, Courtown, and Dunmore. Northerm stations beginning to be affected by vortex. Greatest velocity of wind (north-east) $=50$ feet per second.

Oct. 1, 9 P. M.-Centre north of Portrush. Absolute minimum of pressure $(=28.853)$ at Portrush ; increase of pressure $=0.09 \mathrm{inch}$. At Donaghadee a sudden shift of the wind from S. S. E. to W. took place at $4^{\mathrm{h}} 30^{\mathrm{m}}$ P. M.

Oct. 4, 5.-Distinct cyclone moving from W.S. W. to E.N.E., and passing over (or near) the northern extremity of the island. (Plate IV., figs. 4, 5, 6.) Mean velocity of wind = 35 feet per second. General electrical disturbance.

Oct. 4, 9 A. M.-General current from S. W. ; centre of vortex north-west of the island. Greatest velocity of wind (on west coast) $=45$ feet per second.

Oct 4, 9 P. M.-Centre close to northern extremity of the island. Absolute minimum of pressure $(=29 \cdot 182)$ at Portrush ; increase of pressure $=0 \cdot 11$ inch. Greatest velocity of wind (north-west) $=55$ feet per second.

Oct. 5, 9 A. ..-Centre north of the island; least pressure at Portrush. Greatest velocity of wind (in the north) $=60$ feet per second. 
From the facts above stated, we may draw the following general conclusions :-

1. The occurrence of cyclonic movements in the atmosphere is not infrequent in Ireland, and may be traced even in the case of moderate winds.

2. The rotatory movement is invariably in the same direction, namely, that opposite to the diurnal movement of the sun in azimuth.

3. This rotation is always accompanied by a considerable disturbance of barometric equilibrium, which is greater in proportion to the velocity of the rotatory movement, the pressure being a minimum at the centre of the vortex, and increasing regularly with the distance from that point.

4. The place of greatest velocity appears to have no very definite relation to that of the centre of the vortex, sometimes nearly coinciding with it, and at others being situated in front, or in the rear, on the right hand, or on the left, of the centre.*

5. The vortex itself has a progressive movement, at the rate of from 100 to 300 miles per diem, the average velocity of those observed being 220 miles per diem. The direction of this movement in Ireland is generally from S. W. to N. E.

6. If a line be drawn through the centre of Ireland, in the direction from S. W. to N.E., the track of the centres of by far the greater number of the cyclones, passing over or near Ireland, lies to the north of that line.

7. There is reason to conclude, that these rotatory movements are caused by the conflict of two rectilinear currents moving in different directions.

\section{SToRMs.}

For the purpose of eliminating local irregularities, and (to a certain extent also) inequalities of estimation, I have, in examining the distribution of the higher winds, combined the stations into four groups, omitting Portrush and Buncrana, which lie somewhat apart. These groups are as follow:-

I. North-Eastern.-Donaghadee, Killough, Armagh. Mean latitude $=54^{\circ} 24^{\prime} ;$ mean longitude $=5^{\circ} 57^{\prime}$.

* In the remarkable cyclone of November 18, 19, 1850, the wind raged with greatest violenco on the right hand of the centre (looking in the direction of the progressive movement); and this appears to be the case of most frequent occurrence. 
II. North-Western.-Killybegs, Markree, Westport. Mean latitude $=54^{\circ} 13^{\prime} ;$ mean longitude $=8^{\circ} 51^{\prime}$.

III. South-eastern.-Dublin, Courtown, Dunmore. Mean latitude $=52^{\circ} 43^{\prime} ;$ mean longitude $=6^{\circ} 29^{\prime}$.

IV. South-western.-Kilrush, Cahirciveen, Castletownsend. Mean latitude $=52^{\circ} 2^{\prime}$, mean longitude $=9^{\circ} 37^{\prime}$.

The line joining groups I. and IV. lies, almost exactly, N. E. and S. W. ; and that joining groups II. and III., N. W. and S. E.

The following are the numbers of times in which the average force of the wind, in each of these groups, amounted to a strong breeze: or the average velocity to 35 feet per second, and upwards :

Number of Times in which the Velocity of the Wind was 35 Feet per Second and upwards.

\begin{tabular}{|c|c|c|c|c|c|c|}
\hline Month. & & & North-East. & North-West. & South-East. & South-West \\
\hline January, . . & . . & • & 18 & 19 & 13 & 29 \\
\hline February, . & . & . & 7 & 15 & 10 & 13 \\
\hline March, . & $\cdots$ & . & 5 & 15 & 4 & 14 \\
\hline April, . . & .. & - & 3 & 11 & 3 & 12 \\
\hline May, . . & . & - & 2 & 10 & 1 & 4 \\
\hline June, . . & . & . & 2 & 15 & $\tilde{j}$ & 10 \\
\hline July, . . . & . & . & 3 & 9 & 4 & 9 \\
\hline August, . . & . & . & 0 & 9 & 3 & 7 \\
\hline September, . & . & . & 2 & 11 & 3 & 6 \\
\hline October, . & . & - & 3 & 21 & 4 & 17 \\
\hline November, & - . & . & 0 & 14 & 1 & 6 \\
\hline December, & - . & - & 3 & 9 & 9 & 14 \\
\hline Spring, . . & . . & - & 10 & 36 & 8 & 30 \\
\hline Summer, . & - . & . & 5 & 33 & 12 & 26 \\
\hline Autumn, . & - . & . & -5 & 46 & 8 & 29 \\
\hline Winter, . & . . & . & 28 & 43 & 32 & 56 \\
\hline Year, . . & . . & - & 48 & 158 & 60 & 141 \\
\hline
\end{tabular}


From the foregoing numbers it appears, that high winds are much more frequent on the western than on the eastern coast, the numbers denoting the relative frequency, on the average of the entire year, being nearly as 3 to 1 . This preponderance of high winds on the western coast holds at all seasons of the year, the maximum occurring at the north-xestern extremity in autumn, and at the south-western in winter. The greatest frequenoy is in the north-west, on the average of the entire year.

The following are the eases in which the mean force of the wind, over the whole island, amounted to a gale; or in which the mean velocity was 45 feet per second and upwards :-

Nov. 23, 24, 1850.-Storm along the western coast, blowing at first from S. S. W., and veering through S.W. to W. Least pressure in north-west throughout.

Nov. 23, 9 P. M. - Storm began at south-western extremity of the island ; velocity $=45$ feet per second.

Nov. 24, 9 A. M.-Wind continued to blow in same district; velocity increased to 60 feet per second. Absolute barometrio minimum (north-west) $=28 \cdot 644$.

Nov. 24, 9 P. M.-Storm extended over whole of western coast; velocity of wind $=55$ feet per second.

Dec. 14.-Storm affecting the whole island, but chiefly the western coast. Wind at first from S. S. W., but veering to W.S.W. at 9 P.м. Least pressure in north-west throughout. Electrical disturbance over the whole island.

Dec. 14, 9 A. M.-Velocity on western coast $=65$ feet per second. Absolute barometric minimum (north-west) $=28.952$.

Dec. 14,9 P. м.- Velocity on western coast $=50$ feet per second.

Dec. 31, Jan. 1, 1851.-Storm from S. W. and S., beginning on western coast, and extending over the whole island.

Dec. 31, 9 A. м.- Velocity on western coast $=50$ feet per second. Direction S.S. W. and S. W.

Dec. 31, 9 P. м.-Gale affecting the whole island, except northeastern extremity. Greatest in south-west; velocity $=60$ feet per second. Direction as before. Absolute barometric minimum (north) $=29 \cdot 177$. 
Jan. 1, 9 A. M.-Wind abated.

Jan. 1, 9 P.M.-Gale from S. W. and S. over the whole island, except north-western extremity. Velocity (south-east) $=55$ feet per second. Absolute barometric minimum (north) $=28 \cdot 975$.

In this case, therefore, there were two storms succeeding each other on consecutive days, with a double fall of the barometer. The direction of the wind on Jan. 1, 9 P. м. was remarkable. The prevailing current was from $\mathrm{S}$. W., and extended over the central parts of the island; while there appears to have been an indraught towards it, from the north-western and south-eastern quarters.

Jan. 12, 13.-Storm from S. and S. W., beginning in the northwest, and advancing in the direction from N. W. to S. E. Velocity of wind $=60$ feet per second. Least pressure in north-west throughout.

Jan. 12, 9 P.M.-Gale in north-west.

Jan. 13, 9 A.M.-Storm advanced to line joining north-east and south-west centres. Absolute barometric minimum (north-west) $=29 \cdot 174$; pressure least at Markree at noon.

Jan. 27.-Storm from S. and S. W. in the afternoon of this day, chiefly along the western coast. Velocity of wind $=55$ feet per second. Absolute barometric minimum (north-west) $=29 \cdot 309$.

June 15, 16.-Gale from S. W. and W., on the western coast. June 15, 9 A. M.-Wind from S. W. Velocity on western coast $=50$ feet per second. Least pressure in north-west.

June 15, 9 P.M.-Velocity $=45$ feet per second. Absolute barometric minimum (north) $=29.575$.

June 16, 9 A. M.-Wind from W. Velocity $=50$ feet per second.

July 13, 14.-Storm chiefly in north-west, blowing at first from S.S.W. and veering through S.W. to W. This appears to have been a cyclonic gale, the centre of the cyclone passing to the north of the island; it is not included in the former series on account of this circumstance. The velocity of the wind was greatest in the north-west throughout; the barometric pressure was least 
in the north-west on the 13th, and in the north-east on the following day.

July 13, 9 A. M. - Storm from S. S. W., in the north-west of the island. Velocity $=60$ feet per second.

July 13, 9 P. M.-Gale veered to S. W., and affected a large portion of the island. Velocity of wind $=60$ feet per second, as before. Absolute barometric minimum $=29 \cdot 052$.

July 14, 9 A. M.-Wind veered to W. Velocity in north-west increased to 65 feet per second.

Dec. 7.-Storm began in south-western extremity of the island, and extended thence over the whole. Direction of wind between S. and S. W.

Dec. 7, 9 A. м.-Gale from S.S. W. in the south-west.

Dec. 7, 9 P. м.- Storm over the whole island. Greatest velocity and least pressure in north-west. Velocity $=70$ feet per second. Absolute barometric minimum $=29 \cdot 267$. At Cahirciveen the barometer fell until 7 P. M. ; and the wind shifted from S. to TV. at the same time.

Dec. 9.-Storm from S. W. along the western coast. Least pressure in north-west throughout.

Dec. 9, 9 A. м. - Velocity of wind in west $=50$ feet per second.

Dec. 9, 9 P. м. - Velocity $=60$ feet per second. Absolute barometric minimum $=29 \cdot 632$.

Dec. 20.-Gale blowing from S.S. W., beginning on western coast, and advancing to eastern. Least pressure in north and north-west.

Dec. 20, 9 A. M.-Gale on west coast. Velocity $=55$ feet per second.

Dec. 20, 9 P.M.-Gale transferred to east-coast. Velocity $=50$ feet per second. Absolute barometric minimum (north) $=29 \cdot 457$.

At Markree there was a sudden shift of the wind from S. S. W. to N. W. at $7^{\mathrm{h}} 35^{\mathrm{m}}$ P. M.

From the foregoing facts we may draw the following general conclusions :-

1. The greater gales are much more frequent on the westex'u, 
than on the eastern coast, the numbers denoting the relative frequency being nearly as 5 to 1 . The frequency of storms is nearly the same in the northern and southern portions of the island.

2. The direction of the wind, in all the cases enumerated, was between S. and W. In about half of these cases the wind blew, throughout, from the same point; in half it veered from 4 to 6 points of the compass, the veering being in the direction produced by a cyclone moving from S. W. to N. E., and having the path of its centre to the north of the island.

3. The axis of the gale is in some cases transferred parallel to itself, to the eastward. Remarkable instances of this movement occur in the gales of January 12, 13, and December 20.

4. The least barometric pressure occurs, in almost every instance, in the north-western quarter of the island.*

5. The locality of the highest wind sometimes coincides with that of least pressure, and sometimes does not. In the latter case, the axis of least pressure is generally to the westward of the axis of the storm.

6. On either side of the axis of a storm, the wind appears to blow towards that line. A remarkable instance of this phenomenon occurred in the storm of January 1. $\dagger$

We are now in a position to consider the question, whether all storms are cyclonic? - -and if not, what proportion do rotatory storms bear to the whole? Of the greater storms which have occurred since the commencement of these observations, the rotatory character of five-those of October 6, 1850, November 18, January 15, 1851, March 18, and September 29-has been completely established. We have seen in this section, that the same character may be predicated, with great probability, of five more; while there remain five in which the wind has blown, throughout, in the same direction. In fifteen months, accordingly, there have occurred fifteen storms, of which two-thirds were cyclonic. As respects the remaining onethird, the phenomena are characterized, not only by the absence of any veering of the wind, but also by the fact, that the pressures

* In one case only, the loeality of least pressure shifted from the north-western to the north-eastern extremity of the island. This is consistent with the supposition, that the storm in question was a cyclone, whose centre had a progressive motion eastward.

† The conclusions numbered 3, 5, 6, have already bcen drawn by Mr. Espy, from an examination of the storms in the United States in the early months of the year 1813.-First Report on Metcoro'ogy. 
appear to increașe with the distance from a line or axis of minimum pressure, rather than from a point; or, in other words, that the isobaric lines are parallel right lines, instead of concentric circles. It is true that these facts are by no means decisive in disproving rotatory movement; for they are consistent with a rotation of the wind in a plane perpendicular, or highly inclined, to the horizon. Still we are perhaps not justified in assuming the existence of a rotation of this kind, without further evidence; and it seems more reasonable, in the present state of our knowledge, to admit two different kinds of winds, than to endeavour to reduce all to one by the help of a gratuitous hypothesis.

Hourly Observations.-It has been already stated, that hourly observations were appointed to be made during twenty-four consecutive hours, at the equinoxes and solstices, in the hope that their results might throw light upon the simultaneous atmospheric changes occurring over the island, and especially upon the direction and rate of progress of atmospheric waves. The results of the observations on the first two of these term-days-March 21, and June 21-are here given. During the two latter-September 22 and December 22-no atmospheric change of a marked kind occurred.

March 21.-A gale occurred on this day, accompanied by a marked barometric depression. The minimum of pressure took place during the observations, the time of its occurrence varying considerably at the different stations. At Cahirciveen, there was a sudden fall of the barometer between 9 A. M. and 10 A. M., followed by a sudden rise between 12 and 1 P. M.; the mercury being nearly stationary from 10 A. M. to 12. A similar change took place at Dunmore East, and at the same hours. For these two stations, accordingly, the epoch of minimum pressure may be taken to be $11 \mathrm{A.M.}$; the subsequent changes were small and irregular. At Courtown, the barometer descended very slowly and gradually until 5 P. м.; it then ascended until 10 P. м., after which it descended again. All the changes were, however, very small.

At the northern stations the fall of the barometer was more considerable, and more regular. At Markree, where it was most rapid, it amounted to 0.210 in 6 hours. The minimum at Markree occurred between 3 P. M. and 4 P. M. ; at Armagh, the minimum took place at 6 P. M. ; and at Portrush, at 8 P. M. 
From these results it would appear that the trough of the wave travelled from south to north, nearly, with a velocity of about 22 miles per hour. The barometric depression was greatest at Markree, where the barometer stood at $28 \cdot 689$, when lowest. The lowest pressure increased from that point in the south-easterly direction, being 28.972 at Dunmore.

At Markree the wind shifted from S. S. E. to S. S. W. at the time of greatest depression. The same phenomenon took place at Armagh and Portrush, although not with such precision; the change of direction at the former station being from S.S. E. to S., and at the latter from S. E. to S. No similar change occurred at the southern stations.

The foregoing phenomena are not necessarily to be ascribed to the transit of a rectilinear wave. They are all consistent with the effects of a cyclone, coming from the S. or S. W., the track of its centre lying to the west of the island.

June 21, 22.-The changes of the direction and of the pressure of the wind, on these days, are manifestly the effects of a cyclonic movement, the centre of the vortex sweeping round the north coast of Ireland, in a somewhat curvilinear path, from west to east. It has not been included in the former series, the force of the wind having been below the limit there adopted. At 9 A. M. of the 21st, the centre of the vortex was off the north-west coast, to the west of Killybegs. At 9 P. M. of the same day it had arrived to the north of Portrush; and at 9 A.M. of the 22nd it was to the northeast of Donaghadee.

The veering of the wind was, on the average, about $90^{\circ}$; its duration was very different at the different stations, being shortest for those near the path of the centre of the vortex, and longest for those remote. The wind, which was very light throughout, fell about the time of veering at most of the stations.

The descent and subsequent rise of the barometer were regular, and the minimum well-defined. The time of least pressure coincided at all the stations, very nearly, with the middle of the time of veering of the wind; it was earliest on the western coast, and latest on the eastern, the epoch of its occurrence being between 12 and 1 P. M. at Markree and Cahirciveen, and between 5 P. M. and 6 P. M. at Dublin and Courtown. The barometric depression was small, the mean pressure at the epoch of minimum being $29 \cdot 74$. 


\section{Humidity of tire Air.}

The first two of the Tables which follow give the results of the psychrometrical observations. The first contains the monthly means of the tension of vapour at the several stations, calculated by Regnault's Table ; and the second the corresponding values of the relative humidity, the state of saturation being represented by 100 .

Very few results of a general nature can be drawn from these observations, the distribution of vapour being governed by the proximity of the station to the sea, or by other local circumstances. It will be seen, from the last column of the first Table, that the yearly mean tension of vapour increases, although not in any regular progression, in proceeding from the north to the south of the island. Its mean value for the entire island is 0.326 of an inch ; its greatest value (at Westport) is 0.374 .

The distribution of humidity is still more under the influence of local circumstances, and therefore still less regular. Thus, Portrush and Castletownsend-the one at the northern, the other at the southern extremity of the island-have nearly the same mean humidity; while Portarlington and Athy-places near each other, and both inland-are almost at the opposite extremities of the scale. The driest station is Portarlington; the most humid, Westport. At the latter place, in fact, the air is nearly saturated with moisture, the place of observation being entirely surrounded by water, and but a few feet above the sea. The mean humidity for the entire island, for the year 1851, is 87 .

\section{RaIN.}

Before proceeding to the observations of rain-fall throughout Ireland in the year 1851, it is important that we should know its normal amount at one or more stations, as deduced from the mean of several years. We have, for this purpose, two series of observations, one at Dublin, and the other at Armagh, extending uninterruptedly over eleven and twelve years respectively. The results of these two series are contained in the third and fourth of the following Tables.

The lowest line in each gives the mean monthly fall of rain. It will be seen, from an inspection of the numbers, that there is 
no regular progression in the amount of rain-fall throughout the year, such as is observed in the phenomena of temperature or humidity. In Dublin the greatest rain-fall, in the mean of the eleven years, occurs in October, and the least in February; their amounts are 3.34 and 1.74 inches respectively. At Armagh the maximum is in January, and the minimum in May; and they amount to 3.75 and 2.15 inches.

The fifth and last Table gives the monthly fall of rain in the year 1851, at all the meteorological stations.

It will be seen that the greatest diversity exists in the amount of rain-fall in different localities. To render this more apparent, and to facilitate the examination of the causes which influence the distribution, I have, in the following Table, given the yearly rainfall at the several stations arranged in the order of magnitude, beginning with the smallest :-

Total Rain-Fall in the Year 1851, at the several Meteorological Stations.

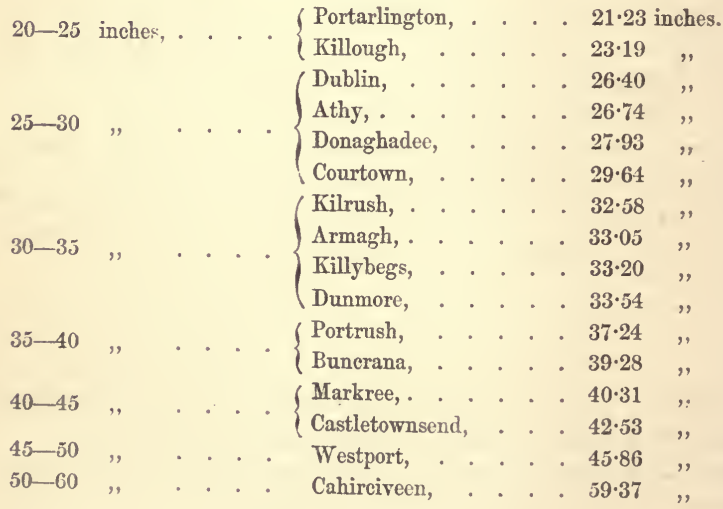

Thus, the greatest rain (at Cahirciveen) is nearly treble of the least (at Portarlington). The mean rain-fall throughout Ireland, in the year 1851 , was 34.50 inches.

If we assume the proportion of rain at the different stations to be constant, or nearly so, the numbers of the preceding Table may 
all be reduced to their mean values, by multiplying by the factor which expresses the relation of the rain of 1851 to the mean at any one station. We already possess two such mean values : viz., at Armagh and Dublin. They are $29 \cdot 14$ and 34.68 inches respectively; and the factors thence deduced are 1.10 and 1.05.

When we examine the results of the preceding Table, taken in connexion with the geographical position and physical circumstances of the stations, we arrive at the following conclusions :-

1. The places of least rain are either inland, or on the eastern coast; while those of greatest rain are at, or near, the western coast. Thus the stations at which the yearly fall of rain exceeds 40 inches are all on the western and south-western coasts; while those at which it is below 30 inches are either inland or on the eastern.

2. The amount of rain is greatly dependent on the proximity of a mountain chain or group, being always considerable in such neighbourhood, unless the station be to the east or north-east of the same. Thus, of the places of least rain, Portarlington lies to the north-east of Slieve-bloom; Killough, to the north-east of the Mourne range; Dublin, to the north-east of the Dublin and Wicklow range; while, on the other hand, the places of greatest rin-Cahirciveen, Westport, and Castletownsend-are in the vicinity of high mountains, but on a different side.

These facts are easily explained. The prevailing wind blows from the S. W., and reaches this island loaded with the vapour of the Gulf-Stream. This vapour is condensed and precipitated in rain, when it first meets the colder air over the land, namely, on the western and south-western shores. But the principal condensing centres are the mountains, in the neighbourhood of which, consequently, the precipitation is more abundant, especially on their western and south-western sides. And the same circumstance which causes the greater precipitation at these points must also protect the region over which the wind next passes (the north-east), the air being thus deprived of a large portion of its vapour before arriving there.

Nots.-A few of the latter Tables of this Paper, as originally printed, have been omitted here as unneccessary. 


\begin{tabular}{|c|c|}
\hline$\stackrel{\varpi}{\check{\varpi}}$ & 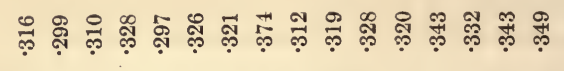 \\
\hline$\dot{\check{̆}}$ & 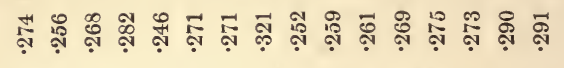 \\
\hline 官 & 令 \\
\hline$\stackrel{\Delta}{0}$ & 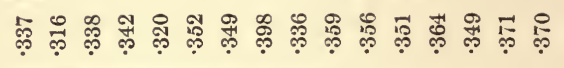 \\
\hline 苨 & 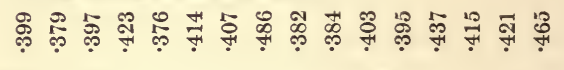 \\
\hline 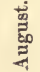 & 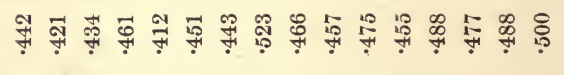 \\
\hline 官 & 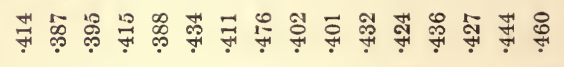 \\
\hline$\stackrel{\dot{\Xi}}{\leftrightarrows}$ & న \\
\hline$\dot{\mathrm{g}}$ & 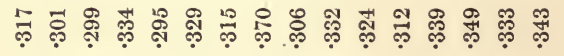 \\
\hline 完 & 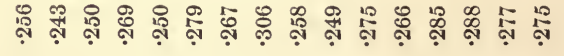 \\
\hline 芦 & 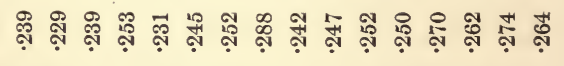 \\
\hline$\dot{0}$ & 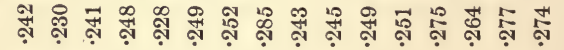 \\
\hline 峞 & 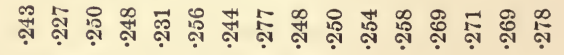 \\
\hline 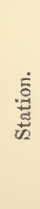 & 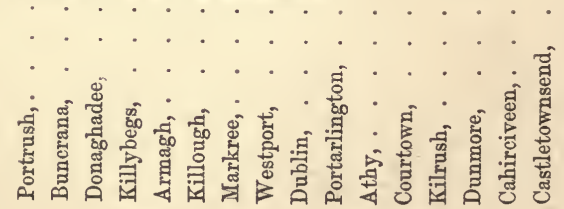 \\
\hline
\end{tabular}


ON THE METEOROLOGY OF IRELAND.

\begin{tabular}{|c|c|}
\hline$\dot{\vec{p}}$ & 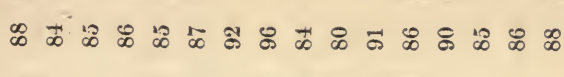 \\
\hline ̊ัँ & ஜ $\&$ வ \\
\hline $2 / 4$ & 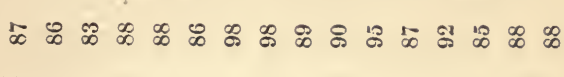 \\
\hline Бे & \& \\
\hline 离 & 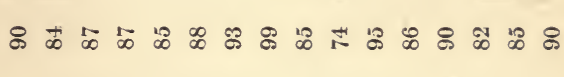 \\
\hline $\bar{z}$ & 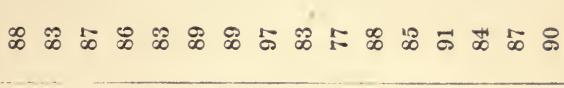 \\
\hline 常 & 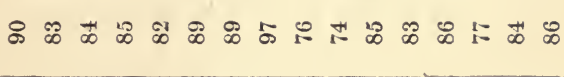 \\
\hline 总 & 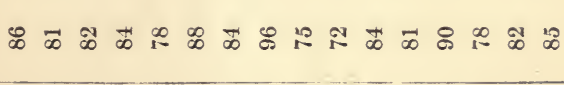 \\
\hline$\pi$ & 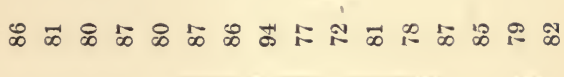 \\
\hline & 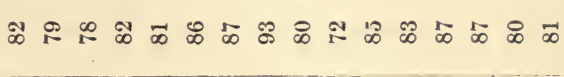 \\
\hline & 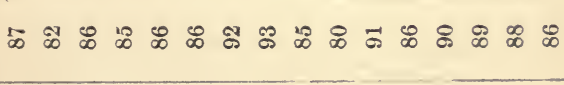 \\
\hline 胥 & 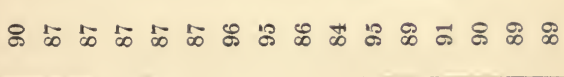 \\
\hline ตี่ & 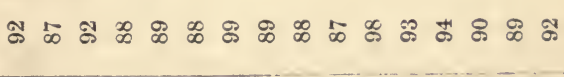 \\
\hline & 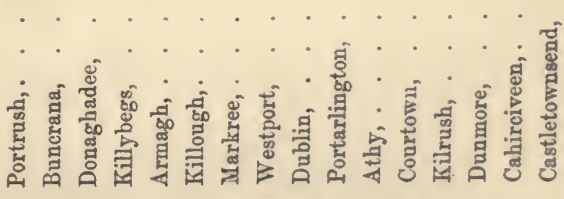 \\
\hline
\end{tabular}




\begin{tabular}{|c|c|c|c|c|c|c|c|c|c|c|c|c|}
\hline 造 & $\begin{array}{l}\stackrel{0}{10} \\
\dot{0} \\
\stackrel{\sim}{N}\end{array}$ & $\begin{array}{l}\stackrel{0}{0} \\
\dot{\infty} \\
\text { o }\end{array}$ & $\begin{array}{l}\text { ก } \\
\text { ก }\end{array}$ & $\begin{array}{l}F \\
\dot{+} \\
\text { क }\end{array}$ & $\underset{\stackrel{\infty}{\sigma}}{\stackrel{\infty}{n}}$ & $\vec{\square}$ & $\begin{array}{l}8 \\
\text { iे } \\
\text { ஸे }\end{array}$ & ت्ञ & $\begin{array}{l}\vec{\infty} \\
\dot{\sigma}\end{array}$ & $\underset{\stackrel{+}{+}}{\stackrel{\infty}{+}}$ & $\begin{array}{l}\stackrel{0}{+} \\
\dot{\leftrightarrow} \\
\text { ํ }\end{array}$ & $\cdot \vec{d}$ \\
\hline ஓ̊ & 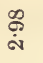 & $\stackrel{\infty}{0}$ & $\ddot{0}$ & $\begin{array}{l}\bigotimes \\
\dot{\omega}\end{array}$ & $\stackrel{T}{\dot{5}}$ & $\begin{array}{l}\mathscr{\infty} \\
0\end{array}$ & $\begin{array}{l}\stackrel{\leftrightarrow}{1} \\
\dot{\text { i }}\end{array}$ & $\begin{array}{l}\infty \\
\infty \\
\dot{\sim}\end{array}$ & 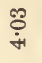 & $\begin{array}{c}\stackrel{0}{\overparen{\omega}} \\
\text { ஸे }\end{array}$ & $\begin{array}{l}\infty \\
\infty \\
0\end{array}$ & $\begin{array}{l}\stackrel{\leftrightarrow}{*} \\
\text { i }\end{array}$ \\
\hline 客 & $\begin{array}{l}\infty \\
? \\
\dot{n}\end{array}$ & $\stackrel{5}{9}$ & $\stackrel{\circ}{\dot{m}}$ & $\begin{array}{l}\text { में } \\
\text { ம் }\end{array}$ & $\begin{array}{l}0 \\
\ddot{n} \\
\dot{\varphi}\end{array}$ & $\begin{array}{l}\infty \\
\dot{\sim}\end{array}$ & $\stackrel{8}{\dot{\rho}}$ & $\stackrel{8}{\stackrel{0}{-1}}$ & $\begin{array}{l}8 \\
\stackrel{\infty}{-1}\end{array}$ & $\begin{array}{l}\text { के } \\
\text { ஸे }\end{array}$ & $\stackrel{0}{\dot{1}}$ & $\begin{array}{l}\mathscr{2} \\
\infty \\
\dot{\omega}\end{array}$ \\
\hline ثீँ & $\begin{array}{l}\infty \\
\dot{+} \\
\dot{+1}\end{array}$ & $\stackrel{\leftrightarrow}{\stackrel{-1}{-1}}$ & $\underset{\dot{n}}{\ddot{H}}$ & $\begin{array}{l}\text { ஸे } \\
\text { ஸे }\end{array}$ & $\begin{array}{l}\stackrel{\oplus}{ } \\
\dot{\infty}\end{array}$ & $\underset{\mathfrak{H}}{\mathfrak{N}}$ & $\underset{\dot{\omega}}{\stackrel{m}{\dot{\omega}}}$ & $\begin{array}{c}\infty \\
\stackrel{\infty}{+}\end{array}$ & ळా & $\stackrel{\text { 乌̈ }}{\stackrel{-1}{1}}$ & 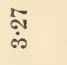 & के \\
\hline 芯 & $\begin{array}{l}\infty \\
\stackrel{\infty}{\sim}\end{array}$ & $\begin{array}{l}8 \\
\dot{+}\end{array}$ & 苞 & $\begin{array}{l}19 \\
\dot{\text { กิ }}\end{array}$ & की & $\begin{array}{l}\infty \\
\stackrel{0}{\omega}\end{array}$ & $\begin{array}{l}10 \\
\stackrel{1}{-1}\end{array}$ & $\begin{array}{l}\stackrel{+}{+} \\
\dot{\sim}\end{array}$ & $\begin{array}{l}\mathscr{\infty} \\
\dot{\infty}\end{array}$ & $\stackrel{\rho}{\dot{-1}}$ & $\begin{array}{l}\overrightarrow{5} \\
\dot{-1}\end{array}$ & \begin{tabular}{l}
$\infty$ \\
\multirow{N}{*}{} \\
$\dot{v}$
\end{tabular} \\
\hline$\stackrel{800}{\bar{z}}$ & $\begin{array}{l}\text { ণิ } \\
\text { ง่ }\end{array}$ & $\stackrel{\mathbb{H}}{-}$ & $\underset{\text { ง }}{\stackrel{N}{ }}$ & $\stackrel{T}{5}$ & ํำ & ஜొ & $\stackrel{\mathscr{9}}{\stackrel{\leftrightarrow}{+}}$ & $\stackrel{ }{\stackrel{1}{10}}$ & $\begin{array}{l}\mathscr{2} \\
\dot{\text { ம }}\end{array}$ & $\begin{array}{l}\infty \\
\stackrel{0}{-}\end{array}$ & $\begin{array}{l}\grave{S} \\
\dot{1}\end{array}$ & $\begin{array}{l}\infty \\
\stackrel{\leftrightarrow}{\text { ஸे }}\end{array}$ \\
\hline$\frac{\dot{B}}{\vec{B}}$ & $\begin{array}{l}\text { ชื } \\
\text { ஸे }\end{array}$ & $\stackrel{\vec{n}}{\dot{\omega}}$ & $\stackrel{\mathscr{\wp}}{\leftrightarrows}$ & $\begin{array}{l}\mathscr{\mathscr { D }} \\
\stackrel{-}{-}\end{array}$ & $\begin{array}{l}\text { 饣ै } \\
\dot{\text { ஸे }}\end{array}$ & $\stackrel{\vec{n}}{\ddot{n}}$ & 㝴 & $\stackrel{\mathfrak{w}}{\mathfrak{\omega}}$ & $\begin{array}{l}\overparen{8} \\
\dot{\sim}\end{array}$ & $\begin{array}{l}\stackrel{\leftrightarrow}{\leftrightarrow} \\
\stackrel{\sim}{N}\end{array}$ & $\underset{+\infty}{\infty}$ & $\underset{\text { ஸे }}{\stackrel{\mathscr{N}}{ }}$ \\
\hline$\underset{\Xi}{\stackrel{\Xi}{ే}}$ & $\stackrel{+}{\infty}$ & $\begin{array}{l}8 \\
\dot{\text { ஸे }}\end{array}$ & $\begin{array}{l}8 \\
\text { ஸ் }\end{array}$ & $\stackrel{8}{Ð}$ & $\stackrel{8}{\stackrel{\leftrightarrow}{+}}$ & 足 & $\vec{\phi}$ & $\begin{array}{l}\mathscr{S} \\
\dot{\text { ஸे }}\end{array}$ & $\stackrel{5}{0}$ & $\stackrel{8}{\dot{-1}}$ & $\ddot{E}$ & $\begin{array}{l}\vec{y} \\
\dot{\sigma}\end{array}$ \\
\hline 葛 & $\stackrel{+}{\dot{H}}$ & $\underset{\dot{c}}{\infty}$ & $\vec{p}$ & $\stackrel{\overrightarrow{0}}{\dot{0}}$ & $\stackrel{+1}{\circ}$ & $\underset{\dot{\sigma}}{\vec{\sigma}}$ & $\begin{array}{l}\vec{S} \\
\dot{\text { v }}\end{array}$ & ஜூ & $\begin{array}{r}\hat{0} \\
\dot{\omega}\end{array}$ & $\underset{\text { in }}{\stackrel{\text { i }}{1}}$ & $\stackrel{\vec{\eta}}{\dot{\eta}}$ & $\underset{\dot{0}}{\stackrel{1}{0}}$ \\
\hline 远 & $\stackrel{\sim}{\stackrel{-}{*}}$ & $\begin{array}{l}\vec{\infty} \\
\dot{0}\end{array}$ & $\begin{array}{l}\text { फ } \\
\dot{\text { ஸे }}\end{array}$ & $\stackrel{0}{\circ}$ & $\begin{array}{l}\infty \\
\infty \\
\dot{0}\end{array}$ & क़े & サै & $\stackrel{10}{\dot{m}}$ & กั & ஜ़ & $\stackrel{5}{\dot{1}}$ & $\overrightarrow{\dot{\omega}}$ \\
\hline 宽 & $\frac{\stackrel{\sim}{~}}{\dot{N}}$ & $\underset{\sim}{\stackrel{T}{*}}$ & $\stackrel{\leftrightarrow}{\stackrel{-}{2}}$ & $\begin{array}{l}\text { के } \\
\dot{\omega}\end{array}$ & $\underset{\leftarrow}{\stackrel{\mathfrak{T}}{\leftarrow}}$ & $\begin{array}{l}\infty \\
\infty \\
\dot{\omega}\end{array}$ & $\stackrel{\text { ণ }}{\dot{-1}}$ & 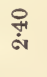 & $\stackrel{5}{\circ}$ & $\stackrel{\vec{\sigma}}{\dot{r}}$ & $\begin{array}{l}\infty \\
\stackrel{\infty}{\omega}\end{array}$ & $\stackrel{\leftrightarrow}{\dot{H}}$ \\
\hline هั & $\stackrel{8}{\stackrel{\circ}{-}}$ & $\overrightarrow{\tilde{s}}$ & $\stackrel{\not}{\stackrel{\leftrightarrow}{-}}$ & $\begin{array}{c}\mathfrak{\sigma} \\
\stackrel{\sigma}{ }\end{array}$ & $\begin{array}{l}-1 \\
0 \\
0\end{array}$ & $\stackrel{\text { f }}{\stackrel{-1}{-1}}$ & @̋ & $\underset{\mathscr{\leftrightarrow}}{\mathscr{\infty}}$ & $\stackrel{N}{0}$ & $\stackrel{\leftarrow}{\stackrel{5}{\sim}}$ & $\stackrel{0}{+}$ & $\stackrel{+}{\leftarrow}$ \\
\hline 离 & $\underset{\dot{\sim}}{\overrightarrow{4}}$ & $\underset{-1}{\stackrel{F}{-1}}$ & $\frac{10}{\dot{\alpha}}$ & $\stackrel{\leftrightarrow}{\stackrel{-}{\circ}}$ & $\stackrel{\infty}{\stackrel{+}{+}}$ & $\underset{\sim}{\stackrel{9}{\circ}}$ & זִ & $\begin{array}{l}\infty \\
\stackrel{\infty}{-}\end{array}$ & $\begin{array}{l}\text { ஜ్ } \\
\text { ம் }\end{array}$ & $\begin{array}{l}\text { ^ึ } \\
\text { ஸे } \\
\text { ஸे }\end{array}$ & $\begin{array}{l}\infty \\
\stackrel{\infty}{1} \\
\text { is }\end{array}$ & $\begin{array}{l}\infty \\
\infty \\
\dot{\sim}\end{array}$ \\
\hline & $\underset{\sim}{\stackrel{H}{-1}}$ & $\underset{\mathscr{N}}{\stackrel{\infty}{-1}}$ & $\underset{\sim}{\infty}$ & $\underset{-1}{\mathbb{\infty}}$ & $\underset{\infty}{\infty}$ & $\begin{array}{l}0 \\
\stackrel{1}{1} \\
-1\end{array}$ & 点 & $\underset{+\infty}{\infty}$ & क્తి & $\begin{array}{l}\stackrel{1}{0} \\
\infty \\
-1\end{array}$ & $\stackrel{\overrightarrow{10}}{\infty}$ & 苋 \\
\hline
\end{tabular}


ON THE METEOROLOGY OF IRELAND.

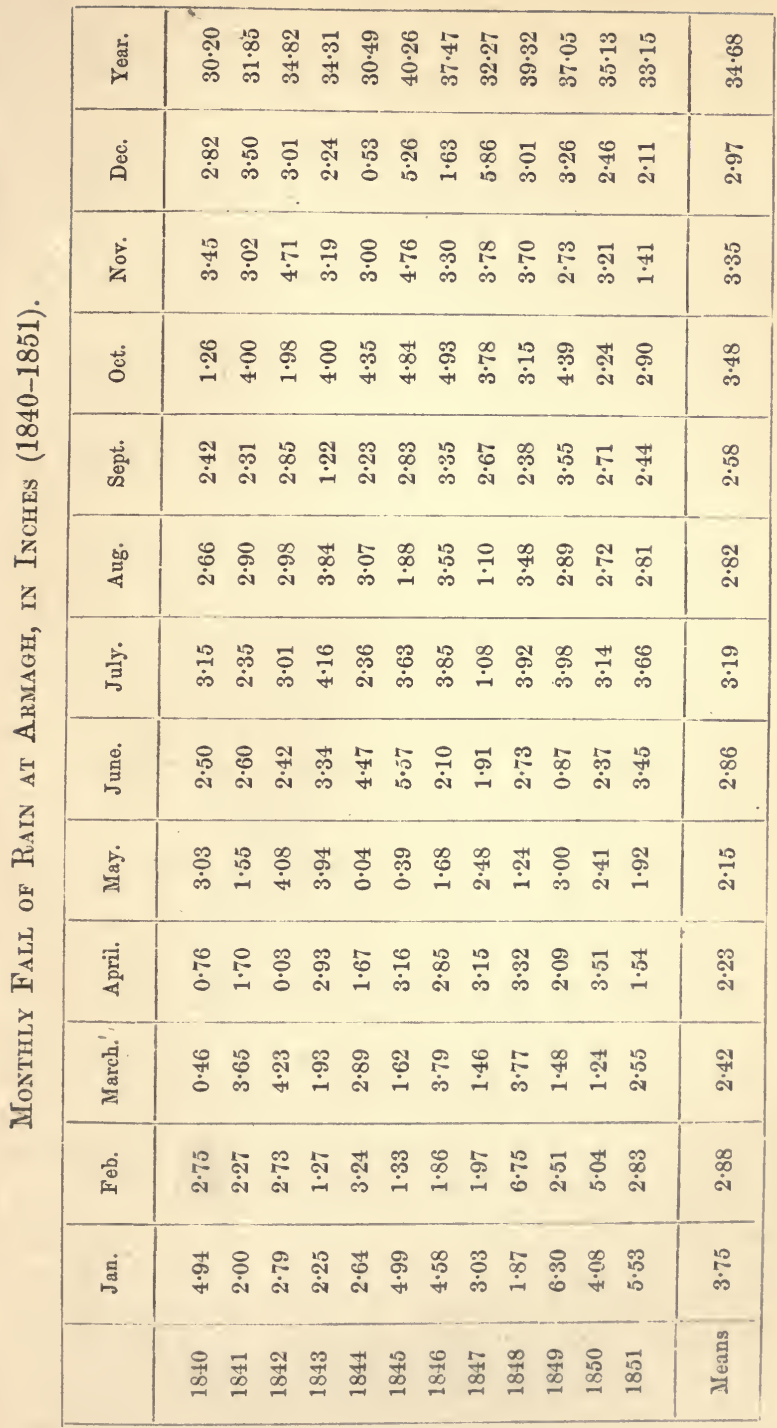




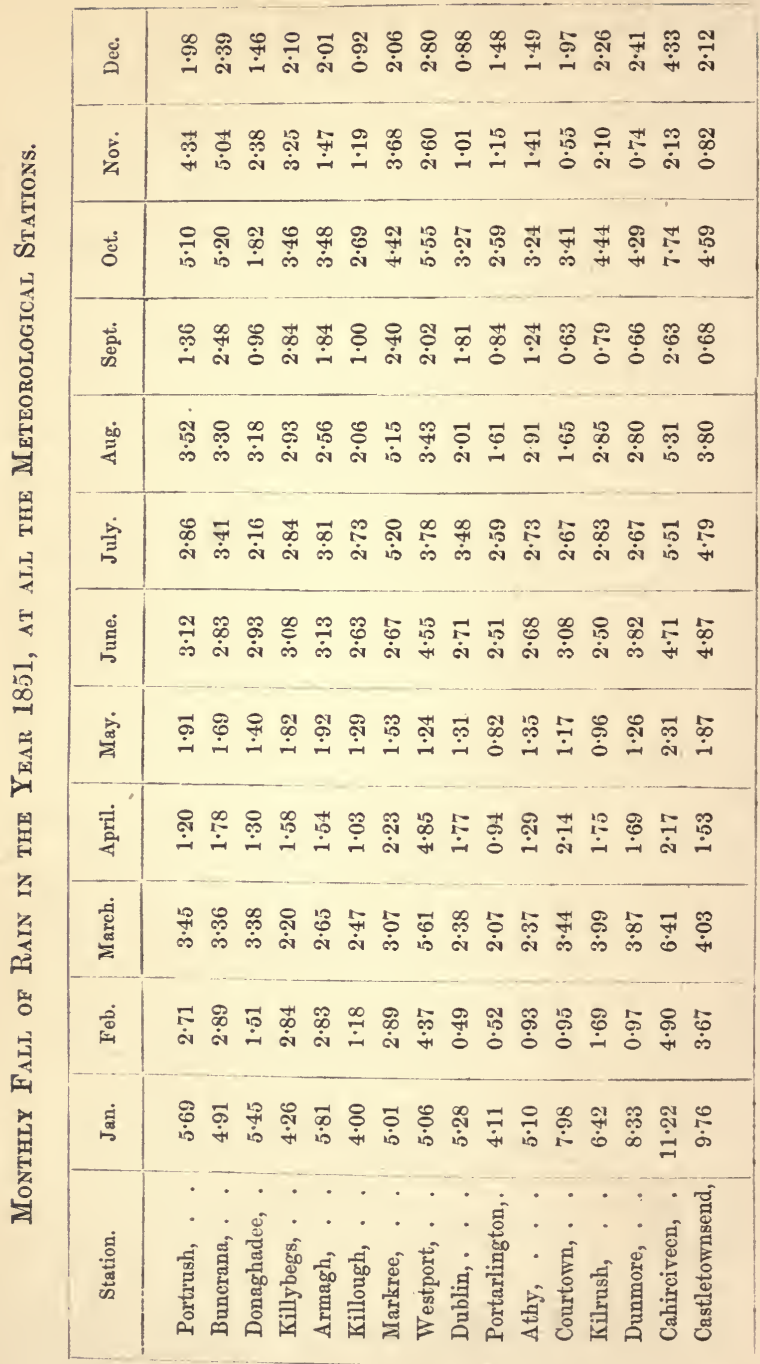




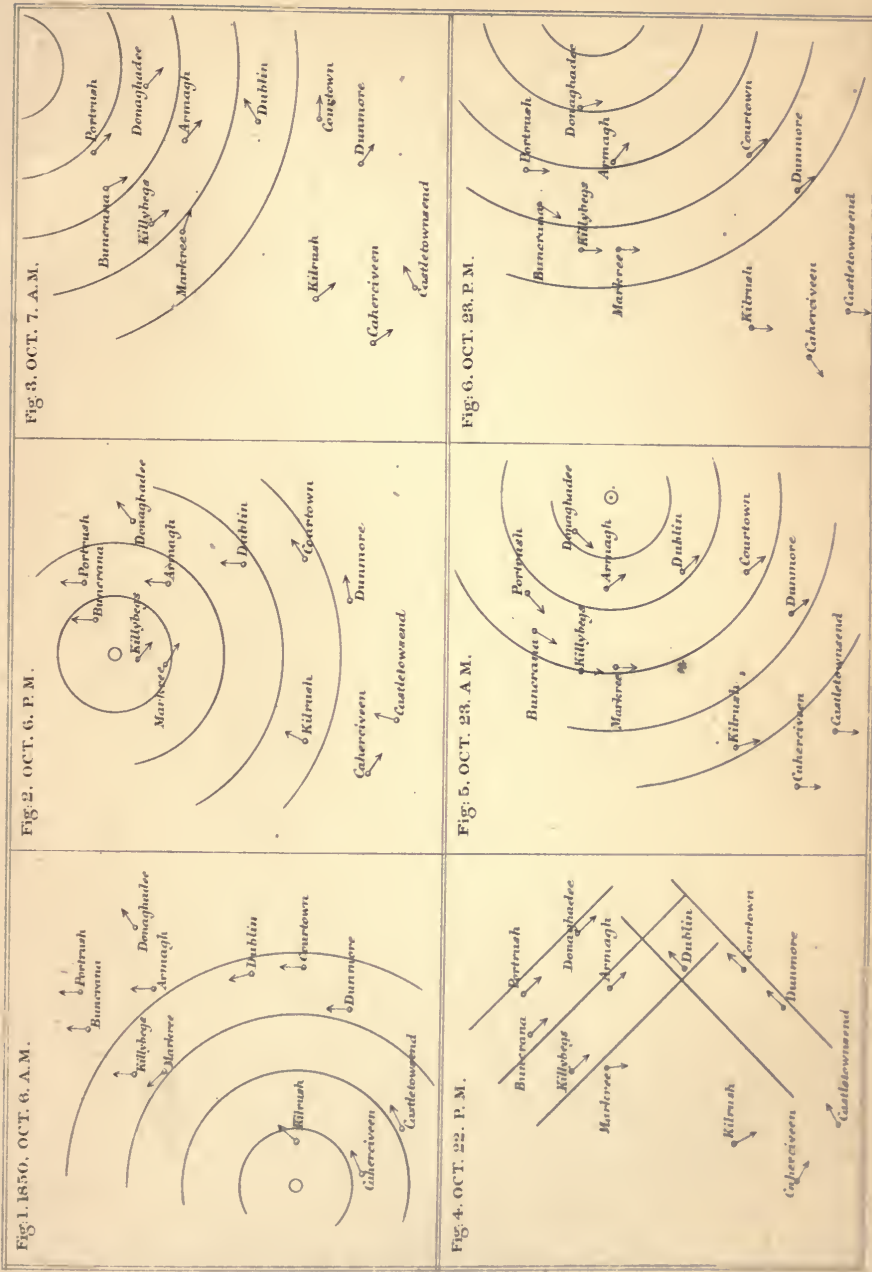



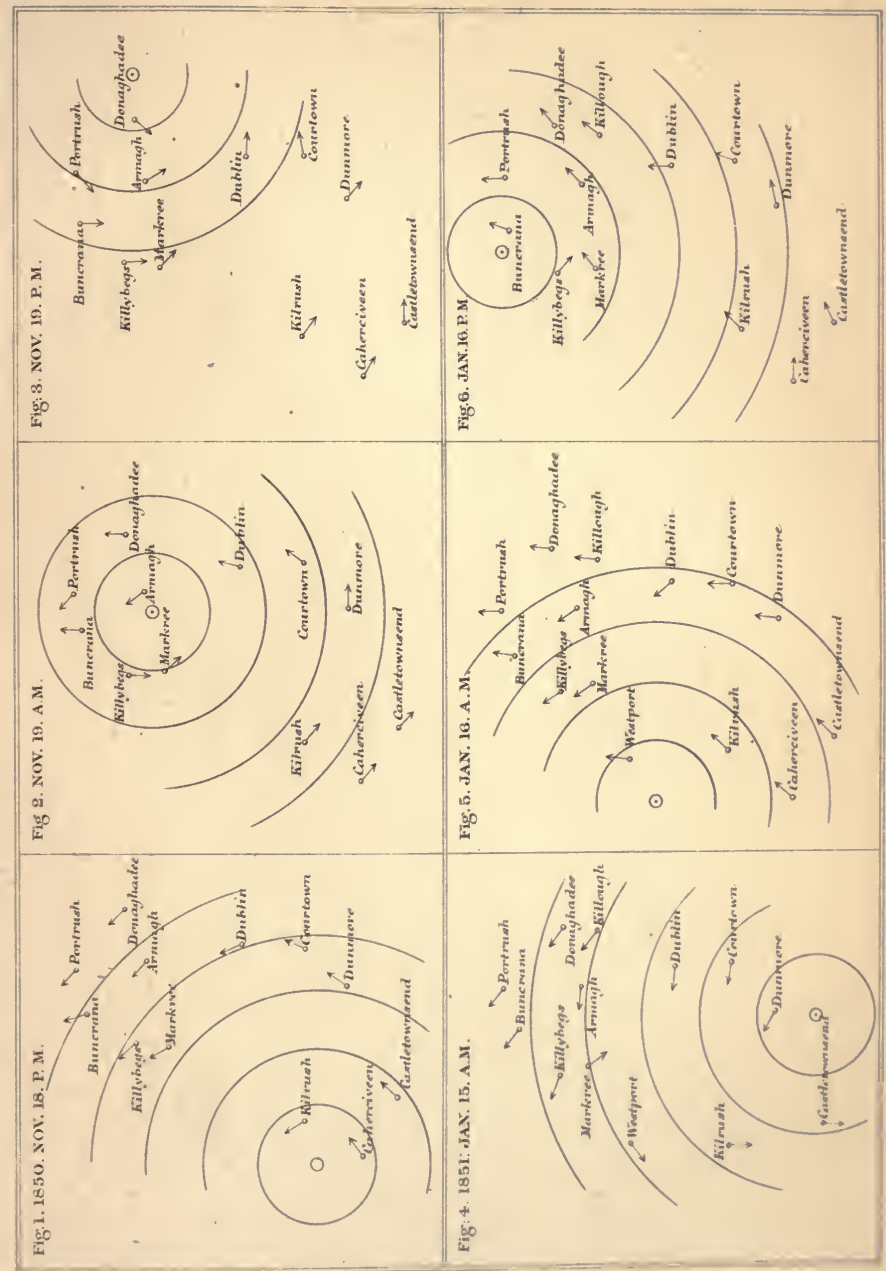





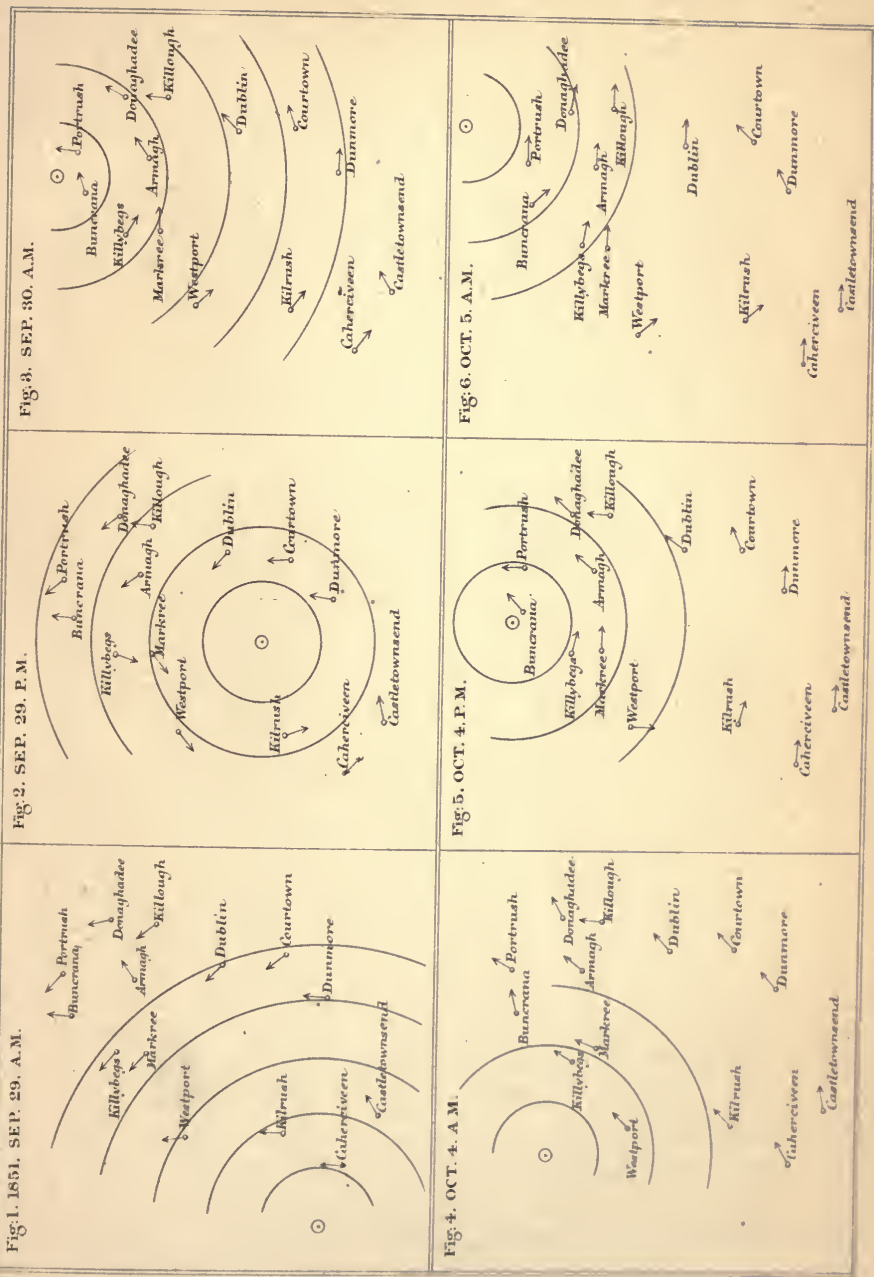



XVIII.-THE CLIMATE OF IRELAND, AND THE CURRENTS OF THE ATLANTIC.

Lecture delivered before the Dublin Young Men's Christian Association, Oot. 25th, 1865.

IT is an old remark of Dr. Johnson, that "when two Englishmen meet, their first talk is of the weather ; they are in haste to tell each other, what each must already know, that it is hot or cold, bright or cloudy, windy or calm." And he ascribes this to the variability of our climate, and to the hopes and apprehensions which constant change is sure to engender; for we naturally congratulate or condole with one another, according as the one or the other is realized.

Now although in this climate the state of the weather from day to day is so changeable as almost to baffle the power of anticipation, there are yet mean laws, and average results, which we obtain from systematic observation, followed throughout long periods, and which teach us what, in the long run, we may expect. To some of the more important of these laws, especially in their bearing on our own climate, I propose to invite your attention this evening; and I trust you will bear with me if, in a subject adapted to instruction rather than amusement, I am ohliged to enter occasionally into what some may consider dry details.

I need not tell you, that the existence, and the continued support, of animal and vegetable life, are dependent upon solar heat. If the sun were extinguished, a few hours would suffice to reduce our globe to a frozen and unchanging mass, and to destroy all living beings on its surface. It has been calculated that the amount of heat received by the earth from the sun in the course 
of a year is such as would suffice to melt a coating of ice, covering the whole globe, and 101 feet in thickness. This great amount is, however, very unequally distributed. The heating power of the sun's rays depends, according to a very simple law, on their obliquity; but an elaborate analysis is required to calculate the total quantity of heat received, during any given time, at a given place. This much, however, it is easy to see-namely, that so far as solar heat alone is concerned, the mean temperature will be greatest at the Equator, and diminish regularly as the latitude of the place increases.

But there are causes on the earth itself which interfere with this regularity of distribution. If the surface of the globe were all dry land, composed of the same kind of rock or soil, and unchequered with vegetation, the law above referred to would accurately hold, and the lines of equal mean temperature-the isothermal lines, as they are called-would be all parallel to the Equator. This, however, is far from being the fact. The land, you know, is in part covered with the waters of the ocean, and in part dry; while the surface of the latter is endlessly varied in its own nature, and in that of its vegetable covering. These causes produce an inequality in the action of the sun's rays in places having the same latitude, which will be readily understood. The same amount of heat which would raise the temperature of the rock, or soil, through any given number of degrees, will raise the temperature of an equal weight of water through a much smaller amount, on account of what is called its greater capacity for heat. And, in addition to this, a very large portion of the heat which is absorbed by the water is employed, not in raising its temperature, but in changing its state into that of vapour. It becomes thus what is called latent, and does not affect the thermometer or the sense. Every pound of water converted into vapour takes in, in a latent form, as much heat as would raise the temperature of 960 pounds through one degree. And all this heat is carried away by the vapour, to reappear at some remote place, where the vapour is converted into rain.

Owing to these and other causes, the surface of the ocean is never raised to so high a temperature as that of the dry land. It rarely exceeds $85^{\circ}$ Fahrenheit, even within the Tropics, while the surface of the soil in the same region is sometimes heated to $140^{\circ}$, and upwards. Captain Sturt relates that, in parts of Australia, a 
lucifer match will take fire, if it chance to fall on the arid and heated soil.

You see then that, owing to the diversity in the physical characters of the earth's crust, the heat developed is by no means proportionate to the intensity of the solar action. But there is another, and a powerful cause, which tends to distribute the heat actually imparted, and to render it more uniform. The air above the Equatorial regions of the earth ascends, and flows over towards the Poles; while the colder air of the Polar regions flows beneath in the opposite direction to take its place. In this manner a double system of aerial currents is established, which tends to mitigate the extremes of heat and cold on the earth's surface, and to reduce the differences which would otherwise exist.

And a similar interchange 'of temperatures is effected by means of the waters which cover so large a portion of the earth's surface. The Oceanic currents, like great arteries, distribute to the more remote portions of the globe the vast store of heat which it receives from the sun in the intertropical regions. To one of these I wish to invite your attention more particularly, because it is to it, mainly, that the peculiarities of our own climate are owing. Let us trace it from its source.

In the Atlantic Ocean, within the Tropies, the waters flow from east to west in a majestic stream, whose breadth occupies $30^{\circ}$ of latitude. It is known under the name of the "Equatorial current." This current breaks into two, or bifurcates, when it encounters the projecting shoulder of the South American Continent at the Cape St. Roque; the main portion sweeping along the northern shore of the Continent, while another portion is deflected to the southward, and follows the eastern shore. The former of these is the parent of the "Gulf-Stream." Continuing its westerly course, along the north coast of South America, it enters the Caribbean Sea, and passes into the Gulf of Mexico through the channel of Yucatan. It then makes the entire circuit of the gulf, elinging to the shore of the North American Continent, until its direction is changed from westerly to easterly; and it passes out of the gulf through the Straits of Florida, and issues into the Atlantic.

It is at this point that the current takes the name of the "Gulf-Stream." Its breadth is here upwards of 30 miles, and its depth more than 2000 feet; and it has a velocity of four miles 
an hour. Following the line of the coast from the Straits, it trends in a north-easterly direction as far as the great bank of Nantucket, off Newfoundland, where its direction is changed to easterly; and it leaves the land near Cape Fear, and crosses the Atlantic. Here reaching the colder water, it rises to the surface by its relative lightness, while, at the same time, it spreads superficially. When it reaches the meridian of $38^{\circ} \mathrm{W}$. longitude, it subdivides, the main portion of the current proceeding in a northwesterly direction to the North Sea, bathing the western coasts of Ireland and Scotland on its way-while another portion bends round to the southward, and forms a great eddy not far from the Azores, in the midst of which is found that vast floating mass of sea-weed known as the sargasso, which lies between the Azores and the Cape de Verd Islands. It thus at length rejoins the waters of the Equatorial sea, and completes the circuit of the Atlantic.

Confining our attention, for the present, to that portion of the eircuit which is more especially designated as the Gulf-Stream, and whose limits are the Straits of Florida and the Azores, we find that it runs its entire course of 3000 miles in 78 days; so that its average rate of progress is 38 miles per diem. Its speed is, however, very different in different parts of its course. In the Straits of Florida it has a velocity of 100 , and sometimes 120 miles in the day; but by the expansion of its waters this is soon lessened. Its average velocity in the first portion of its coursei.e., from its origin to the point at which it leaves the American shore-is 63 miles a day; in mid-Atlantio it is 55 miles; and in longitude $42 \frac{1}{2}^{\circ}$, where it begins to bend southward, it is 35 miles.

And the temperature of the waters decreases with their speed, although much less rapidly. When they issue from the Gulf of Mexico, they have a temperature of $86^{\circ} \mathrm{Fahr}$; and near the termination of their course at the Azores, in $40^{\circ} \mathrm{W}$. longitude, they have a temperature of $74^{\circ}$, having lost only $12^{\circ}$ in traversing a space of 3000 miles. It is to this great mass of heated water, and to the westerly and south-westerly winds which carry to us the heated air above it, that we owe the genial climate of the British islands.

A circuit of waters, similar in its principal features, is performed in the South Atlantic also. I have already mentioned that the great Equatorial current breaks into two at the Cape 
St. Roque, where it first encounters the American shore. The smaller portion descends along the eastern shore of South America, under the name of the "Brazil current," until it arrives at the latitude of $30^{\circ}$ south. It then turns eastward, and, under the name of the "southern connecting current," it crosses the South Atlantic-much like the Gulf-Stream in the North Atlanticuntil it reaches the western coast of Africa. It finally returns along that coast to the northward, under the name of the "Southern Atlantic current," and rejoins the Equatorial current from which it originally broke off.

And the same phenomena are repeated in the Pacific Ocean. In it, also, there is an Equatorial current, which travels in a westerly direction between the Tropics, until it meets the land at Australia, and the barrier of islands which lies to the eastward of the Continent of Asia. A portion is then deflected northward, and recrosses the Pacific from west to east at a higher latitude, to bathe the shores of California and Oregon. Finally, another current detaches itself from the Equatorial current, on the western side of the great basin of the Pacific, and, after travelling for some distance southward, returns eastward to meet the western shore of South America, and finally rejoins its source.

The generally received explanation of these phenomena is that given by Franklin. The Trade-winds, acting on the waters of the ocean on both sides of the Equator, produce a drift of the surface water between the Tropics, the waters north of the Equator being impelled in a south-westerly direction, and those south of it in a north-westerly. At the Equator, or near it, these two drifts combine, and form one great westerly stream, which is the Equatorial current. This current is deflected when it meets with a barrier of land; and the effect is magnified, when that barrier takes the form of a gulf, and so prevents the lateral escape of the waters. These conditions are satisfied in the Mexican gulf. The waters of the Equatorial current, which enter it along: its southern shore, are pent up into narrower limits within it, and increase in depth and speed, until they finally escape along the northern shore through the Straits of Florida. And a similar explanation will apply to the other great oceanic currents which I have briefly described, bearing in mind, of course, the differences due to the different configuration of the land.

I have spoken of these ocean streams generally, in order that 
you might see that they have all certain features in common. But in considering the climate of Ireland, and the influences to which it is subject, we may confine our attention to that branch of the circuit of waters which bathes its shores.

I have said that the Gulf-Stream divides when it reaches the meridian of $38^{\circ} \mathrm{W}$., a portion there turning off to the southward, while the greater part bends to the north-east, sweeping along the western shores of Ireland and Scotland on its way to the North Sea. That these warm waters reach the shores of Ireland is proved, not only indirectly, by their effect on the climate-but also, directly, by the drift-wood and the fruits of the Tropics, which they often carry thither. Even the living inhabitants of the Tropical seas are thus sometimes transported to our waters. It is only a few years since the bonita, and other tropical fishes, entered the English Channel in vast numbers, and destroyed the pilchard, which is the chief support of the fishermen of the southern coast.

But although there can be no doubt of the presence of these warm waters on our shores, it was desirable to obtain some measure of their influence; and accordingly, in the year 1851, when a general system of meteorological observations was carried out in Ireland, the attention of the observers was specially directed to this subject, and measures of the temperature of the sea were taken at several of the coast-stations. When the results were compared with those of the temperature of the air, it was found that the sea was sensibly warmer than the air over the land, the difference amounting, in the mean of the year, to $3^{\circ} \cdot 8$. The excess is, as might be expected, much greater in winter than in summer: in the latter season it is $1^{\circ} .8$ only; in the former, it amounts to $5^{\circ} \cdot 7$.

But this difference is by no means a measure of the whole heating effect of the Gulf-Stream ; for the temperature of the air over the land is elevated by the influence of the heated waters which encompass it. We obtain a juster notion of the magnitude of the effect, by noting the course of the lines of equal temperature in the Atlantic. Looking at the map of the isothermal lines which is before us, we observe that, in the Atlantio, these lines all deviate greatly from the parallels of latitude, as they recede from the Equator, their convex summits falling near the western shore of the Atlantic, while their concave summits are on the eastern. It 
will be noted, also, that the most sudden bending of these curves takes place in the neighbourhood of the British Islands, the greatest deviation from the parallels of latitude being in, or near, the meridian of London. And, in accordance with this, London has the same mean temperature as Philadelphia, although it is $11 \frac{1}{9}$ nearer to the Pole; while Trondjhem, in Norway, has the same mean temperature as Halifax, notwithstanding the difference of $19^{\circ}$ in latitude.

What has been just said relates to the mean temperature of the year; but the influence of the Gulf-Stream is much greater upon the temperature of vinter. Thus the winter temperature of Dublin is considerably higher than that of Milan; and Stromness, in the Orkneys, has a winter temperature greater than that of Paris. In fact, the isothermal line for January, which passes through the Shetland Islands, runs almost exactly from north to south; and thus the winter temperature is nearly the same along the whole eastern coast of Great Britain, while it increases as we proceed from that coast to the westward. In Norway the effect is even more remarkable. Owing to the interposition of the British Islands, the Gulf-Stream is intercepted from the southern portion of the Norwegian coast, while it reaches the northern. The southern limit of the stream thus falls on the town of Bergen; and accordingly, in winter, the temperature actually inereases in proceeding northward from that point.

But the disturbing effect of local causes on the temperature is measured exactly by the method employed by Professor Dove. In this method the mean temperature, corresponding to any parallel of latitude, is deduced from the observed temperatures at 36 equidistant points on the parallel, and is regarded as the normal temperature of all places on that parallel; and the difference between this, and the actual temperature of the place, is obviously a measure of the local influence. This difference is ealled by Professor Dove the thermic anomaly. We thus find that the measure of the influence of the Gulf-Stream, or the thermic anomaly, at the Orkneys is, on the mean of the entire year, $15^{\circ}$; while, in the month of January, it amounts to $34^{\circ}$. The excess of the actual above the normal temperature is greatest between Jan Meyen and the Lofoden Islands, a little above the Arctic Circle. The mean thermic anomaly is there $22^{\circ} .5$; while, in the month of January, it amounts to $45^{\circ}$. 
The mean thermic anomaly for Ireland, or the excess of the mean temperature of the year $\left(50^{\circ} \cdot 3\right)$ above that due to the latitude $\left(37^{\circ} \cdot 7\right)$, is $12 \frac{1}{2}^{\circ}$. The corresponding excess for the month of January is $27^{\circ}$. Our winter temperature is thus raised, by the Gulf-Stream, as much as if our place on the globe had been $15^{\circ}$ nearer to the Equator!

The Gulf-Stream changes its boundaries from time to time; and these changes are sometimes indicated in a singular manner. The Medusce, or "sea-nettles," as they are commonly called, are often found in vast numbers in the stream; and it seems to be owing to the attraction which these creatures have for the whale, who feeds upon them, that the leviathan is so often found hovering on its skirts-for its temperature is too high to permit him to enter it. Now this fact furnishes some curious evidence respecting the boundary of the stream, and its changes. Thus M. Babinet tells us, that in the autumn of 1846 he learned that the whalers had been obliged to run up to a higher latitude in search of their prey. From this fact he concluded that the stream had advanced further northward than usual; and he predicted, in consequence, that the following winter would be one of unusual mildness in Europe. The prediction was fulfilled.

I now proceed to consider, somewhat more particularly, the influence of these and other causes on the climate of Ireland.

I have already adverted to the system of meteorological observations adopted in 1851, under the direction of the Royal Irish Academy. In addition to the observatories of Armagh, Markree, and Dublin, where such observations are made continuously, the observations were taken at eight coast-guard stations, by boatmen belonging to the service, and at three of the lighthouses, by the light-keepers. The instruments were furnished by the Academy. They were constructed on a common plan, and carefully compared with standard instruments by members of the Council of that body, by whom also the men were instructed in their use. The instruments were recorded daily at 9 A.M. and 9 P.м. ; and observations were also taken, on the same system, at two stations in the interior of Ireland, where the task was voluntarily undertaken by private individuals.

The mean temperatures of the several months having been calculated, and reduced to their normal values by means of the Dublin observations, it was found that the temperatures of the 
inland stations were in defect, as compared with the coast stations. The cause of this is obviously their greater distance from the warm waters of the surrounding sea; and it follows evidently from the fact, that the actual isothermal lines in Ireland are inflected in passing from the sea to the land, and must even, in part of the island-as Mr. Hennessy has pointed out-take the form of closed curves, dependent on the position of the places traversed with respect to the coast-line, as well as upon their longitude and latitude. To determine such curves with exactness would require a far greater number of stations than those employed, and would not seem likely to yield results of corresponding value. The most feasible course seems to be to determine, in the first instance, the law of distribution of temperature depending on geographical position alone; the disturbing influence of the land can afterwards be computed approximately, and allowed for.

Dealt with in this manner, the observations show that, on the mean of the whole year, the isothermal lines are inclined to the meridian by the angle N. $49^{\circ} \mathrm{W}$., and that the temperature increases, in a direction perpendicular to these lines, at the rate of $1^{\circ} \mathrm{Fahr}$. for 89 miles.

We learn further, that the mean isothermal lines for the entire year furnish a very inadequate representation of the progression of temperature, and that, when the course of these lines is traced from month to month, they vary within very wide limits. The extreme positions correspond to the months of June and December. In the former, the inclination of the isothermal lines to the meridian is N. $106^{\circ} \mathrm{W}$.; in the latter, it is N. $9^{\circ} \mathrm{W}$. Thus these lines vary in direction through an angle of $97^{\circ}$ in the course of the year, being nearly parallel to the meridian in December, and nearly perpendicular to it in Jume. The rate of increase of temperature changes little.

The mean yearly temperature for the central station, whose latitude and longitude are the arithmetical means of those of the stations of observation, is $50^{\circ} \cdot 3$. It is thus distributed throughout the year:- 


\begin{tabular}{|c|c|c|c|}
\hline Month. & $\begin{array}{c}\text { Mean } \\
\text { Temperature. }\end{array}$ & Month. & $\begin{array}{c}\text { Mean } \\
\text { Temperature. }\end{array}$ \\
\hline January, . . & $41^{\circ} \cdot 7$ & July, & $58^{\circ} \cdot 8$ \\
\hline February, . . & $42 \cdot 3$ & August, . . & $58 \cdot 3$ \\
\hline March, . . & $44 \cdot 1$ & September,. . & $57 \cdot 8$ \\
\hline April, . . . & $47 \cdot 1$ & October, . . & $50 \cdot 2$ \\
\hline May, . . . & $52 \cdot 9$ & November,. . & $48 \cdot 4$ \\
\hline June, . . & $56 \cdot 7$ & December, . & $45 \cdot 4$ \\
\hline
\end{tabular}

These facts are in entire accordance with the general course of the isothermal lines, as laid down by Dove. After what has been said, they are readily accounted for. The heating effect of the Gulf-Stream is greatest about the time of the vinter solstice, or soon after. It then overpowers altogether the effect of solar radiation, which is then weakest; and the temperature increases as we approach the waters of the stream-i.e., as we proceed to the vestward. On the contrary, the effect of the stream is least, and that of the sun greatest, about the time of the summer solstice; and the heat increases as we proceed southward. The maxima of the curves in Europe, at this period of the year, show that the burning soil of the African Continent then influences most the distribution of temperature to the north of it.*

* The following are the mean temperatures of the several places of observation in Ireland, reduced to their normal values, for the four seasons of the year, and for the entire year :-

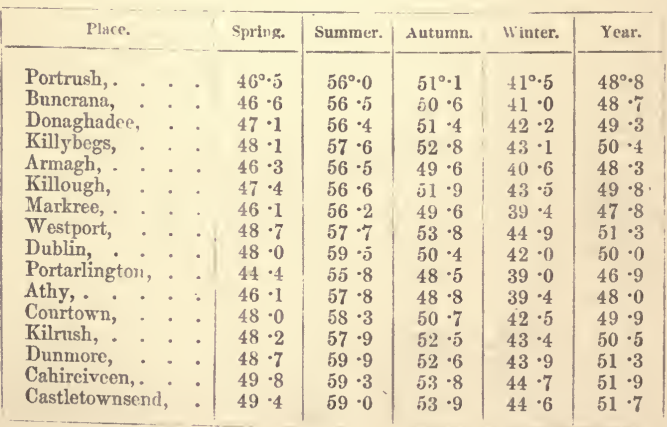


But the climate of a place depends upon the ranges of temperature, whether diurnal or annual, no less than upon the mean ralues. It is a well known meteorological fact, that the ranges of temperature on the globe vary within very wide limits, being least of all at sea, and increasing with the distance of the place from the ocean, until in the interior of the continents they become very great. Thus, the difference between the mean temperatures of summer and winter, or the annual range, is, on the average, $19^{\circ}$ in Great Britain ; $27^{\circ}$ in France ; $36^{\circ}$ in the eastern parts of Germany; $40^{\circ}$ in European Russia; $60^{\circ}$ in Siberia-until at Jakoutsk, in that inhospital clime, it reaches the enormous amount of $101^{\circ}$ ! And these differences exist even among places having the same mean temperatures. Thus Dublin, Prague, and Astrakhan on the shores of the Caspian, have all nearly the same mean annual temperature. But the difference between their summer and winter temperatures are respectively, $17^{\circ} \cdot 5$ for Dublin, $37^{\circ}$ for Prague, and $57^{\circ}$ for Astrakhan. These differences are due to the different effects of solar radiation on water and on dry soil, already explained; and climates have been distinguished into marine and continental, according to the amount of this variation. The annual range in Ireland is the smallest in Europe; on the coasts it falls as low as $14^{\circ}$.

The difference between the mean temperature of day and night, or the diurnal range, follows the same law, being least ou the coasts, and greatest in the interior of the continents. I have already adverted to the principal cause of this. A great portion of the solar heat which falls on the water during the day is employed in changing its state into that of vapour, and does not affect its temperature; and, on the other hand, the heat so employed is, in part, restored and rendered sensible, when the vapours are condensed during the night. Thus the two extremes approach one another. But another reason is, that the vapour itself stops, or absorbs, a large portion of the sun's heat; and the effect, of course, is greatest when the air is most humid. Dr. Livingstone found a difference of temperature, amounting to $48^{\circ}$, between sun-rise and mid-day, on the eastern side of South Africa; while in the valley of the Zambesi, on the opposite side of the Continent, where the air is loaded with moisture, the difference amounted only to $12^{\circ}$.

In accordance with this, the diurnal range of temperature 
in Ireland is small, the mean difference between the greatest temperature of the day, and the least temperature of the night, being only $11^{\circ} \%$. The range is greater inland than on the coast, the mean range of the inland stations being $13^{\circ} \cdot 1$, and that of the coast stations $10^{\circ} \cdot 3$. Among the places of observation, it is greatest at Portarlington, and least at Cahirciveen at the southwestern extremity of Ireland.

Having spoken of the temperature of the air, I must now say a few words of its movement-and, first, of the direction of that movement.

During the period of the simultaneous observations already referred to, the wind blew, on the average of the entire year, most frequently from S. W. and W., and least frequently from N.E. and $\mathrm{E}$. Thus, taking the mean of all the stations, the number of times in which the wind blew from the N.E. is only 7 per cent. of the whole, while the number of S.W. winds is 20 per cent. The ratio of the numbers is greater in winter than in summer. The following Table gives the number of times out of 1000, in which the wind blew from each of the eight principal points of the compass, for the whole island.

\begin{tabular}{|c|r|r|r|}
\hline Direction of Wind. & Summer. & Winter. & Year. \\
\hline N. & 122 & 96 & 108 \\
N. E. & 94 & 52 & 73 \\
E. & 89 & 50 & 69 \\
S. E. & 100 & 86 & 92 \\
S. & 107 & 164 & 136 \\
S. W. & 170 & 234 & 202 \\
.W. & 163 & 176 & 170 \\
N. W. & 156 & 144 & 150 \\
\hline
\end{tabular}

The velocity of the wind varies from 0 to 70 feet per second, and upwards-this last being the speed corresponding to a storm. If we confine our attention for the present to winds whose average velocity is not less than 35 feet per second, which is the velocity of a strong breese, we find that the high winds are much more frequent on the western than on the eastern coast, the numbers denoting their relative frequency being, on the average of the 
entire year, nearly as 3 to 1 . This preponderance of high winds on the western coast holds at all seasons, the maximum occurring at the north-western extremity in autumn, and at the southwestern in winter.

But of these winds, there are two distinct kinds. In one of these, the wind blows steadily in the same direction for a considerable time, and the axis of the gale is a straight line, or a line but slightly curved. In the other, the wind rotates round a centre, while the centre itself has a progressive motion-just as we see, on a miniature scale, in the vortices of dust which sweep along the road in gusty and unsettled weather. These rotating winds are called cyclones. They are from 200 to 300 miles, and upwards, in diameter, and their centres move with a speed of from 100 to 300 miles per diem. The direction of the rotation is invariably the same. In the northern hemisphere the wind revolves in the direction opposite to that of the sun in its daily course, i.e., in the direction N.W.S.E. In the southern hemisphere, the direction of rotation is with the sun, or N.E.S.W. When a cyclone passes over any point on the earth's surface, the wind must veer. If the centre of the cyclone passes directly over the place of observation, the veering is through 180 degrees, or the wind changes to the opposite. The amount of veering is less, the smaller the chord of the circle which passes over the place; until, for places at the circumference, there is no veering whatever.

But there is another remarkable distinction between these two classes of winds. In the case of the cyclone, the barometric pressure diminishes to a point, which is the centre of the vortex. In the non-rotatory gales, the pressures diminish to a line, which is the axis of the storm.

The most remarkable of the cyclonic movements traced in the course of the simultaneous observations was that which occurred on the 18th and 19th of November, 1851. The centre of the vortex passed over two of the principal stations, Markree and Armagh, at both of which all the changes were accurately observed; and the velocity of the wind reached a maximum of 65 feet per second. The lull of the wind during the passage of the central portion of the vortex, and the reversal of its direction, were observed at Markree, Armagh, and Donaghadee. The diameter of the vortex was about 400 miles; that of the quiescent central portion 40 miles. 
The facts collected relating to these winds lead to the following conclusions :-

1. The occurrence of cyclonic movements in the atmosphere is not infrequent in Ireland, and may be traced even in the case of moderate winds.

2. The rotatory movement is invariably in the same directionnamely, that opposite to the diurnal movement of the sun in azimuth.

3. This rotation is always accompanied by a considerable disturbance of barometric equilibrium, which is greater in proportion to the velocity of the movement, the pressure being a minimum at the centre of the vortex, and increasing regularly with the distance from that point.

4. The vortex itself has a progressive movement, at the rate of from 100 to 300 miles per diem, the average velocity of those observed being 220 miles per diem. The direction of this movement is generally from S. W. to N. E.

5. If a line be drawn through the centre of Ireland, in the direction from $\mathrm{S}$. W. to N. E., the track of the centres of the greater number of the cyclones, passing over or near Ireland, lies to the north of that line.

With reference to the first of these conclusions-viz., the prevalence of cyclonic movements in the atmosphere over, or near, this island-I may here state, that of all the greater storms which occurred during the period of observation, no fewer than two-thirds were cyclonic; while several cyclonic movements of smaller velocity were distinctly traced. The area of observation may be thought, perhaps, to be too small to furnish conclusive evidence relating to these great aerial movements. But I have the satisfaction of knowing, that of the cases recorded in the Menoir on the subject published in the Transactions of the Royal Irish Academy, the cyclonic character of most (if not all) has been since confirmed by the late Admiral Fitzroy, from the records of the logs of ships.

The prevalence of such movements is just what we should be led, a priori, to expect, from the vicinity of the Gulf-Stream. The waters of the stream in mid-Atlantic have a temperature of $80^{\circ}$ Fahr., while the air on either side of it is, in winter, at the freezing temperature. We have here all the conditions required for the production of cyclonic storms. Aqueous vapour is much lighter than air at the same temperature, and conse- 
quently ascends in it; and, in its ascent, it drags the heated air along with it, and produces an upward current. There is thus an upward rush of heated air and vapour above the heated waters, and an inward rush of cold air from either side to supply its place; and such a combination of movements will produce a great rotating eddy, much in the same manner as the downward flow of water through a hole in the bottom of a vessel will give rise to a vorticose movement of the whole liquid mass.

Accordingly, the most terrific hurricanes of which we have any record have been generated on the borders of the Gulf-Stream. In the hurricane of 1780 , in the Bermudas, houses were levelled by the gale; forts were washed away by the waves; heavy pieces of ordnance were lifted into the air; and the bodies of animals were carried aloft, and dashed to pieces in the fall. The loss of human life was terrible. It is computed that not fewer than 20,000 lives were lost on shore; and on the water no vessel could stand the gale.

And the Gulf-Stream appears also to exert a marked influence on the course of storms which are engendered in other parts of the Atlantic. From the examination of the logs of ships, Captain Maury has ascertained that the gales, which are produced in the Atlantic to the south-east of the current, usually travel to the north-west until they meet it, after which they turn along with it, and follow its course. And, aecording to Mr. Espy, a similar effect is produced in the case of storms which have their birthplace in the valley of the Mississippi.

There are two facts which have been brought to light by the Irish meteorological observations, which confirm in a remarkable manner this explanation. The main branch of the Gulf-Stream approaches the coasts of Ireland most nearly at the north-western extremity of the island; and it is, accordingly, there, if this account be the true one, that the evidenees of cyclonio movement should be most marked. And such is the fact:- the track of the centres of most of the cyclones whose course has been investigated lies to the north-vest of the island. And in exact aceordance with this fact is another of a different kind. I have said that, in the area covered by a cyelone at any moment, the minimum of barometric pressure is at the centre of the vortex. Hence the frequent passage of cyclones in any particular direction must affect the mean distribution of atmospheric pressure, the pressuro being most 
diminished at those places which are nearest to the track of their centres. It has been found, accordingly, that there is an inequality in the distribution of the atmospheric pressure in Ireland, and that the minimum occurs in the north-western quarter of the island.

I now proceed, lastly, to the effects of the Gulf-Stream on the humidity, and on the rain-fall, in this island.

The amount of evaporation from the surface of water increases rapidly with its temperature. Consequently the air above the Gulf-Stream is loaded with vapour to a much greater degree than that which rests on other portions of the Atlantic. This vapour is borne to the British Islands by the south-west winds, which are the predominating winds in this portion of the globe; and, in consequence, these islands have more than their average share of humidity. The effect is, of course, greatest at those places which the wind first reaches. It is, accordingly, greatest in Ireland, less in England, and still less on the Continent of Europe, diminishing with the distance of the place from the Atlantic. Thus, in Paris (and probably in France generally) the mean amount of vapour in the air is about 77 per cent. of the maximum which it is capable of holding. In London, it is 84 per cent.; and in Dublin it amounts to 88 per cent., which is about the average for Ireland.

The humidity is greatest, as we should expect a priori, on the western coast of Ireland, and least on the eastern-the former being about 90 per cent., and the latter 85 . The following Table gives the mean humidity of the several months of the year at Dublin. It is greatest in December, and least in June :-

\begin{tabular}{|c|c|c|c|}
\hline Month. & Humidity. & Month. & Humidity. \\
\hline January, . . & 92 & July, . . . & 84 \\
February, . . & 88 & August,. . . & 86 \\
March, . . . & 88 & September, . . & 90 \\
April, . . . & 87 & October, . . & 91 \\
May, . . . & 85 & November, . . & 92 \\
June, . . . & 83 & December, . . & 92 \\
\hline
\end{tabular}


The distribution of rain is very unequal. In the east of England, the annual rain-fall is between 20 and 25 inches only; in the west of Ireland, it is between 40 and 50 inches. In Ireland, in the year 1851, the greatest amount of rain fell at Cahireiveen, and the least at Portarlington, the amounts being $59 \cdot 4$ inches, and $21 \cdot 2$ inches, respectively. The mean rain-fall for the whole island, in that year, was 34.5 inches.

The causes already adverted to explain, in part, the distribution of rain. The vapour which is borne to us by the southwesterly winds is partly condensed into rain when the air is cooled by contact with the land. The chief condensers are the mountains, because they interpose the greatest obstacle to the movement of the vapour-laden air. Accordingly the high mountains of Kerry, which offer the first barrier to the progress of these winds, bring down on their flanks the largest amount of rain; and, generally, the rain-fall is greatest on the vestern and southvestern coasts, while the places of least rain are either inland or on the eastern coast. The disparity is greatest in winter, when the elouds are low; in summer, when they are high, they escape the condensing effect of the land over which they pass, and the rain is more evenly distributed.

The amount of rain is always considerable in the neighbourhood of a mountain chain or group, except at places to the eastward of it, where it is small. Thus, the places of least rain in Ireland-Portarlington, Killough, and Dublin-all lie to the north-east of a mountain range; while the places of greatest rain -Cahirciveen, Westport, and Castletownsend-are all in the vicinity of high mountains, but on a different side. All this is readily understood from what has been stated. The prevailing wind is the south-west, which comes to us laden with the vapour of the Gulf-Stream, and this vapour is precipitated chiefly on the flanks of the mountains. And the same cause protects the region over which the same wind next passes-the east and north-eastthe air having been deprived of its excess of vapour before arriving there.

The distribution of rain throughout the year in Ireland will be understood from the following numbers, which give the mean rainfall in each month at Dublin, as deduced from the observations of the eleven years (1841-1851). The mean yearly rain-fall is $29 \cdot 1$ inches. 


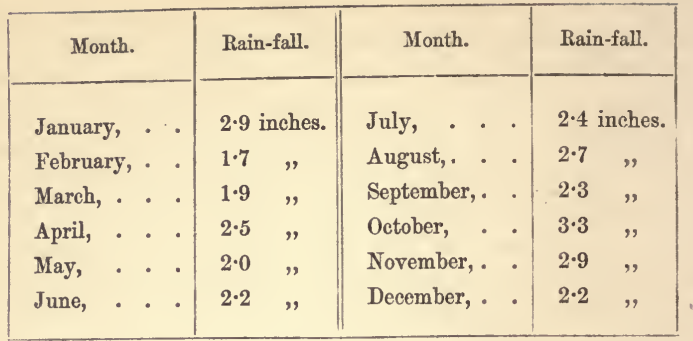

In dealing with the subject of Climate, even in the cursory manner which alone is suited to a discourse such as this, it is impossible to overlook its effects upon animal and vegetable life; and therefore, although no physiologist myself, I will offer no apology for laying before you some broad facts connected with the sciences of life, in relation to Meteorology.

To begin with the vegetable kingdom-it is well known that plants are dependent, in a remarkable degree, upon every change of heat and moisture; and that, accordingly, the genera and species of plants are distributed on the earth's surface so that each shall receive its proper supply. The distribution of temperature being much more regular than that of moisture, the general features of the geography of plants are determined by the former; and thus the several divisions of the vegetable kingdom are found disposed in broad bands on the earth's surface, which are closely connected with the isothermal lines. And the succession of these bands is similar-but of course more rapid, and therefore more readily seen-on the flanks of high mountains. Thus we meet, near the base of the Andes, first the palms; and then the tree-ferns. At greater altitudes there, or at lower heights in the temperate regions, we come to the deciduous trees-among which the oak, the beech, and the birch, are the principal. Then, as we ascend, we reach the pines; next the rhododendrons, and other dwarf shrubs; after these, at still higher elevations, the creeping herbaceous plants; and, lastly, at the very limit of vegetation, the lichens.

But in studying the effects of temperature upon vegetable life, it is important to observe, there are two pairs of limiting temperatures to be considered, one wider, and the other narrower. The former are the limits beyond which the existence and continued 
life of the plant is impossible; the latter are the limits which determine its flowering, and the ripening of its seed or fruit.

It is hardly needful to remind you, that the very existence of plants is dependent upon two extremes of temperature, one higher and the other lower. 'In the temperate regions of the earth, in which we ourselves have the happiness to live, the effect of the higher extreme is seldom felt; for there are few plants which will not bear our highest summer heat. Accordingly, with us it is the winter temperature, or rather the lowest temperature of the year, which determines the existence of most perennials. We are all familiar with this fact in the case of our ornamental greenhouse plunts, which are for the most part natives of warmer climates, and will not bear the rigours of our own without protection; and the same thing is true of many native and acclimatized plants in seasons of unusual severity. On the other hand, in the south-west of Ireland, which has the highest winter temperature of any in the British islands, there are twelve species of wild plants which are natives of Spain.

But within the limits beyond which the plant cannot live, there are two other limits which determine its fruitfulness. It is now well established that, in order to blossom, and to ripen its seed or fruit, each species of plant requires a certain definite amoint of sunimer heat, abore that which is necessary to the continuance of its existence as a vegetable; and that, provided it receives this fixed amount, the time in which it is imparted is not essential. This determines the lower limit to the successful culture of the fruit-bearing plants.

But there is also a higher limit, which is less obvious. Thus in the case of the annuals, which include the plants most useful to man, there is a certain temperature at which the plant becomes perennial, and is propagated by lateral shoots, and not by seed. When the temperature reaches this limit for any plant, its cultivation for seed becomes impossible, and we can use it only for its leaves or root. On the other hand, tuberous plants become seedbearing, by the lowering of the temperature. We have a familiar instance of the latter transformation in our fields. Most of our green crops, such as turnips and mangolds, are cultivated for their roots, and under ordinary circumstances produce neither flower nor seed. But it sometimes happens that certain plants in a ficld have received a smaller amount of heat than is required for this mode 
of growth; and these plants throw up a lofty stem, and flower, while the tuberous growth ceases altogether. This is one of those many beautiful adaptations which compel us to look up from nature to nature's God. When the plant is under such conditions that it must perish, it sends up its spikelet of flowers-as it were by an instinct-and matures the seed, which is destined to continue the life of the species, while the individual fades and dies!

And here I cannot refrain from throwing out a suggestion which, if found to be true, may be of some practical importance. The plant which we chiefly cultivate for its root, and whose life and health is of such importance to this part of the kingdom, produces (as you know) flower and seed, as well as tuberous root. I venture to suggest to physiologists the inquiry, whether this circumstance may not be closely connected with the uncertainty of the crop? and whether, if means could be found to repress the flover, the root might not acquire vigour and strength to resist disease? But however this may be, the transition from the condition of perennial to that of annual is a subject of much importance in connexion with the question of the acclimatization of plants. It is probable that there are many tropical plants, unknown to this climate, which are perennial in their native place, but which would become annuals, with seed and fruit, when transferred to a colder clime. When we consider the acquisitions which have been already made by such transfers, we can hardly over-estimate the importance of future gains, or the benefits which may be thus acquired for the human family.

But the lower limit of summer temperature is by much the most important to us; for it is that which in our climates determines the question of success in the culture of the cereals. I will therefore refer to it a little more particularly.

There is no doubt that the integral of solar heat, taken between the limits of time when the vegetation becomes active in Spring, and when the seed is ripened in Autumn, is the element which determines one of the geographical limits which Nature has imposed to the culture of the plant. To find this, for each important species of plant, would require an amount of observation which has not yet been bestowed. In the meantime, however, a tolerable approximation to its value may be made, by taking simply the mean summer temperature-i.e., the average temperature of the 
months of June, July, and August. Now, if we look to the distribution of the cereals in Europe, as given by Berghaus or Johnstone in their physical Atlases, we observe that they are arranged in belts, or zones, whose limits correspond nearly with the lines of equal summer temperature. Thus we have, first, a belt of barley alone, which extends from the North Cape, in latitude $70^{\circ} \mathrm{N}$. to about midway in Norway and Sweden. This is followed, as we proceed southward, by a belt containing oats and rye, as well as barley. Then comes a belt of rye and wheat, in the south of Scandinavia, and in the north of central Europe. In the southern half of Europe, we have wheat alone; and, beyond this, wheat and maize on the coasts of the Mediterranean. And the boundary lines of these several districts are all related, more or less closely, to the isothermal lines of mean summer temperature.

It is of the first importance to the agriculturist to know these limits in his own district; for on their position will depend the chances of successful cultivation of the particular crop. I shall therefore ask your attention for a few moments longer, while I endeavour to ascertain the limit of wheat, the most important of the cereals, in these islands. The result of the inquiry is somewhat startling, and it deserves to be more fully known.

There is some doubt still as to the native place of the cereal grasses. Most of them have been found growing wild in Persia, on the banks of the Euphrates, and in Tartary. Whatever their original habitat may have been, it was a warmer clime than ours. The mean summer temperature of the British Islands is under $60^{\circ}$, while that of the plains of Lombardy, where wheat is grown in perfection, is $73^{\circ}$; and that of Sicily - "the granary of ancient Rome" -is $77^{\circ}$. We are, therefore, in these countries, probably near the lower limit of the wheat crop, beyond which its successful culture is impossible. It is important that we should know how near.

We have some of the data requisite for the determination of this question, in the long series of observations of temperature made at the apartments of the Royal Society, in London, as compared with the prices of wheat, given for an equally long series of years in "Tooke's History of Prices." From the former we learn how far the summer temperature of each year deviated from the mean; while the latter furnish us with a measure of the abundance 
or scarcity of the years in question.* The comparison has been made by Mr. Whitley, of Truro, in Cornwall, and the result which he has obtained from it is interesting and important.

The observations of temperature to which I refer were begun in the year 1774, and were continued (with only an interruption of five years) to the year 1842, inclusive; they thus extend through sixty-four years. They have been carefully reduced by $\mathrm{Mr}$. Glaisher, and the means of each month and season computed. One of the results which may be inferred from them-and it is a result of some interest in connexion with the effocts of seasonis that, for the most part, the spring and summer of the same year are of the same character, either both above, or both below the mean. In the course of the sixty-four years, the character of the two seasons was the same forty-six times, and opposite only eighteen times; so that the chances that a warm summer will follow a warm spring, or vice versâ, are as two and a-half to one.

A like fact appears also from Dr. Rutty's observations of the weather in Dublin, which extended through forty-one years in the last century (1725-1765). In these observations the character of each season is defined, with reference to the rain-fall, as dry, wet, or variable. The observations have been discussed by Kirwan; and it appears from that discussion, that wet springs are followed by wet summers five times out of six.

But to return to the London observations-the greatest deviation of any particular year, from the mean of all, was $4^{\circ} .8$ in defect; it occurred in the year 1816, which was a year of famine. In the course of the sixty-four years, a deviation of $2^{\circ}$, and upwards, occurred twenty-three times, the summer temperature being thirteen times above the mean to that amount, and ten times below it. Now it is deserving of notice, that there is no appearance of a regular cycle in these good or bad years, such as Luke Howard and many other meteorologists have imagined. On the contrary, both the warm and the cold seasons usually occur in groups, comprising three or four years in succession; and the groups themselves do not recur

* It is not, of course, meant that prices furnish an accurate measure of the abundance or scarcity of the crop; for they are dependent, we know, on other causes also, among which legislative enactments regulating the import of foreign grain are the principal. It is enough for the argument in the text, that prices varied with abundant or scanty harvests, as with their principal cause; and this we know to have been the fact, as long as any restriction on the import of corn remained. 
in any order or regularity. The most remarkable of these groups is that of the years 1809-1817, comprising no fewer than nine years in which the temperature was below the mean. The average price of wheat in these years was 958 . the quarter, the ordinary average being $70 s$; while in the years 1811 and 1812, it reached 122s. The total deficiency of summer temperature in these nine years amounted to twenty-three degrees.

I need not remind you of the incidental confirmation of this law of groups, in the account which we have in Genesis of the seven years of famine, following seven years of abundance, in Egypt and Syria. Our own recent experience at home has instructed us, that years of deficient harvest come in succession. But I wish particularly to point out, that this recurrence of bad years by no means justifies the conclusion which some have drawn from itnamely, that our climate has changed, or was changing, for the worse. On the contrary, we learn from observation that such unpropitious years are not likely to occur more frequently than three or four in succession; and that they will probably be succeeded, at some future time, by three or four years of an opposite character.

Now when these years of low summer temperature are examined, with reference to the price of wheat, it is found that they are, with few exceptions, years of scarcity and high prices. A deficiency of temperature, amounting only to $2^{\circ}$, is most injurious to the wheat harvest in England ; while a deficiency of $3^{\circ}$ is almost destructive. The reverse is the case when the summer temperature exceeds the average by the same amounts.

This result is of considerable importance. The lowest summer temperature at which wheat ean be successfully cultivated in England, is only $2^{\circ}$ below the mean; and, as the mean summer temperature of England is $60^{\circ}$, it follows that the minimum for wheat is $58^{\circ}$. This minimum, however, is not absolute. It varies a little with the soil, and other eircumstances; and thus it is, that we find the culture of wheat in Scotland extended as far as Inverness, where the mean summer temperature is only $57^{\circ}$.

Now taking this lower limit as the most favourable, let us see what we are to learn from it in Ireland. In this island, the mean temperature of the three summer months is $58^{\circ}$; and, accordingly, for places about the centre of Ireland, a deficiency of a single degree of summer temperature brings us to the very limit of wheat culti- 
vation; while a greater deficiency is fatal to the crop. The case is somewhat better in the southern half of the island, and somewhat worse in the northern; and we are therefore justified in concluding, that it is contrary to the rules of all sound experience to attempt the culture of this cereal in Ireland, except in the most favoured localities.

In the preceding discussion I have considered only the relation of temperature to the culture of vheat. I have not adverted to the frequently destructive effect of the autumn rains, which set in with the fall of temperature, and often before the harvest is gathered. Time will not permit me to discuss the corresponding questions for the other cereals; nor to advert to the fact, which is now beginning to gain general acceptance, that the climate of this island, while it is unfarourable to the higher cereals, is adapted in a peculiar manner to the cultivation of root-crops, and of fodder.* I hasten to say a few words, before I conclude, of the effects of climate in general, and especially of our own climate, upon the health of man.

We are all familiar with the command of the physician to the patient, "to change the air ;" and most of us know something, although probably less than the importance of the subject demands, of the salubrity or insalubrity of different districts.

Of all the meteorological elements, that which exerts the most direct effect upon human health is the temperature of the air; and in this respect the two forms of organized life are subject to one law. We all know the effects upon the bodily frame of an extreme cold in winter, and of harsh winds in spring; and some of us are obliged to leave our homes and daily occupations, and to take refuge from these dangers in the more genial climates of the south. $\dagger$

M. Quetelet, in his important work "On Man," has brought together many curious facts connected with the influence of climate upon human life. Dividing Europe into three zones-which we

- The turnip depends chiefly on a proper supply of moisture during the summer months; and it is injured by much heat. Accordingly, moist and cool summers-such as are frequent in Ireland-are the fittest for this vegetable. The same rule applies to grass.

+ The connexion of the mortality with the cold of winter is very distinctly marked in the weekly returns of the Registrar-general. In the severe frost of Christmas, 1860, the increase in the number of deaths in London was forty daily, being greater than the increase caured by cholera. 
may call northern, central, and southern Europe, respectively-he finds that the mortality in the last is by much the greatest, while it is nearly equal in the two other divisions. In this comparison M. Quetelet has considered merely the effect of latitude upon mortality. But as we have already shown that places in the same parallel of latitude often have widely different temperatures, it seems plain that the comparison should be made differently, and that we should gronp together for the purpose places under the same, or nearly the same, isothermal line. Then we compare in this manner the death-rates of the several countries of Europe with their mean temperatures, we find that the mortality is greatest in Italy and Greece, where the temperature is highest; and that it decreases as the temperature decreases, down to a certain limit, after which it appears to increase again.

But this is obviously an inadequate mode of considering the problem. It seems plain that, for animals as well as for plants, the salubrity of a climate will depend on the extremes of temperature, much more than on the mean ; and that, of these extremes, the most influential on life will be that most removed from the normal temperature which is best adapted to the species. Accordingly, we should expect the mortality to be influenced chiefly by the summer temperature in hot climates, and by the winter temperature in cold ones. And this I find to be the case in Europe, where alone we have the data requisite for the comparison.

The following are the principal conclusions which we seem to be warranted in drawing:-

1. In the southern half of Europe the mortality depends upon the temperature of summer, being greatest where that temperature is greatest, and diminishing with it down to a certain limit.

2. In the northern half of Europe, on the contrary, the mortality depends on the temperature of winter, being greatest when that is least.

3. The boundary line between these two regions is not far from the mean yearly isothermal of $50^{\circ}$, which is accordingly the line of least relative mortality.

4. The mortality attains an absolute minimum in the British islands, at the western extremity of this line, the annual range of temperature being there least.

These conclusions will be evident on the inspection of the following Table, in which the mortality is compared with the excess 
of the temperature of the hottest month above $50^{\circ} \mathrm{Fahr}$., or with the defect of temperature of the coldest below the same.*

\begin{tabular}{|c|c|c|c|c|c|}
\hline Countries. & & & $\begin{array}{c}\text { Excess of } \\
\text { Summer } \\
\text { Temperature. }\end{array}$ & $\begin{array}{c}\text { Defect of } \\
\text { Winter } \\
\text { Temperature. }\end{array}$ & $\begin{array}{l}\text { Death rate } \\
\text { per } 1000 .\end{array}$ \\
\hline Italy; Turkey, . & . & • & $+25^{\circ}$ & - & 33 \\
\hline France; Austria, & - & & +18 & - & 25 \\
\hline Central Germany, & . & - & +15 & - & 22 \\
\hline British Islands, . & 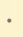 & ${ }^{\circ}$ & +12 & $-13^{\circ}$ & 21 \\
\hline Belgium, . . & - & - & - & -16 & 23 \\
\hline Holland, . . & . & - & - & -18 & 26 \\
\hline Prussia, . . & . & - & 一 & -22 & 28 \\
\hline Russia, . . . & - & - & - & -36 & 37 \\
\hline
\end{tabular}

I believe that this decrease of the mortality, in proceeding from the hotter to the colder parts of Europe, and its subsequent increase, result from the joint operation of different diseases having opposite relations to temperature. Epidemic and endemic diseases appear to diminish indefinitely, as the temperature diminishes; while consumption, and other diseases of the respiratory organs, increase. These are the maladies most destructive of human life; and it will be readily understood in what manner their contrary tendencies may produce a minimum mortality at some temperature far removed from either of the two extremes. $\dagger$

I have already stated that the absolute minimum of mortality is to be sought for at the western extremity of the yearly isothermal of $50^{\circ}-i . e$. , in our own island; and the facts bear out this conclusion. The average mortality of Ireland, so far as it can be

* There is, of course, some uncertainty in comparing numbers, one set of which are dependent on political boundaries, and the other upon physical features. In Russia, the mean winter temperature adopted is that of the January isothermal passing nearly through three of the great centres of population-Petersburgh, Moscow, and Astrakhan. No attempt has been made to include Sweden and Norway in the comparison, the extraordinary flexures of the January isothermals in these countries rendering the deduction of a mean winter temperature uncertain.

† This law appears to be exhibited, even within the narrow limits of our own island. It appears from the valuable statistics of disease collected during the Census, and discussed by $\mathrm{Sir}$ W. Wilde, that deaths by consumption are least numerous in the south-west of Ireland, while deaths from epidemic and contagious diseases are most numerous in the same district. 
determined by the imperfect method of a decennial census, is 21 per 1000, that of England being 22. And here I cannot avoid romarking, that the connexion of these facts with temperature has been strangely mis-stated by the eminent French statist, M. Moreau de Jonnes, by whom these vital statistics were first collected. After observing that, of all the states of Europe, the British Islands are the most favoured by a low mortality, he adds with evident surprise- "It is on the frozen rocks of Ireland (les rochers glacès de l'Irlande) and in the midst of the eternal mists of Scotland, that man reaches the most advanced age !"

But the season of the year, and probably even the hour of the lay, exert their influence upon mortality. The following numbers give the relative mortality of the several months of the year in Belgium, that of the whole year being taken as unity:-

\begin{tabular}{|c|c||l|l|}
\hline Month. & Mortality. & \multicolumn{1}{|c|}{ Month. } & Mortality. \\
\cline { 1 - 3 } January, . . & 1.21 & July, . . . & 0.81 \\
February, . . & 1.20 & August, . . & 0.82 \\
March, . . . & 1.19 & September,. . & 0.89 \\
April, . . . & 1.12 & October, . . & 0.93 \\
May, . . . & 0.98 & November,. . & 0.94. \\
June, . . . & 0.88 & December, . . & 1.03 \\
\hline
\end{tabular}

The regularity of these numbers is very remarkable. The mortality is greatest in January, the coldest month of the year, and least in July, which is the varmest; and the proportion of the former to the latter is that of three to two.

These facts are in accordance with the laws of mortality stated above; for Belgium is to the north of the neutral line, and in it, therefore, the mortality should depend chiefly upon the cold of winter. To the south of that line we should expect an opposite result-namely, that the mortality should be greatest in the hottest months of the year. We do not possess the data necessary to verify, or to disprove, this conjecture. It is worthy of remark, however, that in Ireland, which is traversed by the mean isothermal of $50^{\circ}$, the periods of greatest and least mortality are neither the coldest nor the hottest months, but the intermediate periods of spring and autumn. It appears from the Census of 1851, that the number of 
burials in Dublin was greatest in March, and least in October and November; and in Ireland generally the mortality is greatest in spring, and least in autumn. The following numbers represent the relative mortality at each of the four seasons, that of the entire year being taken, as before, as unity :-

\begin{tabular}{|c|c|c|}
\hline Season. & & Relative Mortality. \\
\hline Spring, . . . . . . . . & 1.31 \\
Summer, . . . . . . & 1.11 \\
Autumn, . . . . . . & 0.57 \\
Winter,. . . . . . . & 1.01 \\
\hline
\end{tabular}

Even for places under the same isothermal line, and at the same season of the year, the difference between the temperatures of day and night, or the diurnal range, exerts an effect upon health. When it is great, the extremes are, of course, more divergent. But, independently of this circumstance, the suddenness of the change of temperature very probably adds to its influence, especially in the case of individuals predisposed to lung-disease. I have already pointed out the fact that, in islands this variation is small, compared with the interior of continents. Througnout Ireland, the diurnal range of temperature is small. It is less on the coast than in the interior of the island; and it is least of all on the coast of Kerry, in the neighbourhood of Valentia. Now it is remarkable that this region is also that most favoured by a high winter teniperature. The mean direction of the isothermal lines in Ireland, in the winter months, is $\mathrm{N} .40^{\circ} \mathrm{W}$.; so that the highest winter temperature is at the south-western extremity of the island, and the lowest at the north-eastern. The former, therefore, seems to be the region most favourable to the patients $I$ have referred to, as well on account of its high winter temperature, as because of its small daily range; while the latter is the least so.

This conclusion (which I ventured to advance some years ago, before the facts were known) has since been fully verified by Sir William Wilde, in his analysis of the Census of 1851. Neglecting the inland districts, for which the meteorological data are insufficient, he finds that the deaths from consumption, at the several 
parts of the coast-line of Ireland, are as follow, the deaths from all causes being 1000 :-

\begin{tabular}{|c|c|}
\hline North-east, from Glenarm to Dublin, . . . & 172 \\
\hline South-east, from Dublin to Youghal, . . . & 161 \\
\hline South, from Youghal to Bantry Bay, . . . & 106 \\
\hline South-west, from Bantry Bay to Galway, . & 76 \\
\hline North-west, from Galway to Donegal Bay, . & 83 \\
\hline North, from Donegal Bay to Glenarm, . . & 127 \\
\hline
\end{tabular}

The proportion of the highest of these numbers to the lowest is nearly that of $2 \frac{1}{4}$ to 1 .

I have hitherto spoken only of the relation of the temperature of the air to health : it remains to say a few words of its moisture.

We all know that the outer covering of the human body is porous, and that these pores are incessantly engaged in the performance of an office, upon the due discharge of which the health is closely dependent; but we are perhaps not all aware of the magnitude of the operation. The experiments of Lavoisier and Seguin show that the quantity of water, which transudes through the skin of an average-sized man, in 24 hours, is 30 ounces; and this, in the ordinary state of things, is earried off by evaporation as fast as it is secreted. Now, it is a well-known meteorological law, that evaporation is less, the greater the amount of moisture actually present in the air; and that it ceases altogether when the air is saturated with moisture. It follows, therefore, that the due performance of one of the most important functions of the animal system is dependent on the dryness of the air. It is not to be inferred from this, however, that the transpiration, as Blumenbach denominates the process, ever wholly ceases, even when the air is saturated with moisture. For the air in contact with the body rises in temperature, and is thus enabled to take up a fresh supply of moisture from the skin.

But besides the effect of humidity in checking the process of the insensible perspiration, it also operates in reducing the animal heat, when the temperature of the air is low. This seems to require some explanation. One of the immediate consequences of evaporation is, we know, the abstraction of heat; and as the evaporation from the surface of the body is less in moist air than in 
dry, it would seem at first sight that the chilling effect should be less also. But this is more than compensated by the increased conducting pover of air when charged with moisture. When such air is much below the temperature of the body itself, the heat of the latter will escape through it, much more rapidly than through air that is dry. The chilling effect of the damp fogs of winter, with all their train of attendant evils, is due to this cause.

On the other hand, extreme dryness of the air produces an injurious effect of a different kind. When the air in contact with the lungs is excessively dry, inflammation of the organ is apt to ensue; and thus, to those who have a constitutional tendency to such disease, a very dry air is often fatal. To this cause are to be ascribed the painful, and even fatal effects of the simoom, the dry wind of the African desert. And it is on this account also that, in rooms heated by iron stoves, which are so common in Germany, a vessel containing water is usually placed at the top of the stove, to give the suitable degree of moisture to the air.

The mortality in towns is much greater than in the rural districts adjoining. Thus in Belgium, where vital statistics have been collected with much care, the death-rate in the towns is 27 per 1000, while in the country it is only 21. This is in part accounted for by the deficiency of pure air produced by over-crowding. Every adult inhales about 13 cubic feet of air in an hour, from which his lungs extract the vital element, and replace it by a noxious gas; and in populous cities-and especially in the dwellings of the poor-this waste is not adequately supplied.* The effects of overcrowding have been clearly shown by Dr. Farr, in his useful analysis of the Reports of the Registrar-general on the mortality of London. The following are the results of his comparison of the mortality with the density of the population, in the three principal districts :-

\begin{tabular}{|c|c|c|c|}
\hline & . & $\begin{array}{l}\text { Square yards } \\
\text { to each } \\
\text { person. }\end{array}$ & $\begin{array}{l}\text { Death-rate } \\
\text { per } 1000 .\end{array}$ \\
\hline I. & Whitéchapel district, & 57 & 33 \\
\hline II. & St. Saviour do. & 78 & 28 \\
\hline III. & Kensington do. & 217 & 22 \\
\hline
\end{tabular}

* It has only lately been brought to light, by the philanthropic excrtions of the 
But the supply of air in towns is not merely deficient in quantity-it is also greatly deteriorated in quality. This is mainly owing to the impurity caused by decaying animal matter. The fluids of the body have a tendency to decompose, which is resisted by the vital power; and when that power is finally withdrawn, we all know how soon the work of destruction is completed. Life is thus a continued struggle between the chemical and the vital powers; and the effect of decaying animal matter is to stimulate the chemical forces, and to a degree which the vital power may be unable to resist. The actual contact of such matter with the blood will destroy life speedily; and every surgeon knows the danger of a wound from the dissecting-knife. But the poison is usually administered more slowly. The effluvium pollutes the air we breathe, and reaches the blood through the lungs; and thus the process of destruction goes on more slowly, indeed, but as surely.

These agents of disease and death may be, and have been, successfully controlled by means within our own power. The deathrate of London was formerly 57 per 1000 -as great as it is in Constantinople now. At present it is only 23 ; while, in some of the London districts, the death-rate has been reduced to 17 , a proportion as small as that of the healthiest of the rural districts. This is very encouraging to the sanitary reformer.

The first attempt to legislate for the sanitary improvement of towns was made as recently as the year 1848 . Since the passing of the "Public Health Act" of that year, the death-rate in the towns to which it has been applied has diminished by 6 per 1000 ; and, in Liverpool, the death-rate is said to have been reduced from 38, which was its amount in 1846, to 24 . Much useful work has also been effected by voluntary associations, and especially by the "Metropolitan Association for Improving the Dwellings of the Industrial Classes." In one of the great lodging-houses of that Society-the "Metropolitan Buildings," in Pancras-road-the death-rate has been reduced to $13 \frac{1}{3}$ per 1000 , which is little more, than one half the average death-rate of London. The effects of these improvements are even more marked in the case of children, who are far more susceptible than adults to the deleterious effects of impure air.* The death-rate of children under five years of

late lamented Lord Herbert, that one half of the barracks of the United Kingdom furnished little more than 400 cubic feet of space to each soldier.

* It appears from the Census of Ireland, that tho number of deaths of children, in 
age, in the buildings of the Association, is said to be only 5 per 1000 , the corresponding death-rate for the whole Metropolis being 46 !

The annual death-rate in Dublin, as deduced from the returns of the Census of 1851, was 29 per 1000 . The Act for the registration of births and deaths in Ireland came into operation only at the beginning of last year; and the death-rate in Dublin, deduced from the returns made under that Act, was 27 per 1000 . Thus there has been some amelioration in our sanitary state, although much still remains to be done. We have every reason to hope that the sanitary measures now in progress in this eity, under the direction of a gentleman so competent to the task as the present Medical Officer of Health, will before long effect the desired improvements.

I have ventured to touch upon these painful topics, on account of their urgent importance at the present time. A fearful epidemic has, in its stern undeviating march, revisited Great Britain, and, if the arm of the Almighty be not stretched forth to arrest its progress, will shortly reach our homes. Whatever be the original source of this formidable disease, it seems to be now generally believed that its proximate cause is the poisoning of the blood; and we cannot doubt that this poison is communicated to others, whose vital powers are unable to resist it, through the air-for we can in no other way explain the fact, that the disease in its progress follows the great highways of human intercourse, while at the same time it is little, if at all, contagious. It is thus referrible to the general cause already adverted to-namely, the power of disorganized matter to generate decay-although the effect bears in this, as in other familiar cases, the impress of the original type. It is true, we have not yet succeeded in tracing the organic element in the air, to which it owes its destructive power. But there are many facts which prove that the air does contain elements which evade all the resources of chemistry, while, nevertheless, their presence is proved by their physiological effects. No chemist has detected the substance which imparts the perfume of the rose to the air; and yet there are individuals so sensitive to the odour of flowers, as to be rendered ill by inhabiting the room in which they lie. It is even stated-I quote from a high French authoritythe first year of their age, is 9 per cent. of the total number in the rural districts, while it is 16 per cent. in towns. 
that the air taken in the great sewer of Montmartre, and that collected in the Place de la Concorde (one of the most open spaces in Paris), have been carefully compared, and found to be undistinguishable by the most delicate chemical tests. But, whatever be the source of the virus, its action is, at all events, powerfully stimulated by the co-operation of decaying organic matter in its ordinary forms ; and as this ally of disease is within our power, we shall be without excuse if we neglect the known precautions for defence.*

I regret to have to close this brief sketch of our atmospheric relations with a disagreeable topic. I have endeavoured to bring before you the more salient features of our climate, so far as they can be exhibited in a popular form; and, on a review of all, we have, I think, no reason to repine. If the humidity of the air by which we are enveloped is at times depressing to our energies of body, or of mind, we must remember that we are indebted to it for the luxuriant crops, which furnish food to our cattle, and through them to ourselves. Our summer temperature may be too low to bring some of our seeds and fruits to perfection; but, on the other hand, it is to its moderated heat that we owe our exemption from some of the maladies which afflict the inhabitants of more southerly climes; while our mild winters press gently on the springs of life, and suffer man to reach an advanced age.

Let us be thankful to the Giver of all good gifts for these His blessings! and if-as all earthly blessings are-they are at times checquered by afflictions, let us remember that these too come from a Father's hand. Let them serve to remind us how far we may have strayed from Him, and bring us back, as sorrowing and repentant children, to His outstretehed arms!

* This seems to be conclusively shown by the effect of elevation on the number of deaths by cholera in London, in the year 1849. The Table contained in the Reports of the Registrar-general gives the heights above the Thames, and the corresponding number of deaths by cholera, out of a population of 10,000. The numbers decrease at first rapidly, and afterwards slowly, until, at heights above 100 feet, the variation in the number of deaths is very small. The facts are justly ascribed to the imperfect drainage of places situated at low levels. 


\section{XIX.-ON THE RISE AND PROGRESS OF MECHANICAL,}

PHILOSOPHY.

Introductory Lecture delivered in the Philosophy School of Trinity College, in Hilary Term, 1834.

Gentlemen,-I have lately endeavoured to lay before you an outline of the methods which have been pursued in the cultivation of Physical Science; and I dwelt in particular on the principles of the inductive philosophy as laid down by Bacon. At present I propose that we should confine our attention to that branch of natural philosophy which is to form the subject of our consideration during the ensuing Term; and review briefly the rise and subsequent advances of Mechanical Science.

The fallacious methods adopted by the ancients in physical science, generally, did not exclude a certain progress in the science of force. The doctrine of equilibrium, as you will soon learn, is altogether independent of experience; and the laws which determine the relations of balancing forces may be deduced wholly by a priori reasoning. In this department, accordingly, some steps were early made. From the metaphysical principle of sufficient reason, Archimedes derived the properties of the lever; and showed that two weights attached to it are in equilibrio, when they are to one another inversely as their distances from the fulcrum. This important and fundamental principle contained the germ of Statical Science : Archimedes himself was able to deduce from it the rules for the composition of parallel forces; and to show that there existed in every body, or system of bodies, a point-now known by the name of the Centre of Gravity-in which its weight might be supposed to be concentred. 
The foundations of Hydrostatical Science were also laid by the same great geometer. His work de humido insidentibus is derived from the principle of the equality of fluid pressure-a principle still adopted as the basis of this science. In this work he has shown that a heavy body, when immersed in a liquid, loses a portion of its weight equal to that of the liquid displaced. It is by the aid of this theorem - a theorem on which the present mode of determining the specific gravities of bodies is made to dependthat the philosopher of Syracuse is supposed to have solved the famous problem of the erown, proposed by King Hiero, and to have detected the fraud of his workman.

A long period of darkness followed the discoveries of Archimedes. The philosophers of Alexandria, Ctesibius, and Hero, Pappus Alexandrinus and others, pursued the inquiries which he had begun, and by their inventions added much to practical mechanics. To the two first of these authors we owe the analysis of the various classes of machines, and their reduction to five

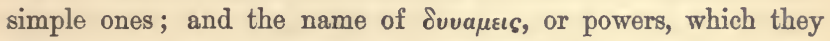
affixed to these elementary machines, is still retained. But yet the theory remained nearly as it had been left by Archimedes, and the doctrine of equilibrium was destined to receive no addition until the close of the 16th century.

Hitherto the theory of the Mechanic Powers was understood only so far as it could be derived from the principle of the lever; and no method was known of determining the conditions of equilibrium of forces whose directions were inclined. It was at this point that the progress of the ancients in Mechanical Philosophy was arrested, and it is here that we are to look for the first important extension of this science. The problem was attempted, but unsuccessfully, by Guido Ubaldi, a mathematician of Italy, in the 16 th century; and was finally solved by Stevinus, a celebrated engineer of the Low Countries, who demonstrated the principle of equilibrium on the Inclined Plane. In this remarkable demonstration, he supposes a chain of uniform thickness to encompass the plane entirely round : part of this will rest on the plane, along its hypothenusal side, part will hang vertically beside its altitude, and the remainder will form a loop below the base. Now the whole is in equilibrio, for if not, a perpetual motion must ensue in the direction of the greater force; and as the part which hangs below the plane draws equally in both directions, its effect 
may be disregarded. There is equilibrium, therefore, between the other two portions - that which is equal to the length of the plane, and that which is equal to its altitude;-and it follows that two weights, which balance by means of the inclined plane, one hanging freely, and the other resting on the plane, must be to one another in the ratio of the height of the plane to its length. This singular solution of the problem was given in the year 1585 ; and from it the author has derived the general conditions of equilibrium among any three forces meeting at a point. Thus, though he does not seem to have been aware of the full value of the principle, he touched the very corner-stone of statical science.

The theory of the Equilibrium of Fluids is also largely indebted to Stevinus; and he was the first to determine the pressure which the bottom of a vessel sustains from the contained liquid.

The second grand division of mechanical philosophy-the Doctrine of Motion-is of much later birth. We are here beyond the circle of abstract truth, and experience alone can furnish the principles of our reasonings. $\mathrm{We}$ are not to wonder, therefore, that no progress was made in this department of science, when the true principles of experimental inquiry were themselves unknown. The school of Aristotle taught that motions are either natural or unnatural. The natural tendency of all terrestrial bodies was said to be, either to fall directly to the ground, or to ascend from it until they reached their place; while bodies that were impelled obliquely were believed to pursue a violent or unnatural course, and the motions thus generated were supposed to tend continually to decay. In the heavens all this was different: the natural motions of the heavenly bodies were pronounced to be circular; and, as their matter was incorruptible, so their motions were eternal and immutable. Amid these reveries, we can still trace throughout the workings of genius; and in some parts of the mechanical speculations of Aristotle, we find very just views respecting the nature of gravity, and of force in general. The principle of the Composition of Motion-that fertile principle which served as the basis of the reasonings of Galileo, and was more fully developed by Newton-is to be found distinctly stated, and reasoned on in Aristotle's writings.

For more than two thousand years the dogmas of Aristotle continued to extort the assent of mankind, and though here and there a bold inquirer might be found, who ventured to doubt the 
authority of the "great master," and, to appeal to nature herself, yet the science of motion was not destined to profit by these glimmering lights, and its very foundations remained unlaid until the elose of the 16th century.

Galileo was born at Pisa, in the year 1564, and at an early age devoted the energies of an ardent and powerful mind to the mathematical and physical sciences;-and here was first formed that union of abstract and experimental reasoning, whose force and value in explaining the phenomena of nature is now so well understood. While yet pursuing his studies at the university, Galileo had begun to make experiments on the laws of falling bodies; and he then discovered the fact, that all bodies, whether light or heavy (the effect of the air's resistance being abstracted), fall to the ground from the same height in the same time. This important law of gravity was in direct contradiction to the principles of the Aristotelian philosophy, then in full repute; and, unaccountable as it now seems, it was believed that two unequal weights, if let fall at the same instant, and from the same height, would reach the ground in times inversely proportional to the weights-that a weight of $100 \mathrm{lbs}$. (for example) would descend in the $\frac{1}{100}$ th part of the time occupied by a weight of $1 \mathrm{lb}$. Galileo tried this very case before many witnesses, from the summit of the famous leaning tower of Pisa. The two weights were found to reach the ground in precisely the same time; and the decisive result of the experiment did more, perhaps, to shake the empire of the Aristotelian philosophy, than any single effort of opposition hitherto made.

Setting out from the principle that bodies, in their free descent towards the earth, receive equal increments of velocity in equal times, Galileo deduced, by the application of mathematical reasoning, the known laws of falling bodies; and having proved that these laws must hold good also in the case of bodies descending on inclined planes, where a portion of the weight is sustained, and where consequently the acceleration is not so rapid, he was enabled to bring the whole theory to the test of experiment. He applied himself, in the next place, to the consideration of the motion of a heavy body projected obliquely; and by the aid of the principle of the composition of motion-a principle tacitly assumed in his reasoning-he proved that the path traced by a projectile was the well known curve of Appolonius-the parabola. 
All these results were confirmed by observation, and thus the nature and laws of terrestrial gravity were fully established.

Galileo seems to have turned his attention at an early period to the motions of pendulous bodies; and he is said to have been conducted to the knowledge of the fact-that all the vibrations of the same pendulum, whether great or small, are performed in the same time-by observing the swinging of the lamps in the Cathedral of Pisa. The tautochronism of the pendulum, however, seems to have been noticed, and even applied, at a much earlier period. The astronomers of Arabia employed the instrument in some of their observations, patiently counting the number of its oscillations during the period of an eclipse, and renewing the motion with a slight push of the finger, when the ares of vibration became too small. The same mode of observation was practised in more recent times by Gassendi, Riccioli, and other astronomers of Europe, when the attention of the scientific world was again drawn to the principle of tautochronism.

By these important discoveries, Galileo has justly earned the title of "father of dynamical science." But to estimate duly the merits of this great man, we must consider the age in which he lived, and the darkness with which he was encompassed. Alone and unsupported, he assailed the Aristotelian philosophy with all the weapons of sound argument and caustic ridicule; and the inveterate spirit of hostility which he thus excited,-the rancour of rivals, and the intolerant persecution of the Church,--ended only with his life. Notwithstanding all this opposition, however, truth was progressive. The proofs which the astronomical discoveries of Galileo brought in support of the Copernican system roused the attention of philosophers; and it may be fairly questioned whether the discoveries of the Florentine philosopher have not had as great an influence on the progress of physical science, as the works of his immortal contemporary Lord Bacon.

While to Galileo we owe the foundation of the doctrine of motion, to his pupil Torricelli we are indebted for the first step which was made in that branch of the doctrine which relates to fluids. The fundamental problem of hydraulics - that which determines the velocity of efflux of a fluid through an aperture in the bottom or side of a vessel-was first solved by this writer, in his work de motu gravium.

As the terrestrial mechanics of Aristotle were overthrown by 
Galileo, so, about the same time, his theory of the heavenly motions was shown to be baseless. According to Aristotle, it has been already mentioned, the motions of the celestial bodies are circular and uniform-because the circle is a perfect figure. Many of his followers, however, attempted to support the doctrine on physical grounds, and asserted with Eudoxus, that the planetary bodies are confined by crystalline spheres, the movements of which gave rise to the motions we perceive. It is somewhat remarkable that the very same bodies, whose motions now tend to establish the existence of a resisting medium in the heavens, should be the first to give evidence against the theory of the crystalline spheres. It was ascertained by Tycho Brahe, from astronomical observations, that the comets were not, as Aristotle supposed, meteors in our atmosphere, but moved throughout the planetary spaces; and it was plain that, as they crossed the orbits of the planets in every direction, they could have encountered no obstacles such as the spheres of Eudoxus. Yet the doctrine of circular motion remained still undisputed, until Kepler, the successor of Tycho Brahe, announced to the world his great diseoveries-the elliptic motion of the planets, the equable description of areas, and the harmonio law.

It is impossible to read the account given by Kepler of these discoveries, without feeling animated with a portion of his enthusiasm. "I have stolen," says he, "the golden vases of the Egyptians, to build up a tabernacle for my God, far from the confines of Egypt." But when from these laws of observation he attempts to ascend to their physical causes, his reasonings are deeply tinged with the mysticism which seems to have gained so powerful an ascendancy over his great mind. Of the existence of gravitation, as the ruling principle of the universe, he entertained very strong and clear views; but the attraction itself he conceived to be of the nature of animal force.- "If the moon and the earth," he wrote, "were not retained in their orbits by their animal force, or some other equivalent, the earth would mount to the moon by a fifty-fourth part of their distance, and the moon fall towards the earth by the other fifty-three parts, and they would thus meet, the substance of both being assumed to be of the same density. If the earth should cease to attract its waters to itself, all the waters of the sea would be raised, and would flow to the body of the moon." The earth itself he supposed to be an enormous living animal, of which the tides 2 E 2 
constituted the act of respiration; and as this vital function was supposed to be produced by the influence of the moon, so its other living energies were dependant on the configurations of the planets. In the Harmonies of the celestial motions, he assigns to Jupiter and Saturn the bass, to Mars the tenor, the counter-tenor to the Earth and Venus, and the treble to Mercury.

The cosmical system of Des Cartes is too important in the history of science to be passed over without notice, although it seems to have been but a refinement on the doctrine of Eudoxus, and was as remote from real existence. Kepler, we have seen, had endowed the great bodies of the universe with vital powers, and chained them together, in their wanderings through space, by the ties of animal sympathy. The astrologers of the day borrowed from the region of spirit itself, and, by a beautiful fiction, assigned to each planet its guardian angel, whose office it was to guide its career through the trackless void. Des Cartes was the first who, with any show of plausibility, attempted to reduce the whole to physical principles, and to derive the phenomena of the material world from the fundamental properties of body. Matter, according to this ingenious philosopher, fills all space; and its parts are endued with motion in every possible direction. By the combination of these infinitely varied motions, matter is supposed to be continually deflected from its rectilinear course; and thus at length to form itself into vortices, in which the denser bodies of the universe floated, and of whose motion they partook. In this manner the earth and planets were supposed to be borne round the sun, in the vortex of the solar system: and each planet itself was the centre of a lesser vortex, which carried its secondaries. Such was the system which preceded the theory of universal gravitation. Mankind looked up with wonder on the symmetrical fabric which the enchanter's wand had raised, and they examined not too narrowly into its foundations; but a mightier wizard soon after came upon the scene, and the rod of Aaron swallowed the rod of the Egyptian.

The actual contributions of Des Cartes to mechanical science are inconsiderable. He was an a priori philosopher, in the strictest sense of the word; and in the barrenness of that philosophyaided as it was by powerful talents, and by the force of these talents gaining an ascendancy, which for a time closed men's eyes to the truth-we have, perhaps, as striking a proof as could be 
given of the soundness of Bacon's views. "We wish," says Des Cartes, "to deduce effects from their causes, and not, conversely, causes from their effects. We appeal to experience only, that out of innumerable effects which may be produced from the same cause, we may direct our attention to one rather than to another." The axioms, to which he endeavoured to reduce all his theories, were grounded on metaphysical notions of the attributes of the Deity ; and we have a remarkable instance of the presumption and error, which characterized most of his reasonings, in the dogma, that the quantity of motion in the universe must remain always the same, because the Divine nature is immutable.

To Des Cartes, however, we are indebted for the first distinct enunciation of the lavs of motion; although his own conception of these laws was far from accurate, and he seems, in particular, to have regarded the inertia of matter as a kind of active force.

The first actual contributions of importance which dynamical science received subsequently to the discoveries of Galileo are due to Huygens. By the aid of the most refined geometry, of which he was so perfect a master, this mathematician discovered the relation which subsists between the length of a pendulum and the time of its vibration; and having developed the theory of the instrument, he realized the conception of Galileo, and applied it to the regulation of the clock. On pursuing his inquiries, however, Huygens ascertained that the property of tautochronism belonged to the circular pendulum only when the ares of vibration are indefinitely small; and he was thus led to inquire what curve possessed the property universally. Finding this curve to be the cycloid, he conceived the idea of making a pendulous body move in it'; and his mathematical skill soon after enabled him to point out the means of accomplishing this object. His account of this eonstruction, and of the principles on which it depends, was published in the year 1670, in a work entitled Horologium Oscillatorium, many years after the date of the discovery. But interesting as the cycloidal pendulum is in theory, it has been long since abandoned, as useless, in practice; and the clocks to which the eircular pendulum was adapted were found far to excel the cycloidal clocks of Huygens.

To Huygens we are indebted also for the next great step which was made in dynamical scienee-the theory of circular motion; and the laws of centrifugal foree were developed by him 
with much clearness and simplicity. Thus was laid the foundation of the doctrine of central forces-a doctrine which, in the hands of Newton, served as the basis of the theory of universal gravitation.

I must pass over the names of Leibnitz and Varignon, Wallis, Wren, Hooke, and Halley, all of whom have made valuable contributions to mechanical science. We have now reached a period, in which the achievements of a single individual outweigh the collected labours of all his predecessors in discovery; and before the lustre of whose genius the lesser lights of science "pale their ineffectual fires." The time had indeed arrived in which one great mind was wanting, to concentrate the rays of knowledge, which were now beginning to dawn in various quarters; and the state of physical science was prepared for a vast revolution. The cumbrous apparatus of cycles and epicycles, with which Ptolemy had deformed the heavens, had disappeared; and the true system of the universe was established by Copernicus. The planetary motions were reduced by Kepler to three general laws; and one of these laws was found by Cassini to hold in the miniature system of Jupiter and his satellites, showing thereby that it was not the result of any accidental congruity, but had its origin in the nature of things. Again, the barrier which the ancient physics had raised between celestial and terrestrial motions had crumbled away, and the dogmas of Aristotle were no longer articles of belief. Lastly, the true road to philosophical discovery was pointed out by Bacon: to precept, Galileo added the more powerful influence of example, and laid the foundations of dynamical science in the laws which regulate the motions of bodies at the surface of our globe. In such a time Newton appeared.

There is not a single department of knowledge approached by Newton, that did not receive the impress of his mighty genius. Some branches of science were his exclusive creation; and when the difficulties of the investigations, into which his physical speculations led him, surpassed the power of the geometry of his day, he at once created an instrument which enabled him to grapple with, and overcome them. But I must confine myself here to those discoveries of Newton which are connected with the subject of mechanical philosophy:-to some of his other discoveries I shall have occasion to draw your attention hereafter.

The theory of equilibrium, in the different simple machines, 
had been hitherto established by separate and independent methods; and the principles of the solution were in many cases obscure and unsatisfactory. Newton traced the whole theory to one fundamental principle, and thus raised Statics to the undoubted rank of a science. The principle of the composition of motions had, it has been said, been assumed by Galileo, though nowhere formally stated in his writings. Newton felt the full force and value of the principle, and combining it with the second law of motion, which states the proportionality of the force to the motion generated, he gave the first formal proof of the celebrated principle of the composition of forces. I shall not dwell upon the objections which have been urged against this mode of establishing the important theorem of the parallelogram of forces, nor upon the attempts which have since been successfully made to assert for this principle the dignity of an abstract truth. It is sufficient at present to observe that the conditions of equilibrium, in the different classes of simple machines, were shown by Newton to flow easily and simply from this fertile principle; and that it has been since generally taken as the basis of statical science.

But much as the doctrine of equilibrium is indebted to Newton, the science of motion has received yet greater accessions from his hand. We have already seen that some great steps were made in this branch of mechanical science by Galileo and Huygens; and many important problems were solved by other writers. Yet these were but so many detached spots in the wide field of truth, reached by independent paths, and cultivated by different processes. No highway had as yet been opened into its fertile territory; and its unexplored recesses were still as diffioult of approach, as if no part whatever had been subjugated to human reason. This great want was supplied by Newton; and, in the first book of the Principia, he has developed, by general and uniform methods, the laws of rectilinear and curvilinear movement under the influence of a central force; the theory of the motion of bodies confined to given curves or surfaces, \&.

But great and valuable as were these additions to dynamical science, they seem to have been regarded by Newton but as steps to that system of the universe, the key of which he had early mastered. The principle of universal gravitation-his grand discovery - is indeed the highest and most comprehensive physieal truth ever reached by man; and we shall not mis-spend our time 
in reviewing briefly the principal steps which led to its complete discovery.

In the year 1666, the visitation of the plague compelled the young philosopher, then about the age of twenty-four, to leave Cambridge and retire into the country : and we are told that as he sat one day in a garden, meditating on the wonders of nature, his attention was arrested by the falling of the apples from the trees; and it at once occured to him that, as the force of gravity appeared to extend from the earth's surface to the tops of trees and of houses, and even to the summits of the highest hills, without any apparent diminution, might it not reach even to the moon itself? And may not our satellite be retained in its orbit by this very force, combined with an original impulse-just as a projectile at the earth's surface is forced to describe a curve under the same influences?

But another question here arose. Although the force of gravity does not appear to vary sensibly in the limited distance from the earth's surface at which we can make observations, yet, at the distance of the moon from the earth, this force (supposing: it to extend so far) may be greatly diminished; and if so, it would be necessary to ascertain the law of the diminution, before we could submit the question to calculation. Here the mind of Newton made another vast stretch. If the moon be retained in her orbit round the earth, by the attraction of the latter body, may not the earth, also, and the other planets, be held in their paths round the sun by the action of a similar force tending to that luminary? Now, it was not difficult to prove that the centripetal forces of bodies, revolving in circles round a common centre, must be inversely as the squares of their distances from that point, provided that (as is the case with the earth and planets) the squares of their periodic times varied as the cubes of their distances from the centre. If this law, therefore, be extended by analogy to terrestrial gravity, at the distance of the moon (which is about sixty semi-diameters of the earth), that force must be less than at the earth's surface, in the duplicate ratio of sixty to one. Is then the space through which the moon is bent in a second, from the rectilinear direction towards the earth, the 3600 th part of that through which a body will fall, in the same time, at the earth's surface?

Here was a question that could at once be submicted to 
calculation. Unfortunately, one of the data necessary to the calculation-the magnitude of the earth's circumference-was hitherto very erroneously determined; and the result, in consequence, appeared to be inconsistent with the assumed law. In the true spirit of philosophy, Newton at once abandoned his hypothesis, the moment that it appeared at variance with facts ; and it was not until some years afterwards that his thoughts seemed to have been recalled to the subject by the remarkable speculations of Hooke. The measurement of a meridional arc, undertaken by Picard, was soon after completed, and the new determination of the earth's radius happening to be the subject of discussion at the Royal Society, one day that Newton was present, he instantly perceived its bearing upon his own inquiries, and noting down the result, he hurried home to resume his calculations. He had scarcely substituted the new value in his formula, when the greatness of the anticipated conclusion overpowered him, and he was unable to proceed : a friend who happened to come in at the moment completed the calculation, and the theory of Newton was verified.

Such was the first step to the theory of universal gravitation. The law was now ascertained, and the analogy which Newton had already remarked, between the moon and earth and the planetary system, led him at once to apply the same great principle to the latter. Accordingly he next inquired what must be the paths, velocities, and periodic times of the planets, on the hypothesis that each of them had received an original impulse in any direction, and was at the same time urged towards the sun with a force varying inversely as the square of the distance. The result of this inquiry was the complete confirmation of the principle. The known motions of the planets, and even of the comets, were shown to flow immediately from the law of gravitation; and the famous laws of Kepler were among its first consequences.

It remained to prove that the forces by which these vast bodies are actuated, and which they exerted in turn, arose from the combined attraction of all their parts; and that the law which was thus manifested among the great bodies of the solar system was, in fact, a universal property of matter. Many strong arguments induced Newton to take this for granted; and assuming that every particle of matter attracts with a force directly proportional to its mass, and inversely as the square of 
its distance from the attracted body, he showed that spheres thus composed must attract according to the same law. Thus the hypothesis had all the certainty that could arise from the agreement of its results with established facts: its full confirmation from direct experiment was reserved for a later period.

That every particle of matter attracts every other, and is itself attracted in turn, is a proposition to which, at first, we find some difficulty in giving credence. We do not see the effects of such attractions, among the smaller masses of matter with which we are surrounded, and therefore cannot easily admit their existence. But the slightest consideration must convince us that these attractions, under ordinary circumstances, eannot be sensible. The force is proportional to the mass of the attracting body; and as the largest mountain on the earth's surface is but as a grain of sand on an ordinary globe, the attraction of the earth cannot, to any considerable extent, be modified by the effects of the irregular masses on its surface. Delicate observations, however, have rendered these comparatively minute forces perceptible. In Peru, La Condamine noticed the effect of the attraction of a mountain, in causing the plumb-line to deviate from the vertical. Maskelyne observed a similar effect produced by the attraction of Schehallien in Scotland; and from the amount of this deviation he was enabled to compare the mass of the earth, and therefore its mean density, with those of the mountain.

The effects of local attractions have been exhibited in another form, in the interesting pendulum experiments of Captain Sabine. This able observer found a difference in the rate of going of his pendulums, amounting to ten seconds in twenty-four hours, and due wholly to the effects of local attraction. The rate was quickest when the soil over which the pendulum ribrated was compact basalt, the heaviest of the known substances composing the crust of our globe; and slowest, when it was alluvial soil, which is among the lightest. From an extensive comparison of these effects with the subjacent strata, he concludes that the nature of the soil may in general be determined, within tolerable limits, and thus the pendulum be rendered a useful instrument of geological inquiry. These important conclusions have, very lately, received the fullest confirmation from the pendulum observations of Captain Foster.

Time will not permit me more than to allude to the decisive 
experiment of Cavendish. By an instrument called the balance of torsion-an instrument of the highest value in the measurement of minute forces-this philosopher was enabled to measure with precision the attraction of large metallic globes, and to compare their forces with that of the earth itself. From these investigations it appeared that the mean density of the earth is about double of that of its superficial parts, and five and half times that of water.

I cannot terminate this subject without noticing the interesting experiment proposed by Mr. Whewell and Professor Airy, in order to determine the law of terrestrial gravity by direct observation. The experiment consisted in comparing the rate of a pendulum clock with that of a chronometer, at the surface of the earth, and at the bottom of one of the deepest mines in England. The rate of the pendulum varying with the force of gravity, while that of the chronometer is independent of it, it is obvious that their comparison would afford the means of determining the relative forces at the two stations. The experiment, I believe, was not completed. But there can be no doubt of its sufficiency; and it is a remarkable fact that Bacon proposed one altogether the same in principle, and having nearly the same objects in view. $\mathrm{He}$ observes, that if the tendency of bodies downwards be the result of the earth's attraction, it must vary with the distance from the earth's centre, and he proposes to determine this by comparing the effects of a weight and of a spring at different heights and depths.

Such are a few of the leading facts by which the universal gravitation of matter was established. But numberless applications of the principle at once suggested themselves to its discoverer. The inequalities in the lunar motions, some of which had been detected by observation so far back as the time of Hipparchus and Ptolemy, were shown to arise from the disturbing attraction of the sun. The evection, the variation, and the amnual equation, were all traced to this cause; and the same cause was found sufficient to explain the principal of the inequalities affeoting the orbit itself, such as its change of inclination to the ecliptio and the motion of its nodes.

It had been observed by Richer, Varin, and Des Hayes, that a pendulum vibrated more slowly near the equator than in the higher latitudes, and the result had been a subject of much 
wonder to the learned. Newton immediately perceived in it a new effect of gravitation: he proved that the earth itself, supposing it to be fluid and homogeneous, would assume the form of an oblate spheroid, whose greatest and least diameters are in the ratio of 230 to 229 ; and that the force of gravity must increase from the equator to the poles, the increment being proportional to the square of the sine of latitude. The attraction of the sun and moon upon the redundant matter encompassing the equatorial parts of the earth was shown to account for the precession of the equinoxes. The phenomena of the tides were proved to arise from the same actions, exerted upon the waters of our ocean; and from a comparison of the effects produced at spring and neap tides (when these two forces conspire or are opposed) Newton deduced the proportion of the forces, and thence determined approximately the mass of the moon, and even its density, as compared with the earth.

This last result is certainly one which cannot fail to strike the uninstructed with wonder. That the mathematician should be enabled to weigh the moon's mass, and even to compute its density, from its effects upon the waters of our sea, is indeed truly wonderful; but it is only one of many results of the same nature reached by Newton in following out the great principle of universal gravitation. By comparison of the orbit of the moon round the earth with that of the earth round the sun, the mass of the earth (relatively to that of the sun) is at once determined; and the same principles are applicable to all the planets which are attended by satellites.

Such are among the more prominent discoveries developed in the Principia of Newton. Its concluding pages are dedicated to the proofs, which the laws of the material world afford, of the existence and attributes of the great First Cause. This is as it should be: and while we admire the genius of the man who could penetrate so far into nature's mysteries, and bind all these varied and complicated phenomena by a single tie, our thoughts are insensibly raised from the creature, to whom it was permitted to gain so large an insight into this wondrous work, to the Infinite Intelligence that planned the whole, and the Infinite Power that ealled it into being.

Of the advances which mechanical science has made, since the time of Newton, I must speak but briefly. The first great step 
was that which clothed the processes of dynamics in the symbolical language of analysis. In order to accommodate his discoveries to the actual state of mathematical knowledge, Newton chose to exhibit them in a geometrical form, and by the aid of a new doctrine which he had invented for the purpose. Thus his results are deprived of that elegance of form which is, in so eminent a degree, characteristio of the conclusions of analysis; and the method of inquiry itself, in the higher and more difficult problems, becomes elaborate and revolting. It must therefore be considered an important era in mechanical science, when it received the aid of the fluxional or differential calculus, the powerful instrument of Newton's own invention, and when its principles were developed by the fixed and uniform processes of analysis. This application of the calculus to mechanical questions was made at an early period, and by several hands; but the systematic development of the science in this new form is perhaps to be dated from the publication of the mecanique of D'Alembert, in the year 1743 .

In what relates to the discovery of important principles, much was also done. So early as the year 1592, in a short treatise on mechanical science, Galileo had reduced the equilibrium of the simple machines to a single principle; and showed that the power and the equilibrating weight are to one another inversely as the spaces which they tend to describe in the same time. This is a limited case of the general principle which has since been assumed by Lagrange, as the basis of the whole of statical science-the principle of virtual velocities. The principle itself was first stated, in all its generality, by John Bernoulli, in the year 1717.

The science of motion was destined to receive a yet greater extension by the accession of a general principle. The dynamical problems, of which we have hitherto spoken, are those in which the several parts of the body acted on are urged alike, and all partake of a common movement. There are, it is true, many and important problems in which this simplification is admissible: but there are many cases, also, in which the parts of the body, in virtue of their mutual connexion, are not free to obey the forces which act externally upon each; and to these the existing theories did not apply. The first problem of this kind that engaged the attention of mathematicians was the famous one proposed by Mersenne-the determination of the centre of oscillation of a compound pendulum; or, in other words, the investigation of 
the length of a simple pendulum which will perform its vibrations in the same time. Huygens was the first to give a complete solution of this important problem. James Bernoulli afterwards treated the subject with great elegance; and derived its solution from the general and self-evident principle-that the motions lost by the different parts of the body, in virtue of their mutual connexion, must be in equilibrio. This important and fundamental principle was afterwards adopted by D'Alembert, as the basis of his treatise on dynamics, and it is now connected with his name. The science of motion was thus reduced to that of equilibrium, and was made to assume the perfect form in which we find it at present. Its application to particular problems is now made by regular and consistent methods, and the success of such applications is limited only by the powers of the analytical instrument which is employed in their development. In the elegance and generality of its methods, the Mecanique Analytique of Lagrange now leaves little to desire; and we cannot hesitate to rank this great work among the most perfect creations of human genius.

But though the general methods of dynamical science may be fixed, and its doctrines complete, yet, when we come to apply these to particular problems, we find abundant room for ingenuity and analytical skill. The cases are comparatively few, in which we can proceed to the direct solution of a dynamical problem by a complete integration of the equations on which it depends. The resources of analysis are, in most cases, unequal to such an attempt, and we are generally obliged to content ourselves with approximate determinations. This is especially the case in celestial mechanics, when we introduce the consideration of even a third body into the system whose motions we seek to determine; and in this department of dynamical science, accordingly, there was abundant scope for the highest exercise of the reasoning powers. We have already spoken of the rise of this science in the mind of Newton, and of the vast steps which he made towards its completion: it now remains to say a few words of the additions which it has since received.

Clairaut was the first writer who made any important addition to physical astronomy, as it had been left by Newton. Observations had shown that the apogee of the moon's orbit was not fixed in space, but moved according to the order of the signs with a motion of about $3^{\circ}$ yearly. Newton proved that such a progres- 
sion of the apogee would necessarily arise from that part of the sun's disturbing force which acts in the direction of the radiusrector of the moon's orbit; but on calculating the amount of the progression due to this cause, it was found to be only the one-half of that observed. Clairaut, Euler, and D'Alembert, attacked the problem with all the resources of analysis, and their first investigations led to a similar conclusion. This remarkable discrepancy between observation and theory was so startling, as to induce Clairaut for a time to doubt the accuracy of the Newtonian law of gravity, and to propose a new and more complex one in its stead; but he soon after abandoned this idea, and found that the apparent deviation arose from the incompleteness of his approximations. There are few things which tend so strongly to confirm our belief in a law, as when a fact, which at first appears at variance with it, turns out on fuller examination to be one of its necessary consequences; and accordingly we find that the explanation of the progression of the lunar apogee, on the law of Newton, dissipated all remaining doubts as to its truth.

The complete solution of the problem of the figure of the earth was also reserved for Clairaut. In Newton's determination of this question, the mass was assumed to be homogeneous; and his method, limited by this hypothesis, was followed up and perfected by Maclaurin. But Clairaut considered the problem generally, and upon any supposed law of change of density; and the result at which he arrived-and which has been distinguished by the name of Clairaut's theorem-is as beautiful as it is comprehensive.

The general methods adopted by Clairaut (and after him by other writers) in the lunar theory were soon found to be applicable, with proper modifications, to the determination of the planetary inequalities also. Since all the bodies of the system attract one another, these attractions must cause each to deviate from the elliptical path, in which it would move under the influence of the sun alone; and the determination of these deviations was obviously of the utmost importance in the completion of the theory of the system. The planetary theory, in this enlarged sense, was the next great addition made to physical astronomy, and is due to the united labours of Clairaut, D'Alembert, and Euler.

Bnt among the perturbations of the bodies eomposing the solar system, there was one which, on account of its magnitude, 
engaged the attention of mathematicians at an early period. Astronomers had long observed that Saturn's mean motion was subject to a slow retardation, and it was discovered by Halley that the motion of Jupiter was in like manner accelerated. It was natural, then, to look for the cause of these phenomena in the mutual action of the two planets; and accordingly the French Academy soon proposed the theory of Jupiter and Saturn, as the subject of their prize-essay. Its difficulties, however, were such as to baffle the genius of Euler, who engaged in the research. Laplace and Lagrange afterwards entered on the investigation, and advanced, almost side by side, to its complete solution. In the course of these researches it was proved that the mean motions of the planets were subject to no secular inequalities; and accordingly the retardation of Saturn's motion, which was supposed to be of that nature, appeared to be at variance with the law of universal gravitation. It was finally shown, however, by Laplace, that the mean motions of Jupiter and Saturn were subject to an inequality of a very long period; and that the retardation of the one planet, and the acceleration of the other, would in time cease, and the effects be reversed. This remarkable irregularity in the motion of these two planets was found to depend upon the near commensurability of their mean motions. A phenomenon of the same kind, depending upon a similar cause, is found to take place in the motion of the earth and of Venus; and these two bodies also are subject to an inequality of a long period, lately brought to light by Professor Airy.

The principle above referred to-namely, that the mean motions of the planets are subject to no secular inequalities-was discovered by Lagrange, who was the first writer to examine with attention the variations of the elements of the planetary orbits. The importance of the discovery is obvious :-if the mean distances and mean motions were under the influence of secular inequalities-if Saturn should continue to be retarded, and Jupiter to be accelerated-the one would recede from the sun, until it finally fell under the influence of some distant body of the starry universe; while the other would approach indefinitely, and at last reach the central mass. This however is not the case : the inequalities which exist are all periodical, and the changes which occur are continually repaired. It is in this sense that the planetary system is said to be stable. 
It was soon after found that the eccentricities of the orbits of the planets, and their inclinations to a fixed plane, in like manner oscillated within certain small limits which they could never transgress. Thus, though the inclination and eccentricity of the orbit of each planet is continually changed by the action of the rest, yet, after certain periods, the same forees which have deranged it from its mean state, will bring it back to that state again, and repair their work of disturbance. To use the words of a distinguished French writer of the present day- "La stabilité du système solaire est donc à jamais assurée: les orbites des planètes dans les ages futurs ne pourront que s'aplatir légèrement en conservant les mêmes grandes axes, et les plans de ces orbites ne feront que de petites oscillations autour d'une position moyenne: immenses pendules de l'éternité, qui battent les siècles, comme les nôtres battent les secondes."

Thus it appeared that the planetary system contained in itself the principle of reparation and permanence. So far as depended on the mutual action of the great bodies which compose it, the system would endure for ever; and would not (as Newton seems to have supposed) ever require the repairing hand of its Divine Author to check the progress of destruction. Late observations, however, render it in the highest degree probable, that there exists an external cause which, in the course of time, must bring all this vast machinery to rest, and precipitate the gigantic masses which compose it into the body of the sun. There are, in fact, strong reasons for believing that a resisting medium is diffused throughout the planetary spaces, the effect of which must be to diminish the velocity and the distance of each revolving body from the sun, until it at length reaches that centre. This medium is of such tenuity, that it has, as yet, produced no perceptible effect upon the motions of the denser bodies of the solar system. Our own gross atmosphere, we know, offers a comparatively feeble resistance to the bullet in its fall; while the descent of the feather may be so retarded, that it may seem almost suspended in the air. If then the spaces which intervene between the great bodies of the solar system be filled with a medium far rarer, as compared with the lower regions of our atmosphere, than these are in relation to the globe they envelop, we are not to look for its effects upon the motions of such bodies as the planets and their satellites.

But there are other mysterious bodies connected with our 
system, which, we have now reason to believe, are less dense than the clouds which float above us; and it is among these that we must seek the effects of resistance. The singular body, now known by the name of Encke's comet, was first observed in the year 1786; and has since that time been frequently seen, its periodic time being only about three years and one-third. It was not until after its return in 1819 , however, that its identity was established by Encke, and its subsequent returns calculated. This comet reappeared in 1822, and on comparing the observed with the calculated places, Encke found that there was a difference, which seemed to be completely accounted for only on the supposition of a resisting medium. $\mathrm{He}$ has been thus led to examine in detail the effects of such a medium on the motion of the comet; and its subsequent appearances in 1825 and 1828 completely fall in with the supposition.

The effect of this resistance is indeed very small : according to the observations hitherto made, the diminution of velocity, in ten revolutions of Encke's comet, has not amounted to the onethousandth part of the entire. And when we proceed from this to speculate upon the probable amount of the effects produced upon the motion of the earth and the other dense bodies of our system, we are compelled to admit it to be so minute, that millions of years must elapse before it becomes sensible. But, however long the time required for the development of its effects, we have here a cause which eventually must put a stop to all the great movements which we observe in the heavens. The earth and the planets must gradually be retarded by the resistance of the medium in which they move: their gravity towards the sun must this preponderate over the centrifugal force; and they will gyrate in nearer and nearer orbits, and in continually diminished periods, until at length they reach the central body, and then all motion will be at an end.

This termination to our system cannot fail to suggest many and grave reflections. Indeed there are few subjects which force so strongly upon the mind the conviction of a ruling Power, as those which indicate the finite nature of all created things- which mark the period when the now order of nature did not exist, and predict the time when it shall cease-which point, in short, to a beginning and an end. But while our thoughts are thus arrested by the probable termination of our system, the astronomer bids us 
look out upon the starry concave, and learn how insignificant a part our sun and all his attendant worlds form of the boundless universe. He tells us that in the deepest abysses of space, the Creator has ordained suns, and assigned to them their appointed courses, which will remain unmoved by our catastrophe. Among these dazzling wonders he points to many that are united into systems, endued with proper movements of revolution, and chained together by some attracting tie:- and we are prompted to ask, are these attractions similar to that which binds together the parts of our own system? Is the law of gravitation obeyed in these remote regions of the universe? If this be the case, we know these bodies should describe conio sections round their common centre of gravity, and round each other: Are then the orbits of the double stars ellipses, like the orbits of our planets, and are their motions in these ellipses governed by the same laws? This inquiry appears at first to involve insurmountable difficulties. The distances of these bodies from one another seldom exceed a few seconds; and the changes in these distances, from which we might hope to compute the orbit, are far within the limits of the errors of observation. But these difficulties have been surmounted by Sir John Herschel. By taking as his data the observed angles of. position, instead of the distances, and by the happy union of graphical with analytical methods, he has obtained the orbits of several of the double stars, and deduced their elements. The result of these investigations has been the complete verification of the assumed law; and thus the grand principle of gravitation which Newton showed to belong to the bodies composing our own system is extended to the remotest regions of the starry universe, and found to be the universal principle of the material creation.

Before I conclude, suffer me to draw your attention for a few moments to an objection often urged, and too easily credited, against the value of the pursuit on which you are about to enter. You will hear theory and practical knowledge contrasted together: you will be taught to despise the former as vain and barren speculation; while the fruits of the latter-its immediate application to the uses of life, and the amelioration of the state of mankind, which are its results-will be paraded ostentatiously before you.

In reply to this it might be enough to ask-has our nature's noblest part no pleasures, no cravings of its own? Is there no part of our complex being which seeks truth for itself? Are the 
pure and disinterested enjoyments which the philosophic mind derives from its exalted speculations-are these unreal? or are they inferior to those which minister to the animal part of our being? But I will quit this high vantage ground, and descend into the field which the utilitarian has chosen for himself. I will assume that the objects of his desire are, and should be, those of all mankind; and I will tell him that science-true science alonecan point out the road to their full attainment. In fact, the objection betrays an ignorance of the real character of inductive philosophy, and of the spirit of its votaries. The true philosophy of nature is founded upon facts, and it is its very essence to admit of practical application: It takes its rise from particulars, and to particulars it is in fine applied. But, unlike that with which it is contrasted, it embraces phenomena-not in their accidental characters-but in their generic and essential ones. It rises from the maze of individual cases, with which alone the man of mere practical detail is conversant,- - but it rises that it may enlarge its view-that it may trace (to use the expressive words of a favourite writer) the spirit of the laws of nature from the letter; and then, and then alone, is it in an attitude to descend, and with all its acquired lights to advance the physical condition of man.

There are some, indeed, whose habits of generalization, and love for the pure abstractions of science, lead them to fix the abode of their thoughts wholly in its loftiest regions-while others ascend into its pure atmosphere, only that they may "steal the fire," and apply it to their earthly uses. It is wisely ordered that it is so; for each of these has his proper part to fill, and both conspire for the good of mankind. Each, too, has his own peculiar pleasures. Those of the former, perhaps, are more remote from sense-more exalted-more near to what we may conceive to be the enjoyments of a purely intellectual being; while those of the latter, on the other hand, are more adapted to our mixed and compound nature. In both, the social and benevolent affections have full room for exercise. But it is from a higher source that the true philosopher-to whichsoever class he belongs-draws his deepest and most exalted pleasures. In the events which pass each moment before the uninstructed eye, and pass unheeded or unseen, he discerns the hand of Infinite Power, guided by Infinite Skill-and surrounded by wonders here, he is brought into close and multiplied contact with the wisdom of God. 


\section{XX.-THE APPLIED SCIENCES, AND THE MIODE OF TEACHING THEM.}

Pralection delivered on the occasion of the opening of the School of Engineering in the University of Dublin, on the 15th of November, 1841.

"Magnum certè discrimen inter res civiles et artes: non enim idem periculum a novo motu et à novâ luce. Verum in rebus civilibus mutatio etiam in melius suspecta est ob perturbationem : cum civilia authoritate, consensu, famâ et opinione, non demonstratione nituntur. In artibus autem et scientiis, tanquam in metalli fodinis, omnia novis operibus et ulterioribus progressibus circumstrepere debent."-BAcos, Novum Organum, lib. $i$.

Gentlemen,-It has been said by one of the wisest of men, that every thing in the institutions and customs of colleges was opposed to the progress of science. We are met this day to exhibit a practical refutation of this vaunted aphorism; - to prove that the universities of the present day, so far from impeding the march of truth, form themselves the vanguard of that intellectual army, whose victories are in the region of the unknown; - and that, while with one hand they are engaged in flinging back the fruits of conquest, with the other they aid in propelling the triumphal car of science into new and remoter regions.

We are met this day, under the auspices of the University, to follow together some of the principal applications of scienee to the wants and to the uses of man. But, in stating that such is the object of the addition which has been recently made to the institutions of this college by its heads, let me guard you against the notion, that it is in anywise intended as a concession to that unphilosophical, but unhappily too popular feeling, which decries as worthless every intellectual pursuit, if unaccompanied by results 
of a practical nature,-which can discern no value in speculation, unless it conducts us to conclusions bearing immediately upon the uses and the necessities of daily life. I feel that there is some degree of humiliation even in the task of defending the pursuit of abstract truth. Yet, though many a voice has been loudly raised in protest against this utilitarian spirit, the feeling, but half smothered, breaks forth again and again. The mind unused to science has a natural tendency to regard it simply as a mean,a mean to an ulterior end; and it is only just so far as this end is attained,-it is only by the direct advantages which science confers upon the physical condition of man, that its value is wont to be estimated.

Must it then again and again be repeated, that man has a twofold nature? that the intellectial and the bodily have each its own aliment, each its own gratifications? and that truth-speculative, abstract truth-is as properly the food of the one, as that whicli ministers to the support of physical life is of the other? And shall we compare the intellectual, and its enjoyments, with the inferior part of our being, and the things which minister to its gratification? Shall we degrade the high and disinterested pleasures that attend the discovery of the links in the chain of a priori knowledge, or those which flow from the contemplation of harmony and order, which reign in the works of God,- - shall we demean enjoyments such as these, by bringing them into comparison with those of sense? No! I trust the day will never come, when a spirit such as this shall take possession of the halls of our venerated universities: I trust that those who occupy their chairs may never be called upon to meet such an antagonist within their walls.

But while we advocate the claims of speculative knowledge, considered apart from its applications, let it not be supposed that we are insensible to the value of such applications, or that our voices will not swell the shout of applause, whenever science yields results bearing upon social well-being, or social progress. Among the heathen nations of early antiquity, the inventors of the sickle and the plough were deemed worthy of a place among their gods: and shall we affect to disdain the richer fruits of modern science, or to look down upon those who have contributed to raise them from her prolific soil ? Reason forbids it: selfinterest forbids it. The useful arts are the results of such applications; modern civilisation is mainly based upon them. And it is 
remarkable that speculative knowledge itself is amply repaid for the time thus lent to practical science ; and that man advances in the scale of intellectual being, not only by the acquisition of new materials for the exercise and development of his intellectual powers, but still more by his release from the thraldom of those corporeal occupations to which his bodily wants coerced him.

It is, in fact, one of the precious advantages of applied science, that its results have delivered man from a great part of that toil to which he was condemned in the infancy of society for the supply of his animal wants;-from that abject condition, in which the body is at once his taskmaster and his slave. This revolution has been accomplished by the knowledge which he has acquired of the forces of nature, and the means which he has contrived to compel them to act on his behalf. The Romans, with whom mechanical science was in its infancy, had recourse to human labour in grinding their corn; in crushing the seeds of plants, and expressing their juices; in removing the earth and the water which covered the treasures of the mine. Slaves, in immense numbers, were condemned to these toils, - - toils so severe, as to constitute the heaviest punishment, next to death itself. But man has since learned the art of employing the forces of nature,- - the action of the wind, and of the running stream,- to aceomplish his will. He has even learned how to develope such forces when otherwise latent; - to call into action the elastic forces of steam, and of the gases; and constrain them to execute his commands.

We shall better learn to estimate the benefits which applied science has conferred upon man, if we reflect for a moment upon the advances in social progress which the application of any of these agents has effected, and the character of superiority that has attached to the nation which has been the first to discover and employ them.

Let us take, for example, the combination of the moving force of air, and the resisting force of water, in propelling and guiding the vessel on the waves. What advantages has not this application of natural forces conferred upon the people of antiquity which was the first to make it? What advantages does it not still bestow upon the nation, whose pre-eminence in nautical skill has earned for her the proud title of "Mistress of, the Seas"? She carries the products of her industry across the ocean, to exchange them for the riches of distant nations. She spreads the terror of 
her arms to the remotest regions of the globe. She peoples the most distant shores with her colonies; and gives birth to nations affiliated to her by the ties of a common blood, a common language, and a common interest. She diffuses in every quarter her science and her arts, - the gifts of civilisation, and the blessings of religion.

Let us take, as another example, the discovery and the application of the power of steam. It was long known that water, in passing into the state of vapour, acquired a great expansive force; yet it is little more than a century since this force has been applied, as a moving power, in the mechanical arts. And yet what has been the progress which civilisation has made, in that short interval, by the aid of this agent? "Survey the metropolis of England, look through her towns; visit her arsenals and her manufactories; explore the depths from which they are raising the riches of the mine, and see the labours executed above the surface; in fine, sail along her rivers, her canals, and the seas which bathe her shores, and you will discover on every side evidence of the benefits which she owes to the inventor of the steam-engine." It is equally available in operations requiring a great expenditure of power, and in those where delicacy and perfection of workmanship are the chief requisites. "The heaviest labours, such as the cutting of wood and of stone, or the raising of water, are accomplished by the same moving force which produces the most delicate pieces of workmanship. The anchor is forged, and the counter stamped, -the metal is rolled out, and the bauble carved,-by this allpowerful and universal agent. And the same giant arms, which twist the cable of the man-of-war, draw out the slender gold and silver wire which form the lightest tissue."**

But we shall better appreciate the value of this agent, if we confine our attention for the moment to what it has accomplished in one or two branches of the arts.

In the year 1789, the first steam-engine was erected in Manchester. The manufacture of cotton goods, which before that epoch depended, almost solely, on animal labour for its moving power, received a new life. The various branches of the manufacture were brought together, to share the energy which the new

\footnotetext{
* Speech made by Sir Humphry Davy, at the meeting held in 1824, for the purpose of paying a tribute to the memory of Watt.
} 
power conferred; and all the operations necessary to convert the raw material into the perfect web were conducted in the same establishment. From that period-and chielly from that sourceriches have overspread the land. Manchester, Nottingham, Preston, and Glasgow, and many towns of less note, owe their wealth and their prosperity to this single manufacture; and millions of inhabitants are indebted to it for their subsistence.* Such, in fact, has been the progress of the cotton manufacture in England, in these fifty years, that, notwithstanding the pressure of the national debt, the heavy burden of the taxes, and the consequent dearness of all the necessaries of life,-notwithstanding these great disadvantages of England, as compared with other nations,- - she purchases the raw cotton of another hemisphere, carries it 10,000 miles to her workshops, transforms it into a web, and sends it back again the same distance, to China and to India, to surpass in beauty, in perfection of workmanship, and in cheapness, the products of the native art, fabricated with all the refinements of manual labour, and with the traditionary experience of many centuries. $\dagger$

But let us look to actual numbers. At the accession of George III., in 1760 , the value of the cotton goods annually manufactured in Great Britain was estimated at $£ 200,000$. In the year 1824, according to the statement of Mr. Huskisson, it amounted to thirty-three millions and a half; and of this sum more than half is returned to the country annually by foreign nations, in the shape of profit and wages. During the war with France, 569 millions sterling was added to the national debt. The sum returned by foreign countries as the price of British manufactures, during the same period-a period, of course, of crippled commercial energyamounted to 548 millions, 400 millions of which was the return for British capital and British labour. And the large share which the steam-engine has had in this manufacturing prosperity is such as fully to justify the assertion often made, that it was the invention of this instrument which enabled England to sustain a

* In the first and last of the above-named towns, the increase of the population in thirty years (from 1801 to 1831 ) amounted to upwards of 150 per cent. The total number of individuals directly employed in the cotton manufacture in Great Britain is estimated at 800,000 .

† "At Calicut, in the East Indies," says Mr. Babbage, "whence the cotton cloth. called calieo derives its name, the price of labour is one-screnth of that in Englaud, yet the market is supplicd from British looms." 
struggle, the most menacing and the most dangerous of any in which she has ever been engaged.

Let us take another example. At the time of the inventions of Watt, the north of England, which supplied by far the greater part of the coal for domestic consumption, and for the arts, had seen all the upper beds of her great mineral treasure exhausted. It was necessary to penetrate to greater depths, in order to supply to Britain a fuel which its forests had long ceased to furnish. But this circumstance greatly increased the cost. The metals, which formed the wealth of whole counties, could no longer be smelted at the same prices as before. The working of the mines thus became more and more disadvantageous; each year brought a sensible diminution in their produce; and all the secondary arts, which depended on them for their material, were menaced with decay and ruin.

The employment of the steam-engine put an end to this downward movement, and the whole face of things was altered. A power was obtained, which, with a comparatively small expenditure of fuel, lifts an enormous weight from the greatest depths; and thus renders the expense of raising the coal a very small fraction of its cost. The average performance, or (as it is technically called) duty of the Cornish engines, is equivalent to the elevation of fifty-five millions of pounds through a foot in height, by the consumption of a single bushel of coal; while that of some of them surpasses eighty millions. And thus it is computed that a weight exceeding one thousand tons is lifted one foot, for the sum of one farthing! In fact, the economical value of the engine, in mining operations, may be judged from the circumstance-that, during the continuance of the patent granted to Watt and Boulton, when they received one-third of the price of the fuel saved, the propietors of a single mine in Cornwall thought it worth while to purchase the right of the inventors for $£ 7500$ per annum.

We have stated that the reduction of the metallic ores was entirely dependent upon the supply of coal, and shared its fortune. But the supply of metal was influenced in another, and more direct way, by the steam-engine. The mining engineer was forced, by the feebleness of his mechanical means, to stop at a comparatively moderate depth in the search of mineral treasures. Independently of the labour necessary to lift the ore to the surface, the power required to clear the mine of water soon exceeded any which, previ- 
ously to the invention of the steam-engine, he could with profit employ.* But, aided by the steam-engine, he has successively doubled, trebled, and quadrupled the depths to which he can descend, in order to return loaded with the riches of the mine. As he penetrates deeper, he discovers richer and more widely-spreading veins of treasure, to which he sends his "captains" and his " companies," and which soon are forced to render up their store. At the head of his well-disciplined army he forces his way through the hardest rock, - he removes the earth and the ore,- and the earth's bosom is furrowed even more diligently than its surface. $\dagger$

And when these sources of national wealth are found combined,- -when the metal and coal occur in juxta-position,-in such favoured localities the application of steam-power effects still greater wonders. The arts spring up and multiply, upon this new and rapid supply of their material, and of the means of fabricating it. An immense population, gathered from all quarters, derives its subsistence from them. Opulent cities arise, as if at the wand of the enchanter; and towns, such as Birmingham and Sheffield, the former of which in the beginning of the last century numbered scarcely thirty streets, are now among the largest, the most populous, and the most wealthy of the empire.

What a gain to our country are such possessions! We lavish wealth, and life itself, upon distant conquests, which may be wrested from our possession in a moment by invasion or revolt, conquests which we are compelled to protect by costly fortifications, and to defend with troops, who perish by thousands, the victims of a pestilential climate,-conquests, whose fruits reach our shores at a vast expense, with great risk, and frequent loss. What service, then, does not that power render, which, by a few mechanical means, and at the cost of a little fuel, brings up upon the surface of our own soil its hidden treasures, and enriches us with the produce of a territory situated beneath our feet! These are triumphs, indeed,--bloodless victories won by the arm of science from unreluctant nature, conquests that no rival can wrest from us, no caprice detach.

I have already alluded, generally, to the moral revolution which

* The removal of the water which rises daily in the galleries of the Cornish mines would require the labour of upwards of 44,000 horses, or of about 300,000 men.

+ In a single mining establishment (the consolidated mines) in Cornwall, which I visited last summer, the total length of the shafts and galleries now measures 68 miles. 
applied science has effected, in delivering man from the thraldom of brute toil, and leaving him more free to cultivate the intellectual part of his nature. The share which the steam-engine has had in producing this effect is pointedly exhibited in the well-chosen illustration of M. Dupin. One of the most wonderful monuments of antiquity, as well for the mass of its materials, as for the height to which they are raised, is, doubtless, the great pyramid of Egypt. Let us compare the labour, which history tells us was bestowed upon this colossal structure, with the calculated steam-power of England. Herodotus informs us that Cheops ordered all the temples of Egypt to be closed, and forbade the sacrifices, in order that the people might devote themselves exclusively to the heavy task which he imposed upon them. Some were employed in working the quarries of the mountains of Arabia ; others in transporting the elevated blocks of granite to the banks of the Nile, and in lading the boats to pass the stream; others, lastly, received them there, and bore them to their intended site. A hundred thousand men, says the historian, were employed for twenty years, in the works of quarrying and building alone. The steam-power of England, said M. Dupin twenty years ago, would have effected the task, - the double task of lifting the stone from its bed in the quarry, and raising it to its place in the structuro-in eighteen hours! In fact, the steam-engines constructed in the establishment of Watt and Boulton alone, in the year 1819, were equivalent to the work of 100,000 horses, or 700,000 men; and the sum of money annually saved, by their substitution for animal labour, amounted to more than three millions sterling. The total number of engines, existing in Great Britain at the same period, exceeded 10,000: their work was equivalent to that of 500,000 horses; and the yearly saving thence arising was from twelve to sixteen millions sterling.

But, notwithstanding this transfer to which I have alluded, the effect of the employment of machinery in the arts has not been, on the whole, to set aside human labour. The severer kinds of toil are, indeed, now transferred from the labourer to the engine; but, from the best calculations of the effects of machinery, it appears that an increased demand for labour, although of a different kind, has, in general, been the result. This remarkable consequence is clearly exhibited in the history of the cotton manufacture. Before the inventions of Watt and Arkwright, the total number of labourers employed in connexion with this manufacturg in Great 
Britain was under fifty thousand ; it now amounts to a million and a half!*

The legitimate-the direct effect of machinery-is indeed to diminish the amount of labour necessary to the production of a given quantity of manufacture. But, in so doing, it lessens the cost ; and the effect of this, again, is to augment the demand, and of course also the production,- to augment it to such an extent, as, notwithstanding the reduction of price, to increase greatly the actual value of the produce. The number of workmen, therefore, so far from being lessened, is in general increased by the introduction of machinery, and by the means which it supplies of rapid fabrication. We are to remember also, that, by lessening the cost of any one article of manufacture, an increased stimulus is given to the production of every other. The restless activity of man, disencumbered of the toil before necessary for the supply of his wants, or enabled to satisfy them at a less expense, bestows the labour or the money thus saved upon some new object. New wants arise, new gratifications are indulged; his physical condition is bettered at every step, and he advances in the scale of civilisation.

Thus the result of the application of machinery, so far from corresponding with the forebodings of unwise economists, and misguided artisans, is, in fact, to increase the demand for labour. And when we turn our view in the other direction, we find that its effect-its much most important effect-is to extend the advantages and the comforts of the products of labour, from the wealthy classes downwards, to the lowest grade in society; to elevate the inferior, without prejudice to the interests of the higher ranks; and thus to smooth down the inequalities of our artificial condition.

In illustrating the benefits which the applications of science have conferred upon the condition of man, and the social progress which has resulted from them, I have dwelt chiefly upon the discovery and employment of steam-power, both on account of the universality of its application, and the gigantio magnitude of its effects. In fact, a powerful and economical agent once obtained,

- In the town of Stockport alone, says Mr. Babbage, the number of hand-looms was reduced to less than one-third, while that of the power-looms increased fivefold, in the ten years ending in 1832; yet the number of workmen, so far from diminishing, increased by one-third during the same period. 
there is almost no one of the arts which it may not subserve. The mechanical arts, the arts of construction, mining, and navigation, are all indebted to it. Every operation, in short, in which motion is to be communicated according to fixed rules, and in a settled order, must be capable of receiving that motion, which is its life, from this universal agent.

But let us turn to those operations in which this condition does not hold,-in which the ever-varying circumstances almost preclude the employment of any agent but one endowed with freewill -or, at all events, one whose effects may be made to vary with the ever-changing conditions, - and we shall still find abundant evidence of the debt which civilisation owes to the applications of science. Let us look to the public works of England, and we shall learn how much the spirit of free enterprise, leaning on the arm of science, can accomplish. We may take on this subject the testimony of an impartial and enlightened foreigner. Until the year 1756, says M. Dupin, England did not possess a single line of artificial navigation; while her land communications were limited to a small number of ill-planned and ill-constructed roads. Then, for the first time, a private individual ventured upon an outlay, which his bold speculations told him must eventually bring a large return; and he constructed a canal, which was to earry to the town of Manchester the produce of his mines. Soon after, Liverpool, seeking a freer passage for her commerce, formed and realized the project of opening a navigable way between the Irish channel and the German ocean. Other canals, more extended still, were by degrees constructed; and, in the short space of half a century, a double system of canals has ramified itself throughout Great Britain, exceeding three thousand miles in length.

The roads of England, adds the same writer, are the subject of yet vaster labours. The existing roads have been enlarged, and constructed anew on better principles; new roads have been opened to commerce, and internal intercourse; and a network of lines of land communication now exists in England alone, measuring 115,000 miles ! Meanwhile spacious harbours are formed to contain her vessels ; piers, embankments and light-houses, augment the security of approach, and of shelter, over an extent of more than 1500 miles of coast; and 22,000 merchant vessels, capable of earrying two millions of tons of merchandize, and manned by 160,000 sailors, are scarce sufficient for her commerce and her carrying trade. 
Now I shall asume it as an admitted truth, that the mode of carrying on these gigantic operations must be tanght. The public will guard itself against incompetency, and stand forward to protect interests of such magnitude; and emulation will play its part, and force the engineer to qualify himself, in some way or other, for his task. The only question is-whether his education is to be allowed to take the direction which chance, or circumstances, have given to it, - or whether, on the contrary, the public bodies (which have been endowed for the professional education of the youth of the country) should step forward, and offer the assistance, which it is their bounden duty to give if required. I think there can be little hesitation in replying to this question. There can hardly be a doubt, that where the practical applications of science are concerned, the sciences themselves must be systematically taught-at least so far as to insure a knowledge of the principles which are thus applied. I am sure, above all, that it is in the universities-the established schools of science-that such applications may be best unfolded; because in them provision has already been abundantly made for communicationg the knowledge of those principles of which these applications are the results.

If it be said (as I know is sometimes said) that many of the most important of the inventions to which $I$ have referred-some of the successive improvements of the steam-engine, for exampleare the results, not of science, but of a happy chance, I do not hesitate to meet the objection by a direct denial. It is true, there are happy moments in the history of every inventive mind, at which new ideas seem to rise as it were by a kind of inspiration. It is even true, that a fortuitous circumstance may have been the means of awakening the train of thought which has ended in great discovery. But it is nevertheless certain that all this is of no avail, unless the mind be already prepared to combine the prolific thought with its existing store,-unless, in fact, the soil be prepared for the seed which the capricious wind has borne to its bosom. The apple might have fallen unheeded from the bough, had not the master-mind of Newton been prepared to connect the fact with the treasures of his existing knowledge. And (to return to the example which I have already so often cited) the circumstances which suggested some of the improvements of the steam-engine might have occured in vain, had not the mind of Watt been prepared, by his acquirements in chemistry and mechanics, to 
seize their bearing, and to embody the idea which they suggested in his machine. These improvements were, in fact, the results of the most assiduous labour, aided by the most extensive knowledge. The amount of the dilatation of water, in passing into the state of vapour,- - the quantity of coal requisite to vaporise a given quantity of the liquid, - the amount of steam expended in each stroke of an engine of known dimensions, - and the elastic force of the vapour itself at different temperatures,-were all the subjects of experimental research, and of rigorous calculation. It was thus that he succeeded, step by step, in diminishing the quantity of fuel necessary to produce a given effect. It was thus that he discovered the means of alternately disengaging and absorbing the steam, and of transmitting, with the smallest loss, the moving power which he had thus developed. Finally, it was by the same knowledge, and the same mechanical resources, that he succeeded in applying the powerful instrument which he had contrived, and modifying it for the accomplishment of the most opposite processes. "Practical mechanics," says Sir John Herschel, "is, in the most preeminent sense, a scientific art; and it may be truly asserted, that almost all the great combinations of modern mechanism, and many of its refinements and nicer improvements, are creations of pure intellect, grounding its exertion upon a moderate number of very elementary propositions in theoretical mechanics and geometry." And the same may be said (though, of course, in ever-varying degrees) of the relative position of the arts, in general, and the physical sciences. The arts, in their highest development, are but the applications of the powers of nature to some practical object; and these powers must be known,- the uncontrollable laws of nature must be understood,--before they can be directed to the uses of life, and to the improvement of our physical condition.

But we have to deal with an objection of more weight. It has been said, and it no doubt will be said, that skill in such operations as we have been speaking of is only to be acquired by practice; that the mechanical arts, and the arts of construction, are still arts, a knowledge of whose processes must be learned by experience, and by experience alone; and that it is only in the engine factory, or in the field, that any solid information can be gained.

Now, we freely grant that there is much that is true in this statement, but, at the same time, we assert that it oversteps the 
truth. We fully admit that no perfect knowledge, in any art, can be communicated by oral teaching alone; - that the mechanical engineer, the mining engineer, the civil engineer, and the architect, must not only see the processes of their respective arts, but also excrcise themselves in them, before they are competent to conduct them on their own responsibility. But is this any reason why the principles of these several arts should not be taught systematically? As well might the physician say- "Away with your schools of medicine! let the student accompany me to the hospital ; he will see there the disease and the remedy in juxtaposition; he will observe the malady and the cure; and he may turn his back for ever on the lecture-room." Would reasoning such as this be listened to, in a profession already acquainted with better modes of teaching,- - teaching in which the principles and the practice are mingled in due proportion? And yet, is not this the very argument of those who ignorantly cry up practice, in opposition-as if it were in opposition-to theory? No! a wise teaching will give practice-practice, without which there ean be no skill : but it will not fail to base practical knowledge upon a knowledge of principles, without which all skill is but the blind performance of a task, as senseless and soulless as that of the engine in its routine of toil.

The extremes of opinion on this question are exemplified in the widely different states of the engineer profession itself in England and in France. Up to the present day, the education of the engineer student in the latter country has been too merely theoretical; while in the former, practice has been insisted on, almost to the exclusion of theoretical knowledge. The Polytechnic School of France,- - the school in which the young engineer commences his education,- - though perhaps the first school of pure science in the world, is decidedly too abstract in the character of its studies, to satisfy the wants of the professional student. And though, after two years of preparation there, he is transferred to the School of Mines, the Naval School, or the School of Roads and Bridges,-notwithstanding this apparently excellent arrangement for imparting special, as well as general information,-it is found, in the end, that his practical acquirements have not kept pace with his theoretical knowledge. In England, on the other hand, the student enters the office of the professional engineer, whom he accompanies in the execution of his publio duties. He thus 
acquires, in the most complete manner, that practical skill which is only to be attained by seeing and handling; but is it not evident that such a mode of teaching alone must be defective in forming the counterpart of his knowledge? His master is too actively engaged in the duties of professional life to attend to the task of oral instruction: the utmost that he can do is to direct his reading, and to superintend his work; and he is almost left to himself, to acquire, or not, the knowledge of those principles which he is afterwards to apply.

The consequences of these widely different modes of instruction are very evident in the opposite character of the professions in the two countries. The French engineer possesses a confidence in his own resources, which he often displays in meeting and mastering a difficulty; while perhaps he fails in the execution of an ordinary task. The English engineer, on the other hand, guided by rule in place of theory, is more successful in the daily walk of his profession, while (unless a man of natural genius) he is often unprepared to cope with new emergencies. The Englishman, from the practical character of his education, and of his mind, goes straightforward to the end proposed; the Frenchman deviates from the road to seek some opportunity of putting forth his skill. The Englishman sometimes commits mistakes, in new and untried cases; the Frenchman is often in error, because he is as likely to err in cases of daily occurrence, as in those which are infrequent and uncommon.

But happily the men of science in both countries now see, and have begun to remedy, the extremes into which this branch of education has fallen,- those of England, by the infusion of theoretical knowledge,- - the French, by the addition of practical acquirement. It is strange, that in a country which has been long forward in raising the intellectual character of the artisan,-in a country where schools and mechanics' institutes have sprung up without number, for the benefit of the working classes, -no regular education should have been provided (until within the last few years) for the master artist, the engineer, or the manufacturer. The University of Durham has been the first to wipe away this reproach, and her example has been ably and successfully followed in King's College, London.

In France, on the other hand, and about the same time, the Central School of Arts was founded, with that profusion of scien- 
tific strength which the capital of France can wield; and the institution has been followed by the establishment of preparatory schools having the same objects. I would gladly, did time permit, have dwelt upon the arrangements of this important school, which I had the opportunity of visiting within the last few months. There is one part, however, of the system pursued there, which I cannot forbear noticing, not only on account of its intrinsic excellence, but because there is nothing in it of a local character,nothing which will not bear transportation to another soil. In the concluding examination, in which is tested the fitness of the student to enter on his professional career, he is, furnished by his examiners with a projet. An engine is to be constructed for some special work ; a suspension-bridge is to be thrown across a certain river; an embankment, or a harbour, is to be constructed under given conditions; or a manufactory of some kind is to be established on a given piece of ground, and with a given capital. Such are the comprehensive queries put to the candidate,- queries addressed, not to an insulated point, but to a large circle of his acquired knowledge. $\mathrm{He}$ is called upon to put himself, for the time, in the place of the professional engineer, to whom such an inquiry is addressed by the capitalist; and he is expected, not only to furnish complete working drawings of all the parts of his design, but also to accompany them by an explanatory memoir, in which the calculations of the dimensions and strength of every part, of the quantity of materials requisite, and of the cost, are all given in full. The best of these plans and memoirs are preserved in the archives of the sehool; and, when corrected by the professors, they furnish the materials for future teaching.

It remains now that I should say a few words of the arrangements which the Heads of this University have made, bearing upon the same objects. I have already stated what seem to be the faults in the existing systems of engineer education ; and have shown, I think, the importance of a systematic instruction, in which theory and practice are attempered in just proportion. Such is the principle which has been steadily kept in view, in the course of education which the University now offers to the engineer student. It is intended to communicate just so much of theoretical knowledge as is indispensable, in order to enable the student to understand fully its applications. The applications themselves will next be 
offered to his attention in detail ; and finally, means will be provided to familiarize him with them, by experience and actual manipulation, as well as by oral teaching.

It has been accordingly arranged, that the first year of the student's course is to be passed in those studies which are prescribed by the University to Students in Arts of the same standing. This course, you are aware, embraces the elements of geometry, trigonometry, and algebra, all of which are necessary to the engineer student-as necessary as arithmetic is to the merchant ; and, in adding to these the amount of classical knowledge required in the Junior Freshman year, it is conceived that the indirect advantages of even a partial classical education will more than outweigh those which might be derived from the employment of the same time in purely professional study. After the expiration of a year, so employed, the engineer student will commence his professional course, under the teaching of the professors connected with the school, and their assistants. In the first year he is required to attend three courses of lectures. The first of these comprises the branches of mathematics which are specially required by the mechanical and constructive engineer,- - as logarithms, the applications of trigonometry, and the elements of solid and descriptive geometry. The second course comprehends the principles of mechanical science; and the third, the elements of chemistry and geology, and the application of these sciences in the arts of construction. The courses prescribed for the second year are more exclusively practical; and are, first, a course of practical mechanics, including the theory and construction of the steam-engine; and, secondly, a course on the practice of engineering itself. Each of these years of study will close with an examination, to be conducted by the professors connected with the school; and at the end of the second, or concluding year, a diploma will be conferred by the University upon those who shall have attended all the prescribed courses, and acquitted themselves with credit at the examinations.

I must pause here; for here terminate the arrangements, so far as they are at present defined. These arrangements, however, will certainly require and receive a further extension, as they are brought into operation; and means will doubtless be provided to instruct the student in drawing, both mechanical and constructive, and to render him familiar with the manipulations of the laboratory, the workshop, and the field. 
Such, Gentlemen, are the provisions which the Heads of the University have made for your instruction. The profession you have adopted,-whose duties were not long since limited to the construction and care of engines, - has now risen to take its rank among the first of the liberal professions. In a country like ours, where public works of such magnitude are ever in progress, the interests committed to its keeping are numerous and weighty; and the knowledge demanded of it proportionably varied and extensive. It is your part, then, to try to profit by the opportunities thrown open to you. Attend with diligence to the instruction which will be given to you in these halls; take notes of what you hear; and endeavour to combine the knowledge thus gained with the results of your private reading. You have every inducement that can be offered to exertion. The path which is to conduct you to the goal of your profession is interesting and attractive; and the career which afterwards expands before you is one in which you may serve your country nobly, and earn for yourselves an honourable independence, and an honourable fame. 
XXI.-ADDRESS DELIVERED AT A MEETING OF THE ROYAL IRISH ACADEMY, HELD APRIL 13тH, 1846, ON THE OCCASION OF TAKING THE CHAIR.

Proceedings of the Royal Irish Academy, 1846.

Gentlemen,-My first-my most urgent duty-on this, to me most solemn occasion, is to thank you for the high distinction you have conferred. It would be idle to attempt to express how highly I esteem the honour. The thought that I had been deemed worthy to occupy the chair which has been filled by Kirwan, by Brinkley, and by Hamilton, might, indeed, well nigh overwhelm me, did I not know that there were other merits, more humble than theirs, upon which you set a value-other qualities less dazzling, which may find here their employment and their use. An institution such as this has been compared to the House of Solomon, in Bacon's philosophical fiction, the New Atlantis, in which the investigation of truth is carried on by labourers of various kinds, to each of which he has assigned a separate task. We have had, in this Academy, the representatives of each of these classes: we, too, have had our "Miners," our "Lamps," and our "Interpreters of Nature." I am content to enrol myself in the lowest class; or if, by reason of the high trust which you have now reposed in me, other tasks should fall to my lot, I am proud to accept a new station among the intellectual workmen, and to perform the part of one whose office it is to harmonize and give effect to the labours of all.

There is another personal consideration, to which I cannot refrain from alluding; and yet it is one upon which I hardly 
trust myself to speak. Among my predecessors in this high office was one whom I am still more proud to follow:-my nearest relative filled this chair. I know how he was valued here; and I cannot but feel that much of the indulgent estimation which you have formed of my fitness for the same station has come to me reflected from his memory, and that you hope to find in the son some of those qualities for which the father was loved and honoured.

But, Gentlemen, whatever qualifications I may want, there is one to which I lay claim: I mean that of deep interest in the welfare of this Body, and zeal for its service. Here I will yield to none; and I console myself with the hope that it may make some amends in your estimation for the many wants which you will hereafter have occasion to observe.

My predecessor in this chair, upon an occasion similar to the present, laid before you some of his views respecting the constitution of the Academy, and the means by which its future interests might be promoted. I am sure that you will permit me to follow this precedent, and to offer a few remarks-firstly, upon the mixed nature of that constitution under which we are here united for the pursuit of truth,- -and, secondly, upon the progress that has been made, or that may hereafter be made, in that high object of our incorporation. It is of the future that it is important to speak: the precept

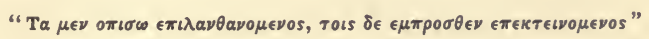

holds good in the pursuit of knowledge, no less than in the advance in piety. But still our hopes of the future, if they are to be more than dreamy visions, must be based upon the history of the past.

The first thing that must strike every one, in considering the constitution of this Academy, is the comprehensiveness of its scheme, and the wide scope of its labours; and we are inclined to ask, whether a constitution so large and so varied,-so opposed to modern precedent,- - can be sound and healthful? When we look into the recent history of Associations for the advancement of knowledge, we see that each division of the wide domain of truth, as it has arisen into prominent view by the labours of those engaged in its cultivation, has claimed for itself the concentrated energy, and the undivided resources, of an exclusive Society. In 
this manner the Royal Society of London, which included originally, and still includes, representatives from every department of philosophy, has seen Society after Society spring up, manned by its own members, and claiming to perform, in a more complete and effective manner, the separated portions of its work.

Such a state of things is the natural result of increased activity in any department, and of the consequent demand which it makes for a larger portion of time, and the other appliances of labour, than can be devoted to it in a body of mixed constitution. and more comprehensive plan. Nor can it be doubted that such a multiplication of the instruments, by which intellectual force is concentrated and applied, is attended with the many advantages which arise from the division of labour, nor that it has actually tended, and in a very important degree, to push forward and to extend the boundary which divides the known from the unknown.

But perhaps these advantages, great as they are, have not been wholly unbalanced. Have we not reason to apprehend that philosophy has suffered, while the portions of her mighty empire have asserted their independence, and erected themselves into separate kingdoms? So far as we insulate any portion of Truth from the rest, by an exclusive devotion to its pursuit-and there can be no doubt that such exclusiveness tends to insulation, - so far we mutilate the fair proportions of Truth itself, and injure and impair the philosophic spirit whose vital power should animate and pervade the whole. And the injury, great as it is, does not end here. There is an evil partaking of a moral nature obviously springing from this exclusiveness, and which, unhappily, we see too often realized, unless where some counteracting power is brought in to check it. I mean its effect in narrowing our views, in rendering us bigots in philosophy, and in causing us to undervalue that which we do not understand.

Now, the mixed constitution of our Society has a manifest tendency to overcome, or, at least, to mitigate, these evils. I do not mean to say that these evils, and these means of combating them, were distinctly perceived by the first founders of this Body. It is an humbling lesson, that human institutions, in which we have learned to find wisdom, have often had their origin in circumstances, and their growth amid the adjustments of conflicting interests. The plan of this Academy took its rise, I believe, in the union of two small Societies, cailing themselves the Paleasophers 
and the Neosophers, starting originally from opposite extremities of the field of Truth. But, whatever may have been its origin, we may now derive from it lessons not only of mutual forbearance, but of mutual instruction. The mathematician may imbibe from the antiquarian the taste which will lead him to explore, with reverence, the early history of the efforts of those masterminds in Science, whose very failures are fraught with philosophic interest, and to trace the progress of discovery up to the first dawn of thought; and he will return from the investigation with clearer views of the human mind itself, and of the means by which it attains Truth. The antiquarian may learn from the man of science those habits of precise thought, and exact reasoning, which, in the mysterious twilight that surrounds the fascinating objects of his pursuit, he is apt to think inapplicable; and both may learn from the cultivator of literature to value and to acquire that magic power which language confers upon thought.

Having said thus much in vindication of the constitution of the Academy, suffer me, in the next place, to consider how far it has been effective in attaining the ends proposed. For this purpose, it will be requisite to take a brief survey of the recent advancement of knowledge in this country, so far as it has been influenced by this Academy. And if, in the brevity with which the necessary limits of this Address compel me to glance over the subject, I should appear to have overlooked, or not to have assigned its due weight to any portion of our labours, you will, I trust, attribute this to its true cause.

The prominent place which the mathematical sciences have occupied in our Transactions may be dated from the time when Brinkley was enrolled amongst our members. But it is to the labours of your late President, and your late Secretary, in this department, that the Academy, in a great measure, owes the high place which it holds among the Scientifie Bodies of Europe. Of these labours, it might, perhaps, be rash to single out any portion as pre-eminent, had not the Academy itself, and the Royal Society of London, by the awards of their highest honours, marked out the researches of Sir William Hamilton and Professor Mac Cullagh, in connexion with the wave-theory of light, as of especial value. The theoretical discovery of conical refraction, by Sir William Hamilton, the theory of crystalline reflexion and refraction, by 
Professor Mac Cullagh, and the general dynamical theory of Light by the same author, mark an era in this branch of science not inferior to that of Fresnel.

Time will not permit me to do more than allude to the new branch of analysis, which has recently engaged so much of the attention of mathematicians, and which originated in the Theory of Quaternions of Sir William Hamilton, and has received an important modification and development in the Triplet theory of Professor Graves. As a Member of the University, I rejoice to be able to add, that worthy successors, even to such men as I have named, are arising there; and that the recent union of the mathematical strength of Cambridge and of Dublin in the Mathematical Journal, which was so long and so ably supported by the former University, is likely to give a new impulse to this branch of science amongst us. And long may these sciences continue to flourish in the University, and in this Academy! Independently of the magnitude and sublimity of their own proper objects,-independently of their direct value in Physical Science, as instruments of research,--they confer a no less important, but indirect service, in disciplining the mind, and correcting those tendencies of other portions of our mental constitution, which, when unbalanced, are sure to mislead.

Turning from the mathematical to the physical sciences,and first of all to astronomy, which stands upon the confines of both,- we cannot fail to be struck by the fact, that in this one Island, with all its disadvantages of climate, there are no fewer than four Astronomical Observatories, each elaiming a high place in the history of European science; and that while, in other countries, these costly institutions have been, with but few exceptions, founded and endowed by their respective Governments, in Ireland (a country not certainly among the foremost in pecuniary resources) they have been erected, equipped, and, with but one partial exception, maintained by the munificence and public spirit of individuals. The names of Mr. Cooper, and of the Earl of Rosse, will henceforward be added to those of Provost Andrews and Primate Robinson, as benefactors of science in this country; and Markree and Parsonstown be united to Armagh and Dublin in the future history of Astronomy.

The Dublin Observatory is the eldest of this noble sisterhood. As respects its connexion with this Academy, I need not, remind 
you that its chair has been filled by two of your Presidents. With the labours of Brinkley the Dublin Observatory will always stand connected in the history of science. I am sure that it is unnecessary for me to remind you of his researches connected with the problem of the "Stellar Parallax," of which your Transactions contain the first results- that great problem, whose final solution has at length been placed beyond question by the observations of Bessel. Of the other and better known inequalities, which affect the apparent places of the stars, all have been illustrated by the observations made with the meridional circle of the Dublin Observatory. In this important class of astronomical investigations, the able director of the Armagh Observatory has had a distinguished share; and the labours of Dr. Robinson have conferred, as might have been expected, increased accuracy upon the result. ing values of the constants.

And here, Gentlemen, you will permit me to pause for a moment, and having named the name of Bessel, to offer a passing tribute to his memory. $\mathrm{He}$, who but a few months since occupied the foremost place in the ranks of living Astronomers, is now no more! He died on the day which followed the last meeting and anniversary of this Body; and those among us who had the happiness to form his acquaintance, during his short visit to England, and to the British Association, four years ago, will be able to sympathize with his personal friends, no less than with the world of seience, in deploring his loss.

Of the astronomical and optical labours of the Earl of Rosse, and of his great reflector-the marvel of astronomical science-it is needless for me to speak. No one who was present when the account of its construction, and of its first achievements, was given in this room by Dr. Robinson, ean readily forget it; and for others, the printed notice of that account, in the last number of our Proceedings, will give the fullest information we yet possess respenting it. Even from this statement of its earliest trials, it is manifest that the astronomical history of the nebulce will, ere long, be re-made; and it must be satisfactory to us to know, that the noble artist has arranged a plan of systematio observation, directed to these remote and mysterious portions of the universe, which promises to reveal all that can be known, until a still higher optical power (if such be practically possible) shall be applied to their examination. The imagination is bewildered when it seeks 
to grasp the possible future which may be opened to this and other departments of Astronomical Science by the application of such means: I will mention but one amongst the many anticipations which press for utterance. The observations of Bessel have detected proper motions in the fixed stars, Sirius and Procyon, which appear to establish the existence of invisible companions, of vast magnitude, about which they revolve. Is the invisibility of these great bodies relative only? and if so, may it not be dispelled before the optical power which Lord Rosse has brought to bear upon the Heavens?

"Astronomy, however," to use the words of one whose philosophic mind, and varied and profound acquirements, well entitle him to legislate for science, "is only one out of many sciences, which can be advanced by a combined system of observation and calculation, carried on uninterruptedly. ${ }^{*}{ }^{*} * *^{*} *{ }^{*}$ in a utilitarian point of view, the globe which we inhabit is quite as important a subject of scientifie inquiry as the stars. We depend for our bread of life, and every comfort, on its climates and seasons, on the movements of its wind and waters. We guide ourselves over the ocean, when astronomical observations fail, by our knowledge of the laws of its magnetism; we learn the sublimest lessons from the records of its geological history; and the great facts which its figure, magnitude, and attraction offer to mathematical inquiry form the very basis of Astronomy itself. Terrestrial Physics, therefore, form a subject every way worthy to be associated with Astronomy as a matter of universal interest and public support, and one which cannot adequately be studied except in the way in which Astronomy itself has been-by permanent establishments keeping up an unbroken series of observation."

Two of the leading branches of Terrestrial Physics - the sciences of Meteorology and Magnetism-have now, as you know, for the last six years, been investigated after one uniform and comprehensive scheme, in more than thirty observing stations scattered over the entire globe; and the very bounds of eivilization itself have been overleaped in order to give a wider development to the system. In order to realize the view which Sir John Herschel has so often and so ably advocated, it is only necessary to give permanence to the more important of these Observatories, and to enlarge somewhat their sphere of labour. All the phenomena of which our earth, its ocean, or its atmosphere, is the seat; the tides aud the 
oceanic currents, no less than the winds; the temperature of the earth and of the sea, as well as that of the air ; the movements of the earth's crust, whether ealm or convulsive, no less than the changes of the mysterious power which animates and pervades its mass; all these, and more which might be easily added, are the proper subjects for continued and systematic observation. We have arrived at a period in the history of these branches of science, when the more obvious phenomena have revealed themselves to our desultory efforts, and when the precise laws, and the quantitative measurements, which must form the basis of exact theory, can be reached only by sustained and systematic exertion.

In these researches, no less than in those of Astronomy, this country has taken its part. The Meteorological Observatory at the Ordnance Survey office in the Phœnix Park, planned and directed by Captain Larcom, has now been upwards of ten years in active operation, and may be taken as a model for similar establishments. Of the Magnetical and Meteorological Observatory of Dublin, founded in the year 1838 by the University, I have already had frequent occasion to speak at these meetings, and I hope before long to communicate some of the ultimate results.

Of the geology of Ireland I have, perhaps, less right to speak, as the subject has been appropriated by another and a younger Society. Yet there are two facts in its recent history, of such importance, that it is impossible not to refer to them in noticing the labours of the members of this Academy. I mean the completion of the Geological Map of Ireland by Mr. Griffith, which, as the work of one man, is certainly one of extraordinary merit; and the recent arrangements for the continuation of the Geological Survey of this country, the first fruits of which are before the world in Captain Portlock's able and elaborate report on the geology of Londonderry.

Passing now from the sciences of Observation to those of Experiment, we here also meet with labourers of our own Body, and our Transactions are enriched with the results of their successful toil. Here are to be found the hygrometrio researches of Dr. Apjohn, which have solved one of the most intricate problems in Meteorology; and the still more refined researches of the same author upon the Specific Heats of the Gases, to which you have awarded your medal. Here too are to be found most of the chemical researches of Sir Rubert Kane, upon the chief of which you have 
conferrer a similar reward; and to this body were communicated the first investigations of Dr. Andrews, upon the heat developed in chemical combination, which have recently been honoured with the Royal Medal.

To remind you of the progress which Natural History has made, and is yet likely to make in this country, I have only to mention the names of Ball, of Thompson, of Mackay, and Harvey, and Allman, whose contributions to the history of the Fauna and the Flora of Ireland are too well known to need any comment here. The researches of Dr. Harvey, indeed, have embraced a wider range; and his latest work, the Phycologia Brittanica, now in course of publication, cannot fail to sustain his high character as a descriptive botanist. As a member of the University, I rejoice to be able to add that, of the distinguished Naturalists just mentioned, four are now connected with her teaching; and that a large portion of the plan contemplated by her late head, with reference to the advancement of those branches of science within her walls, has now been realized.

The contributions to the department of Polite Literature, which in the early volumes of our Transactions occupied a large and conspicuous place, have, I regret to say, been of late years less numerous. To whatever cause this may be ascribed, we are the more indebted to such men as Dr. Wall, Dr. Hincks, and Dr. Kennedy Bailie, who have enriched our volumes with the results of their learning and their research. But it would not be difficult to name others, fellow-countrymen and fellow-members, who are qualified to share with them the honour and the toil. The latest communication that we have received in this department-the paper by Dr. Hincks upon Egyptian Hieroglyphics, the first part of which was recently read-promises to throw much light upon the deciphering of these ancient and mysterious records, and, if the author be right in his theory, to add considerably to the discoveries of Young and Champollion.

The study of Antiquities, on the other hand,-and especially of the Antiquities of Ireland,- - has never been, and, I hope, never will be, out of fashion here. From the time of Molyneux, and of the Dublin Philosophical Society, the earliest of the learned Societies in Ireland from which we can trace our descent, the pursuit of Irish Antiquities has been a favourite one. Of the researches of our living antiquaries, the most conspicuous, undoubtedly, is the 
important work of Mr. Petrie, on the Ecclesiastical Architecture of Ireland, which has been referred to in the recent Report of your Council, and which forms, as you know, the last volume of your Transactions. Of the value of that work we should judge inadequately, were we to confine our view to the light which it has thrown upon the subject discussed; it is, perhaps, still more valuable as an example of the mode of dealing with Antiquarian questions, and of the evidences which may be brought to bear in their investigation.

The study of Irish Antiquities will, there can be no doubt, receive both aid and impulse from the institution of your Museum, a collection well worthy of this Academy, and of this country. It may be rash in one wholly unacquainted with the subject, as I am, to offer any suggestion respecting it; yet I cannot but think that much more may be done, in advancing our knowledge of Antiquities generally, and especially of that higher department of it which borders so closely upon History,-the distribution of the early races of mankind,- by the comparison of our own Monuments, and other relics of early civilisation, with those of other countries. The information we gather from a cairn, a torque, or a spear-head, will then no longer be limited to the light which they may throw upon the arts and manners of our Celtic ancestors. We may obtain from them a knowledge of the geographical distribution of their various tribes, much in the same manner as the geologist recognises the fragments of one of the great formations which compose the earth's crust by the comparison of their imbedded fossils; we may approach the history of their families, and trace them up to the parent stock. Studied with this reference, Antiquities may, perhaps in an important degree, tend to advance the science of Ethnology; and be combined with the study of Language, and of Physiological characters, as a new instrument in its research.

Gentlemen, - I fear I have already trespassed too long upon your time. But I desire, before I conclude, to offer a few remarks upon the future advancement of the objects of the Academy, and upon some of the modes by which it may be accelerated.

The first and chief of these, beyond all question, is rapid publication. It is not to be expected that men, who find a reward for their toils in the sympathy with which they are hailed by those engaged elsewhere in the same pursuits-it is not to be 
expected that they will communicate to us the fruits of those toils, if they should be long withheld from public view. Already there are indications that researches, which should naturally find their place in our Transactions, are about to reach the public through other channels. I trust that this evil may be stayed. The injury that it inflicts is not merely the loss of so much that should add to our credit and our character as a public body, but this very loss itself reacts upon, and augments the evil from which it has sprung. Nor is it necessary for me to urge, that publication is the first,- the main and essential duty of such a body as ours. No matter what may be the interest of our meetings, - no matter how far the study of Science, Literature, or Antiquities, may be aided by our library and our museum,-it is by our published works that we shall be judged, and by which we must stand or fall. I have only to add, that your Council are duly impressed with this feeling; and that your Officers are at present engaged in the consideration of some measures which promise to give not only a speedy, but also an increased publicity to our Proceedings.

Another instrument of progress, to whose efficacy I will advert, and which this Academy may, I think, effectively wield, is the directing power which it may reasonably assume, in pointing out to its members problems of local interest remaining to be solved, and encouraging them to the task by the proposal of Honorary Rewards. The practice of proposing subjects for investigation, and of honouring them by prizes, has existed, you must be aware, from the very origin of the Academy; and it has tended to elicit researches of considerable interest and value. Some years since, indeed, it was generally felt that the system had failed; and that opinion (in which at the time I shared) led to an alteration in the system of Honorary Rewards, with which you are of course acquainted. It may be doubted, however, whether this failure was the necessary result of the system itself, and not rather of the nature of some of the topies selected and proposed. It must be manifest, I think, that no encouragement which such a Society as this can bestow will be likely to stimulate a man of genius to the investigation of an abstruse question, to which he feels no predisposing movement,- that no reward can usurp the place of inspiration itself. But there are problems of a different stamp, whose solutions may be expected as the certain result of well- 
directed labour; to such problems as these, especially when their local character invests them with additional interest, and in some degree prepares men's minds for the research, - to such problems the recommendation of a learned Society may, with full assurance of the result, direct the attention of its Members. We know how much our knowledge of the Antiquities of this country has benefited by the proposal of such questions. Allow me to suggest one or two of a similar character connected with Physical Soience, as examples of what may be done in other departments.

In an interesting paper recently printed in the Philosophical Magazine, Colonel Sabine has suggested that the almost unparalleled mildness of the late winter may possibly be explained by an unusual extension of the Gulf-stream, bathing the shores of these Islands, and carrying with it a portion of the high temperature of the tropical region from which it flows. And the probability of this explanation has been augmented by the fact, that in the winter of 1821-2 (a winter in many respects resembling the last), this great oceanio current, whose force is usually spent when it reaches the Azores, was actually observed in the neighbourhood of our shores. I have long speculated upon the probable influence of the Gulf-stream upon the Irish winters generally, which appear to be much milder, in comparison with those of England, than can be well accounted for upon the principles of insular olimate alone; and I was glad to see, from Colonel Sabine's paper, that my conjectures had some real foundation. Whether or not they will account for the fact may, I think, be easily tested by a series of observations of the temperature of the sea on the eastern and western coasts of the Island, and under the same parallel; and I cannot but think that such a result, throwing so great a light upon the Climatology of this eountry, would, if established, well reward the labour bestowed in the investigation.

The Climate of Ireland, indeed, engaged a large share of the attention of the Academy during the lifetime of Kirwan; and several papers on the subject, by himself and others, are to be found in the early volumes of our Transactions. Should the Royal Irish Academy, as I think it ought, take that subject again under its peculiar care, the knowledge of it might be extended and improved, by the observation of the times of the leafing and flowering of certain plants, after the plan suggested and carried out by M. Quetelet of Brussels, and now extensively followed in many 
parts of Europe. Such observations furnish us with a simple but admirable measure of the total effects of all the influential causes in their combination and union.

Another subject of special inquiry, which might be fitly urged by this Society, is the history of the Tides on the coasts of Ireland. On this subject much has been already done; but probably much yet remains to be accomplished. Of the observations made in the summer of 1842 , by the non-commissioned officers of the Ordnance Survey under the direction of Colonel Colby, Mr. Airy (by whom they have been ably discussed in a paper recently printed in the Philosophical Transactions) observes, that "extent of time alone appears wanting to render them the most important series of tideobservations that has ever been made." Among the results to which Mr. Airy has arrived is the remarkable one, that in the harbour of Courtown, on the coast of Wexford-" "the only place on the earth in which such a result has been distinctly obtained," the Solar Tide exceeds the Lunar. Such a result as this affords not only encouragement to fresh exertion, but also direction as to its application.

Another, and most interesting subject of research, which this Academy might direct, if not itself undertake, is that to which attention has been recently drawn by Mr. Mallet,-the movements of the earth's crust, ,whether convulsive and paroxysmal, or gentle and regular. The phenomena of earthquake shocks in Scotland have been systematically observed for the last five years, at the instance of the British Association, and yearly reports of the results have been made, and published in its Proceedings. Although there appears to be nothing in this country analogous to the local movements at Comrie, in Perthshire, still there is no doubt that earthquake shocks have been felt here; and that more refined methods of observation would detect numberless others, which wholly escape the cognizance of the unaided senses.

These and many other investigations, connected with the physical, the physiological, and the monumental history of Ireland, appear to be fitting subjects, if not for the direct labours of this Academy, at least for its encouragement. Science has a right to demand such histories of local phenomena from the representatives of Science in each portion of the civilized globe, and shall this Academy be deaf to the call? 
Gextlemen, - I have, at the outset of these remarks, noticed the moral, as well as the intellectual benefits which result from the union of different mental powers, such as this Academy presents, combined in the investigation of different portions of Truth. But there is a yet higher principle, to which this union may lead us-a yet holier temper which it may inculcate; I mean the contemplation of Truth itself as essentially oxe, under its many and diversified forms, and the habit of tracing all its varied and refracted rays to its One and Eternal Source. Strengthened by this high thought,-our feelings raised and spiritualized by this habit,there is no danger that we shall give place to the weak apprehension (which is but a subtle form of unbelief itself), that any portion of Truth can ever prove inconsistent with any other. And the same principle, while it saves us from slavish fear, will also guard us from presumption. Standing in the presence of confessed and established truth, we shall feel that we are treading upon holy ground; and we shall demean ourselves, not with the elation and pride of conquest, but with the devotion of worship and of love. 
XXII.-ADDRESS DELIVERED AT A MEETING OF THE ROYAL IRISH ACADEMY, HELD JUNE 26TH, 1848, ON THE OCCASION OF THE PRESENTATION OF THE MEDALS AWARDED BY THE COUNCIL.

Proceedings of the Royal Irish Academy, 1848.

Gentlemen, - We have this night reached the close of a Session of more than usual activity; and I might, therefore, naturally have desired,-before leaving this chair and adjourning the Academy to another winter, - to trespass for a short time upon your attention, and to lay before you a brief summary of the results of our toil.. On the present occasion, however, my duty is narrowed and defined; and the recent award of the Cunningham Medals by the Council renders it imperative on me to submit to the Academy the grounds of their decision. In doing this, it will be necessary for me to present a brief analysis of the results of those labours whose value your Council have thus honourably recognised; and in the execution of this task I must request the indulgence of the Academy, and still more that of the gentlemen of whose discoreries I am to speak, if, in my imperfect acquaintance with them, I should fail to do justice to their merits.

You are aware that, during the past Session, the laws respecting the award of medals have occupied the attention of the Council ; and that certain new regulations relating to it were, upon their suggestion, adopted by the Academy. It is unnecessary for me to recapitulate these regulations, or to state the grounds for the changes therein made, as this has been fully done by the Council in their last Annual Report. It will be sufficient for me on the pre- 
sent occasion to remind you, that the principal alteration in the rules respecting the award of medals under the Cunningham bequest has been to extend the limit within which the Council are enabled to bestow such rewards, and to confine them only to memoirs or works printed and published in Ireland, or relating to Irish subjects.

A considerable interval having elapsed since the last award of these prizes, the Council for the present year, on coming into office, referred the matter to the three Committees of which that body is composed. Upon the recommendation of these Committees, in their several departments, the Council have adjudicated Medals to the following gentlemen :

1. To Sir William Rowan Hamilton, for his " Researches respecting Quarternions," published in the twenty-first volume of the Transactions of the Academy.

2. To the Rev. Samuel Haughton, Fellow of Trinity College, Dublin, for his Memoir "On the Equilibrium and Motion of Solid and Fiuid Bodies," published in the same volume.

3. To the Rev. Edward Hincks, D. D., for his various papers on Egyptian and Persepolitan Writing, also published in the same volume.

4. To John O'Donovan, Esq., for his contributions to the Transactions of the Irish Archæological Society, for his Irish Grammar, and for his edition of the Annals of the Four Masters.

In attempting to lay before the Academy a concise account of the origin of the new Calculus invented by Sir William Hamilton, and of the principles upon which it is based, I shall avail myself of the elucidations and applications of the theory which its gifted author has, from time to time, communicated to the Academy, and of which abstracts have appeared in our Proceedings as also of the series of papers published by him in the Philosophical Magazine upon the same subject. Of the latter, the author's letter to John T. Graves, Esq., written immediately after the discovery, possesses a high value, not only as a fragment of scientifio history, but still more, as laying bare in a new instance that most interesting and instructive of all the mental phenomena,-the actual train of thought which takes place in the creative mind, from the first dawn of truth within it to its full and noon-tide effulgence. 
It is now twenty years since the Rev. Mr. Warren of Cambridge* showed that the ordinary imaginary symbol $(\sqrt{ }-1)$ had a geometrical significancy, and denoted a right line whose length was equal to unity, to be measured, not on the axis of the real units, but on an axis at right angles to it. It followed from this, and from another principle respecting the symbolical meaning of the sign + , as applied to lines, that the ordinary binomial imaginary, whose real parts, or constituents, are multiplied by unity and $\sqrt{ }-1$, respectively, may be taken to represent both the length and direction of a right line in a given plane; the square root of the sum of the squares of the constituents being the length of the line, and their quote, or ratio, the tangent of the angle which it forms with the axis on which the first of them is measured. These quantities have been denominated the modulus and the amplitude of the imaginary binomial.

Now, if two such binomials, or couplets, be added togethər, the sum is a binomial of a similar form, or a couplet whose constituents are the sums of the constituents of the original couplet. And if two couplets be multiplied together, the product is likewise a couplet; and the relation of the product to the factors is such, that the modulus of the product is the product of the moduli of the factors, and the amplitude of the product is the sum of the amplitude of the factors. From these algebraical properties of couplets, combined with their geometrical significancy, it follows that right lines in a plane, having direction as well as magnitude, may be operated upon according to certain simple algebraical conditions, and the direction and amplitude of the resultant lines obtained by certain simple algebraical rules.

It was in the effort to generalize the theory of Couplets, and to extend their properties to right lines in space, that Sir William Hamilton was led to the construction of his theory of Quaternions. "Since," he says, " $\sqrt{ }-1$ is, in a certain well-known sense, a line

* Since the delivery of this Address the attention of the writer has been directed by Sir William Hamilton to the earlier steps of the inquiry. The first appears to have been made by M. Bueé, in a Paper published in the Philosophical Transactions for 1806, in which he lays down the principle that the symbol $\sqrt{-1}$, as applied to lines, denoted perpendicularity. A further step was made by M. Argand, in a memoir published at Paris in the same year, in which he shows that the sum of two lines, estimated in direction as well as magnitude, is the diagonal of the parallelogram constructed upon them. The subject was resumed and more fully developed by M. Francais, in a memoir published in the Annales des Mathematiques for 1813. 
perpendicular to the line 1 , it seemed natural that there should be some other imaginary to express a line perpendicular to both the former; and because the rotation from 1 to this also, being doubled, conducts to - 1 , it also ought to be a square root of negative unity, though not to be confounded with the former."

Starting thus with the conception of triplets involving two distinct square roots of negative unity, and endeavouring to frame laws for their algebraical treatment, analogous to those which hold in the case of couplets, he was soon led to perceive that the existence of the two imaginaries, just alluded to, necessarily involved the existence of a third, which was also a square root of negative unity distinct from either of the former. He was thus led to the conception of quaternions, or quadrinomials whose real parts, or constituents, are multiplied, the first by unity, and the other three by the imaginary roots of negative unity just referred to ; and he determined the conditions which must subsist amongst these new imaginary coefficients, in order that the resulting quadrinomials should be subject to the same algebraical laws as the ordinary imaginary binomials, or couplets.

I. may here observe, in passing, that one of these laws,-namely, the law of the moduli,-is equivalent to a celebrated theorem of Euler, viz. : that the sum of four squares, multiplied by the sum of four squares, is also a sum of four squares. An extension of this theorem to sums of eight squares has been effected, independently, by Mr. John Graves and Professor Young; and the latter writer (whose paper on the subject is published in the last part of the Transactions of the Academy) has proved that the property cannot be extended to higher numbers.

To return to the Quaternion-we have seen that it is made up of a real part, and an imaginary trinomial, using the terms real and imaginary in their ordinary acceptation. The latter of these represents a right line in space, drawn from the origin to the point whose co-ordinates are the three constituents of the trinomial; and it is accordingly designated by Sir William Hamilton by the term vector. The real part of the quaternion, on the other hand, designates number alone, whether positive or negative, without direction in space; and, accordingly, although real in the algebraical sense of the term, it is in some sense imaginary, when contemplated on the geometrical side. This part of the quaternion is denominated by Sir William the scalar. 
Thus we see that a quaternion is reducible to a binomial, the component parts of which-the scalar and the vector-designate, the one a number, the other a line. The whole tendency of the later speculations of the author has been to realize this reduction, and having determined the laws of operation upon scalars and rectors, to dismiss altogether the consideration of the constituents of the vector, and to treat it as a single integral quantity. It is easy to see what amount of simplicity is thus, at one step, introduced into the whole of Geometry and Mechanies. In place of the three co-ordinates (rectilinear or polar) by which the magnitude and direction of a line, or of a force, are ordinarily determined, the theory of Sir William Hamilton deals with the line itself, or with the force, directly; and thus not only is the number of necessary equations reduced at once, in the proportion of three to one, but also the interpretation of the equations themselves is rendered simpler and more direct.

The scalar, or algebraically-real part of the quaternion, thus appearing to have no direct geometrical significancy, geometers seemed inclined to regard it as a sort of intruder in their domain; and I believe it was to the desire to exclude it, that we may, in part, attribute the very elegant and ingenious theories of triplets invented by Professor De Morgan and Professor Graves. The scalar, however, is represented in mechanics by the time; and even in its application to pure geometry, Sir William Hamilton has shown that the introduction of this fourth quantity confers power and generality upon the Calculus of Quaternions, inasmuch as no direction in space is thus selected as eminent above another, but all are regarded as equally related to the extra-spatial, or scalar direction. The calculus thus frequently admits of a simpler and more direct application to geometrical problems than the Cartesian method of co-ordinates, inasmuch as it demands no previous selection of arbitrary axes.

I may observe, also, that in the triplet theories of Professor De Morgan and Professor Graves, the law of the moduli is not preserved, if the term modulus be taken in its ordinary signification,it being not generally true that the sum of three squares, multiplied by the sum of three squares, is a sum of three squares.

But whatever be thought of the principles of the Calculus of Quaternions, its advantages as an instrument of mathematical thought will undoubtedly be judged by the simplicity and ease 
with which it may be applied. In this the author has already done enough to establish its power. He has applied it with great success to many problems of the geometry of Surfaces; and he has given a sketch of its application to the problem of the Three Bodies, and to the Mechanics of the Heavens generally. These instances of its application,-whether we look to the elegance and simplicity of the method, or to the beauty and symmetry of the results,- -are abundantly sufficient to demonstrate the power and pliancy of the instrument.

Still, however, more will be required from its author, before the weapon which he wields with a giant's grasp may be touched by feebler hands. It will be necessary that the principles and fundamental rules of the calculus should be rendered familiar by elementary exposition, and their certainty tested by ordinary applications, before the violation of known analogies which some of them present will be universally acquiesced in; and I am happy to be able to say that the large debt, which Science already owes at his hands, is likely to receive ere long this addition, and that, like a genuine lover of Truth, he will not rest content until the difficult path which he has cut for himself into her tangled and obscure recesses shall become a highway for all.

I now proceed to the consideration of Mr. Haughton's memoir "On the Equilibrium and Motion of Solid and Fluid Bodies."

The object of this memoir, as stated by the author himself, is " to deduce, by the method of the Mecanique Analytique of Lagrange, the laws of equilibrium and motion of elastic solid and fluid bodies from the same physical principles, and to discover by the same method the conditions at the limits." The method of Lagrange (which is so peculiarly adapted to the mechanies of a system composed of an indefinite number of acting molecules, situated indefinitely near each other) seems to have been first applied to the problem of elastie bodies by M. Navier, who determined by that method the laws of equilibrium of a homogeneous uncrystallized solid. The late Mr. Green, of Cambridge, applied the same method to the more difficult dynamical question of the movement of the molecules of the luminiferous ether; in which application he was followed by the distinguished mathematician whose name is imperishably connected with the records of this Academy.

Mr. Haughton has judiciously adopted the same mathematical 
method; and he has determined the form of the function which enters the general equation of Lagrange (and which depends upon the internal forces acting at any point of the medium), from the assumed principle, that the molecules of solid and fluid bodies act on each other only in the direction of the line joining them, and with forces which depend on the magnitude and direction of that line. This function is easily shown to consist of two parts, one of them depending on the first power of the displacement, and the other upon its square; the former of these is assumed to relate to perfect fluids, and the latter to solids, while both must be taken into account in imperfect or viscous fluids. The form of this function, in the case of solids, bears some analogy to (although it is quite different from) that of the function employed by Professor M'Cullagh in his. dynamical theory of light; and the author deduces, from that difference, the important physical consequence that the molecules of the luminiferous ether do not, according to that theory, act on one another in the direction of the line joining them.

The differential equations of motion cannot be integrated generally; but the values of the three component displacements which correspond to the case of plane waves are manifestly particular integrals; and the equations of condition, which result from the substitution of these values in the general equations of motion, lead to a remarkable geometrical construction for the three possible directions of molecular vibration, and the corresponding velocities of the plane waves, by means of six fixed ellipsoids.

The author then determines the equation of the surface of waveslowness (or the reciprocal polar of the wave-surface), the nature and properties of which are analogous to those of the surface of the same name in the theory of light. This surface is of the sixth degree, and has three sheets, corresponding to the three velocities of wave propagation; and, like the corresponding surface in the theory of light, it serves to determine the direction of the refracted waves, in passing from one medium to another, as well as the laws of propagation in the same medium. In the most general case considered by the author,-namely, when the molecules of the medium are arranged symmetrically round three rectangular planes,-it is shown that this surface has four nodes, at which the tangent plane is a cone of the second degree; and thence arises a conical refraction in Sound, similar to that discovered theoretically by Sir William Hamilton in the case of Light. 
That such analogies, and points of correspondence, should exist between the theory of light, and any general theory of vibration in crystalline solids, was, of course, to be expected from the common foundation and the common postulates of the two theories. Notwithstanding this, however, the two theories diverge at a very early point. In both, indeed, the form of the characteristic function is deduced from the assumed molecular constitution of the medium. But that constitution is essentially different in the two cases, - the fundamental molecular property of the luminiferous ether, in the theory of Professor M'Cullagh, being the unchangeableness of its density, while the corresponding basis of the theory of $\mathrm{Mr}$. Haughton is the property that the molecules of the medium act on one another in the direction of the joining line.*

In conclusion, I may observe that the value of Mrr. Haughton's theory-considered on its physical side, and independently of its mathematical elegance-consists in its high degree of generality; which is such as necessarily to embrace all the fundamental conditions of the problem, and thus to leave to future mathematicians the task only of limiting and interpreting his results.

In speaking of Dr. Hincks' philological researches I must pass over those which relate to Egyptian Hieroglyphics, and hasten to his more recent, and (at the present time) more interesting labours connected with Persepolitan writing. And in order to present an intelligible statement of the nature of these labours, and of the additions which have been thereby made to the existing amount of knowledge upon this curious subject, it will be necessary to take a hurried glance at the history of the investigation, and its principal steps.

The cuneiform writing has been generally reduced to three leading divisions, which have been denominated, respectively, Persian, Median, and Babylonian. Many of the cuneiform inscriptions eontain all the three kinds of writing, the first being the principal, and apparently the vernacular record, and the other two

* The theory of Mr. Haughton bears a much closer resemblance, in many of its results, to the wave-theory of $\mathbf{M i}$. Cauchy than to that of Professor M 'Cullagh, although it differs from it wholly in method. The theory of M. Cauchy is, in fact, a theory of the laws of propagated vibration in solids, and is inapplicable (as was shown by Professor M'Cullagh) to light. 
translations. They are found on rocks, slabs, and pillars, at Persepolis, at Behistun, at Ván, at Murgháb, and at Hamadán. These trilingual inscriptions are all, without exception, records of the Achæmenian dynasty; the earliest which has been discovered (the inscription at Murgháb, or Pasargadæ) relating to Cyrus the Great, and the latest to Artaxerxes Ochus.

Of the three kinds of writing found in these inscriptions, the first, or Persian, is the simplest, containing the fewest and least complicated characters. It is also distinguished from the other two by the divisions between the words, which are separated by an oblique wedge; and this circumstance, of course, greatly facilitates the task of the decipherer. The second Persepolitan writing appears to have been co-eval with the first, and to have been employed only in conjunction with it, in the trilingual monuments of the Achæmenian princes; it is accordingly ascribed by the concurrent voice of philologers to the Medes, the people next in importance to the native Persians under the Achrmenian dynasty. The number of characters in this writing is far greater than in the Persian, its alphabet (or syllabary) containing about 100 letters. The third Persepolitan writing belongs to one of a group of languages (distinguished by Major Rawlinson into the Babylonian, the Assyrian, and the Elymoan) written in a similar character. It is ascribed, with every probability, to the Babylonians, legends in a like character being found on cylinders and bricks excavated from the foundations of the primæval cities of Shinar. It is unquestionably the most ancient of the three kinds of cuneiform writing, and was probably the type upon which the other two were constructed. The characters are more numerous and more complicated than those of the first and second kinds.

The process of resolving and interpreting an inscription in an unknown and extinct language, and written in an unknown character, appears to include three distinct and principal steps. The first of these is that of deciphering (properly so called), or determining the phonetic powers of the letters. The next step is the determination of the nature of the inflections, and the grammatical structure of the language itself, and the discovery of its congeners or representatives amongst the living languages. The third and last step consists in tracing from these sources the meaning of its roots, and thus translating the inscription.

The first of these steps was long since taken, with respect to the 
first Persepolitan writing. In the year 1802, Professor Grotefend, of Göttingen, examined two short trilingual inscriptions, which had been copied at Persepolis by the traveller Niebuhr, and succeeded in identifying the names of Cyrus, Darius, Xerxes, and Hystaspes, in all the three characters. The analysis of these names, in the case of the Persian, enabled him to determine the values of eleven out of the sixteen letters of which they were composed, or nearly one-third of the entire alphabet.

The next step was made by Professor Rask, of Copenhagen, in 1826. He recognised the title Achemenide in the inscription of Niebuhr, and thus determined the values of two important letters, $m$ and $n$, which occur in it. But the most valuable contribution made by Rask to this branch of palæography, consisted in his discovery of the resemblance of the extinct language to the Sanscrit in some of its inflections, a discovery which has been justly regarded as the key to its interpretation. Ten years later the inquiry received a fresh impulse by the simultaneous publication of two works, one by M. Burnouf, of Paris, and the other by the distinguished orientalist, Professor Lassen, of Bonn. By the analysis of a trilingual inscription, containing the names of the provinces of the Persian empire, the values of many new characters were ascertained, and the known alphabet was enlarged to twenty-six letters. In the year 1838 the values of five new characters were added to the list,-two by Dr. Beer, of Leipsic, and three by M. Jacquet, of Paris ; and the same writers discovered, independently, the fundamental principle which, strange to say, had hitherto escaped notice, that the Persian alphabet contained but three vowels, $a, i$, and $u_{0}^{*}$

But the most important of the researehes connected with the first Persepolitan writing are those of Major Rawlinson. Hitherto little had been accomplished beyond the first step of the process, - the determination of the values of the letters. Rask, indeed, had observed the similarity of the language to the Sanscrit, and this was confirmed by Lassen and Beer, the former of whom proposed to employ the Sanscrit as a key to its interpretation; but, as yet, little had been correctly done on this head. In 1835,

* This striking similarity of the Persian to the languages of the Shemitic type, in its rocalic structure, has been recently drawn still closer by Dr. Wall, in his ablo paper on the different kinds of cuneiform writing, published in the last volume of the Transactions of the Academy. 
Major Rawlinson eommenced his labours, in the country of the inscriptions; re-discovered for himself the greater part of what had been already done by European scholars; and determined the values of, at least, four new characters. But his chief workin which he has, by one great stride, surpassed all his predecessors -is the translation of the Persian portion of the great trilingual inscription at Behistun, containing above 400 lines of euneiform writing. This inscription had been eopied, in part, by Major Rawlinson in 1837; and a large portion of the translation was made by him, and communicated to the Royal Asiatic Society, in 1839. His philological labours were suddenly interrupted in the following year, by active duty at Affghanistan; but in the autumn of 1845 he succeeded in making a correct copy of the whole of the Persian inseription (together with a considerable portion of the Median and Babylonian), and soon after completed the translation in the form in which it has been recently published. With the contents of this singular record, written more than twenty-three centuries since, and throwing an unexpected light upon one of the most controverted questions of early history, the literary public are now well acquainted.

Dr. Hincks' first paper on Persepolitan writing was communicated to the Academy in June, 1846 , before the publication of the first part of Major Rawlinson's memoir. In this paper he proposes three general principles respecting the orthography of the Persian, in which he corrects Lassen's account of that language. The most important of these consists in the distinction of the consonants into two classes, which he calls primary and secondary, - the former being those which may be used before the vowel $a$, expressed or supplied, the latter such as are only used before one of the other vowels. Dr. Hincks maintains, in opposition to Lassen, that these secondary consonants are phonetically equivalent to their primaries; and he lays it down, "as an invariable rule, that if a primary consonant precedes $i$ or $u$, when a secondary consonant existed of the same value as the primary one, and appropriate to that vowel, an $a$ must be interposed, either as a distinct syllable, or as a guna to the vowel." The Persian alphabet may now be considered to be completely established. Of the thirty-nine letters which compose it, Major Rawlinson and Dr. Hincks are now agreed as to the values of all but one; Dr. Hincks having adopted three of Major Rawlinson's values, and 
Major Rawlinson having taken, independently, nine of those assigned by Dr. Hincks.

The data for the investigation of the Median or second Persepolitan writing are abundant, the trilingual inscriptions of Persepolis and Behistun furnishing more than ninety proper names, together with their Persian equivalents. Notwithstanding this, the progress made in the investigation has been comparatively small. In fact, with the exception of Grotefend, who made the first step, Westergaard is the only writer who has attempted the task of deciphering it with success. Major Rawlinson indeed informs us, in his memoir on the Persian character, that he has made considerable progress in deciphering the two other kinds of Persepolitan writing; and he has given a sketch of his views on the orthography, and the general structure and affinities of the language of the second kind: but none of his results, as to the values of the characters, have been as yet published.

Westergaard held that the Median alphabet had six vowels and sixteen consonants; and that the characters represented first these twenty-two letters, and then syllables composed of the consonants followed by vowels. Dr. Hincks maintains, on the contrary, that there are but four vowels and five consonants; and that, besides the characters representing these nine simple sounds, there are also characters representing combinations of the five consonants with preceding and following vowels, as also combinations of the vowels with each other. Again,-while according to Westergaard the vowels are not all expressed,-according to Dr. Hincks every vowel is expressed at least once, and often more than once; it being customary to write vowels twice over, at the end of one character and at the begining of the next. In accordance with this principle, Dr. Hincks adds vowels, in many cases, to Westergaard's values, thus making the characters to represent syllables instead of letters. Notwithstanding these important differences, however, he confirms, in general, the values given by Westergaard, although he differs from him altogether as to five of the characters, and assigns values to five more, which that writer had not valued at all.

But it is upon his labours connected with the third Persepolitan writing that Dr. Hincks' chief claim as an original discoverer must be founded. Grotefend discovered that the characters, in this writing, were partly expressive of syllables, and 
partly of letters: to a few of them, also, he assigned phonetic values; and he ascertained the fact of the correspondence of certain lapidary with certain cursive characters. To this little has been added by the many archæologists who have written upon the subject, beyond the mere classification of the characters. At an early period of his inquiries, Dr. Hincks arrived at the conclusion that the Babylonian and Assyrian writing agreed with the second Persepolitan in many of the features of the latter already noticed. The chief of the materials upon which he has since laboured are the Achæmenian inscriptions published by Westergaard, and the great inscription of the East India Company, containing 619 lines of lapidary characters. His first step in the deciphering of these documents was, of course, to analyze the proper names which occur in the third columns of the trilingual inscriptions, and to compare them with their equivalents in the other two. The values of many characters were thus determined ; those of others were ascertained by comparing different modes of writing the same words in the inscriptions which commence with the same formula; and, finally, when the equivalence of two sets of characters, lapidary and cursive, was ascertained, more values were determined by comparing the proper names in the great inscription with their representatives in the other languages. By such. means Dr. Hincks has constructed an alphabet, or syllabary, of the third Persepolitan writing, containing the values of ninety-five characters, together with the corresponding lapidary characters; and he has given a series of numbers from the rock inscription at Van, exhibiting the mode of expressing numerals in cuneatic characters.

Before I take leave of this subject, one more remark is necessary. It has been assumed by every writer who has hitherto engaged in the investigation of the cuneiform inscriptions, that the writing of the second and third kinds (as well as that of the first) is alphabetical. This fundamental position, however, has been recently assailed by Dr. Wall, in a very able critical paper read before the Academy; and arguments of much weight have been adduced to distinguish the principle of these two kinds of cuneiform writing from that of the first, and to prove them to be ideagraphic. It is not my duty (even if I were competent to the task) to offer any opinion upon the question thus raised. I have only to observe that what has been said above, respecting the progress 
recently made in deciphering these two kinds of writing, is upon the ordinary assumption, and must be received with the based reserve which necessarily attaches to a controverted position.

With Mr. O'Donovan's archæological labours I regret to say that I possess no direct acquaintance; and, accordingly, in the present notice of them, I am compelled to lean upon the friendly aid of the Secretary of the Academy, who is himself a large contributor to the same department of literature.

Mr. O'Donovan's vast acquirements connected with Irish archæology may' be traced, 'in a great measure, to his connexion with the Ordnance Survey. In the course of the duties which this connexion imposed upon him, he visited every part of Ireland for the purpose of tracing the ancient names of places, and of collecting the local traditions connected with them, all of which he compared with the existing records in the historical manuscripts preserved in the Libraries of the Academy and of the University. The object of these inquiries was to collect materials for the Historical and Antiquarian memoirs, which it was the original intention of the enlightened officers at the head of the Irish Survey to compile and publish,- - an intention which (as the Academy are aware) was unhappily frustrated by the interference of Government. In the researches in which Mr. O'Donovan was thus for many years engaged, he acquired the vast amount of historical and topographical knowledge which his subsequent writings have displayed. He availed himself of the same opportunities to perfect his acquaintance with the dialects of the Irish language; and he has thus been enabled to throw a light on this department of philology, such as probably no other could have done.

The works edited by Mr. O'Donovan for the Irish Archæological Society are the first of his published labours which claim our attention. They are the following:-

1. "The Circuit of Ireland, by Muircheartach Mao Neill, Prince of Aileach. A poem written in the year 942, by Cormacan Eigeas, chief poet of the North of Ireland."

2. "The Battle of Magh Rath (Moira), from an ancient manuseript in the Library of Trinity College, Dublin."

3. "An Account of the Tribes and Customs of the district of Hy-Many, commonly called O'Kelly's Country, in the counties of 
Galway and Roseommon. Edited from the Book of Lecan, in the Library of the Royal Irish Academy."

4. "An Account of the Tribes and Customs of the district of Hy-Fiachrach, in the counties of Sligo and Mayo. Edited from the Book of Lecan, in the Library of the Royal Irish Academy, and from a copy of the Mac Firbis manuscript in the possession of the Earl of Roden."

Mr. O'Donovan has also edited the following minor pieces in the Miscellany of the Irish Archæological Society, viz. : "An aneient poem attributed to St. Columbkille;" "The Irish Charters in the Book of Kells;" "A covenant in Irish between Mageoghegan and the Fox;" and "The Annals of Ireland from A. D. 1453 to 1468. Translated from a lost Irish original, by Dudley Firbisse."

These historical tracts and bardic tales are edited, for the most part, in the original Irish, with translations and notes. In the latter Mr. O'Donovan has brought together a vast body of historical and genealogical information connected with the ancient families referred to; and ho has illustrated the subjects with much curious antiquarian lore, respecting the manners and customs of the times. He has also, in many cases, annexed maps of the districts described, and topographical indexes, in which the etymology of the ancient names is given, together with the corresponding modern appellations.

Among the works of Mr. O'Donovan enumerated by the Council in awarding him the Cunningham Medal, 'is his Irish grammar. This work was undertaken for the use of the senior classes in the College of St. Columba, and was published at the expense of the trustees of that institution. 'The publication has supplied a want long felt by the philologers of Europe; and the Celtic student is now in possession of a grammar compiled by a scholar who has studied the ancient language as it exists in our manuscript literature, and whose judgment and learning have enabled him to discriminate between the original and characteristic grammatical forms, and the accidental peculiarities belonging to particular districts or periods. The vast body of examples which Mr. O'Donovan has collected from Irish MSS., in illustration of this work, contributes greatly to enhance its value.

But Mr. O'Donovan's principal work is his edition of the Annals of the Four Masters, from the autograph manuscript in the Library of the Royal Irish Academy. The publication of this curious and 
important chronicle had been long and earnestly desired by Irish scholars. The language in which it is written was fast becoming obsolete, and another half century would probably have interposed a serious difficulty in its interpretation; while the curious mass of information which Mr. O'Donovan has brought together in illustration of it, - collected, as it has been, in a great part, from oral traditions,- -would, in all likelihood, have been wholly lost. This work will ever remain a monument of the learning and labour of its author, and would suffice alone to place his name in a bigh rank in the list of Archæologists. The three large quarto volumes which have already appeared (contain the Annals from A.D. 1172 to 1616 ; Mr. O'Donovan is now engaged in preparing for publication the earlier portion, which will be accompanied by a complete index of the names of persons and places mentioned in the Annals.

Upon the conclusion of his Address, the President presented the Medals to Sir William Hamilton, Mr. Haughton, Dr. Hincks, and Mr. O'Donovan, addressing them separately, as follows :

Sir William Hamilton,--In awarding you this Medal, the Council cannot have the gratification of feeling that they are contributing to the reputation of a name which is already known wherever Science is cultivated. But they trust that you will value it as a mark of sympathy from the Society, whose scientific character you have raised by your labours, and whose interests you have done so much in other ways to promote. Suffer me, on my own behalf, to add, that the duty which I now discharge, as the organ of the Academy on the present occasion, is to myself, personally, the most grateful of any which have devolved upon me as your successor in this Chair.

Mr. Haughton,-Accept this Medal as a testimony of the high value which the Council of the Royal Irish Academy set upon your researches, connected with a most difficult branch of Applied Mathematics; and as an expression of their hope that the labours in the application of the higher branches of analysis to physical problems, for which you have proved yourself so eminently qualified, and which have been already crowned with such success, may long continue to add to your own honour, and to that of the Academy of which you are a member. 
Dr. Hrxcks,-Accept this Medal as a proof of the high opinion. with which the Council of the Royal Irish Academy regard your researches, connected with some of the most obscure and difficult problems of Archroology. Allow me to add, that the merit of those researches, high as it is in itself, is enhanced in your case by the circumstance, that they have been pursued in the seclusion of retirement, and without any of those aids derived from the intercourse with others engaged in similar pursuits, which are usually so effective in impelling to and suggesting inquiry.

Mr. O'Donovan,-Accept this Medal as a testimony of the high value which the Council of the Royal Irish Academy set upon your labours connected with Irish philology, and Irish history and antiquities. This is the first occasion on which the Council, acting on the laws recently enacted by the Academy, have conferred the honour of the Cunningham Medal for works not published in the Transactions of the Academy. They therefore hope that you (and through you the literary public) will receive this award, not only as a just tribute to the value of your own researches, but also as a token of their sympathy with all who are engaged in the common pursuit of Truth. 
XXIII-ADDRESS DELIVERED AT THE OPENING MEETING OF THE BRITISH ASSOCIATION FOR THE ADVANCEMENT OF SCIENCE, HELD IN DUBLIN, AUGUST 26, 1857.

Report of the British Association for the Advancement of Science, 1857.

Gentlemen of the British Association,-Before I proceed to the task which devolves upon me this evening, in virtue of the position in which your kindness has placed me, suffer me first to thank you for the high honour you have conferred. But, highly as I esteem the distinction, it was not without hesitation that I accepted it; for no one can feel more strongly than I do myself how unfit I am for some of the duties connected with it, or how much more adequately they might have been performed by others. But I knew, at the same time, that it has been the desire of your Council, when practicable, to select your President from anong those local Members who had served in the ranks of the Association, and had shared in its labours: and with such knowledge, and the consciousness that I had, at least, that humble claim, I felt that I had no right to dispute your choice.

I do not know whether I may venture to interpret further your motives, and to assign another reason for your selection. Two-and-twenty years have elapsed since you visited this City. Upon that occasion my nearest relative presided, and I myself had the honour of serving as one of your local Secretaries. Many concurring circumstances contributed to make that Meeting an agreeable one; and if your Council has thought fit, on this oceasion, to associate the present with the memories of the past, the motive is, at least, a pardonable one. 
Gentlemen, this is to me a solemn occasion. Two-and-twenty years áre no inconsiderable portion even of the longest life; and that man's moral nature is not to be envied, who can contemplate the distant past thus vividly recalled without emotion. These two decades have brought with them their own large measure of change. The Body in' which we are associated has grown up from youth to maturity; and many of its honoured names are now sought for only in the imperishable records of their toils. The institutions which welcomed it here, upon its former visit to this City, have all received the impress of the changing times. And yet, amid all this change, we meet once more in the same city,-in the same room,- to enter again on the same labours; our assemblage is now, as it was before, dignified by the presence of the Representative of Majesty; and I see around me, associated for this task, many of those who shared it before; - the men whose sagacity first perceived the want of such a Society as this, whose energy supplied it, and whose wisdom directed its steps while it had need of guidance.

I trust I may be forgiven for dwelling thus far on the peculiar circumstances under which we are here assembled; and I now hasten to discharge the task which the usages of this Chair impose. upon me, and proceed to lay before you, as well as I am able, a brief sketch of the recent progress of some of those Sciences to whose advancement we are pledged by our Institution. In doing so, I gladly follow the practice which has of late become the rule,-namely, that your President for each year should bring under your notice, chiefly, the recent additions to those departments of Science with which he happens to be himself most familiar. It is plainly fitting that he who addresses you should speak, as far as he can, from his own acquired knowledge. Partial views are better than inexact ones; and provision is made for their completion in the annual change of your Officer. In the present instance I derive the full advantage of this arrangement, inasmuch as the subjects upon which I could not thus speak have been, most of them, ably treated by my predecessor in this Chair.

To commence, then, with Astronomy:-The career of planetary discovery, which began in the first years of the present century, and was resumed in 1845, has since continued with unabated ardour. Since 1846 not a single year has passed without some one 
or more additions to the number of the planetoids; and in one year alone (1852), no fewer than eight of these bodies were discovered. The last year has furnished its quota of five; and in the present, three more have been found, one by Mr.Pogson, of Oxford, and the other two by M. Goldschmidt, of Paris. Their known number is now forty-five. Their total mass, however, is very small; the diameter of the largest being less than forty miles, while that of the smallest, Atalanta, is little more than four.

These discoveries have been facilitated by star-maps and starcatalogues, the formation of which they have, on the other hand, stimulated. Two very extensive works of this kind are now in progress, - the Star-catalogue of M. Chacornac, made at the Observatory of Marseilles, in course of publication by the French Government; and that of Mr. Cooper, made at his Observatory at Markree, in Ireland, which is now being published by the help of the parliamentary grant of the Royal Society. It is a remarkable result of the latter labour, that no fewer than seventy-seven stars, previously catalogued, are now missing. This, no doubt, is to be ascribed, in part, to the errors of former observations; but it seems reasonable to suppose that, to some extent at least, it is the result of changes actually in progress in the Sidereal Systems.

The sudden appearance of a new fixed star in the heavens,-its subsequent change of lustre,-and its final disappearance, are phenomena which have at all times attracted the attention of astronomers. About twenty such have been observed. Arago has given the history of the most remarkable, and discussed the various hypotheses which have been proposed for their explanation. Of these, the most plausible is that which attributes the phenomenon to unequal brightness of the faces of the star, which are presented successively to the earth by the star's rotation round its axis. On this hypothesis the appearances should be periodic. M. Goldschmidt has recently given support to this explanation, by rendering it probable that the new star of 1609 is the same whose appearance was recorded in the years 393,798 , and 1203 ; its period, in such case, is $405 \frac{1}{3}$ years.

The greater part of the celestial phenomena are comprised in movements of the heavenly bodies, and the configurations depending on them; and they are for the most part reducible to the same law of gravity which governs the planetary motions. But there are appearances which indicate the operation of other forces, and 
which therefore demand the attention of the physicist,-although, from their nature, they must probably long remain subjects of speculation. Of these the spiriform nebulæ, discovered by Lord Rosse, have been already referred to from this Chair, as indicating changes in the more distant regions of the universe, to which there is nothing entirely analogous in our own system. These appearances are accounted for, by an able anonymous writer, by the action of gravitating forces combined with the effects of a resisting medium,- the resistance being supposed to bear a sensible proportion to the gravitating action.

The constitution of the central body of our own System presents a nearer and more interesting subject of speculation. Towards the close of the last century many hypotheses were advanced regarding the nature and constitution of the Sun, all of which agreed in considering it to be an opaque body, surrounded at some distance by a luminous envelope. But the only certain fact which has been added to Science in this department is the proof given by Arago, that the light of the Sun emanated-not from an incandescent solid-but from a gaseous atmosphere; the light of incandescent solid bodies being polarized by refraction, while the light of the Sun, and that emitted by gaseous bodies, is unpolarized.

According to the observations of Schwabe, which have been continued without intermission for more than thirty years, the magnitude of the solar surface obscured by spots increases and decreases periodically, the length of the period being 11 years and 40 days. This remarkable fact, and the relation which it appears to bear to certain phenomena of terrestrial magnetism, have attracted fresh interest to the study of the solar surface; and upon the suggestion of Sir John Herschel, a photo-heliographic apparatus has lately been established at Kew, for the purpose of depicting the actual macular state of the Sun's surface from time to time.

It is well known that Sir William Herschel accounted for the solar spots by currents of an elastic fluid, ascending from the body of the Sun, and penetrating the exterior luminous envelope. A somewhat different speculation of the same kind has been recently advanced by Mosotti, who has endeavoured to connect the phenomena of the solar spots with those of the red protuberances which appear to issue from the body of the Sun in a total eclipse, and which so much interested astronomers in the remarkable eclipse of 1842. 
Next to the Sun, our own satellite has always claimed the attention of astronomers, while the comparative smallness of its distance inspired the hope that some knowledge of its physical structure could be attained with the large instrumental means now available. Accordingly, at the Meeting of the Association held at Belfast in 1852, it was proposed that the Earl of Rosse, Dr. Robinson, and Professor Phillips, be requested to draw up a Report on the physical character of the Moon's surface, as compared with that of the earth. That the attention of these eminent observers has been directed to the subject, may be inferred from the communication since made by Professor Phillips to the Royal Society on the lunar mountain, Gassendi, and the surrounding region; but I am not aware that the subject is yet ripe for a Report.

I need not remind you, that the Moon possesses neither sea, nor atmosphere of appreciable extent. Still, as a negative, in such ease, is relative only to the capabilities of the instruments employed, the search for the indications of a lunar atmosphere has been renewed with every fresh augmentation of telescopic power. Of such indieations the most delicate, perhaps, are those afforded by the occultation of a planet by the Moon. The occultation of Jupiter, which took place on the 2 nd of January last, was observed with this reference, and it is said to have exhibited no hesitation, or change of form or brightness, such as would be produced by the refraction or absorption of an atmosphere. As respects the sea, the mode of examination long since suggested by Sir David Brewster is probably the most effective. If water existed on the Moon's surface, the Sun's light reflected from it should be completely polarized at a certain elongation of the Moon from the Sun. No traces of such light have been observed; but I am not aware that the observations have been repeated recently with any of the larger telescopes.

It is now well understood that the path of astronomical discovery is obstructed much more by the earth's atmosphere, than by the limitation of telescopic powers. Impressed with this oonviction, the Association has, for some time past, urged upon her Majesty's Government the scientific importance of establishing a large reflector at some elevated station in the Southern Hemisphere. In the meantime, and to gain (as it were) a sample of the results which might be expected from a more systematic search, Professor Piazzi Smyth undertook, last summer, the task of transporting a large collection of 
instruments-meteorological and magnetical, as well as astronomical-to a high point on the Peak of Teneriffe. His stations were two in number, at the altitudes above the sea of 8840 and 10,700 feet respectively; and the astronomical advantages gained may be inferred from the fact, that the heat radiated from the Moon, which has been so often sought for in vain in a lower region, was distinctly perceptible with the aid of the thermo-multiplier.

The researches relative to the Figure of the Earth, and the Tides, are intimately connected with Astronomy, and next claim our attention.

The results of the Ordnance Survey of Britain, so far as they relate to the earth's figure and mean density, have been lately laid. before the Royal Society by Colonel James, the Superintendent of the Survey. The ellipticity deduced is $\frac{1}{299} \cdot \overline{33}$. The mean specific gravity of the earth, as obtained from the attraction of Arthur's Seat, near Edinburgh, is 5.316,-a result which accords satisfactorily with the mean of the results obtained by the torsion balance. Of the accuracy of this important work it is sufficient to observe, that when the length of each of the measured bases-in Salisbury Plain, and on the shores of Lough Foyle-was computed from the other, through the whole series of intermediate triangles, the difference from the measured length was only 5 inches in a length of from 5 to 7 miles.

Our knowledge of the laws of the Tides has received an important accession, in the results of the Tidal Observations made around the Irish coasts in 1851, under the direction of the Royal Irish Academy. The discussion of these observations was undertaken by Professor Haughton, and that portion of it which relates to the diurnal tides has been already completed and published. The most important result of this discussion is the separation of the effects of the Sun and Moon in the diurnal tide-a problem which was proposed by the Academy, as one of the objects to be attained by the contemplated observations, and which has been now for the first time solved. From the comparison of these effects Professor Haughton has drawn some remarkable conclusions relative to the mean depth of the sea in the Atlantic. In the dynamical theory of the tides, the ratio of the solar to the lunar effect depends not only on the masses, distances, and periodic times, of the two luminaries, - but also on the depth of the sea; and this, accordingly, 
may be computed when the other quantities are known. In this manner Professor Haughton has deduced, from the solar and lunar coefficients of the diurnal tide, a mean depth of 5.12 miles-a result which accords in a remarkable manner with that inferred from the ratio of the semidiurnal coefficients, as obtained by Laplace from the Brest observations. The subject, however, is far from being exhausted. The depth of the sea, deduced from the solar and lunar tidal intervals, and from the age of the lunar diurnal tide, is somewhat more than double of the foregoing; and the consistency of the individual results is such as to indicate that their wide difference from the former is not attributable to errors of observation. Professor Haughton throws out the eonjecture that the depth, deduced from the tidal intervals and ages, corresponds to a different part of the ocean from that inferred from the heights.

The phenomena of Terrestrial Magnetism present many elose analogies with those of the tides; and their study has been, in a peculiar manner, connected with the labours of this Association. To this Body, and by the hands of its present General Secretary, were presented those Reports on the distribution of the Terrestrial Magnetic Force which re-awakened the attention of the scientific world to the subject. It was in the Committee-rooms of this Association that the first step was taken towards that great magnetic organization which has borne so much fruit;-it was here that the philosophical sagacity of Herschel guided its earlier career; - and it was here again that the cultivators of the science assembled, from every part of Europe, to deliberate about its future progress. It was natural, therefore, that the results obtained from such beginnings should form a prominent topio in the addresses which have been annually delivered from this Chair; and the same circumstances will plead my excuse, if I now revert to some of them which have been already touched upon by my predecessors.

It has been long known that the elements of the earth's magnetio force were subject to certain regular and recurring changes, whose periods were, respectively, a day and a year, and which, therefore, were referred to the Sun as their source. To these periodical changes Dr. Lamont, of Munich, added another of ten years, the diurnal range of the magnetio declination having 
been found to pass from a maximum to a minimum, and back again, in about that time.

But besides these slow and regular changes, there are others of a different class, which recur at irregular intervals, and which are characterized by a large deviation of the magnetic elements from their normal state, and generally also by rapid fluctuation and change. These phenomena, called by Humboldt "magnetic storms," have been observed to occur simultaneously in the most distant parts of the earth, and therefore indicate the operation of causes affecting the entire globe. But, casual as they seem, they are found to be subject to laws of their own. Professor Kreil was the first to discover that, at a given place, they recurred more frequently at certain hours of the day than at others; and that, consequently, in their mean effects, they were subject to periodical laws, depending upon the hour at each station.

The laws of this periodicity have been ably worked out by General Sabine, in his discussion of the results of the British Colonial Observatories; and he has added the important fact, that the same phenomena observe also the two other periods already noticed-namely, the annual and the decennial periods. $\mathrm{He}$ has further arrived at the very remarkable result, that the decennial magnetic period coincides, both in its duration and in its epochs of maxima and minima, with the period observed by Schwabe in the solar spots; from which it is to be inferred that the Sun exercises a magnetic influence upon the earth, dependent on the condition of its luminous envelope.

We are thus in the presence of two facts, which appear at first sight opposed-namely, the absolute simultaneity of magnetio disturbances at all parts of the earth, and their predominance at certain local hours at each place. General Sabine accounts for this apparent discrepancy by the circumstance, that the hours of maximum disturbance are different for the different elements; so that there may be an abnormal condition of the magnetic force, operating at the same instant over the whole globe, but manifesting itself at one place chiefly in one element, and at another place in another. I would venture to suggest, as a subject of inquiry, whether the phenomena which have been hitherto grouped together as "occasional" effects may not possibly include two distinct classes of changes, obeying separate laws-one of them being strictly periodic, and constituting a part of the regular diurnal 
change; while the other is strictly abnormal, and simultancous. If this be so, it would follow that we are not justified in separating the larger changes from the rest, merely on the ground of their magnitude; and that a different analysis of the phenomenon will be required.

The effects hitherto considered are all referrible to the Sun as their cause. Professor Kreil discovered, however, that another body of our System-namely, our own satellite-exerted an effect upon the magnetic needle; and that the magnetic declination underwent a small and very regular variation, whose amount was dependent on the lunar hour-angle, and whose period was therefore a lunar day. This singular result was subsequently confirmed by Mr. Broun, in his discussion of the Makerstoun observations; and its laws have since been fully traced, for all the magnetic elements, by General Sabine, in the results obtained at the Colonial Magnetic Observatories.

The foregoing facts bear closely upon the debated question of the causes of the magnetio variations. It has been usual to ascribe the periodical changes of the earth's magnetic force to the thermio action of the Sun, operating either directly upon the magnetism of the earth, or affecting it indirectly by the induction of thermoelectric currents. Here, however, we have a distinct case of magnetic action, unaccompanied by heat; and the question is naturally suggested, whether the solar diurnal change may not also be independent of temperature.

The most important fact, in its bearing upon this question, is the existence of an annual inequality in the diurnal variation, dependent on the Sun's declination, recently pointed out by General Sabine. If we deduct the ordinate of the curve, which represents the mean diurnal variation for the entire year, from those for the summer and winter half-yearly curves respectively, the differences are found to be equal and opposite; and the curves which represent them are, consequently, similar, but oppositcly placed with respect to the axis of abscissæ. From this, General Sabine draws the inference, that the diurnal variation is a direct effect of solar action, and not a result of its thermio agency.

The most important step which has been recently taken in this country, to advance the science of Meteorology, has been the formation of a department connected with the Board of Trade, for 
the collection and discussion of Meteorological Observations made at sea. The practical results of a similar undertaking in the United States are now well known. The charts and sailing directions, published by Lieutenant Maury, have enabled navigators to shorten their passages, in many cases by one-fourth of the time, and in some even to a greater extent. The commercial importance of such results could not fail to attract general attention; and accordingly, when the United States Government invited other maritime nations to co-operate in the undertaking, the invitation was cordially accepted. A conference was held at Brussels in 1853, at which meteorologists deputed by those Powers attended; and a Report was made, recommending the course to be pursued in a general system of marine meteorological observations. This Report was laid before the British Parliament soon after, and a sum of money was voted for the necessary expenditure. The British Association undertook to supply verified instruments, by means of its Observatory at Kew ; and the Royal Society, in consultation with the most eminent meteorologists of Europe and America, addressed an able Report to the Board of Trade, in which the objects to be attended to, so as to render the system of observation most available for Science, were clearly set forth. With this co-operation on the part of the two leading Scientifio Societies, the establishment was soon organized. It was placed under the direction of a distinguished naval officer, Admiral Fitz-Roy: and in the beginning of 1855 it was in operation. Agents were established at the principal ports for the supply of instruments, books, and instructions; and there are now more than 200 British ships so furnished, whose officers have undertaken to make and record the required observations, and to transmit them from time to time to the Department. At the present time 700 months of logs have been received, from nearly 100 merchant ships, and are in process of tabulation.

Holland is taking similar steps; and the Meteorological Institute of that country, under the direction of M. Buys Bellot, has already published three volumes of nautical information, obtained from Dutch vessels in the Atlantic and Indian Oceans.

For the purposes of Meteorological Science this system cannot be considered as complete, until observations on land are included. Most of the greater atmospheric changes are due to the distribution of land and water, and to the different effects of the Sun's rays 
on each. Observation alone ean furnish the data from which the effects of these agencies may be calculated; and we can therefore probably make no great advance in the knowledge of the meteorology of the globe, without a concurrent investigation of its two leading departments. Land observations exist in great numbers. In Prussia,-in Russia,-in Austria,-and in Belgium, such observations are organized under Government direction, or at least with Government support. In other parts of Europe, as in Britain, the labour is left to individuals or scientific societies. What is needed is to give unity to these isolated labours-to connect them with one another, and with the results obtained at sea; and the first step to this seems to be, to give them, in each country, that permanence and uniformity of system which can only be insured in measures adopted by the State.

Here, however, we encounter an objection, upon which it is necessary to say a few words.

It has been objected to the Science of Meteorology, as it is usually studied, that it proceeds upon a false method; and that, consequently, it has led-and can lead-to no results. I feel myself in a manner compelled to notice this grave objection, in the first place because it proceeds from men whose opinions on this (or almost any other scientific question) are entitled to the highest deference; and secondly, because this Association must bear no inconsiderable measure of the reproach, if it be well founded.

First, then, as to results. I am free to admit that the number of those engaged in the discussion of meteorological observations is disproportionately small, and that the results obtained probably fall far short of what may be expected from the data already accumulated. But that the methods have led, and can lead, to no results, is, I think, sufficiently disproved by the labours of a single manProfessor Dove of Berlin. And if it be true that the course pursued in the science has yielded much fruit, in proportion to the labour bestowed on the discussion, it will hardly be deemed widely erroneous. Still, as it is possible that the methods pursuedthough not fruitless-may be inadequate, it seems necessary to notice the objection somewhat more minutely.

It is asserted, hen, that the eapital vice of the Science of Meteorology, as at present pursued, is that it has no definite aim ; that it ought to embrace an inquiry into the physical constitution of 
the objects with which the science is concerned, and an investigation of causes as well as laws of phenomena.

It may be admitted, at once, in reference to this objection, that the physical constitution of the bodies whose changes we are investigating is a proper object of study to the physicist; but it does not seem to follow that it should necessarily be conducted by the same individuals who are in search for the laws of the phenomena, or even that the former knowledge is essential to the progress of the latter. The noblest of all the physical sciences, Astronomy, is little more than a science of laus-laws, too, of the simplest kind of change; and the knowledge of these laws is wholly independent of the physical constitution of the masses whose movements it studies. A similar observation may be made regarding the science of Terrestrial Magnetism; and the case is one which brings us still nearer to the question at issue, inasmuch as the laws which have been obtained-and they are numerous-have resulted from a method of inquiry altogether similar to that adopted in Meteorology.

Time will not permit me to inquire whether there is not a misconception of a metaphysical kind at the root of this objection. I may observe, however, before leaving the subject, that there are two modes of studying the sequences of natural phenomena, - one in their relation to time, and which is best accomplished by observations at stated periods, and the other in the relation of the successive phases of the phenomena to one another. Of these, the latter, although not wholly neglected, has not been so much followed as it deserves; and I cannot but think that it would, if more systematically followed, enrich the science of Meteorology with a new harvest of results.

The most important of the recent additions to the theory of Light have been those made by M. Jamin. It has been long known that metals differed from transparent bodies, in their action on light, in this, that plane-polarized light reflected from their surfaces became elliptically-polarized; and the phenomenon is explained, on the principles of the wave-theory, by the assumption that the vibration of the ether undergoes a change of phase at the instant of reflexion, the amount of which is dependent on its direction, and on the angle of incidence. This supposed distinetion, however, was soon found not to be absolute. Mr. Airy showed that diamond reflected light in a manner similar to metals; 
and Mr. Dale and Professor Powell extended the property to all bodies having a high refractive power. But it was not until lately that M. Jamin proved that there is no distinction, in this respect, between transparent and metallic bodies; and that all bodies transform plane-polarized into elliptically-polarized light, and impress a change of phase at the moment of reflexion. Professor Haughton has followed up the researches of M. Jamin, and established the existence of circularly-polarized light by reflexion from transparent surfaces.

The theoretical investigations connected with this subject afford a remarkable illustration of one of those impediments to the progress of natural philosophy, which Bacon has put in the foremost place among his examples of the Idola:-I mean the tendency of the human mind to suppose a greater simplicity and uniformity in nature than exists there. The phenomena of polarization compel us to admit that the sensible luminous vibrations are transversal, or in the plane of the wave itself ; and it was naturally supposed by Fresnel, and after him by M'Cullagh and Neumann, either that no normal vibrations were propagated, or that, if they were, they were unconnected with the phenomena of light. We now learn that it is by them that the phase is modified in the act of reflexion; and that, consequently, no dynamical theory which neglects them, or sets them aside, can be complete.

Attention has been lately recalled to a fundamental position of the wave-theory of light, respecting which opposite assumptions have been made. The vibrations of a polarized ray are all parallel to a fixed direction in the plane of the wave; but that direction may be either parallel, or perpendicular, to the plane of polarization. In the-original theory of Fresnel the latter was assumed to be the fact; and in this assumption Fresnel has been followed by Cauchy. In the modified theories of $\mathrm{M}^{\mathrm{r}} \mathrm{Cullagh}$ and Neumann, on the other hand, the vibrations are supposed to be parallel to the plane of polarization. This opposition of the two theories was compensated, as respects the results, by other differences in their hypothetical principles; and both of them have led to conclusions which observation has verified. There seemed, therefore, to be no means left to the theorist to decide between these conflicting hypotheses, until Professor Stokes, recently, in applying the dynamical theory of light to other elasses of phenomena, found one in whish the effects should differ on the two assumptions. When light is 
transmitted through a fine grating; it is turned aside, or diffracted, according to laws which the wave-theory has explained. Now Professor Stokes has shown that, when the incident light is polarized, the plane of vibration of the diffracted ray must differ from that of the incident, the two planes being connected by a very simple relation. It only remained, therefore, for observation to determine whether the planes of polarization of the incident and refracted rays were similarly related, or not. The experiment was undertaken by Professor Stokes himself, and he has inferred from it that the original hypothesis of Fresnel is the true one; but, as an opposite result has been obtained by M. Holtzmann, on repeating the experiment, the question must be regarded as still undetermined. The difference in the experimental results is ascribed by Professor Stokes to the difference in the nature of the gratings employed, the substance of the diffracting body being supposed to exert an effect upon the polarization of the light, which is diffracted by it under a great obliquity. I learn from Professor Stokes that he proposes to resume the experimental inquiry, and to test this supposition by employing gratings of various substances. If the conjecture should prove to be well founded, it will, unfortunately, greatly complicate the dynamical theory of light. In the meantime the hypothesis is one of importance in itself, and deserves to be verified or disproved by independent means. I would venture to suggest that it may be effectively tested by means of the beautiful interference-refractor of M. Jamin, which the inventor has already applied to the study of the effects upon light, produced by grazing a plate of any soluble substance inclosed in a fluid.

It is well known that the refractive index of bodies increases with their density; and the theory of emission has even expressed the law of their mutual dependence. That theory, it is true, is now completely overthrown by the decisive experimentum crucis of MM. Fizeau and Foucault. It was, therefore, probable, $a$ priori, that this law-the only one peculiar to the theory-would be found wanting. Its truth has recently been put to an experimental test by M. Jamin. Water, it is known, has its maximum of density at about $40^{\circ}$ of Fahrenheit; so that, if Newton's law were true, its refractive index should also have a maximum value at the same temperature. This has been disproved by M. Jamin, by observing the interference of two rays, one of which has passed 
through air, and the other through water; and thus the last conclusion of the emission-theory has been set aside.

It would occupy too much of your time were I to touch, even lightly, upon the subject of the chemical action of light, and the many beautiful and important discoveries of the Art to which it has given rise. I may, however, mention, as one of the latest of the marvels of photography, that M. Poitevin has succeeded in producing plates in relief, for the purposes of engraving, by the action of light alone. The process depends upon the change in the affinity for water, produced by the action of light upon a thin plate of gelatine, which is impregnated with bichromate of potash.

In the whole range of experimental science there is no fact more familiar, or longer known, than the development of Heat by friction. The most ignorant savage is acquainted with it, and it was probably known to the first generation of mankind. Yet, familiar as it is, the science of which it is the germ dates back but a very few years.

It was known from the time of Black, that heat disappeared in producing certain changes of state in bodies, and reappeared when the order of those changes was reversed; and that the amount of heat, thus converted, had a given relation to the effect produced. In one of these changes-namely, evaporation-a definite mechanical force is developed, which is again absorbed when the vapour is restored by pressure to the liquid state. It was, therefore, not unnatural to conjecture, that in all cases in which heat is developed by mechanical action, or vice versa, a definite relation would be found to subsist between the amount of the action, and that of the heat developed or absorbed.

This conjecture was put to the test of experiment by Mayer and Joule, in 1842, and was verified by the result. It was found that heat and mechanical poicer were mutually convertible; and that the relation between them was definite, 772 foot-pounds of motive power being equivalent to a unit of heat-that is, to the amount of heat requisite to raise a pound of water through one degree of Fahrenheit. The science of Thermo-dynamies, based upon this fact, and upon a few other obvious facts, or self-evident principles, has grown up in the hands of Clausius, Thomson, and Rankine, into large proportions, and is each day making fresh conquests from the region of the unknown. 
Thus far the science of Heat is made to rest wholly upon the facts of experiment, and is independent of any hypothesis respecting the molecular constitution of bodies. The dynamical theory of Heat, however, has materially aided in establishing true physical conceptions of the nature of heat. The old hypothesis of caloric, as a separate substance, was indeed rendered improbable by the experiments of Rumford and. Davy, and by the reasonings of Young; but it continued to hold its ground, and was interwoven into the language of science. It is now clearly shown to be selfcontradictory; and to lead to the result, that the amount of heat in the universe may be indefinitely augmented. On the other hand, the identification of radiant heat with light, and the establishment of the wave-theory, left little doubt that heat consisted in a vibratory morement either of the molecules of bodies, or of the ether within them. Still, the relation of heat to bodies, and the phenomena of conduction, indicate a mechanism of a more complieated kind than that of light, and leave ample room for further speculation.

The only mechanical hypothesis (so far as I am aware) which is consistent with the present state of our knowledge of the phenomena of heat, is the theory of molecular vortices of Mr. Rankine. In this theory all bodies are supposed to consist of atoms, composed of nuclei surrounded with elastic atmospheres. The radiation of light and heat is ascribed to the transmission of oscillations of the nuclei; while thermometric heat is supposed to consist in eirculating currents, or vortices, amongst the particles of their atmospheres, whereby they tend to recede from the nuclei, and to occupy a greater space. From this hypothesis Mr. Rankine has deduced all the laws of thermo-dynamics, by the application of known mechanical principles. He has also, from the same principles, deduced relations (which have been confirmed by experiment) between the pressure, density, and absolute temperature of elastic fluids, and between the pressure and temperature of ebullition of liquids.

The dynamical theory of heat enables us to frame some conjectures to account for the continuance of its supply, and even to speculate as to its source. The heat of the Sun is dissipated and lost by radiation: and must be progressively diminished unless its thermal energy be supplied. According to the measurements of M. Pouillet, the quantity of heat, given out by the Sun in a year, is equal to that which would be produced by the combustion of a 
stratum of coal seventeen miles in thickness: and if the Sun's capacity for heat be assumed equal to that of water, and the heat be supposed to be drawn uniformly from its entire mass, its temperature would thereby undergo a diminution of $2^{\circ} \cdot 4$ Fahr. annually.

On the other hand, there is a vast store of force in our System capable of conversion into heat. If, as is indicated by the small density of the Sun, and by other circumstances, that body has not yet reached the condition of incompressibility, we have, in the future approximation of its parts, a fund of heat probably quite large enough to supply the wants of the human family to the end of its sojourn here. It has been calculated that an amount of condensation, which would diminish the diameter of the Sun by only the ten-thousandth part, would suffice to restore the heat emitted in 2000 years.

Again, on our own earth, vis viva is destroyed by friction in the ebb and flow of every tide, and must therefore reappear as heat. The amount of this must be considerable, and should not be overlooked in any estimation of the physical changes of our globe. According to the computations of Bessel, 25,000 cubic miles of water flow, in every six hours, from one quarter of the earth to another. The store of mechanical foree is thus diminished, and the temperature of our globe augmented, by every tide. We do not possess the data which would enable us to calculate the magnitude of these effects. All that we know with certainty is, that the resultant effect of all the thermal agencies, to which the earth is exposed, has undergone no pereeptible change within the historic period. We owe this fine deduction to Arago. In order that the date palm should ripen its fruit, the mean temperature of the place must exceed $70^{\circ} \mathrm{Fahr}$; and, on the other hand, the vine cannot be cultivated successfully when the mean temperature is $72^{\circ}$ or upwards. Hence, the mean temperature of any place, at which these two plants flourished and bore fruit, must lie between these narrow limits, $i$. e. could not differ from $71^{\circ} \mathrm{Fahr}$. by more than a single degree. Now, from the Bible we learn that both plants were simultaneously cultivated in the central valleys of Palestine, in the time of Moses; and its then temperature is thus definitively determined. It is the same at the present time; so that the mean temperature of this portion of the globe has not sensibly altered in the course of thirty-three centuries. 
The future of physical science seems to lie in the path upon which three of our ablest British physicists have so boldly entered, and in which they have already made such large advances. I may therefore be permitted briefly to touch upon the successive steps in this lofty generalization, and to indicate the goal to which they tend.

It has been long known that many of the forces of nature are related. Thus, heat is produced by mechanical action, when that is applied in bringing the atoms of bodies nearer by compression, or when it is expended in friction. Heat is developed by electricity, when the free passage of the latter is impeded; it is produced whenever light is absorbed; and it is generated by chemical action. A like interchangeability probably exists among all the other forces of nature, although in many the relations have not been so long perceived. Thus, the development of electricity from chemical action dates from the observations of Galvani; and the production of magnetism by electricity from the discovery of Oersted.

The next great step was to perceive that the relation of the physical foroes was mutual; and that of any two, compared togegether, either may stand to the other in the relation of causc.

With respect to heat and mechanical force, this has been long known. When a body is compressed by mechanical force, it gives out heat; and, on the other hand, when it is heated, it dilates, and evolves poucer. The knowledge of the action of electricity, in dissolving the bonds of chemical union, followed closely upon that of the inverse phenomenon: and the discovery of electro-magnetism by Oersted was soon followed by that of magneto-electricity by Faraday. With reason, therefore, it occurred to many minds that the relations of any two of the forces of nature were mutual;that that which is the cause, in one mode of interaction, may become the effect, when the order of the phenomena is changed;and that therefore, in the words of Mr. Grove, one of the able expounders of these views, while they are "correlative," or reciprocally dependent, "neither, taken abstractedly, can be said to be the essential cause of the others."

But a further step remained to be taken. If these forces were not only related, but mutually related, was it not probable that the relation was also a definite one? Thus, when heat is developed by mechanical action, ought we not to expect a certain definite propor- 
tion to subsist between the interacting forces, so that if one were doubled or trebled in amount, the other should undergo a proportionate change? This anticipation, it has been already stated, has been realized by Mayer and Joule. The discovery of the mechanical equiralent of heat has. been rapidly followed by that of other forces; and we now know not only that electricity, magnetism, and chemical action, in given quantities, will produce each a definite amount of mechanical work, but we know further-chiefly through the labours of $\mathrm{Mr}$. Joule-what that relation is, or, in other words, the mechanical equivalent of each force.

The first step in this important career of discovery-though long unperceived in its relation to the rest-was, undoubtedly, Faraday's proof of the definite chemical effect of the voltaic current. The last will probably be to reduce all these phenomena to modes of motion, and to apply to them the known principles of dynamics, in such a way as not only to express the laws of each kind of movement, as it is in itself, but also the connexion and dependence of the different classes of the phenomena.

A bold attempt at such a generalization has been made by M. Helmholtz. The science of Thermo-dynamics starts from the principle, that perpetual motion is impossible, or, in other words, that we cannot, by any combination of natural bodies, produce force out of nothing. In mechanical force, this principle is reducible to the known law of the conservation of living force; and M. Helmholtz has accordingly endeavoured to show that this law is maintained in the interaction of all the natural forces; while, at the same time, the assumption of its truth leads to some new consequences in physies, not yet experimentally confirmed. Expressed in its most. general form, this principle asserts that the gain of vis viva during the motion of a system is equal to the force consumed in producing it; from which it follows, that the sum of the vires riva, and of the existing forces, is constant. This principle M. Helmholtz denominates the conserration of force. A very important consequence of its establishment must be, that all the actions of nature are due to attractive and repulsive forces, whose intensity is a function of the distance,- the conservation of vis viva holding only for such forces.

It is usually stated, in mechanical works, that there is a loss of ris riva in the collision of inelastic bodies, and in friction. This is true with respect to the motion of masses, which forms the sub- 
ject of mechanical science as at present limited ; but it is not true in a larger sense. In these, and such-like cases, the movement of masses is transformed into molecular motion, and thus reappears as heat, electricity, or chemical action; and the amount of the transformed action definitely corresponds to the mechanical force which was apparently lost.

In the cases just considered, mechanical action is converted into molecular. But molecular actions of different kinds are themselves in like manner interchangeable. Thus, when light is absorbed, vis viva is apparently lost; but-not to speak of phospho rescence, in which the light absorbed, or a portion of it, is again given out-in all such cases, heat and chemical action are developed, and in amount corresponding to the loss. Hence the apparent exceptions to the principle are in reality confirmations of it; and we learn that the quantity of force in nature is as unchangeable as the quantity of matter.

This, however, is not true of the quantity of available force. It follows from Carnot's law, that heat can be converted into mechanical work only when it passes from a warmer to a colder body. But the radiation and conduction, by which this is effected, tend to bring about an equilibrium of temperature, and therefore to annihilate mechanical force: and the same destruction of energy is going forward in the other processes of nature. Thus, it follows from the law of Carnot, as Professor Thomson has shown, that the Universe tends to a state of eternal rest; and that its store of available force must be at length exhausted, unless replenished by a new act of Creative Power.

Mr. Rankine has attempted, in another method, to combine the physical sciences into one system, by distinguishing the properties which the various classes of physical phenomena possess in common, and by taking for axioms propositions which comprehend their laws. The principles thus obtained are applicable to all physical change; and they possess all the certainty of the facts from which they are derived by induction. The subject-matter of the science so constituted is energy, or the capacity to effect changes; and its fundamental principles are-1st, that all kinds of energy and work are homogeneous-or, in other words, that any kind of energy may be made the means of performing any kind of work; and 2nd, that the total energy of a substance cannot be altered by the mutual action of its parts. From these principles the author has deduced 
some very general laws of the transformation of energy, which include the known relations of physical forces.

I have occupied your time so largely with the sciences of one section, that I cannot do more than advert to one or two topics connected with the others, which have struck my own mind, although, from my limited acquaintance with the subjects, I could not venture to say that they are absolutely the most deserving of notice.

Among the most remarkable of the recent discoveries in inorganic chemistry are those of MM. Wohler and Deville, relative to silicon and boron. Each of these substances is now proved to exist in three very different states, analogous to the three known states of carbon, to which they are thus closely allied,-namely, charcoal, graphite, and diamond. The last of these states is, of course, the most interesting. Crystallized boron possesses a hardness, brightness, and refractive power, comparable to those of diamond; it burns in chlorine, without residue, and under circumstances resembling those of the combustion of diamond in oxygen; it is not acted on by any of the acids, and appears to be the least alterable of all the simple bodies. I have been informed that its powder is already used in the arts, instead of diamond dust; and it seems not improbable that, when obtained by the chemist in crystals of larger size, it may rival the diamond as a gem.

The science of Geology appears, of late years, to have entered upon a new phase of its development, - one characterized by a stricter reference of its speculative views to the principles of those sciences with which it is connected, and upon which it ought to be based. The able memoirs of Mr. Hopkins, on what may be called dynamical geology, afford a remarkable proof of this; and we have another instance of the application of sound physical principles to this science in the explanations which have been recently offered of the phenomena of slaty cleavage. A report on this interesting subject was presented to the Association by Professor Phillips at its last Meeting, and will be found in the volume just published. These sounder views originate, I believe, with himself and with Mr. Sharpe; but they have been enlarged and confirmed by Mr. Sorby, Dr. Tyndall, and Professor Haughton. 
We have an interesting proof of the readiness of geologists of the present day to submit their views to the test of exact observation, in the measurements undertaken by Mr. Horner for the purpose of approximating to the age of the sedimentary deposits. Of the geological changes still in operation, none is more remarkable than the formation of deltas at the mouths of great rivers, and of alluvial land by their overflow. Of changes of the latter kind, perhaps the most remarkable is the great alluvial deposit formed in the valley of the Nile by the annual inundations of that river; and here it fortunately happens that history comes to the aid of the geologist. These sedimentary deposits have accumulated round the bases of monuments of known age ; and we are, therefore, at once furnished with a chronometric scale by which the rate of their formation may be measured. The first of the series of measurements undertaken by Mr. Horner was made, with the co-operation of the Egyptian Government, around the obelisk of Heliopolis, a monument built, according to Lepsius, 2300 years B.c. A more extensive series of researches has been since undertaken in the district of Memphis; but Mr. Horner has not yet, I believe, published the results.

The problems now to be solved in Palcoontology are clearly defined in the enunciation of the problem recently proposed by the French Academy of Sciences as one of its prize questions, viz., "to study the laws of distribution of organic beings in the different sedimentary rocks, according to the order of their superposition; to discuss the question of their appearance or disappearance, whether simultaneous or successive; and to determine the nature of the relations which subsist between the existing organic kingdom and its anterior states." The prize was obtained by Professor Bronn, of Heidelberg; and his memoir, of which I have only seen an outline, appears to be characterized by views at once sound and comprehensive. The leading result seems to be, that the genera and species of plants and animals, which geology proves to have existed successively on our globe, were created in succession, in adaptation to the existing state of their abode, and not transmuted, or modified, as the theory of Lamark supposes, by the physical influences which surrounded them.

I must now pass, from the results of science, to the administrative measures which have been adopted by this Association for its 
advancement, and more especially to those which will be brought under your consideration at the present Meeting.

One of the modes in which this Association most effectively promotes the advancement of science is, you are aware, by the preparation and publication of Reports on the history, and actual state, of its several branches. With the help of these, original investigators may, with little labour, ascertain all that has been accomplished in each department, before they proceed to increase the store; and so not only prepare their own minds for their task, but also aroid the waste of time and toil which has been too often incurred in the re-discovery of the same truths.

To further the same objects, it was proposed by Professor Henry, of Washington, at the Glasgow meeting of the Association, that a Catalogue of papers occurring in the Transactions of Scientific Societies, and in the Scientific Journals, should be prepared by the Association, the Smithsonian Institution undertaking to execute that part of the work which related to American Science. A Committee, consisting of Mr. Cayley, Mr. Grant, and Professor Stokes, was appointed to consider this proposal, and their report was submitted to the Cheltenham meeting. The subject has since been under the consideration of the Council of the Royal Society; and a preliminary Report has been drawn up by a sub-committee of that body, which will probably be brought before your Committee at this Meeting.

A still more important question has been, for some years, under the consideration of this Association, and of the Royal Society-the question, namely, whether any measures could be adopted by the Government, or Parliament, that would improve the position of science, or its cultivators in this country.

The Parliamentary Committee of the Association have taken much pains in the attempt to arrive at a solution of this large and complex question. They consulted, in the first instance, several of the most eminent scientific men of this country; and in their first Report, presented to the Meeting of the Association at Glasgow, they have analyzed the replies obtained, and have recommended certain general measures founded thereon. The most important of these recommendations are, the provision, at the cost of the nation, of a central building in London, in which the prin- 
cipal Scientific Societies of the metropolis may be locatedt ogether; and the formation of a Scientific Board, to have the control and expenditure of the public funds allotted to the advancement of science. This report was brought under the consideration of your Committee of Recommendations at the last two Meetings of the Association; and the opinions of the members of the General Committee have been since invited in reference to its suggestions. The Council of the Royal Society have likewise deliberated on the same question, and have passed certain resolutions on the subject, which accord in substance with the conclusions of the Parliamentary Committee. A copy of these resolutions was forwarded by Lord Wrottesley, as President of the Society, to Lord Palmerston; and motions have been made in both Houses of Parliament for the production of the correspondence.

The first of the objects above referred to-namely, the juxtaposition of the Scientific Societies of London in one locality-has been since accomplished by the grant of Burlington House for the use of the Royal, Linnæan, and Chemical Societies; and the result affords a fresh instance of the readiness of Her Majesty's Government to listen to, and comply with, the suggestions of men of science, when deliberately and carefully made. I cannot but think that this important step is fraught with consequences affecting the promotion of science, and extending far beyond the external and obvious advantages, which it insures to the Scientific Societies more immediately benefited.

Another mode in which this Association has materially aided in the advancement of science is through the instrumentality of its Observatory at Kew. The objects which are at present attained by that important establishment are, the trial and improvement of instrumental methods, and especially of those connected with the photographic registration of natural phenomena; the verification of meteorological instruments, and the construction of standard barometers and thermometers; the supervision of apparatus to be employed by scientific travellers, and the instruction of the observers in their use; and lastly, the conduct of special experimental researches, undertaken by members of the Association at its request. In all these various ways, the labours of the Kew Observatory have tended, in no small degree, to the advancement of the sciences of observation and experiment in this country; and the result is 
due, not only to the sagacity of the Committee under whose management it is placed, but also, and eminently, to the zeal and talents of Mr. Welsh, the gentleman who has the immediate oharge of the establishment.

There is but one other topic connected with the administration of the Association to which I feel it necessary to invite your attention before I conclude,-I mean the change which has been made in the constitution of one of the Sections, and which will come into operation at the present Meeting.

By a resolution of your Committee, adopted at the last meeting, the scope of the Statistical Section has been enlarged, and it now embraces Economic Science in all its relations. I regard it as a fortunate circumstance for the Association, that this important change will come into operation under the Presidency of the distinguished prelate whose talents have been so long devoted to the advancement of this science, and to whose munificence we owe the formation of a school of Political Economy in the University of Dublin, which has already attained a high measure of celebrity. The Section will have the aid, on this occasion, of more than one of those gentlemen who have filled the Chair of the Whately Professorship, as well as of other members of the Statistical Society of Dublin; and its proceedings will receive the countenance and support of many foreigners who have devoted themselves to the cultivation of Economic Science.

Gentlemen,-suffer me now to thank you for the indulgent attention with which you have favoured me. I am conscious that the sketch of the recent progress of the Physical Sciences, which I have endeavoured to present, is but a meagre and imperfect summary of what has been accomplished; but it is enough, at all events, to prove, that Science is not on the decline, and that its cultivators have not been negligent in their high calling. I now beg, in the name of the local members of this body, to welcome you warmly to this city; and I pray that your labours here may redound to the glory of God, and to the welfare and happiness of your fellow-men. 



\section{N 0 TES}

Note to TABLE VI., p. 289.

The values of the Magnetic Intensity at Munich and Prague, and at the four Russian stations, were originally expressed in Gaussian units, in which the unit of weight is the gramme, and that of length the metre. One gramme is equal to $15 \cdot 4426$ grains, and one metre to $3 \cdot 2809$ feet. Accordingly, to reduce the Gaussian measures of Horizontal Intensity to British units (one grain and one foot), the values of $\mathrm{X}$ must be multiplied by $\sqrt{\frac{15 \cdot 4426}{3 \cdot 2809}}=2 \cdot 1695$. This has been done in Table VI.

Note to TABLes V. AND VIII., pp. 286 AND 291.

The unit of Intensity in Tables V. and VIII. is the ten-thousandth part of the unit of Magnetic Force. 


$$
4016
$$







UNIVERSITY OF CALIFORNIA LIBRARY Los Angeles

This book is DUE on the last date stamped below.

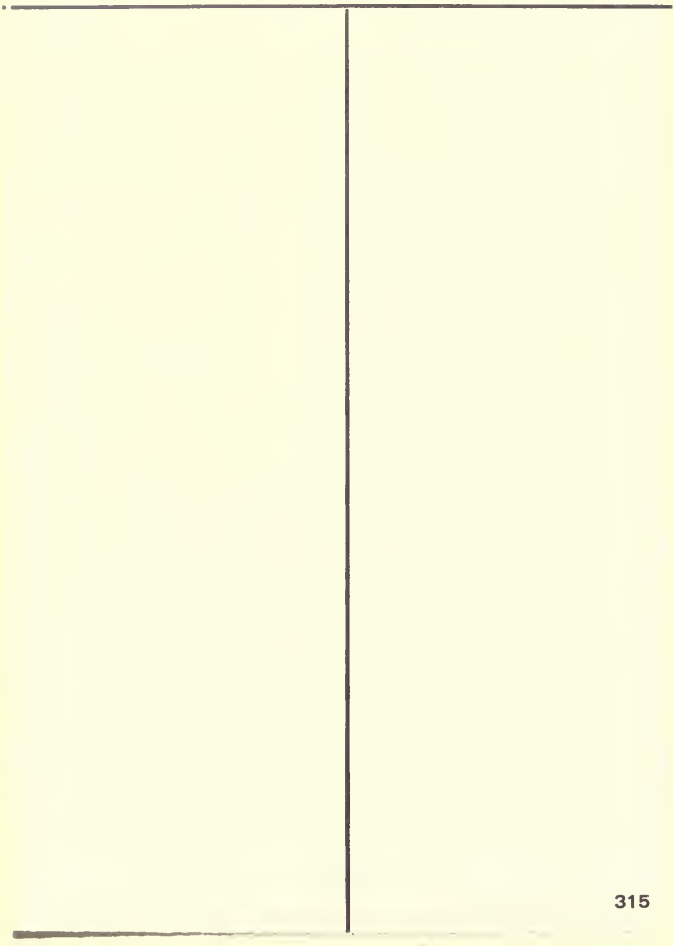




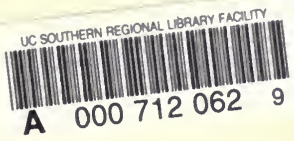


